i:
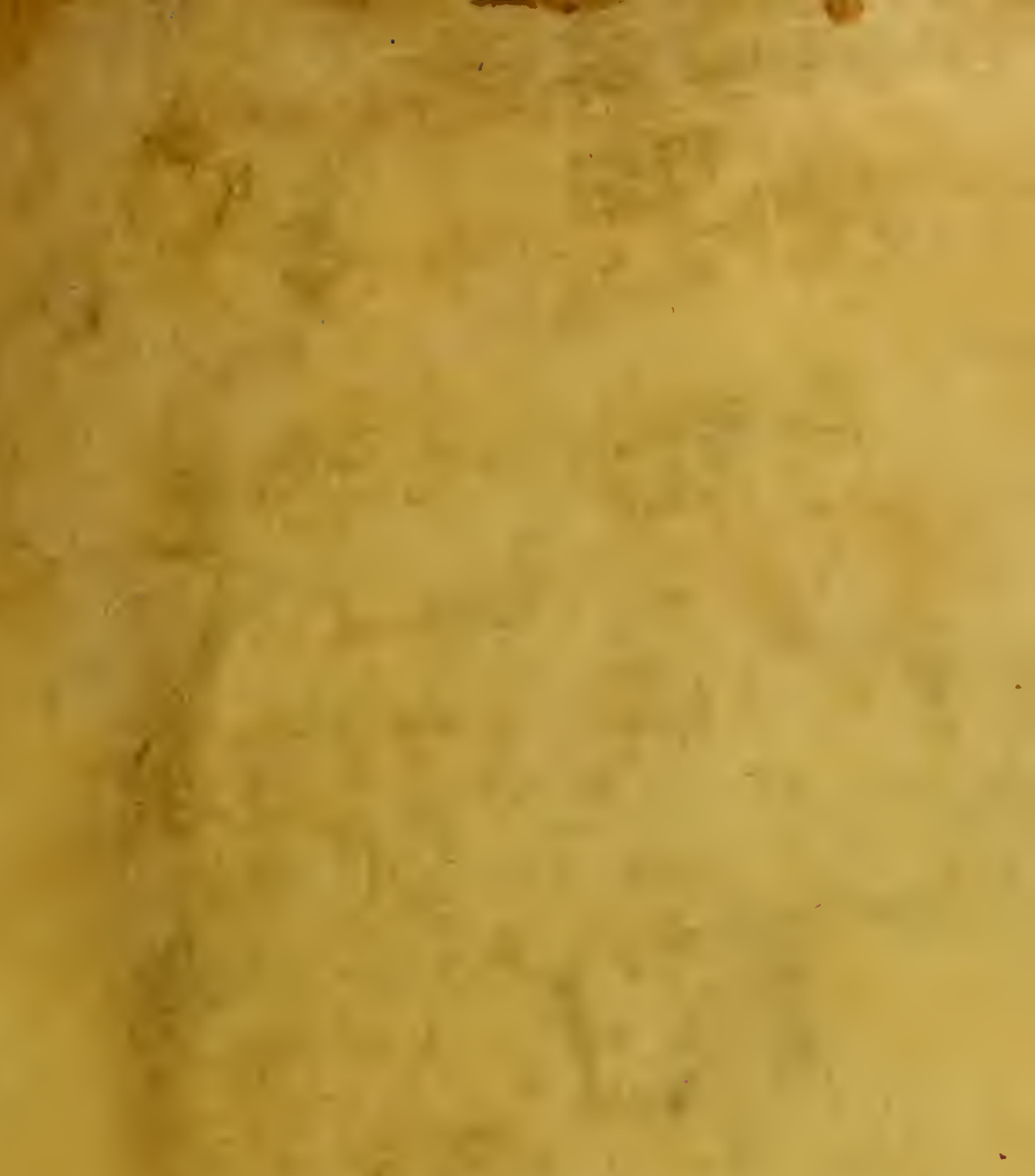

5.1178 


\section{A $\quad \mathrm{T} \quad \mathrm{T} \quad \mathrm{I}$}

D E L L'

\section{ACCADEMIA DELLE SCIENZE}
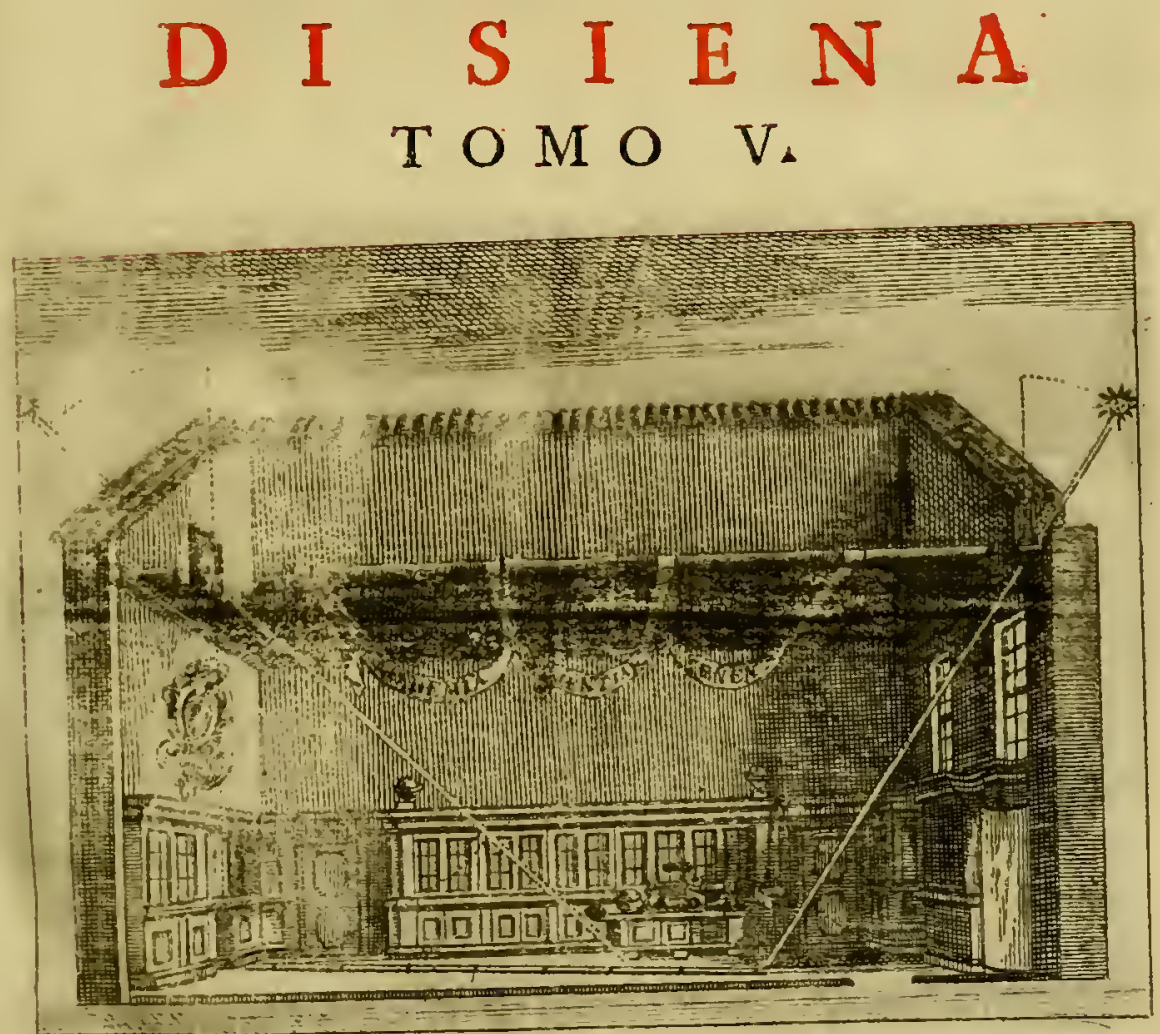

IN SIENA MDCCLXXIV.

NELLA STAMPERIA DI LUIGI, E BENEDETTO BINDI. CON LICENZA DE' SUPERIORI,

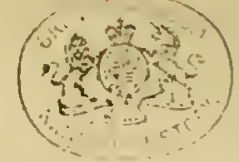





\section{)o( 1 II $) \circ($ \\ $\mathscr{R} \mathscr{B} \mathscr{T} \mathscr{L}, 90 \mathcal{N} \mathscr{G}$}

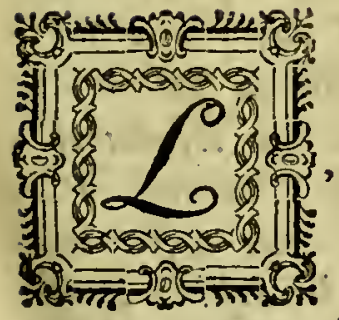

Iftiturione delle fcientifiche Accademie egli e ftota una delle piu gloriofe, e luminoSocietd, abbian mai faputo gli uomini in ogini tempo efeguire. Per il comun confenfo dei Sapienti, quefte Accidemie fono fate quelle, che pit d' ogni altro, a forza déloro: Atudj, ed efercizi, e de lumi ovunque $\int p i r \sqrt{2}$ banno acciefciuta quella cultura d' ingegno, che frid perfone d'ogni fato, $e$ condizione oggidi rifplender vegriamo; che banno arriccbite oncora tutte le facolta, e profeffioni di vaftifime coginizio: sii, 


\section{)$($ I V ) 0(}

ni, e maravigliofe fcoperte; e clo finalmente banno apportata quella buona Filofofia, che gia fembra aver prefo picde nei noftri fortunati tempi, fra ogni culta gente, e Nazione, per cui trionfando ormai la ragione, vive l'ignoran$z a$ nemica, abbattuta, $e$ deprefja.

Cosi manifefte verità radicate nell' animo de' Sovarai; 'fenza l' opra dé quali difficil cofa d' intraprendere cofe grandi, $e$ le intraprefe condurle al loro divetto fine, ban fatto si, che efficacemente banno accordata a quefte Societd lettesavie la loro Sovrana Protezione, $e f i$ jono adoprati ancora con Reale munificenza nello fabilirne delle nioz'e, in quei refpettivi Stati al loro Governo commefs.

La Reale Accademia delle Scienze di Siena ebbe il primo fuo nafcimento per la fomma cura del noftro gloriofo Concittadino 'l' immortal Gabbrielli l' anno r691.; ed il Real Principe Cardinal Francefco de' Medici fu quel Sovrano poi, che amorevolmente l'accolfe fotto la fua Real Protezione. Sul bel principio dilato quefta in ogni parte il fuo nome, ma ficcome tutto cio, $c b^{\prime}$ f fotto il Cielo alle umane vicende é fottopofto, s'indeboli nel corfo di poco tempo quel fuo natural fervore, ed ebbe bifogno per rifquoterfi, $e$ riforgere vie piu gloriofa alla luce, della Clemenza di Cefare; FRANCESCO I. IMPERATORE, che come noftro amorevole Padre; e Sourano, l'anno 1737. lo deftino it fate vor del Cielo al Governo di quefti feliciffimi Stati. Per. lo che l'onorò della fua Special Protezione, e per incoraggirla all interrotto profieguimento 'de Juo 'fudj; ;. e nell' efercizio degli fperimenti fopra le naturali fcienze, le afjegnò con Cefared munificenza Jicuri Aabilimenti.

Riceve poi adeffo a' giomi nofri livghe beneficenze per, li clementifima, e paterna cura del noftro Real Sourano, 


\section{)$\circ(V) x$}

Pietro Leopoldo d'Aufria felicemente regnante; che volendola immediatamente fotto lombra della fuaReal Proteziotie, promuove con indefefla cura $l$ avanzamento dé fuoi ftudj, e l'arriccbifce infieme colla fua Real generofitd di continue, e. fabili rendite; ed affincbe non mancbi in elfa il buon ordine, ba vegolata ogni di lei funzione, e prefcritta ogni norma di procedere, con favia e prudente Legislazione. - Sono erette quefte Accademie, come ed ben noto, a folo oggerto di coltivare le Scienze; é poiché non Sappiamo di quanta efenfione fieno le Scienze capaci, cioe fra quali limiti reftino coftrette, $f_{i}$ proccura percid d'ampliarle, ed illufivarm le con nuovi lumi, ad oggetto, che la sfera delle cognizioni umane fo dilati fempre, e $f_{i}$ accorefca, e perche ancora ogevole vie piu viefca la Arada, per incamminarci delle, Scienze al polfeffo.

It principal fine poi d' ogni noffra cura, e vicerca per coltivarle, ad un fol 'punto é diretto; quaie de di vintracciare il vero ovunque trovifh, in mezzo a quelle sante incertezze, che da ogni parte ci aftringono, e fra $l$ immenfitd dè rapporti, che nel corfo, e varieta delle mondane cofe, ai nofri fguarti l'occultano. Ritrovate pofcia le veritd in qualunque Scienza; o Profeffone, e di qualffifa velazione, o fpecie, l' efperienza ci mofira, che una reagifce fopra dellialsra, e vengona a formar poi tutte inffeme, e'direttameinte; ed indirettamente quel bene, il qual fi diffonde in ogni corm po politico. Ella è dunque quefta conofcenza idel vera un certo bifogno d'ogni ben regolato Stato, d'ogni ordinata famiglia, e d ogni, e qualunque oggetto, cbe la Societd civile compone; poicbe per legge invariabile della Natura, quelto che a feconda delle regole del vero fi divige, e fi opra, divien folo il ficuro mezzo, ch' infuifce nelld univerfal fe- 


\section{)o( VII ) $)($}

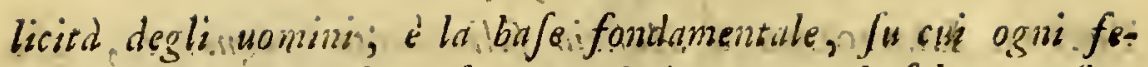
licitd ftabilmente Ji conferva; oed è pure quel folo, e ficu-

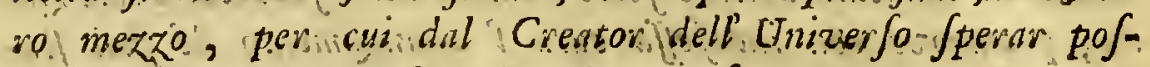
fatmo delle opere noftre la ricompensa:

i: 0 Qualinque veritd perd, bencbe univerfalmente util.

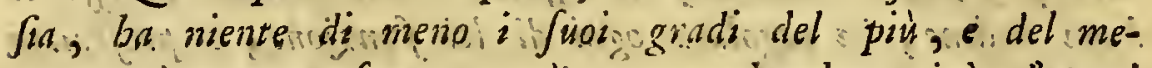
nos; né per confeguenza te vero, che le verita d' ogni fcienzo, o profelfione siano del medefimo ordine, per influire nel comun beners; quindi e icbe necefjario egli era un cerito accordo fra uominis dotti, e Japienti, e del ben comune amatoris per la pxeferenza di quelle foienze, che colle riceribe del vero, cis apportano maggion le utilita, e pii notabili i vantaggi e Queft accorda efprefJo appunto vieve in quelh unione di qualificati, e dotti Soggetti, la quale. Accademia $f_{i}$ nomina; dove reftringendofi unitamente a coltivare alcune particoldri facoltd, ciafcuno degli Accademici $s^{3}$ impegna cominican $\sqrt{2}$;icendevolmente $i$ pensieri, corredare di nyovi, lumi, ed ampliare al pofjibile le dottrine delle faienze propofte, a forza de loro perpetui fudj, e delle non mai interrotte $\int p$ erienze.

Quafi tutte. le Accademic fcientificbe piu ragguardevoli d' Europa banno prefcelte per foggetto de' loro Audj, ricerche le Mattematiche; e le Sciemze della Natura; come quelle, che piu manifeftamente portano in fronte $i$ caratteri del vero, è rbe altrëst racclizudono certe cognizioni, accorm date ai noftri faciols talenti. Imperoccbe loaino bene intefo; cbe quefte Scienze eran quelle, che réfe un poco comini fra $i$ dotti, non Jolo tramandano un certo lisme, chs irradia iutta cio, cb intorno effe aggira, ma clbe eziandio riflettono luminofi riggi, i quali, alle altre: fcienze, ed alle altre opre, e funzioni della vita apportano vivacifjama luce. Quindi 


\section{)o( VII )o(}

Quindi el cbe quei noftri primi Fondatori conofcendone effi puire il merito, e l'eccellenza, foltanto eleffero quelle per Soggetio dei loro efercizj; Ed il noftro Real Sorrano, che non folo con grandiofital le Scienze protegge, ma che al pai d'ogni altro ancora $P$ intende, compiacendofi fecondare gli anticbi fabilimenti, ba ordinato con accademicbe Leggi che fieno quefte Scionze pure ancije il principale oggetto de' noftri Studj.

La fublimità de’ penfievi, alla quale follevano il noAro animo, e l'aggiuftatezza, e precifione de' raziocinj, che quefte fcienze gli fomminiftrano, ed infieme gli innumerabili vantaggi, che feco portano, fono cofe ormai ad evidenza dimoftratc; e l'affenfo d' Uomini celeberrimi, che coll' efcmpio, e col configlio efficacemente le raccomandano, ed il confentimento univerfale delle Societd fcientificbe, qualificano abbaftanza il merito della loro eccellenza; nulla di meno pero mi fia lecito il fare Sopra di effe alcune brevi riAlefjioni, non effendo mai Juperfue allorcbé dirette fono a rilevar maggiormente il pregio di quefte facolta, e per vie pit intereffarci a coltivarle con genio.

Principiando dalla Geometria, la definifce un Geometia di primo ordine del noftro Secolo, una Scienza, cbe prefcrive la mifura piu precifa del noftro Spirito', la mifura det fuo grado d'eftenfione, di fagacitd, e profonditd, e. di giuftezza. Ognun vede pertanto, che la Geometria eftender deve le forze della nofra mente, fino a dove naturalmente ella.' capace, le deve affegnare le giufte mifure del vero, pafcolarla con dati certi, e ficuri, ed alimentarla nel metodo; e col replicato ufo di tali funzioni, convertiv $\sqrt{i}$ dere in una Mente giufta, ed eftefa, mente indagatrice dal vero, che geometrica la diremo; che quanto $d$ a dive, ridot- 


\section{jo( VIII )oc}

vidotta al grado della fua eccellenza. Le veritd, che tratta il Geometra fono femplici, e fenfibili, e fufcettibili d'applicazione per ogni ragionamento, quindi de, $c b$ effer vero Geometra, nient' altro vuol dive, $c b^{\prime}$ effere in pratica un ottimo ragionatore; percbe fenza accorgerfene, col maneggiave le veritd indubitate della fua fcienga, colla dovuta precifione, e cbiarezza, egli avrd per abito apprefa l'arte del giufto ragionave; ficcome per abito ragioniamu a feconda di quelle fcienze, e fecondo l'indole loro, e natura, le quali per avventura da noi $\int i$ profeffano. Quali dunque cognizioni piv utili, fra le cognizioni delle mortali cofe, pud acquifare il noftro Spirito, che di quefte geometricbe? Quali faranno mai pii di quefte adequate per riformarlo? E percid ecco di fatto quali effer dourebbero le cognizioni, che con ogni poffibil cura d' uopo farebbe provvederle; $e$ con altrettanto impegno intereflar ci dovrebbemo per renderle comuni; e famigliari all intelligenza d' ognuno.

Dalla Geometric trapalfando in Seguito all Algebra; fi $f_{a}$ il palfaggio da una perfettiffoma Logica alla pit perfetta, e fublime Metafifica. Ogni fcienza, il di cui aggetto. non verte fopra cofe palpabili, ma bensi immateriali, dicono $i$ Dotti, cbe ba la fua Metafijica ; e s'intende per. effa la collezione di quei principj, fopra cui è fondata la fcienza, ficcome la loro diramazione, ed eftenfione, i quali principj poi fono il germe delle verita, da cui $i$ raziocinj derivano, e da cui pur nafcono altre veritd fecondarie, cbe colla föienza medefima mantengono le necefarie relazioni. L'Algebra dunque, cb'd della Geometria la Metafifica, a mifura dell" indole, e natura di quei principj, della certezza, e perfezione dei medefimi $f_{u}$ i quali fi fonda, deve da quelli portare il pregio della jua perfezione. Non d 


\section{Jo( IX $) \circ($}

noftro iffituto $l$ 'individuare quefi princitj., $e$ ne meno the loro feconditd dal cui compleffo fi forma queft cccellents: matrematica Metafifica; ma per dirne di pafjaggio un esempio, l'Analifi degli, infinitamente piccoli, fin da'tempi degli anticbi. Filojofi conofciuti, 'e dei quali l' Algcbra ne ftabilifce il. calcolo, e la loro applicazione per :moltiffme, e varie invenzioni, folamente fervir potrebbe per qualificare il pregio. della più ingegnofa, e luminofa Mctaffica, cbe mai cader poffa fotto il umana immaginazione. Non folo poi dirige quefta Metaffica per la retta, e ficura ftrada i noftiri penfieri, attefe le teorie fu i ficuri principj della Geometria fabilite; ma ci preffa inoltre tutti i:necefarj mezzi, per agevolmente introdurci in molte altre fcienze, e fpecialmente in quelle, :cbe fotto il general nome di Mattematiche fi comprendono. E per fino la Geometria, elb' impiefta all Algebra i fondamenti, ne ritrae pofcia da quella foprabbondainte ricompenfa; paicbd colle ragionate teorie dell Algebra, alla cbiarezza, e precifione del calcolo Joitopofte, $f_{z} d \dot{d}$ eftenfone maggiore alla Geometria, efiapre altresi ma ftada incomparabilmente piu facile per ifudiaila.

Sarebbe un croppo dilungarci fe partitamente divifar volefsmo i fingolari pregj delle altre facoltd, clbe nel compleffo delle Mastematicbe. Sono annoverate; bafti foltanto il dive, che tutte ci aprono un' inefaufta forgente di luminofe. cognizioni, per le loro amminabili, e Singolari fcoperte, e.e che infsem con effe, apportano altresi nella civil Socictd utili notabiliffrmi. Diremo ancor di piu, che colle lorio dimofrazioni, bencbe ad altro fine fian dirette, mainfeftamense ci dimoftrano quella palpabile veritd, di quanto fiamo mifsvi, ed ignoranti; Verita poco inte $\int a$, ma che vitornar poirebbe verfo noi utilmente, fe ferviffe di virtuofa umilia- 


\section{) $0(X) 0($}

(ease per noi, e per il noftro trattar fragli womind di prus dente regolamento.

Con ragione per tanto in ogni Unjuerfita, ed in ogni Accadimia fcientifica, fra culte genti ftabilita, fono ai di noftri le Mattematiche amorevolmente accolte, con indefoffa applicazione $\sqrt{3}$ coltivano, è profeffano. Né fon pirs quétempi, inei quali era quafi un ribellarfs alla Pasria, at Genitari, agli Anzici, fe alcuno guidato dal buon ganio, prafeffar voleva quefte fcienze ; anzicloe avendo cangiato arpetto le vicende della letteratura, formano adefo le Mata, tematiclse"una parte della moderna educazione.

E per vero dive, dopo che Uomini fommi do ogni NaFione ban principiato di propofato a coltivare le Mattematiche fcienze, banno di fatto rintracciato ih retto cammino per ifdirozzare gla ingegni degli Uomini. Pare cbe lo fpia vito abbia ai di nofiri nuova farma acquiftatio; quindi le fcienze tutte fentendone il benigno influffo, banno quafi mutata in tutto I antica lor faccia. La Filofofia, che ed it primo paffo fabile, cloe fa il noftro fpirito, per incamminarfi dirctiamente a qualunque fcienza, non e gid ogsigiono quel. la degli anticloi, per ari in vece di woanzar paffs, tornavafs indictro. E la barbara inumanitä, $c b^{2}$ in qualcbe Regno delTa noftra Europa; in altri tempi. non malto lontani da' noAri, giunse per fino a tal fegno, di far praibire fotto pena della Galera, di penjar diverfamente da Ariftosile, bai ceduto oggi mai alla ragione; t percid fcolla qual fervil giogo i Filofofr, banno:abbandonate quelle pure, e femplici Speculazions, abe rapparto alcuna non barno colla comune felicita; ed d in tal guifa rimafta fepolta nol nulla la Filofafia delle fole parale, ed altresi quella delle reali cofe, vincitrice, e. lumisnafa, trionfa; Onde el cbe per buo- 


\section{)$\circ(X I) \circ$}

na forte fra $x$ cultori delle fichze, non potbi ne vitrovia. no ai tempi noftri, $i$ quali meritamense chiamar fi polfono. veri Filofofi; ciod Filofofi utili, e necelfari, che at comuin bene cï guidano.

Le fcienze matiematicbe inoltre fon quelle, cibe diresz tamente conducono, allo fooprimento delle veritd, che in $\int_{e}$ ftefla racibiude la modorna Fifica; fcienza parimente; cbe non ba bifogno di pruve, per dimoftrare nel commercio dels

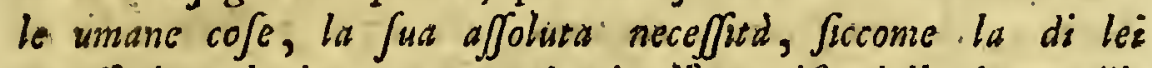
necelfaria relazione, per condurci all acquifto della buona $F i$ lofofia. Por eftender dunque le fue vedute negli arcani del: la Natura, neceffarijforme fono le Mattematiche; la loro wnione colla Fifica forma delle medefime una utilit, nel. tempo fiefso, che frabilifce della Fifica la foliditd, e certEzza; e giornalmente fotto i noftri occbj veggiamo, che quanto piu l' applicazioni dolle Mattematicloe fi moltiplicano verfo i naturali oggetti, altrettanto comparifcono le utilitd nella Fijica, e diremo ancora nell' arti tutte a pro noftro inventate.

Per lo che fono quafi innumerabili le fcoperte, $e$ co. gnizioni, cise zanta nell' ctd noftra la moderna Fifica. Quefo mirabile Spaziofo campo del Ciclo, la vafta fuperficie della Terra, ábitata da prodigiofa quantità di Viventi, $e$ ripiena d' immenfo numero di produzioni, fono ai di noftri note provincie, $o$ almeno non tanto incognite noi. Le sicende ammivabili della Natura. Larte; e fomma maefria scn cui le dirige, non fono piu agli orebj del moderna $F i$ fico fopramaturali prodigj; e quei Jingolari feriomeni, che come difordini in altri tempi erana apprefs, per rifvegliare nell' animo delle Genti vani timori, gli Spiega anzi it modcono Filofofo come natwraliffmi effetti grovenienti dall ore dine 


\section{) $0(X I 1) 0($}

dine dill inmutabili leggi dell' Universo. Sono, egli è vera, debolifsmi $i$ noftri lumi per ijcorgere di quell arte ingegno$f_{a}, e$ dei Fenomeni, $e$ vicende le cagioni tutte in nativa, $c$ la dipendenza, che da effe ne banno gli effetri; niente dis meno perd ne riconofce la moderna Fifica di quefte ifteffe cagioni.non pocbe, e non pocbi eziandio gli efferti, cbe da quelle derivano. E quel nobile oggetto, cbe ella $f i$ propone d' inveftigare le fabili, e non arbitrarie leggi della fagace Natura, di conofcerne i rapporti, che aver poffono nelle di lei regolate vicende, ed il boro infulfo net corpi, accresce almeno, fe altro non foffe, notabilmente le noftre cognizioni, ed a tal fegno le porta, che polfiamo arrifcbiarci a proferire, che $\int e$ non toca il vero il muderno $F i f i c o$, percbe ai mortali non $d$ cid facilinente concelfo, al vero perd, a gran pafji is approljima.

Sembra pertanto, che in quefti nofiri fortunati tempi fi fano alquanto addomefticati colla. Natura i modemi Filofofi, e che le abbiano in parte fcoperto quel velo, con cui si gelofamente fudia nafconder. ir; e quanto: appunto ancora ella ftudia agli occlij loro copringi, altrettanto con induAriofa arte fi munifcono effi di penetrantifjema viflo, per efplorave $l$ opere $\int u e, e$ gli andamenti.

Sono le Efperienze, a l offervazioni non mai interrotte, cbe unite all affiduo, e profondo: fudio ban fatto alla Fifica far dé progrefli ammirabili, nel vafto regno della $N a$. tura. Depofte ormai veggiamo in tutto, e per tutto quelle aftrazioni fterili, e tutti. quanti gli inventati fiftemi; perché efaminando il Fifico foltanto la Narura nei fuoi fenomeni, ba rintracciata cosi, per via dell' efperienze, altra frada incomparabilmente più certa e ficura, per. guidare il nofro animo nelle naturali fcienze, a quel grado d' acquiftar le pit aftrufe, e recondite cognizioni. 


\section{)o( X III ) o (}

Quefa nuova manicra delle fperienze per efaminare diclla Natura le léggi, ed i fenomeni, ficcome ella 'è la fola via, "che aperta rimane per' giugnere' alla cognizione dello natwali cofe, costici prefta ancor dé forcorli per ampliare le noftre vedute fopra lo jpirito. Sembra noftra vifta, rbe l' efperienze, ed efame Jopra del corpo, a nulla conferifcano per l'intelligenza del noftro fpirito, atsefa quella, che fra ambedue vi pafsa efferizialiffona differenta; ma e cbi de' mortali ardirebbe affermave, che quella maniera d'accofsains alla cognizione del corpo; at alla intelligenza dé fenomeni per mezzo dell' efperienze, "non fia pure quella ftef$f a$, clse avvicinar ci faccia alla perfetta cognizione del noftro Spirito, e delle Sue operazioni? Forse per la perfercranza nell' opra, coll andare degli anni, fi diraderanno quelle folte ressebrs g cbe la noftra intellizenza adefo ci ofcurano, e ver. ranno fualmente una volta gli Uomini ad aver magsior comtezza di quefta piu nobil parte di noi.

La Fifica per fane d' quclla fola fcienza, che ci appaga di quella innata curiojitd, cbe por coftante legge di Natura domina in tutti noi; mentre filfa alla medefima; limioi con i dilcttevoli fuidj, e colle curiofe Jperienze; onde d cl'infiem coll' utile, cbe feco porti, e colle forprendenti cognizioni, che ci dilettano, ci fmorza pare una de!le paffioni piu vive dell animo noftro.

Le fopra espofte veritd, che qualificano it merito delIc fcienze Mattematicbe, e delle Fijicbe, fembrerebbe, cbe effer dovelfero bene intefe, e da alcuni.maggiormente; che pur credono d' occupare diftinto pofto inella letteraria Repubblica; nulla di meno perd $\int_{c}$ ne trovan fra quefti, che pocbiffimo fodisfatti fono di tali fcienze, ne perd perfuafi de' Solidi piaceri, ed utilita, clbe ci appreftano; delle

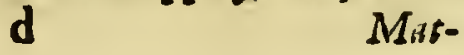




\section{)$\circ(\mathrm{XIV}) \circ($}

Mattenaticbe fpecialmente Son difguftati oltrenodo, verfo le quali apparifcono con ilor detti, d' effere di foverchio indifcreti.

Non intendo di far qui per. quefte fcienze diffefa alcuna, perclbe poco ne ban di bifogno; Se perd mi fia permefto l'e faminar le ragions, por le quali quefti indifcreti gindici le difapprovano, a due fole le ridurrei. Primieramente ed veriffsimo, che quei piaceri, che recedono un poco dal comune degli uomini, fiano fempre meno ftimati, perche giuftamente nel comune delle genti fono poco not; ; vale a dive da pocbi conofciuti. Le menti volgari, ficcome limitate affai fono nell' eftention de' piaceri, Sono cost parimente alfai limitate nell eftenzione degli utili ; ed a poclsifjani og: getti, fuorclie a quelli fra mano di tutti, e triviali, fanno capire, clee dar dẹvafi quefto nome di usilità. Il Soldato per efempio porta un' arme in ifpalla, ed in' arte profella, cise tanto l'invenzione dell asa, che la perfezione dell altra, meditazioni, $e$ fatiche coftano al Filofofo, $e$ Mattenatico; mia non conofcendo egli, cbe dalle due predette fcienze parte ogni conimodo, ogni piacere, ed utilita, che dall' armi, e dalla Guerra intende vitrarre, difprezzerd come oziofe, ed inutili nella Societd, le fcienze gid dette. Ed ecco in prima luogo Sopra di che el foridita una di quelle ragioni del difprezzo, cbe molti ne kanno; dal non conofcer ciod ne l influffo diretto, nd l indivetto, per tutti qué vantaggi, $i$ quali per mezzo di effe- $f i$ godono.

La feconda ragione poi tira direttamente la yua forgente dall amor proprio, che per lo piu forma la tiraninia del noftro cupre; percbe dice I illuftre Segretario Fontenelle = Quello che non $f i$ fa facilmente fi crede inutile, quafi per una fpecie di vendetta; e poichè le Mattematiche, e le Fifiche fono perordinario fconofciute, comunemente fono ftima- 


\section{)$(x$ V )ó}

Itimate inutili; l'origine della loro difavventura è manifeAta, fono fpinofe, e felvaggie, e difficili a confeguirfi $=$. Siccbe' nel non conofeere in che fon utili, e nella vendetta percbet 's igzorano, ftan ripofte le ragioni turte della naufea, \& difprezzo per quefle fcienze.

E qual rimedio mai dunque rinvenir fi potrebbe per difingannare i contrarj? Il gran Ruifchio agli avverfarj della fua si rinomata injezione, iquali oftinatamente negavano, cbe per mezzo di quella comparifjero come viventi i Cadaveri, per convincere la loro oftinazione, proponeva un fol rimedia colle Seguenti parole $=$ Venite, e vedete $=$. Efficacifjimo, e perfua: fivo rimedio di fatri; che $\int e$ i tanto malcontenti delle noAre fcienze, che per lo piu fon quelli, che mai l ban guardate in faccia, veniffero feriamente a vederle, faum Jicuri, cbe $f i$ fmorzerebbe in loro il nijerabile appetito di criticars le, allorclbe vi foftituiffero il bel piacere d' intenderle.

Penfi perd ciafcuno a fua voglia, e faccia quella che pis gli aggrada; noi frattanto non cefferemo mai di ripetere quella nobiliffima pregbiera, la quale uno dè primi Genj del noftro fecolo it Sig. D' Alambert indirizza ai Principi=Sovrani, che governate $i$ Popoli, ai quali defiderate fare fcuotere il giogo dell ignoranza, e fuperftizione; fate nafcer fra quefti dei Mattematici, che quefta razza d'Uomini produrrà col tempo de' Filofofi fenza accorgerfene...... Ben prefto lo ftudio della Geometria condurrà per fe medefimo allo ftudio della vera Fifica, e quefto poi alla vera Filofofia $=$.

Oltre alle fcienze, delle quali fi d finora parlato, abbiamo per legge Accademica clb ogni altra, e qualunque fiafi Filofofica ricerca, formi un Soggetto de' noftri accódentici efercizj, riputandojoggetto ben degno d' un corpo Accademico, che tutto ciò, cbe all im- 


\section{)$\circ($ X V I ) $)($}

all impero della Filofofia vien fottopofto, non refti da una fcientifica Accademia trafcurato. Sotto la ricerca perd di quefte veritd Filofofiche, foltanto s' intendono comprefe quelle, che naturali fi dicono, cioe che dalla ragione, $e$ dalla Sperienza dipendono; vale a dive, cb'efclude ogni, e quallsvoglia ricerca, la quale fondata fia, ovver dipenda dall autoritd. Né il limitare in tal forma la liberta de' Filofofici penfieri degli Accademici, parer deve contro ragione; pere cbe anzi ella é ragione, cbe fian quelli dalla rifpettabile Autoritd dipendenti. Non rimane dall' altro canto st riftretto lo Spazio nella Filofofica Provincia, dove le forze del noftro. Spirito $\sqrt{2}$ polfono eftender gloriofamente, e con frutto. E quefta fola libertà meffa in opra; potrd effer certamente quel mezzo opportuno di fabilive alla sagione fteja un affoluto dominio; che tanto d a dive, d'aficurare all adorabile verith un luminofo trionfo.

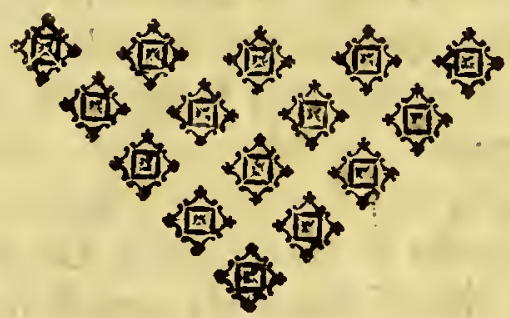




\section{)०( X V II: )०० \\ I N D I C E \\ DE G L I A U T O R I \\ E LORO OPUSCOLI, CONTENUTI NEL TOMO. \\ DANIELIS MELANDRI.}

1 Editationes nonnulla de Machind bujus Mundi, ejufque 1 Conferwatione per vires in initio conceffas.

\section{PAULII FRISII .}

De Problematis quibusdam Geometricis.

\section{ABATE LEONARDO XIMENES.}

Rifeffoni intorno all' Obbliquita dell' Eclitica, e fua Dim:nuzione fecolare di 29 , dedatta dalle Ofjervazioni Siffi. ziali, fatte alla Meridiana Fiorenisna, negle anni 1757 , c. 1756 .

\section{- GREGORII FONTANAE.?}

SCHEDIasma I. Problemata Ajtronomica :

pag. 55:

SCHEDIASMA II." "Theoremata ad Calculum integral.fpectiantia. pag. 6\%

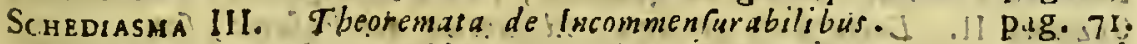
Scuediasma IV. De Problemate quadam Almo/pberico. pag. - Gó. Schediasma V... De Trianguli Spberici dimenjione. . I pag. 83. SGHEDIASMa VI. De Binemj Noutoniani indicem rationalem bubentis Evolutione.

pag. 88. Schroinsma Vil." Del Moro equabilmente accelerain pag. 92. Scisedasba VIII. Del Moto de' Projetiz nella Parabola Apolloniana.

Schediasma IX. Sopra un Fenomeno della Vifta. Scindiasma X. Se l'azione delle forze Luni-Soluri può inpag. 97. pag.103. Auire nello ftato del Barometro, ed alterarae fenfibilnente l'alitzza. 


\section{)०( X VIII )ol \\ DELLO STESSO AUTORE .}

Saggio ropra i Progrefli Maltematici di Girolamo Cardano, e Bonaventura Cavalieri, dopo il rifubslimento delle lettere in Occidenie.

pag.12g.

GIUSEPPE BALDASSARRI :

Ofervacione fopra l' Aido Vetriunlico, trovato naturalmente puro, concreto, non combinato.

pag.140.

\section{FRANCESCO CALURI.}

Relazione fopra un pretefo Ermafrodito.

pag.16\%.

GIOVAN JACOPO FERBER.

Memorie Epiftolari di Offervazioni Mineralogicbe, e Oritto. ligicibe.

Memoria I.

Memoria II.

GIOVANNI ARDUINO.

pag.203.

pag.214.

Saggio Mineralogico, di Lytbogonia, Oragnosa.

pag.228.

Articolo ty.

pag. 242.

Arricola II:

pag.260.

Antríbéro IIL.

\section{DOMENICO BARTALONI .}

pag.281.

Offervationi fopra il Vefuvio.

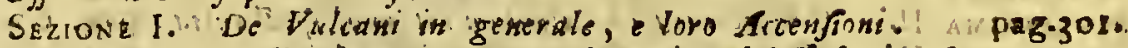

Seziont II. Dello fturo prefente, ed antico del. Vefuvio, fur Aicenfioni, Fenometi

Salion III. Doqu fi efamina fe la Materia cbe ejpelle negli Ino cendj al Vefuoio venga da altrove, fo quella, cbe in fe contrene fia fufficiente per riantenergli. 


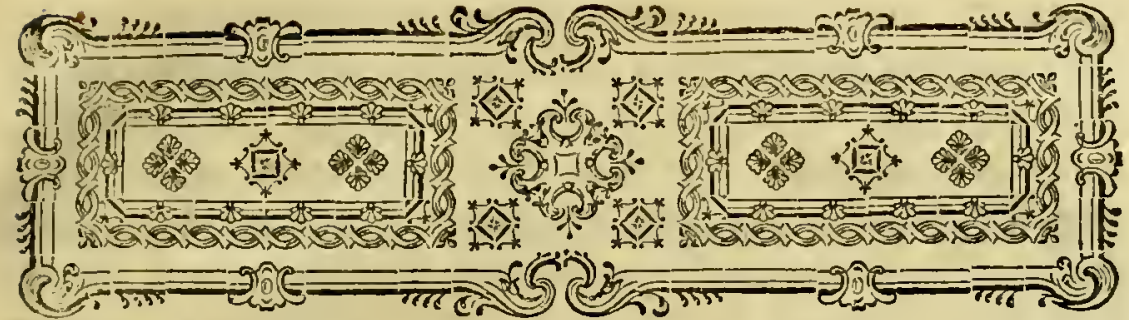

\section{DANIELIS MELANDRI} ASTRON. PROF. UPSAL.

\section{MEDITATIONES NONNULLÆ}

$$
\text { (c) }
$$

De Maclina bujus Mundi, ejufque confervatione per vires in initio conceffis.

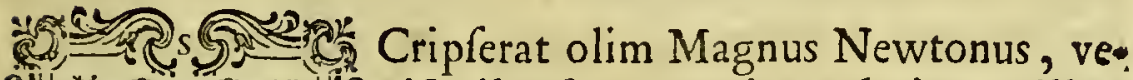
2) (c) 4 are ex mutuis Cometarum, \& Pla(f) dis majores ufque evadant, donec hæc Na: tandem fit defideratura (2ueft. opt.3 I. ) Iftud Newtoni effatum cum fana Philofophia non fatis apte conciliari poffe putabat illuftris Leibnitius, arguens Newtonum machinæ mundanx haud majorem tribuiffe perfectionem, quam ut Deus concurfu extraordinario opus haberet cam poft certa temporum intervalla iterum reficere, haud aliter ac horologii fabricator, qui minori minorique arte inftructus effe cenfebitur, quo frepius ejus horolo- 
rologium correctione egeat. Huic argumento refpondebat Celcbris Clarckius, obiiciendo comparationem a Leibnitio faetam Deum inter, \& horologii fabricatorem, eo minus appofite effe adductam ex Newtoni affertis, quo Deus ipfus materix, ejufque virium auctor effet, dum alter tantummodo materiam, ejufque partes fecundum leges virium ipfi a Deo conceffas fciret componere, nec non quod excellentiam operum Dei adhuc magis extollat ille, qui nil abfque providentia, \& gubernatione fieri divina afferit . Utilius fortaffis fuiffet, $f_{1}$ clariftmi hi Viri loco difputationum hujufmodi methaphyficarum, linquendo lites eruditas de Intelligentia mundana, \& fupramundana, ipfam hujus mundi machinam feveriori fubjeciffent examini; inveftigaffentque, aut utrum illarum irregularitatum, de quibus precipuè loquitur Newtonus, omnes vel aliquæ ejus fint indolis, ut fefe non reftituant interjectis certis temporum intervallis, aut an alix etiam fint in Natura mutationes, qux fucceffione temporis magnas quafdam, \& maximè notabiles producerent turbationes, quæ machinæ mundanæ ruinam accelerare pofent, nifi manus Dei emendatrix adeflet. Ad prius horum argumentorum quod attinet, non animus eft hac occafione in illud inquirere, quod quidem intra limites huic opufculo deftinatos contineri non poffet; de pofteriori autem meditationes nonnullas fubinde natas in chartam coniicere volui.

f. I. Opinio de communicatione lucis, \& caloris per veram emanationem particularum Solis, tam validis fufulta effe videtur rationibus, ut alix quxpiam explicationes illius communicationis abfque ulla probabilitatis fpecie proferantur. Hac ratione Solem, qui continue

tan- 


\section{Dell' Accadenia .}

tantam particularum copiam emittit, que codem modo fpatia fyftematis planetarii, \& ultra taanfeunt, quo in ipfos incidunt planetas, quique hujufmodi jacturam perpetuo facit, oportet fimiliter minui. Neque aliqua ratione contenderetur, materiam ignitam, que Soli ineft, utut firma illa fit, fortifimeque cohæreat, alius effe naturæ, quam omnis alia materia nobis nota, ut dum in flammam eruperit, fumum non emittat, cujus particulie in atmolphxram Solis denfam afcendentes, pro gravitate fua, fimul ac Solis molem minuunt, hanc augent, qux tamen augmenta cum Solis atmofphram denfiorem facerent, quam pro mole Solis, ejufque attractrice vi, efficient atmolphæræ folaris partes continue aliorum coleftium corporum atmofphæras pro illa menfura, \& proportione ingredi, qux ad debitum atmofphæras inter xquilibrium fervandum requiritur. Huic ratiocinio fortaflis opponitur, exhalationes dictas Solis, poftquam in ejus atmofphæram afcenderint more exhalationum terreftrium iterum coalefcere nubium inftar, deindeque in Solem decidere, qua ratione tantum novi alimenti Sol femper acquireret, quantum amitteret Verum præter alia, qux huic argumento regeri poffunt, minus bene ab exhalationibus humidis terreftribus, quæ in guttas aqueas coalefcunt, \& decidunt, ad eandem particularum Solis ficcarum naturam concluditur. Quomodocumque autem hæc omnia fiant, id tamen fatis certum effe exiftimo, materiam Solis dictas ob caufas minui, licet illæ diminutiones ità fint exiguæ, ut non nifi polt longam fxculorum feriem fiant perceptibiles.

s.. II. Negandum effe videretur, Cometas incolis aninalibus vegetalibufque ad vichum neceffariis effe proA 2 ditos, 
ditos, propterea quod nimium calorem uno tempore; nimiumque frigus alio has Nature productiones deftruere concipiamius. Experientia autem eft teftis, productiones unius climatis noftræ telluris fub alio etiam virefcere cœlo, \& maturefcere, incolafque æquatoris in regionibus frigidiffmis valere, \& hæ variationes in cometis tantummodo majoris funt intenfitatis \& gradus. Ille autem horum corporum creatione finis fi non effet intentus, ea tamen fupervacaneas Naturæ moles effe non erit dicendum, fi poft plures circa Solem revolutiones, inque ejus viciniis tanto majorem ab atmofpera Solis refiftentiam paffa, quanto funt velociora, proindeque hifce retardationibus ad Solem appropinquantia; in ipfum ultimo incidant, eaque modo in ejus alimentum inferviendo, frmul ad confervationem reliqui fyftematis planetarii ifta ratione tribuant. Sicut vero Cometie dicto hoc refpectu in fyftematis planetarii confervationem facerent, ita e contra nimius eorum numerus augeret turbationes fyftematis ejufdem, eafque multiplicando produceret fucceflione temporis premagnas fortafis irregularitates; itaque cum decrementa Solis per ejaculationem particularum, de qua agit f.r. fint perpetua, quamdiu aliquid materiæ folaris fuperfit, neque alius crit cometarum ufus, quam ut exitium Solis $\alpha$, proinde fyftematis planetarii, eorum ope, in eo longiora protiahatur tempora, quo piures fint Cometæ in hunc deftinati finem, Solis materia ejufque idcirco vi attractrice abfoluta nililominus decrefcentibus.

6.III. Sole jam decrefcente, ejufque propterea vi abfoluta continue diminuente, oporteret planetas etiam. e Sole continuo recedere, inque figuris quibufdam fpi- 


\section{Dell' Accademia .}

ralibus circa Solem convolvi, \& ab eo in illis figuris continue recedere, donec Sole tandem evanefcente, planet $x$ in infinitum abirent. Invetigatio autem figurarum, quas planetx hoc modo defcriberent, proponi poterit modo fequenti.

\section{P R O B L E M A I.}

Exeat corpus \& loco dato cum velocitate data $\mathrm{g} ;$ fit $\mathrm{h} f \mathrm{f}$ nus anguli projectionis, 6 lex vis contripete duplicata diftantiavm inverfa, augeatur vero vel minuatur vis abfoluta centri quantitate in data ratione crefcente, 6 oportet invenire motum bujus corporis.

Erficuum eft problema folvi non poffe; nifi augmenta, vel decrementa vis abfoluta exponantur per functiones variabilium æquationem ad curvam ingredientium, utpote radii vectoris, \& anguli circa centrum defcripti illis augmentis, vel decrementis proportionales. Itique cum dicta augmenta, vel decrementa dependeant a tempore cujus exponens eft $\int \frac{x^{2} d z}{2}$, dum radius vector $\dot{x} \&$ angulus $z$, oportet invenire functionen temporis; feu ipfus $\int \frac{x^{2} d z}{2}$ proportionalem rationi augmenti, vel decrementi vis abfolutx, qux expreffionis vis centripetæ numeratorem ingredi debet. Sit $\mathrm{P}$ vis abfoluta in initio augmentationis, vel diminutionis, defignet autem $Q$ quantitatem materix, feu vis abfolutx; qua dato tempore materia $P$ fecundum illam rationen functionis temporis, cui proportionalis eft auctio, vel diminutio, augetur vel minuitur. Sit præterea functio dicta 
dicta temporis, cui proportionalis eft auctio vel diminutio; $p \int \frac{x^{2} d z}{2} ; \& \operatorname{erit} \frac{P \pm Q p \int \frac{x^{2} d z}{2}}{x x}$ exponens vis centripetx qua corpus urgetur, \& $d z=$

æquatio differentialis ad curvam. $x x \sqrt{\frac{1}{b b}-2 \int\left(\frac{P_{ \pm} Q p \rho \int x^{2} d z}{g g x x}\right) d x-\frac{1}{x: x}}$

Cafus omnium hujus problematis fimplicifimus eft, dum $p=\mathrm{I}$, feu dum augmenta, vel decrementa funt ut tempora, ex qua tamen ratione diminutio Solis ex dictis caufis oriunda abludet. Analyfis autem pro hoc cafu fequenti ratione inftitui poterit. Equationem etenim expofitam quadrando, \& ordinando habetur $\frac{g g}{b b}-\int \frac{d x}{x x}\left(p_{ \pm} Q \int \frac{x^{2} d z}{2}\right)$ $\frac{-g g}{2 x x^{2}}=\frac{g^{2} d x^{2}}{2 x^{4} d z^{2}}$, in qua xquatione aut $d z$ aut $d x$, aut etiam $d t$, cujus exponens eft $\frac{x^{2} d z}{2}$, ut conftans affumi poffet, reliquis variantibus. Sit $d z$ conftans, \& xquatio una vice differentiata dabit $-P \pm Q \int \frac{x^{2} d z}{2}+\frac{g g}{x}=$ $\frac{g^{2} d d x}{x^{2} d z^{2}}-\frac{2 g^{2} d x^{2}}{x^{3} d z^{2}}$ \& iterum differentiata $- \pm \frac{Q x^{4} d z 3}{2 g g}-d x d z^{2}=$ $d^{3} x-\frac{6 d x d d x}{x}+\frac{6 d x^{3}}{x^{2}}$, ex cujus integratione dependet determinatio motus corporis. Si pofuiffem $d x$ vel $d t$ confans, tam complicata prodiifet æquatio, ex qua nil concludi poffe videretur. Hæc vero æquatio in cafu $d z$ conftantis inventa ponendo $x=\frac{1}{v}$ transformatur in hanc fimpliciorem $v^{2} d^{3} v+v^{2} d v d z^{2} \div \frac{Q^{2} d z^{3}}{2 g g}=0$, in qua fignum + obtinet, dum vis abfoluta minuitur, - vero dum 
dum augetur. Circa hanc xquationem fequentia obfervari pollunt .

I. Illam faciendo $Q=0$, feu ponendo nullam effe auctionem, vel diminutionem vis abfolutx, relinquere $d^{3} v+d v d z^{2}=0$, qux integrata dat $d d v+v d z^{2}+$ $R d z^{2}$, qux æquatio defignat fectionem conicam, ficut etiam debuit.

II. Aquationem $v^{2} d^{3} v+v^{2} d v d z^{2} \mp \frac{Q d z^{3}}{g g g}=0$, fortaffis inter difficiliores, quarum abfolutx integrationes exhiberi queant, numerandam effe. Varios licet tentaverim modos, quibus uti foleo ad æquationes differentiales conftruendas, propofita hæc huc ufque meam effugerat induftriam. Contentus itaque fui methodo $\mathrm{fe}$ quenti illam tractandi, ex qua ad naturam motus corporis hujufmodi vi agitati, aliquatenus concludere fas erit. Equatio in hanc formam difpofita $d^{3} v+d v d z^{2} \mp$ $\frac{Q d z^{3}}{2 v^{2} g g}=0$, ponendo $v=K+t$, ubi $K$ eft diftantia planctx media a Sole, $t$ vero pars radii vectoris recipreci variabilis, que proinde parva crit præ $K, \&$ non nifi longo temporis tractu fat magnam ad $K$ nancifcet proportionem, migrat in hanc $d^{3} t+d t d z^{2} \mp \frac{Q d z^{3}}{2 g g \cdot(K+t)^{2}}=0$, $\& d^{3} t+d t d z^{2} \mp \frac{2 d z^{3}}{2 g g} \cdot\left(\frac{1}{K^{2}}-\frac{2 t}{K^{3}}-6 r c\right)$, in qua xquatione cum $t$ parva eft præ $K, \& \frac{2 t}{K^{3}}$ adhuc magis exi. sulus prex $\frac{1}{K^{2}}$, xquatio hæc $d^{3} t+d t d z^{2} \mp \frac{Q d z^{3}}{2 g g K^{2}}=0$ naturam curve defcribendx proxime exprimet. Hanc xquationem integrando, debiteque corrigendo per additionem conftantis, habetur $d d t+t d z^{2}+\overline{R F N^{2}} \cdot d z^{2}=; 0$ 
ubi $N=\frac{2}{2 g g k h^{2}}$, quæ xquatio eft formx not: \& quam modo fequenti reducere foleo. Sit $t=c^{n z} y$, ubi $c$ numerus, cujus logarichmus eft unitas, $y$ nova variabilis, \& $n$ quantitas arbitraria, debitafque fubstitutiones ex hac fuppolitione faciendo, oriatur $n^{2} c^{n z} y d z^{2}+2 n c^{n z} d y d z+$ $c^{n z} d d y+c^{n z} y d z^{2}+\overline{R F N \approx} \cdot d z^{2}=0$. Fiat jam $n^{2}+1=0$, $\&$ erit $n= \pm \sqrt{-1}, \&$ xquatio fiet $d d y+2 V \overline{-I} d y d z+$ $\overline{R F N z} \cdot c^{-z V^{-1}} d z^{2}=0$. Sit ulterius $d y=q d z, \&$ orietur $d q+2 V \overline{-I} \cdot q d z+\overline{R F N^{2}} \cdot c^{-z V \overline{-x}} d z=0$. Hxc xquatio ducta in $c^{2 V \overline{-1}} \cdot z$, dat $c^{2 V \overline{-1}} \cdot z d q+2 \sqrt{-1} \cdot c^{2 V \overline{-1}} z q d z+\overline{R F N z}$. $c=\sqrt{-2} d z=0$, cujus integralis corrigendo, \& redicendo eft $q=-L c^{-2 V \overline{-1} z}-c^{-2 V \overline{-1} z} \cdot \int R \overline{F N z}$. $c^{z V \overline{-1}} d z, \& \mathrm{ob}$ id $q d z=-L c^{-2 V \overline{-1} \cdot z} d z-c^{-2 V \overline{-1} z}$ $d z \int \overline{R F N z} \cdot c^{2 V \overline{-x}} \cdot d z=d y$, qux integrata, iterumque correcta dat $y=\frac{L c-2 V \overline{-I} z}{2 V \overline{-I}}+G-\int c^{-2 V-\bar{x}} d z \sqrt{R F N z}$.

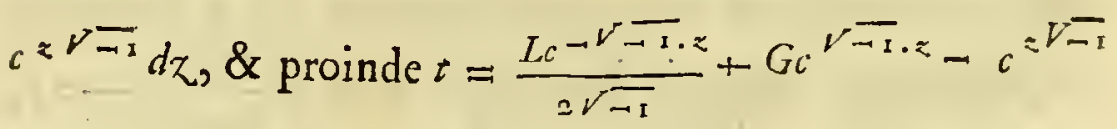
$\int c-2 V \overline{-x} \cdot z d z \int \overline{R F N z} c^{z V \overline{-1}} d z$. Deprehenditur autem, $\int \overline{R F N z} \cdot c^{V \bar{F} \cdot z} d z=\frac{R c^{V \overline{-1}} \cdot z}{\sqrt{-1}}+\frac{N z c V \overline{-1} \cdot z}{V \overline{-1}}+N c^{V \overline{-1} \cdot z}$ $\frac{R}{V-1}-N$, fimiliter ac $\int c^{-2 r-1} \cdot z d z \cdot \int \overline{R F N_{z}} \cdot c^{V \overline{-1} z}$ $d z=R c^{-z V \overline{-1}}+N_{z c}-V \overline{-1} \cdot z-\frac{R c^{-2 V-1 \cdot z}}{2}+\frac{N_{c}-2 V \overline{-1} z}{2 V \overline{-1}}$ $\frac{R}{2}$ 


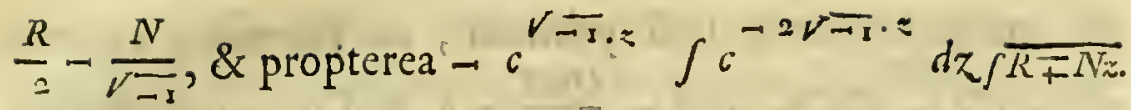

$c^{V=1 \cdot z} d z=-R-N z+\frac{R c-V \overline{-1} z}{2}+\frac{R_{c}^{z V \overline{-x}}}{2}-\frac{N_{c}-z V \overline{-x}}{2 V \overline{-1}}+$ $\frac{N_{c}=V \overline{-x}}{2 V \overline{-1}}=-R-N_{z}+R$ cof. $z+N$ fin. $z$, \& ob id $t=$ $\frac{J_{c}-z V \overline{-1}}{2 V \overline{-1}}+G c^{V \overline{-1} \cdot z}-R \pm N z+R$ cof. $z \mp N$ fin. z, feu debitos valores pro $L \& G$ correctionibus inveniendos fibltituendo $t=q$ cof. $z+\varepsilon$ fin. $z-R \pm N z+R$ cof.zF $N$ fin.z, \& ob id $v=K+t=K-R+\overline{q+R} \cdot \operatorname{col} \cdot z+\overline{\varepsilon+N}$. fin. $z \pm N z$. Ex loc valore radii vectoris reciproci deprehenditur, illum preter terminos indicantes fectionem conicam vi duplicata inverfa defcribendam effe, ingredi cum fignis mutatis, dum vis abfoluta augetur, terminos $-N z, \&+N$ fin. $z$, illos vero cum hifce fignis eum ingredi dum vis minuitur, horum autem terminorum unum $N_{z}$ continere arcum circuli, qui quoniam in reciproco radii vectoris eft negativus, continuo illum augebit, \& efficiet corpus, ut in initio hujus $\theta$ dictum erat, in fpirali a Sole continuo recedere, contra vero fi vis abfoluta crefceret.

III. Majori accuratione radium vectorem hujus orbitx inveniri, $f$ ponatur in xquatione $d^{3} v+d v d z^{2}+$ $\frac{Q d \times 3}{2 g v^{2}}=0, v=y+t$, in qua $y$ defignat reciprocum radii vectoris in figura elliptica, qua ratione xquatio $d^{3} y+d^{3} t+\overline{d y+d t} \cdot d z^{2}+\frac{O d z^{3}}{2 g g} \cdot \overline{\frac{1}{y^{2}}-\frac{5 t}{y^{3}}+\frac{3^{t^{2}}}{y^{4}}} \cdot$ - \&c. prodiret, in cujus termini ultimi ferie, eadem ratione negligenB 
do omnes terminos poft primum, ac fuperius, habetur $d^{3} y+d^{3} t+\sqrt{d y+d t} \cdot d z^{2}+\frac{Q \cdot d z^{3}}{2 g g y^{2}}=0$. Sed quoniam eft $y$ reciproca radii vectoris in Ellipfi erit $y=A+B$ cof. $z$ dum initiun motus fumitur $a b$ aphelio, \& propterea $d y=$ $C d z$ fin. $z, d d y=D d z^{2}$ cof. $z, d^{3} y=\varepsilon d z^{3}$ fin. $z$, quibus valoribus fubftitutis orietur $d^{3} t+d t d z^{2}+(\overline{C+\varepsilon}$. fin. $z+$ $\left.\frac{Q}{2 g g \cdot(A+B \text { cof.z })^{2}}\right) d z^{3}$. Cum vero termini hujus ultimi integralis femper fumi poterit, quatenus eft $d z$ conftans, erit $d d t+t d z^{2}+\int\left(\overline{R+C+\varepsilon}\right.$. fin. $\left.z+\frac{Q}{\operatorname{ggg}(A+B \operatorname{cof} . z)^{2}}\right)$ $d z^{3}=0$, qux xquatio eft ejufdem form $x$, ac cujus integrationem dudum exhibui, fed quem calculum ob prolixitatem perfequi non vacat. Poni etiam potuiffet in xquatione $d^{3} v+d v d z^{2}+\frac{Q d z^{3}}{2 g G v v}=0, d v=p d z, \& d^{3} v=$ $d d p d z$, unde proveniret $d d p+p d z^{2}+\frac{Q d z^{2}}{g_{2}^{2} v^{2}}=0$, in qua pro $v^{2}$ ponendum foret $(A+B \text { col. } z)^{2}$, \& xquatio prodiret formæ prioris, qux daret $p$ in $z$, quo invento xquatio $d v \doteq p d z$ dabit $v$ in $z$.

§.IV. Modo jam defcripto oporteret Planetas e Sole continuo recedere, ita tamen ut radius vector in paullo minori crefcat ratione, quam fecundum calculum jam expofitum, cun ratio diminuticnis Solis fit paullo minor ratione temporis fimplicis. Ne autem hoc modo corpora planetarum, etfr poft longam fæculorum feriem, inutiles fierent Naturx moles, quippe in quibus tum ob perpetuum receffum a Sole, tun etiam ob ipfus Solis continuam diminutionem, omnia animalia, 
$\&$ vegetabilia ultimò deftruerentur, oportuit fimul aliam quandam vim continuo agentem contra directionem motus planetarum dari, qux vis ifta fua actione eorum motus retardaret, eofque ad juftas a Sole diftantias retineret, proindeque loco receffus a Sole efficeret illos ad Solem propius propiufque, dum circa Solem convolverentur, acceffuros fore, ita ut Sole diminuente fufficiens in planetis gradus caloris fentiretur. Hic acceffus planetarum Solem verfus, conjunctus cum diminutione Solis reali illum acceffum ex obfervatione diametri Solis apparentis haud perceptibilem redderet, planetæ nimirum diftantia a Sole diminuta, fimul ac Sol decrefcit. Ad hunc jam defcriptum effectum producendum inferviet xther, feu materia illa fubtiliffima, qux fecundum plurimorum placita philofophorum fpatia occupat coleftia.

§.V. Etheris autem hujus in naturam \& indolem fi inquirere velim, non fat bonas invenio rationes, illum ut ab atmofphæris corporum cœleftium plane diverfum confiderandi fuidum, illumque etfi materian fubtilifiman ut coleftia occupantem fpatia atmofphrras, porofque corporum permeantem habendi. Mihi magis arridet opinio, fpatia coleftia occupare quidem fluidum quoddam rariffimum, verum ab atmolphæris Solis, \& planetarum dependens. Nam cum fxpius iterata experimenta, ficut potiflimum ex obfervatis Domini Bouguer colligere licet, comprobent denfitatem atmofphxra telluris in altiflimorum montium cacuminibus haud minus quam inferius accurate fequi rationem ponderis atmofphæræ incumbentis, nullam video rationem illam natire fibi diflimilitudinem tribuendi, ut 
altius, 8 alcius in atmofphram exfurgendo, illa lex tandem deficiat, utque denfitates atmofphrrarum Solis, \& reliquorum planetarum ab hac alienam fequantur condenfationis legem. Hifce principiis innixo ifte mihi conceptus fubvenit, ut initio rerum omnia occuparet cœleftia fpatia fuidum homogeneum clafticum, \& xqualiter denfum, pofitifque deinde hifce in fpatiis omnibus fixis planetarumque fyftematibus, hæc corpora pro menfura virium gravitatis cuilibet propriam tantum illius fluidi in fe traherent, quantum uniufcujufque vi refponderet attractrici, quo ipfo totum illud fluidum in atmofphæris cingendo omnia hæc corpora confumeretur, ita ut cum affunta in univerfum dicta illa lege condenfationis, \& vi gravitatis in ratione duplicata diftantiarum inverfa, altitudo atmofphrre in infinitum extendi debeat, unius corporis coeleftis atmofphrra ad ufque alterius atnof phæram pertingeret, limefque inter atmofpheras ibi effet, ubi ejus particula ad utruinque corpus æqualiter gravitent, \& loc modo planetz in molibus fuis a. Solis \& mutuorum atmofpheris refiftentias patientur. Hac in opinione eo magis confirmor, quo certior fin xquationem Lunx fecularem, feu cjus motus medii accelerationem maxima!m partem dependere ex refiftentia atmofphare terreftris in diftantia Lunx, exiguam tantumodo tamen aliquam hujus inxqualitatis partem fibi atmofplaæra Solari vindicante. Si hæc omnia ita fe haberent, notatu certe dignum eft, Solem fua atmofphra recefibus planetarum a fe ipfo, ex fua diminutione, ut prius expofitum eft, oriundis remedium adferre. f.VI. Hos preter jam nominatos effectus naturæ ad retardandam accelerationem fux deftructionis, ipfa 
aliis quoque mutationibus obnoxia effe poterit. Jam non præcipue loquor de turbationibus planetarum a $\mathrm{fe}$ mutuo, quippe qux preterea quod peculiaris fit confiderationis, etiam exigux funt, verum alia eft in eo præfertim ratio cometarum, quod hi dum planetæ intra fuos limites ad certas a fe invicem pofitis diftantias convolvantur, in orbibus valde excentricis quaquaverfum pofitis excurrant, quorumque plurimi etiam fuos curfus intra orbitas planetarum eriam Soli propiorum porrigant, quo fieri poflet, ut dum in alicujus planetx orbitam impingerent, dum planeta in loco proximo effet, fi non potentes effent planetam fecum rapiendi, cum plerique nobis noti, non tantæ fint molis, tamen in ejus motu noxios producerent effectus . Ab hac diverfa ratione alias quoque turbationes in fyftemate planetario efficere poffent cometx, fi fcilicet ut in \$.2. dictum eft, ad alendum Solem effent deftinati; Si etenim quidam magnæ molis cometa in Solem impingeret, Solis mafla hoc augmentum adfcifcendo fortius. traheret planetas, unde alix illorum orbitarum fpecies orirentur, ad quas indagandas otii ergo proponi poterit problema fequens.

\section{P R O B L E M A II.}

Sit vis centripeta quadrato diffantice e centio reciproce proportionalis, mutetur autern vis aljoluta per faltus, or requiruntur mutationes motum inde oriundi.

Uoniam lex vis centripetr eadem eft ante, \& pont Tab.r. mutationem quantitatis vis abfolutx, orbita no- Fig. I. va femper crit aliqua fectionum conicarum. Ad illam deter- 
determinandam fit $A$ locus, quem occupat planeta in orbita fua momento mutationis vis, $C$ centrum virium, \& jungatur $A C=a$. Sit $c$ velocitas Planetæ in $A, \&$ $A D$ altitudo illi velocitati debita, quæ $A D$, cum detur fectio conica, \& locus planetx in illa, etiam dabitur. Sit $v$ velocitas planetæa cadentis in loco quovis $E, C E=x, E F=-d x, \& C D=m$. Sit $P$ vis Solis ante mutationem, \& $Q$ poft mutationem, quibus pofitis invenitur $v=\sqrt{\frac{2 P}{i}-\frac{2 P}{2}}, \& c=\sqrt{\frac{2 P}{m}-\frac{2 P}{a}} \cdot$ Cadat rurfus planeta $\mathrm{ab} A$, agente vero jam vi centripeta $Q$, $\&$ fit $A G$ altitudo celeritati $c$ jam debita, velocitas vero in quovis puncto $H=10, C H=y, H K=-d y$, eritque $\mathbf{1 0}=\sqrt{\frac{2 Q}{y}-\frac{2 Q}{a}}$, iterumque $c=\sqrt{\frac{2 C Q}{c g}-\frac{2 Q}{a}}$, unde $\frac{P}{m}-\frac{P}{a}=\frac{Q}{C G}-\frac{Q}{a}, \& C G=\frac{a m Q}{a P+m \cdot \overline{Q-P}}$. Inventa fit $G C$, feu $A G$ altitudine filicet velocitati nova agente vi debita, fectio conica defribenda facile conftruitur. Generaliter autem, fi $C G$ major quam $\stackrel{a}{2}$, figura erit Ellipfis, fi xqualis Parabola, fi minor Hyperbola.

ç.VII. Defcripto fuperius modo etfi Sol continue diminueretur, ultimoque totus difliparetur, planctæque interea temporis ad illum perpetuo accederent, fylematifque planetarii ruina fic prepararetur, fatis nihilominus intelligitur, Solis diminutionem, medii refiftentiam, \& dimenfiones fyftematis ita ab initio fecundum fe mutuo temperari potuiffe, ut ejufdem fyftematis duratio intra quodvis tempus datum protraheretur. Huic autem fini ficut lex gravitatis in ratione duplicata di- 
ftantiarum inverfa, omnium qux quamcunque diftantiarum rationem fequantur, unica eft naturæ confervationi idonea, ita etiam accomodatiffima eft. Nam cum illa lege planeta, jufta cum velocitate projectus Ellipfim defcribet, \& huic figuræ defcribendæ infinitr velocitates projectionis inferviant, quarum quippe limites funt, unus filitet, dum altitido velocitati debita projectionis eft tota a centro diftantia, \& alter, dum eadem altitudo eft hujus diftantix dimidium, facilè concipitur planetam projectum velocitate ad defcribendain ellipfin parve excentricitatis propria, a medio autem refiftente retardatum continue, nihilo tamen minus, ufque dum velocitas ulterius progrediendi fecundum tangentem ab illo medio tota pereat, femper incedere velocitate ad defcribendam ellipfin debita, fi illo momento medium tolleretur refiftens. Licet igitur geometrice loquendo punctum orbitx planetx quodvis fequens non pertineat ad eandem ellipfin, ac punctum antecedens, tamen erit ad aliam figuram ellipticam, qux offervationibus a priori diftingui non poterit, antequam auctio excentricitatis ita notabilis fiat, ut illa differentia obfervari queat. Diminutione itaque Solis, \& refiftentia medii ita fecundum fe invicem temperatis, ut non nifi poft longam fxculorum feriem aliqua variatio excentricitatis exiftat perceptibilis, eandem defcribere ellipfin, abftrahendo ab illis inæqualitatibus, qux a turbationibus planetarum mutuis orientur, planetæ femper deprehendentur. Si vero cun accurationi obfervationum noAtri ævi magis fidendum fit, quam illi prifci, ita ut hæc inæqualitas comparando hujus temporis obfervationes cum illis antiquilinorum temporum detegi nequeat, 
ars bene obfervandi inter deperditas longe fubfequentium xvorum non fit numeranda, \& futurorum illorum temporum Aftronomi inveniant excentricitates planetarum, præcipue Soli proximorum, ut Mercurii, \& Veneris, qui majorem ab atmofphæra Solis refiftentiam patientur, auctas fuiffe, harumque mearum meditationum tum aliquod veftigium fupereffet, ipfre ut meri lufus ingenii tum fortaflis non haberentur. Si docti fuerint Aftronomi parallaxin Solis debita cum accuratione determinandi, per comparationes parallaxium illarum temporum intervallis longiflime difitis inventarum, iidem quoque effectus fefe confpiciendos præberent.

§.VIII. Dictum erat $\$$ præcedenti legem duplicatam inverfam naturx confervandx folam effe idoneam, quo affertun effe velim omnibus aliis legibus corpora projecta aut in infinitum excurrere, aut in ipfum centrum cogi, aut orbitas admodum irregulares defcribere, aut fi plura corpora fyftema componant, ifta fe invicem ita turbatura fore, ut brevi totum corrueret fyftema, quo ipfo fummi opificis fapientia, dum elegerat unicam ex omnibus legibus, quæ quamvis diftantix rationem fequantur, durationi operis fui idoneam, maxime etiam confpicitur. Dum itaque in hifce difquifitionibus occupatus fun, lubet etiam quxdam adferre ad hoc dilucidandum pertinentia, ad quod præcipue inferviet inquirere, ad quas corpus projectum pofita lege vis centripetæ in qualibet ratione diftantix agente, perveniet apfides. Propofitum itaque fit. 


\section{P R O B L E M A III.}

Sit lex vis centripete in quacunque diftantiarum ratione directa; vel inverfa, oportet autem invenire apjides, ad quas

corpus e dato puncto data cum velocitate in data directione egrediens, perveniet.

It $C$ centrum virium, \& corpus data cum veloci- Tab.1. 1) tate exiens e loco $U$ defcribat trajectoriam $U I K$. Fir. .. Sit $A$ locus, a quo Corpus cadendo ad $U$, in $U$ acquirit velocitatem æqualem velocitati projectionis. Ad rectam $C \mathcal{A}$ applicentur ordinatæ rectangulæ $D F, E G$, proportionales viribus centripetis in omnibus diftantiis $C^{\prime} D, C E$, a centro, \& fit curva $O S$ locus omnium punEtorum $F, G$. Sint puncta $D, E$, fibi proxima, \& centro $C$ intervallis $C D, C E$, defcribantur arcus circuli trajectorix occurrentes in punctis $I, \& K$, junganturque $K C$, \& $I C$ occurrens arcui $K E$ in $N$. Hifce conftruCtis oftenderat Newtonus Propp.39, 40, 4 I Libr.r. Princ. Phil: Nat. Math. 1. Corpore defcribente curvam ICK circa centrum virium $C, \&$ alio corpore recta afcendente, vel defcendente verfus $C$, horum corporum velocitates in omnibus diftantiis æqualibus a centro effe xquales, $f($ eorum velocitates in uno diftantiarum æqualium cafu fint xquales. 2. Velocitatem in omni trajectorix puncto $I$ effe ut $V \overline{A B F D} \cdot 30^{\circ}$ Effe $K N$ femper ut $\frac{Q}{I C}, f_{1} Q$ fit quantitas data. Polito jam $d t$ feu elemento temporis conftanti, erit $I K$ ut $V \overline{A B F D}$. Si ergo $Q$ ita eft fumta, ut in uno cafu fit $\frac{Q}{\bar{I}}: V \overline{A B F D}:: K N: I K$, 
obtinebit hxc proportio in omni cafu. Sumta itaque 2 ejufmodi, erit $\frac{Q}{I C}=V \overline{A B F D}$, ubicunque fuerit $K N=I K$. Dum vero $K N=I K$ erit corpus in apfide, ergo xquatio $\frac{Q}{I C}=$ $\checkmark \overline{A B F D}$ dabit valores radii vectoris dum corpus eft in apfide, quorum $f_{1}$ unicus tantum inveniatur affirmativus $\&$ realis, vel plures, dummodo fint æquales, corpus non nifi ad unicam perveniet apfidem, $\& a b$ ea aut perget in infinitum, fi fit ima, vel incidet in centrum, fi fit fum$\mathrm{ma}, \mathrm{f}_{1}$ vero plures fint affirmativi inæquales \& reales, corpus intra certos terminos retinebitur. 2.E.I.

6.IX. In applicatione hujus æquationis duo cafus præcipue erunt attendendi. I. Si diftantia $C I=x, \&$ lex vis centripetæ fit $\frac{\overline{n-1} \cdot P}{x^{n}}$, fitque $n$ major unitate, in quo cafu tres cafus velocitatis projectionis occurrunt confiderandi; effe etenim poterit velocitas initialis aut æqualis ei, quæ acquiritur cadendo a puncto dato $A$ ad punctum projectionis, aut æqualis ei, quæ acquiritur cadendo a diftantia infinita, aut adhuc major. $20^{\circ} \mathrm{Si} n$ fit aut unitas, aut minor unitate, unicus tantum cafus velocitatis initialis eft confiderandus, fcilicet dum velocitas in $U$ xqualis eft ei, quæ acquiritur cadendo a diftantia finita. Sit $C A=a, D E=I N=d x, D F=$ $\frac{\overline{n-1} \cdot P}{x^{n}}$, unde $D E G F=\frac{\overline{n-1} \cdot P d x}{x_{n}}, \&$ ob id $C D F S s=$ $R-\frac{P}{x^{n-1}}, \& A B F D=\frac{P}{x^{n-1}}-\frac{p}{a^{n-1}}$, qui valor eft in cafu primo velocitatis initialis. Si vero velocitati altitudo debita eft infinita, erit $a=\infty, \& O D F o=\frac{p}{x^{n-1}}$. 
In cafu velocitatis initialis adhuc majoris, fit $P$ punctum a quo corpus caderet vi centripeta conftanti ufque ad $U$, ut in $V$ acquireret velocitatem initiali $x$ qualem, fitque $P Q$ xqualis illi vi, completoque paralellogrammo $Q P U R, e=\sqrt{U I^{p} Q R}$. Sit jam $C U=r$, eritque U LFD $=\frac{p}{x^{n-1}}-\frac{p}{r^{n-1}}, \&$ ob id velocitas in omni puncto $D$ erit ut $\sqrt{e e+\frac{P}{x^{n-1}}-\frac{P}{r^{n-1}}}$ Exponens velocitatis in hoc cafu etiam invenitur, faciendo corpus cadere a diftantia infinita ufque ad punctum $H$ centro propius quam $U$, inque $H$ acquirere velocitatem $x$ qualem velocitati initiali in $V$, critque fi ponatur $C H=c$, exponens velocitatis in $D, \sqrt{\frac{P}{c^{n-2}}+\frac{P}{x^{n-1}}-\frac{P}{r^{n-1}}} \cdot$ Exponens hujus velocitatis etiam modo fequenti inveniri poteft. Ut volocitas in $V$ fit major velocitate ab altitudine infinita acquifita, fingatur corpus altitudine infinita cafum fuum incipere volicitate ea ratione acquifita, ut corpus ab altera parte centri, vi contripeta in vim centrifugam verfa, ita ut centrifuga vis in omni diftantia a centro xqualis fit vi centripetx in eadem diftantia, a punto dato afcendat ad diftantiam infinitam, eaque velocitate fic acquifita ab illa diftantia infinita ad partes $U$ centri virium defcendere incipiat ufque ad $U$, ibidemque acquirat velocitatem xqualem velocitati projectionis. Sit $a$ illud punctum, \& $a C=b$, eritque velocitas in omni puncto $D$, vel $I$, ut $\sqrt{\frac{P}{b^{n-1}}+\frac{P}{x^{n-1}}}$. Ad inveniendum 2 , fit $U p$ tangens orbitam in $U, \& C_{p}$ normalis ad $U p$. Sitque $C p=p$, eritque $Q=p \sqrt{A U L B}=$ $\mathrm{C}_{2}$ 
$p \sqrt{\frac{p}{r^{n-1}}-\frac{p}{a^{n-1}}}$, in primo cafu velocitatis initialis, $\& Q=p V \frac{P}{m^{n-1}}$ in fecundo, $\& Q=p e$ in tertio, vel etiam $Q=p \sqrt{\frac{p}{c^{n-1}}}$, nec non $Q=p \sqrt{\frac{p}{b^{n-1}}+\frac{p}{b^{n-1}}}$ in eodem cafu. Pro inventione apfidum igitur habebuntur xquationes fequentes. Si $n$ eft major unitate, \& velocitas initialis æqualis ei, quæ acquiritur cadendo a diftantia finita, erit $\frac{p}{x} \sqrt{\frac{P}{r^{n-1}}-\frac{p}{a^{n-1}}}=\sqrt{\frac{P}{x^{n-1}}-\frac{p}{a^{n-1}}}$. Si velocitas fit æqualis cadendo a diftantia infinita acquifitx erit $\frac{p}{x} V \frac{\bar{P}}{r^{n-1}}=V \frac{\bar{P}}{x^{n-1}}, \&$ fi velocitas eft adhuc major erit $\frac{e p}{x}=\sqrt{e e+\frac{P}{i^{n-1}}-\frac{P}{r^{n-1}}}$, aut $\frac{p}{x} \sqrt{\frac{P}{c^{n-1}}}=$ $\sqrt{\frac{P}{c^{n-1}}+\frac{P}{x^{n-1}}-\frac{P}{r^{n-1}}}$, vel etiam $\frac{p}{x} \sqrt{\frac{P}{r^{n-1}}+\frac{P}{b^{n-1}}}=$ $\sqrt{\frac{P}{b^{n-1}}+\frac{P}{x^{n-1}}}$, unde fequentes formulæ cruuntur, fcilicet pro cafu primo $x^{n-1}+\frac{2 a^{n-1}-p^{2}}{r^{n-1}} \cdot x^{n-3}-$

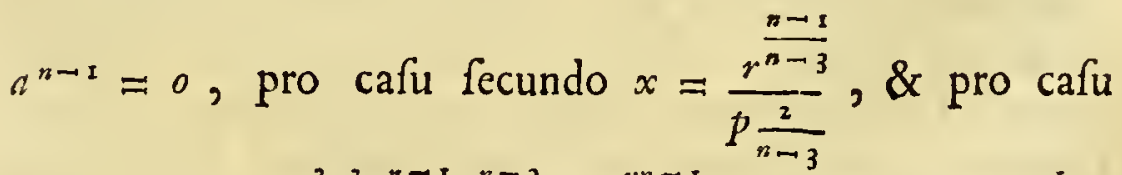
tertio $x^{n-1}-\frac{e^{2} p^{2} r^{n-1} x^{n-3}}{c^{2} r^{12-1}-1}+\frac{y^{n-1}}{e^{2} r^{n-1}-1}=0$, feu $x^{n-1}-$ $\frac{p^{2} r^{n-1} x^{n-3}}{r^{n-1}-c^{n+1}}+\frac{c^{n-1} r^{n-1}}{r^{n-1}-c^{n-1}}=0$, vel etiam $x^{n-1}-\frac{\overline{p^{2} b^{n-1}}+p^{2}}{r^{n-1}}$. $x^{n-1}$ 
$x^{n-3}+b^{n-1}=0$. Si eflet $n=1$, erit exponens in omni puncto $I$ hæc $\sqrt{\log \cdot \frac{a}{x}}, \&$ xquatio apfides indicans $\frac{p}{x} \sqrt{\log \cdot \frac{a}{r}}=$ $\sqrt{\log \cdot \frac{a}{x}}$, ex qua fimiliter conftat non nifi unum velocitatis cafum tum obtinere, fcilicet quæ acquiritur cadendo a diftantia finita a centro, cum fi a effet infinita, etiam velocitas erit infinita. Idem etiam obtinet femper, ubi eft $n$ minor unitate. Ex hifce jam fequentia deducuntur confectaria .

I. - Formulæ pro cafu velocitatis majoris ea quæ acquiritur cadendo a diftantia infinita ctiam in fe continet duos reliquos cafus. Si etenim in rquatione $\frac{p}{x} \sqrt{\frac{1}{r^{n-1}}+\frac{1}{b^{n-1}}}=\sqrt{\frac{1}{b^{n-1}}+\frac{1}{x^{n-1}}}, b$ fit infinita, hoc eft quod corpus in diftantia a centro infinita nullam habeat velocitatem, erit $\frac{p}{x} \sqrt{\frac{1}{x^{n-1}}}=\sqrt{\frac{1}{x^{n-1}}}$, qux eft xquatio pro cafu fecundo velocitatis initialis. Si velocitas fit adhuc minor affici debet $b$ figno - , \& redit ille cafus.

2. Cafus dum $n=3$, eft quafi limes legum virium centripetarum, fecundum quas corpora projecta, quxcumque fit velocitas initialis ab una parte, aut in infinitum abibunt, aut in centrum incident, \& ab altera parte jufta cum velocitate projecta, ab una apfide ad alteram pervenient, \& fic intra certos continebuntur terminos a centro. Pro cafu ctenim primo velocitatis initialis

xqua- 
xquatio $x^{n-2}+\overline{\frac{p^{2} a^{n-1}}{r^{n-1}}-p^{2}} \cdot x^{n-3}-a^{n-1}=0$ fatim in-

dicat quicumque valor integer vel fractus ponatur pro $n$ major numero ternario, radicem $x$ non nifi unicum habere valorem realem, \& affirmativun, proindeque corpus illa velocitate projectum, non nifi ad unicam pervenire poffe apfidem, quæ quidem erit fumma, \& ex qua in centrum cogetur, fi furfum projiciatur, \& abfque omni appulfu ad apfidem in centrum incidet, fi deorfum projiciatur. In cafu fecundo velocitatis initialis æquatio inventa easdem conclufiones indicat. Formulæ autem pro cafu tertio velocitatis initialis indicant quidem duas effe radices reales, \& affirmativas, verum quas has formulas applicando ad plures cafus inveneram effe æquales, \& corpus ifta velocitate projectum furfum in infinitum abire, deorfum vero pervenire ad apfidem imam, ex qua in infinitum excurret, unde etiam ad eamdem hanc proprietatem in reliquis cafibus valoris ipfus n'majoris numero ternario, concludere fas effe duxi, licet regulam generalem 'quandam id ipfum comprobantem ex illis huc ufque non deduxerim formulis.

3. In cafu ipfus $n=3$, \& dum velocitati altitudo debita eft finita, deprehenditur fimiliter corpus non nifi ad apfidem fummam pervenire poffe a quo defcendet, \& infinitis gyrationibus circa centrum convolvetur, antequam in id incidat. Si hæc altitudo eft infinita invenitur $p^{2} x^{2}=r^{2} x^{2}$, cui xquationi duplex valor ipfius $x$ refpondet, fcilicet $x=0, \& x=\infty$, hoc eft corpus hac 
velocitate projectum ad apfidem imam non perveniet nifi dum in centrum poft infinitas gyrationes incidat, \& furfum projectum ad apfidern fummam non pertinget, nifi in diftantia infinita a centro. Hifce cafibus tertius addi poteft qui eft dum $p=r$, in quo corpus circulum defcribet, duabus apfidibus hoc modo coeuntibus.

4. Cum igitur, quemcumque habeat valorem $n$ aut xqualem numero ternario, aut majorem, quævis fit velocitas projectionis corpus aut abeat in infinitum, aut in centrum cogatur, neque ulla omnium harum legum virium centripetarum naturæ confervandæ fuiffet idonea.

5. Dum fecundum hxc eadem principia examinandæ effent leges virium centripetarum, dum $n$ eft minor numero ternario, ufque dum $n=\mathrm{I}$, in applicatione dictarum formularum ad quofvis cafus particulares duorum cafuum velocitatis projectionum, fcilicet dum altitudo velocitati initiali débita eft aut infinita, aut major infinita, deprehenditur illa iifdem difficultatibus premi ac de legibus, dum $n=3$, vel major fupra dictum eft: In tertio autem velocitatis projectionis cafu, præterea dum $n=0$, licet corpora projecta ab apfide ima ad fummam perveniant, \& fic viciffim, orbitas tamen admodum irregulares defcribere, \& qux naturæ confervationi fint minus idonex. Ipro etenim actu, quo magis hæ leges differant a duplicata inverfa, \& accedant ad triplicatam inverfam, eo majores \& majores defcribent hæc corpora iftis viribus agitata angulos fupra ${ }_{3} 60^{\circ}$. circa centrum ab una apfide ad eandem, \& ab altera parte, quo magis recedant hæ leges a duplicata ad fimplicem inverfam, eo minores \& minores corpora hifce 
viribus convolventia angulos infra $360^{\circ}$ ab una apfide ad eandem conficient. Hac ratione hæorbitæ in infinitis punctis non tantum fefe decuftabunt ipfas, \& propterea planetx in illis convolventes admodum irregularibus afficientur motibus, verum etiam fi apfidum diftantix a Sole effent in majori quadam inxqualitatis ratione, ad eas a fe mutuo pervenirent diftantias, ut eorum muture turbationes poflent fieri maxime notabiles, \&. totum fyftema ultimo corruere poffet. Hæ vero irregularitates fient minores, \& minores, quo leges fint propiores duplicati inverfæ, illaque adhibita, pulcherrima illa fabrica mundi motufque planetarum regularis cum fui naturæque confervatione perfiftet, quam intenderat Sapiens rerum Opifex.

6.X. Supereft jam phœnomena ex illis legibus virium centripetarum dum $n=1$, vel minor, oriunda, \& quix prefentem materiam fpectant, paucis confiderare. $\mathrm{Si} n=\mathrm{I}$, preterea quod orbitx planetarum fecundum hanc legem admodum irregulares effent, etiam planetarum mutux turbationes a fe mutuo hac lege virium maxime notabiles, nature effent nimis noxix. Sic Jupiter Martem, \& Tellurem precipue turbaret, \& Luna noftra æquali fere vi Solem verfus ac ad Tellurem traheretur, ita ut illa e puncto conjunctionis exiens in linea paullo incurvata verfus centrum quod prepolleret abiret, donec velocitate ulterius progrediendi deficiente, eandem viam retroiret, \& quafi of cillaret Solem inter, \& Tellurem . liadem hæc incommoda verum majora, $\&$ naturx confervationi magis inimica orirentur, fi $n$ adhuc effet minor. Ut fi $n=0$, oporteret noftram Tel- 
lurem planetas, ipfumque Solem utpote ad omnes fixas ubique difpofitas attractas, \& feniel in motu conftitutas fere in lineis rectis per totum orbem terrarum peragrari. Et fi omnes reliqui Soles; \& planetæ reliquorum fyftematum in motu effent, i. hæc corpora quaquaverfum abfque ullo ordine traherentur a fe mutuo; utpote viribus centripetis magis tum proportionalibus. Nulla autem omnium legum dum $n$ minor unitate, rnaturx confervandx magis effet apta, fi tantummodo motus fpectaretur, quam fi effet $n=-I$, fi tantum unus effet planeta, unufque Sol. Præterea omnia qux non effent affixa huic planetx, \& huic Soli afcenderent a mutuis hifce corporibus, ufque dum pervenirent ad illum locum, ubi ad duo hæc corpora æqualiter traherentur, ibique hrerent .

९.XI. Dum jam defcripferam præeminentiam legis duplicatæ inverfe pre omnibus aliis ad naturam fuftentandam, fubvenit etiam paucis annotare, eamdem hanc legem etiam totius mundi machinæ confervationi haud minus accommodatam effe. In immenfis etenim illis diftantiis, ad quas a fe invicem pofita funt corpora fixarum, per hanc legem attractricem vires quibus hæc corpora fe mutuo turbarent, evanefcunt fere. Deficiente omnino parallaxi orbis annui illam neceffe eft effe minorem $1^{\prime}$. Affumta vero illa parallaxi fixx nobis proxi$m æ$, oportet illam cadendo verfus Solem noftrum, confumere annos circiter 44000. cafu fuo, defcribendo immenfx fux diftantix tantillam partem, que non fupe. raret radium orbitæ Telluris. Dum vero ad id attenditur, quod omnia liæc corpora undique in colis ad 
eafdem immenfas a fe invicem diftantias pofita fint, \& propterea quaquaverfum attrahantur fere æqualiter, fatis intelligitur ipfa in quiete permanfura fore. Quod fi ruina quxdam ex hac caufa effet derivanda, oportęret illas fixas qux juxta mundi limites pofitæ fint, primum e locis moveri fuis, \& fic fucceffive, ufque dum omnis materia creata ultimo in unam concideret molem. Tantum . 


\section{P A U L L I F R I S I I}

PRESB. REG. BARNABITAE

IN MEDIOLANENSI GYMNASIO PUBLICI

MATHESEOS PROFESSORIS.

DE PROBLEMATIS QUIBUSDAM GEOMETRICIS.

\section{(6) \\ P R O B L E M A I.}

Ex puncto quocumque $F$ peripheria circuli $F G$, ad diametrum pofitione datam $A G$, rectam $F C$ ita inclinare, ut que in. ipfius productione ufque al alterm peripberie partem - 1 intercipitur reita $C E$ Jit radio $B G$ requalis.

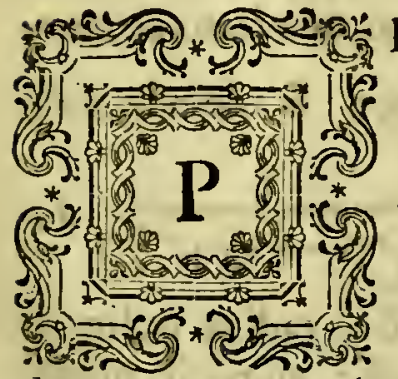

Roblematis hujufmodi, decem fere ab Tab.s. hinc annis, in Hetruria cum effem, Fig. 3. ab amico quodam propofiti, hanc illico confructionem me indicaffe memini . Centro $F$, \& radio $F B$ defribatur circulus alter $B I L$, proa c fuper $B C, B C^{\prime}, B C^{\prime \prime}$ tres radices æquationis, qux fubtenfam arcus $B H$ trifariam fecti exprimit . Puncta $C, C^{\prime}, C^{\prime \prime}$ fimul cum centro $B$ problemati fatisfacient . Ratio conftructionis patet. Cum enim fit $C F, C E \equiv$ $C F . B G=B G^{2}-B C^{2}$, fi ex $F$ in $A G$ ducatur perpendiculum $F D$, erit $C F=V\left(B G^{2}-2 B D . B C+B C^{2}\right)$ : unde quadratis, \& tranfpolitis terminis habebitur æquatio $B \cdot C^{4}-3 B G^{2} \cdot B C^{2}+2 B G^{2} \cdot B D \cdot B C=0$ :

$$
\mathrm{D}^{2} 2
$$

$\mathrm{PRO}$ 
28

\section{A $T$ T I \\ P R O B L E M A. II.}

Si bifectis femper lateribus quadrato oftogonum circumfcribatur;

\& octogono poligona alia $16,32,64$ laterum \&c, invenire radium poligoni unius cujufque, \& circuli in quem ultimo poligona

omnia definunt.

Tab.r. $\quad$ It $r$ radius $A B$ arcus $B D, \&$ cofinus $A C$ anguli $\circlearrowleft B A D$ vocetur $b$, ac fit propterea $B D=V\left(2 r^{2}-2 r b\right)$, $\frac{1}{2} B D=B E=V\left(\frac{1}{2} r^{2}-\frac{1}{2} r b\right), A E=V\left(\frac{1}{2} r^{2}+\frac{1}{2} r b\right)$. Erit is cofinus dimidii anguli $B A D$, eritque fecans $A F=$ $\frac{r^{2}}{V\left(\frac{1}{2} r^{2}+\frac{x}{2} r b\right)}$. Fiat jam $r=1, \& b=V \frac{x}{2}$, ut fit $A B$ radius circuli quadrato circumfripti, \& femilatus quadrati lit $A C$. Erit radius oftogoni quadrato circumfcripti $A F=\frac{1}{V\left(\frac{1}{2}+\frac{1}{2} V^{\frac{1}{2}}\right)}$. Quod $\mathrm{f}_{1}$ deinde fubfituatur $A B$, five I , loco $b, \& \frac{1}{V\left(\frac{1}{2}+\frac{1}{2} V^{\frac{1}{2}}\right)}$ loco $r$ fecans dimidii anguli $F A B$, five radius poligoni circumforipti fexdecim laterum reductis numeris prodibit $=\frac{1}{V\left(\frac{1}{2}+\frac{1}{2} V \frac{1}{2}\right) V\left(\frac{1}{2}+\frac{1}{2} V\left(\frac{1}{2}+\frac{1}{2} k \frac{1}{2}\right)\right)}$, cademque femper ratione inquiri poterit radius poligoni alterius cujufcumque bifectis fimiliter lateribus circumfcripti :

- Facilior autem evadet approximationis totius calculus fi prioribus, radicalibus decimales numeri fubfti- 
tuantur. Ita fi radius circuli quadrato circumfcriptiaccipiatur loco unitatis, calculo intra feptem priores cyphras inftituto, erit radius oftogoni circumfripti $\frac{\mathrm{I}}{0.9238795}$ fatis proxime, \& radius poligoni fexdecim laterum $\frac{1}{0.9061274}$. Ita etiam fcribendo $\frac{1}{0.9238795}$ loco $b, \& \frac{1}{0.9061274}$ loro $r$, ex priori formula eruetur radius poligoni $3^{2}$ laterum $\frac{1}{0.9017^{6+4}}$. Denique eadem femper fubftituendi ratione poligonorum aliorum latera erunt ordine

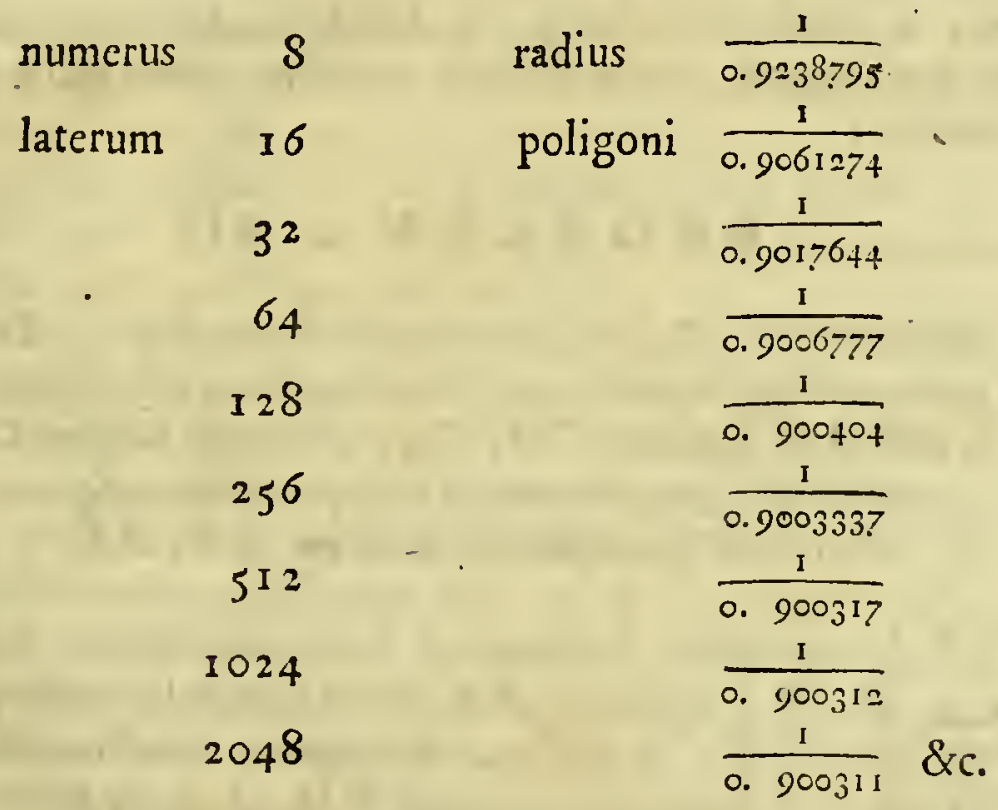

Ita igitur radius circuli quadrato circumfcripti ad radium circuli alterius, in quem definunt omnia poligona quam proxime fe habebit ut $g:$ ro. Elegans hujulnodi 
jufmodi problema binis fere ab hinc annis in Infubria propofitum cum diverfis rationibus folvi poflit, hanc aliis folutionem preponerem, \& quod poligonorum radios ultra quemcumque cyphrarum numerum facile exhibeat, \& quod generalem methodum involvat , qux fimili modo cafibus aliis numeri laterum in ratione qualibet crefcentis aptari poffit. Nuper elegans aliud problema a celeberrimo Klingenftierna refolutum D. Melander Upfalienfis Aftronomus mecum per litteras communicavit, folutionemque ipfam Geometricam exfcripfit fine demonftratione. Ut hac apud noftrates etiam innotefcant, referam primo qux Sueci Mathematici folutio, ac confructio fuerit, \& deinde analyfin exponam, ex qua finguli conftructionis ejufdem cafus facile colliguntur .

\section{P R O B L E M A III,}

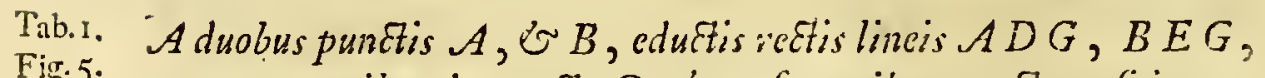
Fir.5. concurrentibus in puncto $G$, 心 auferentibus a recta pofitione data $C H$ Sermenta $C D, C E$, ad datum punetum $C$ terminata, que fint inter fe in data ratione: Qu,cvitur locus concurfus $G$ edustanim $A D, B E$.

Tab.r. C A fus primus. Jungatur $A B, \&$ producatur fi opus Fig.6. $\bigcirc$ eft, $\&$ fi infuper $A B$ fuerit parallela recte pofitione datx $C H$, in recta $A B$ fumatur punctum $F$, ut fit $F A$ ad $F B$ in data ratione $C D$ ad $E C$, utque fit idem ordo punctorum $F, A, B$, qui eft punctorum $C, D, E$. Per puncta $F, \& C$ ducatur, \& producatur recta $F C$, eritque hxc locus quxfitus. 
Cafus fecundus. Si $A B$, occurrat pofitione datæ $C H$ in puncto dato $C$, non vero fuerit $C A$ ad $C B$ Fir.: ut $C D$ ad $C E$, exiftente codem ordine punctorum $C, A, B$, qui eft punctorum $C, D, E$; fumatur in $A B$ punctum $F$, ut fit $F A$ ad $F B$ in ratione $C D$ ad $C E$, utque idem fit ordo punctorum $F, A, B$, qui eft punctorum $C$, $D, E$. Ad $C F, C A, \& C B$ fumatur quarta proportionalis $C N$, ita quidem, ut puncta $F, A, B, N$, cadant vel omnia ad eafdem partes puncti $C$, vel bina ad diverfas. Per punclum Nducatur NI parallela pefitione datæ $C H$, \& erit linea recta $N I$ locus quælitus. Cafus tertius. Si $A B$ occurrat ipfi $C H$ in pun- Tab.r. Eto $O$, quod diverfum fit a puncto $C$, fueritque $O A$ Fiv.s. ad $O B$ ut $C D$ ad $C E$, exiftente codem ordine punEtorum $O, A, B$, qui eft punctorum $C, D, F$; locus quæfitus puncti $G$ erit Parabola tranfiens per. tria puncta data $A, B, C, \&$ habens diametros fuas parallelas rectx pofitione datx $C H$.

Cafus quartus. S: $A B$ occurrat ipfi $C H$ in pun- Tab.1. Eto $O$, quod diverfum fit a puncto $C$, non vero fue- lis. 9 . rit $O{ }^{\prime} A$ ad $O B$ ut $C D$ ad $C E$, exiftente eodem crdine punctorum $C, D, E$; fumatur in recta $A B$ punctum $F$ ut fit $F A$ ad $F B$ in ratione $C D$ ad $C E$, ?tque idem ordo punctorum $F, A, B$, qui eft punctorum $C, D, E$. Ad $F O, F A, F B$, fumatur quarta proportionalis $F M, \&$ ad $O F, O A, O B$ quarta proportionalis $O N$, ita quidem ut puncta $O, A, B, M$, cadant vel omnia ad ealdem partes puncti $O$, vel bina ad diverfas. Per puncta $M, \& N$ ducantur rectæ linex, $M S$ parallela ipf $F C, \& N R$ parallela ipfi $C O$. Et afymptotis $M S, N R$ per punctum $C$ defcri- 
pta hyperbola cum ipfi oppolita erit locus quæfitus; qui ctiam tranfit per data puncta $A, \& B$.

Si $A B$ occurrat ipfi $C H$ in $C$, ut in fecundo cafu, \& fimul fuerit $C A: C B=C D: C E$, exiftente ordine ponctorun $C, A, B$, eodem qui eft ordo punctorum $C, D, E$, educti $A D, B E$, erunt parallelx, puncturn $S$ nullum, \& locus nullus. Similiter fi recta pofitione data $C H$ ponitur tranfire per alterutrum punctorum datorum $A$, vel $B$, problema nullum eft.

Hac ex Mílandri epiftola tranfcripfi. Ut indicem qua analyfi calus omnes exhiberi poflint, fit, in fig.5., $A B=a, B O=b, O C=c$, atque ex $G$ in $A B$ produCtam demiffo perpendiculo $G S$, fit $B S=x, S G=y$. Sit infuper $C E: C D=I: m, \&$ finus anguli $C O B$ vocetur $b$, cofinus $V\left(I-b^{2}\right)$. Quia angulus externus $O E G$ xqualis eft fumm $x$ duorum internorum $B, \& O$, finus anguli $O E G æ q u a l i s$ erit finui fummx angulorum $B$, $\& 0$, five erit fin. $E=\frac{b \cdot B G}{B G}+V\left(1-b^{2}\right) \cdot \frac{G S}{B G}$ : atque erit pariter fin. $D=b \cdot \frac{A S}{A G} \div V\left(1-b^{2}\right) \cdot \frac{G S}{A G}$. Unde fet $E O=\frac{\text { fin. } B}{\text { fin. } E} \cdot B O=\frac{C S \cdot B O}{b \cdot B G+V\left(1-b^{2}\right) \cdot G S}=\frac{b y}{b x+-V\left(1-b^{2}\right) \cdot y}$ $D O=\frac{\sin \cdot A}{\mathrm{fin} \cdot D} \cdot A O=\frac{G S \cdot A O}{\bar{b} \cdot A S+V\left(1-b^{2}\right) \cdot G S}=\frac{\overline{a+b} \cdot y}{b \cdot \overline{a+x}+V\left(1-b^{2}\right) \cdot y}$ Quare cum juxta conditionem problematis efle debeat $E O-c: D O-c=\mathrm{I}: m$, fubrtitutis fpeciebus, \& fractionum numeratoribus ad eamdem denominationem reductis habebitur xquatio $\overline{m-1} \cdot c \cdot\left(y^{2}+b^{2} \cdot \overline{a x+x^{2}-y^{2}}+\right.$ $\left.V\left(I-b^{2}\right) \cdot b \cdot \overline{a y+2 x y}\right)=n b b a y+\overline{b a-a-b}$. $\left(b x y+V\left(1-b^{2}\right) \cdot y^{2}\right)$. 
Primo igitur fi ex $C$ in $A B$ demiffo perpendiculo $C M$ fiat $b=\frac{C B I}{M O}, \&$ punctum $O$ longius abire intelligatur, ut binx $C O, M O$ fiant tandem rectx $B O$ aquales, reductis terminis, xquatio problematis evadet $\overline{n-1} \cdot \overline{x+a y}=a$, quæ eft xquatio ad lineam rectam. Quare clim, pofito $y=o$, fiat $B S=x=\frac{a}{m-1}, \&$; pofito $C E=0$, eadem linea tranfire debeat per punctum $C$, patet prioris Klingenftierne cafus confructio.

Deinde fi fuerit $O C=c=o$, erit xquatio problematis $m b b_{j} a+\overline{m b-a-b} \cdot\left(b x+V\left(\mathrm{I}-b^{2}\right) \cdot y\right) \stackrel{=}{=}$, atfue adhuc locus eiit ad lineam rectam. Pofita autem $y=0$, fiet $x \frac{m b a}{a+b \cdot \frac{m}{1-n}}$ : quod congruit cum fecundo cafu. Nam polita infuper in fig 7.CF+a+b:CF+ $b=m: I$, fiet $C F=\frac{a+b \cdot \overline{1-m}}{m-1}, \&$ pofita denique $C N=\frac{\overline{a+b} \cdot b}{C F}$, fiet $B N=C N+b=\frac{m b a}{a+b \cdot \overline{1-m}}$.

Præterea fi fuerit $O A: O B=C D_{1}: C E$, fig.8. five $m b=a+b$, erit rquatio $\overline{m-1} \cdot c \cdot\left(y^{2}+-b^{2}\right.$. $\left.\overline{a x+x^{2}-y^{2}}+V\left(\bar{x}-b^{2}\right) \cdot b \cdot \overline{a y+2 x y}\right)=m b b a y$, quam illico patebit effe ad parabolam fi ad hanc formanı reducatur $y^{2}+b^{2} \cdot \frac{1}{1+a x-2 y^{2}}+V\left(\begin{array}{ll}\text { I } & b^{2}\end{array}\right)$. b. $\overline{a y+2 x y}=\frac{m b b c y}{m-1}$; ea vero cum tranfire debeat per punĉum $C$, tranfibit ctiam per puncta, $A, \& B$, pofito enim $y^{\prime}=0$ fiet $a x+x^{2}=0$.

Quod fi denique non fuerit $m b=a+b$, erit prior xquatio ad hyperbolam, \& conftructionibus juxta HoE fpita- 
fpitalii regulas peractis manifefum erit in parabola quidem axem, \& diametros parallelas effe reetæ $C D$, atque hyperbolx afymptotos ea ratione determinari, quam Klingenftierna indicaverat .

Tab.r.

Methodus autem, qua in folutione hujus proble.

Fig.10.

matis uf fumus, aliis etiam quam plurimis folvendis facile inferviet. Ut $f_{1}$, dato quocumque angulo $D E C$, $\&$ puncto $A$, ducta $A E$ effe debeat $A L=F E+L E$, fiat perpendicularis $A B=a, B E=b, B L=x, L E=$ $b-x, \&$ finus anguli $D E C$ vocetur $b$. Cum finus anguli $F L E$ fit $=\frac{a}{V\left(a^{2}+x^{2}\right)}$, erit finus anguli $L F E=$ $\frac{a \cdot V\left(1-b^{2}\right)}{V\left(a^{2}+x^{2}\right)}+\frac{b x}{V\left(a^{2}+x^{2}\right)}$, \& cum angulorum finus proportionales fint oppofitis lateribus, fiet latus $F E=$ $\frac{a \cdot \bar{b}-x}{a V\left(1-b^{2}\right)+b x}$, atque, ob $A L=F E+L E$, fiet etiam $(b-x)\left(b x+a \cdot \overline{1+V\left(1-b^{2}\right)}\right)=\left(a V\left(1-b^{2}\right)+\right.$ $b x) V\left(a^{2}+x^{2}\right)$. Hoc pariter in Hetruria problema olim mihi propofitum fuiffe memini.

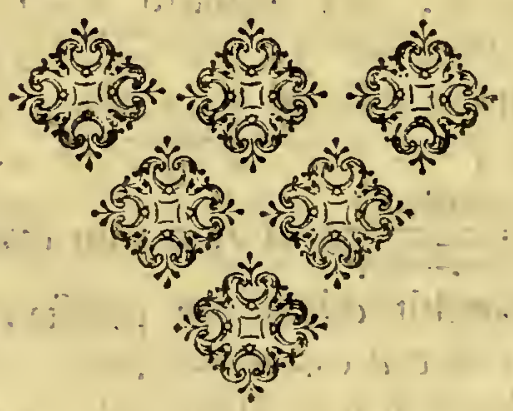

DELL' 
$D E L L A B A T E$

\section{LEONARDO XIMENES}

GE O GR A F O D I S. A. R.

\section{(6) \\ $\begin{array}{llllllllllll}R & \text { I } & F & L & E & S & S & I & O & N & \text { I }\end{array}$}

Intorno! all Obbliquitd doll Eclittica, e Jua Diminuzione "Secolare di $29^{\prime \prime}$ dedotta dalle offervazioni Solftiziali

fatte alla Meridiana Fiorentina negli anini $1755 \cdot 1756$.

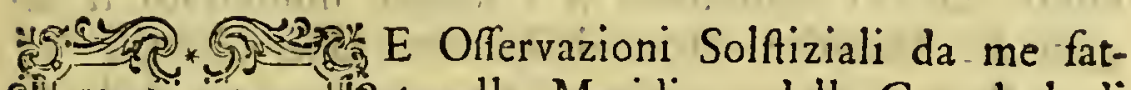
i. (5icengli anni 1755 , e 1756. ef* L 1.0 fendo ftate paragonate coll' antica ofI fervazione del I5:0. ben rapprefen-

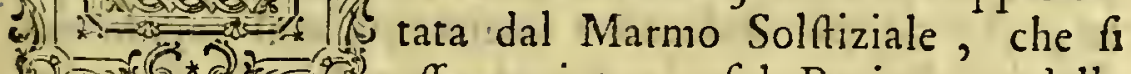

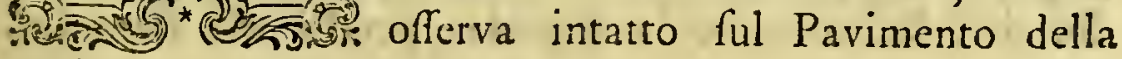
ftefla Cattedrale, portano in confeguenza un Periodo Secolare dell' Obbliquità dell' Eclittica molto minore di quello, che fu fuppofto dal Cavaliere di Loville, e di quello ancora che nel 1755. era accettato da molti Aftronomi. Secondo il primo, la Diminuzione Secolare era di un minuto, e fecondo gli altri, facevali di $47 \frac{1}{2}$. Queto fecondo Periodo fu promulgato dall' Eulero l' anE 2 no 
no 1754 . in una fua Memoria inferita negli Atti dell' Accadamia di Berlino, nella quale egli, colla Teoria dell' univerfal gravità, e con alcune Ipotefi, che premette, viene a dedurre la Diminuzione Secolare di $47 \frac{1}{2}$.

Gli Aftronomi, che ammettono l'I potefi della invariabilità dell' Eclittica, dal 1755 in quà hanno confultate le Offervazioni Solftiziali, che fi fanno alla Meridiana di S. Sulpizio di Parigi nella quale l'immagine Solare cade ful pavimento al foco d'una Objettiva di piedi 80 , che fu già collocata al Centro dello Gnomone l' anno 1745 . E da tale offervazione ripetuta fino all' anno 1767 , pare agli Offervatori, che l' angolo dell' Obbliquità media non fia fenfibilmente diminuito. Di tal fentimento fi $\dot{e}$ fempre dimottrato il Signor Le Monnier autore delle dette Offervazióni.

Pare adunque, che al Periodo Secolare dell' Obbliquità di 29 , fi oppongano le Offervazioni di S.Sul: pizio, e la Teoria dell' Eulero. Le prime, perchè pare, che da effe deducafi l'immobilità dell' angolo' medio dell' Eclittica, e la feconda, perchè effa fomminiftra un Periodo molto maggiore di $47^{\prime \prime} \stackrel{1}{2}$ :

Sarebbe facile a rifpondere alla prima dificclia colla brevità del tempo trafcorfo dal 1745. al 1767 , nella quale dovendo effer la variazione di circa 6 , non è maraviglia, che effa non $f_{1}$ manifefti ancora fenfibilmente. Ma non è così nell' Offervazione Solftiziale del 
Dell' Aceadeim:a.

del $175^{6}$, la quale effendo paragonata a quella del i sio; il corfo di anni 246 ; ha poturo render vifibile la diminuzione, come in fatti l' ha ben manifeftata di I. I" I"

Ma fi! puo rifpondere qualche cofa di più efaminando le dette Offervazioni di S. Sulpizio, riducendolé al momento Solftiziale, e liberandole dali' effetto della Nutazione...Poichè da tali Riduzioni viene a comprovarfi qualche vizio nella ftabilità dello Gnomone Parigino.

Fatté tali Riduzioni paffero poi alla Teoria, e dimoftrero l'incertezza di alcune Ijotefi, fulle quali fi appoggia il Rifultato dell Eulero. Non laferó ancora di produrre altre offervazionis apportate dopo il 1755 , le quali confrontano affai bere colla diminuzione di $29^{\prime \prime}$ per fecolo. In una parola procurero di mettere in chiaro; che nè la Teoria, nèle Offervazioni fi oppongono punto al mio Periodo Secolare di 29 , anzi che le combinazioni fatte da alcuni Aftronomi dopo il 1755. incominciano ad accordarfi collo fteffo Periodo.

\section{§. I.}

Riduzione, Rifeffioni, c confeguenze delle offerzazioni Solftiziali fatte a S. Sulpizio di Parigi.

T Ell anno i 767. della Reale Accademia delle Scien1 ze alla pagina 4 i 7 . fi legge una memoria del Signor Le Monnier intorno, al Soltizio eftivo offervato al foco di un Objettivo di 30 . piedi, nella Chiefa di S. Sul- 
S. Sulpizio di Parigi. Il precifo dell' Oftervazione !è riportato dal dottiffimo Aftronomo colle feguenti parole.

" $\mathrm{Nel}^{1} 767$. il dì 22 di Giugno l'orlö dell Im"magine Solare radeva dalla parte Auftrale la linea fcol-

" pita nel 1745 per la fua parte inferiore, ed avendo 3. leggernente punteggiato colla Matita la traccia del

"detto Orlo, la linea, che congiungeva i due punti mi

" è fembrata diftante dal mezzo del Segno fcolpito m) di $0^{\prime \prime} \frac{\mathrm{I}}{3}$, oppure di ${ }^{\prime \prime} \frac{\mathrm{I}}{4}$ di linea, il che corrifponde " a $5 \frac{1}{3}$, ovvero a $4 \frac{1}{8}$

"Dalla parte Boreale I' altr' Orlo non arrivava fi"no al fegnale fcolpito, e mancava una mezza linea, 9. Ovvero $8 \frac{1}{11}$ lino al mezzo della traccia del 17.45 .

\% Io piglio fempre il nuezzo della tráccia fcolpita. .2 Poichè la groffezza può variare, e vi è luogo di cre"dere, che effa dal 1745. in quà fiafi alquanto indebo" lita a motivo, che il Marmo bianco è fato qualche "volta confricato con una fpugna, per ifcanceliare le "linee della Matita.

"Il Signor di Vaucal, che fi è trovato prefente all'Olfervazione del dì 22 Giugno, ne ha fatta un'al-

" tra il dì $2 \sigma$, della quale egli renderà conto infieme "con quelle del Solitizio hiemale, in una memoria par"ticolare . Egli avea pure offervato il di r g. la trac"cia del Sole ful Marmo, ed io ho concorfo con $\mathrm{A}^{\prime}$ "fo lui a rilevare le diftanze di quefte linee dalla trac" cia principale del 1745 . 
"Il luogo Solare ci viene ad indicare, che nel „ dì 22. Giugno il Centro Solare era al di fotto del "Tropico di 6", laddove effo è frato, trovato a 6 " $\frac{1}{2}$ " in 7 'per l' Offervazione. Così l' ecceffio è di $\frac{3}{4}$ di fei" condo, il quale unito alla mezza groffezza delia trac"cia nera fegnata ful Marmo, fon gis trent' anni, rap"prefenta affai efattamente l' effetto della Nutazione, "O la quantita, di cui l' Eclitrica $f_{1}$ è accoftata al "piano dell' Equatore dopo l'offervazione fatta nel 1764 .

Tali fon le paro!e tradotte fedelniente nel volgare Italiano, colle quali vien rapprefentata l'Offervazione del di 22 Giugno 176.7 .

Or fe tale Offervazione voglia paragonarfi folamente con quella del I 764 - come fa M. le Monnier; a me pare, che la brevità del tempo di foli anni 3 , la qualità dell' Offervazione, che non puó effer certa dentro 4 , e finalmente gli effetti compofti della Nutazione dell' Affe terreftre, e del Periodo dell' Obbliquità, ci tolgano ogni fperanza di un ficuro rifultato, o quefto fra in favore della Diminuzione dell' Eclittica, o fia contro la nedefima.

E cio tanto piu vale, quantochè la riduzione di 6 all' offervazione del 1767 . per ridurla al vero monento folftiziale, non è efattifima. L'ingreffo del Centro Solare nel tropico Eftivo il detto anno 1767 . accadde ore 6.28 dopo il mezzogiono del di 21 . Onde dal mezzo giorno del dì 22 . vi corrono ore $17 \cdot 3^{2}$; alla qual 
qual diftanza Oraria, fecondo la tavola inferita nel mio Gnomone, vi corrono preffo a 7 . Quefto divario di I", benchè paja aflai tenue, con tutto ciò effo paragonato alle differenze, che pofton nácere nello ifpazio di foli anni 3 , o fia per la mutazione del nodo afcendente Lunare, o fia per il Periodo dell' Obbliquità, 'effo non è infenfibile.

$\mathrm{Ma} f e$ in vece di paragonare detta offervazione del di 22 Giugno 1767 . con quella del 1764 , voglia paragonarfi con quella del i 745 , che'è la fondanentale per la Mericiana di S. Supizio, potrebbe effere che il rifulato foffe fenfibile, e fervifie alla famofa queftione intorno all' Obbliquità dell' Eclittica.

Ma per fare utilmente un tal paragone, conviene introdurre nelle due Offervazioni le Riduzioni, che competono alle medefime; che nel calo nofro fon due. La prima è relativa alla diftanza del Momento Solftiziale dal Mezzogiono dell' offervazione. La feconda fi deve al movimento della Nutazione terreitre, che dipende dalla pofizione del Nodo afcendente Lunare.

Incominciando dall' ORerrazione del " 1745 , in er fa è ftato calcolato, che il Centro Solare nel Mezzogiorno del di 23 . Giugno fi era ritirato di so fotto il Tropico; e le due tracce Solftiziali fegnate di nero fono thate collocate ne veri termini Solftiziali", come 3 annunzia lo fteffo Autore nella fua Memoria del 1762 . inferita alla pagina 263 . Onde quelta prima riduzione è fata inclufa in detti termini Solftiziali.

Refta 
Refta adunque in effa l'Equazione della Ntwazione dell' Affe terreftre, per ridurla alla media Obbliquità dell' Eclittica.

Il nodo afcendente Lunare era a $0^{5} \cdot 26^{\circ} \cdot 9^{?}$. Onde il feno del fuo complemento farà di 897 . E così facendo, come il feno totale al feno $S_{97}$, cosi 9 , al quarto termine, quefto tornerà di $8^{11}$. o 7 cent. per 12 Nutazione, che è additiva all' Obbliquità media. Ma nel noftro cafo effendo l' Offervazione affetta di tal $\mathrm{Nu}$ tazione, per ridurla alla media Obbliquità, converrà fottrarla all' Offervazione.

Sia per tanto per chiarezza maggiore il centro del- Tab.s. la Meridiana di S. Sulpizio ful punto $S$. Sia la Sezione Fig. I. del Cono Solare luminofo $S T A$. Sia $T A \mathcal{A}$ 'Afle maggiore dell' Elliffi Solare nel momento dell' Offervazione. Onde ful punto $T$ caderà l'Orlo Solare Boreale del 1745 , e la linea $T T$ rapprefentera la traccia fegnata in nero, la quale è ftata ridotta dall' Autore al vero Momento Solftiziale. Partendofi dal punto $T$ verfo Oftro, fi pigli la linea $T G$ di $8^{\prime \prime} .07$ centef. ed il punto $G$; - la linea $G G$ rapprefentera l' Offervazione del I745. ridotta all Obbliquità Media, effendo già fpogliata dall' effetto della Nutazione terreftre.

Volendo adunque paragonare alla medefima l'altra Oftervazione del $17^{6} 7$, in ordine all' Orlo Solare Boreale, fi dovrà dal punto $T$ pigliare la $T H$ di 8 . 25 centefime, giacchè di tanto fi allontanava l'Orlo Boreale dal termine Solfiziale Boreale del i745. feconF do 
do l' Autore. Sarà pertanto la linea $H H$ la traccia immediata del 1767.

Effendo ftato il momento Solftiziale lontano dal Mezzodì del 22. Giugno di ore 17. 42. l'Equazione farà di ${ }^{7}$, fecondo la mia tavola. Onde pigliando la linea $H F$ verfo Settentrione di 7 , la linea $F F$ rapprefenterà l' Offervazione del 1767 . ridotta al Momento Solftiziale. Togliendo da 8." 25 . la detta Riduzione di ${ }^{\prime \prime}$, refta la linea $F T$ di $" 1$ I. 25 centefime.

Per il nodo lunare, che in detto giorno trovavafi

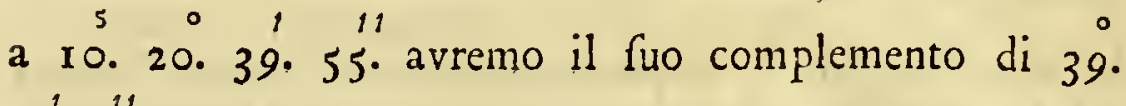
20. ${ }^{\prime \prime}$, il cui feno farà di 633 . Onde colla folita Analogia, farà la Nutazione di 5.69 centefime, fottrattiva nell' Offervazione. Si pigli adunque la linea $F M$ di $5^{11}$ 69 , e ne verrà la linea $M M$ dell' Offervazione del i 767. totalmente ridotta, la qual rapprefenta l'Obbliquità media, che nafcerebbe dall' Offervazione,

Sarà pertanto la linea $T M$ di ${ }^{11}$. 94 . E parágonandola alla linea $T G$, che è di 8. $^{11}$, come è ftato detto, ne rifulterà la linea $G M$ di "I. I 3 centefime, di cui l' Angolo dell' Eclittica farebbe crefciuto dal 1745 . al 1767 .

Una confeguenza fomigliante molto più nafcerd, fe tutta l' Offervazione adatiata all' Orlo Solare Boreale 
Dell' Accademia.

le fia riputata full' Orlo Auftrale $A$. Facciafi adunque l' $A B$ di 8.07 ; e farà la linea $B B$ l' Offervazione del 1745. ridotta per la Nutazione.

Pigliando ura mifura media tra $5{ }^{\prime \prime} \frac{\mathrm{I}}{3}, e_{4} \frac{1}{8}$, avremo 4.73 centefime. Onde $f_{1}$ pigli la linea $A C$ di $4 \cdot 73$ centefime, e la linea $C C$ rapprefenterà l'immediata, e nuda Offervazione del i 767 .

Dal punto $C$ fi alzi la linea $C E$ di $7^{\prime \prime}$, e farà la linea $E E$ per l' Offervazione ridotta al Momento Solftiziale. Effendo la $C A$ di 4.73 , e la $C E$ di 7 , avremo il punto $E$ fuperiore al punto $A$ di 2.27 centefime.

Ora fi abbaffi la linea $E D$ per la Nutazione di " 5.69 , e farà la $D D$ ridotta per la Nutazione. Sottraendo ${ }_{2.27}^{27}$ da 5.69 , refterà la linea $A C$ di $3 \cdot 42$ centefime. Ma la linea $A B$ è di ${ }^{11}$. 07 . Onde il termine Solftiziale $D D$ del 1767 . ridotto totalmente, refterà fuperiore di ${ }^{41} .65$. in confronto dell' altro termine Solftiziale del 1745. fimilmente ridotto. Donde fi dedurrebbe, che in anni 22 . l'obbliquità media dell' Eclittica in vece di femare, come porta la più comune Ipotefi, fia piuttofto crefciuta di 4.65 .

Io non credo, che tal confeguenza $f_{1}$ accordi punto con tante Offervazioni Aftronomiche, e che non piaccia neppure all Autore delle Offervazioni della MeF 2 
ridiana di S. Sulpizio. Poichè egli in molte fue opere foftiene ora l' infenfibilitì della Diminuzione, ora la coftanza, ed invariabilità dell' Eclittica, ma non mi pare, che in alcun fuo paffo egli deduca alcuno incremento dell' Angolo dell' Obbliquità .

Ma non folo la prefente Offervazione riferita a due Orli Solari, ma quella ancora del $\mathrm{I}_{7} 6_{3}$. ripoitata nel tomo dell' Accademia del 1762. accenna un incremento d' Obbliquità .

$\mathrm{Dal}$ I 745. al 1763 . vi corre un intero Periodo del Nodo Lunare. Onde l' Equazione della Nutazione non può turbare il rifultato. Ora nel detto anno $1_{7} \sigma_{3}$. il dì 22. Giugno da' Signori Fauchy, e Tuillier fu- offervato l' Orlo Auftrale lontano dal termine Analogo Solftiziale del 1745 . di linee ${ }^{\prime} \frac{1}{4}$. E ficcome ogni linea nella Meridiana di S. Sulpizio porta $16^{11} \frac{\mathrm{I}}{2}$. così un quarto di linea porterà 4. I 2 centefime.

II Momento Solftiziale del detto anno 1753 . cad. de dopo il Mezzogiorno del di 2 r. $7 \cdot 27$. Onde la fua diftanza dal Mezzogiono del dì 22. fi trova a I 6.33 , e perciò la fua riduzione porta fecondo la mia tavola 6.1111 . O fia 6. 25 centefime. Da cui fottraendo 4. I a centefime, refteri l'Orlo Solare Auftrale piu Boreale di 2 . I 3 centefime, che non era nel I 745 , cioè l'Angolo dell' Obbliquità farebbe crefciuto in anni 22. di ${ }^{11}$. I 3 centenne. La fieffa affurda illazione fi ri- 
fi ricaverebbe da altre Offervazioni riferite l'anno I 762 .

Ma le due gia calcolate par che poffan fervire per dubitare della perfetta ftabilità dello Gnomone di S. Sulpizio. Il Signor de la Lande prudentemente attribuifce l'e:rore a qualche piccolo sbaffamento della Muraglia, fulla qual ripofa il Centro della Meridiana. E benchè tal Muraglia fia fondata ful Maffo, come lo afferifce il Sig. le Monnier, e fia ftata fabbricata circa 20: anni prima della Meridiana, con tutto ciò, non è inpoffibile un fedimento di una mezza linea in una groffa Muraglia, ancora venti anni dopo la fua coltruzione .

Converrebbe inoltre offervare, ed efaminare fulluogo fe la Muraglia fia fufcettibile di qualçhe piccola inclinazione Boreale. Poichè le groffe Muraglie per qualunque piccola inugualità di fondamento, o fia nella qualità del fuolo, o fia per la natura del Muramento, durano a piegar lentifimamente più di un Secolo, non che anni 20.

Va ancora efaminata l' incaffatura dell' Objettiva al Centro della Meridiana. Poichè fe efsa è incafsata o con glutine, o con materia morbida, e pieghevole, vi vuol pochifimo, che il calore de' Raggi eftivi Solari vi produca un cedimento verticale. Qui fi tratta di $\frac{1}{3}$ di linea. Con tal moto verticale il tutto fi fpiega. Poichè fecondo le mie Ofservazioni Aftronomiche fatte a quefto Gnomone Fiorentino, e Atampate in un volume nel $175 \%$, il periodo della Diminuzione dell' Eclitica fard di 29 . in un fecolo. Rifpetto a tal periodo 
riodo, il movimento in anni 22 . farebbe di 5 . in 6 . $\mathrm{E}$ precifamente $\frac{1}{3}$ di linea alla Meridiana di S. Sulpizio porta 5 . in 6 .

Quantunque io fia perfuafifimo dell' efattezza, colla quale il dotto Autore, ed i Signori della Reale Accademia procedono nelle loro Ofservazioni, con tutto ciò io non fo diflimulare un fofpetto, che mi nafce ful metodo di ofservare, e fifsare gli Orli Solari. Per quanto fi dice nelle Memorie, un tal Metodo confifte nel tracciare ful Marmo la linea, che trafcorre l' Orlo Solare, e quefta traccia fi fegna con Matita. Nelle mie OSservazioni Solftiziali in quefta Cattedrale, ful principio fu da me tentato un tal Metodo, ma mi riufciva afsai ambiguo. Primieramente perchè qualche volta in quel momento importante, la Matita forma una linea un poco troppo grofsa. Secondariamente perchè nel Difco Solare in certe giornate fi ofserva una certa of cillazione, che nafce dalla variabilità delle Refrazioni. E finalmente percliè effendo il Marmo di una materia alquanto diafana, l' Orlo Solare vi produce una penombra difficiliffima a riconofcere, perchè rifplende agli occhi non folo la fuperficie, ma ancora una piccola profonditiz del Marmo.

Per tali ragioni io ho tenuto, e tengo un metodo molto diverfo, che confifte nel diftendere, e fpianare con piccoli pefi un foglio di carta bianchiflima, che cuopre la Meridiana, avvertendo però che una linea nera fegnata nel foglio coincida colla ftefsa Meridiana. Così gli Oili Solari fono afsai piu terminati fenza paragone. $\mathrm{Pei}$ 
Per tener dietro agli Orli Solari, ho fatti fabbricare due Archetti finiflimi di Acciajo, che foltengono due fili di fotrilifimo Argento. Dalla metà della corda, parte un altro filo perpendicolare, che fi pofa fulla Meridiana.

Nell accoftarfi l' Immagine Solare, $\mathrm{i}$ detti due fili Auftrale, e Boreale fi fanno radere l'Orlo; e fi vede in 4,05 , fe realmente lo radono per correggerli.

Per trafportare l' ofservazione dalla carta alla fottopofta Meridiana di Metallo, fi piglia con una punta d'Acciajo il punto della carta, dove la Meridiana taglia la Corda dell' Archetto, e tenendola fermiflima in una polizione verticale, fi ftraccia la carta; affinchè colle Sefte pofsa pigliarfi la diftanza della piu proffima divifione dalla punta d'Acciajo, che fi tiene immobile fino alla fine della mifura, che fi può replicare più volte.

Dacchè io ho tenuto un tal metodo, che lungamente non ho potuto defcrivere nel mio Volume, ho trovata nelle mie Ofservazioni Solftiziali una regolarità molto maggiore . Negli anni 17.73. in 74. fi vèdrà dopo un Periodo del Nodo Lunare, fe le Ofservazioni ci fomminiftreranno un fenfibile decremento di circa 5 , quanti convengono al Periodo di 29. per fecolo, fecondo le Ofservazioni del 1755 , e 1756 , paragonate con quella del i 5 Io. efprefsa nel Marmo Solitiziale.

Intanto pero non pare, che a tal fiftema polsano opporfi le Ofservazioni Solftiziali di S. Sulpizio, nella

cui 
cui Meridiana può fópettarfi fondatamente di qualche vizio .

$$
\text { §. I I. }
$$

Delle altre offervazioni efaminate dopo l'anno 1755.

Ltre Ofservazioni combinate dopo il I755. par 11 che fi accoftino più alla diminuzione di 29, clie a quella di $47^{\prime \prime}$ per Secolo.'

Talc è per efempio l' Orservazione comparativa fatta allo ftefso Quadrante Piccardiano nel 1677 . dallo ftefto Picard, e poi nel I739. dal Sig. Le Monnier. La prima ci fomminiftra l'Ofservazione del Picard, ridotta per la Nutazione, dell' altezza Solare Solftiziale all' Ofservatorio Parigino l'anno 1677 . di

64.55 .12 .0$.

Allo Atefso Ofservatorio l'anno 1739. $\mathrm{di}$

$$
64 \cdot 55 \cdot \text { 2. } 5 .
$$

Differenza in anni 62. di

I 6.5 .

Onde in un Secolo farà di circa $25^{\prime \prime}$, che fi fcofta di foli 4 dal mio Periodo.

L'anno I757.' nel Tomo della Reale Accademia delle Scienze, leggefi una Memoria del Signor Gentil full' Obbliquità dell' Eclittica alla pag. I 8o. In efsa l' Autore riporta due Calcoli in favore della Diminuzione. $\mathrm{Nel}$ 
Nel primo egli confronta la fua Offervazione del $1753^{\circ}$ con quella del Valtero l'anno 1487.

L'Obbliquità del . $17.53 \cdot$ totalmen-

te ridotta è di...... 23. 28. I $6^{\prime \prime} .111$ Quella del 1487 . torna di ...23. 29.37.00.

Diminuzione in anni $266 . . . \quad$ I. 20.211 .

Ciò che fomminiftra la diminuzione di un Secolo di 30.02 centefime. Quefta è alquanto minore di quella dedotta dal Signor Gentil, perche pigliando la differenza delle due Obbliquità, effa torna non già di I. 2 I. 2 I. ma bensì di ${ }^{\prime \prime}$ I. 20 . 2 I.

Nel fecondo Calcolo egli paragona l' Obbliquita dell' Eclittica offervata a Lione dal Sig. Mouton I' anno 1662 , con quella del 1753 .

La prima da lui ridotta efprime.

$$
\text { l' Obbliquitá di.......23. } 28^{\prime} .43^{\prime \prime}
$$

La feconda di ...23. $28^{\prime}$ " 11 " 111

La differenza, o la diminuzione in anni $9 \mathrm{I}$. farà di... $26^{11} .211$.

Onde in un Secolo porterà la diminuzione di 28.94 centefime, cioè 29. affai proffmamente, come nol rifultato di tutte le mie Offervazioni allo Gnomone di quefta Catedrale.

Onde per le due combinazioni fatte dal Sig. Gentil nel detto anno 1757 , come nella citata memoria, vien confermato il mio Periodo di 29 . 
Non difcorda gran fatto dallo fteffo rifultato quello che vien regiftrato nella tavola del Signor Gentil alla pag.189. nella quale con più combinazioni di antiche colle moderne Offervazioni, egli ritrova la media diminuzione fecolare di 34.9 . 9 .

Nella detta Tavola quattro Periodi fi trovano, che pochiffimo difcordano dal mio rifultato, e fono

$\begin{array}{ll}\text { Per la Combinazione I. } & 30.111 \\ \text { Per la II. } & 28.57 \\ \text { Per la IV. } & 32.34 \\ \text { Per la IX. } & 30.51 .\end{array}$

Pare adunque; che per le combinazioni aftronomiche combinate dopo il 5755 , nel qual anno fu da me ritrovato il Periodo Secolare di 29 , ovvero di $3^{\circ}$. colle Offervazioni fatte allo Gnomone di quefta Cattedrale, gli Aftronomi abbiano confermato un tal Periodo. Ma le Offervazioni da effi confrontate non pare che abbiano quel grado di certezza, quale rifulta dalla Grandezza, dall' Autenticità, dall' Identità, e da tutte le altre circoftanze dello Gnomone Fiorentino, nel quale alle moderne Ofiervazioni è ftata paragonata quella del 1510 . rapprefentata così efattamente dal Marmo Solftiziale, e dalla fua Ifcrizione, come potrà vederfi nel Volume ftampato fu tale argomento l'anno 1757 .

६. III. 


\section{§. III.}

Qual fia la certezza della Tcoria dell' Eulero.

A Nteriormente alle mie Offervazioni Solftiziali del 1755 . io non ho trovato alcuno Scrittore, il quale abbia tenuto, e fiffato il Periodo di un mezzo minuto per Secolo, deducendolo da Offervazioni Aftronomiche verificate con buon criterio. Ma alcuni fi erano perfuafi della totale immobilità dell' Eclittica, falvo il movimento della Nutazione, altri l' hanno creduta variabile di una quantità affatto infenfibile, 'e tale opi-nione efclude il periodo di un mezzo minuto, che non può mai dirfi infenfibile particolarmente nella precifione della moderna Aftronomì. Ed altri affaiffimi vi fono ftati, e vi fono, che hanno adottato dopo la Teoria dell' Eulero, la Diminuzione Secolare di 47 . Tale Ipotefi è ftata abbracciata dal Signor Abate la Caille nella Sua Memoria del 1757. inferita in detto anno nel Volume dell' Accademia delle Scienze, nel quale però alla pag. I09.' fa menzione delle mie Offervazioni a lui comunicate nelle lettere di corrifpondenza fino dall' anno $1755^{\circ}$ La fteffa Ipotefi c̀ prefentemente adottata dal Signor de La Lande, e da altri Aftronomi, che tuitti feguitano la Teoria dell' Eulero.

Ia. Teoria in queftione fu pubblicata dall' Eulero l' anno 1754. negli Atti dell' Accademia di Berlino.

Le Ipotefi, fulle quali effa fi appoggia non fono di quella certezza, quale farebbe indifpenfabile, per in-

$$
\mathrm{G} 2
$$


trodure nell Aftronomia un Elemento tanto importante, qual' c̀ quello del Periodo Secolare di $47^{11} \frac{1}{2}$.

Egli fuppone la Parallaffi Solare di 10., fecondo l'Ipotefi comunc degli Aftronomi di quel tempo. Prefentemente effa è ftata trovata di $8^{\prime \prime} \frac{3}{10}$ per l' ultimo Paffaggio di Venere, e tal nifura è certa dentro $\frac{1}{4}$ di fecondo.

Il Volume di Vencre fi faceva allora dagli Aftronomi quafi uguale al terreftre, e benchè I' Eulero non $f_{1}$ fpieghi in quefta parte, pure egli non poteva non feguire l' Ipotefi comunemente allor ricevuta. Prefentemente il Volume di quefto Pianeta è diminuito proffimamente della fua quarta parte. Il Sig: de La Lande dopo il paffaggio di Venere del 176 r. ha calcolata la proporzione del Volume terreftre al Volume di Venere, come il 100: 77. Veggafi la fua memoria inferita negli Atti dell' Accademia nel 1762. Siccome 1 'Eulero attribuifce all' Azione di Venere $3^{21}$, a buon conto quelti diminuifcono di $\delta^{i}$, e perciò la fomma non farà più di $47 \frac{1}{2}$, ma bensì di $39 \frac{1}{2}$.

Finalmente confeffa lo feffo Eulero, che le forze affolute di Venere ci fono fconofciute (a) : Quefto Pianeta è privo:di Satelliti, che fono unicamente quelli, che.

(a) Alla pag.316. dell' Accademia di Berlino anno 1754 . 
che ci fanno conofcere la gravità del Pianeta. Così la gravità della Terra, di Giove, e di Saturno ci fón note per il movimento, e diftanza delle refpettive Lune. Ma quella di Mercurio di Veneie, c di Marte, ci fono perfettamente of cure per mancanza di Satelliti:

In tale ofcurita doveva l' Eulero affumere qualche Ipotefi: Egli lia adottáta quella della denfità in ragion reciproca fidduplicata de' tempi Periodici di ciafcun' $\mathrm{P} \cdot \mathrm{ia}$ neta. In favore di tale Ipotefi non vi è altro argomento fe ron quello, che paragonando infieme le gravita della Terra, di Giove; e.di Saturno colle loro refpettive diftanze dal Centro Solare, la proporzione delle denfità torna "all' incirca fecondo tale Ipotefi: Ho detto all' incivia, perchè tal proporzione non è efatta, e per confeguenza dinoftra baftevolmente la fua incertezza :

Se la denfità di. Venere fi diminuiffe di una fola quarta parte di quella affunta dáll' Eulero, : i fuoi effetti in rapporto all' Obbliquità dell' Eclittica diminuirebbero di $\frac{1}{4}$, e perciò la Revoluzione Secolare fi ridurrebbe a foli $33^{\prime \prime}$; giacchè combinando infieme la Di. minuzione del Volume con quella della denfità, l' Azione di Venere femerebbe nella ragion duplicata dell' $x$. al $\frac{3}{4}$, cioè nella ragione di I $6: 9$. Cosi la forza rapprefentata dall' Eulero di $3{ }^{21}$. ridurraffi a $18^{\prime \prime}$, e fatti tutti i calcoli per Giove, la Diminuzione compofta delle fue Azioni, che era di $47^{11}$ fi ridurrà a foli $33^{11}$ Quefto Periodo cosi ridotto per la Teoria con adoperare Ipotefi ugualmente verifimili, che quelle adotta: 
te dall' Eulero; mette una tale affinità tra le immedia= te Offervazioni, e la Fifica Aftronomica, che la differenza di circa ${ }^{21}$ potrà attribuirfi, ugualmente agli errori, inevitabili delle Offervazioni, ovvero all' incertezza delle Ipotefi di tutto il Calcolo Euleriano.

Pare adunque da quanto è ftato detto in quefta Memoria fulle Offervazioni di S. Sulpizio, fulla Teoria Aftronomica, e fulle Offervazioni, o fatte, o combinate dopo il 1755 , che il Periodo dell' Obbliquità di circa un mezzo minuto per Secolo da me dedotto col paragone delle Offervazioni Solftiziali del I 755 , e $175^{6}$. con quella del I 5 ro. fia il più accettabile, finchè la maggior certezza delle I potefi Fifiche dell' Aftronomia, o delle Offervazioni immediate continuate in qualche Secolo, di più poffa coftringere i noftri Pofteri a correggere un tal Periodo, o con aumentarlo, e con diminuirlo fecondo l' efigenza de' Principj, e delle Offervazioni future.

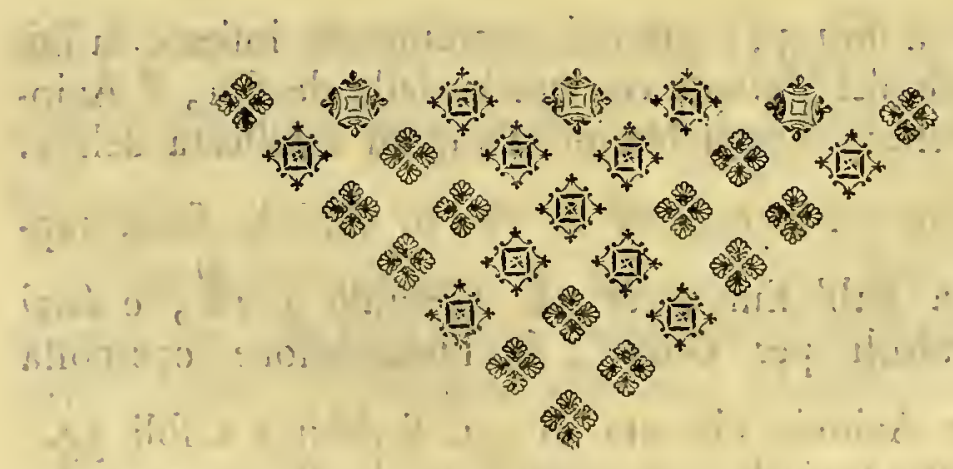

GRE- 


\section{GREGORII FONTAN无 ORD. SCHOL. PIAR.}

IN REGIO TICINENSI ARCHIGYMNASIO PUBLICI MATHESEOS PROFESSERIS

\section{S C HE D I A S M A I.}

PROBLEMATA A S T RONOM ICA

\section{(Gus \\ P R O B L E M A I:}

Data Sideris Declinatione invenive ipfius Jupra Horizontem Altitudinem, in qua fub data Poli elevatione Altitudinis ejufdem Variatio contingit onmium maxima.:'

$$
S \circ \mathrm{t} \text {. }
$$

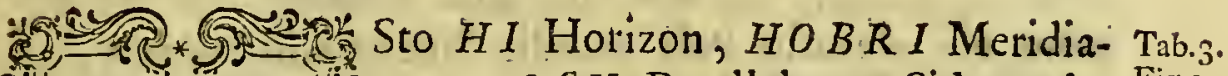
(2) $B$, Polus in $R, \&$ captis $S$, $s$ fum-
fcriptus; fitque Sidus in $S$, Zenit in
me propinquis, ac per $S p$ abfiffo Cætera porro fiant ut in Schemate. Sit jam $R S$ complementum datæ Sideris Declinationis $=a$; complementum elevationis Poli $R B=c$; complementum $B S$ Altitudinis inveniendr $=x$, cujus variatio $p s$, feu $d x$ differentiata, ac nihilo xquata, quxfitam fuppeditabit Sideris Altitudinem. Jam vero fi accipiatur $S_{s}=\mathrm{r}$, fiet $p s$, feu $d x=$ col. $S s p=$ cof. $O S B=$ (ob rectum angulum $O S R$ ) fin. $B S R$. Ex notis autem Trigonometrix 
metrix Spherica formulis, in triangulo $S B R$ habetur cof. $B S R=\frac{\operatorname{cof} c-\operatorname{cof} a \cdot \operatorname{cof} x}{}$, atque inde colligitur fin.

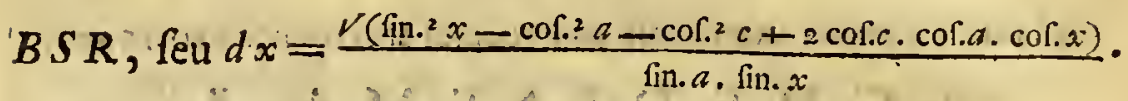
Facta differentiatione, pofitoque $d d x=0$; poft debitas reductiones obtinetur quadratica xquatio $\operatorname{cof}_{0}^{2} x-$

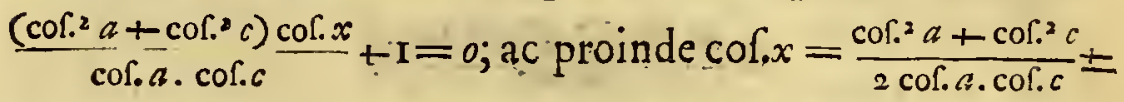
$V\left(\left(\frac{\operatorname{cof}^{2} a+\operatorname{cof}^{2} c}{2 \operatorname{cof} a \cdot \operatorname{cof} c c}\right)^{2}-1\right)=\frac{\operatorname{cof}^{2} a+\operatorname{cof}^{2} c+\left(\operatorname{cof} .^{2} a-\operatorname{cof} \cdot{ }^{2} c\right)}{2 \operatorname{cof} a \cdot \operatorname{cof} \cdot c}=$ vel $\frac{\operatorname{cof} a}{\operatorname{cof} . c}$, vel $\frac{\operatorname{cof} . c}{\operatorname{cof} . a} \cdot Q \cdot E$. $S$.

Schoz. Ex ipfa rei natura manifeftum eft ex binis cof. $x$ valoribus $\frac{\operatorname{cof.a}}{\operatorname{cof} . c}, \& \frac{\operatorname{cof} . c}{\operatorname{cof} . a}$ ufurpandum effe primum ubi elevatio Poli excedit Sideris Altitudinem, alterum ubi illa ab hac fuperatur.

\section{$E X E{ }^{\prime} M L^{\prime} U M$ 。}

Declinatio Syrii eft $=16.24$; Mediolani Latitụdo $=45^{\circ} \cdot 28^{\prime}$.

Itaque $+l$ fin. tot. $\ldots \ldots \ldots .=10.0000000$ $+l$ cof. $q=l$ fin. Decl.

$=$ l. fin. $1 \stackrel{0}{6} .24 \ldots .=9.4507747$

$-l$ cor. $c=l$ fin. $45.28^{l}=9.852993^{6}$

$l \operatorname{cof} x=l$ fin. Altit. $=9.59778 \mathrm{II}$ 
Igitur Altitudo Syrii quxfita $=23 \cdot 20^{\circ}$, ad quam nimirum ubi Syrius pervenit Maximam patitur Variationem.

$$
\text { P R O B L E M A II. }
$$

-Iifdem ut antea manentibus, invenive tenzus; quo maxima contingit Altitudinis Variatio.

\section{$S$ o r.}

D hoc preftandum oportet horarium angulum $B R S$ determinare, Habetur jam ex triangulorum fphæricorum proprietatibus cof. $B R S=\frac{\operatorname{cof} \cdot B S-\operatorname{cof} \cdot B R \cdot \operatorname{cor} . R S}{\operatorname{fin} \cdot B R \cdot \operatorname{fin} \cdot R S}=$ $\frac{\text { cof.x-cof.c. cof.a. }}{\text { fin.c. fin.a }}$.

In hac formula fubrogatus ipfius cof. $x$ valor $\frac{\text { cof. } a}{\text { cor.c }}$ in præc. inventus, offert cof. $B R S=\frac{\text { cof. } a-\operatorname{cof} . a \cdot \operatorname{cof} . z}{\text { cof.c. fin.c. fin. } a}=$

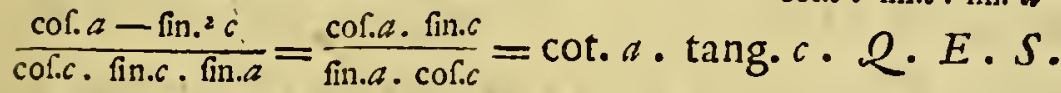

Schoz. Si valor alter ipfrus cof. $x$ in fuperiori formula fuiffet fubftitutus, prodiiffet col. $B R S=$ cot.c. tang.a pro iis nempe fideribus, quorum declinatio Poli altitudinem excedit.

$$
\text { E X E M P L U M. }
$$

In Syrio $+l$ cot. $a=l$ tang. 6. $_{0}$ 24. $^{\prime}=9: 4688 \mathrm{r} 39$

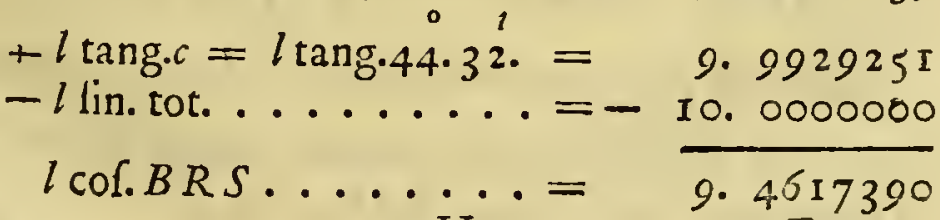
H Ergo 
Ergo horarius Angulus $B R S=73^{\circ}$ : I'; factaque in tempus converfione, invenitur Variationis Maximæ tempus $=4 \cdot 5^{2} .40$; videlicet Maxima altitudinis Syrii Variatio contingit quatuor horis, quinquaginta duobus minutis, quadraginta fecundis, ante, vel poft ipfus culminationem.

\section{P R O B L E M A III.}

Sub üfdem datis invenive Angulum Sideris Azimutbalem Variationi eidem Maxim.e refpondentem.

\section{$S$ o L.}

T $N$ triangulo $S B R$ ex fundamentali analogia fin. $x$ : fin. $S R B::$ fin. $a:$ fin. $S B R$, eruitur fin. $S B R$, feu (ob Angulum Sideris Azimuthalem VBS fupplementum Anguli $R B S$ ) fin. $V B S=\frac{\text { fin.a. } \operatorname{fin} . S R B}{\operatorname{fin} . x}$. Eft autem fin. $S R B=V\left(\mathrm{I}-\operatorname{cof}_{0}^{2} S R B\right)=($ præc. $) V\left(\mathrm{I}-\cot ^{2} a\right.$. tang. $\left.{ }^{2} c\right)$; \& fin. $x=V\left(\mathrm{I}-\operatorname{cof}^{2} x\right)=$ (Prob. I.)

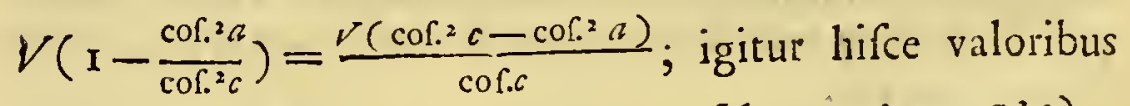
fubftitutis oritur fin. $V B S=\frac{\operatorname{cof} \cdot \cdot \text {. fin. } a V\left(\mathrm{r}-\mathrm{cot}^{2} a \cdot \tan \cdot^{2} c\right)}{V\left(\operatorname{cof}^{2} c-\operatorname{cof}^{2} a\right)}=$ $\frac{V\left(\operatorname{cof}^{2} c \cdot \operatorname{fin}^{2} a-\operatorname{cof}^{2} a \cdot \sin ^{2} c\right)}{V\left(\operatorname{cof}^{2} c-\operatorname{cof}^{2} a\right)}=\frac{V\left(\operatorname{cof}^{2} c\left(\sin .^{2} a+\operatorname{cof}^{2} a\right)-\operatorname{cof} .^{2} a\right)}{V\left(\operatorname{cof}^{2} c-\operatorname{cof}^{2} a\right)}=$ $\frac{V\left(\operatorname{cof}^{2} c-\operatorname{cof}^{2} a\right)}{V\left(\operatorname{cof}^{2} c-\operatorname{cof}^{2} a\right)}=\mathrm{I}$; proindeque $V B S 90^{\circ}$; nimirum ubi Sidus Maximam fubit Altitudinis Variationem, Angulus Azimuthalis rectus eft. Q.E.S.

Schor. 
Schor. Si Declinatio Sideris major eft Poli elevatione, fit cof. $x=\frac{\operatorname{cof} \cdot c}{\text { cofia }}$, feu fin. $x=\frac{V\left(\operatorname{cof} .^{2} a-\operatorname{cof}^{2} c\right)}{\operatorname{cof} \cdot a}, \&$ fin. $S R B=V\left(\mathrm{I}-\operatorname{cof}_{0}^{2} S R B\right)=V\left(\mathrm{I}-\cot _{0}{ }^{2} c \cdot \operatorname{tang}_{0}{ }^{2} a\right)$. Igitur fin. $V B S=\frac{\text { fin. } a \cdot \text { fin. } S R B}{\text { fin. } x}=\frac{\text { fin. } a \cdot \operatorname{cof} \cdot a V\left(1-\cot ^{2} c \cdot \text { tang. } .^{2} a\right)}{N\left(\operatorname{cof}^{2} a-\operatorname{cof}^{2} c\right)}=$

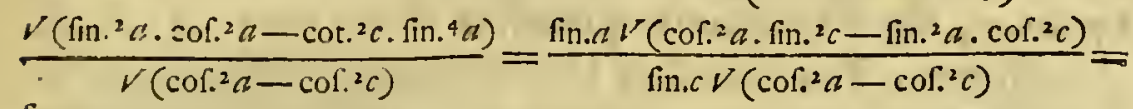
$\frac{\text { fin. } a}{\text { fin. } c}$, nimirum finus Anguli Azimuthalis quartus eft proportionalis ad cofinum elevationis Poli, ad colnum Declinationis Sideris, \& finum totum.

\section{E X E M P I U M:}

Declinatio Stella $d$ Draconis eft $6 \%$ I 4 . Eft itaque: + l fin. tot. ....... = 10.0000000 $+l$ fin. $a=l$ fin. $22.4 \frac{l}{6}=9.5876876$

$-l$ fin. $c=l$ fin. $44.32 .=-9.8459188$

Prodit ergo fin. VBS..... = 9.7417688 Et angulus Azimuthalis $V B S=33^{\circ} 29^{\prime}$.

\section{P R O B L E M A IV.}

lifdem m.nnentibus, Variationis iplus quantitatem invefigare.

$$
S \text { o L. }
$$

It Horarius Angulus $B R S=y$, Azimuthalis $V B S=p$ : Habetur ex Sphxricis cof. $y=\frac{\text { cof.x-cof.c. cof. } a}{\text { fin.c. fin. } a}$; adeoque cof. $x=$ fin. $c$. fin. $a$. cof. $y+\operatorname{cof} . c$. col. $a$. Inftituta $\mathrm{H} 2$ hujus 
60 A $T$ T I

hujus æquationis differentiatione, fit $d x$ fin. $\bar{x}=d y$ fin. $\bar{c}$. fin. $a$. fin. $y, \& d x=\frac{d y \text { fin.c. fin. } a \text {. fin. } y}{\text { fin. } x}$. Eft autem fin. $p=$ $\frac{\text { fin.a. fin.y }}{\text { fin. } x}$; ergo $d x=d y$ fin. $c$. fin. $p$; cumque Maximx Variationis tempore (prec.) fiat fin. $p=\mathrm{I}$, fupereft $d x=$ $d y$ fin. $c$, feu Variationis quantitas $=d y$ fin.c. Q.E.S. $\mathrm{Schoz.}$ Pro Sideribus Declinationem habentibus Poli altidudine majorem Variationis quantitas $d x$ oritur $=$ $d y$ fin. $a, \mathrm{ob}$ fin. $p=\frac{\text { fin. } a}{\text { fin. } c}$.

Cor. I. Sub eadem Latitudine, feu in eadem $\mathrm{Pa}$ rallelo, Variatio Maxima Altitudinis Siderum omnium Declinationem habentium elevatione Poli minorem, eft eodem tempore eadem.

Cor.II. In hifce Sideribus eo magis crefcit Variatio Altitudinis, quo propius ad Equatorem itur : \& fi nulla fuerit Sideris Declinatio, nullaque Loci Latitudo, Variatio fit Maximarum Maxima.

Cor. III. Minor contra fir jugiter ipfa variatio, quo major eft Loci Latitudo, ac fub Polo penitus evaneficit.

Cor. IV. In Sideribus, quorum Declinatio Latitudinem Loci excedit, major fit Variatio Altitudinis quo minor eft ipforum Declinatio.

Cor. V. Ejufdem Sideris Declinationem habentis Latitudine Loci majorem, Maxima Altitudinis Variatio fub diverfis Latitudinibus eft jugiter eadem. 


\section{P R O B L E M A V.}

Anni tempus determinare, quo Difcus Solis citiffime fupra. Horizontem attollitur, feu tempore quamminimo contactu inferiori ad fuperiorem tranfit.

\section{$S$ o $L$.}

Umpto xquationis cof. $y=\frac{\text { cof. } x-\operatorname{cof} . c \cdot \text { cof.a }}{\text { fin.c. lin. } a}$ differen: $D_{\text {tiali, }}$ obtinetur $d y=\frac{d x \text { fin. } x}{\text { fin.c. fin. } a \text {. fin. } y}$. Porro perfpicuum eft, accipi fat tuto poffe diametrum Solis $D=d x, \&$ fin. $x=1$. , fole in Horizonte verfante. Hinc fiet fin. $y=$ $V\left(\mathrm{I}-\frac{\operatorname{cof}^{2} c \cdot \operatorname{cof}^{2} a}{\operatorname{fin}^{2} c \cdot \operatorname{fin}^{2} a}\right)=\frac{V\left(\operatorname{fin} .^{2} a-\operatorname{cor}^{2} c\right)}{\text { fin.c. fin. } a} ; \&$ confequenter $d y=\frac{D}{V\left(\operatorname{fin}^{2} a-\operatorname{cof}_{0}^{2} c\right)}$ : Evidens etiam eft, per $d y$, feu d. $B R S$, tempus defignari in partibus Æquatoris expreffum, quo Solaris Difcus fupra Horizontis planum attollitur. Pofita igitur in hac hypothef Declinatione Solis variabili $a=z$, fiet $d d y$, feu $d d . B R S=0$, nimirum $\frac{D d z \operatorname{cof} z \cdot \text { fin. } z}{\left(\text { (fin. }{ }^{2} z-\operatorname{cof}{ }^{2} c\right)^{\frac{3}{2}}}=0$. Hinc ftatim colligitur fin. $z=0$; hoc eft Declinatio Solis omnino nulla. Igitur Solaris Difi Horizontis planum pertranfeuntis velocitas Maxima eft tempore Equinoctiorum. Q.E. S.

Cor. I. Tempus in hoc tranfitu infumptum reprxfentatur per $\frac{D}{V\left(1-\cos 0^{2} c\right)}=\frac{D}{\text { fin. } c}$ in partibus Æquatoris : Sic æquinoctiorum tempore cum fit $D=32 \cdot 5^{11}=1925^{\prime \prime}$, 
62

A $T$ T :

habebitur $+l D \ldots .=3 \cdot 2844307$

+ l fin. tot..$=10.0000000$

$-l$ fin.c $=l$ fin.

$$
44 \cdot 32 .=-9.8459188
$$

Ergo $\quad l \frac{D}{\text { fin. } c} \ldots=3.43^{85119}, \& \frac{D}{\text { fin. } c}=$ $2745^{\prime \prime}=45^{\prime} \cdot 45^{\prime \prime}$, quibus Equatoris partibus refpondent 3. 3: temporis. Igitur diebus xquinoctialibus Solis difcus in Horizontis plano traiiciendo, tempus trium minutorum, ac trium fecundorum in hac noftra Mediolani Latitudine infumit.

Cor.II. Quo minor eft Latitudo Regionis, eo brevius eft tempus in hoc tranfitu elapfum, atque ubi nulla eft Latitudo, five fub Aquatore eft Minimorum Minimum, videlicet 2. ${ }^{\prime \prime}$. 20 .

Cor.III. Quo major eft Regionis Latitudo, eo diuturnior eft diebus xquinoctialibus Difci Solaris afcenfus trans planum Horizontis, quippe cum tempus hujufce afcenfus inverfam rationem jequatur Cofinus Latitudinis. Ita ex. gr. in Latitudine 89 .

habebitur $l$ fin. tot. ......... = 10.0000000

$$
\begin{aligned}
& l D=l_{1925 \ldots \ldots . . .} \ldots=3 \cdot 2844307 \\
& l \text { fin.c }=l \text { I. } \ldots \ldots \ldots=\text { S. } 2418553
\end{aligned}
$$

Propteren $l \frac{D}{\text { fin. } c} \ldots \ldots \ldots . . .=\overline{5.0425754}$

Hinc 
Hinc vero fit $\frac{D}{\text { fin. } c}=110300$. in Equatoris partibus, quibus in tempus converfis, invenitur quæfitum tempus $=2 \cdot 2 \cdot 33 \cdot 20$.

Itaque prope Polum in Latitudine $89^{\circ}$. Solis Difcus æquinoctiorum tempore, dum fupra Horizontis totus attollitur plus duabus horis impendit. Cxterum perfpicue patet, in majoribus Latitudinibus, in quibus trajectus Difci Solaris trans Horizontis planum, non ita parvam habet rationem ad diem Solarem, calculum minus cum veritate confentire.

Cor.IV. Ex tempore $T$ velociffimi tranfitus Solaris Difci per Horizontis planum, infertur Latitudo Regionis, cum fit fin. $c=\frac{D}{T}$. Ponamus ex.gr. effe $T$. alicubi $=4$, hoc eft in Equatoris partibus $T=\dot{I}$. Jam erit $+l$ fin. tot...... = 10. 0000000

$$
\begin{aligned}
& +-l D \ldots . . . .=3 \cdot 2844307 \\
& -i T=l 3600 \ldots . .3=-3.5563025
\end{aligned}
$$

Inde vero oritur $c=32.20^{\prime} ;$ ac proinde Latitudo Loci $=57.4{ }^{\circ}$. 


\section{P R O B L E M A VI.}

Data elevatione Poli, of Sideris Declinatione invenire bujus. Supra Horizontem Attitudinem, in qua ipfius. Azimuth lenti]Jsme variat.

\section{S O I.}

A Ngulus quamminimus $S B s$ exprimit ipfius Azimuth A variationem, qux cum ex hypotefi Minima fit, ejus iccirco differentiale nihilo xquabitur. Jam in triangulo $S s B$ latufculum $S s$ coftans, quippe tempori proportionale, dicatur $d t, \&$ fequens habebitur analogia fin. $S B:$ fin. $S S B(=$ cof. $B s R)::$ fin. $d t(=d t)$ :

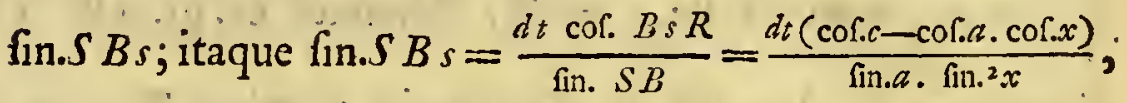
cujus differentiale nihilo xquatum dat cof.a. fin. $\alpha$ fin. ${ }^{3} x-2$ fin. $a$. fin. $x \cdot \operatorname{cof} x(\operatorname{cof} c c-\operatorname{cof} a \cdot \operatorname{cof} x)=o$, hoc eft cof. $x-\frac{2 \operatorname{cof} c \cdot \operatorname{cof} x}{\operatorname{cof} a}+\mathrm{I}=0$; adeoque cof. $x=$ $\frac{\operatorname{cof} c \pm V\left(\operatorname{cof}^{2} c-\operatorname{cof}^{2} a\right)}{\operatorname{cof} a}$. Nota igitur erit Sideris Altitudo $=90^{\circ}-x \cdot 2 \cdot E \cdot S$.

Schor. Simpfonius in eleganti Opere The Doctrine and Application of Fluxions folvendum fibi proponit pro Sole Problema hujufmodi, The elevation of the Pole and the Declination of the Sun being given, to find ad wobat Time of the Day the Azimutls of the Sun increafes the sloweft: verum ejus folutio ex aliis principiis deprompta nihil habet cum hac noltra commune. Rem 


\section{Dell' Accademia :}

meditanti occurrit, ex binis cof. $x$ valoribus alterum

tantummodo $\frac{\operatorname{cof} c-V\left(\operatorname{cof}^{2} c-\operatorname{cof}^{2} a\right)}{\operatorname{col} a}$ qurfito fatisfacere :

\section{$E X E M P L U M$.}

Arcturi declinatio eft $=20^{\circ} .26^{\prime}$. Itaque

$$
\begin{aligned}
& l \text { cof. } c=l \text { fin. } 45.28^{\prime} . .=9.852993^{6} \\
& l \text { cof. }^{2} \text { c........... }=9.7059872 \\
& l \text { cof. } a=l \text { fin. } 20^{\circ} .26 . .=9.5429713
\end{aligned}
$$

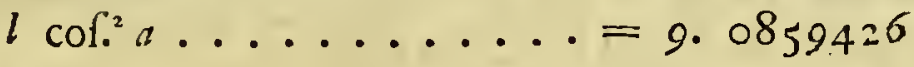

$$
\begin{aligned}
& \operatorname{cof}^{2} \text { c............ } \\
& \operatorname{cof}_{0}^{2} \text { a........... }=0.1218693 \\
& \operatorname{cof}_{0}^{2} c-\operatorname{cof}_{0}^{2} a \ldots \ldots=0.3^{861703} \\
& l\left(\operatorname{cof}_{0}^{2} c-\operatorname{cof}_{0}^{2} u\right) \ldots \ldots=9 \cdot 5^{867 \Omega_{35}} \\
& l \vee\left(\operatorname{cor}_{0}^{2} c-\operatorname{cof}_{0}^{2} a\right) \ldots .=9.7933917 \\
& V\left(\operatorname{cof}^{2} c-\operatorname{cof}_{0}^{2} a\right) \ldots \ldots=0.62 \mathrm{I} 3757 \\
& \text { col.c........... o. } 7128426 \\
& \text { col. } c-V\left(\operatorname{cof}_{0}^{2} c-\operatorname{cof}_{0}^{2} a\right) \cdot=0.0914669 \\
& l\left(\operatorname{cof} . c-V\left(\operatorname{cof}^{2} c-\operatorname{cof}^{2} a\right)=8.96 \mathrm{I}_{42} 88\right. \\
& l \frac{\operatorname{cof} . c-V\left(\operatorname{cof}^{2} c-\operatorname{cof}^{2} a\right)}{\operatorname{cof} a} \cdots=9.4184575
\end{aligned}
$$

Itaque $x=74^{\circ} \cdot \AA^{\prime}$; ac proinde in Arcturo tunc Minima Azimuth Variatio contingit, cum ad altitudinem I5. I2. pervenit. 


\section{P R O B L E M A VII.}

Iifdem manentibus, tempus, quo Minima Sideris Azimutb Variatio contingit aftimare.

\section{S O $\mathrm{r}$.}

T $\mathrm{N}$ triangulo $B S R$ Angulus horarius $S R B$ notus fiet 1 per formulam cor.S $R B=\frac{\operatorname{cof} . x-\operatorname{cof} . c \text {. cof. } a}{\text { fin.c. fin. } a}$, in qua fubrogato ipfus cof. $x$ valore in prxc. Probl. invento, obtinetur cof. $S R B=\frac{\operatorname{cof} . c_{0}-\operatorname{cof} . c \cdot \operatorname{cof} .^{2} a \pm V\left(\operatorname{cof} .^{2} c-\operatorname{cof}^{2} a\right)}{\text { fin.c. fin. } a \cdot \operatorname{cof} . a}=$ $\frac{\text { cof. } c \text {. fin. } 2 a+V\left(\operatorname{cof}^{2} c-\operatorname{cof}^{2} a\right)}{\text { fin.c. fin. } a \cdot \operatorname{cof} a}=\cot \cdot c \cdot$ tang. $a \pm$ $\frac{V\left(\operatorname{cof}^{3} c-\operatorname{cof}^{2} a\right)}{\text { fin.c. fin. } a \cdot \operatorname{cof} a} .2 . E . S$.

Schor. Palam eft, ipfius col. $S R B$ alterum tantummodo valorem cot. $c$. tang. $a-\frac{V\left(\operatorname{cof}^{2} c-\operatorname{cof}^{2} a\right)}{\operatorname{fin} . c \cdot \operatorname{lin} \cdot a \cdot \operatorname{cof}^{2} a}$ ad rem effe. Sit igitur.

$$
\text { E X E M P L U M. }
$$

In ipfo Areturo habetur

$$
\begin{aligned}
& l \text { cot. } c=l \text { tang. } 45.28 . .=10.0070749 \\
& l \text { tang. } a=l \text { tang. } 69.34 . .=10.4288049 \\
& l \text { cot. } c . \operatorname{tang} a \ldots \ldots \ldots=10.4358798 \\
& \text { cot. } c . \operatorname{tang} a \ldots \ldots \ldots=2.7277102 \\
& l \text { fin. } c=l \text { fin. } 44.32 \ldots=9.8459188 \\
& l \text { fin. } a=l \text { fin. } 69.34 \ldots=9.9717762 \\
& l \text { cof. }
\end{aligned}
$$


$l$ cof. $a=l$ fin. $20^{\circ} .26 . .=9.5429713$

$l$ fin.c. fin. $a . \operatorname{cof} . a \ldots . .=9.360666_{3}$

$l V\left(\operatorname{cof}_{0}^{2} c-\operatorname{cof}_{0}^{2} a\right) \ldots=9.7933917$

$l \frac{V\left(\operatorname{cof}^{2} c-\operatorname{cof}^{2} a\right)}{\operatorname{fin} c \cdot \operatorname{cin} a \cdot \operatorname{cof} a} \ldots \ldots=10.4327254$

$\frac{r\left(\operatorname{sof}^{2} c-\operatorname{cof}^{2} a\right)}{\operatorname{fin} c \cdot \operatorname{fin} a \cdot \operatorname{cof} a} \cdots \ldots=2.7081923$

cot.c.tang.a $-\frac{V\left(\operatorname{cof}^{2} c-\operatorname{cof}^{2} a\right)}{\text { fin.c. fin } a . \text { cofla }^{2} a}=0.0195179$

Igitur Angulus Horarius $S R B=88^{\circ} .53^{\circ}$, quibus de more in tempus converfis, prodit quxfitum tempus $=$ $5 \cdot 55^{1} \cdot 2^{\prime \prime}$; tot nempe horis, ac minutis ante, vel port culminationem Minima accidit in Arcturi Azimuth Variatio.

\section{P R O B L E M VIII.}

Variationis ejufdem Minimæ quantitatem Supputare.

$$
\text { S } 0 \text { L. }
$$

Inima ifta Variatio reprefentatur per fin. $S B s=$ $\frac{d t(\operatorname{cof} . c-\operatorname{cof} \cdot a \cdot \operatorname{cof} \cdot x)}{\sin . a \cdot \text { fin. } .^{2} x}$. Cum autem inventus fit cof. $x=\frac{\operatorname{cof} c \pm V\left(\operatorname{cof}^{2} c-\operatorname{cof}^{2} a\right)}{\operatorname{cof} . a}, \&$ confequenter fin. $.^{2} x=$ $\frac{2 \operatorname{cor}^{2} a-2 \operatorname{cof}^{2} c \mp 2 \operatorname{cor} . c V\left(\operatorname{cof}^{2} c-\operatorname{cof}^{2} a\right)}{\operatorname{col}^{2} a}=$ $\frac{\left(\mp 2 \operatorname{cof} c c-2 v\left(\operatorname{cor}^{2} c-\operatorname{cof}^{2} a\right)\right) v\left(\operatorname{cof}^{2} c-\operatorname{cof}^{2} a\right)}{\operatorname{cof}^{2} a}$. His valoribus fubftitutis in priori formula, poft reduI 2 ct io- 
68

A $\quad T \quad r \quad I$

ctiones omnes rite peractas, affequemur fin. $S B s=$ $\mp d t$ cot. $a$. cof. $a$ $\overline{\mp 2 \operatorname{cof}_{c}-2 V\left(\operatorname{col}^{2} c-\operatorname{col}^{2} a\right)} \cdot 2 . E . S$.

Schoz. Perfpicuum eft, valorem alterum ipfius fin. $S B s$, nimirum $\frac{d t \cot a \cdot \operatorname{cof} a}{2 \operatorname{cof} c-2 \sqrt{ }\left(\operatorname{cof}^{2} c-\operatorname{cof}^{2} a\right)}$ ufurpandum effe. Sit in ipfo Arcturo.

\section{E X E M P L U M.}

Accipiatur $S s$, feu $d t=1 "$, quibus refpondet unicum temporis fecundum.

$l$ cot. $a=l$ tang. $20.26 . .=0.57$ II $95 \mathrm{I}$

$l$ cof. $a=l$ fin. $20.2 \% \ldots=9.5429713$

$l$ cot. $a . \operatorname{col} . a \ldots \ldots \ldots . . .=9$. I I 41664 cof.c...................

2 cof.c....................... 4256852

$\checkmark\left(\operatorname{cof}^{2} c-\operatorname{cof}_{0}^{2} a\right) \ldots .=0.6213757$

$2 V\left(\operatorname{cof}^{2} c-\operatorname{cof}_{0}^{2} a\right) \cdots=1.24275^{14}$

2 cof.c $-2 V\left(\operatorname{cof}^{2} c-\operatorname{cof}^{2} a\right)=0.1829338$

$l\left(2 \operatorname{col} c-2 V\left(\operatorname{cof}^{2} c-\operatorname{col}^{2} a\right)\right)=9.261994 \mathrm{I}$

$l \frac{\cot a \cdot \operatorname{col} a}{2 \operatorname{cof} . c-2 V\left(\operatorname{cof}^{2} c-\operatorname{cof}^{2} a\right.} \cdot \cdots=0.8521723$

$l d t-l 11 \ldots \ldots \ldots=$ I. $17609 \mathrm{I} 3$

$l \frac{d t \cot a \cdot c o f \cdot a}{2 \operatorname{cof} c-2 V\left(\operatorname{cof}^{2} c-\operatorname{cof}^{2} a\right)} \cdots=1.0282636$

Itaque $\frac{d t \cot \cdot a \cdot \operatorname{col} a}{2 \operatorname{col} i c-2 V\left(\operatorname{col} .^{2} c-\operatorname{cof}^{2} a\right)}=$ 10. $67, \&$ confequenter fin. $S B s$, five angulus ipfe $S B s= \pm 0.40$. Tempore igitur unius fecundi Minima Anguli Azimuthalis in Aretnro Variatio I $0^{11}$ 40. complectitur.

SCHE- 


\section{SCHED I A S M A I I}

Tbeoremata ad Calculum Integralem Spectantia.

$\mathrm{N}$ Euleriano Opere abfolutiffimo De Calculo Integra$l_{i}$, in quo tot eximia, ac prope divina occurrunt de Formularum Logarithmicarum, \& exponentialium Integratione, nec non de Integratione Formularum angulos finufve angulorum implicantium, videtur aliquid defiderari circa Formulas illas differentiales, quæ poteftatem anguli ductam in finum, aut cofinum anguli ipfus, vel in finum aut cofinum Logarithmi anguli ejufden complectuntur. Prafto funt mihi hac de re Theoremata fex, qux neque inutilia fortalis, neque inconcinna videbuntur. Demonftrationes in pofterum dabo, a Geometris cateroquin facillime inveniendas.

\section{THEOREM A I.}

$\int \cdot x^{n} d x \operatorname{lin} x=-x^{n} \operatorname{cor} x+n x^{n-1}$ fin. $x+n \cdot n-\mathrm{I}$. $x^{n-2} \operatorname{cof} x-n \cdot n-$ I $\cdot n-2 \cdot x^{n-3}$ fin. $x-n$. $n-\mathbf{I} \cdot n-2 \cdot n-3 \cdot x^{n-4} \operatorname{cof} x \ldots \ldots \ldots \ldots+$ $n \cdot n-\mathrm{I} \cdot n-2 \cdot n-3 \cdot \cdot \cdot \cdot n-m+2$. $\int x^{n-n+2} d x \mathrm{X}$ in col. $x$, fi terminus precedens continet cof. $x$, vel in fin. $x$ fi antecedens terminus complectitur fin. $x$.

Cor. Formula elt integrabilis quando $n$ numerus eft affirmativus integer. 


\section{T H E O R E M A II.}

$\int x^{n} d x \operatorname{cof} x=x^{n}$ fin. $x+n x^{n-1} \operatorname{cof} x-n \cdot n-\mathrm{r}$. $x^{n-2}$ fin. $x-n \cdot n-\mathrm{I} \cdot n-2 \cdot x^{n-3} \operatorname{cof} x \ldots \ldots$ $\pm n \cdot n-1 \cdot n-2 \cdot n-3 \cdot \cdots \cdot n-m+2 \cdot \int x^{n}-m+?$ $d x X$ in cof. $x$, vel in fin. $x$ pro uti terminus præcedens involvit col. $x$, vel fin. $x$.

Cor. Hæc quoque formula integrationem admittit quoties $n$ eft numerus integer aftirmativus.

\section{T H E O R E M A III.}

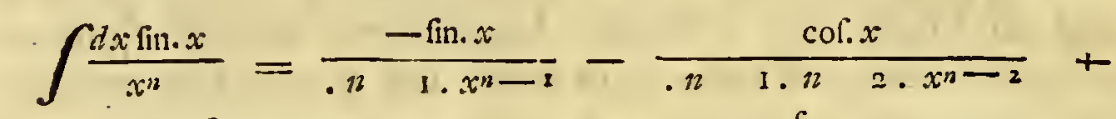
$\frac{\text { fin. } x}{. n-2.12-2.12-3 \cdot x^{n-3}-3}+\frac{\operatorname{cof} x}{. n-1 . n-2 \cdot n-3 \cdot 12-4 \cdot x^{n-4}} \cdots$ $\ldots \ldots \mp \int \frac{d x \text { fin. } x}{n-1 \cdot n-2 \cdot n-3 \cdot \cdots \cdot n-m+1 \cdot x^{n}-m+1}$ vel $\mp \int \frac{b \cdot x \text { cof.x }}{\mathcal{E}_{c} .}$, prouti antecedens terminus continet ex adverfo cof. $x$, vel fin. $x$.

Cor. Formula integrabitur femper, fi $n$ fuerit numerus integer affirmativus .

\section{T H E O R E M A IV.}

$$
\begin{aligned}
& \int \frac{d x \operatorname{cor} x}{x^{n}}=-\frac{\operatorname{cor} x}{\cdot n-1 \cdot x^{n}-2}+\frac{\text { fin } x}{\cdot n-1 \cdot n-2 \cdot x^{n}-2}+ \\
& \frac{\operatorname{cof} x}{. n-1 \cdot n-2 . n \quad 3 \cdot x^{n}-3}-\frac{\text { fin. } x}{. n-1 \cdot n-2 . n-3 \cdot n-4 \cdot x^{n}-4} \cdots \\
& \ldots \mp \frac{1}{. n-1 . n-2 . n-3 \cdots n-n+1} \int \frac{d x}{x^{n}-n+1} \times \text { in }
\end{aligned}
$$


cof. $x$, vel in fin. $x$ prouti terminus proxime antecedens implicat inverfo ordine fin. $x$, vel cof. $x$.

Cor. Integratio femper continget ubi $n$ fuerit affirmativus integer.

$$
\text { THEORE M A V. }
$$

$$
\begin{aligned}
& \left.\int x^{n} d x \text { fin.l } l x=\frac{x^{n+1}}{n^{2}+2 n+2} \overline{(n+1} \cdot \text { fin. } l x-\operatorname{cof} . l x\right) \text {. } \\
& \text { T H E O R.E M A VI. } \\
& \int x^{n} d x \operatorname{cof} l x=\frac{x^{n+1}}{n^{2}+2 n+2}(\overline{n+1} \cdot \operatorname{cof} \cdot l x+\text { fin. } l x) \text {. } \\
& \text { SCHED I A S A III. }
\end{aligned}
$$

Theoremata de Incommenfurabilibus.

uadrati Latus effe Diagonali incommenfurabile, ufque adeo fuit apud veteres Geometras pervulgatum \& celebre, ut Platonis, cxteroquin mitiffimi, iracundiam excitaret, fi quis illud ignoraret . Poft accuratas elegantiffimafque, fed longas illas quidem atque prolixas Euclidæas hac fuper re demonftrationes, novis fubinde detectis methodis rem totam de Incommenfurabilibus nifi elegantius, preflius certe, \& utilius tractarunt Geometre recentiores, quorum ftudio atque induftrix nova quamplurima in hoc argumento ingeniofe excogitata ac fubtiliter enucleata debentur. Nos hic pauca damus ad eam hujufce argumenti partem fpectantia, qui in vulgaribus Libris non videntur ea qua par eft brevitate, vel fimplicitate explicata. Con- 
fule, fi lubet, inftar omnium unum, Caillium in egregiis Prælectionibus Mathematicis a Cl. De Marie editis, atque infigniter locupletatis. Confer . 6. 23 I. \& feq. ubi id ipfum ab Auctore celeberrimo propofitum, \& explanatum deprehendes: Nefcio quid profecto majoris Geometricæ feveritatis in illius demonftrationibus defiderabis, nec fine fcrupulo ac fufpicione latentis Pavalogifmu ab illo difcedes. Cæterum quicquid fit de aliorun conatibus $\&$ inventis, quibus nihil detractum volumus, tria fequentia 'Theoremata rem declarabunt. Sit igitur.

\section{T H E O R E M A I.}

Si numeri alieujus $A$ potcfas $\mathcal{A}^{n}$ aucatur in mumcrum alterum

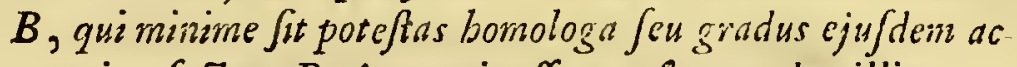
prior; factum $B A^{n}$ nequit effe potefias gradus illius.

\section{E M.}

Onftat, ex poteftate numeri cujufvis divifa per poteftatem homologam numeri alterius 'oriri quotientem, qui rurfus fit poteftas homologa numeri alicujus; fractionis enim cujuscumque (licet autem dividendum, ac diviforem uti fractionis numeratorem ac denominatorem fpectare) fit poteftas aliqua cum nusnerator ac denominator ipfius attolluntur ad poteftatem ipfam. Igitur fi $B A^{x}$ eft poteftas gradus $n$, divifa per poteftatem $A^{n}$ ejufdem gradus, prabebit quotientem poteftati homologx xqualem $\frac{B A^{n}}{A^{n}}=B$; ergo $B$ erit poteftas gradus 2 ; quod cum lypothel adverfetur, patet 
patet iccirco; factum $B A^{n}$ nunquam effe poffe poteftatem gradus $n . Q . E . D$.

Cor. Hinc perfpecte liquet, cur duplum, triplum, quintuplum \&c. numeri quadrati, aut cubici \&c. nequeat effe numerus quadratus, aut cubicus; cur in Quadrato geometrico ratio, quam habet Quadratum Lateris ad Diagonalis Quadratum nullis exprimi poffit numeris, quorum ambo fint quadrati; cur in Problemate Deliaco ratio dupla Aræ unius cubicx, ad alteram per numeros fimul cubicos nequeat defignari \&c.

\section{TH E O R E A II.}

Si numeri alicujus $A$ poteftas $A^{n}$ divifibilis of per bomo: logom poteftatem. $B^{n}$ alterius numeri $B$, ip $\int e$ quoque $A$ divifibilis erit per $B$.

\section{E M.}

I $\mathrm{N}$ hac hypothefi $B$ erit factor ipfius $\mathcal{A}^{n}$, ac proin: de $A^{n}$ xquabitur ipfi $B^{n}$ ducto in factorem alterum; qui (Theor. I.) fit poteftas ejufdem gradus 12, nimirum in factorem $E^{n}$. Itaque $A^{n}=B^{n} E^{n}, \& A=$ $B E$. Eft autem $B E$ divifibilis per $B$. Igitur \&c. $\mathcal{L}$. E. D.

Cor. Quotiefcumque numerus aliquis $A$ non eft per rumerum alterum $B$ divifibilis, neque illius poteitas $A^{n}$ per hujus poteftatem homologam $B^{n}$.divifíbilis crit: \& In fractio aliqua $\frac{A}{B}$ fit minimis terminis expreffa, poteftas $A^{n}$ minime divifibilis erit per denominato: 
74

A T T I

rem fimplicem $B$, vel per ejus poteftatem quamcumque $B^{m}$.

\section{THEOR E A III.}

Frattionis $\frac{F}{G}$ poteftas qualibet $\frac{F n}{G n}$ nunquam fit numero integro aqualis.

\section{E M.}

I $F<G$, theorema convertitur in axioma ; f $F>G$, $\checkmark$ cum numerator. $F$ per denominatorem $G$ dividi minime poffit, quemadmodum fractionis natura requirit; neque poteftas $F^{n}$ (Cor. præc.) divifibilis erit per $G^{n}$, hoc eft $\frac{F^{n}}{G^{n}}$ femper fractio erit, feu nunquam fiet numero integro xqualis $Q . E . D$.

Cor.I. Nunc itaque luculenter invicteque innotefcit (quod a plerifque per longas ratiocinationum ambages operofe demonftratur), ex binario, ternario, quinario \&c. radicem quadratam aut cubicam \&c. educi minime poffe, feu nullis effe numeris five integris; feu fractis accurate affignabilem; inde vero fit, ut incommenfurabiles fint magnitudines Quadrati Latus, \& Diagonalis, earumque ratio nullis numeris utcumque ingentibus exprimenda.

Cor II. Hinc luculenta \& facilis deducitur demonftratio Theorematis in Analyfi longe utiliffimi, videlicet. 


\section{T H E O R'E M A I V.}

Si Fquationis cujuslibet $x^{n}+a x^{n-1}+b x^{n-2}+\ldots$ $e x^{2}+f x+g=0$ nullus fit terminus fractionarius; nullia aquationis radix realis, o rationalis vere fractionaria effe poteft.

- Ujus demonftratio, qux valde operofa, \& imple$x a$ afferri vulgo folet, fic nitide breviffimeque abfolvitur: Ex fuperiori Æquatione habetur $x^{n}=-a x^{n-1}-$ $b x^{n-2}-\ldots . .-c x^{2}-f x-g$ : Jannero fi ponas effe $x$ numerum fractionarium, minimifque terminis expreflum in hac hypothef vel fecundum $x$ quationis membrum - $a x^{n-1}-b x^{n-2}-\& c$. numerus integer erit, vel fractus; Si integer, jam nequaquam æquabitur prinio membro $x^{n}$, quod (Theor. prec.) neceffario eft fractionarium, fi fractus, ejus denominator poft reductionem erit numeri alicujus poteftas ordinis $\overline{n-1}$, primum autem xquationis niembrum $x^{n}$ denominatorem habebit xqualem ejufdem numeri poteftati ordinis $n$, cumque $x^{n}$, hoc eft ex. gr. $\frac{p^{n}}{p^{n}}$ xquetur $\frac{2}{p^{n-1}}$, nimirum membro alteri ad terminum unum reducto, erit $\frac{r^{n}}{p^{n}}=\frac{Q p p}{p^{n}}, \& i^{n}=$ $2 p$, adeoque quemadmodum $2 p$ ita $\& r^{n}$ divifibilis erit per $p$, quod repugnat Corollario Theorematis I. Igitur \&c. $2 \cdot E . D$. 


\section{SCHEDIASMA I VA}

\section{De Problemate quodam Atbmospluevico.}

L. rerum Phyficarum Speculator J. A. De Luc, elaboratiffimo Opere nuper edito, Recherches fur les Modifications de l' Athmofphére, cum primis illuftris, datis ad me anno præterito litteris prid. Kal. Nov. Probl. quoddam a celeberrimo doctiffimoque conterraneo fuo Le Sage propofitum, illius poftulatu mecum communicavit, cujus ego Solutionem ftatim ad ipfum mifi eidem Cl. le Sage, qui illan petierat, exhibendam. Litterarum exemplar erat hujufmodi : Monfieur Le Sage, "l'un de nos Savans les plus diftingués tant par l'é-

"tendue de fes connoiffances en Phyfique, que par fon "génie, me charge de vous préfenter fes refpects, \& "de vous demander une faveur au cas qu' elle ne vous 2) foit pas incommode. Il fouhaiteroit une formule " par la quelle on pût évaluer la Maffe de l'Atmo" fphére depuis une hauteur donnée quelconque jufq' à "La furface de la Mer, dont l' expreflion fut par exem" ple en pieds cubes d'air de la denfité ou il fe trou" ve au niveau de la Mer quand le Baromètre eft à 28. " pouces, ou en géneral à une denfité donnée. Je " dis Maffe, \& non Poids; parce qu' il ne faut avoir „ égard dans ce calcul, ni à la diminution que fubit " le poids des couches fupérieurs, par la diminution "que reçoit toute pefanteur felon la Loi Newtonienne, " ni à la prètendue diminution que reçoit le poids des couches'de l'Atniofphére à caufe de leur forme de 
"voute. Il fuffit donc d' avoir ègard à la diminution " de la denfité de l'air ; fuivant la Loi de Halley, \&

"à l'aggrandiffiment des couches fphériques à mefure "que leur rayón augmente. Monfieur Le Sage ayant "vû votte Livre, Monfieur."

Delle Altezze Barometriche is \& compris que la "folution de ce Probleme ne feroit qu'un jeu pour vous, "il a efperé que vous voudriez bien lui faire le pläifir "de l'entreprendre. Si la folution devient courte, il "fe propofe de l'employer dans un Ouvrage; au quel ; il travaille, en l'attribuant, comme il eft jufte, à ce"lui de qui il la tiendra. "

Hujus itaque Problematis fequentem ftatim inveni folutionem, quam iffem verbis, quibus illam cum. $\mathrm{Cl}$. De Luc per litteras communicavi, hic ob oculos pono.,",

\section{P R O B L E M E:}

Trowver la Magle de I Atmofpbére depuis une bauteu. donnéc jufqu' d la furface de la Mer.

\section{S O L.}

4 $U^{\prime}$ on nomme $x$ la diftance du centre terrefte à un hauteur variable de l'Atmofphére; i : c le rapport du rayon à la circonference circulaire. On aura donc $2 c x^{2}$ pour la furface de la couche atmofphérique, dont $x$ eft le temidiametre, \& en multipliant par $d x$, on aura $2 c x^{2} d x=$ au volume de la couche infiniment mincé. Pour obtenir la Mafte de cette couche elementair il faudra multiplier „fon 
\#. Ton volume par la pefanteur fpecifique de. l'air à

"l'endroit de la meme couche, eft à dire à la di-

„A Atance $x$ du Centre de la terre.

Or en nommant $H$ la hauteur du Barometre au

" niveau de la Mer, $z$ la hauteur du nême Barome-

" tre à la diftance $x$ du Centre terreftre, $f$ la pefan-

" teur fpecifique de l'air au niveau de la $\mathrm{Mer}$; la loi

, de Mariotte, ou de. Halley fournira l'analogie $H$ :

2) $:: f: \frac{f z}{H}=$ à la pefanteur fpecifique de l'air de la

is dite"couche. Donc la Maffe de cette couche elemen-

taire fera $\frac{2 c f z x^{2} d x}{H}$. Pour integrer cette expreffion

$\Rightarrow$ il faut retrouver $z$ donnée en $x$; \& pour cet effet

") je remarque, qu' en multipliant la denfité $\frac{f z}{H}$ de l'air

" par la groffeur infiniment petite de la tranche de la

" colonne acrienne, c'eft a dire par $d x$, on a $\frac{f z d x}{H}=$

a la depreffion fuxionaire $-d z$ du barometre (on

" comprend aifément que $-d z$ elt multiplié ici par

"la pefanteur fpecifique di Mercure fuppofée $=1$,

3) etant à peu-prés $f=\frac{1}{10800}$; \& qu on prend $-d z$ ne-

$\because$ gatif parce qu' en montant le baronetre baiffe). Donc

$\because \frac{f d x}{H}=\frac{-d z}{z} ; \& \frac{f x}{H}=-l z+$ conf., \& parceque $z$

\%) devient $H$ lorfque $x$ devient egal au femidiametre. $r$ de

"la terre, on tirera conft $=\frac{f^{\prime}}{H}+l H, \&$ par; confequent

,l $\frac{z}{H}=\frac{f r-f x}{H}$, d' cù fi l'on prend $E$ pour le nom"bre 
; bre dont le logarithme $=I$, l'on tire $\frac{z}{H}=E^{-H}$, $\underline{f r-f x}$

.) \& $z=H E S$; ; quelle valeur fubftituée darisil ex-

"preffion differentie!le $\frac{2 c f x^{2} d x}{H}$ de la Maffe da la cou"che fphérique de l'atmof phére, donne $2 c f x^{2} d x \frac{f y-f x}{H}-$.

,

Or $\int 2 c f x^{2} d x \frac{f r-f x}{H}=2 c f E^{\frac{f r}{H}} \int x^{2} d x E_{-f x}^{\frac{-f x}{H}}=$

$\leadsto$ (en integrant par parties) $-2 c f E^{\frac{f r}{H}} \cdot \frac{H}{f} x^{2} E^{-f H^{-}}$ "2cf $E^{\frac{f x}{H}} \int \frac{H}{f} \cdot 2 x d x E^{\frac{-f x}{H}}=-2 c H E^{\bar{H}} x^{2} E^{\frac{-f x}{H}}$

$\Rightarrow \frac{4 c H z E^{\frac{f r}{H}} x E^{-f x}}{f}+\frac{4 c H^{2} \frac{f r}{E^{H}}}{f} \int_{d} d x E^{\frac{-f x}{H}}=-\frac{f^{\prime}-f_{x}}{E}$

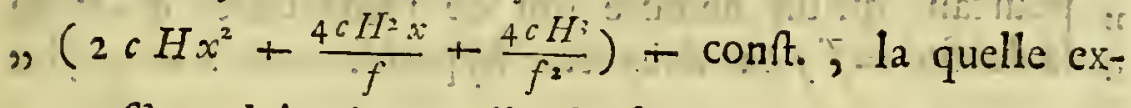

"preflion doit étre nulle lorfque $x=r ; l$ on aura

$"$ doric conft. $=2 c H \cdot r^{2}+\frac{4 c H^{2} r}{f}+\frac{4 c H^{3}}{f^{2}}$ Donc Ia

" Maffe de l' atmofphere depuis la furface de la "mer jufq' à un hauteur indeterminée $x-r$; fe$\Rightarrow \mathrm{ra}=2 c H r^{2}+\frac{4 c H^{2} r}{f}+\frac{4 H_{3}}{f^{2}}-E \frac{f r-f x}{H^{2}}\left(2 c I I x^{2}+\right.$ ", $\left.\frac{4 c H^{2} x}{f}+\frac{4 c I^{3}}{f^{2}}\right), \&$ fi on voudra la Málfe de l'at"mofphere jufqu' à une hauteur donnée a depuis lạ fur"face de la mier, dans cette hypothefe, $x$ deviendra $r+q$, , \& l'ex- 
2)

2

э9

99

29

23

9)

ว

25

29

2

ว9

3

2)

97

29.

35

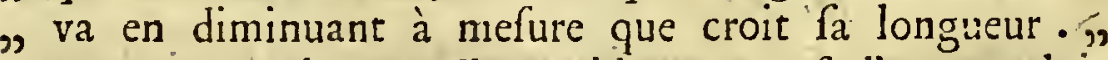

\& l'expreffion précedente fera $=2 c \mathrm{Hr}^{2}+\frac{4 c \mathrm{H}^{2} r}{f}+$

Ce qu'il falloit retrouver.

"Maintenant il fera fort aifé de réduire cette expreffion analytique en pieds cubes d'air de la denfité ou il fe trouve au niveau de la mer, étant données en pieds de Paris les quantites $r, a, H ; c^{\prime}$ eft un calcul numerique, qui ne peut pas aréter perfonne. "

Cor. "En examinant la formule précedente l'on tire un Theoreme qui prefente un efpece de' paradoxe affez fingulier, \& peut-etre nouveau, c' eft-a-dire qu' en fuppofant l' atmofphére étendue a l'infi. ni, ou d' une hauteur infinie, fa maffe ne renferme pourtant qu' un nombre fini de pieds cubes d'air de la denfité $=f ;$ car dans cette hypotefe étant $a=\infty$, les

termes de la formule, qui font multipliés par $E^{H}$, deviennent $=0, \&$ ne reftent que les autre termes tous finis. On entrevoit quelque raifon metaphyfique de ce myftere phifico-mathematique, en reflechiffant, que la Maffe de chaque couche aerienne diminue à mefure qu' augmente $f a$ diftance de la furface de la terre. Ainfi dans la Geometrie, le folide hyperbolico-afymptotique infiniment long ne comprend "qu' un volume fini; à caufe que la groffeur du folide Rem. "Il eft affez evident que fi l'on vouloit „ fuppoler connue la hauteur du Barometre à un ele- 
2) vation quelconque au deffus du niveau de la $\mathrm{Mer}$,

"la folution du Probleme n'auroit alors la moindre

"difficulté; car il fuffiroit de multiplier le poids de

" $2 S$. pouces cubes de mercure par le nombre connu

"'de pouces quarrés de la furface de la Terre, \& l' on

"auroit un produit égal au poids de toute l' atmo-

"fphére; on multiplieroit de mème a l'elevation donnée

" au deffus de la Mer, ce poids des pauces cubes de

" mercure du mème Barometre en ce lieu, par le nom-

"bre aufli connu des pouces quarrés de la furface fphé-

"rique, dont le femidiametre feroit la diftance de ce

"lieu au centre de la terre; il faudroit ôter ce fecond

" produit du prenier, \& la difference égaletoit le poids

" de la couche aerienne, dont la grolfeur eft egal à

"l'elevation donnée. Poul trouver maintenant combien

"de pieds cubes, renfermeroit l' air de cette couche fi

"on le reduiroit tout à la denfité qu'il a au niveau

"de la Mer, il faudroit dire, comme le poids d' un

"pied cube d'air de la denfité, qu'il a au niveau de

"Ia mer, au poids de la couche retrouvé, ainfi $I$, au

"quatrieme proportionnel, qui eft le nombre des pieds

"cubes que l' on cerche."

"Mais dans le Probleme il ne faut pas fuppofer " connue la hauteur du barometre dans l'elevation don-

"née, \& dans ce cas le feul Calcul Integral en four.

"nit la folution." 


\section{A $\quad T \quad T \quad r$ \\ SCHEDIASMAV. \\ D. Trianguli Spbarici Dimenfione.}

DImenfo fuperficiei Trianguli Sphrrici Problema eft recentioris Geometrix præclarum, \& nobile, in quo complurium excellentium Geometrarum ingenium fe exercuit, \& laboravit induftria. Præ cxteris magnus Eulerus, cui in univerfa Mathefi nihil invium \& inacceffum, in Actis Accademiæ Berolinenfis, Trianguli Sphærici aream per circuli rectificationem elegantiflime dimenfus eft. Cum autem nec inutile nec incuriofum lit plures vias, quæ ad eandem veritatem ducunt, cognofcere, novam hic tradimus methodum idem affequendi, indeque porro Eulerianum Theorema de æqualitate inter aream Trianguli Sphrici, \& exceffum trium ejus angulorum fupra rectos duos ductum in fphxræ femidiametrum, perfpicue deducimus.

\section{P R O B L E M A. I.}

Trianguli Spbstrici rectanguli $F A B$ aream invenire.

$$
\text { S O I U T I } 0 \text {. }
$$

Tab.3. It rectus angulus ad $F$, ducaturque circuli maximi Fig.2.

$D$ arcus $B a$, lateri $B A$ infinite propinquus. Sitque $A N$ arcus minimus paralleli habentis polum in $B$. Dicatúr $c$ latus $B A, b$ latus $B F, I$ radius phæræ. Areola $B N A$, quæ elemertum eft trianguli fphærici $B A F$ xquatur, juxta inventa Archimedra, rectangulo ex finu yerfo ar- 
cus $B A$ in arcum minimum $P p$ circuli maximi, cujus polus eft $B$. Eft porro, ex Trigonometria Sphærica, fin.e: I : : $N A: P p=\frac{N A}{\text { fin.e }} ; \&$ in triangulo infinitefimo, ac rectilineo $A N$ a rectangulo ad $N$, eft I : tang. $a=\operatorname{tang} . A:: a N=d e: N A=d e \operatorname{tang} . A$; hinc $P p=\frac{\text { detang.A. }}{\text { fin.e }}$. Jan vero ex eadem Trigonometria, fe habet fin. $A:$ fin.b: : I : fin.c, feu fin. $A=$ $\frac{\text { fin. } b}{\text { fin. } e}, \& \operatorname{cof} . A=V\left(\mathbf{I}-\frac{\text { fin. } b^{2}}{f \operatorname{fin} . e^{2}}\right)=\frac{1}{\text { fin. } e} V\left(\right.$ fin. $e^{2}-$ fin. $\left.b^{2}\right) ; \& \frac{\text { fin. } A}{\cot A}$, feu tang. $A=\frac{\text { fin. } b}{V\left(\operatorname{fin} \cdot e^{2}-\text { fin. } b^{2}\right.} \cdot$ Ergo $P p=\frac{d e f \ln . b}{\operatorname{fin} e V\left(\operatorname{lin} \cdot e^{2}-\operatorname{lin} \cdot b^{2}\right)} ;$ ac proinde areola $B N \mathcal{A}=$ $\frac{d e \text { fin. } b \text { fin. verf.e }}{\text { fin. } e V\left(\text { fin. } e^{2}-\operatorname{fin} . b^{2}\right)}=\frac{d e \text { fin. } b}{\text { fin.e } V\left(\text { fin. } e^{2}-\operatorname{fin} . b^{2}\right)} ;$ ac proinde $=$ $\frac{d e \text { fin. } b}{\text { fin. } e V\left(\operatorname{fin} . e^{3}-\sin . b^{2}\right)}-\frac{d e \operatorname{cof} e \text { fin. } b}{\sin . e V\left(\sin . e^{2}-\operatorname{lin} . b^{2}\right)}$ : Igitur area $B A F=\int \frac{d e \text { fin. } b}{\text { fin. } e V\left(\operatorname{lin} e^{2}-e_{\text {fin }} b^{2}\right)}-\int \frac{d e \text { cor.e fin. } b}{\text { fin. } e V\left(\operatorname{lin} . e^{2}-\text { fin. } b^{2}\right)}+C$. Ad hæc integralia obtinenda, prioris termini numerator ac denominator ducantur in col.c $c$, ut prodeat $B A F=$ $\int \frac{d e \text { cof.e fin. } b}{\text { din. } e \text { cof.eV }\left(\text { fin. } e^{2}-\text { fin. } b^{2}\right)}-\int \frac{d e \text { cof.efin. } b}{\text { fin. } e V\left(\text { fin. } e^{2}-\operatorname{fin} . b^{2}\right)}+C=$ $\int \frac{d \cdot \operatorname{fin} . e \operatorname{fin} . b}{\operatorname{fin} . e \operatorname{cof} e V\left(\operatorname{fin} . e^{2}-\operatorname{fin} . b^{2}\right)}-\int \frac{d \cdot \operatorname{fin} \cdot e \sin . b}{\operatorname{fin} . e V\left(\operatorname{fin} . e^{2}-\operatorname{fin} \cdot b^{2}\right)}+C=$ $\int \frac{d \cdot \text { fin. } e \text { fin. } b}{\text { fin. } e V\left(1-\operatorname{fin} \cdot e^{2}\right)\left(\text { fin. } e^{2}-\operatorname{fin} \cdot b^{2}\right)}-\frac{d \cdot \operatorname{fin} \cdot e \operatorname{fin} . b}{\sin \cdot e V\left(\text { fin. } e^{2}-\operatorname{fin} \cdot b^{2}\right)}+C$. Fiat $V\left(\mathrm{I}-\mathrm{fin} \cdot c^{2}\right)\left(\right.$ fin. $\left.c^{2}-b^{2}\right)=\left(\mathrm{I}-\right.$ fin. $\left.c^{2}\right) u$; critque fin. $c^{2}-f_{1 n} \cdot b^{2}=\left(1-\ln _{0} e^{2}\right) a^{2}, \& z=$ fin.e d. fin.e $=$ L. 2 is 
$8_{4}$

A $T$ T $\mathrm{I}$

$2 u d u\left(\mathrm{I}-\mathrm{fin}_{.} e^{2}\right)-2 u^{2}$ fin.ed. fin.e ; fin.e d. fin.e $=$ $\frac{u d u\left(\mathrm{I}-\operatorname{fin} \cdot e^{2}\right)}{\mathrm{I}+u^{2}} ;$ fin. $e^{2}=\frac{u^{2}+\operatorname{fin} \cdot b^{2}}{1+u^{2} .} ; \frac{\text { fin. } e d \cdot \operatorname{fin} \cdot e}{\operatorname{fin} \cdot e^{2}}=\frac{d \cdot \text { fiu. } e}{\text { fin. } e}=$

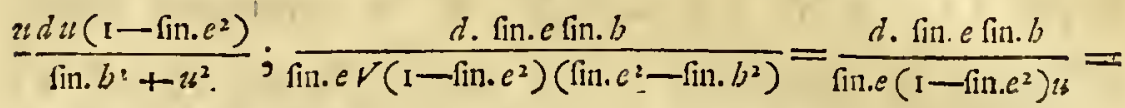
$\frac{d u \sin . b}{\sin . b^{2}+u^{2}}=\frac{\frac{d u}{\operatorname{fin} . b}}{I+\frac{u^{2}}{\sin \cdot b^{2}}}$. Pro telmini alterius reductione ponatur $V\left(\right.$ fin. $e^{2}-$ fin. $\left.b^{2}\right)=z$, fin. $e d$. fin. $e=z d z$;

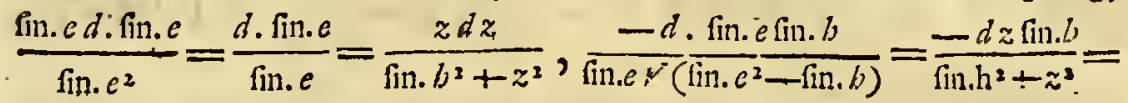
$-\frac{d z}{\text { fin. } b}$

$\frac{z^{2}}{\mathrm{I}+\frac{z^{2}}{\operatorname{fin} b^{2}}}$. Igitur triangulum Sphæricum $B A F=$ $\int \frac{d \cdot \operatorname{fin} \cdot e \text { fin. } b}{\text { fin. } e V\left(1-\text { fin. } e^{2}\right)\left(\text { fin. } c^{2}-\text { fin. } b^{2}\right)}-\int \frac{d \cdot \text { fin. } e \text { fin. } b}{\left.\text { fin.eV (fin. } e^{2}-\operatorname{fin} \cdot b^{2}\right)}+$ $C=\int \frac{\frac{d u}{\sin \cdot b}}{1+\frac{u^{2}}{\operatorname{fin} \cdot b^{2}}}-\int \frac{\frac{d z}{\operatorname{fin} . b}}{1+\frac{z^{2}}{\operatorname{fin} \cdot b^{2}}}+C=$ Arc. tang. $\frac{u}{\operatorname{fin} . b}-$ Arc. tang. $\frac{z}{\sin \cdot b_{2}}+C=$ Arc. tang. $V\left(\frac{\text { fin. } e^{2}-\text { fin. } b^{2}}{\operatorname{cof}^{2} c^{2} \text { fin. } b^{2}}\right)-$ Arc. tang. $V\left(\frac{\text { fin. }^{2}-f^{2} \cdot b^{2}}{\text { fin. } b^{2}}+C\right.$. Conftans $C$ invenitur nihilo $x$ qualis, evanefcente videlicet area $B A F$ ubi latus $B A$ congruit cum $B F$, feu $c=b, \&$ evanefcentibus fimul Arcubus binis per integrationem inventis. $Q . E . I$. Schol. Bini arcus inventi revocari commode poffunt ad unicum, per vulgatifimum Trigonometrix Theo-

rema, 
rema, quo demonftratur datis arcubus $C, D$, fore tang. $(C-D)=\frac{\text { tang. } C-\operatorname{tang} . D}{\mathrm{I}+\operatorname{tang} \cdot C^{\prime} \operatorname{tang} . D}$. Si itaque $C, \& D$ exprimant arcus binos antea erutos, prodibit tang. $(C-D)=$

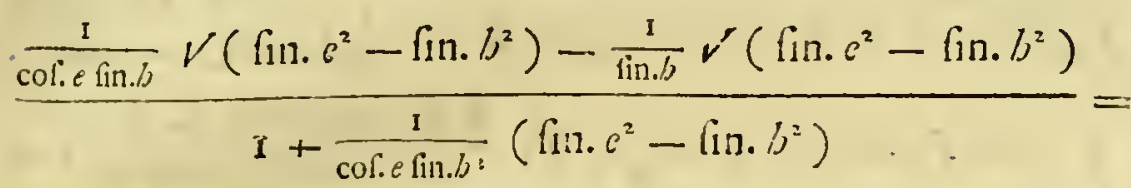

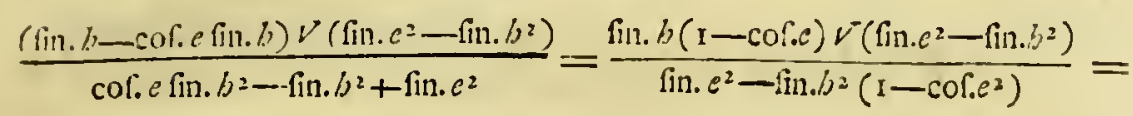
$\frac{\text { fill. } b \text { fin. verf.e } V\left(\text { fin. } c^{2}-f i n . b^{2}\right)}{\operatorname{lin.} e^{2}-\sin . b^{2} \text { fin. verf.e } e}$. Igitur $C-D$, feu Triangulum

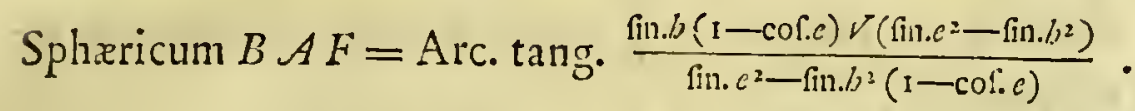
Inftitui poteft examen hujufce noftre folutionis in exemplo fat luculento ac perfpicuo, quo bina trianguli latera $D A, A F$ fuerint quadrantalia, ac latus tertium $B F$ femiquadrantale, quo in exemplo (Archimede demonftrante) Trianguli area exæquat fuperficici hamiphericæ oftantem. Erit igitur in hac hypothefi $c=90^{\circ}$, $b=45^{\circ}$, fin. $e^{2}=\mathrm{I}$, cof. $e=0$, fin. $b=V \frac{1}{2}$, fin. $b^{2}=\frac{1}{2}$; ac proinde Arc. tang. fin. $b \frac{(1-\operatorname{cof} . c) r\left(\operatorname{fin} \cdot e^{2}-\operatorname{cin} \cdot b^{2}\right)}{\text { fin. } c^{2}-\sin \cdot b^{2}(1-\operatorname{cof} \cdot c)}=$ Arc. tang. $\frac{\frac{1}{2}}{\frac{1}{2}}=$ Arc. tang. $I=45^{\circ}=$ rectangulo ex femiquadrante in radium $I=\frac{1}{4}$ circuli maximi $=\frac{1}{8}$ fuperficiei hxmifphricx. 
Aliud iniri poteft examen, ponendo $e=90^{\circ}, b=$ $60^{\circ}$, qua in hypothefi palam eft, Trianguli dati aream xquari parti fextæ fuperficiei hæmifphericæ. Idipfum vero eruitur ex formula noftra; eft enim fin. $b=V \frac{3}{4}$, fin.e $=$ I, col. $e=o, V\left(\right.$ fin. $e^{2}-$ fin. $\left.b^{2}\right)=\frac{1}{2}$, fin. $b(\mathrm{I}-$ cof.e $)$. $V\left(\operatorname{fin} . e^{2}-\operatorname{cof} . b^{2}\right)=\frac{1}{4} V_{3}, \frac{\operatorname{fin} . b(1-\operatorname{cof} e) V\left(\operatorname{fin} . e^{2}-\operatorname{fin} . b^{2}\right)}{\operatorname{fin} \cdot e^{2}-\sin \cdot b^{2}(1-\operatorname{cof} e)}=$ $\frac{\frac{1}{4} \sqrt{3}}{\frac{1}{4}}=V_{3}$. Igitur Arc.tang. $\frac{\operatorname{fin} . b(\mathrm{~s}-\operatorname{cof} . e)\left(\operatorname{lin} \cdot e^{2}-\mathrm{fin} . b^{2}\right)}{\operatorname{fin} \cdot e^{2}-\operatorname{lin} \cdot b^{2}(1-\operatorname{cof} e)}=$ Arc. tang. $V_{3}=60^{\circ}=$ rectangulo ex triente femiperiphæriæ in radium $=\frac{1}{3}$ circuli maximi $\frac{1}{6}$ fuperficiei hęmifphæricæ .

\section{P R O B L E M A II.}

Trianguli Spbarici cujufcunque $B A F$ aroam invenive.

Tab.3. Fig.2.

Iant omnia uti prius, dicaturque propterea $q$ angu-
Ius conftans $F$. Erit ut antea $P p=\frac{d e \tan . \cdot A}{\text { fin. } e}$. Et quia finus angulorum funt uti finus laterum oppofitorum, erit fin. $A$ : fin. $b::$ fin. $q:$ fin. $c$, feu fin. $A=$ $\frac{\text { fin. } b \text { fin. } q}{\text { fin. } e}$; cof. $A=\frac{1}{\operatorname{lin} . e} V\left(\right.$ fin. $e^{2}-$ fin. $b^{2}$ fin. $\left.\frac{n^{2}}{L}\right), \&$ tang. $A=\frac{\operatorname{fin} . A}{\operatorname{cof} . A}=\frac{\operatorname{fin} . b \sin . q}{r\left(\operatorname{fin} . c^{2}-\operatorname{fin} \cdot b^{2} \sin \cdot q^{2}\right)} \cdot \operatorname{Ergc} P p=$

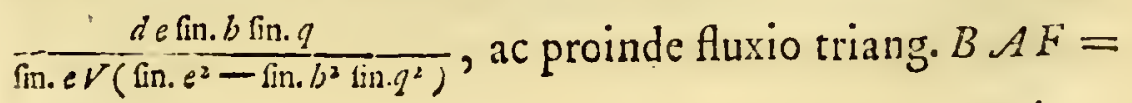
de 
$\frac{d e \text { fin. } b \text { fin. } q}{\sin . e V\left(\text { fin. } e^{2}-\sin . b^{2} \text { fin. } q^{2}\right)}(1-\operatorname{cof} . e)=\frac{d e \text { fin. } b \text { fin. } q}{\text { lin.eV }\left(\text { fin. } e^{2}-\operatorname{lin} . b^{2} \text { fin. } q^{2}\right)}-$ $\frac{d e \operatorname{cof} e \text { fin. } b \operatorname{lin} . q}{\text { fin. } e V\left(\operatorname{lin} . c^{2}-\sin . b^{2} \sin \cdot q^{2}\right)}$. Eodemque modo quo fupra invenietur area $B A F=$ Arc.tang. $\frac{\text { fin. } b \text { fin. } q(1--\operatorname{cof} . e) r^{r}\left(\sin \cdot c^{2}-\sin \cdot b^{2} \text { fin. } q^{2}\right)}{\operatorname{fin} \cdot e^{2}-\sin \cdot b^{2} \sin \cdot q^{2}(1-\operatorname{cof} \cdot e)}$ Q. E. D.

\section{THEOR E M A.}

Cujufcumque Trianguli Splacerici area cquatu" exceffui trium ejus angulorum fupia rectos duos.

\section{E M.}

CIt ${ }^{\circ}$ Triangulum Rectangulum: Anguli $F B A$ flu- Tab.3. $D$ xio eft Angulus $A B a$, five ejus menfura $P p$, hoc Fig.s. eft (per Probl. Sol.) $\frac{d e \operatorname{lin} . b}{\operatorname{lin} . e V\left(\operatorname{lin} . e^{2}-\sin . b^{2}\right)}$; \& fluxio anguli $\mathcal{A}$ abeuntis in alterum a eft $a-\mathcal{A}$, five (uti notum aliunde ) $\frac{d \cdot \ln \cdot A}{\operatorname{cof} \cdot A}=\frac{d \cdot \frac{\operatorname{fin} \cdot b}{\operatorname{fin} \cdot e}}{V\left(\mathrm{I}-\frac{\operatorname{fin} \cdot b^{2}}{\operatorname{fin} \cdot e^{2}}\right)}=\frac{-d e \operatorname{cof} \cdot e \text { fin. } b}{\operatorname{fin} \cdot e V\left(\text { fin. } e^{2}-\operatorname{fin} \cdot b^{2}\right)}$ : Jam vero area Triang. rectang. $F B A=\int \frac{d e \sin . b}{\text { fin.e } \sqrt{\left(\operatorname{lin} \cdot e^{2}-\sin \cdot b^{2}\right)}}-$ $\int \frac{d e \text { cor.e efin. } b}{\text { fin. } e V\left(\text { fin. } e^{2}-\text { fin. }^{2}\right)}+$ Conft. $=B+A+$ Conft. $=($ evanefcente area quando $\left.B=0, \& A=180^{\circ}-F\right)$ $B+A+F-180^{\circ}$.

Sit $2^{\circ}$ triangulum obliquangulum ut $A C F$; tum $\mathrm{T}_{\mathrm{ab} . \hat{\jmath}}$. duBto latere perpendiculari $C E$, elt area $A D B=A+$ Fig.3. $B+D-\mathrm{r} 80^{\circ}, \&$ area $E C F=E+C+F-\mathrm{r} S 0^{\circ}$. Er- 
go area $A C F=A+B+C+F+E+D-180^{\circ}-180^{\circ}=$ $A+B+C+F-\mathrm{I} S 0^{\circ}=$ tribus angulis trianguli $-\mathrm{I} 80^{\circ}$.

Tro.s. Si triangulum fuerit $M N S$, ducto arcu perpendiculari lig.t. $T P$ in $M N$, productum crit area $M P T=M+S+$ $T+P-\mathrm{I} 80^{\circ}, \&$ area $O T P=O+T+P-180^{\circ}$; Ergo area $M N S=M+S-O=M+S+N-$ I $80^{\circ}$; feu exceftui trium angulorum fupra rectos duos.

\section{SCHEDIASM A VI.}

De Binomii Newtoniani indicem iwationalem babentis evolutione.

Tolutio Binomii Newtoniani habentis indicem, vel exponentem rationalem quemcumque five integrum, five fractum, ab omnibus fere Algebre Scriptoribus demonfratur. Id ipfum vero fine Calculi Differentialis fubfidio demonftrari minime poffe, quotiefcumque Binonii index irrationalis fuerit, opinio eft clarifimorum virorum, qui hac de re data opera egerunt. Hujufmodi autem demontrationes, quas videre hactenus licuit, non Infinitefimali tantum Calculo, fed Logarithnorum etiam Hyperbolicorum Doctrine innituntur, quo fit, ut imple$\mathrm{x} 2$ paullulum \& indirecte videantur. Nos itaque binas hic afferemus hujus Theorematis demonftrationes, alteram quidem ab Infinitefimali Calculo, fed fine ullo Logarithmorum fubfidio divefte depromptam, alteram ex fcla quantitatum irrationalium indole, fine ullius Calculi ope deductam. Sit igitur. 


\section{T H E O R E M A.}

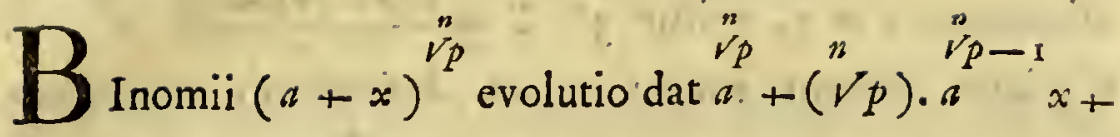

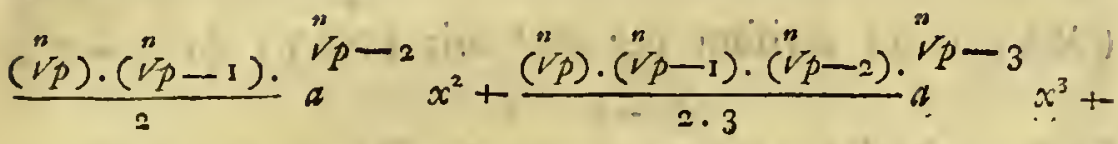

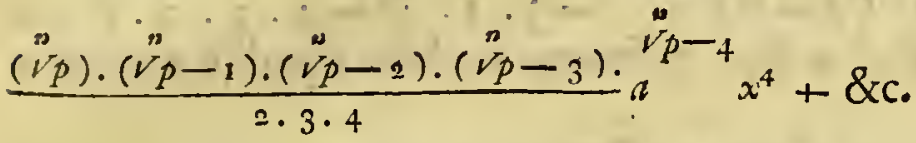
D. E.M O N S T R A T I O - I.

P irp

$F x^{5}+\& c .$, refque eo redacta erit, ut coefficientium indeterminatorum $A, B, C$, \&c. valores xquales oftendantur valoribus terminorum refpondentium in propofita formula: id vero ita affequi licet. Evanefcente $x$.in

$$
\stackrel{n}{\sqrt{p}}
$$

ifta $x$ quatione oritur $\mathrm{I}^{\circ} . A=a$. Sumatur porro æquationis ejufdem differentiale; prodibit æquatio $(U)$

(

$(V P) \cdot d x(a+x)=B d x+2 C x d x+3 D x^{2} d x+$ $4 E x^{3} d x+5 F x^{4} d x+\& c$., qua divifa per $d x$, factoque $x=0$, prodit $2^{\circ} . B=\left({ }^{n} p\right) \cdot \stackrel{n}{V p-s} \cdot$ Capiatur rurfus differentiale xquationis $(U)$, affumpto $d x$ conftanti;

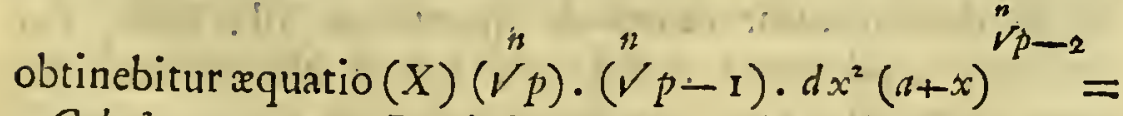
$2 C d x^{2}+3 \cdot 2 \cdot D x d x^{2}+4 \cdot 3 \cdot E x^{2} d x^{2}+5 \cdot 4$. $F x^{3} d x^{2}+\& c$., qux fi dividatur per $d x^{2}$, ac deinde $\mathrm{M}$ affu- 
Capiatur iterum eodem modo differentiale æquationis $(X)$, quod divifum per $d x^{3}$ erit $(\stackrel{n}{V} p) \cdot\left({ }^{n} p-I\right)$. $(\sqrt{ } p-2) \cdot(a+x)^{V p-3}=3 \cdot 2 \cdot D+4 \cdot 3 \cdot 2 \cdot E x+$ $5 \cdot 4 \cdot 3 \cdot F x^{2}+\& c^{2}$, pofitoque $x$ nihilo xquali, fiet $4^{\circ} \cdot D=$ $\frac{(n p) \cdot(\sqrt[n]{V p}-1) \cdot\left(v_{p}-2\right)}{2 \cdot 3} a^{n} a^{n}-3$ Eodem calculi progreffu

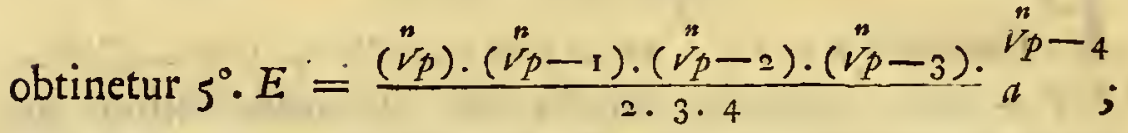
tum $6^{\circ} . F=\&$ c. Igitur affumptæ indeterminatx $A, B$, $C$, \&c. tales funt, quales exigit Theorema. 2. E. D.

\section{E M O N S T R A T I O II.}

Ndex irrationalis $V^{n} p$ eft limes, ad quem fine fine 1 accedunt fracti rationales $\frac{n}{n}, \frac{q}{r}, \frac{s}{t}, \&$ c. ultra quamlibet datam differentiam. Conftat id ex magnitudinum irrationalium conftitutione atque indole: Quod autem demonftratur de quantitatibus ad quantitatem datam magis magifque accedentibus, \& in eam tandem definentibus, vel potius ab ea ina/fignabiliter deficientibus, id ipfum demonfratur etiam de quantitate illa data, feu de limite, ad quem acceditur. Igitur Newtoniani Binomii formula, quæ pro indice quolibet fracto rationali locum habet, obtinebit etiam pari jure ubi illius index 
irrationalis fuerit, feu limes fractorum rationalium. 2. E. D.

$$
\text { S C H O L I O N. }
$$

T Demonftratione $\mathrm{I}$. alterutram Binomii partem va1 riabilem fingimus facultate. Geometris late conceffa, uti notum eft. Utraque pars fingi variabilis poffet per transformationem Binomii $(a+x)^{V p}$ in $a\left(1-\frac{x}{a}\right)^{V p}$, "vp $\quad v p$

feu in $a(1+y)$, pofito $\frac{x}{a}=y$. Evoluto autem bi$\overrightarrow{v p}$

nomio $(\mathrm{I}+y)$ per methodum ante traditam, ductifque terminis fingulis in $a$, , \& fubftituto $\frac{x}{a}$ pro $y$, eadem oritur expreflio qux prius. Si quis demonftrationen hanc I. conaretur infringere obiiciendo, inter

$(a+x), \& A+B x+C x^{2}+\& c$. xqualitatem affu$\mathrm{mi}$ haud aliter poffe, quam per meram petitionem principj, is fane inductionis perfettæ jura ignoraret, Algebramque ipfam, qux tota inductione nititur, ludibrio haberet. In exemplo non multum diffimili principj petitionem Cheynæo objecit acutus Geometra Moivræus, qui jure vapulat fummo Johanni Bernoullio, ut videre eft in hujus Operibus Tom.IV. Obfervat. in Moivr.

Cæterum vel me non monente liquet demonftrationem hanc illi etiam hypothef accomodari, qua Binomii exponens non irrationalis tantum, fed tranfcendens accipitur. 


\section{SCHED I A S M VII}

\section{Del Moto equabilmente accelerato:}

T $\mathbf{N}$ arcano geometrico s'afconde nella dottrina del moto accelerato: Un corpo "fpinto e follecitato in tutti gli iftanti da nuovi urti della gravità coftante, defcrive ne' fucceffivi tempetti infinitefimi degli (pazj, che confervano la proporzione de' numeri naturali, I, 2 , $3 ; 4,5,6,7$; \&c.; e per lo contrario ne' fucceffivi tempi finiti, il medefino corpo trafcorre fpazj proporzionali, ai numeri difpari $\mathbf{1}, 3 ; 5,7,9 ;$ I I , I $3 ; \& \mathrm{c}$. Come e perchè nel paftaggio che fi fa da'tempetti infinitefimi a tempi finiti, debba nafcere una sì grande varietà negli fpazj trafcorfi, non può negarfi eflere alduo, e malagevole a concepirfi. Le Geometriche dimoftrazioni d'una tal verità ottengono imperiofamente l'affenfo da chiunque ne fente la forza, e riducono i contraddittori al filenzio, ma lafciano la mente nello, ftato penofo di non poter intendere il come. Determiniano prima bene la natura, e lo ftato della Queftione: $\mathrm{E}$ evidente che nel primo tempetto infinitefimo, il corpo follecitato dalla gravità coftante, defcrive lo fpazietto infinitesmo I, nel fecondo tempetto infinite/imo. eguale, defcrive lo fpazietto 2 , nel terzo lo fpazietto 3 , \&rc. $;$ giacchè nel primo tempetto pel primo urto della gravità, la velocità generatrice è $\mathbf{~}$, nel fecondo tempetto pel nuovo urto della gravitì, la velocità generatrice è 2 , nel terzo tempetto è 3 , \&c. dal che fi. vede, che gli fpazietti infinite/mi forfi ne' fucceftivi infi- 
iinfinitefini tempufcoli, feguitano la proporzione de' numeri naturali: In ciò non vi lia ombra di difficoltà. Ma qual cangiamento di fcena quando ai tempetti infinitefimi fi foftituifono i tempi finiti! Allora gli fpazj. finiti fucceffrvamente defcritti non fi trovano più proporzionali ai numeri naturali, ma ai numeri difpari. Onde nafca in quefto paffaggio una tale diverfità, non $f i$ arriverà mai a comprendere, fe non fi efamina la natura medefima, e l'indole delle ferie de' numeri: Egli è manifefto, che gli fpazietti infinitefimi defcritti ne' fucceflivi infinitefimi tempufcoli, venendo rapprefentati dalla ferie de' numeri naturali $1,2,3,4,5,6$, 7, \&c.; e gli fpazj finiti trafcorfi ne' fucceffivi tempi finiti refultando dal fommare un numero infinito per volta de' fuddetti fpazj infinitefimi, è manifefto, dico che gli fpazj finiti fuccelfivamente defcritti verranno rapprefentati da una ferie di numeri, $i$ quali nafcono dal fommare una moltitudine infinita per volta dei numeri naturali $1,2,3,4,5,6,7$, \&c. Ora fe nella ferie de' numeri naturali fempre continuata, fi fomma fucceffivamente una moltitudine infinita di termini per volta, fi ottiene appunto una ferie di numeri proporzionali ai numeri difpari: il che io credo di poter dimoltrare generalmente e femplicemente così

$$
\text { D I M O S T R A Z I O N E. }
$$

1 Noto che in qualunque progreflione Aritmetica crefcente $f_{1}$ ha l'ultino termine, agginngendo al primo il prodotto della differenza moltiplicata pel numeto de' termini, diminuito dell' unità; licchè chiamando il pri- 
il primo termine $P$, il numero de' termini $N$, e la differenza $D, l$ ' ultimo termine $\grave{e}=P+(N-\mathrm{I}) D$; ed è noto ancora, che la fomma dei termini di ogni progreflione aritmetica fi ottiene multiplicando la fomma del primo, ed ultimo termine per la metà del numero de' termini, ond'è efpreffa per $(2 P+(N-I) D)$ $\frac{1}{2} N$. Nel cafo noftro effendo $D=1$, l'ultimo termine è efpreffo per $P+N-I$, e la fomma de' termini per $(2 \mathrm{P}+\mathrm{N}-\mathrm{I}) \frac{\mathrm{I}}{2} \mathrm{~N}$. Ciò pofto, nel primo tempo finito per avere lo fpazio trafcorfo, fi fomma per la prima volta una moltitudine infinita di termini della ferie naturale, la quale in quefto primo tempo folamente ha per primo termine l' unità, oflia $P=I$; onde fatta la foftituzione di $\mathrm{I}$ per $P$ nell' efpreffione della fomma, fi ha lo fpazio defcritto nel primo tempo finito, efpreffo per $(N+\mathrm{I}) \frac{1}{2} N$. Nel fecondo tempo finito eguale per avere lo fpazio nuovamente defcritto, fi fomma per la feconda volta una moltitudine infinita di ternini della ferie naturale, il di cui primo termine (com'è evidente) è l' ultimo della moltitudine prima antecedente, crefciuto d' un' unità, o fia $N+\mathrm{I}$; onde foftituito $N+\mathrm{I}$ per $P$, lo fpazio defcritto nel fecondo tempo finito viene rapprefentato da $(3 N+$ I $) \frac{1}{2} N$. Nel terzo tempo finito eguale fi ricava lo fpazio corrifpondente trafcorfo, fommando per la terza volta una moltitudine infinita di termini della 
ferie natutale, il di cui primo termine è l'ultimo della moltitudine antecedente feconda, crefciuto d' un' unità, il qual ultimo termine della moltitudine precedente $f_{1}$ ha foftituendo nell' efpreffione generale $P+N-I$ in luogo di $P$, il fuo valore $N+\mathbf{I}$, che produce $2 N$, a cui aggiungendo l' unità fi ottiene $2 N+$ I per primo termine di quella terza moltitudine, e quefto poi foftituito per $P$ nell' efpreffione generale della fomma, fi ottiene lo fpazio defcritto nel terzo tempo, efpreffo $\operatorname{per}(S N+\mathrm{I}) \frac{\mathrm{I}}{2} N: \mathrm{E}$ con un fimile ragionamento $\mathrm{fi}$ troverà lo fpazio defcritto nel quarto tempo finito, efpreffo per $(7 N+\mathrm{I}) \frac{1}{2} N$, nél quinto tempo per $(9 N+$ I $) \frac{1}{2} N$, \&c. Dunque nel moto equabilmente accelerato, gli fpazj fucceffivamente defcritti in tempi finiti eguali, fono rapprefentati da $(N+\mathrm{I}) \frac{\mathrm{I}}{2} N$, $(3 N+\mathrm{I}) \frac{\mathrm{I}}{2} N,(5 N+\mathrm{I}) \frac{\mathrm{I}}{2} \mathrm{~S},(7 N+\mathrm{I}) \frac{1}{2} N$, $(g N+\mathrm{I}) \frac{\mathrm{I}}{2} N, \&$ c., i quali termini divifi pel primo $(N+1) \frac{1}{2} N$, diventano I, $3-\frac{2}{N+1}, 5-\frac{4}{N+1}, 7-$ $\frac{6}{N+1}, 9-\frac{8}{N+1}, \& c . ;$ ed effendofi fómmato un numero infinito di termini per volta; e però $N$ effendo infinito, le frazioni $-\frac{2}{N+1},-\frac{4}{N+1}, \& c$. fvanifono. Nel moto adunque equabilmente accelerato, gli fpazj 
fpazj in tenipi eguali finiti fucceffivamente defcritti fono proporzionali ai numeri difpari I , 3, 5, 7, 9, \&x. Il che era \&c.

\section{S C O I I O.}

$F$ Inutile per l'avveduto Leggitore di quì avvertire; Lalle parole numero infinito, moltitudine infinita, \&c. non deve attribuirfi altro fignificato, fuor di quello che a fomiglianti efpreffioni attribuito viene generalmente da que moderni Geometri, che più addentro penetrarono nella vera metafifica del Calcolo Infinitefimale, quali fono Maclaurin, Simplon, Emerfon, Kaeftner, $D^{\prime}$ Alembert, \&c. Nella procedente dimoftrazione fi è fuppofto colla comune de' Geometri, che la gravità operi per impulf, ovvero che la di lei azione fia interrotta per intervalli di tempo infinitamente piccioli, ipotefi, che adottafi in grazia della brevità, che quindi rifulta nelle dimoftrazioni di fimil forta; febbene anche nella vera ipotef della natura, che l'azione della gravità fia continua, e fcorra e fluifca col tempo, non, farebbe nè lungo nè difficile il dimoftrare col metodo delle efauftioni, o colla dottrina de' Limiti i Teoremi Galileani full' accelerazione de' gravi, ficcome potrà accorgerfi di leggieri chiunque non è of pite, o novizio in fiffatte fpeculazioni. 


\section{SCHEDIASMA VIII.}

Del Moto de' Projetti nella Parabola Apolloniana:

T Na difficoltà, che da me propofta a qualche illuftre Geometra fembro meritare una feria rifleffrone, e la di lei foluzione da me immaginata in ap. preffo, potrebbero qui per avventura non inutilmente occupare l'attenzione de' Leggitori. Ecco la difficoltà .

$$
\begin{array}{llllllllll}
D & \text { I } & F & F & I & C & O & L & T & A
\end{array}
$$

E rette indefinite $A M, A N$, verticale l' una, Tab.3. 1 orizzontale l'altra rapprefentano le direzioni del- Fig.5. la gravità terreftre (che qui vuolf fupporre coftante, e di direzioni parallele), e dell' impeto primitivo comunicato al projetto lanciato orizzontalmente da $A$. Suppofto ora, che nel primo momento per l'impulfo della gravità il corpo cafchi da $A$, per uno fpazio eguale alla lineetta verticale infinitamente piccola $R B$, mentre per l' impeto di projezione fcorre lo fpazietto orizzontale $A R$, è manifefto, che per l'azione fimultanea delle due forze combinate defcriverà la diagonale $A B$. Nel fecondo momento, fe ceffaffe l'azione della gravità, andrebbe il corpo per la fua inerzia lungo la retta $B S$, eguale e continua alla $A B ;$ ma ricevendo in $B$ un nuovo urto dalla gravità, per cui in quell' iftante cafcherebbe verticalmeute da $B$ per uno fpazio eguale alla verticale $C S, \circ B R$, fara coftretto a muoverfi per la diagonale $B C$; e prodotta la verticale $C S$ in 2 ; $\mathrm{N}$

tro- 
trovafi $C Q$ tripla di $B R$, poichè nel triangolo $A S Q$ per le parallele $S Q, B R$, effendo $A S$ doppia di $A B$, ed $A Q$ di $A R$, è in confeguenza la $S Q$ doppia della $B R$; e quindi la $C 2$ tripla di $B R$. Nel terzo momento, fe il corpo non ubbidiffe alla gravità, in virtù della fua inerzia $S_{1}$ porterebbe per $C V$ eguale, e continua a $B C$, ma per l'urto ricevuto in $C$ dalla gravità, in virtù del quale da $C$ difcenderebbe per uno fpazietto eguale alla verticale $D V$, o CS, oBR, defcriverà di bel nuovo la diagonale $C D$; e prodotta, come dianzi, la verticale $D V$ in $P$, trovafi la $D P$ feftupla della $B R$, effendo la $V T$ doppia di $C S, O B R$, e T $P$ tripla di $B R$. Per fimil modo nel quarto momento il corpo per la fola inerzia defcrivereobe la retta. $D F$ nella diritcura di $C D$, ed uguale a $C D$, ma follecitato in quel momento dall' azione della gravità, che da $D$ lo fpinge all' ingiu per uno fpazietto eguale alla verticale $E F$, o $B R$, torce nuovamente, e fi muove per la diagonale $D E$; e qui pure ritrovafi, che prolungandofi la $E F$ in $I$, rifulta la retta $E I$ decupla di $E F, \circ B R$; Imperciocchè nel triangolo $F C G$ effendo la $C F$ doppia di $C D$, anche la $F G$ farà doppia della fua parallela $D V$, $\circ R B$, e nel triangolo $G B H$ effendo $G B$ tripla di $B C$, farà $G H$ tripla della parallela $C S$, o $B R$, e nel triangolo $A H I$ la $A H$ quadrupla di $A B$, rende eziandio la $H I$ quadrupla di $B R$; dunque l' intiera $E I$ è decupla di B.R. Collo fteffo difcorfo $f i$ dimoftrerà nel quinto momento l'ordinata $L K$ uguale a quindici $B R$, nel fefto a venti $B R$, e così profeguendo. Dal che ì manifefto, che prendendofi le afciffe $A R, A \cap, A P, A I, A K, \& \mathrm{c}$ fecondo 
do I' ordine de' numeri natturali I, $2,3,4,5$, \&c. ; le ordinate $B R, C 2, D P, E L, L K, \&$ c. della Curva $A D L$ fi vanno fuccedendo fecondo la ferie de' numeri triangolari I , 3, 6, 10, 15. \&c. Ora qui appunto è ripofta la difficoltà ; imperciocchè e chi non fa, che nella Parabola Appolloniana $A D L$ defcritta da' projetti nel vuoto, o in un mezzo non refiftente תell' ipotefi della gravità coltante, e delle direzioni di effa parallele, alle afcifte crefcenti, fecondo la progreffrone de'numeri naturali corrifpondono le ordinate alla conveffità crefcenti, fecondo la ferie de'numeri quadrati $1,4,9$, 1 6,25 , \&c. ? E come potrebbono due ferie tanto fra lor differenti adattarfi ad efprimere le medefime quantità?

..... Nimirum bace illa Cbarybdis.

Hos Helenus fcopulos, bac faxa borrenda canebat.

\section{$S \circ I U Z I O N E$.}

Imoftreremo prima, che fe ne' tempufcoli infinim tefimi crefcenti fecondo l' ordine de' numeri naturali, e rapprefentati dalle afcife $A R, A Q, A P$, $A I, A K, \& c$. le ordinate corrifpondenti fi vanno fuccedendo fecondol' andamento de' numeri triangolari, nei tempi però finiti, e quanto vogliafi piccioli crefcenti cone dianzi le ordinate feguitano il tenore de' numeri quadrati. In fatti l' efpreffione generale de' numeri triangolari fi fa effere $\frac{n(n+1)}{2}$, denotandofi per $n$ l'indice o efponente del termine della ferie. Se ora fupponfi $\mathrm{N} 2$. deno. 
denotare $n$ un infinito numero di momenti, o tempufcoli infinitefimi eguali, vale a dire un tempo finito, $2 n$ il doppio, $3^{n}$ il triplo, \&c., egli è vifibile, che ai tempi finiti $n, 2 n, 3 n, 4 n, 5 n$, \&c. corrifponderanno le ordinate $\frac{n(n+1)}{2}, \frac{2 n(2 n+1)}{2}, \frac{3 n(3 n+1)}{2}$, $\frac{4 n(4 n+1)}{2}, \frac{5 n(5 n+1)}{2}$, \&c. dove annullandofi I nel fecondo fattore pel valore di $n$ infinito in ordine a quella unità, fi trasformano quelle ordinate in $\frac{n^{2}}{2}$, $\frac{4 n^{2}}{2}, \frac{9 n^{2}}{2}, \frac{16 n^{2}}{2}, \frac{25 n^{2}}{2}, \& c_{\text {. }}$, ovvero procedono vilibilmente fecondo l' andamento de' numeri quadrati $I$, $4,9, \mathrm{r} 6,25,8 \mathrm{c}$.

Ma perchè nè tempetti infinitelimi accade l'oppofto? perchè le ordinate trovanfi allora non ai numeri quadrati, ma ai triangolari proporzionali? perchè finalmente quefto falto nel paffaggio da' tempi infinitefimi ai finiti? Tutto nafce, come in un cafo analogo lì è fatto vedere nella precedente Memoria, dall' ipotefi che fi affume della gravità operante pet impulfi momentanei, e oziofa per intervalli infinitamente piccioli. In quefta ipotefi, che non è certamente quella della natura, non dce far maraviglia fe incontrafi un rifultato, che non è conforme alla natura, Si tẹrà però fempre più indietro il paradoflo, e da' tempi infinitefimi di primo ordine fi confinerà a quelli di fecondo ordine, qualora ciafcun tempetto infinitefimo di primo ordine vorrà concepirfo divifo in un infinito numero di altri tempufcoli fra fe eguali di fecond' ordine, e in ciafcuno di quefit u'ti- 
ultimi s' immaginerà un impulfo della gravità ; imperciocchè in tal fuppofto collo fteffo ragionamento di prima fi moftrera, che, le fole ordinate corrifpondenti ai tempi infinirefini di fecond'ordine procederanno fecondo i numeri triangolari, ma negl infinitefimi, di primo ordine feguiteranno il progreffo dé numeri quadrati . E così pure da' tempi infinitefini di fecond' ordine fi potrà confinare l'antinomia agl' infinitefini di terz' ordine, e cosi fempre piu indietro. Dal che fi-rende manifeno che ammeffa, ficcome è giufo, la perfetta continuita cosi del tempo come dell' azione della gravità , ed elfendo una tale continuita il Limite, a cui fempre fi avvicina oltre qualunque affegnabile differenza l' interruzione fempre via via decrefente della gravita e del tempo, feguiteranno le dette ordinate in ogni tenpo, o tempufcolo comunque finto o infinitefimo di qual fiafi ordine la ferie de numeri, quadrati, ficcome dalla Dottrina de' Limiti fi raccogliẹ.

\section{$S \subset O$ L I O :}

¿U propofto nel Gionale di Trevoux l'anno 1701. a tutti i Geometri il Problema di ritrovare la Curva, nella quale effendo prefe le ordinate alla concavità fecondo la ferie de' numeri naturali, le afciffe corrifpondenti fono come i numeri triangolari. 'L' Anonimo proponente faceva eftremamente valere la Curva incognita, la decantava dotata di proprietà importanti per la pratica delle Arti, ed infinuava forfe con arte, non effer quella la Parabola. Ma non vi eta bifo- 
gno di gran geometria per accorgerf, che la Curva cercata non poteva effer altro che la comune Parabola d' Apollonio; imperciocchè effendo $\frac{y^{2}+y}{2}$ la formula generale de numeri triangolari, dove y rapprefenta fucceffivamente $i$ numeri naturali, fi avrà per l' ipotefi del Problema $\frac{y^{2}+y}{2}=x$, equazione come ognun fa alla $\mathrm{Pa}-$ rabola Apolloniana, defcritta col paiametro $=2$, nella quale prendefi per linea delle afciffe la retta, che tirafi parallela all affe ad una diftanza uguale ad un quarto del parametro, e piglianfi per ordinate le perpendicolari a quefta linea Ma quel Sig. Anonimo poteva rendere molto più generale il Problema, con fupporre le afciffe crefcenti non fecondo i foli numeri triangolari, ma fecondo i numeri poligoni di qualunque Claffe; ed averebbe fempre ritrovato, che la fua pretefa Curva incognita è fempre la ftella Parabola Apolloniana: avvegnachè in fatti la formula generale di tutti $\mathrm{i}$ numeri poligoni è $\frac{(n-2) y^{2}-(n-4) y}{2}$, nella quale $n$ efprime il numero de' lati del dato poligono, e y rapprefenta fucceffivamente i numeri naturali; 'ond' è $\frac{(n-2) y^{2}-(n-4) y}{2}=$ $x$ per l'ipotefi ; e però la Curva ricercata una Parabola, per defcrivere la quale bafta trasformare quefta equazione nell'altra $y^{2}-\frac{(n-4)}{n-2} y=\frac{2}{n-2} x$, dalla quale a un tratto $f$ fcorge, che il parametro $\grave{e}=\frac{2}{n-2}$, che la li- 
nea delle afciffe $x$ è una parallela all' affe, lontana per un intervallo $=\frac{n-4}{2 n-4}$, che l'origine delle afciffe $x$ è nel concorfo di quefta retta colla Curva, e che in fine le ordinate $y$ fono le rette perpendicolari alla fteffa.

\section{SCHEDIAS M A IX.}

Sopra un Fenomeno della $V i f f a$.

T L Signor Buffon nella fua Iftoria Naturale in quel 1 luogo, dove parla del fenfo della vifta, per if́piegare onde avvenga, che un oggetto rimirato con un occhio folo, ceteris paribus apparifca illuminato una fola tredicelima parte meno, che veduto con ambedue gli occhi, coficchè la chiarezza, o illuminazione d'un oggetto offervato con un occhio folo ftia all' illuminazione del medefimo oggetto veduto con ambi gli occhi, in parità di tutte le altre cofe, come 12 a 13 , e confeguentemente $i$ caratteri d' un libro illuminato da-r 2. fiaccole uguali, i quali fi leggono con ambi gli occhi. precifamente in una certa diftanza, non poffano leggerli alla diftanza medefina con un occhio folo, fenza aggiungere una tredicefinia fiaccola alle prime dodici, come con replicate fperienze ha ritrovato il famolo Jurin; per ifpiegare, diff, quefto maravigliofo fenomeno, immagina un' ingegnofa ipotefi, a cui non manca, che l' appoggio dell' efperienza, e la verificazione dei fatti, per effer vera. Ei concepifce, che $i$ due nervi ottici, o le loro filamenta fi unifcano nel fenforio co-

risune, 
inune, o prima di giungervi, interfecandor fotto un angolo di I I $4^{\circ}$.

Ciò pofto fi può difcorrer così: I raggi lucidi riflettuti dall' oggetto nel fondo degli occhi fcuotono con ugual forza ed energia in ambe le parti le fibre nervofe delle duc retine, e quindi gli fpiriti animali, i quali o incontrandof coilloro movimenti nella congiunzione dei nervi ottici, o fpingendo da due bande gli fpiriti, che nella medefima unione fi ritrovaro, vellgono a comporre un terzo movimento rifultante dai due primi moti, e ad effi equipollente, il qual terzo movimento farà efpreffo per la diagonale del parallelogrammo, i di cui lati rapprefentano i due moti componenti ed eguali, 'e comprendono $1^{\prime}$ angolo di I $14^{\circ}$; e quefto terzo movimento portato quindi al fenforio comune, o alla fede dell' Anima rifreglia in effa una fenfazione corrifpondente, o fia la vifione dell' oggetto, che fi rimira. Qualora poi queft oggetto con un fol occhio fi riguarda, l' impreffione o il moto prodotto full' organo della vifta, e propagato al comun fenforio è uno dei due moti uguali, che dal-medefimo oggetto nei due nervi ottici fi producono, quando con ambi gli occhi fi contenipla, dai quali due moti combinati nafce il terzo moto compofto. Dunque l' impreflione eccitata nell' organo da un oggetto rimirato con un fol occhio, ftarà all' impreffione fatta dal medefimo oggetto veduto con tutti e due, come fta il lato di un rombo, che ha un angolo di I I $4^{\circ}$, alla fua diagonale, la quale paffa pel vertice di queft: angolo; ed effendo, come volgarmente fupponefi, la vividita delle fenfazio- 
ni proporzionale alla forza delle impreffioni organiche corrifpondenti, farà parimente l'illuminazione, o chiarezza apparente d' un oggetto, che fi guaràa con un fol occhio, all' illuminazione o chiarezza del medefimo offervato nella fteffa diftanza con tutri e due, come il lato del rombo mentovato alla diagonale, ed -effendo quefto lato alla diagonale in un rombo, che abbia un angolo di $114^{\circ}$ gradi, come 12 a 13 (perchè i feni di due angoli di 57 , e 66 gradi ftanno appunto come, 12 a 13 , ed a quefti. feni fono proporzionali i lati oppofti nel triangolo, i quali fono il lato e la diagonale del rombo); ne verrà, che l'illuminazione. dell' oggetto nel primo cafo, ftará all' illuminazione nel fecondo calo, appunto come I 2 a I 3 .

Si concepifca in fatti, che $M C B$ fia l'angolo formato dai due nervi ottici $M C, C B$ concorrenti in $C$, nell' allonTab.3. Fig. 6 . tanarfi dagli occhi $x, y$. Compito il rombo $M B$, i lati $C M, C B$ eguali ef primeranno i movimenti eguali, eccitati nei due nervi ottici dai raggi dell' oggetto, e la diagonale $C A$ rapprefenterà il moto compotto, il quale dovrà ftare a $C B$ come 13 a $: 2$. Ora fe nel triangolo ifofcele $A C B$, fi fuppone $A C$ di 13 parti, $C B, \circ B A$ di I 2 , fi rinviene fubito $\mathrm{l}$ angolo $A C B$, e per confeguenza il fuo doppio ricercato $M C B$. Si conduca in fatti dal punto $C$ una perpendicolare $C Q$ alla retta $B A$, la qual perpendicolare caderà verfo l'ángolo $B$ acuto, per effere il quadrato di $C A$, offia 169 , minore de due quadrati di $C B$, e $B . A$, ollia di 288 . Quindi col centro $C$, c coll' intervallo del lats minore $C B$, fi defcriva il cer-, chio $B M R$, il quale, con'è evidente, tagliera in $F$, c. in $S$ i due lati $B A$, ed $\Lambda C$. Producafi il lato $A C$, 
finchè concorra colla periferia del cerchio in $R$. Ciò fatto fi avrà $R A \times A S=B A \times A F$, ovvero $\overline{B C+B A} \times$ $\overline{C A-C B}=B A \times A F$; donde fi ricava $B A: B C+C A:$ : $C A-C B: A F$, oflia I 2:25:: I : $\frac{25}{12}=A F$; е $B F=$ $12-\frac{25}{12}=\frac{144-25}{12}=\frac{119}{12}$; e però $B Q, 0 Q F=$ $\frac{1}{2} B F=\frac{119}{24} ;$ e $Q A=2 F+F A=\frac{119}{24}+\frac{25}{12}=$ $\frac{119+50}{24}=\frac{169}{24}$, il di cui logaritmo è uguale a $l_{1} 69-$ l $42=2.2278867-$ I. 3802 I I $2=0.8476755$. Il logaritmo poi di $C A$, oflía di I 3 , è I. I I 39433 . Ora nel triangolo rettangolo $C A Q$, fta $C A$ ad $A Q$, come il feno tutto al feno dell' angolo $A C Q$, e però dalla fomma del logaritmo del feno tutto, e del lato $A Q$, offia da 10.8476755 fottraendo il logaritmo del lato $C A$, ovvero I. I I 39433, fi avrà 9.7337322 , che farà il logaritmo del feno dell' angolo $A C Q$, del qual logaritmo il proflimamente minore 9.7335693 , nelle Tavole dà l'angolo di $32.47 \cdot$, del qual angolo volendofi fapere anche $i$ fecondi, fi faccia come $196 \mathrm{I}$, ecceffo dẹl logaritmo del feno di 32.48 fopra il logaritmo del feno di $32^{\circ}, 47^{\prime}$, a $60^{\prime \prime}$, ecceffo dell arco, così 1629 , ecceffo del noftro logaritmo fopra il fuo proffimamente minore, al quarto proporzionale, che efprimerà i fecondi da attribuirfi all' angolo di 32.42, i quali fecondi fono $49 \frac{1651}{1961}$. Dunque l'angolo $A C 2$ fara 
farà di 32. $47 \cdot 49^{\prime \prime} \frac{1651}{1965}$. Per ritrovare ora il feno dell' angolo $Q C B$, fi ha $B C$ a $B Q$, come il feno tutto al feno dell' angolo $B C Q$; e perciò prendendo il logaritmo di $B Q$, che è uguale a $l$ I $19-l_{24}=2$. 0 $755470-1.3802112=0.6953358$, e fommandolo col logaritmo del feno tuttó 10.0000000 , e dalla fomma 10.6953358 fottraendo il logaritmo di $B C$, offia di 12 , che é 1.0791812 , il refiduo 9.6161546 farà il logaritmo del feno dell' angolo ricercato, del qual logaritmo il proffimamente minore 9.6160598 , nelle Tavole dà l' angolo di 24.24 . A ritrovare i fecondi fi faccia, come fopra, 2784 , differenza de' due logaritmi proflimi nelle Tavole, a $60^{\prime \prime}$, così 948 , differenza fra il noftro logaritmo, e il fuo proflimamente minore, al quarto proporzionale, che è $20 \frac{1200}{2784}$; e però l'angolo 2CB farà di $24.24 \cdot 20^{\prime \prime} \frac{1200}{2784}$, ovvero di $24 \cdot 24 \cdot 20^{\prime \prime} \frac{25}{5^{8}}$.

Dunqué l' angolo $A C B=A C Q+2 C B$ farà di 57. I 2. $9 .+\frac{165^{\prime \prime}}{1961}+\frac{25^{\prime \prime}}{5^{8}}$; offia di $57 \cdot 12.9^{\prime \prime}+\frac{49025^{\prime \prime}+9575^{\prime \prime}}{11373^{8}}$; o finalmente di $57.12 .10 \frac{31045}{11373^{8}}$, onde l' angolo $M C B$ doppio di $A C B$ farà di I I $4.24 \cdot 20 \frac{62090}{11373^{8}}$. Il che era da ritrovarfi.

$$
\mathrm{O}_{2} \quad \mathrm{Per}
$$


Per dimoltrare lo Ateffo, facendo ufo delle note formole trigonometriche, fi proceda così : L'angolo. $M C B$ fi chiami $X$, e però $A C B \frac{1}{2} X$; l' angolo $A B C$ avrà lo ftefo feno del fuo complemento $M C B$, offa $X$. If Problema fi riduce a trovare un tal angolo $M C B$, che G abbia $C B: C A:: N: M::$ I2: $_{13}$. Sia dunque

$$
\begin{gathered}
\text { L E M MA. } \\
\text { Sin. } \frac{1}{2} x=V\left(\frac{1-\operatorname{cof} x}{2}\right) \\
\text { D } 1 \text { M. }
\end{gathered}
$$

F Noto, che dati due archi $k, q$ fi ha fin. $(k+q)=$ fin. $k$ cof. $q+$ cof. $k$ fin. $q$; onde fuppofto $q=k=\frac{1}{2} x$, fi ottiene fin. $x=2$ fin. $\frac{1}{2} x$ cor. $\frac{1}{2} x=2$ fin. $\frac{1}{2} x \sqrt{1-\left(\text { fin. } \frac{1}{2} x\right)}$; $(\text { fin } x)^{2}=4\left(\text { fin } \frac{1}{2} x\right)^{2}-4\left(\text { fin } \frac{1}{2} x\right)^{4} ;$ dalla qual equazione trattata fecondo il metodo delle quadratiche, fit ha (fin. $\left.\frac{1}{2} x\right)^{2}=1 \pm V \frac{\sqrt{1-(\text { fin } x)^{2}}}{2}=\frac{1 \pm \operatorname{cor} x}{2}$, Ora il primo di quefti due valori di (fin. $\left.\frac{1}{2} x\right)^{2}$ è affurdo, perchè quando è $\frac{1}{2} x=0$, diventando $\left(\text { fin. } \frac{1}{2} x\right)^{2}=0$, cof. $x$ diventa eguale ad I, onde farebbe $\frac{1+1}{2}=o$; il che è affurdo. Dunque (fin. $\left.\frac{1}{2} x\right)^{2}=\frac{1-\operatorname{cof} x}{2} ;$ fin. $\frac{1}{2} x=V\left(\frac{1-\operatorname{cof} x}{2}\right)$. II che ec. 


\section{Deli' Accademia . \\ P R O B L E M A :}

Ritrovare qual debba effere langolo $M B C$, affuctbe $C B$ a $C \mathcal{A}$ fria comie $N$ ad $M$.

\section{$S$ o $x$.}

$C B, \circ B A$ tha a $C A$, come fin. $A C B$ a fin. $C B A, \underset{\text { Tab. }}{ }$. offia come $V\left(\frac{1-\operatorname{cof} x}{2}\right)$ a fin. $x$; dunque $n$ fin. $x=$ $m V\left(\frac{1-\operatorname{cor} x}{2-1}\right) ; n^{2}(\text { fin } x)^{2}=\frac{m^{2}-m^{2} \operatorname{cor} x}{2} ; 2 n^{2}-2 n^{2}$ $(\operatorname{cof} x)^{2}=m^{2}-m^{2} \operatorname{cof} x ; \frac{2 n^{2}-m^{2}}{2 n^{2}}=(\operatorname{cof} x)^{2}-\frac{m n^{2}}{2 n^{2}}$

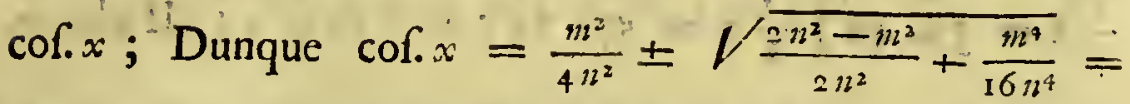
$\frac{m^{2}}{4 n^{2}} \pm \sqrt{\frac{16 n^{4}-8 m^{2} n^{2}+m^{4}}{16 n^{4}}}=\frac{m^{2} \pm 4 n^{2} \mp m^{2}}{4 n^{2}}=\frac{m^{2}}{2 n^{2}}-$ I . II valore $=$ I non ha luogo, perchè l' angolo ricercato farebbe nullo. L'altro poi è $\frac{\overline{3}^{2}}{2 \cdot \overline{12}^{2}}-\mathrm{I}=\frac{169}{288}-\mathrm{I}=-$ $\frac{119}{288}$, che fi deve fupporre riferito al raggio 1, come è evidente. Ora per ritrovare nelle tavole l'angolo corrifpondente al cofeno, bifogna fare come il raggio:i al cofeno $-\frac{119}{288}$, cosi il raggio 10000000 al cofeno $4131944 \frac{128}{288}$. Si fa, che cof $x=$ fin. $\left(90^{\circ}-x\right)=-1$ 4 I 3 I $944 \frac{128}{288}$, e fi trova fin $(24 \cdot 24)=4$ I 3 I 944 
110

$$
\text { A } \mathbf{T} \mathbf{T} \mathbf{r}
$$

$\frac{128}{288}$, offia fin. $-(24 \cdot 24)=-4131944 \frac{128}{288}$. Dunque fin. $\left(90^{\circ}-x\right)=$ fin. $-(24 \cdot 24 \cdot) ; 90-x=-24 \cdot 24 ;$ $x=90+24 \cdot 24=$ I I $4 \cdot 24$. Il che \&c.

\section{Qucfto Problema fi pud fciorre più fpeditamente} in queft altro modo.

\section{S O I U Z I O N E II.}

CIn. $\frac{1}{2} x: 2$ fin. $\frac{1}{2} x \operatorname{cof} \frac{1}{2} x:: n: m ; m$ fin. $\frac{1}{2} x=2 n$ 1 fin. $\frac{1}{2} x \operatorname{cof} \frac{1}{2} x ; \operatorname{cof} \frac{1}{2} x=\frac{m}{212}=\frac{13}{24} \times 10000000=$ $5416666 \frac{2}{3}=$ fin. $\left(90-\frac{1}{2} x\right)=\operatorname{lnn}\left(3_{1}^{2} \cdot 4_{1}^{8}\right) ; 90 \div$ $\frac{1}{2} x=32.48^{\prime} ;-\frac{1}{2} x=57$. I $2 ; x=1$ 1 $4.24 \cdot$ Il che ec.

\section{SEOLIO.}

T Opo è quì di offervare, che ficcome l' ipotefi dell' illuftre Signor-Buffon intorno al cóncorfo de' nervi ottici fotto l'angolo di $14^{\circ}$, non pare dalle offervazioni anatomiche confermata, fi è peró penfato di retrificarla con foltituire al concorfo de nervi quello delle loro minime, edultime filamenta, due delle quali fi concepifcono partire dal primo punto della loro origine nel comune fenforio, fotto un angolo determinato; fuppofizione, che non effendo, nè potendo mai effere contraddetta dalla più fottilé, e recondita Anatomia, e fervendo altronde mirabilmente a fpiegare uno de' più arcani, e portentofi fenomeni della Vilta, viene quindi ad 
ad acquiftare uno certamente non piccolo gradu di probabilità - Quanto poi ai feguenti Corollarj, effi compariranno fenza dubbio al primo afpetto molto ftrani, e paradoffi; ma forfe non parrà meno ftrano l'intendere, che vi fono de' fatti nella Storia Medica, i quali confermano là verità di alcuni de' mentovati Corollarj, e che la mia fteffa offervazione fatta fopra $\mathrm{i}$ miei occhi affai difuguali nella potenza vifiva, e fopra quelli di alcuni miei conofcenti, è venuta con mia grande forprefa molto acconciamente all' appoggio di taluno de' medefimi Cornllarj, come del VI. \&c., di che parleraffi più ampiamente in altra occafione, in un Opufcolo intorno a quefto ftefto argomento.

$$
\text { C O R O L L A R I O I. }
$$

C l'angolo de' nervi ottici $M C B$ fard di $120^{\circ}$, l'og$\checkmark$ getto apparirà ugualmente illuminato, guardandolo con un occhio, con due, perchè allora $A, G$ ùguale a $C B$; e però coloro, nei quali $i$ nervi ottici fi unifcono fotto un angolo di $120^{\circ}$, non fanno alcuna perdita diventändo monocoli, perchè confervano anche in quefto ftato come prima l'ifteffo grado di forza, e d' intenfione nella poténza vifiva .

$$
\text { COOR:OLI:A R IO II. }
$$

Uelli poi, che avranno l' angolo $M C B_{\text {maggio- }}$ re di $120^{\circ}$, in luogo di perdere, diventando monocoli, vi guadagneranno, effendo in quefti tali la forza vifiva femplice in un fol occhio, maggiore della potenza 
tenza vifiva compofta in ambedue gli occhi, mentre in quefto cafo il lato $C B$ è maggiore della diagonale. $C \cdot A$.

$$
\text { Cor O L L A R I O III: }
$$

(Uanto maggiore di $120^{\circ}$ farà l'angolo $M C B$, tanto maggiore farà l' acquifto fatto da'monocoli nella potenza vifiva; e per l'oppofto quanto minore di $120^{\circ}$ farà queft angolo, tanto maggiore farà lo fvantaggio; e la perdita de monoculi.

\section{O R O L I A R I O IV.}

C E l'angolo $M C B$ farà infinitamente piccolo, i mo$D$ nocoli perderanno la metà della vifta; mentre fta $C B, \circ B A$ a $C A$, come il feno dell' angolo $A C B$, al feno dell angolo $C B A$, o del fuo complemento a due retti $M C B$; e il feno dell' angolo infinitefimo $A C B$, fta al feno dell angolo infinitefimo doppio $M C B$, come fta I a 2, come fi dimoftra nella Geometria degl' Infiniti.:

$$
\text { COROLI A RIO V. }
$$

E l'angolo $M C B$ differifce da due rette per un angolo infinitefimo, allora diventando infinitamente piccola la diagonale $C A$, i Binocoli perderanno tutto, e i. Monocoli per l'oppotto guadagneranno tutto; i primi faranno ciechi, e gli altri veggenti. 


\section{COROLIARIO VI.}

CE la potenza vifiva farà minore in un occhio; che $S$ nell altro, anche la vifione compofta fara minore, che non farebbe, fe tutti e due gli occhi forfero di ugual forza e portata; perchè effendo in quefto cafo $G M$, o $B A$-minore di $C B$, fi diminuifce, com'è vifibile, anche- la diagonale $C A$.

\section{COROIO I A R I O VII.}

CE la forza vifiva d'un occhio ftarà alla forza vifiva $\$$ dell' altro, come I I 9.3 I 44 . la forza vifiva compofta farà uguale alla forza vifiva femplice dell' occhio fano, e niente fi perderà nella facoltà del vedere, perdendo l'occhio più debole. Imperciocchè fta $B F$ a $B A$, offia $B C$, come $\frac{119}{12}$ a I 2 , ovvero come II9. a I44., e allora la diagonale $C A$ incontrando la retta $B A$ nel punto $\leftleftarrows$, diventa raggio del Cerchio, e però uguale a $C B$.

\section{O ROILARIO VIII.}

Uindi fe la forza vifiva d' un occhio ftarä alla forza vifiva dell' altro, in minor ragione di ing. a r.4., la vifione congiunta di ambedue farà fempre minore della vifione femplice dell' occhio fano; e perciò fi fcapiterà a fervirfi di ambi gli occhi, e fi vantaggerà a fervirf d' un folo, cioè del più forte: ed ecco un nuovo cafo di vantaggio per li Monocoli, e di dan- 
no ai Binocoli. Tutto ciò $f i$ vede manifefto dall' effere in quefto cafo la retta $B A$ minore di $B . F$, e però la diagonale $C A$ minore del raggio del cerchio, ovvero di $C B$.

\section{O R O L I A R I O IX.}

C Tando fempre in quefta fuppofizione di difuguaglianz $\checkmark$ za nella forza e portata degli occhi, il maflimo fvantaggio per li Binocoli fuccederà allor quando la forza vifiva d'un occhio avrà a quella dell' altro, la proporzione di I I g. a 288 , la qual proporzione è fubdupla di quella, che produceva la vifione compofta, eguale alla femplice (Coroll. VII:) .. Di fatto la diagonale $C A$ diventa minore di qualunque altra, quando coincidendo con $C Q$, cade perpendicolarmente fopra: $B A$, come è evidente; c allora $B Q$ fta a $B C$ come $\frac{19}{24}$ a I2, ovvero come II g. a 288 . Per ritrovare in quefto cafo il rapporto della vifione compolta alla femplice, fi cerchi il valore della diagonale $C Q$, e $f_{i}$ paragoni con $C B$. Si ha $C Q=\sqrt{C B^{2}-B Q^{2}}=\sqrt{\frac{144-\frac{14161}{576}}{176}}=$ $\sqrt{ } \frac{68783}{576}=$ al numero, che corrifponde al logaritmo di $\sqrt{68783} \frac{1}{576}$, il qual logaritmo è uguale a $\frac{1}{2} l 68783-\frac{1}{2} l 576$. Per ritrovare poi nelle tavole ordinarie, che non vanno oltra 10000 , il logaritmo di 68783 . fuppongafi divifo per ro. il numero propofto 68783 , e fi avrà $\overline{6878+\frac{3}{10}} \times$ I0 $=68783$; e peró $l 68783=l$ ro + 
$7 \overline{6878+\frac{3}{30}} \cdot$ Ora il logaritmo di 6878 è 3.8374622 ; quindi per ritrovare il logaritmo di $6878+\frac{3}{10} \mathrm{fifc}$ cia come I, ecceflo di 6879 . fopra 6878 . a $63 \mathrm{I}$. ccceffo de' logaritmi, cosi $\frac{3}{10}$ al quarto $189 \frac{3}{10}$, il quale aggiunto al logaritmo di 6878 . darà $3 \cdot 83748$ I I $\frac{3}{10}$ per il logaritmo di $6878 \frac{3}{10}$, e a quefto logaritmo aggiungendo quello di ro, e poi dividendo la fomma per 2 , fi otterrà 2. $4187405 \frac{13}{20}=\frac{1}{2} l 68783$, e fottraendo $\frac{1}{2} l 576=\mathrm{I} \cdot 3802 \mathrm{II2} \frac{\mathrm{I}}{2}$, fi avrà finalmente I. 03 85293 (nègletta la frazione $\frac{\mathrm{I} 3}{20}$ ) $=\frac{\mathrm{I}}{2} l 68783-\frac{1}{2} l_{5}$ $7^{6}=l V_{-57^{6}}^{688^{8}}$. Cercando nelle tavole quefto logaritmo, fi trova il fuo proflimamente minore 1.0000000 , a cui corrifponde il numero 10 . $\mathrm{Per}$ aver dunque il numero corrifpondente al noftro logaritmo, $f_{1}$ faccia come 413927: ecceffo del logaritmo di 1 r. fopra quello di Io, ad I; cosi $3^{8} 5293$. ecceffo del noftro logaritmo foprà lo fteffo logaritmo di Io, al quarto $\frac{3^{85293}}{41392 ?}$, da aggiungerfi al numero $r O$. per ottenere il numero corrifpondente al noftro logaritmo. Dunque fatta $C B=$ I 2, farà C Q proflimamente $=10+\frac{385293}{413927}=\frac{45^{2} 4563}{413927}$, e confeguentemente ftarà $C$ Q a $C B$, come fta $\frac{45^{2} 45_{6}}{413927}$ a I 2, ovvero come $45245^{6} 3$. a 4967124 . proffimamente; dal $\mathrm{P}_{2}$ che 
che $f$ raccoglie, che qualora la forza vifiva $d$. un occhio avrà a quella dell' altro la proporzione di I I g. a 288 , la forza vifiva compofta, che in tal cafo è la minima, avrà alla facoltà vifiva femplice dell' occhio più forte, la ragione di $45245^{6} 3 \cdot$ a 4967 1 24 - proffimamente.

\section{SCHEDIASMA X.}

Se iazione "dclle Forze Luni-Solari pud infuire fullo fato del Barometro, ed alterame fenfibilnzente $l$ altezza.

T A bella Opera di frefco pubblicata dal ch. Sig. Toaldo, illuftre Profeffore d'Aftronomia nell' Univerfità di Padova, Opera che comprende la più ampia ed efatta raccolta, che fia mai ftata fatta, di Tavole calcolate circa le altezze della Marea, e del Barometro, rifvegliar dee l'attenzione de' Matematici fopra un punto de' piu intereffanti della Fifica Celefte, cioè fopra l' azione, e l' efficacia delle Forze Luni-Solari nell' alterare lo ftato del noftro Pianeta. Non vi voleva meno d'una lunghiffima ferie di offervazioni, continuate per lo fpazio di 48.anni, per poter con qualche fondamento fofpettare, che quella attrattrice forza de' Luminari, Ia quale per confenfo oggimai unanime di tutti i FificoMatematiciagita cotanto, e fconvolgc le acque dell' Oceano, eferciti pur anco una qualche azione ful mercurio del Barometro, alterandone fecondo le circoftanze un tal poco, 'ma però fenfibilmente l'altezza : ed era rifervato alla fagacità del Sig. Toaldo nell' accurata difamina, e ne ${ }^{\prime \prime} a$ delicata analifi delle indicate offervazioni, il fa- 
pere fra la moltitudine delle caufe accidentali, che concorrono ad alterare il Barometro, quelle circoftarze trafcegliere, che manifeftar fembrano meno ambiguamente l' azione della Forza Lunare. Gettando l' occhio fulla feconda 'Tavola di queft' Opera, dove trovanfi regittrate le mezzane altezze del Barometro in tempo del paffaggio della Luna pel fuo. Apogeo, e Perigeo, e pofcia in tempo delle Sizigie, e Quadrature, li fcopre primieramente, che le altezze apugee del Barometro nel corfo di 48 . anni fono fate $3 \mathrm{r}$. volte ecceflive, e 17 . volte difettive; che l'altezza apogea la media'delle medie è di 29,3 12. pollici di mifura di Londra s e la perigea di 29,659 , e l'ecceffo della prima fi riduce a una mezza linea: Si fcopre in fecondo luogo, che le altezze barometriche delle Sizigie hanno variato in quell'in. tervallo di tempo 27 . volte per ecceffo, e 2 I. volte per difetto, refpettivamente alle altezze delle Quadrature; e che finalmente l' ecceffo giornalicro delle prime giunge ad uguagliare un fefto di linea. La terza tavo. la rapprefenta le altezze del Barometro per li 12 . fegni del Zodiaco relativamente alla Luna; fcorgefi, che l'altezza del Capricorno è maggiore, che non è quella del Cancro, effendo l' ecceffo d'un quarto di linea per giorno. Per ultimo la quarta tavola rapprefenta il confronto delle altezze barometriche de' fegni meridionali, con quelle de' fegni fettentrionali; e trovanfi le prime 26 . volte ecceffive; e fatto del pari il paragone delle altezze barometriche dei tre fegni intorno all' A pogeo, con quelle dei tre fegni intorno al Perigeo, fi oflerva, che quefte ultime fono per 29. volte fuperate dalle altre: fenomeni tutti, che pajono concorrere a ftabilire la rea- 
Jità dell' azione Lunare ful Barometro, in quanto riguardafi quella come una concaufa delle variazioni di quefto. Intorno a tali offervazioni merita d' efler letta, oltre la lodata Opera del: Profeffore di Padova, una Differtazione Soprá l' Influffo della Luna nel Pefo dell. Atmofplora, inferita dall' infigne Filofofo e Geometra Signor Enrico Lambert nelle Nónveaux Mem.de l'LAcud. de Berlin, ftampate queft anno: 1.773 .

Il Signor D'Alembert nelle fue profonde Rifefjoni Julla Caufa Generale déVenti Gi propone il quefito, perchè quell' azione, la quale fa follevare tant'alto le acque dell'Oceano, non produca altresi un tal cambiamento nel pefo dell' arią, che debba ferfibilmente manifeftarfi nel Barometro; e dalla differenza delle denfità dell' aria, e dell' acqua ripete la fpiegazione di queito fenomeno: imperciocchè fupponendo, che l' acqua in alto mare fi follevi all' altezza di 6o. piedi, qualunque altro fluido nelle medefime circoftanze fi leverebbe ad un' altezza uguale, perchè l' azione Luni-Solare, la qual produce ne' due fluidi una forza proporzionale alie loro maffe o denfità, dee far si, fecondo i principii Meccanici, che la velócità, e l' elevazione di quelli fieno le ifteffe. Per tal modo una colonna d' aria omogenea (riflette il Signor D'Alembert): d' una denfità uguale a quella, che noi refpiriamo, fi folleverebbe all' altezza di 60 . piedi, e varierebbe da un giorno all' altro, nel montare e difcenderé, di r 20. piedi. E quindi il mercurio per effere undici mila volte piu pefante dell' aria che refpiriamo, non potrà variare fe non fe di due linee, quanto appunto avuto riguardo alle denfità differenti, corrifponde alla variazione di $\mathbf{2} 20$. piedi nell' altezza dell' atmosfe-

ra. 
ra. Quefta é an dipreffo (foggiunge l' A utore) la quantita dell' alzhta gionalieraldel Barametro fotto l' Equatore, nella fuppofizione che il vento d' Eft fcorra cold 8. piedi per fecondo- Ma ficcome bavvi un' infinitd di. caufe accidentali, che fanno bon Jovente alzare, é abbalfare it Barometro di molto piu che due linee in un giono, non dee recarmeraviglia, che le ofcillazioni-le-quali poffono in effo eccitarli dall"azione del Sole, e della Luna, "non fieno facili a diftinguerfi: io eforto perd gli offervatori a farvi atcenzione. La qual efortazione ha avuto il fuo adempimento, nella pubblicazione dell' Opera del Signor Toaldo:

Sembra per tanto (lafciando anche da parte l'opinione dell' immortal Geometra Signor Diniello Bernoul$\mathrm{li}$, che $\mathrm{fa}$ afcendere fino 'a' 20 . liné la variazione del Barometro, prodotta dall' azijne Luni-Solare), che la maggior parte de' piu illuttri, Fifici, e Matematici fi accordi nel riconofcere un qualche fenfibile influffo-della forza Lunare ful Barometro. Prefentemente che le recenit fcoperte aftrononiche ci fomminiftrano dati più ficuri, per calcolar quefta forza, e ci mettono fulla dritta via per innoltrarci con maggior fiducia di prima nell' efame di quefto đ̈ilicato Problema, propor vogliamo un particolar metodo per giungere con eftrema facilità, $e$ fpeditezza alla foluzione; e fe i rifultati del noftro calcolo fi troveranno contrarj a quanto i fuddetti. Valentuomini hanno creduto e ftabilito; non intendiamo di proporre quefta difcrepanza, fe non fe come un dubbio da effere ulteriormente efaminato, e difcuffo. Ecco pero il proceffo del noftro calcolo.

E Teorema noto preffo gli Scrittori dell' Alta Idro-' ftatica, fegnatamente preflo il piu grande dei Geometri 
ne' fuoi ammirabili Principii generali del moto de' Fluidi nelle Memorie dell'Accademia di Berlino per l'anno $175 \dot{5}$, che $f e$ in qualfivoglia Fluido una qualunque particella di. denfità $=D$, e foftenente una preflioné $=P$, follecitata venga nello fteffo tempo verfo due o piu centri di forze acceleratrici $Q, Q, 2, \&$, dipendenti comunque dalle rifpettive diftanze $q, q, q$, \&c. dai centri fuddetti, fi dee fempre verificare la feguente equazione differenziale $\frac{d P}{D}=-2 d q-2 d q-2 d q-\& c$; Teorema maravigliofo per la fua eleganza e fecondità, e per.effere il fondamento di tutte le più ardue, ed arcane ricerche della Dottrina de'Fluidi.

Ciò premelfo, fia $T$ il centro della terra, di cui $T B$ è il femidiametro $=r ; L$ la Luna o il Sole alla diftanza $T L=g$ dal centro della Terra; $N$ la particella del Fluido di denfita $=D$, e la di cui preflione, ovvero l'altezza del Barometro che la rapprefenta, $\grave{\mathrm{e}}=P$, la qual particella è fituata alla diftanza variabile $T N=x$ dal centro terreftre, e alla diftanza $N L=y$ dal lunare. Dicafi $T$ la maffa della Terra, $L$ quella della Luna, 0 del Sole. Suppofta ora la legge Newtoniana della Terra Attrattrice in ragion reciproca duplicata delle diftanze, convien indagare le forze, dalle quali è inveftito e follecitato l'elemento $N$ del Fluido. Tre per tanto fono quefte forze follecitanti ; colla prima tende il fuddetro elemento al centro $T$ della Terra; colla feconda al centro $L$ della Luna, o del Sole; colla terza verfo $N S$ in una direzione parallela, e contraria a $T L$, giacchè effendo il centro $T$ della Terra (che qui vuolf́ 


\section{Deli' 'Accademia.}

riguardar come immobile) (pinto verfo $L$, con una for$\mathrm{za}=\frac{L}{\mathrm{~g}^{2}}$, dalla fteffa forza, $\mathrm{ma}$ in direzione contraria fi debbono concepire animati tutti i punti terreftri. Ora la prima di quelte forze $\grave{e}=\frac{T}{x^{2}}$; la feconda $=\frac{L}{y^{2}}$; la terza $=\frac{L}{g^{3}}:$ e condotta $T M$ perpendicolare a $T B$, $a$ $N S$, pongafi $N S=z$. Dunque moltiplicandofi la prima forza per $d x$, la feconda per $d y$, la terza per $d z$, $f_{1}$ avrà pel Teorema Euleriano dianzi ftabilito, l' equazione $\frac{d P}{D}=-\frac{T d x}{x^{2}}-\frac{L d y}{y^{2}}-\frac{L d z}{g^{2}}$. Se per tanto, per integrar quefta formula, $f_{i}$ addimanda, $a$ la nota preffione dell' aria, o l'altezza del Barometro nel luogo terreftre $B$, ed $f$ la denfità dell' aria in quel luogo, fuppofta la denfità del mercurio $=$ I., la nota Legge Halleyara ci fomminiftra l'analogia $D: f:: P: a$, e però $D=\frac{f P}{a}$, che foftituito nella formula antecedente fi ottiene mediante l'integrazione $\frac{a}{f} l P=\frac{T}{x}+\frac{L}{y}-\frac{L z}{g^{6}}+$ coft., e diventando nel luogo $B$ la quantità $P=a, x=r$; $y=g-r$, e $z=r$, fi raccoglierà il valore della coft. $=$ $\frac{a}{f} l a+\frac{L r}{g^{2}}-\frac{L}{g-r}-\frac{T}{r}$, e però $\frac{a}{f} l \frac{P}{a}=\frac{T}{x}-\frac{T}{r}+\frac{L}{y}-$ $\frac{L}{g-r}-\frac{L z}{g^{2}}+\frac{L r}{g^{2}}$, ovvero $l \frac{P}{a}=\frac{f T}{a x}-\frac{f T}{a r}+\frac{f L}{a y}-\frac{f L}{a g-a r}-$ $\frac{f L z}{a g^{2}}+\frac{f L r}{a g^{2}}$. Quindi è manifefto, che fe fi fuppone il punto $N$ nella fuperficie terreftre, dove in confeguenza $x=r, \mathrm{i}$ due primi termini del fecondo membro fi eliQ dono 
dono, e nafce per tutti i differenti luoghi della fuperficie della terra l' equazione molto femplice $l \frac{p}{a}=\frac{f L}{a y}-$ $\frac{f L}{a g-a r}-\frac{f L z+f L r}{a g^{2}}$. Per indagare prefentemente il valore di $P$ quando il punto $N$ ritrovafi in $M$, cioè $90^{\circ}$ lontano dalla Luna, o dal Sole, che faranno allora confeguentemente all' Orizzonte del luogo propofto, offervifi che quivi diventa $y=\sqrt{\mathrm{g}^{2}+r^{2}}$, e $z=0$; e percio la formola riducefi a quefta $\downarrow \frac{p}{a}=\frac{f L}{a \sqrt{g^{2}+r^{2}}}-\frac{f L}{\| g-a r}+$ $\frac{f L r}{a g^{2}}=\frac{f L}{a}\left(\frac{1}{\sqrt{g^{2}+r^{2}}}-\frac{1}{g-r}\right)+\frac{f r L}{a g^{2}}$. Svolgendo ora in ferie le due efpreffioni $\frac{1}{V \frac{1}{g^{2}+r^{2}}}$, e $\frac{1}{g-r}$, e pel valore affai grande di $g$ in paragone di $r$, trafcurando $i$ termini, che contengono nel denominatore le potenze di $g$ al di là della terza, fi ha la prima efpreffione $\frac{1}{\sqrt{g^{2}+r 2}}=$ $\frac{1}{g}-\frac{r^{2}}{2 g^{3}}$; e l'altra $\frac{1}{g-r}=\frac{1}{g}+\frac{r}{g^{2}}+\frac{r^{2}}{g^{3}}$, i quali valori furrogati nella formola precedente, la trasformano in quefta fempliciffima, e comodiffima $l \frac{p}{a}=-\frac{3 f r^{2} L}{2 a g^{3}}$; prefo pui $E$ pel numero che ha per fuo logaritmo iperbolico l' unità, frottiene $\frac{P}{a}=E^{\frac{-3 f r^{2} L}{2 a g^{3}}}$ e e $P=a E^{\frac{-3 f r^{2} L}{2 a g^{3}}}$; ed effendo, come ì noto, $E^{\frac{-3 f r^{2} L}{2 a g^{3}}}=1-\frac{3 f r^{2} L}{2 a g^{3}}$, ommefli gli altri termini divifi per le potenze di $g$ piu alte della terza, fi ritrarra fubito $P=a-\frac{3 f r^{2} L}{2 g^{3}}$, efprefío- 
ne templicifima, ed elegante dell' altezza del Barometro ne' due luoghi $B, M$, cioè quando la Luna o il Sole è nel Meridiano; e nell Orizzonte, viene rapprefentata dalla quantità $\frac{3 f r^{2} L}{2 g^{3}}$.

Trattafi ora di ritrovare il valor numerico di quefta efpreffione in una mifura analoga a quella di $a$. A tal effetto, per procedere con chiarezza, fi offervi primieramente, che effendofi fuppofta la preffione, o il pef́o $P$ rapprefentato da una colonnella di mercurio di conveniente altezza, ed effendofi pofto $P$ uguale a quell' altezza, $f$ veniva in confeguenza a fupporre tacitamente, che detta altezza folfe moltiplicata per la gravità terreftre alla fuperficie della terra, il qual fattore non comparendo nell' efpreffione, veniva perció a concepirfi implicitamente uguale all' unità. E' adunque la gravità terreftre, ovvero $\frac{T}{r^{2}}=\mathrm{I}$, e quindi $T=r^{2}$. Sia in primo luogo $L$ la maffa del Sole, in vece della quale farà opportuno d'introdurre nell' efpreffione $\frac{3 f r^{2} L}{2 g^{3}}$ la forza centrifuga qual fi fperimenta fotto l' Equatore, la quale; come è noto, è $\frac{1}{289}$ della gravità de’ corpi terreftri. Per introdur quefta forza, ed eliminare la quantità $L$, fi ricorre al noto Teorema, che le forze centrifughe, o anche centrali fono in ragione compofta della diretta femplice de' raggi, e della duplicata inverfa de' tempi periodici; onde effendo proffimamente il tempo periodico della Terra intorno al Sole di 365 . giorni, e intorno a fe fteffa Q 2 di 
di un giorno, fi ha l'analogia $\frac{L}{g^{2}}: \frac{1}{239}:: \frac{g}{3^{65^{3}}}: r$, dalla quale fi ricava $L=\frac{g^{3}}{289 \cdot 3^{6} 5^{2} \cdot r}$, e quindi $\frac{3 f r^{2} L}{2 g^{3}}=$ $\frac{3 f_{r}}{0.289 \cdot 3^{65^{2}}}=\frac{3^{r}}{0.089 \cdot 10800 \cdot 3^{65^{2}}}$, per effere $f=\frac{1}{i 0800}$. Siavrà dünque $\frac{3 f r^{2} L}{2 g^{3}}=\frac{3 r}{83^{1643740000}}$; e prendendo fecondo le accurate ricerche del Sig. De la Lande nella fua eccellente Aftronomia \$. 3592. il femidiametro r della terra uguale a 1974 I 200. piedi france $f_{1}$, fe ne raccuglie $\frac{3 f r^{2} L}{2 g^{3}}=$ $\frac{59223600}{831643740000}=\frac{1}{1404^{2}} \cdot d^{\prime}$ un piede $=\frac{1}{97}$ di linea. Da ciò a chiare note fi fcorge, che l'attrattrice forza del Sole non puó variare l' altezza del Barometro al di là di $\frac{1}{97}$ di linea, quantita picciolifima e onninamente infenfibile, e di lunga mano eludente le più diligenti ricerche di qualunque attento, e fagace Offervatore. Sia fecondariamente $L$ la maffa della Luna, la quale fuppofta la nuta-

zione dell' affe terreftre di $" 9$, trovafi fecondo il calcolo del Sig. D' Alembert nella fua Preceffione degli Equinozi ellere $\frac{1}{80}$ della maffa terreftre, ovvero $L=\frac{T}{80}=\frac{r^{2}}{80}$. Se ora quefto valore di $L$ fi foftituifce nella quantità $\frac{3 f r^{2} L}{2 g^{3}}$, quelta trasformafi in $\frac{3 f r^{4}}{2.80 . g^{3}}$. E perchè già fi fa effere proflimamente $g=60 \mathrm{r}$, e $g^{3}=216000 \mathrm{r}^{3}$, la preceden- 
te efpreffione fi cangia in $\frac{3 \mathrm{fr}}{2.80 .216000}=\frac{59223600}{2.80 .10800 .216000}=$ $\frac{59223600}{373248000000} d^{\prime}$ un piede, cioè $=\frac{1}{44}$ di linea. Dinque nè tampoco la Luna ifteffa, con tutto che più attiva del Sole per riguardo al noftro Pianeta, puo alterare fenfibilmente lo ftato del Barometro, giacchè la variażione di $\frac{1}{44}$ di linea nell' altezza di quefto ftromento è troppo picciola, e troppo impercettibile, per poterfi mai manifeftare al piü paziente, e al più oculato Offervatore.

\section{$S$ C O I I O I.}

F Di per fe evidente, che quefto fteffo metodo $f_{1}$ adatta alla foluzione del Problema di indagare l' altezza della Marea. In fatti riaffumendo la formola differenziale $\frac{d P}{D}=-\frac{T d x}{x^{2}}-\frac{L d y}{y^{2}}-\frac{J d z}{g^{2}}$, e fupponendo, che $D$ rapprefenti la denfità uniforme dell' acqua $=I$, fi ha l'integrale tutto algebraico $P=\frac{T}{x}+\frac{L}{y}-\frac{L z}{g^{2}}+$ Coft.; e ficcome nel luogo terrettre $B$ alla fuperficie del mare diventa $P=0, x=r, y=g-r, z=r$, nafcerà Coft. = $\frac{L r}{g^{2}}-\frac{L}{g-r}-\frac{T}{r} ;$ e però $P=\frac{T}{x}-\frac{T}{r}+\frac{L}{y}-\frac{L}{g-r}-\frac{L z+L r}{g^{2}}$, ovvero $\frac{T}{x}=P+\frac{T}{r}-\frac{L}{y}+\frac{L}{g-r}-\frac{L r+L z}{g^{2}}$. Offervifi prefentemente, che nel punto $M$, cioè quando la Luna, o il Sole fono nell' Orizzonte, $x$ rapprefenta la diftanza della fuperficie del mare dal centro della terra, laddo- 
ve $\because$ efprime quella diftanza pel punto $B$, quando cioè trovafi la Luna nel meridiano; riflettafi in oltre, che nello fteffo punto $M$, le quantità $P_{2}$ e $z$ fvanifono, ed $y$ cangiafi in $\sqrt{g^{2}+x^{2}}$, coficchè la formula diventa $\frac{x}{x}=$ $\frac{T}{r} \frac{L}{\sqrt{g^{2}+x^{2}}}+\frac{L}{g-r}-\frac{L r}{g^{2}}=\frac{T}{r}-\frac{r L}{g^{2}}+L\left(\frac{1}{g-r}-\frac{1}{\sqrt{g^{2}+x^{2}}}\right)$, dove effendo $\frac{1}{g-x}=\frac{1}{g}+\frac{r}{g^{2}}+\frac{r^{2}}{g^{3}}$, ed $\frac{1}{\sqrt{g^{2}+x^{2}}}=\frac{1}{g}-$ $\frac{x^{2}}{2 g^{3}}$, fatte quefte foftituzioni ritrovafi $\frac{T}{x}=\frac{T}{r}+\frac{r^{2} L}{g^{3}}+$ $\frac{L x^{2}}{2 g^{3}}$, e finalmente $x^{3}+\left(2 r^{2}+\frac{2 g^{3} T}{r^{2} L}\right) x-\frac{2 g^{3} T}{L}=0$ : Se per tanto alla rifoluzione di quefta equazione cubica fi applicano i noti metodi di approflimazione colla indicata avvertenza per riguardo alle potenze di $g$, fi ricava la radice $x=r-\frac{3 r^{4} L}{2 g^{3} T}=r-\frac{3 r^{2} L}{2 g^{3}}$, per effere $T=r^{2}$. Di qui è manifefto, che l' altezza della marea in $B$, quando 1 Aftro trovafi nel Meridiano, viene rapprefentata dalla quantità $\frac{3 r^{2} L}{2 g^{3}}$.

Suppongafi primieramente, che $L$ efprima la maffa del Sole ritrovata poc $^{2}$ anzi $=\frac{g^{3}}{289 \cdot 3^{65^{2} \cdot r^{2}}} ; l^{\prime}$ efpreffione $\frac{3^{r^{2} L}}{2 g^{3}}$ fi muterà in queft' altra $\frac{3 r}{2.289 \cdot 365^{2}}=\frac{59223600}{7700405^{\circ}}=a^{\prime}$ poco più di $\frac{5}{7} d^{\prime}$ un piede.

Pongafi fecondariamente $L$ uguale alla maffa della Luna già ritrovata dianzi $=\frac{r^{2}}{80}$; e la quantita $\frac{3 r^{2} L}{2 g^{3}}$, per cffere 
effere in tal cafo $g=60 \mathrm{r}$, diventera $\frac{3 r}{2.80 .603}=\frac{59233600}{34560000}$, vale a dire poco più di un piede, e due terzi. Dal che fi raccoglie, che le forzé combinate del Sole e della Luna follevar poffono te acque dell' Oceano, poco al di là di due piedi ed un terzo. Si tralafciano le rifleffioni; che naturalmente fi prefentano fu quefto punto, e come aliene dal noftro iftituto, e come troppo facili a farfi perchè fiavi bífogno di fpecificarle.

\section{S C O I I O II.}

E adunque le forze perturbatrici della Luna, e del Sole non fono baftanti, come fi è dimoftrato, ad alterare fenfibilmente lo ftato del Barometro; onde avvien poi, che le Offervazioni per l'oppofto pajono indicare una qualche azione fenfibile delle forze ifteffe fit quell' iftrumento? E donde puo derivare quefta fpecie di contrafto tra il fatto, "la teoria", fra l'offervazione, e il calcolo? Noi non ofiamo definire il precifo grado di probabilità, che dalle mentovate offervazioni rifulta, e molto mero fino a qual fegno dovrebbero efic favorevolmente combinarfi, e in qual numero, e fino a qual termine eftenderfi, perchè fi poteffe fondatamente afferire, ovvero con ragionata probabilità congetturare, che per qualche delicata sfuggevole circoftanza, per qualche occulto elemento, e arcana inacceflibil forza, la tcoria effer debba difettofa e imperfetta, e quindi neceffariamente difcorde dall' offervazione. In fimil cafo pare, che la divifa del Fifico-Matematico debba effer quel- 
128

A $\quad r \quad T \quad r$

la dell' Oratore, e Filofofo Romano, ut potero explicabo: nec tamen ut Pytbius Apollo, certa ut fint 'es fixa quae dixero: Sed ut bomunculus probabilia conjectura Jequens. E appunto in fiffatte queftioni inviluppate, e complicatiffime di Fifica Particolare dee piu che altrove avverarfi il fenfato proverbio del Poeta Inglefe

Tis with our judguments as our watches, none. Go juft alike, but each believes bis own:

$$
\begin{aligned}
& *^{*} *^{*} *^{*} *^{*} *^{*} *^{*} * *^{*} * * \\
& *^{*} *^{*} *^{*} * *^{*} * *^{*} * * \\
& \text { - ********* } \\
& *^{*} * * * * * * \\
& *^{*} * *
\end{aligned}
$$

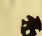




\section{DELLO STESSO AUTORE \\ W}

Saggio Sopra i Progreffi Matematici di Girolamo Cardano, e Bonaventura Cavalievi dopo il riftabilimento delle Lettere in Occidente.

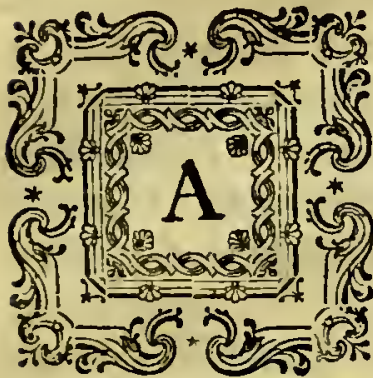

Due univerfalmente fi riducono, lafciando da parte i tempi favolofi ed incerti, le Epoche piu memorabili, in cui le Scienze, le Lettere, e le Arti dalla Grecia, dove per tanti Secoli sì felicemente allignarono, vennero ad ingentilir l' Occidente. La prima, quando i Romani ftanchi per le continue guerre, e ricchi delle fpoglie delle già vinte Nazioni nel feno dell' ozio e della pace prefero da' Greci, loro Schiavi e Maeftri, il fiore di ogni gentilezza e di ogni maniera di letteratura ( $I$ ): la feconda, quando dalle ar-

(1) Hor.Lib.II.Ep.I. Grecia capta ferum Victorem copit, EO Artes Intulit agrefti latio......

Serus etuin Grecis admovit actunina chartis:

Et poft Punica bella quietus querere capit, Outid Sopbocies, E Tbelpis, \& Aefcbilus utile ferrent.

La Grecia però col tirolo di Domicilio delle Scienze e delle Arti confervò fempre fopra gli altri Paefi una fuperioritz, che niuno le ha mai potuto contendere. Il Genio Latino nato per comandare, e dertar Leggi, per perdonare a'Sogreetri e debcllare i Superbi fece dei progreffi ammirabili nell' Eloquenza, nella grande e furte Poefia, e produffe dei capi d' ope$\mathrm{ra}$ in quefte maeeric: $\mathrm{ma}$ fi vide fempre retare alla Grecia la Commedia, la Mufiea, la Piecura, la Scolcura, l'avvenenza, la dolcczza, lia grazia delha favella, la fertiva urb:unict, $i$ leggiadri Sali, gli argutti motri, la dclicata fenfi- 
mi vittoriofe di Mrometto II. prefa e occupata Coftantinopoli, e diftrutto l' Impero d' Oriente, que' Greci Eruditi, che quivi formata aveano lar dimora, corfero in Italia a cercare un Afilo contro alla barbarie de'Turchi. La decadenza dell' Impero Romano avea già involte nella propria rovina tutte le fcienze, e le Belle Arti dalla Grecia trapiantate nel Lazio. I fecoli, che feguiron da preffo, erano non folamente immerfi nelpiù cieca ignoranza, ma ancora in una general corruttela, fi per il Luffo fmifurato, che is introduffe in Roma, e cangiò tutta la faccia dell' Impero, depravando i coftumi, deprimendo gli ftudi, falfificando il gufto, degradando la retta ragione, fi per le guerre fanguinofe fralle Nazioni foggiogate e le Colonie trafportate al fondo dell' Afia, e dell' Africa, fi finalmente il diluvio di Barbari, che difcendendo dal Settentrione a guifa d'impetuofo torrente inondò tutta l' Europa. Allora $\mathrm{fu}$, che fra le uccifioni, gl' incendj, $\mathrm{i}$ faccheggi, fra la Religione profanata e avvilita, e il rovefciamento generale di tutte le buone regole e di tutte le Leggi niuno fu più in iftato di penfare, di riflettere, di ragionare: imperciocchè fono umili a forza e languidi, e invo.

fenfibilith, in una parola la cintura di Venere, che i Romani non poterou mai togliere ai Greci. Quindi è, che Anchife prefra Virgilia Acn, Lib.VI. vaticinando la futura grandezza di quel Papolo Re, il quale imperium Terris animos aquavit Olimpo; fi efprime con que' maeftofi verfi.

Excudent alii pirantia mollius cera;

Credo equidem, viros ducent de marmore vultus:

Orabint caufas melius, coolique meatus

Defcribent radio, EN furgentia fidera dicent.

Tu regere imperio populos, Romane, memento,

(Hoe tibi erunt artes) pacique imponere morem:

Parcere fibjectis, Es debellare fuperbos. 
involuti i concetti di una mente, che penfando né colpi afhitti, ed oppreffi è dal grave péo de' mali a liberamente follevarfi impedita. Un tale deplorabile ftato e quafi deliquio dell' Umana Ragione, che fofprefe per così dire tutte le fue facoltà, durò per molti fecoli, e fi diffufe per tutte le Contrade d'Europa (2). Non gia che la Natura non faceffe di quando in quando qualche sforzo per produrre degl' Ingegni nobili, ma per difetto. di coltura abbandonati a fe fefli perivano fra gli theipi, e le fpine d'un Suolo ingrato e lilveftre: Il dotto P. Mabillon nella Prefazione del quinto fecolo di San Benedetto ne fomminiftra gli efempj. Ma finalmente i tempi cangiarono: Quella Grecia, che avea fervito altra volta a ripulire l'antica Roma, e ad infpirarle il gufto delle Buone Arti, fu per la feconda volta la Rithoratrice delle Lettere in Occidente. Coftantinopoli venne al foccorfo dell' Europa già da tanto tempo, e fi cru$\mathrm{R}_{2}$ del-

(2) Tanta era l' ignoranza, e la rufticirà di que' fecoli tenebrofi, che lo Refro Carlo Magno, Uomo anche nelle Lettere dei più illuminati del fuo tempo appena fapeva fcrivere. Eginardo fuo Secretario e Intendente delle Fabbriche, nella Vita di queft Imperadore dice efpreffamente cosi : Tentabat E foribere, Tabulafque $\mathrm{E}^{2}$ Codicillos ad boc in Lectulo fub cervicalibus circunferre folebat, ut cum vacuum tempus effet, manum effingendis. Litieris adfuefcerct; fed parun fucceffit labor prepoflerus ac fero incboatus. II P. Pagi apud Baroniuliz ad an. 792 .num. 8. $s^{\prime}$ ingegna di dare un altra iuterpetrazione alla fopr.llegata teftimonianza di Eginardo, feguitando in ciò il Lambeccio nel Lib. II. Biblioth. Ces.peg. $a_{4}+;$ Ma le parole degl' Iltorici Umani hanno un folo fenfo, al che non ha forfe riffettuto il Teologo Pagi. Senza che è troppo noto, che i Principi di quel tempo, quando volevano fcriverfi, erano obblizati di ricorrere a qualche Cherico, c fervirfi della fua mano, mettendo folamente il loro monogramma o figillo nel fondo della lettern. I Fefcovi fieffi, che occtpavano le prime fedi (dice il Deflandes Ilift. Crit. de la Phil. zom. Ill. Chap.XXXIX. n. IX.) potecalio appena balbetare le parole farrabientaib. 
delmente sfigurata dall' ignoranza $e$ dalla barbaric, $e$ rifvegliò dall' antico letargo il genio, il gufto, le Arti, l'induftria. I Letterati Greci fuggitivi dinanzi alla ferocia Ottomanna ebbero tantofto un gran numero di Difcepoli, e d'Imitatori in Italia. Non bifogna per altro credere che gl' Ingegni paffaffero tutto in un colpo dalle tenebre alla luce: Si dovette paffare per un lungo crepufcolo, che durò per un fecolo e più ; e il tragitto fu afpro, intricato e f́pinofo. Ognuno fi applicò da principio allo ftudio delle Lettere Umane, alla Lingua Greca e latina, ai Tefti Originali, alla Critica, alla correzione degli antichi Codici e Manofcritti; e fi attefe piu allora a fcrivere bene e pulitamente in latino, che a fcrivere giudiziofamente, a cercare $i$ fiori della Rettorica, che a ftudiar la Natura, ad ordinar un difcorfo ed abbellirlo con arte, che a fcoprire una verità intereffante. Ma tutti quelti preliminari erano d'un' affoluta neceffità : Gli Uomini avevano difimparato a penfare: Perdendo la traccia de' buoni Autori, che friffera nei bei giorni di Atẹe, e di Roma, nei fecoli di Pericle e di Augulto, fi perdette il fentimento, e fino quafi l' abito di meditare: I bifogni dello fpirito, quando per un certo fpazio di tempo non fi fon fuddiffatti, diventano preffoche irreparabili : Perciò allorchè verfo la fine del quindicefimo feccolo il buon Gufto portato in Italia dai Greci rifvegliò gli Uomini Penfatori, - li fece accorti della propria miferia e imbecillità, la neceffità li coftrinfe a dirizzarfi agli antichi, a fludiarne le lingue, a rilevarne lẹ bellezze per ricominciarne di li dove fi era finito. Bifognò rigiıardare i fecoli precedenti come tempi, in cui fi era fimarrito il filo del vero, del 
bello, $e$ in cui la memoria delle produzioni ammirabili de' Greci e Romani fi era interamente perduta : Bifogno dunque prender le moffe dallo ftudio delle lor Lingue per ritornare ful diritto fenticro: E facendo poi tutti a gara d' imitare que' perfettifrmi Modelli deli' Antichità, fi cominciò a prender la tintura del loro fpirito, $c$ quell' aria nobile e femplice congiunta alla più paffionata eloquenza, che tanto grandeggia nelle Opere degli Antichi. Così il più gran merito del quindicefimo e fedicefimo fecolo fu di aver letti e comentati i più eccellenti fcrittori Greci e Latini, d' aver, analizzate le loro bellezze, e di aver faputo imitarli nella profa e nel verfo.

Ma finalmente quefto difcernimento dell'ottimo nelle Belle Lettere, quefto ftudio della Bella Natura fatto fu i gran Modelli di Atene, e di Roma doveva infenfibilmente influire nelle Scienze e nella Filofofia. Era impoflibile, che ad Uomini ingentiliti dallo ftudio di quanto vantar può di più graziofo e leggiadro l' Antichità piaceffe il lezzo e la barbarie degli Scolaftici, i quali parlando dal Tripode, e tralle ofcurita ricoprendofi con una fpecie di tirannia efigevano la venerazione dovuta agli Oracoli. Ariftotile cacciato già da Atene dagli antichi Sacèrdoti, accolto da' noftri con qualche varietà però di fortuna, avea prefo già da quindici fecoli, benchè confufo e maltrattato dagl' Interpetri Arabi e ingarbugliato dagli Scolaftica, un tal afcendente fopra tutti gl Ingegni, che era delitto di fellonia il fottrarti al giogo della fua autorità; e il far ufo della propria ragione, e oltrepaffare i confini dell' Arabefca Filofofià egualmente era pericolofo, che il voler cangiare $\mathrm{i}$ Termini del Pomerio dell' antica Roma, alla cultodia de' quali religiofamen- 
te dagli Auguri fi vegliava. S' inneftava eziandio piu che mai colla Religione la Filofofia, e i Teologhi di quell' età febbene fi attenti a tener lontano l' errore confermavano col loro efempio quefta ftrana condotta, e nelle loro opere fácévano dipendere una gran parte delle priove della Religione, altronde fi ftabilmente appoggiata, dall' autorità di Ariftotele. Ond'è, che il dotriflimo Iftorico del Concilio di Trento ébbe a dire colla fua ordinaria franchezza, che fenza Ariftotele noi man-. cavamo di molti Articoli di Fede; la qual prepolizione ridotta al fuo vero fenfo, non altro. fignifica fe non che i venerabili. Padri e Teologi di quel Sacro Augufto Confeffo fi fervirono alcuna volta delle frafi, e degl' Infegnamenti Ariftoticelici (a).

$\mathrm{Ma}$ già fi acioftava il tempo della rivoluzione. Il Gufto delle Belle Lettere generalmente introdotto avea lentamente preparati e difpofti gli Animi Penfatori alla ribellione contro l' illegitima Autorità di Ariftotele (3).

I pri-

(a) Gli Intendenti dell' Ecclefnfica Hiftoria dicevano, che in tuti i Concilj tenuti nella Cbiefa dal tempo degli Apoftoli flno a quell'ora, pofti miti infiene, mai erano fati decifi tanti articoli, quanto in quella fola feflone, in che baveva una gran parte Arifotele coll baver difinto efattamente tutti i generi delle Caufe, a cbe fe egli non fi folfe adoperato, noi nancavamo di molti articoli di Fede. Frì Paolo Stor. Ec.

(3) Anche prima del fedicelimo Secolo, e verfo il fine del quindicefimo alcuni Ingegni fublini già cominciavano ad accorgerfi della rozzezza della Filolofia dominante. Balti per turti la teftimonianza di due Uomini infigni di quel rempo, cioè Niccolo Lconico, il primo che bandiffe dalle Scuole Pubbliche la barbarie degli Averroifti, e introduceffe la lezione de'refti Greci, e Giovanni Pico Mirandolano, del quale il Conringio De Scriptoribus Saculi $X V$. Cap.II. parla cosi : Ex Grecorum Exfir!tum Scbola profecti funt Foannes Picus Mirciudula, ac Concordice Comes, ingenii bumani Pbonix, ac plane ftuperdum miraculum Ec: Ora il primo in un fuo Dialogo intitolato Peripatetico cosi rngiona: Tanlam cibeft, wh ab ifinfmodi bonubibus cxculti aliquid crudi- 
I primi tentativi non riufcirono; ma $f_{1}$ accrebbe fempre più la forza e il coraggio. Finalmente ful principio de! diciaflettefimo fecolo furfero Vindici della Ragione tre Uumini originali, e fublimi, nati per far epoca nei faAti dello : fpirito umano, e per cangiar la faccia delle cofe, Bacone di Veruliamo in Inghilterra, Cartefio in Francia ; $e$ Galileo in Italia, Uomini degni dell' ammirazione di tutti $\mathrm{i}$ fecoli, e per dir tutto in una parola degni di ftare a fianchi, di Newton; i quali dopo aver rovefciato l' Arabefco edifizio della Filofofia Ariftotelica, e piantati $\mathrm{i}$ fondamenti del folido Tenipio, che Newton poi innalzò e confacrò alla Verità, pagarono il tributo, folito pagarf dagli Uomini grandi, all' ingratitudine e malignità de'loro contemporanei (4). Ora pri-

ma

eruditique difcas, ut e comtrario fi quid cruditi frius excultique didiceris, cum ad illos accefferis, comm contagio procul dubio dedocearis oportent. An non ego decem integros annos borum auditoria, ne dicam luftra, adfidzic coirtrivi opera? onnefque illorun ineptias, \& futiles captionum tricas, ficcis, ut ajunt, auribus ebibi? anxie femper quacitans, fi quid inde excerpere, poffem, ne vacuis, quod dicumt, manibus E of $^{\circ}$ orans domum redirem: I $c$ rum, Dii. immortales, quam revum inanitatem apud illos, quantani bontrun litterarum folitudinen reperi! In quo tamen, ut in malis, nibil mibi magis fopere rifus fum, quan quod cum illis defipere aliquando deffiti; neque per onme vitce fpatium, ut plerique folent, in conofa illa ignorantiae :oragine demerfus contabui. E il fecondo in una fua lettera ad Ermolano Barbaro così fcrive: Dum barbaros bos Pbilofophos infectaris, quos dicis baberi vulgo fordidos, rudes, incultos, quos nec vixiffe viventes ne dum extinchi vivant, E fi munc vivant, vivere in panam E contumelian; ita bercales fun commotus, ita me puduit, piguitque foudiorum meoruin ( $j a n$ enim fereminu apud illos serfor') ut nibil minus me feciffe velin, quam in tan? nibili facienda re tan laboriofe contendiffe. Perdiderin ego, inquam, apud Avcrroem melioves annos, tantas vigilias, quibus potucrim in bonis littcris fortafis nomibil effe.

(4) A tutti fon noti $\mathrm{i}$ difatri e $\mathrm{i}$ rovefcj di fortuna accaduti a quefti tre grand' Uomini, $c$ forfe meritati dal primo; ma non è forfe noto egualimente, che i Perfecutori del Galilco Martirc della Ragione, e dell' Aftronomì 
ma eziandio che l'agitazione, $e$ il fermento eccitato nel Regno delle Lettere dai mentovati Triumviri della Filofofia fi propagaffe da un' eftremità all' altra d'Europa, e tutto fi rifentiffe della gran rivoluzione prodotta nelle idee degli Uomini, mentre nella noftra Italia le Univerfità di Bologna, di Pavia, di Pifa, e di Padova fi contendevano a gara il primato nel far fiorire gli ftudj, e aprire l'entrata al vero metodo di filofofare, e afterger lo fquallore dell' anticha barbarie, erano già forti fotto il Cielo Lombardo, ad immortale onore di quefté felici Contrade due fommi ingegni, Girolamo Cardano, e Bonaventura Cavalieri, che occuperanno fempre un ragguardevoliffimo pofto nella Storia delle matematiche, e poffono a gran ragione pretendere un diftinto luogo nel picciol Catalogo degl' Inventori.

E primieramente per riguardo al Cardano, non v'è chi non fappia la parte ch' egli ha avuta grandiffima nel promuovere, e ajutare il più gran parto del ingegno Italiano, cioè l'Algebra allora nafcente: Egli fu il primo a fcoprire la moltiplicità dei Valori del incognita nelle equazioni, e la loro diftinzione in pofitivi, e negativi; la quale fcoperta con un' altra di Francefco Vieta fopra i coefficienti dei termini delle e. q iazioni è ftata di poi il germe, e il principal fondamento di tutte quelle di Harriot, e Defcartes fopra l'analif, e rifoluzione delle ifteffe equazioni: Ed una fiffat-

furono alcuui Membri di quell eftinto Corpo fempre nemico dei progreffi della Ragione, del quale un bizzarro Ingegno Napoletano ne ritrovava l'origine negli Accufatori di Socrate. Si può vedere fu ciò Ugone Grozio contemporanco del Galileo in una fua Lettera a Ifacco Voffio: dove dice Galileus Galilei, Vir in ommi Matbematum parte fummus Ëc. 
fiffatta invenzione di Cardano trovafi efpreffa in termini chiari e precili nella fua Ars Magna. E' ben veró però, ch' egli lafciò la fua fcoperta imperfetta, ed avendo in mano il filo d'Arianna, pare nonabbia faputo trovare la via per ufcire dal Laberinto: imperciocchè dell' ufo delle radici negative egli non parla, il che fa fofpettare averle egli riguardate come inutili; delle radici poi eguali, e affette del medefimo fegno non ne conta mai che una fola in qualfivoglia equazione, $e$ così fembra diftruggere con una mano quello che edificava coll' altra. Se poi nella rifoluzione delle equazioni cubiche, ritrovato importantifimo di Scipione Ferreo, e di Niccolò Tartalea, il Cardano non può pretendere alla gloria d' Inventore, benchè le formole di quefta rifoluzione portino il fuo nome, per effere ftato il primo a pubblicarle comunicategli dal Tartalea, puó però con ogni diritto arrogarfi il titolo d'Illuftratore, sì per avere dimoftrato il metodo di Tartalea, sì per averlo eftefo a tutti i cafi poffibili delle equazioni cubiche, limitato prima a quei foli, in cui il fecondo termine mancava; la qual eftenfione di metodo, benchè faciliffima oggigorno che l'Algebra da tanti eccellenti Ingegni è ftata illuftrata, efigeva però a' tempi di Cardano non mediocre acume e deftrezza. Fu bensi il primo il Cardano ad accorgerfi di un cafo delle equazioni cubiche, in cui l' eftrazione della radice quadrata, che entra nella formola, diventa impolfibile, e contraddittoria; nel che confifte il tanto famofo Cafo Irreducibile, che è ftato, e farà fempre l'experimentum crucis, e il nodo gordiano degli Algebrifti: Era per altro facile ad accorgerfene, ed è affai forprendente, che quando Car$S$

dano 
dano comunicò la fua offervazione al Tartalea, quefti l'abbia potuta riguardare come un gingillo, con cui $f_{1}$ voleva ofcurar la fua gloria, e diminuire il merito delle regole da fe, e dal Ferreo ritrovate. Intantó fard fempre gloriofo (dice Fontenelle nella Storia dell Accademia dell' anno r705.) ai primi Autori, i quali banno lavorato intorno all' Algebra, cbe quelle difficolta", cbe effi non poterono vincere, non - fi fieno ancora formontate. Il cafo irreducibile del terzo grado d ancor tale come lo eira - tempo di Cardano, giaccbé l' Algebra non é propriamente conofciuta clie da dugento anni in quad, e noi la abbiamo riceruta dalle mani degli Italiani. Quetti fono i paffi fatti dal Cardano in una Scienza allora nuova ed a pochiffimi eletti accellibile, e cinta d' ogn' intorno di triboli e fpine, che il Cardano colla penetrazione del fuo ingegno ha faputo in parte fvellere, ed eftirpare. Nelle altre fue Opere poi, che formano una collezione immenfa di dieci volumi in foglio, non fi conofce più il Cardano. Algebrifta: Sembra, che il fuo fpirito fiafi come rarefatto ed eftenuato nell' ampiezza e vaftità-di tante e tanto varie fue produzioni, come quei fiumi- reali, che tanto più perdon di fondo quanto più fi dilatano e acquiftano in fuperficie. Brillano è vero di 'gran lampi d'ingegno tutte le Opere di queft' Uomo, ma fono lampi pafraggieri e momentanei in una notte tenebrofa. Il fanatifmo, la fuperftizione, la credulita furmano il principal carattere di tutti i fuoi Libri. Nın vi è femminuccia tanto imbecille, la quale poffa credere o fognare inezie maggiori di quelle, che a fangue freddo e tranquillamente frive il Cardano in ogni pagina delle fue Opere, le quali faranno un eter- 
no monumento dei delirj, e delle deboleźze, in cui può cadere l'ingegno umano non regolato da un retto giudizio. Nè fi può dire, che quefti foffero vizj del fecolo, non della perfona; imperciocchè i dotti, e avveduti Uomini di quel tempo avevano già cominciato a rinvenire dalle fuperftiofe fcempiaggini dei fécolli tenebrufi; oltrecchè niun fognatore vi fu mai $f_{1}$ vaneggiante, il quale ne fpacciaffe tante e tanto ftrávaganti, "come fece il Cardano. Bafta leggere la fola fua vita fritta da lui medefimo, per intendere che il fuo capo era lavorato ful torno della pazzia; dico la fola fua vita, giacchè lo ftudio delle altre-fue Opere io non avrei cuore di configliarlo, nè anche ai piu intrepidi e agguerriti nelle letture fonnifere. In mezzo però a tante ftravaganze non ' $f$ può negare che Cardano non moftri uno fpirto vatto, e una grande fottigliezza d'ingegno: E s'egli aveffe potuto imbrigliare la fua immaginazione ftemperata e folleggiante, e contenerfi nei limiti che prefcrive un buon metodo di ftudiare, avrebbe refo degl' importanti rervigj alle Matenatiche, alla Filofofia, e alla Medicina. Dai femi di alcuni penfieri grandi originali e fublimi, fparfi in diverfi luoghi delle opere fue fi raccoglie quello, che egli era capace di fare, fe (come dice di Ovidio Quintiliano Inft. Orat. lib: X. Cap. I.) ingenio fus temperare quan indulgere maluiffet. Scrivafi dunque appiè della fúa Statua così : velles eum fur ingenio diaiffi, alicino juticio (s).

$$
\mathrm{S}=
$$

OSSER-

(5) Ita Quint. loce cit. de Suneca Inquirut. 
I 40

OSSER V A Z I O N E $S O P \quad R A$

\section{L'ACIDO VETRIOLICO}

TROVATO NATURALMENTE PURO, CONCRETO,

E NON COMBINATO

\section{GIUSEPPE BALDASSARRI}

PUBBLICO PROFESSORE D' ISTORIA NATURALE, E DI CHIMICA NELL' UNIVERSITA' DI SIENA.

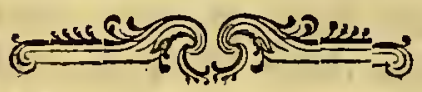

egen 5) (C) ralifi, e da iChimici non folo quel2i. S $\mathrm{S}$. lo, che per mezzo della diftillaziofi. ne fi eftrae dal Vetriolo, o colla all combuftione dallo Zolfo, ma un' a-

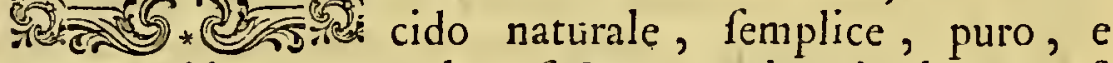
non combinato con altra foftanza, ed un' tal nome fi è dato a queft' ultimo, perchè tale appunto fi eftrae dal Vetriolo, quale acido fi ritrova nel maggior grado di purità, che poffa averfi, per effere affolutamente fenza alcun' odore, e colore, raffomigliandofi per quefto Capo all' acqua.

I più dotti, e profondi Chimici, e fopratutto l' Illuftre Stahlio, riguardano queft' Acido Vetriolico naturale la fola foftanza per fe fteffa effenzialmente falina, e che per l' unione, che contrahe con differenti altre foftanze non faline, è capace di formare la ferie innu- 
innumerabile di altre materie faline meno femplici, che ci prefentano l' Arte, e la natura. Un numero di fenomeni particolari, le proprietà delle foftanze faline, ed $i$ principj della chimica moftrano un grande accordo con quefta idea generale, la quale moftra d' avere una grand' aria di verità, ma fe fi efamina diligentemente, fi troverà mancarle molti fatti, ed efperienze per coftituirla del Carattere d' una verità dimoftrativamente provata.

Ma comunque fiafi, è certo, che quefto Acido naturale per altro femplice, e puro, trovafi ordinariamente combinato con altre fortanze, per la gran' facilità, che hà di fcioglierle, e di unirfi, e legarfi con effe. Quindi è che fi accoppia col Flogiftico, con i fali Alcalici fiffi, e volatili, colle terre afforbenti, con l'Argille, col Ferro, col Rame, col Zinco, di dove rifultano lo Zolfo, i Sali terzi, i Spati, i Gefli, le Seleniti, le Stalatiti, i Vetrioli, l'Allume.

Da quefto principio deriva il fentimento coftante de i più accreditati Chimici, che il predetto Acido Vetriolico non fi trovi giammai naturalmente puro, e femplice, cioè a dire folo, e fenza effere intimamente combinato con qualche altra foftanza: Nullibi autem, quantum fcimus, purum, five in fuida, five in folidefente forma occurrit diffe il Juachero (Confpect. Chym. Tom. 8. Tab. 58.) parlando dell' Acido univerfale, o Vetriolico, e poco dopo foggiunge Acidum univerfale, licet nunquam purum, \& Sancerum quantum adbuc innotuit, in terrie vifceribus reperiatur, fod unice Sulpburis mixto inditum fit, agnofimus tamen illud pro fempliciffmo $心$. Il dotrifimo Autore del Dizionario chimico nell Articolo 
142

ticolo Acide Vetriolique cosi fi fpiega, On ne trouve "point dans la nature d' acide Vitriolique pur, c' et , a dire, feul, \& nullement combinè avec aucune aú"tre efpece de Corps ; ce qui vient de la grande " quantitè des fubftances differentes, qu', il eft en etat , de diffoudre; \& de la facilitè avec la: quelle, il fe " conftituè ávec les diverfes corps, a mifure qu' il les "rencontre, Poi foggiunge, Il eft clair, pur ce qui "vient d' etre dit, de l' etat, ou fe trouve naturelle, mente, \& abitualmente l' acide Vitriolique, qu' on , ne peut l'obtenir feul, \& pur, que par des opera"tions particulieres de l'art, c'efta dire, en decom2pofant ceux des corps, qui en-contiennent le plus, "\& dont on peut le retirer plus facilement; ces corps "font le foufre, \& les vitriols, Lo fteffo fi conferma dal celebre Sig. Beaumè nel fuo Manuale di chimica pag. 8 r. ove dice "Cet acide (parla del :Vetriolico) "ne fè trouve jamais pur dans la nature a caufe de la "grande difpofition, qu'il a pour s'unir, \& fe combiner avec tous le corps, dans les que!s il eft com"binè "ed il Sig. Bucquet nella fua Introduzione allo "Atudio dei corpi minerali Tom. I. pag. 273. cosi fi efprime, on ne rencontre en aucun endroit l'acide vi"triolique pur,

Dalle addotte autorità adunque, e da altre, che averei poffuto addurre ad evidenza rifulta il fentimento, che comunemente corre intorno all' acido Vetriolico, cioè che quefto non fi trovi giammai naturalmente folo, e fenza effere combinato con qualche altra foltanza e che tale fi ottenga folo per mezzo della diftillazione del Vetriolo, e dell' Allume, o della combutione dello Zolfo. 
In mezzo per altro a quefta comune, e profondamente radicata opinione, mi avanzerò ad efporre qualmente in congiuntura di alcune mie offervazioni fatte nell' Anno fcorfo intorno alle Acque Termali dette di S. Filippo nel Territorio Senefe, mi è fortito di ritrovare in una grotta incavata negli Ammaffamenti di Tartaro depofto dalle medefime un'vero Sale vitriolico puro, fincero, naturalmente concreto, e fenza alcuna minima combinazione con altre foltanze, e perciò effer quefto una eccezione alla regola generale fiffata da i Chimici. Intanto ad oggetto di caminare col maggior' ordine, e chiarezza poffibile, credo neceffario il cominciare dalla defcrizione del luogo, dell' Acqua, delle depofizioni tartarofe, e della Grotta già mentovata.

Dalla parte Meridionale di Siena, \& in diftanza della medefima di trenta, e più miglia in circa, s'erge un'altiflimo Monte, chiamato Monte Amiato, e volgarmente Montagna di Sunta Fiora, in cima del quale in fecoli da noi remotiffimi, apparifce effere ftato accefo un' Vulcano, già da tempo eftinto, conforme ad evidenza raccogliefi dalle Lave, Pomici, vetrificazioni, ed altri avanzi Vulcanici, che fi offervano ammaffati nella fuperficie del medefimo, fcoperta dovuta all' immortal' Bottanico Fiorentino Pier' Antonio Micheli. Il monte fuddetto nell' abbaflarfi dalla fua Cima verfo la Valdorcia per quella parte, che riguarda il Greco a Levante rialza il fuo fianco in un' altro piccolo monte, chiamato comunemente il Zoccolino. Alle falde di quefto fecondo monte forga un' acqua Termale, chiamata il Bagno di S. Filippo, la quale quantunque di 
prefente fcaturifer in una Collina molto al di fotto delle falde fuddette, nondimeno moftra di effere ufcita anticamente all' aperto dalle falde medefime, mentre un' continuo non interrotto ammaffamento di un' Tartaro bianchiffimo depofto da detta Acqua Termale fi ftende da quefte falde fino al luogo dell' odierna forgente. Quivi ancora $f_{1}$ offerva, che al giorno d'oggi fi chiudono fuccefivamente le aperture dalle quali fgorga 1' Acqua a motivo del Tartaro dalla medefima depofto, onde è coftretta ad aprirfi fempre più baffo nuove ufcite, dalle quali poffa sboccare. Deriva queft' effetto dall' aggrumarfi immediatamente il Tartaro, tofto che l' Acqua giunge al contatto dell' aria efterna, conforme fi offerva ancora in altre Acque Termali, mentre in altre poi, che fono parimente Termali, non fi forma l'ingrummamento del Tartaro nel luogo dello sbocco, ma doppo effere l' Acqua fcorfa per qualche tratto ne' fuoi canali, come in quelle di Vignone, del Bagno di Santa Agnefe di Chianciano, ed in altre.

In un' fito di quefta depofizione Tartarofa pofto tra le falde del Zoccolino, ed il luogo dove prefentemente sboccano le varie polle di queft' Acqua Termale, e dove detta depofizione pende verfo Tramontana, fi offerva un' ampia Grotta incavata nel Tartaro; nella quale per due aperture fi può entrare, ed egualmente ufcire all' aria aperta .

Vaghiffimo fpettacolo rapprefenta agl'occhi di un Naturalifta l' interna cavità di quefta Grotta, poichè $\mathrm{fi}_{1}$ oferva il fondo tutto ricoperto da una bellifima crofta gialla di Zolfo minutamente criftallizzato, quale incroftatura fi rialza attaccata alle pareti per l'al:- 
tezza di un'braccio, e mezzo incirca, formando in tal guifa una bella fafcia gialla, che a tale altezza circonda la parte più baffa di tutte le pareti della Grotta. I Corpi eftranei poi, che fi trovano in detto fondo, come Legni, Foglie di Alberi, Erbe, Offa di animali, e Pietre, tralportatevi dal Vento, o da altra cagione, fi rinvengono tutti coperti da una crofta di Zolfo di maggiore, o minor' groffezza a proporzione del tempo, per cui detti Corpi vi anno foggionnato.

Terminata quetta Zona Sulfurea, fi fcorge nel rimanente delle Pareti, e nella volta della Grotta una congerie d'innumerabili gruppi di una bianchiffima fioritura lanugginofa, quali gruppi, per fervirmi di una volgare fimilitudine, anno qualche raffomiglianza alle palle del Cavol' Fiore. Accoltate alla lingua quefte fioriture, vi producono un' impreffione di fapore acido, ma di un' acido perfettamente fimile a quello dello Spirito cavato per diftillazione dal Vetriolo, e non lafcia in bocca quella fenfazione di auftero, o di aftringente, che vi lafciano i Vetrioli, o l'Allume. Se attentamente fi offervi quefta fioritura, o fi guardi con una Lente, fi ritrova effere un' aggregato di minutiffimi Fili, e Criftalli Salini ramoli, trafparenti, e compofti di varie faccette, ma non puó per altro determinarfene la precifa figura.

Lavati più volte con Acqua quefti gruppi fi fcioglie agevolmente la parte falina, e rimangono $\mathrm{i}$ medefimi con una fuperficie afpra, ed ineguale a motivo d'innumerabili punte, piramidi, e criftallizazioni pietrofe totalmente infipide, e indiffolubili nell' Acqua ufa ta non in dofe eccefliva.

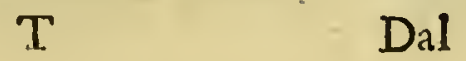


Dal fondo della Grotta efala un' caldo vapore, che fpande un'ingrato odore fulfureo, e che fi alza in diftanza di un'braccio, e mezzo in circa, civè a quella fteffa altezza a cui fi eftende la fafcia, o incroftatura dello Zolfo. Comparifce detto caldo vapore in forma di un' fottiliffimo fumo, o nebbia ; ed è fuffocativo degli Animali, qualora fi trovano inviluppati in quella Atmosfera, e in fatti vi trovai morti diverfi infetti come Farfalle, Mofche \&c. Alla predetta altezza fi eleva il mentovato vapore allora quando fpirano i venti Meridionali, ma allo fpirare de i Settentrionali tofto fvanifce, e ftando in piedi nella Grotta non $f_{1}$ fente alle gambe il nominato calore, conforme per replicate prove $\mathrm{mi}$ fono afficurato. In vicinanza di detta Grotta, ve ne fono alcune altre, ma molto più piccole, nelle quali feguono preffo a poco gl' ifteffi fenomeni.

Ma foprattutto merita di effere confiderata una lunga fenditura aperta in quefta gran' maffa di. Tartaro, che paffa in poca diftanza avanti l'imboccatura di detta Grotta, la cui profondità farà di trenta, e più braccia, e guardando dall' alto fi fcorgono le pareti di quefto gran' Cretto; pure incroftate di Zolfo, e al di fopra di quefto vi è la mentovata bianchiffima fioritura :

Quelta breve, e fuccinta defcrizione credo, che farà più che baftante per quanto hò in animo di provare; e chi bramaffe una più eftefa notizia di quefto luogo, potrà leggere la bella defcrizione del Cafale, e Bagni di S. Filippo diftefa dall' eruditifimo Signor Dottor Leonaido Vegni, e dirette al chiariffimo Signor Dottor Gaetano Monti Profeffore d'Iftoria naturale nell' 
nell' Iftituto di Bologna; ed io intanto pafferò alla confiderazione di quanto hò finora notato.

L'Efalazione, che come diffi, s'alza dal fondo di quefta Grotta, deve per ogni riguardo confiderarf per una emanazione di ciò, che da i moderni Chimici fi chiama col nome di Spirito Sulfureo Volatile, quale confifte in un' compleffo di Acido Vetriolico univerfale acquofo, unito debolmente ad una porzione di Flogiftico, quale li compartifce alcune proprietà che non ave$\mathrm{va}$ in ftato di puro Acido Vitriolico. Sono diverfe quefte proprietà, poichè l' odore dello Spirito Sulfureo Volatile, ch'è di Zolfo bruciato, è sì vivo, e sì penetrante, che può in un' fubito foffogare qualunque animale. Quefto appunto fuccede nella noftra efalazione, poichè, come diffi, trovai nel fondo della Grotta molti infetti morti, e vi gettai vivo quel infetto, chiamato dal Vallifnieri Ragno-Locufta, \& immediatamente vi morì. Un mio compagno, che inavertentemente fi era abbaffato col capo dentro alla sfera di attività di quefta efalazione, fenti penetrarfi nella trachea un' alito foffocativo, che l' obbligò a rialzare prontamente il capo.

Altra proprietà pofliede lo Spirito Sulfureo Volatile, che è quella di diftruggere il colore de i Corpi. Gettai nel fondo della Grotta un' pezzo di Carta turchina, $\mathrm{e}$ in breve tempo fi fcolorì, e divenne cenerina; fi fcolorì parimente una Fettuccia di feta di colore cremifi, che ivi avevo collocata . Tutto l'Argento, che avevamo indoffo tanto io, che due miei compagni, confiftente in Fibbie, Caffa d' Orologio, e Denari, perdette lo fplendore metallico,

$$
\mathrm{T}_{2} \text { fi fco. }
$$


fi fcolori, e divenne nero con qualche mefcuglio di color' d'oro, in quel tempo, che fi dimorò dentro la Grotta. Da tutto ciò dunque chiaramente rifulta, che la defcritta efalazione altro non è che una emanazione di ciò, che dai moderni Chimici chiamafi col nome di Spirito Sulfureo Volatile, cioè di un' compofto d'Acido Vetriolico univerfale, d'Acqua, e di Flogiftico.

Siccome in tempo della noftra permanenza dentro la Grotta, fi fperimentava un' certo vapore caldo a $i$ piedi, ed alle gambe, che $f_{i}$ eftendeva fino a $i$ ginocchi, volli offervare a qual' grado fi follevaffe il Mercurio nel Termometro, perciò pofto quefto nel fondo della Grotta, in cui il Mercurio all' aria aperta ftava dodici gradi fopra il punto della congelazione fecondo la Scala di Reaumur, e avendovelo tenuto per lo fpazio di mezz' ora in circa, $f$ ritrovò, che il Mercurio era falito fino a gradi venti fopra il detto punto della congelazione. Il Sig. della Condamine ancora offervò nella Grotta del Cane di Pozzuolo, che il Termometro dello Spirito di Vino di Monfieur Reaumur collocato nella Grotta in una mezz' ora da $\mathrm{i}$ gradi 12 . fali fino a i 30 . fopra il punto di congelazione, ficcome può vederfi dalla defcrizione del fuo viaggio in Italia, inferita nelle memorie dell' Accademia Reale delle Scienze di Parigi per l'Anno $1757^{\circ}$ quefti fatti difcordano da quanto afferifce il Signor Bucquet nella fua Introduzione allo ftudio dei Corpi minerali, il quale nel Tom. 2. pag. 20. parlando di quefte efalazioni, dice che con tutto fiano calde, non inducono alcu- 
alcuna mutazione nei Termometri, ed ecco le fue precife parole, Cette vapeur quoique chaude eteint les lu" mieres, \& empêche la detonation de la poudre a "Canon: d'ailleurs elle ne fait eprouver variation aux "Thermometres, \& Barometres, qu' on y plonge."

Quefto Spirito Sulfureo Volatile, cioè quenta efalazione compofta d'Acido Vetriolico, d'Acqua, e di Flogiftico giunta all aria aperta nel vano di quefta Grotta, che puo confiderarfi come un'maravigliofo Lambicco appreftato dalla Natura, fi offerva Comporfi, ed entrare in nuove combinazioni. L' Acido Vetriolico, che ftante l' interpolizione della quantità dell' Acqua eccelliva per la naturale fua compofizione, non avevia poffuto a vanti contrarre una intrinfeca combinazione colla parte infiammabile, quivi poi fi unifce colla medefima in ftato di ficcità, e forma lo Zolfo, che fi attacca, e fi criftallizza nel fondo, e nelle pareti della Grotta; e fopra gl'altri Corpi Solidi, che incontra difperfi nel tondo fuddetto. Si fa che l'Acido Vetriolico ha maggiore affinità col Flogiftico, che con qualunque altra foftanza, conforme cofta dalla tavola delle affinità di Geoffroy, nella quale fi offerva che il Flogiftico ottiene il primo luogo frà le affinità, che anno diverfi corpi con quefto Acido. Lo fteffo confermafi dalla Tavola delle affinita corretta, ed eccrefciuta in qualche parte da Monfieur Rovelle; ed il Sig. Filippo Limburgo nella Tavola delle affinità, da effo notabilmente ampliata nella fua bellifima Differtazione fopra le Affinità Climiche, dimoftra, che non folamente l' Acido Vetriolico, ma anche gl' altri Acidi Minerali, e gli ftefli Acidi 
Acidi Vegetabili, anno maggiore affinità col Flogitico, che con tutte le altre foltanze.

Ciò fuppofto qui chiaramente ci fa conofcere l' offervazione, che in quefta circoftanza l'Acido Univerfale, o- Vetriolico efercita la fua grande azione fopra il principio infiammabile, fi fepara dalla fortanza acquo$f a, e, f i$ riduce in ftato di ficcità, condizione neceffaria, ed effenziale acciocchè l' Acido col Flogiftico poffa coftituire lo Zolfo. Che la parte acquofa refti feparata dagl' altri due principj, me lo fecero apertamente conofcere tutti quei Corpi eftranei, che diffi avere ritrovati incroftati di Zolfo nel fondo della Grotta, cioè foglie d'Alberi, Legni, \& Erbe, quali tutti erano bagnati, e grondanti di umido, ed accoftati : alla lingua mi dimoftrarono effere queft' umido un' acqua affatto infipida, e fimile all' acqua commune, e niente acida conforme fi farebbe a prima giunta creduto. Il Cannello fteffo del Termometro, che conforme diffi, tenni per mezzora fopra il fondo della Grotta; era grondante di-detta infipida umidità, ficcome lo era ancora, la Crofta dello Zolfo attaccata al fondo, ed alle pareti della Grotta. Quefta natural feparazione dell' acqua dallo fpirito acido tofto che giunge all' aria aperta nel vano di quefta Grotta; fi comprende ancora dal faperfi, che l' acque che fgorgano dalla terra ripiene di quefto fpirito, in breve tempo lo perdono, e ne reftano fpogliate, fe fi tengono efpofte all' aria.

Nel profondiffimo Cretto poi, che diff paffare avanti le imboccature della Grotta, fi offerva l'incroftamento Sulfureo eftenderf ad una altezza incompara- 
bilmente maggiore, ma per effere quefto inacceffibile, e pericolofo a motivo del foffocante vapore, non $\mathrm{mi}$ fu poffibile il determinarla con qualche precifione; offervai bensì che a detto incroftamento fuccedevano "al di fopra le fteffe fioriture faline. Sembra molto verifimile, che per ritrovarfi lo fpirito Sulfureo riftretto tra le Pareti altiffime di quefta gran' feffura abbia campo di entrare in nuova combinazione, per un'tratto maggiore, dove che nella Grotta, deve in parte diffiparfi a cagione delle due ampie bocche, che danno l'ingreffo nella medefima, e per confeguenza deve ancora lo Zolfo aggrumarfi a minore altezza .

Una porzione dunque di quefto Flogiftico fi unifce con altra porzione di acido, e forma lo Zolfo; altra porzione bifogna credere, che fvanifca per, le bocche della Grotta, argomentandofi dall? odore Sulfureo, che dalla Grotta medefima fi tramanda all' aria aperta. Qui in tanto $f i$ vede, che una porzione di acido, che non fi è combinata col principio infiammabile, ne fi è diffipata nell' aria, incontrando le pareti; e la volta della Grotta, che foro formate dal Tartaro depofto anticamente dalle Acque, che è di natura Calcaria, mentre lo Spirito di Nitro, ed altri acidi bollono vivamente con effo, detto acido fcioglie quefto Tartaro, vi fi unifce; $e$ forma le defcritte concrezioni, e coftituifce in tal guifa una fpecie di Sale, O Concrezione Selenetica : Che quefte tali Concrezioni frano un' Sale Selenitico, cioè un' compofto di Terra Calcaria, e di Acido Vetriolico combinati a perfetta faturazione, chiaramente apparifce dal non fufcitarfi alcuna ebullizione allor' quando vi fi verfa fo-

pra 
pra l'Acquaforte, dove che verfata la medefima topra il Tartaro, in cui è incavata la Grotta, vi bolle con molta veemenza, ficcome ancora bolle moltiffimo con alcune Concrezioni alabaltrine, che fi trovano - mefcolate col Tartaro.

Dopo, che queft' Acido Vetriolico ha faturata perfettamente la Terra, e coftituita una Concrezione felenitica, continua a deporfi fopra la medefima, ma per effere effa perfettamente faturata, non può ulteriormente combinarfi colla Terra, ma fi offerva attaccarvifi fopra in qualità di puro Acido, ed in figura di minutiffimi Criftalli, e di filamenti a guifa di una lanuggine, o di una muffa. Or quefto è appunto l'Acido Vetriolico puro, concreto, e non combinato, e che mi lufingo di avere, trovato tale naturalmente, il che è mio affunto adeffo il dimoltrare.

In primo luogo fe $f \mathrm{i}$ accofta querta fioritura falina alla lingua, fi fperimenta fopra di effa una impreffione, e fapore di Acicio totalmente fimile a quello; che vi produce lo fpirito cavato per diftillazione dal Vetriolo, e dall' Allume, o per combutione dallo Zolfo, ne rifveglia quella fenfazione di aftringente, di auftero, 'o di dolce, che vi produce detto Acido combinato colle foftanze metalliche, o terree, ciò̀ il Vetriolo; e l' Allume,

In fecondo luogo la predetta fioritura bolle furiofamente con l'Olio di Tartaro, come appunto accade con 1 Acido Vetriolico puro, dove che allora quando è combinato $f$ unifce placidamente colle foftanze alcaline, fenza alcun' indizio di tumulto, o di effervefcenza : 
Oltre a ciò le predette fioriture Acido-Saline efpofte all' aria umida $s^{\prime}$ inumidícono; fi rifolvono in in un liquore, e vanno, come diceli, in deliquio, il che non accade "quando l' Acido' Vetriolico è combinato. Si fa che l'Acido Vetriolico puro, particolarmente quando è concentrato tira avidamente l'Acqua dall' aria; dove che i Vetrioli, cioè l'Acido combinato, tenuti efpofti all' aria non fi rifolvono giammai in umore, ma piuctofto vi fi calcitano per l' evaporazione dell' Acqua, che concorre al loro criftallizamento.

Una Carta turchina, in cui era involta una porzione di detta fioritura, . tenuta efpofta all' aria umida contrafle un vivaciflimo color' roffo per la rifoluzione in umore di quell' Acido, ma dalla foluzione del Vetriolo non If produce un tale effetro. E' offervabile intanto che quefto Acido quando era in forma di Acido Sulfureo Volatile, cioè di Acido benchè leggiermente combinato col Flogiftico, efalando dalla terra, diftruggeva il colore della Carta turchina, feparato dalla parte infiammabile, ed acquiftando il carattere di puro Acido Vetriolico, tinge la detta Carta di un colore roffo molto vivace, altra riprova, che ad evidenza dimoftra effere la predetta fioritura falina un puro Acido Vetriolico non combinato.

Sciolf una porzione ben grande di quefte Acide fioriture in Acqua di pioggia, a fegno di averne una foluzione ben faturata, quale feci feltrare per carta grigia ad oggetto di ripurgarla da qua unque mefcuglio di foftanza terreftre, che nel raccoglierle fi foffe con effe rimefcolata. Quefta foluzione aveva lo fteffo fapore, che hà lo Spirito di Vetriolo Acquofo. Verfai fopra

una 
una porzione di quefta l'Olio di Tartaro per deliquio, fi fufcitò l' effervefcenza, e fi formò un' vero 'Tartaro Vetriolato di fapore falfo, ed amaro, fenza che fifcor. geffe alcun' indizio di feparazione terreftre, o metallica. Altra parte di quefta foluzione la pofi ad evaporare in una tazza di Vetro lotata a fuoco di arena, ed a mifura, che fi avanzava l' evaporazione fi accrefceva l'Acidità; a fegno tale, che compita la medefina, e ridotto il tutto a ficcità, reftò un' Sale di Color' nero, intenfamente Acido, ed infoffribile alla lingua.

Pofto quefto Sale in un' vafetto di Vetro, ed otturatane la bocca in maniera che I' aria aveffe libero acceffo nella cavità del medefimo, il Sale in poco tempo attrafle l'umidità dall'aria, e fi riduffe in un' liquido nero intenfamente Acido, e fimilifimo all' Olio di Vetriolo.

Quefte due ultime proprietà convengono parimente all' Acido Vetriolico puro, quale fappiamo, che per.il contat to del principio infiammabile diviene, nero, mentre nell' atto dell' evaporazione, fi è unito il noftro Sale alle parti infammabili fomminiftrate dal fottopofto fuoco ; e fappiamo altresi che l'Acido Vetriolico puro attrahe avidamente l' Acqua dall' aria.

Giacchè dunque da quanto ho io notato, e fperimentato sù quefto Acido; chiaramente rifulta, che le divifate proprietà; ed effetti offervati convengono all'Acido. Vetriolico puro, e non combinato, fi potrà ficuramente inferịre effere il medefimo della divifata natura.

Ma ben' comprendo, che mi fi potrebbe objettare un' fentimento del Signor Rovelle dottifimo Chimico Franzefe. Quefto in una memoria prefentata all Accademia 
demia Reale delle fcienze di Parigi, ed inferita trà quelle dell' Anno I754. avanzó che piu Sali terzi quando fono giunti al punto di una perfetta faturazione, fono ulteriormente fufcettibili di un' ecceffo di Acido nella loro compofizione, e che quefto Acido ecceffivo, quantunque dimoftri tutte le proprietà di un'Acido, tuttavia egli vuole, che debbafi riconofcere come Acido non libero, ma combinato. In riprova di quefta fua opinione adduce in primo luogo più : combinazioni di foltanze Metalliche con 'gl' Acidi, come quella del Mercurio con l' Acido del Sal' Marino, e del 'Vetriolico, quella dell' Antimonio con l' Acido del Sal' Marino, e quella del Cobalto con l'Acido nitrofo, e pretende che ciafcuna di quefte foftanze metalliclie poffa formare con gl' Acidi due Sali Neutri differentifimi; uno dei quali fia imbevuto di Acido al punto di una perfetta faturazione, e l'altro contenga un' ecceffo di Acido. Produce ancora quefto dotto Chimico l' efempio di un'Sale terzo nato dalla combinazione di un' Ácido Vetriolico con lin' Sale fiffo Alcalico Vegetabile, cioè del Tartaro Vetriolato. Quefto Tartaro Vetriolato con ecceffo di Acido, ma di Acido combinato, hà un'fapore Acido, tira l'umidita dall' aria, va in deliquio, tinge di roffo la tintura di Viole, fa effervefcenza con gl' Alcalici tanto fill, che volatili, efi criftallizza rimanendo Acido. In fequela dunque della dortrina del Signor Rovelle, mi fi potrebbe objetrare; che l' Acido Vetriolico, di cui io parlo, non è puro, ma bensì combinato, guantunque dimoftrı tutte le proprietà di Acido furo, e libero, e che in foftanza altro non è il compleflo delle Grume colla fioritura Acida che 
un' fale Selenitico naturale con ecceffo di Acido Vetriolico, ma combinato.

Per : rifpondere; a quefta difficoltà, che potrebbe efferni promoffa; farò avvertire che la dottrina Rovelliana sù quefto particolare non è così certa, e ftabile, che debba ammetterfi per ficura, e indubitata, anzi moftra effere più tofto dubbia, e vacillante, n'per meglio dire affolutamente falfa. Il celebre Chimico Signor Baumè la combatte vigorofamente, e ne fa vedere l' infuffiftenza, e la debolezza, in piu Memorie lette all' Accadeniia Reale delle Scienze, come ancora nel Giornale, e nelle Gazzette di Medicina, e dimoftra gli sbagli, e il poco fondamento sù ciò del Signor Rovelle. Io foggiungerò, che queft' Acido per ecceffo, che $f_{1}$ vuole combinato, non dimoftra in qualunque prova fatta alcun' indizio di combinazione, nè in quanto al fapore nè in quanto alle altre proprietà, ma coftantemente fi dimoftra per Acido libero, e fciolto. Oltre a ciò queft' Acido fi fepara dalle concrezioni di Tartaro fulle quali fiorifce meccanicamente, e fenza foccorfo di fuoco, o di altro agente intermedio. Io l'ho feparato ftaccandolo colle dita, o con una penna; oppure lavando con acqua pura le fuddette Concrezioni, le quali doppo la lozione fono rimafte infpide, come appunto doveva rettare un' fale Selenitico, e I'Acido creduto per ecceffo, ma combinato, ha dimoftrate tutte le proprieta di Acido puro Vetriolico. Dovrafli dunque conchiudere, che quefto Acido dopo di avere efattamente faturate le grume Calcarie nella volta, e nelle pareti di quefta Grotta, vi fi è foprapofto in forma di filamenti, e di piccoli criftalli libe- 
ro da ogni vincolo, e che per confeguenza trovafi l' Acido Vetriolico naturalmente concreto, puro, e fenza alcuna combinazione.

Tali efforefcenze Sulfuree, e Vitrioliche fono fate da me offervate in altri luoghi, ne quali efalava dalla terra lo Spirito Sulfureo Volatile. A S. Albino in vicinanza di Monte Pulciano, dove fgorgano certe acque fredde, e Vetrioliche, al margine di certe buche, dentro le quali fi fentiva rumoreggiare l'Acqua, trovai attaccati alcuni filamenti a guifa di una lanuggine, in parte Sulfurei, e in parte Vetriolici. Parimente a i I agoni di Travale, de i quali l'acque tramandano confumili efalazioni, incontrai in certi rami d'Albero gettati al di fopra di una di quelle forgenti; alcune concrezioni in parte di Zolfo, e in parte di Vetriolo. Ma la fcarfa quantità di effe, che in quefti due cafi mi fi prefentarono, non mi diede il comodo nè di raccoglierle, e per confeguenza nè anco di efaminarle. Il Chiariflimo Signor Dottor Targioni nelle commendabiliflime Relazioni de $\mathrm{i}$ fuoi Viaggi per la Tofana Tom. 3. pag. 433. Ediz. 2. parlando di un' croftone prefo dalle ripe de $\mathrm{i}$ Lagoni di Monte Rotondo, dice che era tutto quanto inzuppato di fugo Vetriolico pretto, che fa allegare $\mathrm{i}$ denti. Lionardo di Capoa ancora nella prima lezione intorno la natura delle Mofete, afferifce, che nella Mofeta di Telefe nel Sannio, fi trova. atticcato alle pietre a guifa di mufta un' fale Acetofo, che con molta probabilità può crederf Vitriolico : Sarebbe fuperfluo poi il diffonderfi in dimoftrare; che dalle efalazioni delle Mofete, delle Acque Minerali, de i Bulicami \&c. fi forma frequentemente lo. Zol- 
fo fopra i Corpi che li fono foprapolti, o fituati lateralmente, mentre ognuno che fiafi aggirato con qualche attenzione intorno alle forgenti di fimili emanazioni, averà bene fpeffo avuta occafione di rilevare.

Da quefte offervazioni chiaramente rifulta un'metodo, che tiene la natura nel produrre la foftanza dello Zolfo, cioè, che nello Spirito Sulfureo Volatile, che efala dalla terra, ed in particolare da buona parte delle Acque Minerali, l' Acido Vetriolico unendofi al principio infiammabile, e feparandofi dall' Acqua, e perciò ridotto in ftato di ficcità, coftuifce lo Zolfo. Quindi ne deriva che quantunque certe Acque Minerali tramandino un' ingrato odore Sulfureo, o per meglio dire un' odore fimile a quello, che tramanda il Fegato di Zolfo difciolto nell' acqua, nulladimeno non dover$f_{1}$ inferire, che in tali acque $f_{1}$ contenga lo Zolfo difciolto, ma folamente i materiali neceffarj alla fua coftituzione.

Non nego però, che in qualche Acqua di tal' natura non poffa trovarfi lo Zolfo difciolto, poichè può darfi il cafo che l'Alcali Minerale combinato collo Zolfo, ve lo tenga in diffoluzione; ma quefto cafo è molto raro, nè tra molte Acque efalanti odore Sulfureo, che hò efaminate, mi è giammai accaduto di ritrovarne alcuna. Il Sig. Monnet nel fuo Trattato delle Acque Minerali nel Cap. 3. nel quale parla delle Acque Minerali Sulfuree, afferifce, che fe per Acque Sulfuree noi intendiamo quelle, che contengono realmente del Zolfo, quelta Claffe di Acque Minerali farà piu piccola di tutte le altre, e che niente è pii raro, che di vedere Acque, che contenghino un' vero Fegato di Zolfo, 
fo, e che per mezzo degl' Acidi lafcino precipitare uno Zolfo reale, e che $f i$ fono fatti molti inutili tentativi per cavare lo Zolfo, che non vi efifte. Soggiunge poi, che vi fono certe Acque Sulfuree, come quelle di $B a$ rege, - e della valle di Montmorency, che realmente precipitano le foluzioni metalliche a guifa del Fegato di Zolfo, ma che il Signor Macquer nella relazione fatta all' Accademia Reale delle Scienze delle Acque di Montmorency, offerva, che quantunque quefte acque a primo afpetto fembrino contenere lo Zolfo, ciò non oftante per l' efame, ch' effo ne ha fatto non ne contengono nè poco, nè punto.

Un erudito, e dotto Scrittore moderno, da me fommamente riverito, e ftimato, il quale ha dato al pubblico un Opera eccellente fopra le Acque della Porretta nel Bolognefe, chiama Acque Sulfuree tutte quelle, che tramandano !'odore del Zolfo, e chiama Zolfo Volatile il vapore producente l' odore fuddetto. Ma quefo poco importa, trattandofi puramente di nomi. Il più effenziale fi è qualmente afferifce, che quefto Zolfo Volatile contenuto in quelle Acque fi puo da effe precipitare, coll' infondervi una porzione prefro che eguale di Aceto fillato, afficurando, che in tal guifa fi precipita una porzione di Zolfo in fondo del vafo, e che altra porzione refta fofpefa nell' Acqua, argomentandofi ciò dal colore lattiginofo, che l' Acqua ritiene. La brama d'apprendere nuove cognizioni, e di fpogliare la mia mente di qualche pregiudizio, $m$ ' induffe a tentare una tale efperienza in queft' Acqua Termale di. S. Filippo, la quale tramanda un' ingratifimo odore Sulfureo, o per meglio dire del vapore del 
Fegato di Zolfo, e che fecondo il noftro Autore deve contenere il Zolfo Volatile. Pofta dunque una porzione di queft' Acqua attinta alla forgente in un gran Vafo di Criftallo, e aggiuntavi una quantità eguale di Aceto Atillato alla prefenza di più perfone, nè l' Acqua divenne lattiginofa, nè fi precipito cofa alcuna, ma fi nantenne il mefcuglio limpido, chiaro, e trafparente, con tutto che teneffi il tutto in quiete per non poco fpazio di tempo. Replicai più volte il tentativo, ma fempre con l' efito medefimo. Che che fia delle Acque Termali della Porretta, io non voglio negare, che un tale effetto in quefte fucceda, nè derogare all' autorità del Chiariflimo Scrittore, diro folo, che in varie Acque Termali da me efaminate, e che fpirano un fpiacente odore di Zolfo, e che fecondo l' Autore devono contenere uno Zolfo Volatile, non mi è .giammai fortito di vedere precipitarfi lo Zolfo per l'affufione degl' Acidi. In oltre io rifletto, che fe quefto Zolfo è Volatile, non dovrebbe precipitarfi al fondo del Vafo per l'affufione dell' Aceto Atillato, ma dovrebbe diffiparfi nell' aria, e fpargere per effa un veemente odore Sulfureo, il che realmente non accade. Le foltanze volatili quando fi feparano dai Corpi, ai quali ftanno unite, non fi precipitano al fondo dei Vafi, ma fi follevano per l'aria. Quando alla foluzione del Sale Ammoniaco fatta nell' Acqua fi aggiunge un' Alcali fiffo, il Sale Alcalino Volatile non fi precipita, ma $s$ 'inalza per l'aria, conforme chiaramente apparifce dall' odore urinofo, che fi fa fentire.

Di fopra accennai, che tra l'informe ammaffamento del Tartaro depolto da quelte Acque Termali di 
di S. Filippo, fi ritrovano alcune Concrezioni AlabaItrine, intorno alle quali mi occorre il dire qualche cofa. Sono quefte femidiafane di un colore bianco fudicio, ed alle volte tendente al giallaftro; la loro figura poi non è coftante, variando quefta moltiflimo. $\mathrm{Si}$ offerva alle volte quefto Alabaftro difpofto in una maffa inregclare, alle volte rarprefenta un' gruppo di Concrezioni emisferiche, e manımillari di varie grandezze, e qualche volta confifte in una congerie diglobuli della grandezza più, o meno di un' Pifello, quali globuli fi offervano o ricoperti da una corteccia fottile di bianco Tartaro, o incaftrati come in una Nicchia del Tartaro medefimo. Sono quefti a maraviglia rapprefentati dalla Figura dei Pifoliti nella Metalloteca Vaticana del Mercati, la qual Figura non ècertamente alterata, nè caricata, ma naturalifima, come ancora ricavo da altre moftre di Pifoliti, che confervo preffo di me. Quefta Concrezione viene denominata dal Chiariflimo Signor Linneo Sift. Nat. Tum.3. pag. m.1 89 . Toplous Calcarius globulis pifformibus cruftatis congeftus, chiamafi anco Topbus Calcarius glomeratus ex granis globofis. Cruftatis Muf. Teff. 74. n. 3. dal Wormio nel Mufeo pag. 52. dicefi Pifa Carolina; nella defcrizione poi foggiunge $=$ Lapis integer mađam ovorum $P$ ifcium majorum exprimit, continentur vero in ea Pifa plurima, fuis queque inclufa favis, feu loculis, ex quibus levi negotio extrabuntur = Non entrerò adeffo nella difcuffione qual' fia precifamente l'origine di tante pietre compofte di un'aggregato di Corpi rotondi, che anno indotta una certa confufione tra i Naturalifti, e che da effi fono riconofciute fotto il nome di Pifoliti, di Ooliti, di Cen- 
chriti, di Meconiti, di Ammiti, Faciti, Orobiti \&c. Non ftaro dunque a ricercare fe quefti Corpi rotondi fiano o piccole Stalagmiti; o grani di arene, o.femi di piante impietriti; oppure Ova di Pefci parimente impietrite. A me bafterà effermi afficurato, che le Pietre dette Pifoliti fono un' prodotto di Acque Minerali pietrificanti.

Ritornando intanto a parlare delle noftre concrezioni alabaftrine dirò, che non è cofa nuova, che tra gl' ammaffamenti di Tartaro, e di Grume depofti dalle Acque Minerali pietrificanti fi trovi framifchiato l'Alabaftro, poichè di ciò ne diedi manifefte riprove nella mia Relazione dell' Acque Minerali di Chianciano, parlando degli Alabaftri di Caftel' Nuovo dell' Abate nel Sanefe, dove notai ancora, che tra $\mathrm{i}$ - Tartari dell' Acque Termali di Rapolano avevo ritrovato l' Alabaftro. Spezzai diverfi de i fopradetti globuli, e Concrezioni Emisferiche, ed offervai che parte erano pieni, ed altri con una cavità nel mezzo; offervai altresì nel luogo della rottura una congerie di filamenti, che dalla fuperficie anno una direzione, ed una. tendenza verfo un' centro comune. Quefta ftruttura filamentofa, e diretta ad un centro unita ad una fpecie di trafparenza, mi fece congetturare, che il predetto Alabaftro fia una Criftallizazione pietrofa, e. che la materia che lo coftituifce fia la fteffa terra Calcaria, di cui è compofto il Tartaro, mentre tanto quefto, che l'Alabaftro bollono furiofamente quando vi fi infonde l'Acqua forte. Da ciò fono portato a credere, che la compolizione del Tartaro, e dell' Alabaftro fia la medefima, cioè che fiano ambedue concrezioni formate da una terra Calcaria non 
perfettamente faturata dall Acido Vetriolico, che debbano confiderarfi come pure varietà, e che la fola differenza dipenda da qualche accidente occorfo in tempo della loro formazione, Non mancai di fare qualche offervazione, e di riflettere fopra gli Accidenti, che potevano aver contribuito a difporre la predetta materia Calcaria ora in forma di un' tumultuario ammaffamento, ed ora in forma di una Pietra Criftallizata; ma tra le diverfe idee, che mi fi prefentarono niuna ve ne $\mathrm{fu}$, che intieramente $\mathrm{mi}$ foddisfaceffe a motivo principalmente di alcune offervazioni, . che parevano direttamente oppofte fra loro.

Nel paffeggiare intanto all' intorno di quefte forgenti, non mancai di notare alcune piante, che fpontaneamente nafcono o in quell' ammaffamento di Tartaro, o all' intorno del medefimo. Tra quefte mi pare di non dover' tralafciare lo Smirnio Cretico, che trovai nafcere, e vegetare copiofamente in un' luogo molto frefco, ed ombrofo alle falde appunto del Zoccolino, dove trovafi il principio della depofizione Tartarofa. E' ftata quefta Pianta creduta efotica, e foreftiera, e fu chiamata dal Mattioli Snyrnium Creticum, e dice nafcere nell' Ifola di Candia, e feminarf in Italia. Dal Cefalpino de Plant. Lib. 3. Cap. 43. pag. 303. fu detta Olufatrum alterius generis, e la dichiara per fo. reftiera, dicendo di effa Alierum genus peregrinum nuper fervi capit. Dagl' Autori dell' Iftoria delle Piante di Lione 791. Perfoliata altera. Da Gafparo Bauino nel Pina: ce I54. Smyrnium peregrinum rotundo folio, e da Gio: Bauino Hift. Plant. 3. 1 25. 2. Smymium Creticum perfoliatum. Il Morifone Hift. Oxon. 3. 277. parlando di que$\mathrm{X}_{2}$ Ata 
Ita pianta dice Biennalis, aut trima ef bac Planta. Infule Cretenfis, nec non Sicilice Alumna eft. Il Chiariffimo Signor Linneo nell' Orto Cliffort. I04. la nomina Smyrnium foliis caulinis fimplicibus, amplexicaulibus, $\mathrm{e}$ foggiunge crefcit in locis bumentibus juxta rivulos; In montibus Eiquicolorum, Valvenfum, o A Aprenfium, in $S a-$ binis; In Sicilia propé Punto Cerciola, juxta Prozzalu; in Creta; e Spec. Plant. m. 376. dopo averla chiamata collo fteffo nome dell' Orto Cliffortiano, le da il nome triviale di Smyrisum perfoliatum, e foggiunge Habitat in Italia, in Creta. A tutto quefto potrò io aggiungere, che nafce ancora in Tofcana, e fegnatamente nello Stato Senefe a i Bagni di S. Filippo.

Degna di offervazione è in quefta pianta la varie: tà delle foglie, imperocchè quelle, che nafcono immediatamente dopo la radice, attaccate ad un lungo piede, fi dividono di tanto in tanto in ale oppofte, e quefte di nuovo in altre con foglie profondamente intagliate, di maniera tale, che le fuddivifioni particolari poffono affomigliarfi alle foglie dell' Apio paluftre. Le foglie poi, che ftanno fotto la divifione de $\mathrm{i}$ rami, fono differentiffime dalle prime, poichè quefte fono rotonde fenza alcuna divifione, ma foltanto leggiermente intagliate nel contorno, ed abbracciano i rami a guifa di alcune foglie dell' Ariftolochia rotonda. . Dette foglie non fono forate da $\mathrm{i}$ rami a guifa della Perfoliata, o fia Bupleuro perfoliato, come nella Defcrizione fuppone Gio: Bauino dicendo, che dette foglie fono Perfoliatre foliorum modo à caule, ramifque transfoffa; La figura però da effo recata moftra che dette foglie abbracciano il fufto, e non fono traforate da effo, come nel Bupleuro. Perfo- 
liato. Il Mattioli viene giuftamente cenfurato da Gafparo Bauino in ordine alla figura di quefta pianta, per averla efpreffa folamente colle foglie fuperiori, tralafciando di rilevare le inferiori, che fono, come ho detto, differentifime. Il fuddetto Bauino però nell Opera del Mattioli da effo fatta imprimere in Bafilea con copiofe giunte, e annotazioni, ha fatto aggiungere le foglie inferiori alla figura della mentovata pianta.

Ofervai ancora vegetare copiofamente fopra quei Tartari una pianticella, che in molte maniere è fata nominata da i diverfi Scrittori Bottanici Siftematici, ed ecco i fuoi differenti Sinonimi

Alcbimilla Linarice folio, calyce forum rubro J.R.H.509. Linaria montana fofculis albicantibus C.B.Pin.2 I 3 . Linaria Similis J.B.3. $46 \mathrm{r}$.

Anonimos Lini folio Cluf. Hift.324.

Sefamoides procumbens montanum Linarice folio flovious albicantibus Moris. Hift. Oxon.3.6or. Sect.5.Tab.I. Fig.3.

Knavel montanum calyce Speciojo lacteo Raj. Sinop.202. Linoployllum alpinum latifolium majus Ponted.Anth.262. Tbefium panicula foliata, foliis Lineari-lanceolatis Linn. Spec. Plant.30x. Thefium Linopibyllum n. Triv.

Tbefium Linn. H. Cliff.4 I.

A propofito della varietà dei nomi dati a quefta pianta foggiunge il Sig. Linneo H. Cliff. l. cit. Rifum moveat obfervafje quod ne quidem duo fyftematici de nomine bujus plantic convenere, fed tot nova nomina, quot ipfifuere, impofueie, ut fané fi Clufius boc die e fatis revocari poffet eandem Anonyman nunquam diceret.

Oltre 
166

A $\quad \dot{r} \mathbf{r} I$

Oltre a ciò fu da me offervato nafcervi copiofamente.

Sopbia Cbirurgorum Lob. Icon. 738. Che chiamafi ancora Sifymbrium annum Abjinthii minoris folio. J.R. H.276.

After Pannonicus, lanuginofus, luteus J.R.H.484. Polium montanum luteum C. B. Pin.220.

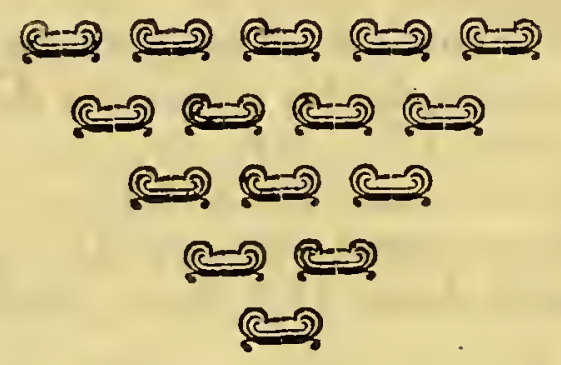




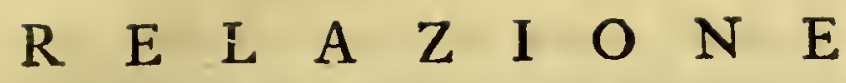
$S O P R A$ U N PRETESO

\section{E R M A F R O D I T O \\ DEL DOTTOR \\ F R A N C E C O C A L U R I}

TUBBLICO PROFESSORE DI FILOSOFIA NELL' UNIVERSITÁ

DI SIENA.

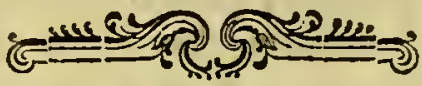

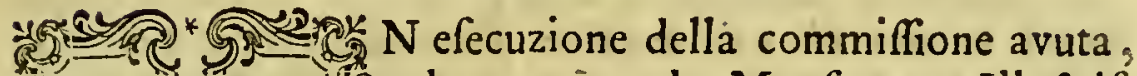
8. 13 ed accetata da Monfignore Illufrif(Simo, e Reverendiffimo, Orazio Ban*. 1 in dinelli Vicario Capitolare dell' Arcifor vefcovado di Siena, come apparifce din $2=-1$ dagli Atti della Cancelleria Arci-

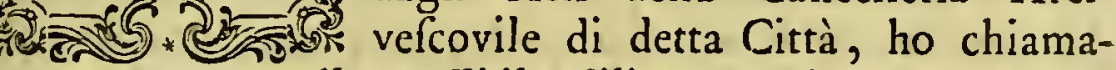
to a me, e colla poffibile diligenza vifitato un certo uomo chiamato Agoftino Broli di profeffione contadino, il quale volendo ammogliarfi gli è ftato in Curia oppofto effere il medefimo incapace al matrimonio: cioè aver' egli, come dicono i Canonifti, un impedimento dirimente, ovvero fia impedimento d'impotenza .

Sicchè in vigore di detta accettata commiffione vifitatolo, ed offervatolo attentamente, ho l'onore con tutto il rifpetto d' umiliare a fua Signoria Illuftriffima, e Reverendiffima la foria in primo luogo del cafo, o fia fatto; in fecondo luogo l' efame del predetto cafo; ed in terzo luogo il mio fincero, qualunque fiafi, giudizio, 
dizio: il qual giudizio mi lufngo di dare per la pura verità, e fenza alcuna parzialita di partito.

Agoftino Broli è un uomo dell' età incirca di trentaquatro anni, così egli mi ha riferito, di ftacura mediccre, non è graffo, ficcome non lo fono i contadini, ben proporzionato di tutto il fuo corpo, fano, e molto vergeto: d'una carnagione bronzina, quale apptinto è quella de $\mathrm{i}$ noftri contadini : di un taglio di corpo quadro, e virile: la voce parimente di uomo: fenza barba nelle guance, quantunque il vifo fia d'uomo, e folamente gli fi offerva un fegno di barba d' un colore piuttofto nero ne $\mathrm{i}$ baffi, che in qualche lontananza ancora dà nell' occhio: poco gli crefce; è flofcia, e di rado è obbligato a raderfela. Ha l'agilità del corpo, e la difinvoltura propria de' mafchj, ficcome ha il camminar franco, e fpedito de i medefimi.

- Fattolo etiamdio difcorrere ho potuto rilevare aver egli il penfare robufto, ed il coraggio de $i$ medefimi. In fomma, fe fi eccettui quel poco, o niente di balba, che lo ftefo ha nel vifo, alle efterne fembianze mi fembra, ch' ei fia un vero, e perfetto mafchio ; ficcome tale ciafcun che il vede, lo dee al parer mio giudicare.

Avendolo in feguito fatto nudare per confiderarne più d'appreffo in lui la proporzione, e conformazione in genere, relativa a tutte le fue membra unitamente prefe, e poi di ciafcuna in particolare; ho con facilità rilevato, fenza che cofa veruna mi abbia fofpefo, e trattenuto nel giudicare, che tutto $l$ infieme pel di lui corpo è virile; poichè le fue membra fono mufcolate, e non di quella rotondezza, e delicatezza comune al 
bel felfo; ficcome ancora l'offatura del medefimo non mi è fembrata minuta, come nella Donna. La carnagione, o per meglio fiegarmi, la cute forte, c denfa, nè delicata, corre in quelle. Le mani, ed $i$ piedi della grandezza, e proporzione degli Uomini. Finalmente tutto l'infieme del di lui corpo mi è fembrato di perfetto uomo.

Quello però, che ho notato fi è, ch' ei non ha' nel petto, nè in altre parti del fuo corpo quel pelo"; che in molti uomini fi trova fovente, e nafce; è folamente ornata del folito pelo la pube, della qual parte è d' uopo fare qui appreffo una più minuta, e circoltanziata defcrizione; Imperciocchè la Natura nel produrre un tale individuo in quefta parte ha fcherzato, e non poco eziandio variato, conformandogli con bizzaria ftraordinaria l' efterne parti, che coftituifcono, e fanno conofcibile, e vifibile il diverfo Seffo nella fpecie Umana.:

Parimente di pochi peli fono riveftite le afcelle : ficcome pelo, e nella pube, e nelle afcelle fi trova fempie, benchè poco, tanto nell' Uomo, che nella Donna.

Paffato in feguito alla confiderazione del bufto, l'ho ritrovato quadrato, ed il petto confimile in tutto, e pier tutto al Torace d'un Uomo; cioè non ho rinvenuto certe varietà nell' attaccatura del collo al petto, nelle fpalle, e nelle clavicole; che offervanfi nel corpo, e bufto della Donna. Imperciocchè il di lui collo non ha la rotondezza che in quelle; comparifce quel che volgarmente fi chiama. Pomo d'Adamo; nè come in quefte il medefimo è continuato uniformemente al petto; le clavicole fono rilevate, ed inarcate, Y 
le fpalle sfogate. Le Mammelle poi fono attaccate, e ftefe tutte nel pettorale maggiore, nè hanno niente di quella foftanza, che le porta in fuori dal petto nelle Donne, e le rotondeggia: In fomma effe fono tali, quali apparifcono nell' Uomo

In fimil modo ho veduto convenire interamente alla proporzione che $f_{1}$ offersa nell' Uomo, il Ventre, - fia Corpo; i fianchi, le natiche, e tutto il refto delle gambe ; ficchè in lui non fi vede quella larghezza, e prominenza in fuori degli ofli de' fianchi, e del bacile, e delle reftanti parti, come nella Donna gli Anatomifti hanno notato; le quali piu minutamente defcriverle credo fuperfluo.

Quello poi, in cui più differifce da fuoi confimili queft' Uomo, del quale quì ora favello, confifte nella configurazione ftrana, e moftruofa della difpofizione delle parti efterne, che fervono alla generazione, ed allo fcarico fuori del Corpo del fluido efcrementizio delle orine: Che appunto come le ho offervate, e ritrovate, o per meglio dire mi fono fembrate, io ora avanti gli occhj del fapientiffimo Giudice ne fottopongo la minuta efatta defcrizione ; fupplicando lo fteflo a compiacerfi di condonare, e permettere, che effendo io forzato a nominar quefte parti mi ferva talvolta d'alcuni termini, e parole, che in altri argomenti potrebbero a ragione chiamarfi parole difonefte, e difdicevoli ad ogni ben nata, e culta perfona; ma che nel mio difcorfo è forza permettere che fi ufino, acciocçhè piu netta, e precifa fi renda la idea della cofa, che devo defcrivere; protêtandomi, che non le ufarò, fe non nel folo cafo d'indif́penfabile neceflità, perchè il 
fapientifimo Giudice poffa comprendere fenza equivoco veruno quello che a me importa, e maflimamente preme, che con evidenza fi conofca; effendo che l'articolo principalmente della queftione, e cafo, che mi è fato commeflo d'efaminare, dipenda dalle dirette deduzioni, che nafcono dalla feria, e rigorofa confiderazione di quefte parti, le quali ora defcriverò.

La pube adunque, come chiamano l'A natomici quel. la parte del corpo, che è fopra le parti vergognofe; detta nel noftro volgare Petrignone, è come l' ho $\mathrm{fl}_{1-}$ gnificato, ricoperta di pelo, ma non molto folto. $\mathrm{Da}$ una parte, e dall' altra della pube quafi fopra all' attaccatura ordinaria del canale, per mezzo del quale l'uomini orinano, offervanfi, due gonfi, de i quali il deftro è maggiore del finiftro : Efaminati, ho trovato primicramente, che effi hanno una figura ovale, l'affe maggiore de $i$ quali è verticale, o perpendicolare all' Orizzonte; e mi è parfo, è credo che non fieno corpi, che appartenghino agl' integumenti comuni, poichè da' medefimi mi fembrano ftaccati, e pare che fi muovano: Quando detto Uomo fatiga, ei mi ha affermato, che $\mathrm{i}$ medefimi un poco calano, e rifalgono nella quiete; ficcome ancora è parfo a me, che un poco fcendano, e fi muovano di: fito, quando egli toffifce, effendomene afficurato col farlo; toffire; e parimente ho offervato, che qualche giorno è più patente, e vifibile il gonfio deftro, e qualche altro giono il finiftro. In mezzo, ed a piè di detti gonfi, come negli altri tomini, nafce la verga virile, bene all' efterno confor. mata, piuttofto corta, e di una mediocre aggiuftata groffezza, ed in nulla, a primo ápetto, differifce dal. Y 2 


\section{I' 2 A $r$ T I}

le ordinarie verghe. E' riveftito il fuo Glande della folita pelle, e cappuccio, chiamata Prepuzio; non è però in cima forata, come lo dee naturalmente effere, $\mathrm{e}$ folo nel luogo dove fi dovrebbe trovare quefta apertura, vi fi fcorge un leggerifimo fegnale, o fia folco; nè in tutta la lunghezza della Verga fi comprende il canale detto Uretra, che va a finire in quella apertura, che dovrebbe effere nell' eftremo del Glande. Alla Verga gli manca intrafatto la borfa, chiamato Scroto; ed invece di quefta borfa comparifce fotto la medefima una fiffura per lungo, o come la vogliamo chiamare apertura co $\mathrm{i}$ labbri leggermente rilevati, e ricoperti di pelo, la quale a prima vifta rapprefenta l' efterno delle pudenda delle Donne. Ho detto, che a prima vifta rapprefenta le nominate parti; poichè attentamente confideratala non vi fi rinvengono quei ordinarj contrafegni, co i quali gli Anatomici diftinguono l'efterno delle medefime - Slargati quefti apparenti labbri comparifcono due corpicelli, che hanno della fomiglianza si nella direzione, e figura, come nel colore, e confiftenza a quella carne, o parte, che nel feno muliebre fi chiamano Ninfe, ma fono affai più gracili, piccoli, 'e meno eftefi di quel che fi vedano in quelle, e pare che fiano una propagine, e continuazione del frenulo.

Quefta rima non ha nel noftro foggetto alcuna co: municazione, per quello che ho potuto comprendere, coll' interno; ed a me pare un vero canale cieco; nè vi fi conofce, che da effa trafudı untuofità, ed umidi. tà alcuna. Nella parte inferiore di quefta rima, cioè verfo l'ano, fi ritrova un piccolo foro, per il quale il nedefimo orina, e dice che da quefto ftefio forame ne 
i fuoi trafporti venerei efce con faglio; e direzione lungo la verga, lo Sperma, o fia l'umore fecondatore. Non ha mai provato, nè da altra alcuna periodica, o menfuale evacuazione di fangue, come fono folite avere le Donne. Quando orina, bafta che folo egli alzi colle dita la fua verga, e l'orina fe n'efce in arco, $e$ con forza come negli Uomini, e come gli altri, otina al muro, giufta la efpreffione, e frafe ufitata del $S_{6-}$ cro $\mathrm{T}$ cfto dicendofi, quando ffi vuol fignificare un mafchio mingentem ad parietem ; nè ad effo è d'uopo d'affettarfi in quell' attitudine, nella quale comunemente le Donne deono comporfi quando orinano.

Quefta adunque è la defcrizione, e quelle cofe, che ho offervate in quefto foggetto, e come le ho efpofte, e mi fono fembrate effere, tali quali ora mi pregic, ed ho l'onore di prefentar fedelmente fotto gli occhj, ed al purgatiflimo difcernimento di Sua Signorìa Illuatriffima, e Reverendiffima. Rifcontrata quefta mia defcrizione con altra benche affai concifa, e riftretta ftata fatta anni fono fopra quefto medefino uomo dal Chiariflimo Anatomico, e Profeffore pubblico di quefta Scienza nella noftra Univerfità di Siena, Sig. Dottore Pietro Tabarrani, riportata nel Tom.3. degli Atti della noftra Accademia delle Scienze, a cui il medefimo ha eziandio anneffe due tavole, che rapprefentano le parti efterne della generazione del detto Agoftino Broli, ritrovo che la mia defcrizione combina, ne è contraria a quella, ficcome è facile il riconofcere: lo che è neceffario, ed è d'importanza che a Sua Signoria Illuftriflima, e Reverendifiña fia noto. 
Premeffa pertanto la deferizione, rimane ora da efaminarfi in primo luogo, a quale de i due Seffi nella fpecie Umana dea il noftro Soggetto Broli effere annoverato, ovvero fe il medefimo poffa effer tenuto per un Andiogino.

Egli non fi può dire Ermafrodito, o Androgino, effendo che la Natura nel Genere Umano quefta terza clafe d'individui ella mai abbia prodotto; come lo faremo vedere, fe fi riguardi queft' opinione con occhio imparziale, e filofofico, nè con mente prevenuta da chimere, e da tradizioni ciecamente invalfe, ed introdotte. Per Androgino, o Ermafrodito, ambo nomi, che nella lingua Greca hanno un ifteffo fignificato, ficcome è noto, $s$ intende quell' ideato individuo nella fpecie umana, che ha, ovvero fi crede avere in un fol corpo ditinti, ed attivi $i$ due Seffi. Così Suida alla parola Ans drogynos, o Hermaphroditos fpiega $=$ Hermapbroditus eft qui utraque or mafculorum, of faminarum organa babet, turpiter \& faciens, \& patiens =e l'Autore delle difinizioni at-. tribuite a Galeno, con più riguardo parlando dice $=$ Her mapbroditus eft mixtio mafculi, of faminei $\int_{\text {igni }}=$.

In queft' ifteffo fenfo la favolofa Teogonia degli Antichi Greci, e Ronani, e molti Filofofi, ed Iftorici hanno parlato degli Androgini. E quefto è il comun fentimento, ed il comune fignificato, che fi dà a que fte voci.

Dice Ia Favola, che Ermafrodito, nome che nel Greco fpiega figliuolo di Mercurio, e di Venere, divenne per potenza de' Numi Androgino, cioè partecipante de i due Seffi: la Favola è nota. 
L' Antichità ha creduto, che la nafcita degli Androgini, o di altri portentofi, o ftraordinarj parti foffero fegnali d'augurio calamitofo per li Stati ; laonde nel primo libro della Divinazione Cicerone riferifce, che la Religione, e lo Stato riguardava quefti portenti per trinti $=$ Quid ortus Andiogyni ? Non ne fatale quoddam monfrum fuit ? = E Plinio il Naturalifta afferendo quefta medefima cofa, dice però che ai fuoi tempi fi teneano per delizie. Leggiamo in Livio che per legge folevano $\mathrm{i}$ Romani un si fatto creduto Androgino fommergerlo fubito nel mare. E l'ilteffo Plinio racconta, che nel Confolato di P. Licino Craffo, e Cajo Caflio Longino, come emenda il Padre Giovanni Harduino, effendo una fanciulla divenuto mafchio, per comandamento degl' Indovini, fu portata in un' Ifola deferta.

Quando però fi riflette alla barbara, e vergognognofa fuperitizione di quefto antico popolo d' Eroi, noi non fappiamo combinare colla medefima come poi effi credeffero che nell' Affrica fi truvaffe, ed efintefte un popolo intero di Androgini, al riferire del medefimo Plinio = Qui utriugque nature funt participes: dexteram manmam virilem isabent, lavam mulicbrem, of inter fe vicibus coeunt $=$ la quale ultima particolarità delle Mammelle da lui fi dice per teftimonianza di Ariftotile: a noi però non pare probabile, che Ariftotile abbia creduto quelta favola; imperciocchè, come lo diremo, ei degli Androgini ha rettamente giudicato. Pare però che la favola abbia ancora alle volte intefo per Androgino un Uomo compofto di due felfi, di due tefte, quattro braccia, e due piedi, al che in qualche 
maniera allude Ovidio, quando racconta che la Ninfá Salmace tenendo ftrettamente abbracciato Ermafrodito, nè con tutto ciò potendolo indurre alle fue voglie, pregaffe gli Dei, che di due corpi uno fe ne faceffe, reftando diftinti, e feparati a quefto novello corpo i due feffi:

"Noc dro funt, fed forme duplex, nec fomina dici

"Nec puer ut polfit, neutrumque, \& utrumque videtur: A quefto propolito ci fia ancora permeffo l'avvertire di paffaggio, che molti Rabini pretendono, che Adamo forfe da Iddio infieme creato, e un uomo da un lato, e femmina dall' altro, e poi li feparaffe. Tale è la opinione del Celeb. Rabino Mamonide, di Manaffe, di Ben-Ifdrael, e di altri molti.

Parimente ancora alcuni antichi poeti, e filofofi hanno fuppofti gli fteffi loro Dei Androgini; così li ha creduti, ed immaginati Orfeo, e Mercurio 'Trifmegifte: Zenone poi, e Zenofane li hanno dipinti non folo Androgini, ma hanno dato loro ancora una figura rotonda, ed altri altra. Platone medefimamente nel fuo Banchetto, facendo Ariftofane parlare, raccontache in origine l'uomo era Androgino, di figura rotonda; e che parimente in principio tre erano le fpecie dell' Uomo, che Ia terza fubbito fi perdetze, e folo ne rimafe il nome. Spiegando poi come dall' Androgino ne fia nato il mafchio, e la femmina, racconta che quefto Androgino era un tutto bizzarro d' una ftraordinaria forza, e d' un animo elevato; ed altiero, per cui refofi infolente, pensò di far la guerra agli Dei. Giove irritato lo volea diftruggere, ma avendo pena di far perire il Generc Unano, onde gli Dei perduto avreb- 
bero il loro culto, ed il loro divertimento, ordinò per indebolírlo cle l' Androgino" foffe divifo in due parti cioè in malchio, e feminina cie degli Androgini; della quale folamente è reftato nel mondo, linfame nome?.

La, origine dunque di quefta terza fpecie nell' Uo. mo riconofce prinçipalmente la fua derivazione dalla. Favola, dalle invenzioni de i Poeti, e da i placiti de i Filofofi non bene = intefr, ed in appreffo mantenuta da qualche equivoco préfol nella nafcita de $i$ moftri, come fáremo vedere; fe diré non vógliamo, che la favola tra l' origine da quelto medefimo equivoco $\mathrm{La}$ Religionelpofcia né, i Greci; e ne i Romani, lo Stato, prima per ignoranza, e di dopo per necelfaria, ce giufta regola di buon governo ha dovuto adottare gli Androgini, li ha inferiti ne i Fafti de i tempi i li ha confegrati come tante verità, onde vie più nella mente-del Popolo portato fempre al favolofo, al forprendente, e allo frádinario, queft $a$ opinione è invalfa, ed ha trovato nella mente de i piü una cieca, e Atabil credenza

Per la fteffa cagione la ignoranza, e la fupertizione ha adottato i parti de il Serpenti negli Uomini, l'Ippocentauro, e tant altre partentofe nafcite; e per prodigio accádute, o prodorte idal commercio infame d' uomini con beftie, riguardate peró fempre meritamente tra loro da i più faggi per vere menfogne, e come tali derife; onde Lucrezio:

9). Sed neque Centanri fuerunt, nec tempore in wllo.

„2. Ele queat duplicilninura, o corpore bino

"Ex alienigenis inembris compieta poteftas

$$
\text { Z }
$$


Cosi ha penfato ancora Galeno, il quale con forti argomenti nel terzollibro dell ufo delle parti, combatte tali errori; e prima di lui anco Ariftotile apprefe fimili ftorie per incredibili, é favolofe.

Lo feffo anche ha penfato Cicerone, quando nel fecondo della Divinazione combatte gli ftorici, e fa vedere il ridicolo, e la falfita d'ogni genere di divinazio ne, e d'augurio; e menfogne ancora le hanno timate l'Imperator Giuftiniano inel titolo = De inutili Stip. $=$

Come poi alcuni dorti Rabini fi fiano immaginati, ed abbiano fatto Adamo Androgino, fi potrebbe con qualche fondamento fofpettare, o che effi fi fiano offufcati nel volere fpiegare il paffo della Genefis $=$ Quefro dofjo delle mie offa, e Carne della mia Carne $=$ ovvero per volere dare una ragione fpeciofa dell' amore fcambievole dell' Uomini verfo le Donne, e viceverfa delle Donne verfo l'Uomini .

Riguardo a Platone è facile por, ch ei ricavaffe la fua ingegnofa invenzione della forma de i primi Uomini da qualche vago racconto che ne fuoi viaggj gli fia tato fatto delle opinioni particolari di "qualche Ebreo, fopra la origine del primo Uomo, d'onde ne ha poi cavati quei nobili penfieri fopra l' amore.

Se finalmente vorremo rintracciare da che poffa effer nata la falla fama de i Popoli Androgini; pare non lontano dal vero, che a quefta favola abbia dato origine la frequenza di trovarfi in certe Regioni Orientali, e calde, le Donne con la Clitoride affai più grande, e vifibile di quello che avvenga in altri climi; e perciò fífrano con facilità potuti fpacciare, e frano ftati tenuti per Androgini quei Popoli, tra i quali maf- 
fime quelto calo fuccede ; imperciocchè le Donne così coftituite mentifcono facilmente il doppio Séffo, comé agli Anatomici è noto, come eziandio quì fotto fá remo vedere.

$\mathrm{Ma}$ ficcome fono fempre le Donne la metà incirca d'ogni Popolazione; ficchè le Donne in quefti luoghi per la sfrenata loro libidine; effendó cósì coftituite, fi doveano reciprocamente fra loro abbracciare, coll' abufare d'una parte; che ha tanta fomiglianza, e ha tante proprieta comuni alla Vérga virile. La quale cofa alle eftere Nazioni ha potuto imporre, che fi diano Popoli di Androgini:.

Che una tal parte fia folito alle volte nelle Donne crefcere Atraordinariamente, egli è un fatto, che niuno nega ; e che ogni giorno per così dire fe ne prefentano degli efempj. Ed ora fono già due anni, che viddemo in Siena una certa Michel-Anna, che era una di quefte Donne cosi conformata .

Aczio, ed Egineta ambo Medici Greci de' Secoli balli hanno infegnato la maniera di recidere quefta parte, chiamata da i medefimi; e da altri Greci Ninfa, che vuol dire germoglio, protuberanza, come fi ricava da Suida . Nè fenza motivo quefti Medici hanno trattato di quefta operazione; poichè una tale parte può crefcere a fegno, che faccia un inciampo, ed un oftacolo al mezzo, per cui fi ottiene il concepimento.

Del pari ancora lappiamo, che una volta in Egitto, dove fi dice effer ftata frequente quefta Atravaganza, fi fuoleva alle fanciulle tagliarla, e chiamavafi quefta operazione circoncidere. Nè Vergine, dice il Vallifnicri, va a marito, fe prima ella non fia ftata cir- 
concifa; che anzi, come ancora lo conferma il Barto:lini, queft' operazione alle Donne Orientali : non folo. è neceffaria per renderle atte alla generazione, ma di più fi chiama onefta, e decorofa.

Preffo altri Popoli dell' Oriente, dove pure fi vuole quefto vizio familiare, non ti ufa toglierla col ferro ; ed a ciò fi adopera il fuoco.:

Finalmente che le Donne cosi, fatte fiano folite d'abufarne, e prendere col fuo Seffo de i divertimenti; fono a baftanza note le Storie delle Tribadi, benchè ancora con quefto nome fi appellino le comuni Donne, che per un depravamento di coftume fi diano in braccio tra di loro a confimili piaceri.

Di quefte parla l' Apoftolo delle Genti, a i Romani dicendo loro $=$ Preterea illos Deus, dedit in paffionem ignominice. Nam Fomince eorum inmutaverunt natusralem ufum in eum ufum, qui eft contra naturam =; e contro delle quali anche gli fteff Pagani hanno inveito, ficcome ha fatto Luciano, ed altri non pochi. E' no-' to che Marziale d' una di quefte diffe.

"Commenta es dignum Thebano enigmate monftrum

"Hic ubi vir non efl, ut Jit adulterium

"Inter fe geminos audes committere cunnos,

"Mentiturque virum prodigiofa Venus.

Sappho parimente quella celebre tanto, ed ammirata Donna da tutta l'Antichità, si per i fuoi fublimi e teneri verfi, quanto per i fuoi amori, chiamata da Strabone miracolo della Natura, e comunemente appellata la decima Mufa, Sappho dunque quefta tanto illutre Donna è fimata contaminata da quefto vizio per l'amori colle Donne di I.esbo. Onde Ovidio le fa dire. 
\# Non me Pyrriades, Mentlyymniadefve Puello

"Nec me Lesbiadum catera turba juvant

"Vilis Anactone, vilis mibi candida Cydno

"Non oculis grata eft Attbis, ut ante meis

"Atque alice centum, quas non fine crimine amavi

Improbe, multarum quod fuit, unus babes

Pertanto da tutto ciò, che fin qui fi è detto ci fembra d' avere fufficientemente dimoftrato, che dal teftimonio favolofo degli Antichi fopra l'Androgini, non fi può fondare alcun' argomento certo della loro efiftenza.

Quando però dagli Anatomici, e fopra tutto da:i Fifici, fcoffo alla fine il gioco alle antiche credenze, fi è cominciato a dubitare di ciò, che nelle Scienze naturali fi prefumeva fapere, e che un pirronifmo giudiziofo, e lodevole ha trionfato, ed ha coperto di tenebre, e velato di dubbj, e d'incertezze le vecchie opinioni: e quando nella ricerca della verità hanno gli Uomini principiato a fervirfi delle loro forze, e della propria loro ragione, nè più fchiavi delle Scuole, non con gli altrui occhj; ma co i proprj hanno contemplato la Natura, accompagnandola fedelmente, ed offervandola in tutti $\mathrm{i}$ fuoi andanenti, e ne' fuoi varj fenomeni, gli Androgini ancora fono ftati pofti a rigorolo efame, fono fati menli in dubbio, c finalmente combattuti. E. fe qualche cofa ha dato motivo a quefta favola, fi è comprefo, che è ftata la nafcita de i moftri, clic fono ftravaganze affai ordinarie, e frequen$t i$ in tutte le produzioni della Natura. E nella claffe maflime degli Animali fovente fi fono ritrovate tali, e fi fatte moftruofita nelle parti, che diftinguono il lo- 
ro Seffo, e fopra tutto nelle femmine, che a primo afpetto poffono ingannare, fomminiftrando con facilità maffima a i poco avveduti, agli amatori del prodigio, alla pigrizia de i più, e finalmente agli ingannatori delle fpeciofe, e falfe prove dell' efiftenza di quefti. ideati Androgini; onde tra il popolo fi è perpetuata la favola de i medefimi.

Quale poi fia la origine, e principal cagione della nafcita de $\mathrm{i}$ moftri, quì non è di meftieri lo efaminare, effendo queftione affai agitata da $i$ più infigni Anatomici del prefente fecolo; imperciocchè a noi giova folo fapere, che i moftri nelle produzioni della Natura fogliono accadere ; intendendo per Moftro quel prodotto, che degenera dal fuo fimile ordinario per qualche cofa, o di più, o di meno, ovvero diverfamente difpofta. Nè occorre a noi per il noftro fine indagare, fe i germi fiano originalmente moftruóí, ovvero fi produca il moftro, come conghietturano molti, dal difordine accidentale della naturale ftruttura de $\mathrm{i}$ germi originali, frano quefti per confufione di due, o più ger$\mathrm{mi}$, o frano per diftruzione, cangiamento, aderenza, - altro di alcuna porzione di germi naturali, nediante $\mathrm{i}$ differenti gradi, e le diverfe maniere di compreffione delle parti vicine, opinione ora rinnovata, ed in antico, come fi ricava da Ariftotile, abbracciata, e tenuta da Democrito, fopra la quale il Wislow, ed altri infigni Anatomici trovano graviffime, ed infuperabili difficoltà; onde fembra che prefentemente i più fiano inclinati alla ipotefi del Doverney, che al fiftema de $i$ montri per difordine accidentale aderifce, foftenuto dal - Lemery. 
In fatti che cosi fempre fia accaduta la faccenda, e che mediante qualche moftruofità nel Seffo degli Animali, i volgari abbiano precipitato nell' errore, lo avverte eziandio-il grand' Ariftotile nel gravifimo Libro della Storia, e Generazione degli Animali, e perciò ci piace il riferire le fue fteffe parole della verfione di Teodoro $\mathrm{Gaza}=$ Pueris etiam quibufdam, genitalis poftremum, \& meatus, qua excrementum vefice tranfit, non eodem tetenderunt: Sed infra meatus ille tranfegit. Quamobrem demifis clunibus mingunt, qui teftibus pauld in Jupeviorem partem retractis, videntur of maris babere genitale, of femince $=$.

Parimente il Divino Ippocrate non leggiamo mai, che abbia parlato di quefta terza fpecie, ed il vocabolo Androgino dal medefimo ufato, lo ha prefo per fignificare un uomo, che partecipi delle maniere, del temperamento, e de $\mathrm{i}$ coltumi delle Donne $=E t$ funt bi Androgyni, ideft viri effominati, atque fic recte appellan$t u r=$. Della Dieta Lib.I.

Che nelle piante, ed in varj infetti, e fopratutto in molti aquatici fi diano, e fi trovino l'Androgini, egli è un fatto che non fi pone in dubbio. Ma ciò non fegue per un accidentale concorfo, ed accade per una legge coftante della Natura, che cosi quefte Piante, e tali Animali fin da principio ha organizzati, e nella fpecie de $\mathrm{i}$ quali ella non ha diftinto, come nelle altre fpecie il mafchio, e la femmina, e fempre folo lo fteffo Animale, ed il medefimo fiore genera, ed è generato. Negli altri Animali poi, nei quali con altre leggi la Natura fi è diportata, quefta terza fpecie neghiamo che fi dia, e polfa darfi anche per acciden. 
dente, perchè farsbbe contraria al maccanifmo de $i$ medefimi, e non neceffaria al fuo fine.

E fe qualche animale, come per offervazione di Lionet riferifce l'Haller nelle note a Boerhaave, abbia Ja proprictà di generare in fe, e di generare fuori di $\mathrm{fe}$, quelta proprictà in efto è coftante, e per legge di Natura, e folo dimoftra, che la Natura ne i diverfi Animali, varie ftrade ha tenuto per la loro propagazione.

$\mathrm{Di}$ già Ariftotile aveva avvertito, che fe nafcono animali col fegnale di due Seffi, uno folo degli fteffi prevale, rimanendo l'altro inerte, e vano: lo che $\mathrm{f}_{1}$ gnifica, che due Seffi in un medefimo corpo non fi poffono congiungere, e che la Natura mai li ha congiunti : ecco cone quefto Filofofo offervatore $f_{1}$ efpri$\mathrm{me}=$ Quibus autem gemina babere genitalia accidit: alterum maris : alterum famine: iis Semper alterum ratum; alterum irritum vedditur. $=$

In vero fembra che noin poffano in conto veruno. riunirfi due Seffi perfettamente in un medefimo animale; di quei intendiamo che fono diftinti feparatanente in Mafchio, e Femmina, fenza che l'uno non fia d'impedimento, nè faccia oftacolo alla neceffaria ftruttura, conneflione, e fimetria dell' altro, conforme offervano gli anatomici, e lo ha ancora confeffato il chiarifimo Haller, il quale con tutto ciò opina, che poffa effer poffibile, che un debol Seffo di Mafchio unifafi ad un' angufta vagina; quantunque ancora egli comprenda, che non fi trovano efempi precifi, e baftanti per confermare quefta fua poffibilità.

L'opinamento però di quefto gran Filofofo non indebolife la noftra afierzione; poiche in primo luogo è una 
\& una fua fuppofizione immaginata per non negare la fede iftorica d'alcuni fatti, e per non fupporre, che alcuni anatomici fi fiano potuti ingannare; ed in fecondo luogo effendo parti così fiacclie, e deboli, effe altro non indicano, che una moftruofità, per avanzo d'un germe originalmente mal conformato, onde per neceffaria confeguenza dee unicamente effere una, e fterile organizzazione, ficcome lo fono in realta le altre confimili moftruofità del corpo, come ricaviamo dalla fevera confiderazione de i moftri.

Tale per modo d'efempio è la moftruofità del giovane Veneziano, veduto, ed efaminato dal Wiflow, a cui da un lato del petto fcappava fuori un' altra tefta un poco più piccola, che altro non era, che un' inerte, e cafuale conformazione del tutto, a cui apparte. neva. In fimil modo, tale dee reputarfi un fimigliante moftro defcritto dal Pareo, ma dal medefimo non veduto, e fi nega a lui, che da quefto fecondo capo $f_{1}$ cibaffe, effendo il Pareo, come fi conofce in tutto il fuo libro de'moftri, affai portato a credere $\mathrm{i}$ prodigj, che fi fpacciavano.

Di quefta claffe era la moftruofità della Fanciulla notomizzata dal Wislow morta in Parigi, dal ventre della quale efciva un'altro più che mezzo corpo. Tale finalmente, per non allungarci con affai più d'efempj, è l' interno Ermafrodito ritrovato dal Medico Petit di Namur; come fi legge nell' Iftoria della Reale Accademia delle fcienze di Parigi, effendo al di fuori interamente uomo, a cui folo mancavano i Tefticoli, i quali fi ritrovavano nel ventre; e tale era il bambino deicritto dal Mery nato fenz' alcun fegno al di fuori di

$$
\text { A a Selfo, }
$$


Seffo, e medefimamente a quefte moftruofità dee riferirfi l'Androgino, di cui parla, ed ha portato anche la figura il fopradetto lodato Signore Tabarrani, defcritto nel Lib. = Medicorum Silefiacorum Sative $=$

$\mathrm{Mi}$ pare adunque da quanto fin qui ho riferito; che il buon fenfo, e la ragione ci guidins affolutamente a concludere, che l'Androgino fia folo un capricciofo nome. Così hanno fempre penfato in tutti $\mathrm{i}$ tempi i piu illuminati Filofof, ed anche lo fteffo Platone, Autore ingegnofo della Favola citata; imperciocchè egli dice, che la razza degli Androgini fi fpenfe, e fu del tutto diftrutta, tolto che Giove lo dimezzò.

$\mathrm{Ma}$ poichè gli Uomini abbandonati al loro femplice ragionamento poffono con facilità ingainnarfi, perciò $f_{a}$ di mettieri il vedere quello, che la ragione ci perfuade effer conforme a ciò che gli Anatomici piu illuminati hanno offervato, e rigorofamente dedorto, allora che effi richiamarono ad efame la defcrizione de i creduti Androgini, fatta da quegli Anatomici, che li hanno preceduti, ed a quello poi che i medelimi hanno offervato, quando loro fi fon prefentate occafioni di efaminare, ovvero notomizzare tali fuppolti Individui : Quantunque abbiano alcuni Medici, ed alcuni Anatomici creduto, ed anco altri fapienti Filofofi non abbiano giudicato per cofa impoftibile la nafcita degli Androgini, nel fenfo in cui abbiamo prefo quefto $f_{1}$ gnificato, è del pari ancora evidente, che quando i medefimi hanno defcritto quefti Efferi, chiamati Androgini, dalla loro fteffa relazione $f$ è manifeltamente dedotto il loro sbaglio, eflendofi i medelimi ritrovati o Femmine con la Clitoride moltruofamente accrefciuta, 


\section{Deli' Accademia .}

ovvero Mafchi, con qualche apparente moftruofità nel efteriore del Seffo.

Femmine erano i due Androgini defcritti dal Vefalio, fe fi faccia attenzione alla fua defcrizione, come riflette ancora il Sig. Tabarrani.

Donna parimente era l' Androgino tagliato l'anno 1615 . in Parigi da Giovanni Riolano, di cui quefta è la defcrizione $=$ Hermaploroditam puellam multis priefentibus diffecui. Penis parvus prominebat, abfque Scroto, e Teftibus, fed ab orificio duplici externo ufque ad fundum Uieri, duplex erat Matrix, mediano pariete fecreta, reliquie partes genitales fimplices erant ac $\int i$ fuiffet unicus uterus $=$

Isbrando Diemerbroeck ha lafciato parimente la defcriżone di due Androgini da effo offervati, ed erano due Donne con apparenza d' uomo: onde" egli dice. $=$ Ex quibus omnibus fatis patet bujufmodi Hermaploroditos non vere utritifque fexus participes effe, fed revera faminas, quibus genitalia "yunt male conformata.

Del primo però di quefti due Androgini fi può con qualchè ragione forpettare, che foffe piuttofto un' Uomo, che una Donna, come pare da ciò ch' ei dice.

Parimente il fimile fi deduce de tre Androgini veduti, e defcritti da Realdo Colombo, ed il mede? 1 mo confeffa che uno di effi era una. Donna, ed il terzo un vero Uomo, l'altro poi che egli ha ftimato un' Androgino, e che fu da lui notomizzato, dalla fteffa relazione, ch' ei ci ha lafciata di quelta fezione, fi conofce ad evidenza ch' effo non era un' Androgino, com'ei lo credeva, ma era una Donna, ficcome a chiun-

$$
\text { A a } 2
$$


que farà facile il chiariri, fe leggerá la fuddetta de-, fcrizione .

Noi non fappiamo, fe altri prima del Colombo abbia notomizzato cadaveri di creduti Androgini, e ci. fupponiamo, che fia que!to il primo Androgino taglia-: to, e dalla diffezione del medefimo, rileviamo fubbito la fallità dell' Androgini.

Androgino fimilmente non era la pecora, di cui parla il Celebre Feder Ruifchio, come il medefimo afficura di avere trovato, e riconofciuto.

Nè Androgini ancora fono $\mathrm{i}$ due defcritti dal Chefelden, de i quali parimente ancora parla il Sig: Tabarrani, fupponendo il medefimo, che il Chefelden facilmente fi fia potúto ingannare da certe apparenze efteriori,$=$ argumentandolo appunto ( com' ei dice) dalle parti clbe fcorgevanfi all' efterno, fecondo che dimoftran le figure, come a me pare, e la Jpiegazione ancora, che di effe lafcio lo Claegelden =.

Nè i Tefticoli, de i quali Chefelden dice effere corredati quefti fuoi Androgini, fono una prova convincente del Seffo dell' Uomo, unito all' altro Seffo, poichè tutt' altra cola potevao indicare.

Così il Ruifchio trovò, che quei due corpi credu: ti Tefticoli nell Androgino pecorino, altro alla fine non erano, che due tumori adipofi. A quefto propolito parimente riferifce, e confeffa il medefimo Ruifchio d'avere a primo afpetto prefo per mafclio uno di quefti creduti Androgini, nel pube del quale fi vedevan due gonfij, che mentivano $i$ Tefticoli, quando poi diligentemente efaminatolo, lo ritrovò una vera Donna, onde giuftamente dubitò, fe folfero Tefticoli, o piut- 
tofto due tumori adipofi, come nell' Adrogino pecorino; afferendo, che mai egli ha veduto un vero Androgino di tanti, che per tali gli furono moftrati; nè crede ciò poterfi dare, nè che altri mai abbiali ficuramente veduti, dicendo = propterea quod illud recederet longifime ab infituto Nature = Thef. Anat. VIII.pag. 17 . num.53. E negli Avverf. Anat. Decade 1. pag.22. parlando parimente degli Androgini fi efprime cosi:

- Interim tam varios perfcrutatus toties, numquan inveni Hermaploroditum verum; tamen plurima fabulofa de eo referre ubique experior $=$

Quantunque tra le fue rarità egli confervaffe molti feti, le parti genitali de' quali abbiano ftupende moftruofità .

Giovanni Ernefto Habenftreit Antropol. Forenfis De Divortiorum Argumentis, ancora egli francamente dice che non $f_{1}$ danno gli Androgini=Attamen veluti iftis Hiftorias legentibus inventus baud eft qui organis utri-: ufque Jexus ita infructus, quo de utroque fexu participare diceretur, effet $=$, foggiungendo, che la maggior parte di quefti creduti Androgini erano folo Femmine, e tale effere l'Androgino del Bauhino; e quelli all' oppofto, che erano veri Uomini, = nibil faminei preter bilum babuerunt, cui nullus refponderet Uterus $=$, e riferifce, che egli fece vedere a i fuoi Uditori uno creduto Androgino, che in foftanza era un'vero uomo.

Ma poichè la defcrizione che fa di queft' Uomo in tutto confimile al fatto di queft' Agoftino Broli, ftimiamo neceffario riferirla interamente $=$. Demonftravi Auditoribus Hermaphroditi, cum viveret, vifi organa, in quibus clarum eft aliquo conformationis vel morbi vitio, evanuife 
muiffe illi tefticulos, frotumque contractum fuifje, ut figna Iamine adeffe vidcrentur, glans illi imperforata evat, \& urina ex rima bilum muliebrem referente exiverat, binc Frmaploroditus vifus, illo titulo ab uxore femotus fuit, cujus funus cum perfpicerem, nibil quidquan, quod fomineum, preter illam externam bili Jpeciem, effet deprebendi $=$.

La ragione pertanto, l' efame de' più particolari Androgini, la fezione anatomica de i medefimi, e l'autorità de i più alluminati Medici, e Filofofi di tutte l'età ci convincono delle falfità de i medefimi; perciò racconti vani, ed inganni, o per motivi politici, o per altro, diciamo che fono $i$ decantati Ermafroditi, regiftrati ne i libri di molti Scrittori, i più fingolari cafi de i quali fi leggono in Riolano, in Bauhino, nel Pareo, ed in altri, che poi da $i$ più fenfati, ed illuftri Filo. fofi, e Medici fono ftati confutati.

Dunque Agoftino Broli non è, nè può effere Androgino. Ma ficcome egli è uno di quelli Individui, in cui la Natura ha fcherzato con qualchè ftravaganza nelle parti efterne della generazione, però con il Ruifchio lo appelleremo Pfeudo-Ermafrodito.

Refta ora che veggiamo a qual Claffe di quefti falfi Androgini il medefimo fi dea riferire.

A me non fono incognite le difficoltà, che talvolta $s^{\prime}$ incontrano in quefta determinazione, mediante la capricciofa, e fallace conformazione delle fteffe parti del Seffo; perchè nell' efame di quefti falfi Androgini viventi, cosi fcherzati, non pofliamo indagare nelle ulteriori parti de i medefimi, folo mezzo per decidere tante liti, nate tra gli Anatomici piu cofpicui.

Quanto fia ftato facile lo errare nell' affegnare il Seffo 
Sefro a qualchè individuo nato con qualche moftruofità nelle parti efterne della generazione, c quale reciprocamente lia perciò l'obbligo, e la vigilanza de i Medici, quando di fimili cafi, di cui a loro unicamente $s$ appartiene il giudizio, devono decidere, fi deduce non folo da quanto fin' ora abbiamo detto, ma anche da ciò che efporremo.

Un fingolare efempio di sbaglio prefo nell' affegnare il Sello, fi ritrova in Regnero Graaf, riportato dall' Autor Francefe nel Libro intitolato. Rifleflioni fopra gli Erma froditi relativamente a Anna Grand-Jean, qualificata talein una memoria di M. Vermeil, Avvocato al Parlamento.

Dice adunque l' Autore di quefte rifleffioni; che il Graaf ha veduto una Bambina di nafcita con una clitoride fomigliante $f i$ bene alla verga virile, che $f u$ battezzata per un mafchio: e l'errore non fi fcoprì che dopo la morte della Bambina, per mezzo della fezione anatomica .

Ma con più frequenza fimile errore fi commette ne $\mathrm{i}$ piccoli aborti, come ha avvertito il gran Morgagni, effendo facile, e comune pigliar per Mafchio un' aborto, quando efaminato fcrupolofamente fi ritrova ef. fere una Femmina; ma in quefti piccoli aborti, ne $i$ quali la vita fi fpenge nella fteffa nafcita, fimili errori ron hanno alcuna confeguenza; che di gran memento all' appofto potrebbero effere in altri tempi.

E celebre il fatto de i quattr' uomini di Siberia, riportato eziandio dal fopraddetto Signor 'Tabarrani, i quali nell' efterno delle parti pudende tutti egualmente avevano l'ifteffa deformità ; e tre celebri Nutomilti della Reale Accademia delle Scienze di Pictroburgo, non 
fi accordarono nella determinazione del loro Sello dominante, a $i$ quali tre anni dopo comparvero, e fi manifeftarono $i$ tefticoli, onde parve la lite decifa in favore del Weitbrecht, che avanti gli aveva giudicati tutti e quattro Mafchi. La opinione del Weitbrecht in feguito fu autorizzata da una offervazione di Abrano Kaav Boerhaave, che avendo notomizzato una Pecora creduta Androgino, la quale aveva le ftefle moftruofità che i quattro detti giovani Siberiti, fu ritrovata del tutto Mafchio.

Parimente il medefimo Kaav ritrovò Mafchio un fanciullo tenuto fino a i fette anni per Androgino, ficcome riferifce il fopracitato Sig. Tabarrani.

Similmente l'Autore delle Rifleffroni fopra gli Ermafroditi, relativamente ad Anna Grand-Iean fa evidentemente vedere contro ciò che aveva avanzato Monfieur Vermein, che la medefima è una Donna, la quale non ha altro difetto di conformazione, fe non fe il folo prolungamento della Clitoride, effendo in tutto il refto fimile a tutte le altre Donne perfettamente organizzate, ed in confeguenza capace ancora di aver figliuoli.

Così Donna, e non uomo, e molto meno Androgino io Ptimo effere Michel-Anna, di cui fopra abbiamo fatta menzione, da me nella fua dimora in Siena più volte attentamente efaminata, contro l'opinione di Monfieur Morand, che l'ha creduta, e foftiene effere un' Uomo, e contro ancora il parere del chiariffimo Haller, e di altri; Imperciocchè il taglio tutto della fua perfona è di Femmina, ficcome ancora le fue maniere, e la fua voce fono di Donna. Ma fopra tutto mediante le offervazioni fatte nel Seffo, ella fi è dimoftrata fenza 
veruna difficoltà tale, ritrovandofi nel medefimo tutte quelle parti, che fi trovano nell' altre Donne, benchè l'ingreffo nella vagina fia affai angufto. E quella parte, che mentifce in lei il Seffo dell' Uomo, è una verga di Mafchio in apparenza ben formata, affai più groffa che lunga, pofta nel luogo ordinario, ma però è imperfetta, nè è pervia, che eflendo pendente ricopre la rima agli inguini, ed a $i$ lati del feno non vi fi trovano fegnali di tefticoli; ed efaminato col tatto intorno alle aponeurofi de $i$ mufcoli del ventre, di dove efcono $i$ vafi fpermatici, niun fofpetto, ed indizio ho avuto, che $\mathrm{i}$ medefimi fiano dentro il ventre, come in molti uomini alle volte accade. Innoltre la medefima Michel-Anna $\mathrm{mi}$ ha afficurato aver' ella periodicamente, come le altre Donne, il fuo ordinario, il quale avanti che fpunti, le cagiona de $\mathrm{i}$ dolori alle mammelle, ed anche qualche leggiera colica. E fe ancora fi roglia fupporre impoftura lo fpacciarfi che fa per neftruata, il che per molte ragioni, noi non $f$ può fupporre, non nè viene perciò, ch'ella fia un mafchio; poichè non fi trovano forfe delle Donne bene organizzate, alle quali manca naturalmente quefto menfuale fgravio?

Se dunque alle volte in qualche cafo non fi fono accordati i Medici nell' affegnare il Seffo: nella moftruofità però del Broli non vi può cadere queftione; ed è chiaro che il detto Broli è un' Uomo: ed uomo parimente lo la giudicato il Sig. Tabarrani, conforme ed evidenza fi deduce dall' efame della defcrizione, che del medelimo, c di quefte parti ho fedelnente fatta. $B$ b E dico 
$\mathrm{E}$ dico che $\mathrm{i}$ due gonfi nominati, fono $\mathrm{i}$ fuoi tefticoli, nè altro poffono effere; imperciocchè ho offervato, che quefti falgono, e f́cendono, e qualche volta pare ancora, che nel vantre fi ritirino, che hanno la figura, e la forma degli fteffi, che al folito uno è maggiore dell' altro, e pigiati colle dita, fanno al médefimo fentire del dolore; le quali cofe nei tumori adipofi non così facilmente fi poffono offervare.

Dico ancora che quel piccolo foro che fi trova dentro quella cieca rima è la fola Uretra virile, in quefto luogo aperta, è terminata, ficcome di ciò abbiamo altri efempj nella foria Medica, riportati fpecialmente dal Morgagni, e da altri; E finalmente congetturo, che un principio di Scroto aperto, e divifo faccia quell apparenza di rima, e di labbri, che abbiamo defcritti. Ed i due piccoli corpi carnofi, che hanno una certa fimglianza alle Ninfe, fembra che fiano produziore bizzarra del frenulo; e tali fono tutte le altre cofe che in lui fi offervano, dalle quali fi dichiara Uomo: Onde credo, che fü quefto articolo non poffa nafcere alcun dubbio al fapientiffimo Giudice.

Sicchè tutta la queftione adunque nel detto Broli fi riduce a quefto: cioè fe egli poffa effere indoneo al Matrimonio, e fe lecitamente gli poffa effer permeffo di pigliar moglie : e fe egli poffa effer' atto alla generazione, quando in lui fi ritrovano le parti efterne del Seffo ftranamente configurate

A me non appartiene it difcorrere per qual meccanifmo, e per quali altre forze $f 1$ operi negli Animali la generazione: ed è forza il confeflare che non fi fa; 
fa; quantunque fi conofcano i mezzi, e i materiali, che a ciò devono neceffariamente concorrere, e fenza de $i$ quali la generazione non fi ottiene.

Ognuno fa che primieramente fi ricerca che il Mafchio fi congiunga colla Femmina; ed a fine che quefto congiungimento abbia il flio effetto è d'uopo, che da una parte, e dall' altra fi fomminiftri quel tan: to, che è neceffario a tale effetto.

In fecondo luogo $f_{1}$ fa, che il corpo della Fem: mina è il folo luogo, dove il germe fi fabbrica, s'alimenta, crefce, e prende la fua determinata maturazione.

Contribuifce a quefta maravigliofa opra il mafchio dal canto fuo, introducendo, e depofitando nel feno della Femmina l'umore feminale, ch' in lui fi fabbrica nel téffuto de i Tefticoli, col mezzo di quella parte, che chiamafi Verga.

Acciocchè egli fia valevole a tale officio fi richiedono neceflariamente tre condizioni. I. Che quefta medefima parte fia in erezione. II. Che nel congiungimento $s$ introduca nel feno muliebre. III Introdotta, depofiti dentro della Femmina il liquore feminale.

Se dunque in quefto reciproco congiungimento per il lato dell' Uomo (giacchè non d'altri animali, ma dell' Uomo qui fi ragiona ) fi adempiranno quefte tre condizioni, mediante le quali fi porta il liquor fecondante nell' Utero della Donna, non farà per difetto dell' Uomo, fe la medefima non refta feconda, nè quegli potrà effere accufato d'impedimento dirimente. Ma.ficcome la erezione, e fopratutto la introduzione intanto per parte dell' Uomo, contribuifcono principalmente all' opera della generazione, in quanto che

$$
\mathrm{B} \text { b } 2
$$

fono 
fono i mezzi, pei quali fi verfa l'umor feminale entro la Vagina, e penetra, ed è raccolto nell' Utero., che delle Vifcere della Donna è quella deftinata al lavoro della generazione; e che l' umore feminale nell' Uomo è folo quello, che fa la generazione; c parimente del medefimo una piccola porzione è quella, che il tutto opera; Perciò è manifefto, che fe avvenga che una parte della femenza nell' ufcire vibrata dall' Uomo poffa penetrare, ed effere attratta fenza il mezzo della introduzione, è manifefto dico, che potrà produrre l' ifteffo effetto, cioè che la generazione, anche in quefto da. to cafo può nella Donna effere operata.

Dunque fe faremo vedere, che non è impoffibile ad Agoftino Broli congiungendofi con una Donna, che egli poffa introdurre una parte d'umor feminale dentro del feno muliebre, quantunque ei non abbia il glande all' ordinario perforato, $e$ che in lui, come abbiamo veduto, l' umor feminale efca da un forame apertofi per moltruofità naturale alle radici della fua verga, mi lufingo di poter' avere giufta ragione di credere, che egli fia atto al Matrimonio, e che la moftruofità defritta delle fue efterne parti genitali non è un' impedimento reale, per impedirli d'ammogliarfi.

Si fa, che l'umor prolifico quando per la contrazione venerea è efpreffo dalle veffichette feminali, dove Ata raccolto, ed è obbligato a efciŕ fuori dell' Uomo, riceve un momento tale d'impulfo dalla fopradetta contrazione, che efce con lancio, e mantiene per un dato tempo una direzione di moto rettilineo, $c$ quefto lanciamento farà più, o meno forte in ragione della maggiore, o minor coltrizione, che riceveranno quefte 
quefte parti; quefta poi c̀ il prodotto della organizzazione delle medefime parti, della fenfibilità e del vigore della perfona :

Cio polto è evidente; che il Broli eflendo giovane, e robufto, benchè conformato, come fi è detto; quando farà nel congiungimento, e che avrà ottenuta la introduzione totale, e che net fervore dell' atto lancerà fuori di fe l'umor feminale, queft' umore dovendo camminare per una linea retta, dee andare ad invadere fubito, e con impeto le parti genitali efterne della Donna, e quefta direzione, che dee prendere il lancio del medefimo, farà parallela all' affe della fua verga ; camiminerà rettamente lungo la medefima; e perciò nell' eftro venereo della Donna potrà effere attratta dalla vagina la parte fua più mobile, e firitora, e fi potrà portare, ed attraere dall' Utero, e parimente per il meccanifmo della verga; quando entra in erezione; dovendo più in fuori fporgere quefta parte, dee effer maggiormente proffimo il foro dell' Uretra alla vagina, e più facile l' ingreffo dell' $u=$ mor feminale dentro alla medefima. Una fimil cofa ha rilevato il Morgagni, quando favella del cafo riferito del Vallifnieri, che noi in apprefso riporteremo; onde ci pare di potere avanzare che dal Broli.fi potra ricavare quello che li ottiene dagli altri Uomini perfettaniente in quefta parte-coftituiti; e fani. Queft ifteflo giudizio lo ha fatto anche il più volte non mai abbaftanza da noi lodato Anatomico Sig. Pietro Tabarrani, corroborandolo col fentimento del Kaau, e d'altri celebri Scrittori. $\because$. La poffibilità di quefto ragionamento dedotto da principj fifrologici certi, credo, che non dea porfi: in dubbio dalle perfone fienziate nell cofe mediche, purchè 
chè non fiano diftratte da alcun partito di fcuola. E quantunque fia fempre difficile imprefa di conciliare $i$ difcrepanti pareri degli altri al proprio, e.il pretenderlo. farebbe troppä prefunzione; $e$ dimoftrerebbe poca conofcenza del cuore umano, fono però perfuafo che fi convenga agli amatori della verità di ufare ogni diligenza, e fare ogni sforza per mettere in buon lume quella verità, che fpero d'avér dimoltrata .

E' innegabile, che tutto ciò che efifte è poffibile: ma tutto ciò che è poffibile non è fempre neceffario, che efifta. Co i fuperiori ragionamenti ho creduto di dimofrare, che il Broli è idoneo alla generazione; ora $m$ ' ingegnerò di porre fotto gli occhj del perfpicaciffimoGiudice alcuni fatti che fufficientemente confermano quefta medefima verità ; lo che è neceffario, perchè i Medici, ed $\mathrm{i}$ Chirurghi antichi, ed anche alcuni moderni hanno avanzato, che chi non ha il canale dell' Uretra condotto fino all' eftremità : del Glánde; non è idoneo al matrimonio.

Molti fono gli efempj di Donne reftate feconde fenza aver fofferto la introduzione dell' Uomo; i libri de $\mathrm{i}$ più claffici Scrittori di Medicina abondano di fimili efempj; onde di alcuni folo faremo eziandio menzione, e fono pochi anni che in Siena un cafo confimile fucceffe, e che fu d'uopo venire al taglio, perchè la Donna partoriffe..

La: Storia antica ci ha tramândato, che Cornelia Madre:de-i. Gracchi era imperforata, lo che fu creduto un "augurio funefto: il fucceffo, e l' azzardo favorirono in feguito con illuftre, e memorabile efempio quefta fuperftizione; i Gracchi, che preffo del Popolo Ro-

mano 


\section{Dell' Accademia .}

mano erano in fomma reputazione, fomentarono, e furono maftime Autori ditumulti, e funefte fazioni nella Repubblica, ed impreffera nella coltituzione di-quel gran Governo una immedicabile piaga, ché in feguito terminò colla deftruzione intera della forma Republicana, e fpargímento idel fangue di tanti ambiziof, e generofi Eroi, figlj di quefta ftéfa Repubblica da loro lacerata.

Giovanni Riolano afferifce di aver veduto in $\mathrm{Pa}$ rigi una Donna gravida, che avea l'orifizio della vagina talmente angufto, che appena vi entrava uno fpecillo, alla quale ne i dolori del parto fu d'uopo far dilatare la vagina, e felicemente partori un feto fano.

Racconta parimente il medefimo, che accadde in Parigi, che una Donna imperforata accufaffe in giudizio il fuo marito d'impotenza di frigidità! ma nell êa me fu ritrovata la Donna imperforata, éd éziandio gravida

Fabbrizio 'd'Acquapendente racconta, che un Confeffore lo avea confultato, perchè una Donna éra reAata gravida per avere abbracciato un A mante, quantunque il medefimo $\therefore$ fi foffe aftenuto dall' introduzione.

Pietro Diony riferifce: ancora un fatto finile a quello dell' Acquapendente, e innoltre dice aver veduto, che molte fanciulle, anche con quefta avvertenza, fono reftate ingannate, $\mathrm{e}$ fono trovate incinte.

Benchè il difetto fia per parte della Dónna-negli efempj citati, i medefimi peró confermano, che fenza. l'introduzione della verga puó accadere la fecondazione. Ma ficcome quefto non è appunto il cafo a - me propofto, e parimente non efferido infrequente leggere 
ne i Libri Medici, Storie di Cafi a quefto del Broliz fomiglianti, per maggiore fchiarimento, e per conferma di quanto ho detto, dico, che Fabbrizio d'Acquapendente dice di aver veduto generare de $i$ Figlj. perfone con tali vizj e che lo fteffo ancora hanno veduto altri, come riporta il Morgagni, ed il Signor Tabarrani ; e Federigo Ruifchio, che un tempo è ftato di parere contrario, dipoi mutando opinione dice, che di rado.coftoro poffono fecondare le Mogli . Ma mi pregio di porre in vifta principalmente del Sapientiffmo Giudice una rimarcabile Storia, che fi legge nel. Vallifnieri. Vifito egli in compagnia del Morgagni un giovine Contadino di anni diciotto, sfigurato nelle parti genitali alla maniera del Broli, e variante folo in una fpecie di folco, che fi trovava nella parte inferiore della lunghezza della yerga "; a cui dal fuo $\mathrm{Pa}$ roco effendogli ftato oppofto un impedimento dirimente, per poterfi unire in matrimonio con una, la quale confefsava d'aver fecondata ei con tutto ciò la ottenne in moglie per decreto della Curia Arcivefóvile di Padova. E poichè la foria di quefto Contadino, e la decifione in fuo favore ottenuta puo efsere di grave autorità per la caufa del detto Broli; perciò trafcriverò.le parti più interefsanti di quefta narrazione, che il detto Vallifnieri, fa nelle fue Ofser. Tom.; I I. P.336. della raccolta di tutte le fue Opere ofs. XXVIII. = Penis cum knentu ad radicem biante Invifimus una cum praftentiffmo Collegaj noftro. D. Morgagno rifticum antocum decem \& octo, qui juvenem quamdan in uxorem ducere, avet animso, quamiple $\int e$ pregnantem reliquide profitetwr. opportune, venerat in dubium, an id verum foret, cum $g c^{-}$ 
nerationis organum imperfectum, ac male conformatum babeat. Quare a fuo Parocbo rufficus ad banc noftram Epifcopalem Aulam miflus. Infpecto membro invenimus ipfum ad inferiona infculpto meatu male conformatum, ideft ad radicem Penis

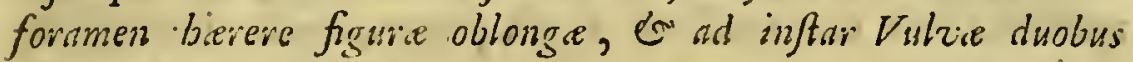
parios labiis concludi.......

Non multo temporis Spatio pricterito compertum eft reie mu'ierem utero gerere, initifque aliis ad rei opportunitatem periculis, variifque animadverfionibus adbibitis, tandem 'viro in matrimonimn dimifja $c f t=$.

$\mathrm{Da}$ tutto ciò adunque, che fin qui fi è detto $\mathrm{mi}$ fembra, che al Broli gli poffa effere accordato di prender moglie, e che è molto probabile ch' ei poffa ancora avere dal canto fuo de' figlj, non oftante la deformità detta delle parti efterne, ferventi alla generazione, e che impedendolo, e trovandofi aftretto a foffirne una dolorofa repulfa, fi potrebbe pregiudicare afsai alla fua falute, e quefta afflizione potrebbe portarlo a funefte confeguenze, ed alla morte ancora, come accadde di morire, al dire del Celebratiffmo Morgagni, ad un giovane in quefta maniera conformato, a cui ( racconta ei per fatto fedelmente riferitogli da Giovanni Giannella ) era fato impedito il Matrimonio. = Vir evat, dice lib.V. de Cauf. \& fed. morb. per Anat. indagatis. Epift.67. art.6.; ex Lemnincenfi agro, qui tametfi Hermapbroditus effe dicebutur, tamén a millice, qua fe gravidam ab ipso factam ajcbat, in judiciun vocatus, non fe defonderat, eamque dotarerint. Is cum liki poftew uxornan quaveret, a fratie autem, a's aliifque tamguam matrimonio mimime aptus deferctur, e medici proxime ciritatis non ignobiles, qui genitalia illius infpereerant, delationem confirmas- 
fent, repulfe dolore incenfus, litibufque cum fratre implicitus, ob bas curas in adeo gravem morbum incidit, ut vivendi finem fecerit $=$.

Quefto è quanto che in efecuzione, ed adempimento della commiffione avuta, con fincera imparzialità ho creduto poter dire, dopo aver maturamente efaminata quefta fuppofta impotenza, e tutto fottopongo col più profondo rifpetto al purgatiffimo difcernimento di Sua Signoria Illuftriflima, e Reverendiflina, alla quale mi dichiaro per fempre Umilifimo Servitore.

France fo Caluri mano propria :

$E$ d'uopo ora di Soggiungere, che dopo prejentata que. fa Relazione e Parere, dal predetto Monfignore Illuftriffimo $e$ Reverendiffimo Vicario Generale fu fatto il Decreto, col quale fi abilițva il detto Agoftino Broli a poterfi ammogliare, come apparife dagli Atti efiftenti nella Curia Arcivefcovile di Siena. Ed in fatti poco tempo dopo il medefimo prefe moglie, e la Donna cbe ei Sposo era una Donna dell' età in circa di trentạcinque anni. Non é peró noto che lo ftefjo abbia per anco avuto figliwoli.

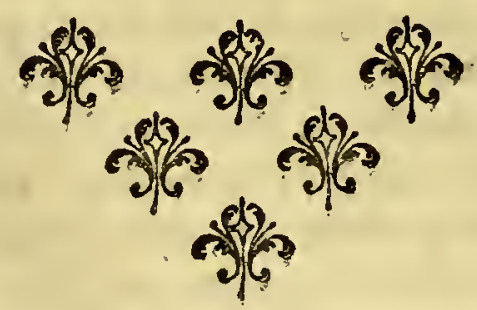




\section{3 \\ MEMORIE EPISTOLARI}

DI OSSERVAZIONI MINERALOGICHE, E ORITTOGRAFICHE DEL SIGNOR

\section{GIOVAN JACOPO FERBER}

DEL COLLEGIO METALLICO DI SVEZIA SCRITTE DALLA BOEMIA AL CHIARISSIMO SIGNOR

\section{GIOVANNI ARDUINO}

PUBBLICO SOPRINTENDENTE ALLLE COSE AGRARIE IN VENEZIA.

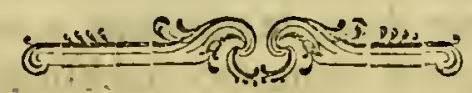

\section{E M O R I P R I M A.}

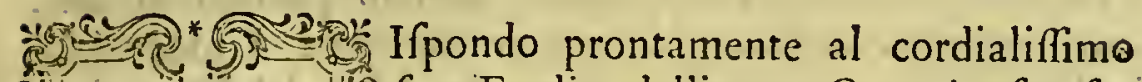

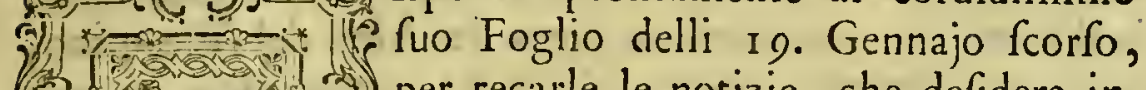
(5): $R$ per recarle le notizie, che defidera in2.: $\mathrm{R}$.

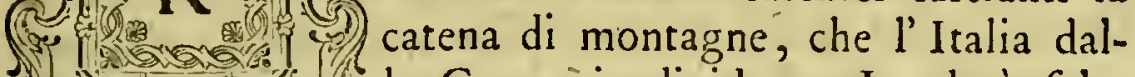

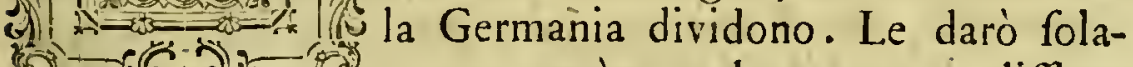

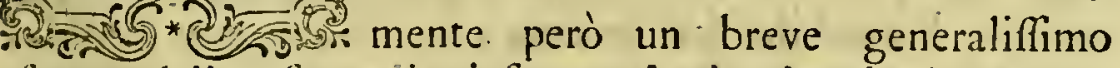
eftratto delle ofservazioni fatte nel mio viaggio da Verona pel Tirolo; di quelle cioè, che in fiffatta materia fono a propofito. $\mathrm{Ci}_{\text {a }}$ ggiugnerò qualche cofa dei monti del Salisburghefe, e degli Svizzeri, e di altre Provincie, ommettendo le ofservazioni d'altro genere, che gia fono vicine ad efcire da' Torchj. Il Signor Cavaliere Ignazio de Born, Configliere delle minere di S. M. R. I. A., mio grande amico, ha voluto ad ogni modo rendere

$$
\mathrm{C} \mathrm{C} 2 \text { pub- }
$$


pubbliche le mie Lettere, ferittegli dall Italia, tra le quali fonori pure le accennate mie ofservazioni. Io veramente avrei voluto, che fi differifse a ftamparle fino a tanto, che l' Illuftre, e Dottiffimo Sig Guettard aveffe dato alla luce il fuo viaggio per molti dei paefi da me vifitati; perfuâfo efsendo, che parecchie cofe da me forfe neglette, da efso farano dottamente efpofte, con giudiziofe rifleffioni, e con quella eleganza, che gli è particolare: ma troppo debbo al Sig. De Born, per non dovermi opporre al fuo volere. Mi lufingo però, che il Sig. Guettard, efsendo mio cordiale Amico, non fia per recarfi ad offefa ch' io abbia trattato un Argomento, fopra cui fta egli per darci interefsanti notizie.

Efse mie lettere vengono pubblicate in lingua $\mathrm{Te}$ defca, ma nondimeno le ne manderò un' efemplare, ftampate clie frano; moltiflimo avendo parlato di Lei, caro Signore Arduino, dando in riftretto I' idea delle bellifine fue ofservazioni fopra le Alpi del Vicentino, e di altri luoghi. Spero ch' ella fra per gradire le mie cordiali efpreffioni intorno al fuo diftinto merito, e la Jibertà, colla quale ho appalefati quei finceri affettuofi fentimenti, che l'amicizia m' infpira, ed infpirerà fempre per l' amabile fua perfona:

$\mathrm{Ma}$ venendo alle ofservazioni da me fatte nel viaggio da Verona in Boemia : lafciata quell' amena bellifJima Città, fi va nel piano fino ai monti, tra quali è fituata la Veneta Fortezza della Chiufa; cioè pel cammino d'una Polta. Viaggiando tra effi monti, $f_{1}$ veggono compofti. di pietre calcarie, che, pafsato Volarni, apparifcono a ftrati groffi di color bianco; poi roffi con Ammoniri, indi di color bigio a letti di molta grofsezza,

e oriz- 
e orizzontali, di grana dura, compátta é nön crifallina.

L'Adige tra que' monti, col lungo giro de' Seco. li, fi è fprofondato, ed ha fcavato un Vallone, dentro cui fcorre, tra rupi calcárie fino a Neunuak, due Pofte fopra Trento:

Dopo Neumark, principiafi a vedere, non piu pietra calcaria, ma montagne di Porfido, prima nero, poi rofso a macchie bianche, di quella forta, che nel: Bergamafco chiamafi Ceriffo, e di Ciottoloni del qual copia. grandiffina fi oferva per tutta la ftrada da Bergamo a Brefcia, Verona, e Vicenza. 'Tal forta di Porfido è affatto fimile, anche nella fruttura, a parecchie Lave vulcaniche; $\mathrm{e} \mathrm{mi}$ ha recato grande ftupore il vedere lunghiflima eftenfione di efse montagne, molto alte, rompofte in gran parte di colonne prifmatiche regolari, per lo più tetraedre romboidali e e talvolta ad angoli retri; c lunghe da due in tre piedi: Parigini, e di circa un palmo di diamétro .. A riferva del numero dei lati, raf-? fomigliano alle colonne dei Bafalti, che d' ordinario fono pentaedre. Siffatti fenomeni rendöno afsai probabile l'opinione, che quelle montagne di Porfido frano grandiffimi ammaffi d'una fpecie di Lava ; na nón. ofo però di affermarlo con certezza, non vi avendo veduto, nè pomici, nè cratéri, nè ceneri vulcaniche. La loró eftenfione ad ambi i lati della ftrada è di circa fei miglia, giugnendo fino quafia Biandfol, che è una Pofta di fopra di Neumark; ma fopraggiunta la notte, inon potei vedere con precifione il loro termine.

Nella feguente matcina mi s'affacciarono a BiantJol, montagne, non piu di Porfidi, maidic Hoinf.chifer $j$ 
cioè di Schifti Cornei, compolti di Quarzo, e di Mica, dove intimamente combinati, dove più, o meno. mifti di Schifto argillofo; e'le ofservai êftenderfi fino alla Città di Brixen, dopo la quale fuccedono altre montagne di Graniti, o granitelli bigj, alternati di Schitti Cornei, e di Schifti argillofi. Quefte giungono fino a Sterzing, dopo il quale apparifcono lungo la ftrada montagne di pietre calcarie a fottili Atrati, dove fole, dove mefcolate di Schifto Corneo, partecipante della natura calcaria. Sonovi pure dei monti onninamente calcarei, e di quelli formati di puro Schifto; e paffato Brenner, eranvi lungo la frada, ciottoloni di marmo; fimile a quello di Polzevera del Genovefato, verde, e nero.

Oltrepaffato In prucb, principiano Colline di pietra calcaria bigia, e nericcia, piena di filoni, e di rilegature di Spato; e quefte continuano a farfi vedere, via facéndo per Dirfcbenbach, e Barbis, cammino di due Pofte, dove cominciano ad alzarf in modo che tra-Nafereit, e Lermas, s'ergono in Alpi altiflime di pietre calcinabili, compatte, a groff ftrati, congiunte con quelle dell' ifteffa natura della Baviera, fulle fommità delle quali la neve mai intieramente non fciogliefi. Nelle Alpi medefime fopra le quali la mia. curiofità mi conduffe, feci copiofa raccolta di Piante Alpine, per accrefcere la mia collezione di naturali. produzioni :

Nei contorni di Hiterwang, e andando a Fuefen, Città dell' Impero Germanico, le Alpi Atefe gradatamente $f_{1}$ abbaffano fino ad occultarfi fotto le pianure, che fi trovano tra Eueffen; e Augsbourg, o fia Augufta, cammino di circa fei Pofte. 
Affinchè V.S., che ha pure viaggiato per le montagne del Tirolo, comprenda precifamente quale fia ftata la via da me tenuta per cui feci le accennate offervazioni, - pafto a aindicarlela, da luogo a luogo, col numero delle Pofte di, un buon tratto.

$\mathrm{Da}$ Veruna giunfi a Volarni, viaggio di una Pofta, e mezza ; indi a Peri, a Ala, a Roveredo, paefi diftanti una Pofta l'unó dall'altro; poi ra Trento, lontano da Roveredo due Pofte Continuai: il cammino da Trento a. Welfobmiclbel; a Neumark, a Braindfol, a Botzen, Deutfchen, Colman, Brixen; Mitterviald, Stcrying, Brenner, Steinach, Scboenberg, e a Infpruch, luoghi tutti diftanti una Pofta l' uno dall' altro. Dopo In prucb vidi Dirfchenbacb; Barbis, Nafereit, Levmas, Haiterwang; e Fueffen, dove termina in larghezzá la: grande catena di montagne, che le ameniffime.Campagne Italiane da quelle di Germania difgiungono.

Dalle fuddette offervazioni rifulta chiaramente effere veriffimo ciò, ch' Ella, ftimatifimo Signor Arduino, nii prediffe, che averei veduto nella parte Settentrionale di quella montuofa catena. Le Alpi interne, e per così dire, centrali del Tirolo, componte di Schifti, di Porfidi, di Graniti, e di altre pietre congeneri; ficcome fono fafciate verfo l'Italia da montagne di pietre calcarie, effe lo fono di pari nel loro lato Settertrionale. Lo fteffo fenomeno fi: offerva nel Salisburghefe, nell Auftria, e Stiria, enegli Svizzeri, ed in altri Paefi. Le mie offervazioni concorrono a confermare il di Lei fiftema, e parlando della catena di montagne anzidetta, che, come ognuno $f a$; lunghifimamente fi eftende dal Levante al Ponente, Higrande fuo nucleo 
cleo longitudinale di pietre Schiftofe, di Porfidi, e Graniti, e di altre tali materie dell' ordine vitrefcente, tro'vafi cinto d'uná fchiera di Alpi, e di monti di pietre calcarie ftratofe nel fuo, lato Meridionale, e di altra $f$ mile nel fuo lato Settentrionale, fotto alle quali effo fi eftende co' fuoi lati, e profonde radici, in modo, che ci dà chiaramente a conofcere effere le medefime allo fteffo fopraffabbricate, e di pofteriore formazione.

La fopra indicata pianura, :che principia alla Città di Fueffen, continua finos a Ratisbona, dove altre montagne Schiftofe, granitufé, continuazioni di quello di Boemia, Saffonia \&c. alzanfi di nuovo dalle campagne.

Ella ha chiaramente dimoftrato con efatte offervazioni che nei monti Bellunefi, Feltrini, Vicentini, Brefciani, Bergamafchi, e del Principato di Trento, e di altre Province d'Italia, li monti calcarei ftanno appoggiati fopra: lo Schifto corneo, e di altre qualità, ovvero fopra altre pietre delli ordine vitrefcente. Io ho veduta la cofa ifteffa: in moltiflimi Paefi da me vifitati; e ne fa prova la relazione del mio viaggio: da Vienna: d'Auftria a Venezia da Lei fatta pubblicare nel Giornale. d' Italia, éd in ina Raccolta di fue memorie, e del Chiarifimo Signor Dottor Giralamo Feftavi di Váldagno, colle itampe di cotefto Librajo Milo co. A quefto propófitc, mi lufingo farle piacere coll' aggiugnere qui altre mie offervazioni, e riflefli alla fcienza Orografica appartenenti.

Le dirò in primo luogo effere perfuafo, che lo Schifto micaceo, e quarzolo, da Lei confiderato una delle pietre primigenie; e quello argillaceo fenza mica,

come 
come pure quello nero bituminofo, quale fi è quello di Idria, e di certi monti degli Svizzeri, fimile di efterna apparenza, ma non di origine, alli Schifti carbono$f_{1}$, ed a quelli contenenti Rame di Mansfeld, non fiano effenzialmente differenti, ma fole varietà d'una medefima fpecie. Ho di più offervato, che quefti tali Schifti fono foprappofti al vero Granito; cioè a quello compofto di Feldf́pato, di Quarzo, e di Mica (Cronftedt. Mineralogie); e lo fteffo ha veduto il Signor Guettard nei Pirenei, ed altrove.

In confermazione di quefta Teoria: li monti, che dividono Ia Boemia dalla Saffonia fono di Granito, int cui trovanfi le minere di Stagno, rariffme in altre fpecie di pietre; e quelli argentiferi della Saffonia fuperiore fono di Schifto Argillofo. Avvi una Minera di Ferro tra il Granito, e lo Schifto appreffo Iobangeorgenfadt in Saffonia, fulle Frontiere della Boemia. Il filone ferreo ivi è fopraftratificato al Granito, e lo Schifto fuperiormente lo cuopre. Effo difcende obbliquamente, incallato tra dette due fpecie di pietre, la prima delle quali è verfo la Boemia, la feconda verfo la Saffonia: locchè dimoftra effere il Granito inferiore allo Schifto.

Li monti Schiftofi della Saffonia, dentro ai quali cavanfi le minere di Argento, veggonfi appoggiari a ridotfo delle montagne Granitofe di Boemia ; e verfo la pianura della Turingia, dove effi monti Saftoni, a poco a poco abbaffandofi, if occultano, fono fafciati da quelli di pietre calcarie a ftrati, che a quelli di Schifo topraggiacciono. Oltrepaffata quella pianura, verfo le montagne dell' Hartz, rialzanfi di nuovo Colline cal-

$D d$

carie, 
carie, indi dal diffotto delle medefime forgono le mon: tagne Schiftofe argentifere dello fteffo Hartz, le più alte fommità delle quali, intorno Andreasberg, fono di Granito. Cosi pure dall' altro lato; andando a Settentrione, verfo. Brunfwic, dove effe montagne fi vanno a poco a poco abbaffando, fuccedono alle granitofe quelle di Schifto, ricche di minere di Argento, e d'altri metalli; e finalmente incontranf pietre calcarie; e arenarie, marghe, Schifti carbonofi, e Schifti metalliferi bituminofi, contenenti Rame, e Argento, e pefci metallizzati: materie tutte difpofte a Araci fopra ftrati, addoffo alle radici delle fopraddette montagne Schiftofe. Nel modo ifteffo, da qualunque lato, che fivoglia falire dalle circoftanti pianure fopra l'Hartz, le prime alture, a Colline, e monti, veggonfi compotte delle anzidette pietre calcarie ec, $;$ indi fuccedono le montagne Schiftofe metallifere, e fittalmente quelle di Graniti, che a guifa di grandiffimo nucleo, forgono dalle profonde vifcere della terra, e s'ergono a grandi altezze.

Il fu Signor Lebmann, Chimico celebre dell'Accademia di Petersbourg, pubblicò in Tedefco moltiffme fue offervazioni in quefto propofito fopra $\mathrm{i}$ monti dell' Artz, come può leggerfi nel fuo Trattato di Flötfgebirige, tradotto in Francefe, ed inferito nel Tomo terzo delle di Lui Opere, ftampate in Parigi ncl 1759. ; ed io fteffo ho rifatte le medefime offervazioni, ed altre molte.

Gli fteffi fenomeni vide il Signor Lclamam nella Slefia, il Signor Gucttard nei Pirenei, ed io medefimo gli oflervai andando dall' Inghilterra verfo la Scozia, 
e negli Svizzeri, dove le Alpi più eccelfe fono Granitofe, come per efempio il famofo monte $S$. Guettard. Le orride Alpi della Lapponia, e quelle della Norwegia fono pure di Graniti; come parimente li monti Carpazj piu alti nell' Ungheria, e quelli della Tranfilvatiia; ma gli altri meno alti, e metalliferi di dette due ultime Provincie fono compofti di Schifto di una fpecie particolare; e li meno elevati di tutti, formati fi veggono di pietre calcarie, e fafciano gli altri dalla parte dell' Auftria. Simili fenomeni ci prefentano anche le montagne del Tirolo, e del Salisburghefe, come rifulta dalla fopraefpofta narrazione delle offervazioni ivi fatte nel mio viaggio.

Quanto ho detto finora bafta a provare; che lo Schifto fta appoggiato fopra il vero Granito, e che le pietre calcarie fono fopraftratificate allo Schifto. Da ciò Ella ben comprende cofa io intenda di fignificarle in rapporto al gran nucleo granitofo, e quarzofo delle montagne Tirolefi, fopra le quali mi ricercò di fcriverle. Effo è ad ámbi i lati cinto da Alpi, e Monti, e Colline di natura calcaria, abbondantifime quafi in ogni luogo di reliquie dei viventi dell' antico mare, che mancano onninamente nei Porfidi, nei veri Schifti coltituenti montagne (*), nei Graniti, Bafalti, ed in altre fimili materie vitrefcenti, propriamente, e rigorofamente appartenenti: alli di Lei monti primitivi:

Quindi fembra rifultare, che il Granito, di cui le montagne più profondamente radicate, dell' Europa almeno, fono formate, fia il materiale di prima origine,

D d 2

cui

(*) Berg-gcinifer. 
cui fuccede lo Schifto, e l'altre pietre dell' ordıne compleflivamente primitivo; ed a quefte le pietre calcinofe delle fue montagne fecondarie, fopra alle quali fono in ultimo luogo collocate le colline, e piccioli monti da Iei nominati Terziarj, compofti di pietre fabbiofe calcinabili, di Arene, di Crete, e di Marne; e contenenti corpi marini, di fpecie molto più numcrofe, ed in maggior copia di dette montagne fecondarie, e talvolta dei Carboni foftili ec.

Ecco Stimatiflimo Amico, la Teoria rifultante da tutto ciò, che rapporto all' Orittografia, ho yeduto viaggiando per la noftra Europa ho con gli occhj proprj offervato in Italia, che $\mathrm{i}$.Vulcani fonofi aperta via in molti luoghı, rompendo, e fquarciando li monti calcarei. Forfe in altri Paefi hanno fatto lo fteffo in quelli di Schifto, e di Granito ec; e s'è pur vero, che le Cordeliere, montagne Granitofe altiffime dell America, abbiano Vulcani, può darfi, che le loro bocche fiano nel Granito.

Rifpetto poi all' origine dei Graniti, degli Schift, e dell'altre pietre vitrefcenti, difcordi effendo di parere li Naturalifti, io non entrerò a dirne opinione. La maggior parte di quelli di Germania, di Svezia, degli Svizzeri, e della Francia ripete dall' Acqua la formazione delle pietre, si vitrefcenti, che calcarie. In Italia ora fi penfa da molti diverfamente; e V. S. fu il primo a dottamente proporre così il fiftema, che le vitrefcenti fiano produzioni del fuoco. Benchè però io mi contenti d' effere unicamente offervatore dei Fenomeni del Regno fotterraneo ; con tutto ciò, nelle mie Lettere fopra l'Italia, mi Cono dichiarato a favore del fuo fenti-. 
fentimento, parlando del Bafalte, e di altre foftanze follili. Le tante offervazioni da Lei comunicatemi mentre io era feco.in Venezia, e le tante giudiziofe prove del fuo fiftema d'Orogenefia, m' hanno fatto chiaramente comprendere la folidità de' fuoi fondamenti, e gli eftefi fuoi lumi nella Scienza orittologica, e orittografica. Quando avrò tempo opportuno a poter difporre ordinatamente tutte le mie offervazioni fu tale argomento, ho intenzione di pubblicarle in un Trattato latino; ma fratfanto, s' Ella caro Amico, trova cofa degna di fua attenzione nella prefente lettera, e Le piaccia renderla atta alla ftampa, potrà farlo correggendo $i$ difetti dello ftile. Gradif́a fe non altro, il mio defiderio di darle contraffegni di mia gratitudine di tante gentiliffime cortesie ufatemi. Se mai trovaffe qualche cofa di ofcuro, favorifca indicarmelo, prima della ftampa, aftinchè poffa meglio fpiegarmi, e ridurre più chia-

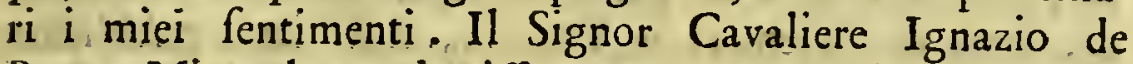
Boin, Mineralogo dottiflimo, appreffo cui mi trovo, molto la rifpetta; ed io divotiffimamente riverendola, c pregandola volermi continuare la cara fua amicizia, e corrifpondenza, finceramente mi dico.

Di, Altzedlitz in Bocmia li I s. Dicembre 1772.

Suo Divotifs. Obbligatijs. Affezionatiss. Amico Giovanni Giacomo Ferber. 


\section{E M O R I A E C O N D A.}

TRADOTTA DAL FRANCESE.

S'Io prefentemente aderifco, mio cariflimo e rifpetta$\int$ to Signor Arduino, al fuo fuggerimento di frriverle in Francefe, ciò -non è certamente per volerlo far fempre. Lo fo folamente in quefta occafione ad oggetto di poter efprimere le mie idee con piì di chiarezza; ma non creda, ch'io voglia abbandonare la più bella delle Lingue da me apprefe; nè che fia in avvenire per rifparmiarle la pena di leggere, di tempo in tempo, un cattivo Italiano:- bifogna ch ella ufi meco un poco di pazienza in quefto propolito.

Non fo immaginarmi come frami ufcito di mente, nelle antecedenti mie lettere, di ringraziarla per la pena, che fi è data di porre in netto le mie offervazioni, fatte tra Vienna, é-Venezia , e di farle pubblicare nel Giornale d'Italia, del quale il Signor Delortbe, Confole Svedefe in cotefta Capitale, $m$ 'ha trafmeffi i Fogli, che le contengono. Non manco però di farlo prefentemente colla piu fenfibile riconofcenza, e col piacere di afficurarla finceramente ch' Ella ha giuftiffimamente efpreffi li miei fentimenti, e con termini della più defiderabile proprietà

Rilevo, che anche l'altre mie offervazioni, comunicatele con Lettera delli 15 . Dicembre dell' anno fcorfo, hanno avuta la fortuna di fembrarle degne di rifleffo, e di effere pubblicate: effe fono a fua libera difpofizione, e può farne quell' ufo, che troverà a propofito. La fua Amicizia le ingrandifce il mio merito; ma non 
perciò perderò io iquella moderazione, che conviene ad un Giovine come me; che conofce beniffimo i difetti di fue cognizioni.:

Dalla fua rifpoftars a detta mia lettera comprendo efferle fembrato, che alcune delle mie offervazioni alle fue fieno contrarie. Nella fuppofizione che noi fiamo difcordi, locchè non è certamente, Ella $m$ ' ha rifpofto con fentimenti veramente degni di un Naturalifta del primo ordine, come è infatti, fenza adulazione. Prende a difendere con nobiltà d'animo li fuoi fentimenti, fenza che la fuppofta diverfità di pareri niente influifca contro l'amicizia, che tra noi farà perpetua, nè finirà, che al terminare della vita. Sia ugualmente di me perfuafa, e fia certa effere affolutamente impoffibile; $c^{\prime}$ is poffa perdere di vifta $i$ riguardi di un' amicizia la più cordiale, e rifpettofa ; movimentó fincero del mio animo; che non ha rapporto alcuno colle noftre offervazioni, le quali, fe anche foffero dif; cordanti, farebbono da ambi candidamente efpofte, fenza reciproca offefa, e fenza viltà, 0 fini indiretti di occultare, o mafcherare il yero.

Ma tanto è lontano che fravi tra di noi difcrepanza, che anzi l'afficuro colla, più femplice verità di non avere fino a queft'ora veduta cofa, che fi opponga in fatto alle offervazioni da Lei fatte . Conofcendo a fondo la fua efattezza, lé fue cognizioni, ed il fuo amore del vero; fe mai $m$ accadeffe di vederne, prima di darne notizia, diffidando degli occhj proprj, uferei la più efatta diligenza, rettificando le offervazioni ; ed allora folamente Le direi con tutta ingenuita ciò, che aveffi bene veduto. 
Nel calo prefente, le rifleflioni da Lei fattemi fopra alcune particolarità dell' anzidetta mia Lettera, mi pongono in dovere di più chiaramente fpiegare i miei fentimenti; donde poffa conofcere, che li medefimi alii fuoi non " $\bar{f}$ oppongono.

Quando V.S.; nelle fue Memorie inferite nella nuova raccolta di Opufcoli del P. A. Calogerd; ha detto, che quel genere di Schift, ivi da Lei con precifione defcritto, è uno almeno dei generi di pietre primigenie, e relativamente a cert' altri materiali dei monti primitivi; non ha già pretefo che, in fuppofizione già impolfibile, che fi ef́cavafé un pozzo fino al centro della terra, altro mai, cheirtale Schifto non veniffe a foprirfi. Si è anzi dichiarata di non intendere di parlare che delle fole materie del Regno foffle, alle quali può giugnere 'l' umana vifta. Il fuo fchifto primigenio fu così da Lei nominato, perchè nei monti, che ha offervati, to ha coftantemente veduto fottopofto, nella fotterränea fua eftenfione, agli altri materiali dei monti compleffivamente primitivi, ed a quelli calcarei, componenti le montagne fecondarie ec., ed in confeguenza più antico di tutte l' altre materie allo fteffo foprappofte. Effendofi dunque fpiegata di confiderare tale, fuo fchifto per una almeno delle pietre primigenie a noi vifibili, non ha efclufe 'i' altre, che, per ragioni univoche, o anche maggiori, efigono la fteffa denominazione di primigenie.

Io, averido vedute le vafte catene di montagne Granitófe nelle principali parti d' Europa, eftendere le loro profonde radici fotto quelle di Schifti, ho conclufo, che il vero Granito è inferiore allo Schifto, come que- 
quefto lo è per rapporto alle montagne calcarie ec. Se poi effo Granito fia d'origine anteriore a quello dello Schifto, ofe frano pietre coeeve, io non fo deciderlo, ed il faperlo non mi pare di grande importanza. Bafta fapere ben difcernere quali frano in compleffo li pietrofi generi componenti li di Lei monti primitivi, e le rifpettive loro collocazioni, andamenti, ftrutture, caratteri, e fenomeni; e non confonderli con quelli de' fuoi monti fecondarj, che a tutta evidenza ne differifcono. Quefto è ciò, che è d'uopo, e giova di co: nofcerfi; non folamente in riguardo alla Mineralogia, e Orografia, ma anche per norma, e guida dell' Arte metallurgica; tanto neceffaria, ed utile all' umana Società .

Suppofto ch'io abbia bene veduro, e che la mia offervazione fia vera, come pretendo; ora non fi tratta, che di bene intenderf fopra il termine Primitivo; - Primigenio, che in effetto fignificano una cófa ifteffa, ciò̀ il rapporto tra pietre, e pietre, in riguardo alle rifpettive naturali loro fituazioni. Dunque il fuo Schifto fara fempre di un genere primigenio in confronto delle pietre calcarie, e di altri generi al medefimo foprappofti, e giammai non avrà Ella bifogno di cangiare una parola di ciò, che fu tale argomento ha pubblicato. Forfe qualche altro Offervatore potrà dimoftrare, che fotto il mio Granito fi trovino altre fpecie di materiali : e chi può dire al contrario ? noi punto non conofciamo l' interiore del Globo noftro. Le noftre più profonde minere non s'internano che nella corteccia, fu cui noi formicoliamo. Ciò non oftante, io fofterrò, che il mio Granito, ed il fuo Schifto foE e 
no pietre primitive in rapporto alle pietre calcarie, e a tutte quelle, che coftantemente fi veggono ellere fopra li medefimi collocate.

Non ho dunque levato certamente alle fue offervazioni, anzi parmi di averle accrefciute, e confermate; aggiugnendovi ciò, ch'Ella ftefla vi avrebbe aggiunto, fe aveffe viaggiato nei medefimi Paefi da me veduti, Se io non vado, come-credo, in America, chi potrà da me pretendere che dica come le montagne di quelle Regioni fono formate?. Non potrei farlo fenza entrare nelle Ipotefi, come più d'una volta è ftato fatto fu tale foggetto.

Ma nella fuddetta mia lettera avvi in apparenza, ma non in foftanza, un altra contraddizione: ed eccola. Io pretendo, che il mio Granito fia radicato inferiormente allo Schifto, ed Ella al contrario ha offervato nel Vicentino, nẹl Trentino, in certi luoghi del Tirolo, ed in altri Paefi, che vi fono dei Graniti, Granitelli, Porfidi ec. fopra al fuo genere di pietre Schiftore; locchè è veriffimo, come io fteffo ho veduto in più luoghi. Le ho già detto nella mia Relazione del viaggio, che ho fatto dall' Italia pel Tirolo, di avere offervato tra Neumark, e Brandfal, delle vafte montagne di Porfido, le quali fecondo ogni apparenza, non fono che Lave veramente fuperiori allo Scliifto , o fopra di effo colate. Egli è anche credibibile, che li Graniti, e Granitelli, che: ha veduti' fuperiori allo Schifto, nei luoghi fopraddetti, effi pure frano Lave, avendo io trovato fopra il Vefuvio delle materie perfettamente fimili a fiffatte pietre, vomitate $\mathrm{d} i$ quell' orribile 'Vulcano. Se qualche Oltramontano ne 
dubita, e vuole fpiegare la loro formazione per'l' Acqua, c̀ arbitro di farlo a fuo talento, che a me poco importa; ma fempre farà vero, che fimili Graniti fopraggiacciono allo Schifto.

D'uopo è dunque neceffariamente di coftituire nell' Orittografia due forti di Graniti, e Granitelli ; non gì in riguardo alla compofizione, $e$ differenza interiore di tali pietre; ma bensi in rapporto alla loro età, dimoftrata dalle rifpettive lor fituazioni fopra, o fotto allo Schifto. Quefta diftinzione da fe è manifefta', nel modo ifteflo che il di Lei Schifto primigenio effenzialmente, per origine, $f_{i}$ conofce diverfifimo da quello efiftente fopra li Carboni foffili, ed in altri fiti ftratofi ; quantunque ambi tengaño per loro bafe una terra argillofa .

Concludiamo dunque, che il mio Granito, efiftente fotto al di Lei Schifto, è come quello una pietra primitiva; I. in comparazione alle pietre calcarie an: che delle Alpi; II. in rapporto ai Graniti, e Granitelli ec. foprappofti a detto Scnifto, li quali certamente a fuo confronto non fono pietre primitive, ma fecondarie.

Ecco appianata, fe male non $\mathrm{mi}$ appongo, ogni difficoltà; e tolta ogni differenza. S'Ella ne refta foddisfatta, come non poffo dubitare, ne avrò molta compiacenza.

Se il ragguardevole Soggetto, che mi-nomina, profeguirà le fue ricerche nelle montagne degli Svizzeri; e s' egli vede con occhi di vero offervatore, cioè a dire fenza pregiudizi; troverà certamente dei Graniti, e delli vafti monti di tal pietra compofti. Anche E e 2 del- 
dello Schifto Corneo, e di altre qualità, ne vedrà co: pia immenfa in numerofe fituazioni di quelle Alpi.

Io ho per alcune fettimane abbandonata la villeggiatura del Signor de Born, e mi trovo in Praga, capitale di quefto Regno di Boemia, ove to difponendo il Gabinetto di effo Signor Born, che vuol far trafportare nella fua Signoria di Altzedlitz. Oh quanto mi farebbe cara la prefenza di V.S. affinchè poteffe contemplare quefto Teforo, veramente ineftabile, di curiofi minerali! Effendo vicino a compire il mio lavoro, ritornerò in breve appreffo detto mio fimatiffmo Amico, donde partirò poi, dopo qualche mefe. di ritorno alla mia Pátria.

Reftituito, che mi faro in Altzedlitz, ho inten. zione di fare una gita orittografica in compagnia del Signor de Born, per efaminare particolarmente un monte di quefto Regno, che dalle pietre portateci da uno de' noftri Amici, abbiamo argomento di crederlo un antichiffimo eftinto Vulcano $\left(^{*}\right)$.

Di quanto ci riufcirà fcoprire, ed offervare d' intereffante per l'Iftoria fifica del Regno Plutonico, non mancherò informarla; ma frattanto le do notizia, che numerofiffimi, fono $\mathrm{i}$ monti di Bafalte nero colonnare e quì in Boemia, ed in Saffonia, Lufazia, e Haffia. La maggior parte degli Orittologi Tedefchi lo crede formato dall' acqua; ma oggimai la noftra opinione, che anche i Bâfalti, non oftantẹ la loro prifmatica forma

(*) Il Signor Cavaliere de Bom, piz volte nowinato in quefte Memnrie, ba gid firitia una Differtazione in Tedefo fopra detto Vulcano, della quals to data notizia al Signor Arduino, con Lettera 16. Ottobre $17 \% 3$. 
forma, fiano produzioni di fotterraneo incendio, $\hat{e}$ pofta fuori di dubbio. Li célebri Naturalifti Inglefi, Banks, e Solander, che unitamente al Signor Uno Troilo di Svezzia hanno vifitata l'Ifola di Staffa, e quella d'Islanda, hanno forprefa la natura, per così dire, ful fatto - Lave di frefco eruttate dall' Hecla, famofiffmo, e orribile Vulcano, aveano precifamente la for$\mathrm{ma}$, la prifmatica regolare figura delle colonne $\mathrm{Ba}$ faltine, abbondantiffime in quelle Ifole, ed in altri innumerabili luoghi del Mondo.

Non mi ftupifco però della contrarietà de' pareri tra tanti che fcrivono, e parlano di Litogenefia, edella Geografia fotterranea. Io ne' miei viaggj, ho conofciuti moltiffimi Mineralogi, veramente dotti, ed. eccellenti, ma mancanti, pochiffmi eccettuati, di quelle offervazioni, e cognizioni, che a poter giudicare con fodi fondamenti delle diverfià di origine; di natura, e delle rifpettive fituazioni e andanienti delle pietre, $c$ dell' altre materie Foffili, fono neceflarie. Chi non ha avuta occafione di contemplare localmente, ed a lungo, gli effetti, e prodotti infinitamente variati de' fuochi fotterranei, delle metallurgiche fufioni, de' vitrificamenti ec. chi non ha ben bene confiderati li diverfiffimi lavori delle Acque; non è in grado di poter penfare, e produrre, nel propofito preaccennato, che mere immaginazioni, credibili folamente a quelli, che, com' effi, non hanno della Terra che idee fuperficiali, ollervazioni vaghe, e slegate.

Per conofere con qual ordine fucceffivo li diferenti pietrofi generi fotterraneamente $f_{i}$ corrifpondono; come gli uni, rifpettivamente agli altri, trovanfi col- 
collocati, e difpotti, e quali fono i fenomeni, e fegni Caratterifici, che la diverfità di tempo, e dicaufe della loro formazione ci additano; bifogna aver molto vifitate le profonde minerali Efcavazioni, i cupi valloni, le frane, le roccie, ed avere anatomizzate, per così dire, le montagne di varie Provincie.

Quefte fono le vie da Lei calcate, amatiffimo Signor-Arduino, per internarfi nella conofcenza delle cofe foffili, e per le quali io fteflo mi fono da molti anni incamminato, e fpero di profeguire.

Ma paffando ad altro: io defidero moltiffimo di vedere pubblicate le tante offervazioni di Iftorià naturale del celebre Inglefe, Sig. Giovanni Strange ; e debbo ben credere ch' egli- non vorrà lafciare inedite notizie, che molto poffono giovare ai progreffi di una buona Orittografia, e della Scienza foffile.

Molto poi La ringrazio del dono fattomi del Libretto, contenente-le fue Memorie fopra le Acque minerali di Recoaro, e di Arzignano, le oflervazioni del Signor Dottore : eftari di Valdagno fopra quelle Alpi, e due delle mie lettere. Efro Sig. Feftari mi ha gentilmente onorato molto più che non merito; ed ho fpiacere di non averlo perfonalmente conofciuto. Lo fteffo dichiarafi meco perfuafo, che le Argille de'luoghi..Vulcanici poffano efferfi prodotte per fcioglimento delle Lave, come in fatto offervai alla Zolfatara di Pozzuolo, nel Regno di Napoli ec.

Benchè però identica lia la bafe di quefto genere di terra, dire non oferei, che qualunque Argilla del noftro Globo fia una produzione-Vulcanica; febbene: ognuna rifulti dall' intima unione dell' Acido Vetriuolico sol- 


\section{Dell' Accademia .}

colla terra vitrefcente, come ha dimoftrato il Sig. Baumè. Imperciocchè non può negarfi; che ogni acqua ftagnante; come anche d'acqua del Mare, non deponga naturalmente un vero fedimento argillofo: e le offervazioni, chimiche delli Signorib Murggraf, Eller; e WalIsxio, e di altri, ci dimótrano, che qualunque acqua), anche della piu pura, ogni volta, che fi diftilla in vafi di vetro, dà fempre una porzioné 'di fimile terrá, fe ancora la diftiltazione foffe mille volte ripetuta 1

Per altro voglio comunicarle uh' oflervazione da me fatta in quefto Regno, le che mi pare degna dé fuoi rifleffi. Buona parte di quefti monti.di Granito, e di Schifto, fra puramente micacéo, corneo, cióè comporto di Quarzo, o di Micá, dalla fuperficie fino alla profondità dilqualcheipiede, tróvafi infignemente cangiata dal primo originale! fuo ctato., pel contato dell' Aria 'Tale efterna corteccia di effi monti è affatto bianca, e fciolta in modo', che più 'non poffono difcernerfi li primi componenti materiali; come a dire, il Quarzo, Feldfpato, e la mica, fortanze coltituenti il Grạnitọ. Ciò che primà erá dura pietra!, per fiffatta trafmutazione, raffomiglia ad un' Argilla di color bianco, dove compatta, e dura, dove molle, e plaAtica. Tutto il tratto del circolo di Pilfén è coperto di detta feconda qualità di Argilla : lefla förma il terreno fertile, mefcolata con altre eterógenee materie, che ne diverfificano $i$ colori, e le proprietà. Dove la medefima trovali pura, e bianca ir ferve a Figuli per la formazione, de' loro vafi, li quáti, ćotti che fiano, hanno un colore rofficcio, fegno che la fteffa Argilla par- 
recipa di terra marziale. Li muratori parimente la inpiegano a dare il bianco alle pareti delle Fabbriche.

Quefte pietre Granitofe, e Schiftofe, all'aria fcrepolano, e a poco a poco fi attenuano fi difciolgono, trafmutandofi in Argilla Il Granito, e Schifto Corneo ec.; fono pietre vitrefcenti; ma qui lazione dell' Atmosfera produce fopra le medefime, effetti analoghi a quelli dell Acido sulfureo foprai le Lave alla Zolfatara . L aria di Bóemia niente o pochiffímo contiene di acido, il quale alla Zolfatara efala di continuo in copia grandiffima dal profondo della Terra, fu cacciato dall occulto fuoco, da cui viene grande quantità di Zolfo fcompolta . Ciò che produce quì liccennato fcioglimento dei Graniti, e degli Schifti fi è, a mio credere, I Acido Vetriuolico originalmente con tali pietre combinato; e l'azione dell Atmosfera non vi contribuife, che collo svincolare, e porre in movimento l'Acido nelle fteffe imprigionato ed occulto, mediante la continua infinuazione di particole umido-aeree.

Le alterazioni, che foffrono all aria le marcaflite, - Piriti, e le materie, che ne fono impregnate; mi fembrano prova evidente di quefto mio fentimento. Quelle marziali-fulfuree, e non arfenicali, o prefto, 0 . tardi vi fcrepolano; la coerenza de'loro componenti fi fcioglie; il Flogifto fulfureo a poco a poco fi diffipa; e l'acido, dal medefimo fvincolato fotto forma falina col ferro, e colla terra non metallica intimamente fi: congiugne, onde rifulta il Vetriuolo; mifto anche fpef-. fo di Allume, e di altri fali a bafe terrofa, fecondo 
Ia natura delle terre, che nel compofto piritolo $f i$ contengono. In cotal modo, ciò, che nello ftato nativo era in folide mafte, dure, pefanti, e di fplendore metallico, $\complement_{1}$ vede cangiato in minuta polvere; e tratti, che ne frano detti fali con l'acqua, rimane terra fangofa.

Se dopo la prima lifcivazione, detta materia, efpofta di nuovo, e per lungo tempo all' aria, ancora fi vitriolizza, egli non è già perchè attiri Acido dall' Atmostera, come gli antichi Chimici hanno creduto; ma perchè non effendo prima feguita una perfetta foluzione della Pirite, lo Zolfo, che vi rimane, viene a fubire le alterazioni già indicate,

Il Quarzo; ed il Feld/pato contengono gran porzione di terra argillofa, come ha dimoftrato il Sig. Baw$m i^{\prime}$, infegnando che fe tali pietre fi fondano con quantità fufficiente di fale alcali fiffo per farne il Liquor. Silicum, e fatto effo liquore, fi precipiti la fua terra con Acido Vetriuolico, vienfi ad ottenere vero Allume; prova manifetta della prefenza dell' Argilla Dette pietre f disfanno inoltre all'aria in certi Graniti, e vi fi cangiano in terra Argillofa : d'uopo è dunque credere che ne fiano compofti. La Mica parimente di effi Graniti fi è dimoftrato dallo fteffo Baumé effere una criftallizzazione argillofa; e cosi pure gli Schifti cornei, e argillacei contengono la medefima terra : dunque l'Argilla cfitte nelle pietre Granitofe e Schiftore della Boemia, delle quali ora parlo. Che anclie detto Acido, uno dei due effenziali principj coftituenti le Argille, vi fia intrinfecamente combinato; che nelle accennate circoAanze fi fviluppi, reagifa nel principio, terreo, ed allo Atato fpecifico argilloro feco fi riduca, rendefi mani-

$$
\mathrm{F} f \text { fefto }
$$


efto dagli effetti fopraindicati, che vi produce. S' effo non vi foffe, quefte pietre non potrebbono mai prendere la forna, e le proprietà di vera Argilla, rifultando effa dall' intima unione dello fteffo. Acido colla terra vitrefcente, con certa particolare proporzione, e combinamento; come dal fopraddetto Autore è ftato dimoftrato. Dall' avere io detto che nel Quarzo, nel Feldfpato, ed in fomma, nell' aggregato dei Graniti, e degli Schifti, vi efifte l'A rgilla, non vorrei fi credeffe che intenda effervi la medefima fotto la fpecifica forma, per la quale le terre argillofe dagli altri terrofi generi fi diftinguono. Effa vi fi trova realmente, ma forto altre modificazioni; anzi ne è, fe non la totale, almeno la maffima foftanza. Allora folamente apparifce fotto la fpecifica forma argillofa, quando dall' opportuno concorfo delle caufe folventi, difgregate le molecole integranti di dette pietre, e fvincolate dalla forte loro prima coerenza, viene a rifultarne un nuovo corpo, ridúcibile con l'acque in pafta tenuiffma, vifcida, e plaftica, indurabile al fuoco, falva ogni fua artificiale, o cafuale figura, e finalmente vetrificabile, e non mai calcinabile .

Così teorizzando, non credo di punto difcoftarmi dalle fue dottrine: Ella, che per lungo tempo ha fatte diligentiffime offervazioni, e chimiche, e metallurgiche fperienze, e che ha pure veduti frmili fenomeni in cotefte parti, ne è giudice competente; e però ne ftaro attendendo il fincero fuo giudizio.

Terminerò dicendole che di molto piacere mi è Atata la notizia ch'Ella abbia ricevuti dal mio Amico Signore Haquet d'Idria della Carniola i faggi di tutte le 
le varietà di quella ricchiffima minera d'Argento vivo, e delle pietre, tra le quali trovafi generata. Spiacemi però molriffimo che li curiofi minerali della Tranfilvania, a Lei inviati dal Signor Cavaliere de Bom, fianf nel viaggio fmarriti; ma fia certa che ne avrà degli altri, infieme con quelli di ftagno, e di altri metalli ec, della Boemia, e colle noftre opere pubblicate. Ella non dubiti punto della coftanza di noftra corrifpondenza: effa deve cotinuare fe anche io andaff, nelle, Regioni più remote della Terra. Continui pure a confegnare le fue Lettere al noftro Confole Delorthe, comune Amico, che avrà cura del loro ficuro indirizzo: e colla più fincera ftima, e cordialità mi dichiaro.

Di Praga il r. Marzo 1773.

Suso Divotifs. Obbligatifs. Affezionatifs. Amico

Giovanni Giacomo Ferber.

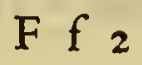

SAG. 
228

\section{$S \quad A \quad G \quad G \quad I \quad O$}

FI S I C O - M I N E R A L O G I C O

D I LY THOGONIA,E OROGNOSIA DEL SIG NOR

\section{G I O V A N N I A R D U I N O}

PROFESSORE DI MINERALOGIA, E DI CHIMiCA METALLURGICA, PUBBLICO SOPRINTENDENTE ALLE COSE AGRARIE DELLO STATO VENETO.

\section{(5)}

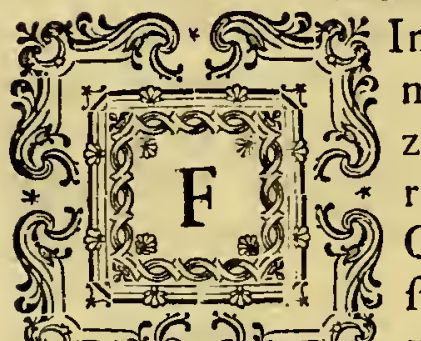

Ino dall' Anno 1759. manifeftai le mie congetture intorno alle differenze di origine, e di natura dei materiali del Regno Lapideo, cofpicui nel Globo nottro, in due Memorie epiftolari, dirette al Chiarilfimo Sig. Cavalier Antonio Vallifneri, pubblico Profeffore d'Ittoria Naturale nell' Unive fi.d di Padova, ch' egli fece putblicare (I).

Offervate lungo tempo le fituazioni montuofe, e le pianure di quefti nottri, e di altri paefi, e attentamente contemplate le differenze di modi, e di tempo della loro formazione, manifeftamente apparenti nelle diverfità di fituazione e difpofizione, e di natura, e di fenomeni de' rifpettivi loro materiali, parvemi di potere

(1) Nuova raccolita di Opufcoli Fiblologici ec., del P. Abb. Calogeris T. VI. Vekezia 1760. 
re ragionevolmente comprendere fotto quattro generaliffimi ordini, o divifioni la Terreftre fuperficie, dalla fua efterna faccia, fino almeno a quella profondita, cui giugnere poffono le umane indagini.

Quindi in tre ordini diftinti tutti li monti, nominando primarj, o primitivi quelli del primo ordine, fecondarj qualli del fecundo, terziarj quelli del terzo, ed affegnando al quarto le pianure. Indicai pure, benchè fuccintamente, le ragioni di fiffatta divifione, e le note caratteriftiche, per le quali effi ordini l', uno dall' altro fi diftinguono, ed $\mathrm{i}$ tempi varj, e fuccellivi delle loro origini fi conofcono.

Furono da me comprefi nell' ordine de primitivi quei monti, che per effere formati di quelle folfili materie, ch' effere fogliono gli ordinarj originali ricettacoli delle minere metalliche, vengono da' Pratici col nome di Monti Minerali diftinti da quelli di altre qualità : ed ho confiderate fecondarie le montagne di marmi, e pietre calcarie a frati, nelle quali tali minere fono fommamente rare, e forfe fempre avveniticcie, $c$ accidentali; ma.che di pietrificate organiche produzioni del mare comunemente fono ripiene (r) . Al terzo ordine ho riferiti quei baffi monti, e colli, che fi ravvifano effere compolti di Ghiaje, di arene, e di terre limofe, argillacee, marnofe ec.; materie quafi fenpre con immenfa copia di marine Quifquilie mefcolate. Fe-

ci an-

(1) Fra le accennate minere metallichs, io non intendo di comprende. re turte quelle del ferro: effo è comune, e fparfo in tutts la Terra; e cer. se fpecie di minere ferrec trowanf talvolta anche nei monti del feconto, $e$ del terzo ordine: come altrest varie forti di Piviti Sulfureo-guarziali, di Figfili bituminofi, ec. 
ci anche cenno delle materie vulcaniche da me offervate nelle noftre montane fituazioni, delle quali, e degli effetti di antichiffimi fotterranei incendj ho poi parlato piu precifamente in altre Memorie, che ho polteriormente fritte ( $\mathrm{I}$ ).

Siccome però anche le pietrofe, e terree materie di ciafcuna di dette quattro divifioni fono tra fe molto differenti, e che confiderando $\mathrm{i}$ modi, e luoghi delle rifpettive loro fituazioni, conofconfi non effere coetanee, ma effetti fucceffivi, prodotti in tempi varj, e col concorfo di circoftanze diverfe, dalle rifpettive loro caufe effettrici; così parlando dei monti primitivi, confiderai primigenj quelli de' loro materiali, che pel fito ch' eff occupano, e per altri rifpetti fembrati mi fono di origine più antica : non già però in riguardo alle contituenti foftanze di ciafcuno; ma unicamente alle modificazioni, fotto le quali ora efiftono .

Che le parti vifibili di quefto Globo non fieno primordiali, rifpetto alla prefente loro forma, e ftruttura; ma che dipendano da varie rivoluzioni e metamorfofi dallo fteffo fofferte, apparifce talmente chiaro agli occhj degli intendenti Offervatori, che moltiflimi tra gli antichi, e moderni Filofof, fonofi applicati ad ifpiegare, con ingegnofe, e talvolta anche con affurde ipotefi, le caufe produttrici dei tanti, e si diverfi fenomeni, ch' effo nella fua fabbrica ci prefenta.

Negli ultimi trafcorfi fecoli pero, e nel corrente, la Teoria fifica del Terraqueo Pianeta ha, molto più che

(1) Trovan/s inferite nel Giornale d' Italia, fampato dal Librajo Milocso, in Venezia, dal T. I. fino al IX; ec. 
che in altri tempi, occupati $\mathrm{i}$ Fifici : quafi in ogni fua parte fonofi fatte penofe ricerche, e diligenti offervazio$\mathrm{ni}$ : molto fi è meditato, e tutt' ora $f_{1}$ ftudia per indovinare le caufe effettrici della prefente fua forma; e numerofi Trattati, Differtazioni, e Memorie fono ftate pubblicate fopra così ofcuro, e difficile argomento.

L'erudito Orittologo Elia Bertrand ha confiderato poterfi riferire a tre Claffi le ipotefi, che fono ftate immaginate per rendere ragione della riformata ftruttura del noftro Mondo (I):

Alla prima egli riferife le fuppofizioni di una riformazione della Terra fopra le ruine della primeva . Dice che Bernardo Paliffe, o della Paliffe, credefi autore di fimile ipotefi, che Tomaso Burnet nella fua opera intitolata Theoria Telluris Jacra, efpofe fiftematicamente, trattane l'idea da Platone, dal quale l'aveva già prefa Francefco Patrizio.

Nella feconda comprende li fentimenti di quelli, che gli accennati effetti actribuifcono, chi al lungo foggiorno dell' Acqua fopra l'intera faccia del Globo, ed al fubitaneo, o lento abbaffamento della medefima; chi al giro fucceffivo del mare intorno allo fteffo; e chi a detto univerfale inondamento, ed infieme alla caduta in parte del primo Mondo ; all' ignea fufione; ai Vulcani ec. (2). Indica, che tali penfamenti íno ftati diverfamente efpofti da Leibnitz, da Vallifneri, da Jufieu, da Reummur, da Celfius, da Maillet, e Buffon; come pu-

re

(1) Memoires fur la fruture interienire de la Terre, a Zuric 1760.

(2) Bcrrand, dove parla di detro feconda Clape, non Specifica né fufone, nd Pulcani, ma envano peró in diverfi dei fiftemi, cb'egli nella inedefina comprende. 
re da Stenone, Wbifton, Hallcy, Harftoeker, Buttner, Gautiev, e dal $P$. Caftel. Tra quefti conviene annumerare Agoftino Scilla (1), Fabio Colonna (2), A. Lazzaro Movo (3), Giovan Giacomo Spada (4), Giovanni Gejnero (5), Giovan Gottlob Lebmann (6), Micbele Lomonofow (7), Giovan Battifta Pafferi (8), Jacopo Odoardi (9), Giovami Targioni Tozzetti(10), Giufeppe BaldafJarri.(1 I), e moltiffimi altri dotti uomini, che lungo farebbe di nominare. Le idee di primitiva fufione, di fotterranei pofteriori incendj, di vulcaniche eruttazioni, di follevamenti, di avvallazioni, rovine, e fubbiffamenti fono tate da molti Geologi combinate in diverfi modi con quelle di univerfale, e di parziali innondazioni.

(1) e (2) De corporibus marinis lapidefcentibus ec. Auctore Auguftno Scilla, addita Differtatione Fabii Columne de Gloffopetris. Rome. 1759.

(3) De' Croftacei; e degli altri Marini Corpi, cbe fi trovano fu' monti. Di Antcn Lazzaro Moro. Venezic, 1740:

(4) Differtazione, ove fi prova, che li petrificati corpi narini, che mei monti adjacenti a Verona $f_{2}$ trovano, non fono fcherzi di natura, ne diluviani, ma antediliviani. Verona' $1737^{\circ}$

Giunta a detta differtáione. Verona 1737.

Corporzm lapidefactorum Agri Veronen/is Catalogts, Ec. Verone i 744 .

(5) Johannis Gefneri Ec. Tractarus plyyficus de Petrificatis. Lugduni Batavorum, $175^{8}$.

(6) Effai d une Hifoire waturelle des Couches de la terre Éc. a Paris 1759 .

Specimen Orograpbice generalis Ece. Petropoli 1762 .

(7). Oratio de generatione metallorm a terre'motu. Petropoli $1757^{\circ}$

(8) Dell' Iftoria de' Foffiti del Pefarefe, 'cc.

Nuova Raccolta di Opufcoli ec. del P. A. Calogera T. V.

(9) De corpi marini, che nell' Feltrino diftretto fi trovano nel T. VIII. di. detta Raccolta.

(10) Relazioni di alcuni viaggi, Ecc. T. VI. Firenze 1752. ec.

(11) Delle Acque di Cbianciano. Siena 1756 .

Saggio di offervazioni intorno ad alcuni prodoti naturali ec. Negli Atti dell" Accademia delle Scienze di Sicni. T. II. 
La conofciuta grande analogia di una parte dei Terreftri materiali con quelli de moderni Vulcani, e dell' altra parte colle note produzioni, pietrificamenti, e ftratificazioni del mare, tanto nelle effenziali rifpettive loro proprietà, che nei caratteriftici loro fenomeni, ̀̀ il fondamento, fu cui. li fiftemi di quefto genere fono appoggiati.

L' Ipotefi della terza Claffe è di Giovami Woodzard, il quale immaginò una totale diffoluzione del primo Mondo, per ifpiegare il Diluvio, e la riproduzione della Terra nella prefente fua forma . Gio: Jacopo. Scbeuczer, Monti, Bourguet, Tommafo Gabrini (1), e tutti quelli, che ad un tale avvenimento hanno attribuita la formazione degli ftrati Terreftri, ed i Prodotti Marini, che nei monti fi trovano, alla fteffa Claffe poffono riferirfi.

Emanuele Swedemborgio, celebre per le fue opere Filofofiche, e Metallurgiche, perfuafo dalle offervazioni, che la forma della Terra fra l' effetto di diverfe fucceffive viciflitudini, cosi fi efpreffe: Regnum minerale eft.effigies viciffitudinum circa formationem Telluris exiftentium; ubicumque penetratur Tellus, documentis abundantifima patet: fs ad jpfun Cimeliarcbam Plutonem o tartara ejus ives, nullibi in via quid finillimum invenies; fed feimper aliquid novum o diverfum, \& quod nova o diverfa; tot viciffrudinum indicia (2). Anche il celeberrimo Linneo, che la ftratificata flruttura della. Terra, giudica effere lavoro G $g$ dell'

(1) Della fuccefiva prodizione de' monti. Nuova Raccolta di OpufioIi ec. del P. A. Colegcrin.

(a) Emanuclis Suedemborgj Opera Pbilefofica \& minialia T.primus

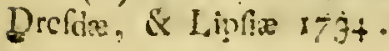


dell': Oceano, cioè prodotta con fucceflivi fuoi fedimenti; dopo averne indicati $\mathrm{i}$ modi, conclude : ideo \& Rupes faxeas altiffanas cevi veros flios effe dum omnia obmutriere, ipfi loguantur lapides. Pofcia egli replica : Jaxa non primava, fed temporis flios effe, abunde evincunt frata montium: ed a propofito dei marini Teftacei, che dentro le pietre de monti veggonfi impaltati, e fepolti, fi efprime: ubi Teftacea, of Litbopbyta folfilia exiftunt in magna copia, ibi quondam fuere maris littora, o abyfjus, cum fint mera veftigia maris, omni biftoria antiquiora, Diluvium vero non demonfrant, fed tantum longioris revi rudera (I). Il confentimento di tanti Filofofi fapienti, e rifchiarati dalle offervazioni, nel credere non primitiva l'odierna forma del Regno foffile; ma per fucceffione di tempo, e con diverfità di avvenimenti, e di modi riprodotta, d'altronde non deriva certamente, che dall' evidenza rifultante daifatti, e fenomeni della natura, e dalli caratteri, co' quali ha effa marcate le fue produzioni.

L'autorità, e l' efempio di quelli, che mi precedettero, $m$ 'accefe, fino dalla prima gioventù, di defiderio d'iftruirmi praticamente nella Fifica fotterranea, e di confrontare i loro fentimenti con ciò, che la natura fteffa ci moftra nei diverfiffimi fuoi mirabili effetti - Le occafioni, che ho avute, di vifitare varie montagne minerali; di dimorarvi lungo tempo; d'internarmi frequentemente dentro efcavazioni, anche profondiffime, di minere; di dirigerne in diverfi luoghi, e per più anni; di efperimentarle chimicamente, e di trattarle nelle grandi fufioni;

(1) Caroli a Linnè, EC. Syft.nat. T. Bertiks. Hohnize 1768. 
fioni; furono circoftanze favorevoli, e combinazioni, alli naturalifti rariffine, che hanno fecondata la mia curiofità. Quanto piùil numero delle mie of?ervazioni fi $\grave{c}$ andato aumentando, tanto maggiormente fono reftato convinto che le materie, e parti coftituenti la croAta frutabile di quefto Globo, tutte in fatti non fono d'una ftefla data ; nè dipendenti da una medefima cagione; ma diverfe di tempo, e di caufe Le offervabiliflime differenze, che certi tratti montuofi da cert' altri diftinguono; tanto in riguardo alla loro ftruttura, ed alla forma effenziale, e fpecifical de' loro materiali, che ai modi, co' quali gli uni fono relativamente agli altri collocati, lo danno efpreffamente a conofcere.

Molte congerie di monti, e catene, anche vaftiffime di mortigne veggonfi compolte di pietre quarzofe, filicee, talcofe, fteatitiche, argillofe, micacee ec; tutte vitrefcenti, e da pyrofi apparentemente originate, e onninamente prive di reliquie d'efferi organici Terreftri, e Marini, de' quali le altre parti montuofe, di natura da quefte diverfé, fono in moltiflimi fiti ripiené. I luoghi, chefle occupano, ed i loro modi di alzarfi dall imo della Terra, e di emergere dal diffotto dei monti di altra natura, fembrano chiari caratteri di primogenita origine. Effe fono le fole, che a rigore, confidero le parti coftituenti l'ordine primario: e perciocchè fono le matrici più comuni dei metalli; e minerali, dei Criftalli, di molte Gemme, e di moltiflime altre produzioni foffili ; io foglio nominarle indiftintamente, ora montagne primitive, ora montagne minerali, o metalliche.

$$
\text { G g } 2
$$


Altri montuofi tratti fono mifti, cioè compolti di materie vitrefcenti, e di calcarie, marnofe ec; difpofte in alcuni luoghi a ftrati; ora feparate le calcinofe dalle vetrificabili; or 'l' une all' altre alternatamente foprappofte; or infieme confufamente mefcolate. In alcune altre fituazioni veggonfi maffi di pietre calcinabili, e. fcogli, anche d'infigni grandezze, irregolarmente fparfi tra materiali di vetrofa natura; talvolta dentro li medefimi immerfi tal' altra lateralmente agli fteficongiunti, o fopraggiacenti .

In quelli di tali monti, che a ftrati fono formati, trovanfi, benchè molto di rado alcune fpecie di Teftacei, di Pefci, di Vegetabili, e di, altre foftanze al Regno minerale non proprie, ma d'altronde derivate.

Siffatti monti, tutti infieme confiderati, effere non fogliono molto atti, nè di grandiffime eftenfioni; ma - formano delle ferie ifolate tra quelli di altra natura; o tramezzano; o fafciano a qualche lato le fuddette prime montagne, fopraftando a' loro piedi e lati, dove feco fi congiungono. Tale fituazione, e la difformità di natura de' loro componenti, ed i fenomeni, clie vi fi offervano, fembrano indizj, che formati fi fieno, efftenti le prime montagne; del che dubbio alcuno non lafcianci quelli a ftrati coftrutti . Con tutto quefto però, effendo effi, come le dette montagne, le matrici e ricettacoli di minere metailliche ec:, ed avendo colle medefime montagne molti rapporti, io gli ho nello fteffo ordine primitivo compleffivamente collocati: locchè principalmente ho fatto in rifleffo della metallurgica, da me prediletta, e per molti anni efercitata. Altri 
Altri monti poi, e lunghiffime e valte catene di Alpi quali d'altro non fono formate, che di marmi, e pietre calcarie a frati fopra ftrati, tra fe cor molta regolarità paralleli; fe fe n' eccettuino certe Lave, ed altre bruciate materie, eruttate da antichiflini fpenti vulcani, tra le fquarciature, e crateri, che tratto tratto vi fi fcuoprono, e le Breccie, le rotture, e fconvolgimenti dalli medefimi caufati . Le quifquiglie.e gufcj di marini Teftacei, di note ed ignote fpecie., vi fono frequenti, e fpeffo in quantità grandifima $e$ forprendente.

Quefte montagne, che, coll' efpertifimo Orittografo Signor Gio: Giacomo Ferber mio cordiale Amico, nominerò Calcarie fratofe, fafciano quafi tutto all' intorno quelle del fopradetto ordine primitivo, fopra le radici, e lati delle quali, dove fono feco congiunte, veggonfi effere appoggiate, ed anche talvolta fopra le loro baffe, o mediocri elevatezze. Effe hanno colpicui caratteri, pe'quali s'abbiano a riconofcere per vere tiglie dell'antico Oceano, nate pofteriormente alle anzidette, ed in ftato, e circoftanze di cofe totalmente diverfe: e quindi fecondarie le ho giudicate.

Hannovi finalmente numerofiflimi baffi monti, $e$ colline, parimente a ftrati, ma compofti di fabbie calcinabili, di arene vitrefcenti, di ghiaje, di argille, di crete, di marne, di ciottoli da lunghi movinenti delle Acque rotondati, e d'altre fimili materie; dove più o meno rimpietrite; dove di tofacea confiftenza, dove ancora fciolte. Immenfa è la quantità, e quafi innumerabili fono le fpecie di Teftacei, di Croftacei, e di Zoofiti Pelagici, che interi, e franti, ed anche 
in minuti trucioli, e polvere, vi fono preffochè fenpre mefcolati, e fepolti.

Molto quefti tali monti, e Colli differifcono dalle fuddette montagne primitive, e fecondarie, $i$ rottami delle quali entrano fpeffiflimo nella loro compofizione. Bafta bene offervarli, per conofcere che in confronto delle medefime, effi fono opere del Mare molto meno antiche, coftrutte con ftratificate depofizioni d'innumerabili eterogenee materie, fortuitamente aggregate, in modi affatto fimili a quelli de' moderni perenni fuoi lavori .

Per quefti rifleffi, e perchè coftantemente fi ravvifano fopraggiacenti alli materiali coftituenti le parti fecondarie della Terra, gli ho tutti, benchè di differentiffime qualità effi liano, nell' ordine terziario comprefi, infieme colle materie Vulcaniche, che in quelli almeno delle noftre Provincie, fono frequenti, ed in certi luoghi abbondantiflime a fegno di coftituire dei tratti non piccioli di bruciati Poggi.

Rifpetto poi alle Pianure ; cTendo evidentemente formate dalle alluvioni delle acque, difcendenti da tanti fecoli, giù dalle parti montuofe, colle materie faffofe e terree dalle medefime condotte, triturate, e fparfe; ed in parte anche dal Mare colle fabbie, e materie limofe ec; da eflo di continuo refpinte ai lidi, e dentro le falfe Paludi, e fopra le terre baffe, che frequentemente inonda, depofitate; ho delle fteffe un quarto ordine divifato.

Ma ritornando ai monti primitivi, ficcome per quanto ho potuto offervare in diverfe Provincie del Tirolo, di quefto ftato, e della Tofcana ec., ho coftan- 
temente veduto, che lo Schifto rupeftre, o montagno. fo (Berg-Scbifer), compofto di Quarzo, e di Mica, o Talco, e non arenofo, occupa, confiderando la fua derivazione dall' imo della Terra, il fito piu profondo, rifpetto agli altri materiali componenti li medefimi monti, fenza che alcuno di altro genere fi faccia vedere allo fteflo inferiore; così ho creduto di poterlo ragionevolmente confiderare primigenio, o uno almeno tra li pietrofi generi primigenj; cioè di origine più antica relativamente agli altri tutti, che all efame degli occhj noftri fi prefentano, e che d'effere fuperiormente fituati manifeltano.

Tale fu, come ho accennato, il mio. Orografico divifamento, fondato fopra le mie offervazioni, e fopra le notizie alle fteffe conformi, datemi da foggetti di fimili cofe efperti, che molto più lungi hanno eftefe le loro indagini. Ma benchè mi lufinghi di avere a fufficienza fpiegate nelle fopraccitate mie Memorie, le ragioni, per le quali mi fono a così penfare indotto; piacemi non per tanto di nuovamente parlarne, ad oggetto di aggiugnere qualche maggiore precifione, e chiarezza alle idee allora efpofte.

Il gufto, che domina oggidi, e : che va fempre piu aumentandofi in tutta Europa, ed in altre Parti per l'Orittografia, per l'Orogenesìa, e generalmente per la conofcenza degli efferi, e Fenomeni del fotterraneo Impero; e la confiderazione dei lumi importantiffimi, che fiffatti Atudj apportano alla Filofofia, ed all' Arte metallurgica; come altresi gli eccitamenti datimi da diverfi Orittologi miei Amici, fono morivi, che a difcutere nuovamente quefto argomento m'indu- 
cono. La contemplazione della natura, e le cognizioni, che quindi derivano, non fono certamente inutili: occafonem fubminiftraie queunt, aliis tacentibus, in prifca tempora retrospiciendi, ac formam terre antiquam, ejus incrementum of metamorplofin confsiderandi (1). Effe fono il fondamento della fcienza minerale, dell' Arte metallica di tanto profitto a moltiffimi Stati, e di tanto bifogno, e comodo all' Umana Società . Molti, per effere ftati privi di tali cognizioni, fonofi dannofamente ingannati, o cercando con grandi fpefe vene metalliche, e di altri Folfiti di pregio, in quelle qualità di monti, che dagli intendenti fe ne conofcono affatto fterili; o ufcendo di ftrada nell' infeguire le vene icoperte, per non conofcere le traccie dei loro canali, e le materie, che loro fervono di matrici; o defiftendo dal lavoro all' incontro di qualche femplice interrompimento dei filoni; oppure oftinandofi a profeguire gli efcavamenti dentro quelle certe fpecie di pietre, e di terre, che non di rado le vene minerali troncano, e terminano totalmente.

Egli è ben vero, che nelle indagini, e nei lavori delle minere, mancano non di rado di buon fucceffo i temativi anche delli piu éperti nella conofenza de' Foffili, e nella fotterranea Geografia; non effendo.gli efterni indizj ficura norma per indovinare con certezza le qualità, le quantità, e'l' eftenfioni dei minerali, che la Natura ha nelle vifcere de' monti occultati. Accade fpeffo di fcoprire nella fuperficie, o poco fotto alla corteccia de'monti, vene abbondanti delle

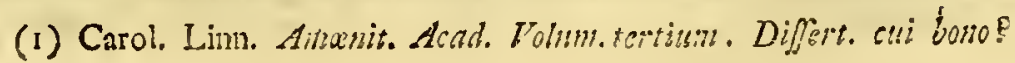


delle foftanze, che da' Metallurgifti fi cercano; ma che prefto, internandovi gli efcavamenti, veggonfi, fvanire, o divenir fterili, o di mefchina fottigliezza; oppure tra maff durifimi inçaffate, o tra materie frali e rovinofe, che neceffiterebbono ad incontrare troppo ecceftivi e dannofi difpendj . Poffono anche incontrarfi nel profeguimento, acque interne talmente copiofe da non poterfi fuperare, o che il farlo non poffa riufcire vantaggiofo. Certe fpecie di pietre e di altri, Folfili non metalliferi; quantunque della loro efitenza non diano efternamente alcun forpetto, troncano talvolta e terminano totalmente i filoni; e diverfi altri imprevifibili accidenti riducono vane le ragionevolmente concepite $\mathrm{pe}$ ranze. In fomma la fcienza minerale è analoga né fuoi pronoftici alla fcienza medica : dovendo arguire da' $\mathrm{Ce}$ gni efterni le cofe occulte nel gran corpo delle montagne, va, come quella, rifpetto al corpo animale, foggetta inevitabilmente ad errori. Li numerof infelici efempi, anche de'luoghi, nei quali la Metallurgia da piú fecoli, e con grande ftudio, e folerzia fi coltiva, troppo evidentemente lo provano.

Con tutto ciò evii moltiffma diftanza dal Metallurgo guidato da una pratica rifchiarata dai lumi fomminiftrati dall' Orografia fifica, e dalla mineralogì, a quello che quette fienze ignora. L'uno dall' altro differif́ce, come il Medico nella fua facoltà eccellente dall' Enpirico ignorante: il primo pronoftica, e fi determina, in ogni particolar cafo, fcortato dalle cognizioni, tratte da' migliori fonti della Medicina, e dalle pratiche offervazioni,; ed il fecondo opina, ed opera fempre a cafo: e fortuna fe non fempre a fipropofito $\mathrm{H} \mathrm{h}$

$\mathrm{Ma}$ 
Ma entrándo nell' argonento, che di trattare mi fono propofto, dividerò il mio difcorfo in tre diftinti Articoli j diffondendomi principalmente fopra ciò; che alle montuofe parti, compréle nélla prinäria mia divifione, appartiene: sì perchè effe forio lè vere native fedi de' metalli, e degli altri minerali, e Folfili piu preżiófi: sì anché perchè di quelle fpettanti alle altre due divifioni, ho date, a quello che mi fembra, indicazioni baftevoli e qüi fopra, é nelle accénnate Memorie. Nel primo Articolo indichero li generali materiali, e caratteri, e prodotti foflili di dette primitive parti della Terreftre fuperficie : nel fecondo mi farồ a fpiegare, quantó faprò-meglio, quelli del genère di pietre fchiftofe ec.; cui diedi il nome di primigenie, ed in che propriamente lo Ateffo dágli altrí genéri differifca : e nél terzo éforrò alcune inie ófervazioni fopra la matrice fchiftófá, nera, bituminofa della minera di Argento vivo d'Idria nella Carniola, e varie rifleffioni fópra la medefima, e fopra gli altri Foflili di Bitume partecipi, ad oggetto di dar a conofcere le ragioni, per le quali io non fo perfuadermi che fiffatti materiali fiano da annoverarfi tra quelli di primogenita origine.

\section{A R T I COLO I.}

T Ell Ordine, o Divifione, de nionti primitivi, io 1 ne comprendo, ccme ho precedentemente accennato di due generali qualità, in relazione ai tempi , ed alle Cáufe fifiche della loro formazione; e percio confludero l'Ordine iftefto in due Serie fuddivifo; cioè in quelle montagne, che impreffi portano caratteriftici indizj 
indizj di primaria antichità, e di pblogofica origine; $e$ certi altri monti, apparentemente di coftruzione alle medefime montagne fucceffiva, e dipendente in parte anche dall' acqueo Elemento; ma pero alle prime analoghi per molti rapporti, e da'monti dell' ordine feconir dario, e terziario molto diverfi, e cofpicuamente più antichi.

Parlando delle prime montagne, ho già detto che effe fono formate: di pietre, e terre vitrefcenti : qualità, che fenza voler qui entrare in chimiche difcuifioni, dichiaro attribuire a tutte le lapidofe, e terree materrie, che non fono d'indole calcaria. Gli Schifti cornei, o fia talcofo-quarzofi, e li femplicemente micacei, e gli argillofi; li Graniti, e Granitelli, e Porfidi, Serpentini, Diafpri Rupeftri, Selci Cornee, e quarzole ; e certi Bafalti, e Trapp (I), e Pietre argillofe, fteatitiche, e talcofe, ed altri diverfi Foffili d' indole vitrefcente; fono li materiali coftituenti tali montagne. Le medefime occupano talvolta riftrette eftenfioni; ma più fovente grandi, ed anche vaftiflimi tratti : e confiderandole a parte a parte, alcune veggonfi formate di un genere unico delle pietre teftè nominate; altre di due, o di tre, e altre di molti, in differenti modi'gli uni, rifpetto agli altri, difpofti, e modificati.

La natura effenzialmente vetrofa di fiffatte materie; le bizzarre forme de' loro impafti, delle loro difpofizioni, venature, e sfendimenti; la totale affenza di reliquie di Corpi organici; e l'interna ftruttura de' loro aggregati, fpeffiflimo in ftrane guife confufa, $e$ $\mathrm{H} \mathrm{h} 2$ fenza

(1) Cronfted. IMineralgg. 
fenza alcuna regolare fimetria di frati; e dove ftratofa fi moftra; da quella dei monti calcarj molto diverfa; fono fenomeni, che agli efperti, e non pregiudicati. Ofervatori prefentano idea, non affatto ofcura, di fofferta fufione, di tumultuarj bollimenti, di accidentali mefcuglj, e di fconcerti accaduti in tempi varj, ed a quelli de' moderni Vulcani non poco fomiglianti. Le vene metalliche, e degli altri minerali, che di frequente in tali montagne efiftono, vi fi trovano comunemente difpofte a filoni, e talvolta a maffe, ed a cumuli di varie grandezze, dentro ampie fenditure, or verticali, ora in varie guife inclinate; le quali diconfi Canali, e Condotti delle minere : e che fono i nidi, e recipienti, o matrici, dentro alle quali tali foftanze fonofi generate, $o$ almeno in folidi aggregati congiunte.

Quefti fono in fuccinto li caratteri generali, che le montagne dell' anzi detta prima fuddivifione, o qualità dalle altre diftinguono. Il celebre Lclmann (r) le ha chiamate montagne a filoni, e le ha egli pure giudicate le piu antiche; indicando anche le fpecie di minerali, che alle medefime in proprio appartengono.

Rifpetto poi alla feconda fuddivifione, o qualità di monti, da me nell' ordine primario comprefa; ho già dichiarato che quantunque abbian effi diverfi rapporti di analogía colle prime montagne; ciò non pertanto, dal vederfi che dove alle fteffe fono congiunti, fopraggiacciono a'loro lati; e profonde radici ; $c$ clie

(1) Effai d" une Hifoire naturelle dies Coucbes de la Terre ec. De Monfieasr Jean-Gotdob Lchmann. P'aris 1759 . 
che dalle medefime notabilmente differifcono per ftruttura, e mifta origine, e per altri fenonieni f fo haindi fondato argomento di crederli meno antichi. Ho anche accennato di averli comprefi nell' Ordine primitivo in rifleffo precipuamente alla metallurgia: perciocchè, contenendo effi pure di frequente varie minere metalliche, e di altri generi, non m è piaciuto di diftinguere in due ordini la ferie, o Claffe generale delle montagne minerali, o fra metalliche.

La compofizione, e ftruttura di tali monti, e le differenze di natura, e di fenomeni, che tra $i$ loro materiali fi offervano, fembrano chiari indizj di mifta formazione, rifultata da Vulcanici, e pelagici effetti; dove difgiunti; dove in varie guife alternati ; dove fenza ordine bizzarraniente confufi. Alcune parti di effi monti fono compofte di pietre, e terre vitrefcenti, in modi analoghi a quelli delle fopradette prime montagne, e fenza forma Atratofa! Altre fono fabbricate a Atrati fopra Atrati tra le paralleli; ora di un folo genere di pietre, o di terre variamente indurite, ora di più generi. Le pietre arenareo-micace, le calcarie le argillacee, e marnofe, gli Schifti argillof, limol, e di altre fimili qualità, e le terre variamente colorate, e di diverfa natura, e confiftenza, fono, compleffivamente parlando, le Coftanze, delle quali effi ftrati fono formati La Aratificata regolare difpofizione di tali materie, li vegetabili, e Pefci, che ridotti bituminofi, o mineralizzati ec., in qualche luogo, benchè raro, vi fi trovano dentro confufamente fparfi, e le marine pietrificate Conchiglie, che negli ftrati, particolarmente di pietre calcarie, più di frequente apparifcono, i- la- 
vori dell antico mare ci moftrano. Li confufi ammaffi di materiali d'indóle vetrofa, de' quali molti di fiffatti monti fono compofti, or interamente, or nelle fole loro radici, e baffe parti, ed inferiormente alle porzioni Atratofe; ora fuperiormente alle medefime, e che anche tramezzano talvolta gli Arati, e riempiono certe verticali larghe fenditure, difgiunzioni, e rompimenti delli tratti ftratificatamente coltrutti, portano impreffi, nelle ftrane loro modificazioni, e fenomeni, li caratteri, benchè talvolta ofcuri, della plologofica violenta forza, da cui la loro formazione verifimilmente deriva. I maffi, e fcogli di varie accidentali figure, e grandezze, rotti, e ftaccati dalle montagne calcinofe fecondarie, alli monti Ateffi contigue, che in diverfi luoghi vi fi veggono quà e là fparfi fenza ordine alcuno, ed immerfi con Atrane, e non originarie pofizioni, dentro i loro vitrefcenti materiali, fono fegni molto meno equivoci del Vulcanico concorfo alla loro conftruzione.

Da quanto ho premeffo, in aggiunta a ciò che fu quefto argomento efpjif nelle fopra menzionate Memorie, $m i$ lufingo fia per riufcire meno difficile agli Offervatori il comprendere quali delle parti montuofe del Terreftre Globo frano precifamente quelle da me all' ordine primitivo, o primario affegnate, e che foglio anche nominare monti, o montagne minerali, o. metalliche.

Che che fia della fuppotta, e forfe anche con qualche ragionevolezza provata loro anzianità di formazione, e delle loro caufe produttrici; egli èperò al. meno vifibilmente certo ch' effe molto fono diverfe da quel- 
quelle calcarie a ftrati, quafi fempre regolari, e molto uniformi, e fpefflfimo ripièni di pietrificati marini Teftacei ec., da me nell'ordine fecondario collocate ; é piú ancorá da quelle del terzó ordiné . Impérciocchè enormemente re differifcono; si in riguardo alla natira ; ed alle proprieta fpecifiche dè loro componenti materiali, é ágl' infinitamente variati loro inodificámenti, e fenomeni, si anche rifpetto alle tante foecie di metalli, di minerali, e di altri Foffili, che dentro le medefime, e nón nellè rimanénti, nativamente efiftono. Effe fono per quanto $\mathrm{mi}$ è noto, le fole; nelle quali poffóno $\mathrm{i}$ Metallurgi occuparfi con fucceffo néllé indagini, e nelle efcavażioni delle minere. Nétratti montuof, che à fécondo te térzo ordiné appảrtengòno, farebbono inutili le liro ricerche, edañón li tentativi, ad eccezione però di quélli riguardanti certé fpecie affai comuni di minerali ferriferi, e piritof, ed i Carboni Folfit:, e altre bituminofe materie, ed i niarmi; êrie altre forti di pietre calcarie, e di fabbie e di terre argillofe, margacee, eretofe ec., atte a diverfi ufi, e lavori Le ininere d'Oro, di Argento, di Rame ; di Stagno, di Plombo, e di Argento vivo, Bifmuto, Zinco, Antimonio, Cobalto, Arfeñico, e altre diverfe particolari fpecie di Minerali, e di Forfili, appartengono in proprio, é originalmente älle fuddette primitive parti del Regno fotterraneo Il Ferro iftefo, quäntunque fotto innumerabili fembianti metamorfolato; tròvifi commifto; e fparfo nella maffma parte delle terrè, e delle pietrè, sì dé mónti, chê delle pianure ec; ha nelle medefime parti anzidette le principali, e veramente native, é più formofe, e piü 
ricche fue vene: e vi fi trova fpeffo in molta copia; fino a formare talvolta filoni, e maffi grandiflini, ed in qualche raro luogo degl interi monti .

Importa dunque moltifimo a chi f applica alla vera Mineralogia, ed all' Arte metallurgica, di ben conofcere, e di fapere precifamente diftinguere quelle porzioni della Terreftre corteccia, ch io nomino primitive, e minerali, da quelle di altre qualità Efre confiderate in pieno, fono $i$ nafondigli de piu ricchi, e più ricercati tefori del Plutonico Impero; ma le caufe, effettrici, che le hanno cosi bizzarramente, come ho accennato, edificate, non furono nella produzione, e diftribuzione de' medefimi molto più metodiche. Egli è in ciò particolarmente, dove il celebre Woodward ebbe molta ragione di dire che nel Regno minerale niente v? ì di certo, e di regolare.

Numerofifimi fono i monti, che quantunque, in riguardo alle materie, delle quali fono compofti, all' ordine primario, e minerale appartengano, mancano non pertanto di quegli efterni indizj, da' quali argomentano i pratici lefiftenza d interne minere ed al contrario, diverfi altri, alli medefrmi fimili, ne fono, or più, ora meno copiofi. Certi metalli a minerali fono nel Mondo rari, ed in poche Regioni folamente fonof finora foperti altri a naggior numero di paefi fono co. muni ; red altri allai volgari, dove monti minerali efr ftono. Le minerali produzioni, ora fono alla fuperficie proflime, ora piu o meno profonde, or raccolte, e continue; o poco interrotte; ora fparfe, ed erranti nelle loro vene, o ne' pietrofi maffi, o tra certi, Folfili, $e$ terrofe materie di generi particolari.

Effe 
Efre trovanfi d'ordinario incaflate tra grandi fenditure verticali, o variamente declivi dei monti, le quali fono delle minere metalliche ; $!$ e di altri generi li più comuni ricettacoli : :e quando ne contengono in poca, o molta quantità, oppure almeno di quelle fpecie di Foffili, che di tali futtanze fogliono effere in. dizj, nomanfi condotci, canali, o vene minerali; c filoni fi dicono li continuati aggregati delle materie nelle fteffe vene rinchiufe. Daffi anche, benchè impropriamente; il nome di vena a qualunque forta di filoni; e. aggiugnendofi a ciafcuno quello dei materiali, de'quali è compofto, fe ne fpecifica la qualitä. Ufanf pure, e dicevolmente, le generali diftinzioni di vene, o filoni fterili, fe fuftanze di pregio non comprendono; di fecondi, fe ne contengono; di poveri, fe ne fono rcarfi; di ricchi fe ne abbondano; ed altre fimili, alle particolari qualità de' medefrmi adattate.

Indicibili fono le diverfità foftanziali, ed i feno. meni, che! nelle vene fuddette fi offervano. In certi monti fono folitarie; in altri o doppie, o moltiplici. Talvolta, dove ve n' ha più d'una, confervano quafi paralleli andamenti; tal altra in varie guife o s'incrocicchiano; o convergendo fi unifcono, o dividendofi, in due, o più parti $f_{1}$ diramano. Nelle loro eftenfioni verfo l'Orizzonte, e verfo il profondo della Terra, alcune fono a un di preffo rette, o poco fleffuole; altre tortuofe, oppure angolofe. Ve ne fono di continuate, d'interrotte, ed anche di allontanate, per via, dalla prima direzione, per slogamenti primordiali, o almeno antichiffimi, accaduti tra parti, e parti delle montagne. Molse dall' alto de'monti alle profonde lo- 
ro radici difcendono tra pietre di un iftefo genere; e non poche tra due, o piu generi di materiali fi veggono eftefe: ma tante fono le varietà di dette vene minerali, che il volerle tutte accennare farebbe un vano tentativo.

Rifpetto alle foltanze coftituenti li filoni, fono fenza numero le loro differenze, irregolarità, e cangiamento; non folo da monte a monte, o da filone: a filone, ma anche da fito a fito, identro una medefima vena. Moltiffimi fe ne veggono, che quafi d'altro non fono formati, che di Quarzo, o di Spati, o di Manganefe, o di Selci Cornee, Diafprine ec. oppure di Piriti, o di altre fpecie di Folfili inutili, o di poco pregio. Avvene purein grande quantità di quelli compoAti di due, o di piü fpecie di dette materie; e talvolta anche di tutte infieme, in modi diverfifimi mefcolate e congiunte. Anche le foftanze metalliche, quaf fempre dallo Zolfo, o dall' Arfenico; o da ambi infieme mineralizzate, trovanfi nelle loro:vene, per: lo: più, con tali Föflili confufe; e non coftituifcono forfe mai filoni, che ne frano totalmente privi : anzi fpeffifimo entro vi fono a guifa di glandule; o minutamente, e come a fpruzzi diffeminate, e fparfe, oppure a sfoglie, e falde, e maffe d'indeterminabili figure, éftenzioni, e groffezze.

Le accennate non fono peró le fole irregolarità e diffiniglianze, che parlando delle vene metallifere, nelle loro interne parti, fi fcuoprono. Li metalli, $c$ l'altre fuftanze a' medefimi affini, 'non fono in ogni fito delle loro eftenfioni uniformemente diftribuite: in alcuni tratti ne fono ripiene, in altri più, o meno fcarfe, ed in altri onninamente prive. Qualche volta 
vi domina un folo mineralizzato metallo; ma più fovente due, o tre; o molti infieme, fotto diverfe modificazioni, e con dofi varie, or intimamente commi$\mathrm{Ati}$; ora in diftinti aggregati congiunti; dove con forme fpecifiche di ogni particolar mifto criftallizzati, dove fenza alcuna determinata fimetria delle rifpettive integranti molecole concreti.

Non è raro a vederfi, che venendo a cangiarfi di qualitä le pietre, o terrofe materie dei monti, tra le quali le vene minerali fr eftendono, variano di pari le fpecie de' metalli, :e dell'altre foftanze, che feco loro li filoni compongono, o che li filoni ftefl divengono fterili, oppure in Foffili inutili fi trasformano; e riduconf anche ralvolta a fole of cure traccic, io fvanilcono, e terminano interamente.

Non ferbano inoltre le vené nuinerali alcun ordine coftante nelle loro direzioni, nè rifpetto all' Orizzonte, trovandofene di ditette ad ogni Plaga del Mondoll'nè in riguardo al céntro della Terra effendovene d'inclinate per ogni verfo co'loro profondamenti. Meno pure ne ferbano nelle loro dimenfioni di groffezze; largliezze; e profondità; perciocchè: ve ne fono di efilifi fime, di molto groffe, e di ogni grado intermedio: di longitudinalmente, rifpetto all' Orizzonte, poco eftefe; e di vifibilmente prolungate a grandi eftenfioni : di quelle che giu per le vifcere delle montagne poco sinternano; altre chetdifcendono la profóndita inarrivabilio

Le fuftanze minerali, sì metalliche, éhe di altri generi, non fempre giacciono dentro $i$ monti fotto la forma di filoni. Efle vi fono anche fovente a pezzi, ed a modo di arnioni; cioè a mafte affettanti globofe fiI i.: $2 \cdot 3$. wa. gure, 
gure, tra diverfe fpecie di Foffili avviluppate. Vi fi trovano pure raccolte a cumuli, talvolta grandiflimi, nell' interno de'canali, fpecialmente dove piu vene confluifcono; o fuori de' medefimi, in certe nicchie, e ripoftigli ; oppure in particole di varie gróflezze difeminate $e$ fparfe entro diverfe terree, e lapidofe materie, coftituenti non già filoni, ma porzioni di monti.

Quanto ho detto delle minere, appartiene peculiarmente a quelle efiftenti nelle montagne, che apparenza non' hanno di origine marina, le vene delle quali dall' alto al profondo fi eftendono; nominate perciò dall' efimio Orittologo Giorgio, Agricola vene profonde quelle a filoni; e vene cumulate quelle a grandi congerie ammaffate (1). Le minere di que'monti Aratificatamente formati, de' quali, come di dette montagne, ho già data qualche idea, fogliono avere direzioni per ogni verfo orizzontali, o non moltiffimo all' orizzonte: inclinate, e ferbare li medefimi andamenti de' pietrof, o. terrei Atrati, tra quali fi eftendono: e quindi dallo fteffo Autore furono col nome di vene dilatate dall'al-' tre diftinte. Quefte tali fono di gran lunga meno delle prinie irregolari; ma fono però, anche in generale, di metalli molto più fcarfe, ed a poco numero di fpecie metalliche limitate. Le piu famole, a mia notizia, fono-quelle di Mansfeld, e di altri luoghi, ne contorni delle metallifere montagne dell'Hartz nella Germania; minere confiftenti in letti; o ftrati di limo tituminofo, indurito, e sfogliofo, contenente poco Rame, e- pochiffimo: Argento, e Pefci mineralizzati; e Vegetabili

(i) De re metallica Lib.III. 
bili ec: fegni evidentiffimi d'effere fabbrica delle acque. Io ne ho trovate di analoghe, ma di poco conto per la troppa loro fottigliezza, in quelle porzioni dei monti minerali della Valle de' Signori, e di Pofena, nel Vicentino, che alla fopra fpiegata feconda qualità, o fuddivifione dei montuof tratti della mia prima Claffe. appartengono: ma le più comuni però di fiffatte ftratofe vene, fono quelle di Schifti bituminofi, e piritofi, che Vetriuolo, e Allume fomminiftrano; e di Carboni? foffili, e di certi ferrei minerali; delle quali ne ho anche vedute in molti luoghi, tra gli Atrati delle pietre calcarie, e delle materie vulcaniche della mia feconda, e terza Claffe.

Le fopra efpofte confiderazioni intorno alla quadripartita mia generalifima diftinzione delle folide parti vifibili del Globo noftro, in montagne primitive, in montagne fecondarie, in monti, e colli terziarj, ed in un quarto Ordine comprendente le pianure; mi lufingo, che quantunque non fieno che folamente abbozzate, e con Jineamenti forfe troppo imperfetti, effer poffano nondimeno baftevoli a far comprendere alli veramente dotti ed efperti in timil forta di offervazioni, in qual fenfo, e con quali fondamenti io $\mathrm{mi}$ fia a fiffatto divifamento determinato; come pure a far loro difcernere li proprj diltintivi caratteri di ciafcuna delle dette mie Claffi, Serie, Ordini, o Divifioni, come più dire $f$ vogliano.

Se oltrepaffando, e di molto i limiti delle mie proprie offervazioni, ho univerfalizzate le mie congetture, io non ho avuta, nè certamente ho prefunzione di fpacciare arditamente fiftemi. Conofco pur trop- 
troppo da quali ardue difficoltà fia quefto argomento avviluppato, e quanto ci vorrà arcora di tempo, di ricerche, e di efami per folidamente dilucidarlo. Altro non è lo fcopo cui miro, fe non fe quello di rendere noto ciò, che in quefto propofito ho offervato, e le idee, qualunque fienfi, che offervando per molti anni, e attentamente contemplando monti, e piani, ho concepute intorno alle claffiche loro differenze; onde altri di me più perfpicaci, ed a portata di poter fare maggiori, e più eftefi efami, polfano o rettificarle, fe alli fatti, e fenomeni del Regno foffile le troveranno in qualche modo conformi; c rifiutarle, fe le conolceranno infuffiftenti, producendone di piu fondate, e ragionevoli.

Nella nota fuppofizione di Leibnitz, Filofofo efimio (1), e di altri parecchj dottilfimi Uomini, che quefto noftro Mondo più non abbia la prima fua faccia; e che quella, che ha prefentemente, tra un rifultato d'innumerabili complicati effetti dell'igneo, e dell' acqueo Elemento; cioè di bollimenti, e fufioni, di univerfali, e parziali inondamenti, di Vulcaniche eruttazioni, di Terremoti, di follevamenti ; abbaffamenti, fquarciature, rovine, fubbiffamenti, e del confeguente ritiro da' luoghi elevati, e caduta delle acque nei fiti profondi, ora dalle niedefime occupati : in quefta fuppofizione dico confifte a mio credere la più verifimile Ipotefi, e la piu adatia ad ifpiegare ragionevolmente i fenomeni, che nella Terra apparifcono. 'Tutte l'altre, da quefta difformi, che ho finora confiderate, incontrano evidentifime, ed informontabili oppofi- 
zioni di fatto, quando nel feno ifteflo della Natura, colle di lei opere feriamente, e come c̀ d'uopo, fi confrơntano.

Io certamente non fo d' avere ravvifato effetto, o fenomeno alcuno, che data fimile ipotefi, in tutta l'ampiezza di fua eftenfione, non $\mathrm{mi}$ fia paruto dicevolmente fpiegabile: e parlando de' luoghi montuofi da me vifitati; ho coftantemente veduto che li materiali, ed aggregati, de'quali fono compotti, tutti fono infigniti di caratteri, talora ofcuri, ma anche fovente affai chiari, dove di vulcanica, dove di marina origine, dove di effetti dell' uno, e dell' altro Elemento, o $f_{1}$ multanei, o alternati, o in varie altre guife, $e$ con $\mathrm{d} i$ verfi modificamenti difpofti.

Le relazioni, e notizie, che $f_{1}$ hanno di quafi ogni Regione della Terra, e la fimiglianza de' Foflili efcici, che ci vengono trafportati, con gli analoghi dei noftri Paefi, m'hanno portato a credere, che detti due Elementi abbiano anche nelle altre parti operati effetti. a un di preffo conformi a quelli da me veduti : con quelle diverfità, e variazioni però, che fono proporzionate alla loro rifpettiva natura, $e$ modi di agire, ed alla lunghezza di tempo, alle qualità de' materiali, e gradi di forza, in ogni particolar luogo impiegati, e ad innumerabili altre combinazioni, e circoftanze. Le offervazioni comunicatemi in quefti ultimi tempi, dal Signor Gio: Giacomo Eerber del Collegio metallico di Svezia ec. Soggetto degno di grande eftimazione, da effo fatte ne'lunghi giri delle fue peregrinazioni per l'Inoria Naturale, come pure da parecchj altri chiariflmi Viaggiatori naturalifti, e folertillimi offervatori. m'han- 
m'hanno vie piì che mai confermato in quefta opinione.

Ecco additate le Fonti, dalle quali derivano le Geologiche mie fantafie, che di buon grado fottometto al fincero giudizio di quelli, che hanno giufto diritto di giudicarne: non avendo io altra vifta che di cooperare, per quanto fo, e pofto, all' avanzamento di una fcienza così effenziale all' Iftoria fifica della Terra, e tanto utile per altri rifpetti, ma fatalmente troppo ancora bambina.

Non vorrei però fi credeffe, che avendo diftinti li monti in foli tre ordini, poffa effere perfuafo che fienfi anche in tre foli tempi formati. Egli è troppo chiaro a vederfi, che tante debbon' eflere le Etadi, quar:ti fono gli effetti diverfi, che nelle montagne gli uni agli altri foprappofti li fuccedono. Nelle lunghiflime, ed altiffime Alpi nottre calcarie, per efempio, compofte a ftrati fopra ftrati, dalle loro più baffe vifibili radici, fino alle più eccelfe cime, è forza concepire tanti periodi fucceffivi di tempo della rifpettiva loro formazione, quanti fono in numero effi ftrati, tutti patentemente compotti di calcinofi fedimenti delle Acque marine; le quali in contraffegni autentici di quelle fterminate loro fabbriche, vi hanno dentro impaftate Conchiglie in copia immenfa, e di moltiflime fpecie, che per lo più fono diverfe da ftrato a ftrato. Lo fteffo debbe pure intenderfi delle altre forte di monti, rifpettivamente alla varia natura e fenomeni de' loro materiali, ed ai modi delle rifpettive loro collocazioni .

Le mie divifioni fono generali generaliffme, e non rifguardano che le grandi diftinte Epoche di ciafcuna fe: 
rerie di quagli avvenimenti, da' quali il producimento, le native effenziali proprietà, modificamenti, e fenomeni de' materiali, e aggregati, coftituenti compleffivamente, ed all' ingroffo, ognuno di detti Ordini in particolare, moftrano di dipendere.

Terminero quefto Articolo col fare qualche rifleffo intorno al fentimento di quelli, che col celeberrimo Linneo ( $\mathrm{I}$ ) credono dall' Acqua generati tutti gli Efferi del Regno Foffile. = Fuiffem bumorem ( così detto. Autore fi efprime) rerum initium, aquamque Terie primoidium, e quo furrexere omnia, tefte MoJe, Tbalete, Seneca, manifeftum eft, dum cboticum Nucleum inundans, maie genitum, in fe fatum, lente enititur in Contineritem, quam, exalando continue Rorem, in nebulas elevindum, imbribus atbereis rectifucatis deciduis, quotamis lactat. Catacbyjmi uinivery falis certa rudera ego nondum attigi, quoufque penetravi; minus etiam veram teiram adamiticam, fed ubique vidi factas ex Aquore teiras, o in bis mera Rudera longinqui, fenfim pretarlapfi avi=. Effo affegna alla Cote il fito piu profondo (2); il fecondo, fuperiormente allo Schifto; il terzo al marmo con Pictrificati pelagici; il quarto ancora allo SchiAto; il quinto e fupremo al fafto rupeftre. Spiegata in$\mathrm{K} k$ di

(1) Carol. Linn. Sy/t. nat. T. IlI. Holmice $\mathrm{I}-68$.

(a) I.c Coti, o pietre arenarie, (Lapis molaris Agricols) da me tons fi cideso mai occupare, nei monti, il fito piit profondo; ma le bo fenpre ofjerate fopraforatificate ad alsri dieer $\sqrt{2}$ generi di pictre. Nel Trensino, o nei monti minerali Vicentini, ed in alcuni altri Luogbi, gli frani delle medefme fopraggiacciono immediatamente allo Scbifto quarzofo-sicasco. I'ogr. le inie Mitemorie fopraccirale.

Lo Scbilo, di cui parla il Signor Linneo, numerandone tredici frecic, è da quel genere di pietre Schiffofe, cbe bo confiderate tra le prit migenie, molio dicerfo, come cofia dalla' definizione, $c b^{\prime}$ egli ne dit reb Tomn firddetro Clafs. I. Ord. I. 
di l'origine di dette materie, conclude; ideoque \& $R$ upes faxeas altiffinas avi veros filios effe, dum omnia obmutuere, ipfi loquantwr lapides.

Io Ateffo ho già detto che le montagne, e. altre parti Terreftri, appartenenti al mio fecondo, e terzo Ordine, prefcindendo dalle materie Vulcaniche che in molti luoghi vi efiftono, moftrano ad evidenza d'effere opera delle Acque; sì per la regolarità e parallelifmo de' loro Atrati, che per le tante, e sì differenti reliquie di Corpi marini, che dentro di fe frequentiflimamente contengono. Non trovo pero di potermi confermare al parere di quell' infigne Naturalifta, e degli altri, che così penfano, rifpetto alli materiali de' miei monti primitivi, ad eccezione foltanto delle porzioni Atratofe di quelli della fopra defcritta feconda qualità, o fuddivifionę,

Quel genere di pietre Schiftofe, che per le ragioni già indicate, e che addurro piu diffufamente nel feguente Articolo, ho confiderato tra le materie primigenic apparenti alla noftra vifta, di acquea produzione a me punto non fembra. Lo ftêfo è pure dei Graniti, Porfidi, Serpentini, e di tutti gli altri Foffili pietrofi e terrei di natura vitrefcente, che detti monti primarj compongono; e di quelle materie, anche dei fecondarj, che fi conofcono dai Vulcani fufe, o bruciate. Tutti li Corpi, e Aggregati folfili di quefta fatta, non raffomigliano punto, in qualunque vifta voglianfi contemplare, alle marine moderne pietrificazioni ; come fanno quelli, componenti, a ftrati a Atrati, le montagne, e colline calcarie, marnofe, fabbiofe ec. Eni per ogni riguardo, ed effenzialmente ne differifco- 
no: ed al contrario molto corrifpondono co' loro caratteri, dove identifici, dove analoghi agli odierni Vulcanici producimenti, come parmi di avere a fufficienza anteriormente f́iegato.

Egli è con la fola fcorta della pratica conofcenza di quegli Agenti fifici, che veggiamo attualmente impiegati ne' continui lavori della Natura, e di quella degli effetti rifpettivi da'medefimi prodotti, che noi polfiamo, con fondamenti ragionevoli, indovinare quali fi foffero le caufe effettrici di quelli de tempi, anche più rimoti. Se ciò non vale: qual mai altra via ci relta per, potere fanamente filofofare in quefto propofito? Duplex origo intelligitur firmorum corporum; (diffe, feguendo quette traccie, il gran Leibnizio) una. cum $a b$ ignis fufione refrigefcerent, altera cum reconcrefcerent ex folutione aquarum ( $\mathrm{I}$ ).

Gl'ingemmamenti e concrezioni criftalline agatacee, c di altre fimili fpecie vitrefcenti, formate vifibilmente dall' Acqua dentro le cavità, e fenditure di certi monti, per lo più dell' ordine primario, non poffono dalli veramente efperti, e conofcitori confonderfi colle foprammentovate vetrofe Roccie, in riguardo alle caufe. e modi di formazione. Bafta bene offervarle ne' loro luoghi natij, per avvederfi, ch'effe fono faliformi criftal-: lizzamenti, coaguli, unioni di molecole vitrefcenti; d'altronde rapite, e difciolte dalle Acque, che giù per ogni meato, feffura, e fcrepolo de' monti difcendono; e là, dove efiftono, coadunate. La mutuá attrazione delle conftitutive particole le ha così, come fi trovano, $\mathrm{K}$ k 2

con.

(I) Protegáa Pag. 7 
congiunte, e confolidate; nell' ifteffa guifa, che nella criftallizzazione, e nei coaguli delle foltanze faline, fi offerva; e nelle materie fpatole, ie falattitiche dentro gli sfendimenti, e caverne delle montagne calcaric ec.

\section{A. R T I C O L O II.}

T. A confufione che fovente $s$ incontra, leggerdo le opere, che trattano di Cofe Foffili, circa le des nominazioni di cerii materiali; ora diverfe in una medefima fpecie, ora univoche per molte, che tra di loro non poco differifcono; cagiona ofcurità ed equivoci agli ftudiofi dell' Orittologia. Per-togliere dunque tale ambiguità, quanto meglio poffo, da ciò che ho fcritto nel particolare di quel genere di Schifto, che ho confiderato o primigenio, o almeno uno tra quelli di prima formazione, che i monti primarj compongono; credo ben fatto di nuovamente parlarne. Il nome di Schifto è ftato affegnato, parlando in generale, a quafi ogni forta di Folfili lapidofi, che in sfoglie, o fottili lame fono per natura loro divifibili; fenza riguardo alle loro differenze di origine, e di natura. Così trovanfi tra gli Schifti numerate le pietre Foffili, compofte di Quarzo, e di Talco, o Mica; quelle puramente fteatitiche, o talcofe, o micacee; e le granitofe, porfi: ritiche, argillofe, micaceo-arenofe, e le fabbioniccie; come pure quelle calcarie marmoree, cretacee, marnofe, limofe, e bituminofe ec. Di tante forti di Schilti, non $v '$ 'e che la prima unicamente, 'ch' io abbia per primigenia confiderata. 
Li principj vifibili, coftituenti cal forta, o genere di pietre fchiftofe, fono il Quarzo, . come difli ; cioè la felce candida, o jalina (I), ed il Talco, o Mica; cioè una materia, che quando è pura, e non col Quarzo intimamente mefcolata, ha tefficura fottilmente sfogliofa, o fquamofa, o in qualche modo fibrofa, ed una certa Jubrichezza con fplendore e colori metallici, o di fquame di pefci. Talvolta, benchè di rado, effa materia talcofa ha una precifa forma di vera Mica (2), teffuta a fotriliffime foglie, pieghevoli, e diafane, o di fuperficie nitida, opaca, e di colore di Argento, o di qualche altro metallo.

In molti luoghi la foftanza talcofa è il maffime ingrediente di effo Schifto; e vi fi trova feparata, e diftinta dal Quarzo, il quale, in tal cafo, vi è per entro a vene, a maffe, ed a filoni, fenza regola alcuna, e con varietà fenza numero, di groffezze, di figure, e di andamenti. In altre fituazioni, detti due coniponenti fono infieme confufamente mefcolati; ora in dofi a un di preffo uguali; ora con ecceffo dell' uno, o dell' altro.

Quando effa talcofa foltanza è pura, o poco milta di Quarzo, ha teffitura molto sfogliofa, tenera, lubrica; ed è agevolmente fendibile longitudinalmente all' an. damento de' fuoi letti; ma quanto piu il Quarzo vi abbonda, e vi è intimamente commifto, tanto la fteffa è piu folida, e meno feffile. Ma fovente la fuftanza quarzofia talmonte vi eccede, e la Mica, o Talco; nella mede-

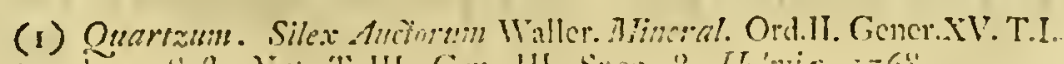

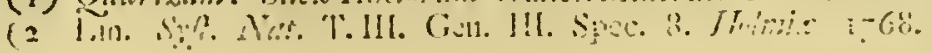


medefima trovafi cosi intimamente mefcolato, ed occulto, che folamente dalli colori, e opacità dell' impaito, la prefenza di effo 'Talco può conofcerfi. Le pietre di quelta fatta non portano il nome, femplice di Schifti, ma di Schifti cornei dal Tedefco Horn-Scbiefer: fe però confervino teflitura sfogliofa. Quando poi effe hanno forma di folidi maffi duriflimi, ed alle percofle di Acciajo fcintillanti, perdono il nome di Schilto, e diconfi Selci cornee, dal Tedefio Hom-Stein .

Il principio terreo del Ferro, ed il flogiftico, che variamente combinati, fono la caufa generale della maffima parte de' colori delle pietre, e delle terre; lo fono di pari anche di quelli infinitamente variati, del genere di cui parlo. La foftanza marziale, della quale il Quarzo puro, cioè bianco, o jalino, e privo affatto di altri colori, non partecipa ; è coftantemente, or vifibilmente, or invifibile, nella fuddetta materia talcofa, o micacea commifta in dofi varie: eccettuata però quella, che ha vera forma di Mica jalina, e trafparente, oppure di colore e fplendore di finiffimo Argento. Di ciò io $\mathrm{mi}$ fono con varie fperienze chimiche afficurato. Ad onta degl' innumerabili differenti modi, di miftioni, di colori, di ftruttura, di durezza, e di altri fenomeni offervabili nelle moltiffime fpecie e varietà di pietre di quefto genere; effe hanno fempre tra di loro quei generici effenziali rapporti, che dalle altre lapidofe materie chiaramente le diftinguono. L'impafto e modificamenti delle due principali loro coftitu, tive foftanze fopra indicate, fono nelle pietre medefime moltiffimo diffimili da quelli, de' quali le foftanze ifteffe fono infignite negli altri pietrofi aggregati, che ne 
partecipano: tali che fono $\mathrm{i}$ Graniti, e diverfe pietre arenarie, e Breccie ec.

Io non vidi mai dentro le pietre di quefto genere nè Breccie, nè Arene, nè altri rottami di materiali dimoftranti folida efiftenza dello fteffo più antica; come ne apparifcono talvolta nei Graniti, Granitelli, c Porfidi, ed in altri fimili lapidofi Foffili dell' ordine primitivo, e moltiftsmo piu in quelli degli altri due ordini. Effe fono inoltre totalmente prive di reliquie di animali, e di vegetabili, e di bituminofe materie; ccme lo fono ugualmente anche gli altri faffofi aggregati dei monti primitivi della foprafpiegata prima qualità, - fuddivifione.

I letti, e falde irregolari dello Schifto primigenio; li fuoi bizzarri venamenti, fpeffo in mille otrane guife contorti, arricciati, ondeggianti, brevi, interrotti, e confufamente intralciati; ci prefentano l'idea di materie in cotal modo modificate, e difpofte da vitriforme fufione ; da tumultuarj pigri movimenti ; da inuguale lento condenfamento. Il Quarzo ha in fe. fteffo $i$ caratteri, e l'effenza di Vetro; ma Vetro della Natura, refrattario, e durifimo. Il principio talcofo, o micaceo del medefımo genere, è parimente una foftanza effenzialmente vetrofa, ma del Quarzo affai men pura, ferruginofa, e molto più fufibile. Il fuoco, convenientemente applicato, cangia tali vetri naturali in vetri artificiali. La Porcellana ferva di efempio: effa non è, che un impafto di Quarzo finamente polverizzato, c di Argilla bianca, ridotto dalla forza del fuoco nel la Fornace, ad un certo grado di artificiale vitrificamento. 
La parte talcofa o micacea di detto Schifto, neile Fornaci fuforic, paffa in nera vetrofa fcoria: e tanto effa, che il Quarzo, quando fi tengono baftevolmente efpofte alla percullione di viviffima fondente fiamma, dentro adatta Fornace, fondonfi almeno fuperficialmente, fenza aggiunta di Sali.

Le fufroni delle minere in grande, e quelle operate dalla Chimica, ci danno a vedere talvolta delle vetrificazioni fmili, in certo modo, alle Selci Quarzofe; e di quelle pure che imitano li criftallizzamenti, ed anche le Miche e Talchi con la loro sfogliofa, o fibrofa, o altrimente figurata interna fruttura (I). La propricti inol-

(1) Il celeberrimo Joh. Gotfchalk Wallerius, Sommo Cbinnico-Orittologo, è forfe il primo che abbia differtato fopra le Criftallizanzioni ignee, e cbe ne abbia data la Teoria. Egli infegna cbe = Cryftallifationes ignex duplici fitut modo: I. ( per fufionem, qua appellatur cryltallifatio ignen filforia \&c. 2.) per volatilifationem \& fublimationem, quæ nuncupatur cryftallifatio ignea fublimatoria $=I n$ prova di tal fatto di crifallizzamenti, al duce l' efperimento delle figure criffalline friate, prefe dallo Zolfo, trattato al fuco in certo particolar modo: e le criftallizate forme, che varie Scorie metallicbe prendono nel congelarfi : talume in tutta la boro malfa; talaltre folanente dentro le loro feffure, e cavite. Dice che niffuno dei Cbimici ne ba parlato prima di lui: indi foggiugne $=$ Nos vero ab experientia jam convieti, 'Cryftallos, feu figuras determinatas corporum folidorum, non pracipitationibus folum, verum etian \& fufionibus, \& fublimationibus produci, noftram fuperftruere conabinur Theoriam, in fequentibus $=$ Chemix Phyfice . Part. pr. Stockholmix 1760.

Numerolifimi fono gli elempj, cbe poffino addurfi di criftallizanuem si di varie forme prodotti dal fuoco, per firfione, per vetrificamento, per fublimazione; ni li manca di quelli di retrofe produsioni di teflatura sfogliata come quella delle MTicbe. Vegg. Pott Litbogeognofie, E⿱乛. Il Signor Klinghammer ba ottenutio finile vetro a sfoglie da certa mifura di Feldefparo, di Spato fuffbile, e di ana particolar Jpecie di terra, col mez di violentifinin fuoco, in vali cbiust.

Le fofanize metallicbe; li metalli puri, non eccettuato neppur lOro, concenientenente trettati con fuoco di fufione, e lafciati lentifimamen- 
irolite, che hanno certe materie di diverfa natura, quando unite fi fondono, di fepararfi, e di ritenere ciafcuna nell' addenfamento, fiti diftinti; può farci credere clse il Quarzo foffe in origine la foftanza piu pura del mifto Schiftoío, ed in iftato di quafi acquea Huiditi, feparatafi, in certe foftanze, dalla materia talcufa, meno fluida, e di vifchiofa confiftenza. Il fale nuriatico, per efempio, mifto nelle vetrofe fufioni, ci moftra come ciò poffa effere addivenuto, poichè fi fepara in fimil modo da tutte l' altre materie, colle quali nell'atto di fluidità trovavafi congiunto. Fenomeno molto analogo a quello apparente in alcune varietà di detto Schifto, nelle quali il Quarzo è dal Talco; o Mica diflinto; è cofpicuo in un Vetro Vulcanico, ftato$\mathrm{mi}$, non ha molio, portato da una delle Ifole dell'Alcipelago, dove chi lo ha di là tolto m'afficura effervi dei monti, ohe non fi veggono d'altro, che di tal Vetro formati.

A propofito dell' accennata effenza vetrofa dei Talchi, e delle Miche; Fonfli di un ifteffo genere, e folo in apparenza diverfi parmi offervabile la trasformazione del Vetro artificiale, operata dal tempo, col mezzo di naturali Solventi. Ne ho trovati dei pezzi più volte, che reftati lungamente fotto la terra fepolti, crano nelle loro fuperficic alterati, difciolti, e riL 1

dotti

te raffreddar $\sqrt{2}$, prendono figure fimatricke, e fpecifiche. vegg. Chymie experimentale \& raifonnce par Monfieur Baumè. Paris 177ĵ. To fleffo bo fpeffe fate veduto di fiffati fenomeni nelle fulsoni di sarie minere, $e$ nelle Docimattiche, c Chimiche fperienze. Ala quale mareciglia je l'Arte non puv

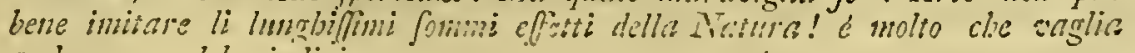
co dame qualche indizio. 
dotti di una forma alle vere Miche fimiliffina; sì nello sfogliarfi in fottili lubriche fquame alquanto fleffibili, che nella metallica rifplendenza di colori; dove di Oro, dove di Argento, dove di Ferro, o di Piom. bo, ed in altri modi variati. Il medefimo fenomeno $\mathrm{mi}$ ha fatto pur offervare S. E. Signor Giacomo Nami, Cavaliere, e Senatore Veneto Preftantifimo, in certi antichiffimi Vetri Sepolcrali della preziofa fua Raccolta di Antichità.

Io non fo darmi a credere, come alcuni penfano, che l'Acido vetriuolico, in certi modi coll' Argilla dalla Natura combinato, abbia generato nè Talchi, nè Miche, nè Asbefti: e fono perfuafu che fiano produzioni dal Fuoco originate. Le fquamette lucenti, e lubriche, che formanfi trattando le Argille con detto Acido, anche prendendo delle più pure, altro non fono che Selenite; come ho già col fondamento di mie fperienze fpiegato nella mia Memoria fupra l' Acque minerali-di Arzignano ( 1 ). Le Seleniti, tanto naturali, che artificiali perdono nel fuoco l'Acqua di Crifallizzazione la coerenza, lo fplendore, e trafparenza; lo che non fanno i Talchi, e Miche, le quali confervano nel fuoco calcinatorio la loro nitidezza, anzi vi divengono più rifplendenti. Effe non fono, niente affatto, diffolubili dall Acqua come le Seleniti; $c$ ne differifcono effenzialmente per altri riguardi. La fopraddetta fuftanza micacea, nata nella deftruzione del Vetro, è dotata di fimili proprietà, e refifte ugualmente che le Miche Forfili all' azione del fuoco, e degli Acidi.

$$
\text { L'ef- }
$$

(i) Giarnale of Iralia T. IX. Fenezia 1773 . 
L'eflere le Argille frequentemente ripiene di Mica a minurifirne fquamette lucenti, e di colori metallici, non è per me una prova che fiafi dentro le medefime generata. Le offervazioni m' hanno anzi dato a conofcere, che dalla diltruzione degli Schifti, cioè dalla loro talcofa o micacea foftanza, varie Argille fi formano: nel qual cafo fono di Mica, non ancora affatto difciolta, ripiene. Tali micanti fquamette fono ugualmente eftranie, ed avveniticcie alle terre argillofe, e di ogn'altra qualità, nelle quali $\mathfrak{f}$ trovano; come lo fono alle pietre arenarie, ed alla maggior parte delle Sabbie.

Ma ritornando alle pietre dell' anzidetto genere Schiftofo, effe, come diffi, occupano in tutti li luoghi da me offervati, dove però allo fcoperto apparifcono fempre il fito piu profondo, rifpettivamente agli altri; pietrofi generi: perciocchè dal diffotto de'medefimi emergono dall'imo della Terra, fenza moftrare di avere fotto di fe materiali di differente natura. Mancano onninamente di qualunque indizio, che atto fia a farci credere ch' effe abbiano tratta la loro forma da fedimenti e concrezioni nel feno delle Acque. Le vene, i floni, le maffe, e cumuli di minerali contenenti metalli, Zolfo, Arfenico, ad altre fiffatte foltanze, a li folitarj ingemmamenti di quafi puro Ferro, di Piriti, di Granati, di Scbuerl (I) di Criftalli, ec., che fovente nelle $\mathrm{L}_{2}{ }_{2}$ me-

(1) Cid che li Minerifti Tedefcbi dicono Schærl, e gli Svedeft Skiærl, potrebbe cbiamarf $f$ Granato colonnare, o prifinatico, con l' agsiunto del colo.e rifpettivo di cinfcheduna Specie: nominnudolo per efempio Granato ne-

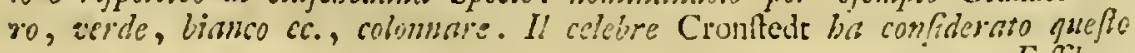


medefime efittono, moltrano di effere corpi o feco congeniti, c paraffitici, e di origine pofteriore.

Egli è bensì vero che vi fi veggono in molti luoghi, intarfiate certe pietre, or nere, or di nerezza più, o meno verdeggiante, o rugginofa ec., tutte di Ferro impregnato, che lo Schifto in mille guife dividono, ed apparentemente penetrano a grandiffima profondità; e vi ftanno interpofte a filoni, ed a cumuli, e malli, anche grandiffimi. Ma a me fono tutte fembrate vulcaniche eruttazioni, fu dal profondo fpinte dalla forza di fotterranei incendimenti, e tra le voragini, crateri, e fquarciature da' medefumi caufate nei monti Schiftof, introdotte. Effe alle Lave molto raffomigliano, si per l' intrinfeche, ed efterne loro forme, che per gli accidentali e Atrani loro andamenti.

Da ciò, che ho detto finora, mi lufingo fiano a fufficienza fpiegati gli effenziali diltintivi caratteri dello Schifto, da me riputato uno dei generi di Foftili della più antica origine, tra quelli, che all' umana vifta apparifcono: ma conviemmi di accennare anche quelli delle altre forti di pietre Schiltofe, che confidero di potterior formazione; onde conofcere fi poffa in che

con-

Foffile dello feffo genere dei Granati: ed il Sig. Linneo lo definifce-Borax la. pidofus columnaris politus, pyramidibus triquetris. Bafaltes, Skiærl. Il mone di Bafalce mi fembra molto improprio in quefto cafo; percioccbe il Schærl $E$ un vero mincrale pietrofo-metallico, crifallizato a minuti ingenmanenti, e totalmente divesfi dalli Bafalti rupeftri, a gran prifmi, e fpeffo compo"zinti porrioni di monti, fooglj, e roccie; li quali non fono da annoverar it tra le foftanze minorali, ma tra le pietre vitrefcenti, formate da vulcanicbe fufioni: come le recenti offervanioni delli celebri Naturalifi, Bankes, Solander, e uno Troillio, fatie intorma alli Vulcani dell' Iflanda, banno pofo fuori di dubbio. 
confiftano le loro differenze, e le ragioni per le quali da me tutti coevi non fi credono.

Le lapidofe materic, connumerate tra gli Schift, che a detto genere primigenio non appartengono, hanno efpreffi caratteri, che dal medefimo le diftinguono. Quelle; che maggiormente allo fteffo fi approffimano, e che piu dell altre gli raffomigliano, hanno per uno de' loro coftitutivi vifibili principj la foftanza talcofa, o micacea, ma fono prive di Quarzo, in vece di cui, contengono un vero Spato calcario, oppure un Marmo falino calcinabile, non diffimile dallo Spato, che nei modi di criftallizzamento. Certi marmi di patta faliforme, a fottili Atrati, e venamenti alternati, bianchi, e cinerei, o piombati, o verdeggianti, qui conofciuti col nome di Cipollino, di Agatato ec; di Grecia, fembrammi appartenere a. quefta forta di Schifo. Le loro vene colorate, tramezzanti le bianche alomorfe, moftrano, non di rado, a chiara vifta che i loro colori dipendono dalla Mica dentro il marmoreo cemento avviluppata. Quelli alli predetti analoghi, delle Alpi Appennine fopra Serravezza, e Maffa di Carrara, ec., nella Tofcana, come pure altri fimili nella Montagnuola di Siena a Montarenti, e di altri Luoghi di quel Gran Ducato; gli ho veduti Atratificati immediatamente fopra lo Schifto primigenio, di cui le parti di quei monti, inferiori a tali ftrati marmorei, fono formate.

La fimilitudine però delle pietre Schiftore, teftè indicate, collo Schifto primigenio, confifte unicamente nella foftanza talcofa o micacea, maffimo, e predominante loro componente; per la quale anche detti narmi hanro collo fteflo qualche, benchè molto diftante, 
analogia, quando della foltanza medefima partecipano: locchè è ben raro, effendone d'ordinario affatto privi. Per altro l'une, e gli altri ne differifcono nella totale affenza del Quarzo, e nella diverfità de' rifpettivi loro fenomeni, e prodotti minerali: e moltiffimo piu per la loro forma regolarmente ftratofa, e per la loro fituazione, a detto primo Schifto fempre fuperiore; e perciò patentemente meno antichi, e da altra caufa fifica dipendenti.

Nelle montuofe Maremme dello Stato Sanefe, ed in altri Luoghi della Tofcana abbonda un' altra forta di Schifto; ed è forfe ciò, che il celebre Signor Giovanni Targioni-Tozzetti chiama Galeftro, e Galeftrino, - qualche cofa di fimile (I). E' quefto d' indole Argillofa, e di colori ofcuri, or quafi neri, o cinericcj, o cileftrini; ora verdognoli, o gialliccj, o roffeggianti o rugginofi: ed ha teflitura groffolanamente sfogliofa, arricciata d' ordinario; e letti, e andamenti ftranamente confufi. Niente partecipa di Quarzo, ma bensì di Spato calcario, che per entro vi fcorre a venamenti ondeggianti, e fenza alcuna regolarità; ma però folamente in certe fituazioni, mancandone in altre.

Siffatto Schiftofo materiale è molto ferrugginofo, e fufibile in nera vetrofa fcoria: e dove confina con monti di Schifto primigenio, vedefi coftantemente effere al medefimo foprappofto. Ciò puo vederfi alle Carbonaje di Montieri, ed al Torrente Merfa di Boccheggiano; come pure nel monte, fu cui il Caftello di tal nome è fituato; e per lunga ferie di què poggi, andando

(1) Relazione di ulcuni viaggi per la Tufcana. 
dando verfo Rocca Strada. Colà effo fafcia, tratto tratto interrottamente collegato con pietre calcarie, e terre marnofe a fottili ftrati, li monti di Schifto primigenio, dal quale non è feparato, infieme con dette pietre, e terre, che da ampia vena minerale; la quale principiando a farfi vedere al giorno nei monti tra Gerfalco, e Prata, fcorre quafi fempre vifibile, lungo le pendici meridionali dell'alta montagna di Montieri, famofa per le fue antiche minere di Rame e di Argento; indi traverfate le due Merfe, giù tra monti dalla Maremma fi eftende ( $\mathrm{I}$ ).

Dentro tale qualità di Schifto, che non ho mai veduta altrove, furono cavate in più luoghi dagli antichi Etrufchi minere di Rame, e di Piombo. L' Arte delle fotterranee efcavazioni, a quello che appare dai Pozzi, alcuni de' quali fono ancora aperti, era preffo que' Popoli ancora bambina. Si profondavano giu nelle vifcere dei monti con Pozzi perpendicolari, o poco declivi, rotondi, e murati, e non foggetti a ruvine. Se fcoprivano metalli, gl infeguivano a profondo folamente, e non ardivano di eftenderfi fotterraneamente con trafverfali Cunicoli. Quando o la troppa profondità

(1) Nel 175., troondomi alle carbonaje di Monteri col Sig. Giufeppe Calcabigi di Livorno, foggetto dotto, ed Arvocato ai detra Cittri. rapprelestai in wha IITappa, buon tratto di quella lena, e ne feci is defcrizione, unn frammento della quale, con detta Mappa, e cont cil, che dellia feffa Vena ba fcritto il Sig. Targioni-Tozzctti, fit pubblicato in titha Relazione fampata in Livorno nel 1755. da Anton Santini, e Comp.

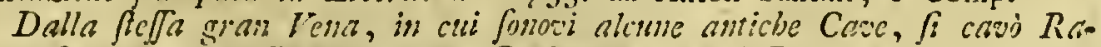
me. Forto la mic dirczione, alle Casbonaje, e nel Botro di Sant' Anfans c ncilla Merfa di Brocibeggiano, da detto ainno 1755., fino 1767., ma tali lavori furono poi totalmente abbandonati, qualche tempo dopo il mio ritorno dilla Patrith. 
dità de' Pozzi, ora colì nominati Buttini, loro era di oftacolo; o fe minerali più non trovavano, ufcivano al giorno, ed in poca diftanza da' primi cavavano nuovi Pozzi. In cotal modo ne hanno aperti in grandiffimo numero nella fuddetta nontagna di Montieri; nei monti di Gerfalco, di Monte Rotondo, e di Prata, ed a Pozzoja, e Serrabuttini, a Poggio al Montone, ed in altre varie fituazioni del Territorio di Maffa di Maremma .

Ma quantunque detti Popoli ignoraffero, come chiaro apparifce da' loro lavori, li modi ufati ne' fecoli a noi vicini, e prefentemente nelle minerali efcavazioni; e non ardiffero, come ora $f \mathrm{fa}$, di eftenderle fotterraneamente per qualunque verfo, a norma delle occorrenze: reca pero ftupore, che effi abbiano avita l'abilità di fcuoprire moltiflime minere in luoghi, che di averne non danno alcun' efterno indizio. Pare che dal folo afpetto dei monti, e dalle fpecie di pictre e di terre, delle quali fono componti, la profonda efiftenza dei metalli argomentando, a fiffatte impreic laborioliffime e pericolofe s'arrifchiaffero; molte delle quali però fono andate a vuoto, come le materie eftrattene, che ancora in diverfi fiti fi veggono, danno a conofcere.

La fpecie Schiftora di fingolar forma poco innatzi defcritta, dentro cui il maggior numero di dette antiche Cave minerali efifte, ha bensi collo Schifto primigenio qualche affinità; ma ne difierifce non pertanto: ed a confronto del medefimo pare un materiale fecondogenito; non gli effendo mai inferiore, ma fempre fuperiormente appoggiato. 
Rifpetto poi alle altre maniere di materiali Schi. ftofi, o feflili, da me vedute, tutte fono moltiffimo più delle predette, dal primigenio lontane di origine, $e$ di forma, e per altri diverfi riguardi. Niente dirò di quelle di natura calcaria, e marnofa, perchè con lo fteffo nón hanno alcuna raftomiglianza. Parlando delle rimanenti fpecie di Schifti "cioè di quelli arenof,; é dei limofi, e bituminofi ec. (I); effi da quello che nomino primigenio differifcono, in primo luogho, per le loro fituazioni, fempre fuperiori al medefimo, relativamente alla di lui derivazione dal profondo della. Terra; donde arguifo che fieno di meno antica forma: Ne fono inoltre totalmente diffimili di componimento, e di difpofizione; perciocchè quelli arenofi chiaramente ci moftrano d'effere pietre ricompofte di fabbie, per lo piu vitrefcenti, e fpeffo anche di minutiffimi frammenti di Mica: e quelli limofi, e limofo-bituminofi ebbero origine da fangofi fedimenti, de' quali jportano ancora efpreffi caratteri.

La difpolizione a Atrati paralleli, e fovente quafi Orizzontali, di tal fatta di Foffili fchiftofi; e gli acquatici Animali, e Vegetabili, che talvolta vi fi trovano dentro impaftati e. fepolti, particolarmente in quelli di. hitume impregnati, fono autentici contraffegni ch' effi furono dalle Acque fabbricati.

Efpofte le offervazioni, e le ragioni, per le quali ho confiderate primigenie le fpecie e varieta di pietre, M m

che

(1) Di tal fatra fono le fpecic del genere Scbiffofo del Sig. Linneo, 77 quale defuifce il fuo Sebifto-Lapis cx. Ilumo Vegetrbilium; fragmenta fiffilia, horizontalia, plans opaca, raflia, igne coubuftibilia. Sỵt. nat. T.Iil. Claff. I. Oru. I. Gen. I. 
che al fopraddefcritto primo genere di Schifo appartengono; oppure almeno tra quei generi di lapidoli, e terrei materiali della fuperficie di quefto Mondo, che hanno fegni di priorità di formazione, relativamente ad altri moltifimi:: debbo manifettare, e lo fo di buon grado, che fecondo le offervazioni del foprallodato Signor Ferber, recentemente da eflo comunicatemi, il vero Granito moftra un' antichità, probabilmente fuperiore a quella di detto mio Schifto.

Per vero Granito egli intende quello compofto di Quarzo, di Mica, e di. Feldfpato (I); quale è quello conofciuto in quefti Paefi col nome di Granito roffo di Egitto. Di tal forta egli ne ha veduto in molte delle principali, e più alte catene di montagne di Europa; e $\mathrm{m}$ afficura di averlo ovunque offervato forgere da profondità maggiore di quella di detto Schifto. Quindi argomenta che fiffatta maniera di Granito fia più annofa, 9 almeno di pari età delle pietre da me dette primigenie. Non avendo io vedute Montagne compofte di quẹl Granito indicatomi dal Signor Ferber; e conofcendo molto bene la fua efattezza; e le vafte fue orittologiche, e orografiche cognizioni ; non poffo dubitare della verità di quanto mi fcrive di avere offervato. Li Graniti, efiftenti in diverf dei Paefi da me vifitati fono di altre fpecie; e non $\mathrm{mi}$ hanno prefentato fimile fenomeno. Potrebbe peró fupporfi, che tanto il di lui Granito, quanto il mio Schifto, fieno effetti prodotti dalla Natura in pari tempo, 0 non guari differente:

come

(1) Spatum campefire. Linn. Syft. nar. T. III. Clarr.I. Ord. II. Ger. 5 . 5p. x 2. Spatums Scintillans. Cronfed. Min. 66. n. r. 3. 
come egli fteffo fi fpiega. Ciò mi fi rende credibile dalle notizie, che ho avute in quelto propofito dal Signor Giovanni Stange, Cavaliere Inglefe, attentifimo, e perfpirace Offervatore, che poco fa ha vifitate, e diligentemente efaminate le Alpi de' Grigioni, e degli Svizzeri ec. Egli mi fcrifle da Ginevra che la fituaziose del Granito, rifpettivamente allo Schifto, non è coftantemente uniforme; poichè talvolta ha veduto il primo inferiore al fecondo; talvolta il fecondo al primo; ed in qualche luogo gli ha offervati fenza ordine infieme confufi (I). Simili informazioni ho pure avute dal Signor de Sanfure, Chiariflimo Profeffore di Fifica in detta Ginevra; e dal Signor Criftiono Klingbamimer, fotto Direttore della Fonderia Elettorale delle minere a Freyberg nella Mifnia, metallurgo, Offervatore, e Chimico.di grande efperienza.

Ma comunque fia dell' anzianità di origine delle pietre fchiftofe in queftione, egli è almeno certo ch' effe fono uno de'principali materiali, componenti, in generale, quelle montagne, e parti della Terra, che all' anteriormente defcritta prima qualità del mio Ordine primitivo, o ninerale appartengono. Sono del pari tra li pietrofi generi, che più abbondano di metalli, e di altre fimili foftanze minerali. Le minere d'Orodi Zillertbal, e di Fufcb, nel Salisburghefe, fono in fiM $\mathrm{m} 2$ loni

(1) Lo Aeffo mi ba poi confermato con altra Lettera da Lyone, con ia quale mi fa fapere de elfere meco comiorde nel ricufare allo Schito Ar. gillufo un liogbo tra le pietre primigenie; avendo offersato, ne' fusi ainggi Alpini, cbe tale fpecie di Scbifto trovafs, per lo pit nelle montrgne alte, addofo alla mia pietra primigenia, chè allo Scbifo cornso, ét al Groiha. 
loni di Quarzo, dentro monti di fiffatto Schifto; effendo l'altre due del Goldsberg, e del Ratbausberg, in fimili filoni quarzofi, dentro altifime montagne di Granito: come il Sig. Klingbammer, che le vifito net $177^{2}$., me $n$ ' ha a voce afficurato. In una 'fpecie cornea, a Mica di colore argentino, delle Alpi Pannie fopra Serravezza nella Tofcana, ho veduta incaffata la vena di Argento, detta del Buttino di Rofina; e quelle di Argento vivo nativo, e di Cinabro a Levigliani, ora abbandonate. La grandiffima minera di Rame di Agordo nel Bellunefe; e quella forfe non meno grande di Pirite fomminiftrante ottimo Vetriuolo, nel monte di Vignola, tra Levico, e Pergine nel Trentino, ambe fono nel medefimo Schifto. Altre molte ne ho pure nello fteffo vedute nel Tirolo, e nei monti di Schio nel Vicentino; e nelle montagne di Brefcia, e di Bergamo: e fo effervene nella Carintia, nell' Ungheria, ed in varj Paefi della Germania ec.

Dell'ifteffe pietre veggonf monti, or in parte; or totalmente formati; e delle vafte ferie di montagne, anche di grande altezza, che quafi d'altro non fono compofte: ma fempre, come diffi, ho veduto che o agli altri montani materiali foffili fottogiacciono, o dove co' medefimi confinano, fotto di efli eftendendofi, al profondo fi occultano.

Per addurre un efempio delle mie offervazioni in quefto propofito, accennerò fuccintamente quelle fatte bei monti minerali del Vicentino, fino a quelli del Principato di. Trento, dove lo Schifto fopraddetto in molti fiti moftrafi allo fcoperto. Effo, alla parte AuArale, principia a farfi vedere nelle radici de monti di Schio, 
Schio, di Torre, di Pieve, e di San Quirico; e forgendo a poco a poco dal diffotto di altri generi di pietre che vi ftanno fopra appoggiate, $f \mathrm{f}$ va alzando con dolce acclività verfo il Settentrione, formando Poggi di varie altezze; di dove pure pietre Schiftofe del fuo genere; dove con altre diverfiffime fpecie di pietro$f_{1}$, e terrei aggregati, fopra allo ftefo Schifto in varie guife giacenti. Fafciato all' Oriente dalli monti minera: li detti le Guizze, e dalle pietre calcarie ec., della montagna di Lavegno; ed al Ponente, ed in parte a Tramontana, delle altiflime Alpi, per lo più marmoree, di Recoàro (I); va fempre più elevandofi fino ad una catena di mediocri monti granitofi, detti il Collicello di Pofena, fuperiori alla Villa della Valle dei Signori, verfo il Nord. Quindi, fotto li medefimi occultandofi, li trapaffi; $\mathrm{e}$ di nuovo ne forge nel loro pendio fettentrionale, preffo a detta Villa di Poffena : e progredendo nei modi a un di preffo fopraccennati, va a nafconderf fotto le ftràtofe Alpi calcarie del Trentino.

Le Alpi fteffe, ripiene di Marini Teftacei di var rie fpecie, lo cuoprono allora; e feco anche le altre forti di pietre dell'ordine primitivo, o minerale; e lo occultano per lungo tratto. Ma effe terminano final-

mente

(1) Le Alpi di-Recoarò' fono calcaric 'Stratofe. Contengono' Brecdie, - sarj alvi Marmi di molia bellezza. Fi fi casnno anche Mlacini da Grano, $c$ Mole da aguzare Ferranenti. Itorie fpecie di Concbiglie mavine

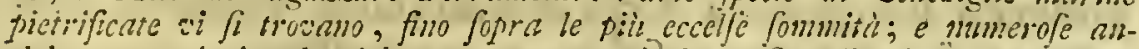

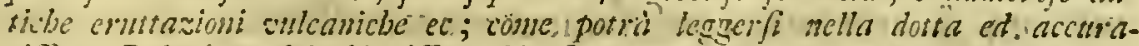
tiflima Relanione del chinrifimo Sig. Jottore Girolamo I"edtari di Valdagno,

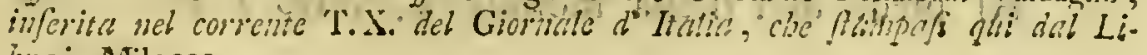
irajo Milocco. 
mente nelle vicinanze di Caldonazzo, e di altri Paefi dello ftato di Trento; e li monti minerali riforgono di nuovo, ed in particolare quelli di detto Schifto: e contornando i Laghi di detto Caldonazzo, e di Levico; il Borgo di Vallugàna, e iPergine, e Civezzano, fu pel Tirolo ifi effendono arandi montagne, tutte vitrefcenti, e totalmente prive di pietre calcarie, e di marini pietrificati; ma di diverfifime fpecie di minerali abbondanti.

Che lo Schifto, di cui ragiono, paffi effettivamente fotto le pietre calcinabili ftratofe della mia feconda Divifione, ecco alcune offervazioni, che me ne fembrano non lievi indizj. Nella fommità dell' alta montagna di Lovegno, poco fa nominata, evvi un Cratere grandiffimo, di figura conica inverfa, e molto profondo, chiramato le Vaccareccie. L'eftremo di fua profondità è ricolmo di Lave vulcaniche antichiflime, ofcure, ferrigne, e tutte confufamente ripiene di frammenti piccioli, e grandi di detto Schifto, e della medefima qualità vifibile nei vicini, molto più baffi, monti mineralí; de' quali ho già parlato.. Vi fono anche, fenza ordine alcuno, mefcolati pezzi di Selci focaje, delle quali certi fiti delle circonvicine "Alpi calcarie abbondano, ed altre varie forti di pietre. Effa montagna è tutta compolta di pietrofi ftrati calcinabili, di un bianco fudicio; ed i fuoi materiali fommantente differifcono di natura, di Atruttura, edi colore; ed in tutti gli altri fenomeni, da dette nere Lave, tumultuariamente fu dal profondo fpinte dalla forza di fotterranei incendj, e come ora fi trovano confufamente ammaffate.

Al piede dell'ifteffa montagna, verfo l'Oftro, fo novi 
novi i Colli del Tretto, già fecondi di minere di Argento e pieni di antiche Cave, da fecoli, non fi fa bene per qual cagione, abbandonate: Sono abbondantiflimi di finiflima Argilla porcellanica, che tutt' ora vi fi efcava in molta copia; e ferve per le noftre. Porcellane; e per altre varie nanifatture, dentro, e fuori dello Stato"; "paffando in comimercio col nome di Terra bianca di Vicenza rof In fito alquanto fuperiore a'me: defimi Colli, veggonf Lave nere, e di altri colori in grandiffima quantità sboccate fuori dalle vifcere di detta montagna : ed i Colli fteffi moftrano 'd' effere rifultati da Vulcaniche eruttazioni, se fonvoglimenti di diverfiffimi materiali, fenza alcun ordine, ce confufamente ammonticchiati L'anzidetta Argilla bianca ha li caratteri più efpreffi di Vulcanico acquofo eruttamento; e di effere figlia del prefato Schifto, nata dalla di lui perfetta foluzione. Effa è piena di frammenti di varie grandezze del medefrmo, e di diverfr altri Foffli, con ogni maniera di accidentali mefugli feco impaftati . Tali Schiftofi rottami, e l'infinita quantità di fquamette di Mica, emule nel colore, e rifplendenza all Argento, che nella Ateffa Argilla fono mefcolate, fono chiari fegni, ch' ebbe il fuo nafcimènto dalla deftruizione dello Schifto; efiftente, profondamente occulto; fotto quelle montagne. Ho detto, che la fteffa ha caratteri di acquofa ecuttazione; perchè nella fua difpofzione, e andamenti, dimoltra d' effere ufeita in forma di liquido fango dall' ime interne parti della foprạeminente montagna di Lovegno $\mathrm{c}$ idi avere Aluidamente. fcorfo giù per le Juperficie declivi de' faftofi ammaffi 


\section{A $T$ T $:$}

de' mentovati Colli ; tra le concavità de' quali fi è, moltiffimo più che altrove, fermata, e raccoltá.

Anche nei monti Brefciani, vicini al Lago d' Ifeo; vidi preffo alla ftrada, per cui : fi va da Brefcia alla Terra di detto nome, un tratto montuofo, tutto compofto di pezzi e rottani di Schifto quarzofo-micaceo; ivi apparentemente alzati da profonde fotterranee ignizioni, che con ampio fquarciamento tra gli frati delle pietre calcarie formanti li medefimi monti, fi aperfero la via. Ciò mi fembra favorire non poco la mia opinione, quì fopra accennatá, perciocchè monti di Schifto mon moftranfi allo fcoperto, che alla ditanza da quel fuogho di venti, o più miglia, andando verfo la Germania. Simili fenomeni ho pure offervati in a!tri diverfi Luoghi, più o meno lontani dalle fchiltofe vifibili parti: ma quanto ho detto nii pare baftante al mio fcopo:

Dalle offervazioni, e dalle ragioni, qualunque fianf, che ho finora efpofte, mi do a credere che li. praticamente, e fcientificamente intendenti delle cofe foffili potranno, almeno all' ingroffo, rilevare quale fieno li fondamenti della mia diffinzione delle parti, fenza fine diverfe, della crofta fuperficiale del noftro Globo in quattro foli generalifimi Opdini ed in che univerfalmente confiftano li caratteri generici de' nateriali di ciafcun Ordine; e quelli pure delle pictre. Schifto-? $f e$, che ho confiderate primigenie, o tra quelle almeno di primaria antichita, rifpettivamente alle modificazioni, fotto alle quali prefentemente ci moltrano di efiftere. Quefto però è un argomento capace, come ognu- 
no può riflettere, di grandiffima eftenfione: ma per una Memoria, io $\mathrm{mi} \mathrm{ci}$ fono forfe troppo lungamente trattenuto. Ma perciocchè ho promeffo di aggiugnere alcune offervazioni fopra lo Schifto bituminoto d'Idria, c qualche rifleffo fopra il medefimo, e fopra gli altri fotterranei materiali di simile natura, onde fi conoícano le ragioni per le quali non fono perfuafo, che veruno dei Folfili bituminofi fia da confiderarfi tra li rigorofamente primitivi, palfo a farne cenno nel feguen. te Articolo.

\section{A R T I C O L O. III.}

T A ricchiffima famofa Minera di Argento vivo d'Idria 1 nella Carniola, Provincia dello Stato Auftriaco, per le relazioni, che ne ho da Soggetti delle Cofe follili intendenti, che l' hanno vilitata (I), trovafi in un cupo Vallone, dove le montagne di certo Schifto Argillofo e nero, per quelle ftratofe di pietre calcarie fi congiungono. Il Signor Haquet, Mineralogo molto riputato, e che in quei metallurgici Lavori ha particolari infpezioni, mi ha trafmeffa ferie numerofa delle differenti fpecie, e varietà di minerali, e di pietre, e di altri Folfili, che ivi fi efcavano, unitamente alla defcrizione di ciafcuna: dono procuratomi dall' Amicizia cordialiffinıa del Signor Ferber.

Il Mercurio, o Argento vivo, colà fi trova in copia grandiffima dentro lo Schifto anzidetto; dove nu$N \mathbf{n}, \quad$ do,

(1) Se ne pud vedere th defcrizione nella Memoria del Sig. Ferbcr delle fise offersazioni Oristografiche da Vicnna a Venezia, inferita neb corresite T.X. del Giormale d"Italid, di quefo anio 1-73. 
do, e puro; dove dallo Zolfo mineralizzato fotto la folida forma di Cinabro; dove anche invifibilmente $a v-$ viluppato, e fparfo tra bituminofi, ed infieme fulfurei materiali. Nella ferie accennata vi fono dei pezzi di tali materie talmente di Bitume abbondanti, che a nera Pece, ed a Carbone foffile raffomigliano; e prendono fuoco, e ardono, anche alla fola fiamma di Candela : per locchè il Signor Haquet gli ha caratterizza$\mathbf{t} \mathbf{i}=$ Minera Hydrargyri pblogifica, nigricans, ad Candelam ardens $=$. Anche lo Schitto, che la minera mercuriale in te racchiude, $e$ che $f e$ ne può dire la matrice, è, dove piu, dove meno, della fottanza bituminofa partecipe: e proporzionalmente alla quantità della medefima effo yaria nei gradi di fua nerezza (I). Quello, che per abbondanza di Bitume è molto nero, e di pingue apparenza, nel fuoco efala denfo fumo, e odore bituminofo; e vi fi accende, producendo chiara fiamma, la quale, confunto il Bitume, fvanife, reftando i. pezzi nella prima loro figura; come fuccede in tutte l' altre fpecie di terrofe materie, di tale foftanza non moltiflimo impregnate.

Perde effo Schitto nel fuoco la fua nerezza nella fuperficie, divenendo bianchiccio roffeggiante, e finile

a ter-

(1) Diverfi faggi delle varieta di detta Minera d" Idria, $e$ della fua Ilatrice. $f$ veggono anche nella molto pregiabile Raccolta di Cofe Naturali di Sura Focellenza Sig. Giacomo Morolini, al-Ponte detto di San Polo. Quefto colpicuo Feneto Patrizio, Fratello di S. E. R: a Monfiguor Giovanni Morofini, Vefcoro della Città e Diocefi di Verona, mia Patria, con zelante impegno dell avanfamento, anche tra noi, della Nineralogia e Litologia, ufa ogni diligenza e Spefa, per aumentare fempre pizi detta fua Collezione; per cui, e pel fuo Orto Botanico, Ii d refó ormai molto noto a cegevie, fino nelle piis lontane Parti di Europa. 
a terra cotta; ma fi fa di un nero carbonofo nell interno. Cosi abbruciato che fia, l' eterogeneo fuo nativo impafto rendefi cofpicuo: e fi conofce allora, ch'efto è un aggregato di particole argillofe, limofe, marziali, e micacee, alle quali, avanti il brucciamento erano intimamente congiunti, il Bitume, e l'Argento vi: ro, e lo Zolfo: parlando però di quei pezzi, che delle due ultime foftanze cuntengono. Il principio terreo del Ferro flogifticato nella combuttione dal Bitume; fi metallizza, e diviene attirabile dalla Calamita. La Mica vi è immerfa, e dentro impaftata a fquamette minucilfime, le quali, dopo detto brucciamento, chiaramente fi diftinguono. Il fuoco loro leva la mafchera bituminofa : e come fuccede a tutte le vere miche; ne efalta la rifplendenza, ed il colore metallico, che in quella di cui parlo, è l'argenteo.

$\mathrm{Da}$ quanto ho detto nel precedente Articolo, e da quefte offervazioni, parmi fia agevole a comprenderfi che lo Schifto della Minera d'Idria è molto diverfo da quello, che tra le pietre primigenie ho annumerato. Senza confiderare il Mercurio, e lo Zolfo, déquali tutto non partecipa, ne differifce per l' interna fua forma, patentemente ricompofta, pel numero, e diverfiti delle coftituenti terrofe particole, e fpecialmente in riguardo al Bitume, che vi è dentro mefcolato.

Dubbio però alcuno io non ho che detto Schifto non fia uno di quei materiali, che alla Claffe generalifima dei monti primitivi appartengono: 1' indole fua; il. Mercurio, e gli altri minerali, che dentro lo ftefo efiftono, lo moftrano ad evidenza. Solamente argomento da quanto ho premeffo, ch' effo non fia di quelli,

$$
\mathrm{N} n 2 \ldots \text {.ch' }
$$


ch' entrano a comporre le montuofe parti della Terra di primaria origine, fecondo il rigorofo fignificato di quefta efpreffione; cioè a dire, che alla fuperiormente fpiegata prima fuddivifione, o qualità di monti primitivi propriamente fpettano, ma bensi alla feconda. Lo Ateffo opino parimente di tutte l'altre materie folfili dei monti minerali, che Bitumi in fe racchiudono; non avendo mai potuto ravifire de' medefimi veftigio alcuno dentro quelle di detta prima qualità.

Non vidi mai Schitti montagnofi, e rupeftri (I), a quel genere, che per primigenio ho indicato, riferibili; nè Graniti, nè Porfidi, nè alcuna fpecie di quell' altre lapidofe materie, che dei veri caratteri dell anzidetta prima fuddivifione fono infignite, le quali di eflere bituminofe, nè poco, nè molto dimoftraffero. Gli Schifti, e l'altre pietre, e terre di Bitumi infette ; li Carboni forlili, ed i Vegetabili, e Pefci divenuti bituminofi, non fo che fieno fati fin'ora fcoperti, fe non fe in certe parti dei monti minerali ftratofi della mia feconda fuddivifione della Clafle primitiva, e moltiflimo più di frequente nelle montagne calcinofe fecondarie, e nei Monti, e Colli terziarj, nei quali vi fono fpeffo in grande abbondanza. Gli Antraci bituminofi a ftrati, e quelli quinci, e quindi fenza ordine fparfi, e confervanti ancora l' arborea, o erbacea loro nativa forma; e le terre carbonarie, laminofe, o polverülenti, fo-

(1) Montagnofe, oppure rupeftri cbiamo quelle forti di pietre, che formano monti, ed Alpi, per diftinguerle. da quelle, cbe non fi trovano, fe non fe in certi fiti a frrati Solitarj, o in poco numero; tali che fono gli Scbifti bituminof:. Il primo Aggiunto \& ufato dab Sig. Klinghammer; il feando dal Cavaliere Linneo, ec. 
fovente ripiene di vegetabili, e di animali acquatici ancora riconofcibili, veggonfi non di rado anche dentro li montuofi anumaffi di terre Vulcaniche, ftate fmoffe, ed in mille modi rimeftate dalle onde marine, che vi hanno quafi fempre depofitate, e fepolte varie fpecie di conchiglie \&c.

Di fiffatte maniere di Corpi, : c Aggregati forfili bituminofi, anche quefto Stato in diverfifimi Luughi abbonda ; particolarnente nella lunga ferie di monti mediocremente alti, e di Colli, che venendo dal Friuli, e fcorrendo la Marca Trevigiana, e la Veneta Lombardia, ed al Milanefe inoltrandofi, fafciano le noftre Alpi, fopra alle loro Auftrali pendici appoggiati, e dalle Campagne le difgiungono.

Degli Arati di Carboni minerali, e di Terre calbonarie, cioè nere, e pel Bitume, di cui partecipano, infiammatili; come pure di tronchi, di radici, e di rami d'Alberi, quà, e là fotterraneamente ,parfi, ed in Antraci dalla medefima combuttibile foftanza trastormati, ne ho trovati in numerofe fituazioni : e particolarmente in que' montuofi tratti del Vicentino, e del Veronefe, che all' Ordine Terziario riferifco.

Prefcindendo dalli detti rami, radici, e tronchi di piante, per lo piu arboree, che trovanfi in varj Paefi anche di quefto Stato, ed in particolare, nei Colli di Braganze, di Fara, e di Lugo, nel Vicentino; e nei monti di Trifino, e di Arzignano dello Ateflo Territorio, confufamente fparfi tra montuofi cumuli di ciottoloni di pietre nere, e duriffime di Ponici, o 'Tufi Vulcanici, e di diverfflime altre fimili produzioni, slegate, e tumultuariamente riunite, tutte l' altre materie 
bituminofe, da me offervate, fono a frati difpofte, con fegni manifefti di marine depofizioni. Imperciocchè o dentro di effe, o contigue, o nelle loro vicinanze, vi fono refti di pelagici Animali, e Vegetabili; anzi qualche Atrato di Limo bituminofo vedefi pieno di Alghe, e di altre Erbe acquatiche, e di Gufcj di Conchiglie: fenomeno offervabile in Recoàro, in Pofena, e nei fopraddetti Colli, ed in altri molti del Vicentino; come pure nei monti Veronefi della Badia di Callavena, di Saline, di Piegara, e di Grezzana ec. Uno ftrato affai groffo di terra carbonaria fchiftofa, ed in parte di vero Carbone foffile, fi vede pose al diffotto della Villa di Lonedo, di detto Territorio di Vicenza, dentro cui, oltre alle impreffioni delle Piante accennate, ed alle pietrificate Conchiglie, dal medefimo poco diftanti, vi fono picciole Coclee di Mare, in Marcaffita, o Pirite, trasformate: e nella vena di Carbone bituminofo a Monteviàle, coperta da groffo Atrato di Marna faponaria, piena zeppa di frantumi di calcinati Teftacei, trovanfi Scheletri di Pefci, de' quali uno ancora confervo nella Collezione di Minerali ec.

In fomma, l'afpetto de' Foffili di Bitume o poco, - molto impregnati, che ho veduti ne' monti, quali per tutto annuncia l'opera del Mare; e mi ha dato argomento di credere, che le foftanze bituminofe non fieno originalmente proprie del Regno minerale, ma acci-. dentali, e derivate compleffivamente parlando, dalli Regni, Vegetabile, e Animale.

$\mathrm{Mi}$ c̀ ben noto che in molte Provincie vi fono e intere Selve, ed Alberi quà e là fparfi, da gran tempo inabiffati, o in altri modi profondamente fepolti; 
e letti torfacei, compolti di Limo, pieno di putrefatti Vegetabili, apparentemente di Laghi, e di Paludi, non più efiftenti; materie quafi fempre in Antraci bituminofi trasformate: e fo, per le relazioni di Neumamn, e di Juncker, che l'A Ambra, o Succino di Pruffia trovafi dentro terra, fotto un fimile letto, o ftrato di Torfa, pieno di legni bituminofi : ma nondimeno fono perfuafo che l'antico Mare debba riguardarfi come il maffimo univerfale Magazzino, d'onde i Bitumi, almeno li piu comuni, e più copiofi fono derivati, e che fempre l'Acido muriatico fia concorfo, qualunque fi foffero i modi, alla bituminofa produzione.

L'immenfa copia di Corpi organici di fpecic quafi infinite, che con perenne, e non mai interrotta fucceffrone, dentro le Acque marine nafcono, crefcono, e perifcono; e quelli che inceflantemente loro portano i Fillmi, o paffano in putrefazione, e difciogliendofi, con le Melme, e fangofi fedimenti fi mefcolano e confondono; o da' flutti vengono in certi particolari luoghi ftratificatamente accumulati . Con l'andare de'Secoli, vengono, a ftrati a Atrati, da varie depolizioni altamente coperti; le materie a pietrificamento difpofte fi lapidificano, e le pingui foftanze vegetabili, e animali, con gli Acidi minerali, muriatico e vetriuolico combinandofi, in Foffli bituminoli fi convertono: ma diverfi, fecondo la differente natura delle medefime foftanze, e fecondo la varietà, il numero, e le dofi delle altre materie che feco trovanfi commifte:

L'accennata Teoria della formazione de' Bitumi, non è unicamente dedotta dalle mie offervazioni, delle quali, per non riufcire foverchiamente prolifo, non ho 
ho che appena fatto cenno: effa ha anche l'appoggio di quelle di Naturalifti efpertiflimi, fatte nelle minere di Carboni foffili, e di altri analoghi materiali della Francia, dell' Inghilterra, dêlla Germania, e di diverfe altre Regioni ; e quello altresi delle Chimiche analitiche, e fintetiche fperienze fu tale foggetto.

Per ciò che riguarda le minere di materie bituminofe delle Provincie teftè accennate; effe fono, come quelle da me vedute, comunemente a ftrati, ed hanno altri chiari fegni d'effere pelagici lavori. Le montuofe fituazioni, entro le quali fi eftendono, fono Atratificatamente formate, e d'ordinario abbondanti di marine produzioni, che non di rado anche nelle minere fteffe s'incontrano. Quindi a me fembra che la fopra indicata origine dei bitumi, e dei Foffli, che ne contengono, porre in dubbio, o negare non $f_{1}$ poffa, fenza dimentire la Natura in ció, che ne' fuoi fatti chiaro a fufficienza ci moftra.

Quefta dottrina non è però unicamente foftenuta dai lumi fomminiftrati dall' Iftoria naturale: la Chimica come ho accennato, ne dì prove non meno convincenti. I Bitumi, ed i Foffili che ne fono impregnati, analizzandoli, ci offrono delle tofanze analoghe a quelle che nel modo ifteffo fi traggono da' Vegetabili, dalle Refine, ec. Da tutti fi ricava vero Olio, che anzi è uno dei principali coftitutivi principj di ogni Bitume: e quefto folo è chiara prova, fenza eftenderci fopra l'analogia delle altre foftanze rimanenti, che tutti derivano dal Regno Vegetabile, e talvoita anche dal Regno Animale, o da ammendue unitamente.

A propofito di tale nativa derivazione delle Specie bitu- 
bituminofe, ecco come fi fpiega il Signor Lomonofor, chiarifimo Profeffore dell Imperiale Accádemia delle Scienze di Petersbourgo, nell anteriormente citata fua Orazione $=$ de generatione metallorum a Terve motu $=S_{e-}$ cundum locum inter abforta (in interiora Terre) obtinent bituminofa corpori, it funt Aidefia, Lytbantrax, PixJuidaica, Petrolewm, Napbtba, o varia Succinorum genera. Hec - omia ex Vegetabili potiffmum Regno 'prodiaffe, ex Jequentibus patet = Segue indi provando che l'Ardefia'; ed i Carboni foffili ec. provengono dal mefcuglio, fpecialmente. de' Vegetabili; che li Bitumi folidi, e liquidi, fono trafudamenti delle medefime foftanze, per opera del fuoco, o calore fotterraneo, che il Succino è una: Refina di Legni foffili; e che in Pruffia fi trova fotto uno Atrato di fiffatti Legni putrefatti (I).

$\mathrm{Ma}$ ritornando all' Olio; eflo punto non appartiene in proprio, e nativamente al Regno Minerale: almeno quefto è il fentimento di Filofof Chimici dei più fapienti, e rinomati, che non può fupporfi lo atbiano apertamente pubblicato, fenza il fondamento dei neceffarj efami. Uno di quefti è il celeberrimo Monfieur Macquer, il quale così infegna nella fua Chimica (2), e nel fuo Dizionario Chimico, in più Articoli (3).

0

Egli

(1) Nei monti di Modena, di Regio, e di Parma, dove il Petrolio ebbordatitemente raccogliefi, vi fonzo copiofe le Produzioni, e Quifquiglie del mave ec.

Veggafi la Raccolta di varic offervazioni del defonto Celeberrimo Pubblico Profefore Antonio Vallifneri Venezia 1728. Theor. 1756 .

(2) Elemens de Chymie praique T.II. Paris 1754. - De Chym:

(3) Difionaire de Chymic. a 25idon 1767. 
Egli nel detto Dizionario, all' Articolo olio, cosi fi efprime = Quefta foltanza è uno dei principj proflimi di פ" tutte le materie Vegetabili, e Animali; ed è anzi per " le loro parti oliofe, che tutte quefte fortanze diffe" rifcono effenzialmente da quelle del Regno minerale; g. perchè al contrario, non havvene alcuna di queft'ul2) timo, nella quale poflafi dimoftrare un folo atomo "d'Olio $=$. Nella fcompofizione (dice lo fteffo all' Ar" ticolo Regnes) di tutti gli Efferi veramente viventi "organizzati, e che portano in fe ftefli un germe di "riproduzione, tali che fono li Vegetabili, e gli Ani" mali, fi ritragge coftantemente una foftanza infiam" mabile, graffa, oppure oliofa; ed al contrario, non " trovali neppure il menomo veftigio di quefto princi" pio in alcuna delle foftanze puramente minerali, "nemmeno nella più infiammabile di effe, che è lo , Zolfo $=$.

All Articolo Bitumi, egli efpofe la queftione fe fiano foftanze proprie del Regno minerale, come hanno creduto li Fifici, e Chimici antichi; e forle molti credono ancora; oppure fe dalli Regni Vegetabile, e Animale originalmente provengono. Dimoltrandofi effere del fecondo fentimento, ne adduce le feguenti ragioni. I. Perchè fi vede che li Bitumi, venendo fottomeffi alla diftillazione, danno principj analoghi a quelli de' Vegetabili, e Animali; e che fono compoti, come tutte l'altre materie oliofe concrete di detti Regni, Animale, e Vegetabile, d' Olio e di Acido. II. Perchè le foftanze folide bituminofe non differifcono in generale dalle Refine, che nella loro maggiore durezza, e pel loro odor grave, che punto all' aromatico refinofo non raffomiglia; 
per la loro indiffolubilità nello Spirito di Vino; per il loro Acido fulfurofo volatile; e per l'Acido concreto, che fi ottiene nella loro Analifi. III. Perchè non vi è corpo alcuno di origine precifamente minerale; in cui trovifi un folo atomo d'Olio, non accettuato neppure lo Zolfo, che più di qualunque altro minerale alla natura de' Bitumi fi approffima : e che perciò da tutti gli antichi Chimici co' medefimi è ftato confufo. IV. Perchè è verifimiliffimo che le qualità, per le quali li Bitumi differifcono dalle Refine, e dalle altre materie oliofe Vegetabili e Animali, fono naturale effetto o della grande antichità delle materie oliofe divenute Bitumi, o dell' alterazione loro caufata dagli Acidi minerali, o da ambe dette caufe infieme. V. Perchè. combinando degli Acidi minerali con degli Olii vege. tabili, pervienfi a formare dei compofti, che molto ai Bitumi naturali s'approflimano, ed a' quali forfe non manca, che una digeftione baftantemente lunga per effere veri Bitumi.

Oltre alle ragioni fopraddette, paffa ad altre confiderazioni favorevoli all' efpreffo fuo fentimento, del quale fi moftra effere anche nei fuoi Elementi di Chimica teorica, ed in quelli di Chimica pratica, dove infegna l'Analifi de'Bitumi, fpiegandone ivi pure, ma in termini più riftretti, li fondamenti. Anche l' infigne Chimico Monfieur Baume fi appalefa effere della medefima fentenza; perciocchè dichiara, fenza riferve, nel fuo Manuale di Chimica (1); che li Bitumi fono Concrezioni oliofe fotterranee, originalmente appartenenti al Regno Vegetabile. $\quad \mathrm{O}_{2} \quad$ Rifpet-

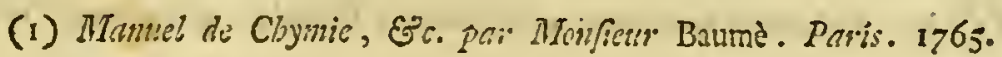


Rifpetto poi alle prove, che della fpiegata origine delle foftanze bituminofe fi defumono dalla chimica imitazione per Sintefi, delle medefime, di cui ho già riportato il cenno fattone da Monfieur Macquer; aggiugnerò qui le rifleffioni in tale propofito del celebre Spielmann. Egli nelle fue Intituzioni di Chimica (I), dove parla della miftione dell' Acido Vetriuolico con l' Olio di Trementina, Efperimento trigefimo fecondo, rendendo ragione degli effetti, che ne fuccedono, dice: = Experimentum boc, Oc.... ip forum etiam aleorum or Acidi Vitriolici mixtionem "docet egregie, "declarat quoque $\circlearrowleft$ refinarum, o bituminum originem.... idem denique refinas of bitumina ex unione Acidi cum oleis odoris furgeve per Syntefin probat, id quod infra per analyfin quoque patebit = Quindi paffando all' Analifi; dove tratta di quella del Succino, Efperimento fettantefimo quarto, argomenta che il Sale acido volatile, che fe n'eftrae facendo con gli alcalini effervefcenza, e feco paffando in Sale neutro fogliato, fimile a quello compofto di Acido vegetabile con gli ftefli Alcali, come ha dimoftrato il Celebre Pott, non debba perciò alli Folfili riferirfi ; ma bensì al Regno Vegetabile. Quefta fentenza non è punto fmentita dalla fcoperta di Monfieur Bourrdelin dell' Acido marino nẹl Sale fuddetto; avendofi ogni fondamento di giudicare, confiderando l' indicata genefi de' Bitumi, che l'Acido muriatico, ed anche il vetriuolico, fienfi fotterra intimamente congiunti con gli Acidi vegetabili ec., delle fortanze, che ivi fonofi in Bitumi trasformate.

Io

(1) Jac, Reinboldi Spiclmann, Irfitutiones Chemie. Argentorati 1766. 
Io non mi eftenderò più oltre fu quefto argomento, lufingandomi di averlo a fufficienza provato, non folamente qui fopra, ma anche nella feconda delle anteriormente citate mie Memorie epiftolari, pubblicate nel 1760 . Allora però non potei parlare fu tale materia, che quafi unicamente coll' appoggio delle mie offervazioni e fperienze; perciocchè gli Autori, de' quali ho qui riferiti li fentimenti, eranni affatto ignoti. Non eravi in quel tempo nella Città, in cui io abitava, alcun altro, che delle Cofe folfili fi dilettaffe; ed in confeguenza, li moderni Libri Oltramontani, che ne trattano, non vi fi conofcevano, e non erami agevole di procurarmene. Parmi nondimeno di avere addotte, anche in quella occafione, delle buone ragioni di fatto, e di analogia, di quelle idee, che fenza efterni fuffidj, furono da me concepute al folo afpetto, e locale contemplazione, e Pyrotecbnici efami dei Carboni foffli, e dell' altre fimili materic, intorno alla loro derivazione dal Regno Vegetabile, ed alla loro trasformazione in foftanze bituminofe nell' interiore della Terra.

Se da quanto ho efpofto e fpiegato relativamente alla genefi, e natura delle bituminofe foftanze, è dimoftrato, come mi do a credere, ch' effe nativamente provengano in generale, dalli Regni Vegatabile, e Animale, e che proprie non fiano del Regno Minerale, ma avveniticcie, e accidentali: e s'è vero, come moAtrano le offervazioni, che l'antico Mare fia ftato l'univerfale principaliffimo edificatore delle loro minere, degli ftrati, tra quali trovanfi eftefe, e dei monti e Colli ftratofi, che le racchiudono: anche la mia opinione, che le medefime non efiftano ne' pietrofi e terrei materiali, 


\section{A T T I}

riali, componenti quelle montagne, che alla fopraddefcritta prima qualità, o fuddivifione dell' Ordine primitivo appartengono, non fara da confiderarfi mancante di ragionevoli fondamenti. Imperciocchè le ftrutture di tali montagne, l'indole, e le proprietà delle loro coAtitutive materie, e le loro minerali produzioni, e fenomeni, non moftrano punto, come ho già dichiarato, fegni del pelagico concorfo alla loro formazione: anzi portano impreffi caratteri di Pyrotecbnici effetti della Natura, anteriori alla genefi delli rimanenti montuofi tratti.

Non avendo mai ravvifata alcuna bituminofa materia nei monti, che non fofle o in quelli evidentemente dal Mare edificati; oppure tra quelle materie, variamente da Pyrofi modificate e difpofte, che pe' loro fenomeni, fi conofcono effere ftate da fottomarine focofiffime effervefcenze fu tra l'onde con fomma violenza fofpinte; fono portato a credere, che una delle caufe effenziali della bituminofa metamorfofi degli Olii, e Refine Vegetabili, e delle pinguedini Animali, fra il liquido-falfo Elemento . Forfe il folo Acido vetriuolico non bafta a produrre nelle medefime foftanze tale alterazione: e lo argomento dai Legni impiegati dagli antichi Metallurgi per fortificare e foftenere le loro fotterranee efcavazioni, e che cafualmente reftati fotto le frane delle montagne fepolti, vi fono per fecoli dimo: rati. Io ne ho veduti, in più occafioni, di fcoperti con nuovi efcavamenti, ed anche dalla violenza de' Torrenti; e quantunque molti, per lungo foggiorno dentro materie piritofe, e di vetriuolo abbondanti, divenuti foffero neriffimi, e di foftanza vetriuolica impregnati, 
nel fuoco non efalavano odore alcuno di Bitume, ma unicamente empireumatico-fulfurofo.

Per lo contrario ho offervato che il Legname reftato nel fargo marino lungamente fotto l'Acque falfe fommerfo, ha acquiftato, con la nerezza, un principio bituminofo. Quefto fenomeno $\mathrm{mi} f \mathrm{i}$ è prefentato, efaminando certe Quercie eftratte dal letto fangofo di quefte Lagune, dove da fecoli eranfi, per varj accidenti, fmarrite e fprofondate. Cosi ho fimilmente rilevato facendo efperienze di varie Torfe : bituminofe non ho conofciute quelle formatefi nelle Paludi di Acque pusamente dolci; ma bensì quelle de' falf luoghi paluftri.

L'Acido marino, fcoperto, come ho detto dal Signor Bourdelin nel Sale di Succino, del qual Sale, per afferzione del Signor Macquer, tutti li Bitumi concreti partecipano, maggiormente nell' accennato fentimento mi conferma.

Non fanno obbiezione alla mia conghiettura gli Alberi ec. divenuti Antraci bituminof, che probabilmente giu precipitati dalle alte vicine montagne, veggonfi confufamente fepolti dentro grandiffimi cumuli di varie forti di vulcaniche materie, poco tra fe coerenti, e tumultuariamente ammonticchiate, in particolari fiti de'fopraddetti Colli del Vicentino ec. Imperocchè tutte le parti de' medefini Colli, e baffi monti, che $f_{1}$ conofcono effere di origine vulcanica, hanno fegni di derivare da eruttazioni di Vulcani fottomarini; come dichiarai nella mia Differtazione orittoJogica fopra le Pietre Obfitiane, e altre Gemme, colà da me rinvenute (I). Lo fteffo oftervò pure il valen-

tifi-

(1) Giornale en Italia. T. I. pag. $36 \mathrm{r}$. e feg. Fenewia 1-65. 
tifimo Orittologo Signor Abbate Fortis ; come ha fpie.: gato nella fua dotta, e molto ftimabile Defcrizione fifica dei Colli, Marini in parte, ed in parte Vulcani ci, di Montegalda (I). Rifpetto poi agli effetti e fenomeni da fiffatti Vulcani caufati, io ne ho data qualche idea in due Rélazioni di varie mie offervazioni, fatte nei monti della Villa di Chiampo, e di altri luoghi del fuddetto Territorio (2).

Gli ftrati calcarei marini, fquarciati, difordinati, e fracciati, e molto, in certi luoghi, dalla prima loro continuità allontanati, e fovente follevati da un lato, all'altro fprofondati, e refi molto declivi, e non di rado ertifími, o pur anche rovefciati; le $\mathrm{La}$ ve intrufe tra le ampie loro apriture, e tra ogni maniera di sfendimenti, e talvolta tra ftrato e ftrato; i letti di pietrificate Conchiglie, rotti, fcompaginati quinci molto all' alto fofpinti, quindi meno, e variamente, a gran pezzi, e falde, dalle native loro pofizioni follevati, ed alcuna fiata dentro le voragini, tra le allora fufe materie innabiffati ; le limofe depofizioni a fottili sfoglie, con vegetabili, ed altre reliquie del Mare tra le bruciate materic quà e là depofitate; ed i Ciottoli marini, con Ghiaje, Sabbie, e Gufcj di 'pelagici Animali, che fopra que'monti Vulcanici, in certi fiti, ftratificati, o confufi $s$ incontrano: fono in generale, i fegni, che ho poco fa accennati, del fottomarino Vulcanico nafcimento.

Quefto

(1) Nel Tomo predetto padg. 83 . e leg.

(2) Una è nel detto Giornale. T.5. pag. 409. e feg.

L'alira in uni Raccolta di Opufcoli delli Sigg. Ferber, e Feftari, e di me, di cui non is ancora terminato it primo Tomo, e fi fampa qui da? Librajo Milocco. 
Quefto penfamento intorno lalla formazione dei fopraddetti Colli'; é Monti, oltre alle offervazioni, dalle quali è dedotto pj di fatto 'Varie Iftoric, Relazioni, e Fifici trattati, ci additano molte Ifole ; Scogli, che fono in diverfi tempil; e Luoghi apparfi; e fuore fpuntati dall' Onde imarine, per opera di profondi fotterranei incendimenti Ma bafti per tutti gli avvenimenti di tal fatta l' Ifola nata nell' Arcipelago, appreffo quella di Santorine, fu cacciata dalla vulcanica forza ; dal mefe di Marzo 1707.; e gradataniente refa da fucceffive eruttazioni fempre più alta, ed eftefa; fino al i 7 Ir. Le Relazioni di Soggetti, che all' orribile fpettacolo fi trovarono prefenti, dando contezza degli ftrani fenomeni di fua apparizione, la defcrivono di un altezza di circa duecento piedi fopra Ja fuperficie del mare; della larghezza di una Lega Inglefe; $e_{\text {e }}$ del circuito di fei (i).

Il creder dunque che li noftri monti compo: fti. di Lave ' di Lapilli, di Tufi ignei, di Pomici, di Ceneri, e di altre fimili materie dal fuoco o fu$\mathrm{fe}, \mathrm{o}$ bruciate, $\mathrm{o}$ in altre guife alterate, non $\mathrm{mi}$ pare un' Ipotefi chimerica; ma anzi appoggiata a folidi fondamenti.

Ma quanto ho detto, bafti per ora: forfe in altre occafioni mi eftenderó molto più a lungo fo$\mathrm{P} p$

pra

(1) De' Croftacei ec. di Anton Lazzaro Moro . Venezir 1740.

De corpi mariti, che fu $i$ monti fi troceno. Di Antonio Vallifieri - Venezia 1728 . peg. 48 .

E nella Relazione di detro arevimento. Nell Operce predetta a p.117, e fig. 
298

$\begin{array}{llll}\mathrm{A} & \mathrm{T} & \mathbf{I}\end{array}$

prale fituazioni, gli effetti, e fenomeni de' numerofi anticamente eftinti Vulçani di quefti noftri Paefi. Avendone io offervati o pochi, o molti, o almeno diverfiffimi fconcerti, e rovine da'medefimi caufate in quafi tutte le parti montuofe da me vedúte; e fapendo che ne fono ftati fcoperti chiari fegni in altre moltifime Regioni del Mondo ( 1 ) ; e dove dei tempi de'loro incendj, e della loro efiftenza non refta tradizione 0 memoria alcuna: fono perfuafiffimo che il bene offervarli, contemplandone gli effetti e fenomeni, fia importantiffimo all' avanzamento della Fifica del Regno foffile,

Senza i lumi, che quindi poffono derivare, non è da fperarfi, per mio avvifo, una Teoria fondata, ed in iftato di fciogliere le tante, e mafficcie obbiezioni, alle quali la maflima parte, almeno di quelle finora divifate, vanno foggette; e ad ifpiegare, con ragioni corrifpondenti alla natura delle Cofe, le tante ftrane forme, fquarciature, slogamenti, ed altri bizzarri fenomeni, che non di rado nelle montagne alla vifta degli Offervatori fi prefentano. Le frequenti ftravagan-

- (1) Il folertiffuno Offerzatore del Regno folib Signor Giovanni Stmn ge, ora definato da S.M. Britanrica Juo Refidente prefo quefta Sereniffim Repubblica, con fua Lettera data di Lyone li 30 . Noregrbre dell' anno corrente 1773., mi aveifa di avere viaggiats da quattro in cinquecento miglia empre fopra Lave vulcanicbe, nelle Provincie Francefi di Auvergne, $e$ di Velay; e che le snontagne formate di colonne bafaltine prifmatiche ei fi contano a dozzinie in eftenfioni di pocbe miglia. Dice cbe tali colonme (anticbifine produzionj del Fuoco fotterraneo) moftrano varietì forprendenti no loro fenomeni; e che vi Jono delle intere Citti fondate fopra effi Bafaiti clue fpeffo occupano eftenfioni efterminate. Detra Lettera è talmente erudita e piena di pregiabili ofereazioni, che merita di elfere pubblicata; e in fari, fe mi farà da! colebre Autore permefo. 
vagantiflme contorfioni, giri ondeggianti, enormi erezioni, e profondi avvallamenti de' pietrof Atati ; e le grandi rotture, che fpefto sfendono o per lungo, o per traverfo, ec. le intere montagne; le Caverne, e voragini, e le materic Vulcaniche, differentiffime dalle pietre, tra le quali fono interpofte, che in varj fiti vi fi trovano; come pure gli fcogli, $\mathrm{i}$ maffi, e le porzioni di monti, faccate dalle rimanenti, allontanate, ed anche rovefciate in verfi contrarj alla comune direzione, o vifibilmente fprofondate, e innumerabili altri accidenti; a me non pare che poffanf ragionevolmente fpiegare, fenza ammettere il concorfo della fomma forza di fotterranee infiammazioni, effervefcenze, fufioni', eruttamenti, impetuofiffime rarefazioni dell' Elemento acqueo, e dell' aereo, e de' confeguenti Terremoti, follevamenti, efplofioni, e fubbiffamenti.

Queti fono $i$ mezzi principali impiegati dalla natura anche nella noftra età, per diverfificare, or quinci or quindi, e dare nuove forme alla faccia della Terra; dove alzando nuovi monti, dove gli antichi orizzontali ftrati di marine depofizioni in mille guife alterando; dove follevando da' profondi Abifli Ifole, $e$ Scogli, o quelle efiftenti fommergendo; dove pianure e montagne innabbiffando, e formando nuovi Laghi, c profonde Voragini. Di fimili avvenimenti pur troppo abbiamo recenti orribili memorie : e di quelli accaduti ne' Secoli remotiffimi, veggonfi gli effetti, e copiofiffimi, in ogni parte del noftro Globo. Effi fono tali, e così copiofi, e univerfali, che il grande ed efpertiffimo Offervatore Lelbmann, poftofi a farvi ri-

$$
\mathrm{P} \mathrm{p}_{2} \text { Alto, }
$$


300

A $\mathbf{T}, \mathbf{T} \mathbf{I}$

flefto, efclamò $=$ que veftigia, nunc borrenda, nune admiranda, revolutionum innumerarum, quas. Tellus nofire variis modis experta eft! $=(1)$.

(1) In Ppecim Orographie s762. Typis Acad. Petropolitano.

* * * * * * * *

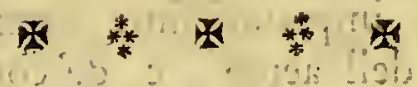

* * *

雨

OSSER: 


\section{1 \\ O S S R VA Z I O N I \\ S. O P. R A. \\ I L V E S U V I $\quad \mathbf{V}^{r} \quad$}

DELDOTIOR

\section{O M E N I C O B A R T A L O N I}

PUBBLICO RROFESSORE DI FISICA NELL' UNIVERSITA' DI SIENA;

E SEGRETARIO PERPETUO DELL' ACCADEMIA.

$$
\begin{aligned}
& \text { (E) } \\
& \text { S E I O N E I. }
\end{aligned}
$$

De' Vulcani in geñerale, e delle loro Accenfioni.

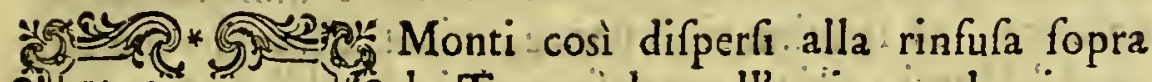
9. 1 (a) Terra, che nell' anime volgari non (S: rifvegliano altra idea, che d' un am* 1 . I. mafo di terra fopra terra, o di pie(19) tra fopra pietra diverfamente poi (4) 2 nella mente del Filofofo, il qual ne -2(3) (3) ch' ammira la fomma Maeftria, con cui nel Terraqueo Globo vengon difpofti, e chi infiem confidera la mifteriofa interna ftruttura, rifvegliano la giuftiffma idea d'effer la formazione loro, una delle più ammirabili opere del divin Creatore.

Pare di fatto, che la Natura: quei difponeffe come tanti punti d'appoggio, o' fortegni ftabili per le altre-parti, che la Terra compongono; e percio ancor deftinati come neceffario mezzo per l' efecuzione delle 


\section{2}

A $\quad$ r $\mathbf{T} \quad 1$

invariabili naturali leggi, affinchè fi manteneffero nell'Univerfo le perpetue vicende, e funzioni, che quotidianamente fotto il Cielo apparifcono, e che le veggiamo a vicenda perire, e porcia di nuovo nafcere, e riprodurfi.

Cosi realmente addiviene; l' innumerabili vantaggi , e commodi, che dalla fuperficie terreftre ricavanfi, tirano la prima loro forgente dalla pofizione de'Monti. L'opra più perfetta della Creazione, chè è l'Uomo, la prodigiofa quantità d'Animali ; ogni pianta, e minerale qualunque; e tutto quanto in fomma, che popola quefto Mondo, riconofce il fuo vivere, e vegetare, e la neceffaria natural confervazióne dall' efiftenz2 de'Monti; e bafti il dire, che quefta vafta eftenfione della Terra; abitata da innumerabile moltitudine di animate, ed inanimate foftanze, nient' altro farebbe fenza de' Monti, ch' un arenofo, ed inabitabil Deferto, o an Globo intiero, d'ofcuro, e lugubre Mare coperto.

La converfione de'Monti in Vulcani egli è un rifultato delle meccaniche leggi ftabilite nella Creazione, e perciò non è già ella un' opra indrizzata dall'ultrice mano d'Iddio per punire i noltri errori, confor-: me il comune delle Genti lo crede; anzichè sfe l' efame della parte fifica non ci poneffe in dovere di abbandonar le rifleffioni fulle caure finali, farebbe facile il dimoftrarvi, ch'i Vulcani fono una Maeftria della Natura, per cui maravigliofamente alla ficurtà noftra, e del pari ai noftri vantaggi provvedef, quantunque vero fia, che quelle proffime regioni efpofte alle loro ardenti furie, fieno nel primo impeto degli incendj fommamente dannificate

\section{Mon-}




\section{Deli' Accademia .}

I Monti convertiti in Vulcani prefentano in fatti fotto degli occhi noftri un bizzarro, e particolar Fenomeno, che giuftamente per non effer cosi comune nell'ordine delle create cofe, a coloro, che per addentro gli Arcani della Natura non mirano, ha in ogni tempo rifvegliata la maraviglia, come di cofa fuor de' limiti delle naturali. leggi, e riftretta foltanto nella sfera 'de'fatti Atraordinarj del Cielo.

Comunemente allora mutano il nome di Monti in quello di Vulcari, o Monti ignii-vomi, comecchè dentro il lor feno un infernal fucina nafcondono, e manifefan quindi alla faccia della Terra, di tanto in tanto alcuni frepitofiffimi incendj:

Moltiffime fon le. Storie, le quali partitamente defcrivono i Fenomeni de' Vulcani, e fpecialmente del Vefuvio, di cui è noftra intenzion favellare; ma ficcome più del bifogno a me pare; che fi fiano adoprati gli Autori nel raccontar femplicemente $\mathrm{i}$ fatti, c defcrivere unicaniente $\mathrm{i}$ Fenomeni; e poco eftefi nel cercare dei fatti, e Fenomeni le ragioni; o fivvero alcuni groffolanamente cercandole, han poi adottati incongruenti fiftemi; ho creduto non fuperfluo far fopra a quegli incendj le mie qualunque fiano offervazioni, ed infieme indagar de' Fenomeni le vere, e reali cagioni; perchè oltre ad effere $\mathrm{i}$ Vulcani un oggetto di femplice ftoria, e di ftoria, clie la noftra curiofità mirabilmente intereffa, intereffano pur anche una parte fcientifica della fifica, avvegnachè dai loro Fenomeni inferir polliamo, confermare, e porre in maggior lume molte fifiche verità. 
Se d'un Vulcano parliamo, fi parla di tutti; poi. chè gli effetti de' loro incendj fono affatto confimili, , $c$ provenienti dalle fteffe cagioni. Or dunque prendendo ad efaminare il Vefuvio, Vulcano ormai in Europa si celebre, fituato poche miglia diftante da Napoli, e fu cui per molti: anni ho avuta la comimodità d'offervare; potremo con ragione applicare a qualunque altro Vulcano del Globo gli ftefi argomenti, e ragioni. Prima però di paffar oltre, fia lecito premetter le feguénti nozioni fopra la Terra, fu il meccanifco delle accenfioni, e fu i Vulcani in generale, a fine d'opportar chiarezza maggiore a' noftri ragionamenti :

I Naturalifi dividono quefta groffa maffa di Terra fu cui abitiamo, in tre diverfe parti, o regioni. La prima è la parte efteriore, dove gli Animali ${ }_{1}$ nutrifcono, e vivono, e dove vegetano, e crefcon le piante. La feconda è l' altra, che: fegue dopo la prima, cioè la più inferiore, e pofta nel mézzo, e la quale può confiderarfi la fede, o regno de' foffili, e mineraIi, che ivi ftan difperfi in varie, e differenti altezze. La terza regione è la parte più interna, o centrale del Globo, che per effer fra tutte l'altre la meno nota, o per meglio dire affatto incognita", ha dato luogo a bizzarre oppinioni, fulla natura del fuo compofto, fu la fpeciale di lei ftruttura, e fu i diverfi corpi, ch' in quefto cieco abiffo hanno fede. Vi è chi ha creduto, ch'ella fia una fterminata mole di Calamita; altri una maffa vetrificata dal fuoco; altri un globo, o sfera infuocata; ed altri a contrario, che fia piuttofto dell'acque un cupo abiffo; e finalmente vi è pure chi vuole, che 


\section{Dell' Accademia.}

che colà fiavi un vacuo, ovvero fpazio con altra Telra popolata, a fimiglianza della noftra, dominata dagli Aftri, e dalle altre vicende confrmili a noi.

La parte prima, o fuperiore, quale è la fola perfettamente a noi nota, $f^{2}$ deve riguardare come una crofita, o corteccia del Globo, che s'eftende in profondità dove piu, e dove meno, ma generalmente partardo da dodici, o tredici piedi all' incirca. Viene querta ordinariamente chiamata terra comune, o materia terreAtre, ed intefa futto l'ufitato vocabolo di fuperficie. Il di lei compoits è un aggregato di particelle d'ogni fpecie di corpi; cioè di terra pura, di parti metalliche, di fpiriti oleofi, e bituminofi, e d'ogni altra forta di íali, e particelle di tutti gli altri elementi in Natura; e vi ha maffimamente una prodigiofa, e quafi immenfa quantità di femi d'ogni forta. Tutte quante quelle parti di differenti foftanze affumono fra di loro una tal qual determinata coefione, e coftituifon quindi come un depofito comune, dove la Natura conferva i materiali, per dare l'eftere, e la forma a moltiflime delle fue produzioni.

Ella è per appunto quefta cosi diverfa compofizione, ed affociazione di parti, che genera poi quella varietà, che fcorgefi nel fuo tutio; la diverfa quantità, le qualità differenti de' componenti, fanno confeguentemente varin della medefima il pefo, diverfo il colore, c diverfifima la fertilita per la vegetazion delle piante, e dello fviluppo défemi. Manifeftifima cofa è dunque, che quefto terreftre fuolo, il quale fotto i piedi calchiamo, effer debba affolutamente il germe da dove fcaturifono tutti $\mathrm{i}$ prodotti, e la potifima cagion in-

$$
\text { Q } q
$$

ficme 


\section{6 \\ A $T$ T $T$ T}

fieme delle fucceffive mutazioni, a cui foggetti fono tutti gli Efferi, che vi nafcono, e vi foggiornano. La varietà parimente nella quantità, e qualità delle produzioni, la loro confiftenza, e mollezza, le varie affezioni, e ftati d'ogni Eflere organizzato, fucceder devono a feconda dell' terreno, che produce, e foltiene. La natura dell' aria atmosferica, mifchiata colle efalazioni terrene, e con $\mathrm{i}$ vapori, $f i$ modifica anch' effa in gran parte, a feconda della natura del fuolo. E cosi le mutabili ftagioni de' refpettivi Paefi, i diverfi fenomeni, ed apparenze nel Cielo, i tempi varj dell' ambiente nafcono in parte da quelle efalazioni, e vapori, da que'principj volatili, e da tutto in fomma, che la fpeciale indole del fuolo continuamente efalta nell' atmosfera. Ne deve ancora da ciò feguire, che lo fteffo Clima, o vogliam dire la parte celefte, che domina ful terreno, benchè dipenda eziandio dal caldo, e dal freddo, $\mathrm{i}$ quali da altre cagioni nafcer poffono, nulla dimeno rilevar deve la maggior fua dipendenza dalla coftituzione del fuolo; ficcome poi a vicenda reagifce il Clima, nel formare di quel medefimo fuolo la fpeciale coftituzione.

Ed ecco quali fono i veri influff , che hanno almeno la principal parte ed azione fu $i$ movimenti, e funzioni dei corpi, e fopra tutte le produzioni, e confervazione degli Efferi, e che affoggettano gli Animali tutti a certe affezioni, temperamenti, e malattie, e che influifcono in fomma fopra tutto ciò, che ha relazione nelle naturali vicende, La nutrizione, che mantiene $\mathrm{i}$ Viventi, e che muta, altera, $\mathrm{e}$ modifica gli flati attuali del noftro corpo, ha la fua forgente nella terra, che calpeftiamo, e nell' indole di quel Clima, che 
che ci fovrafta; e perció feco porta gli effetti connaturali alle cagioni, le quali fon ripofte e nella Terra, e nel Clima.

Quefta fuperficie dunque, ch' appena riguardiano come un punto d'appoggio per farvi camminar fopra la nottra macchina; o al più per quel folo fine, che ai Viventi prefta la fuffiftenza, fe con lo Spirito filofofico la prendiamo ad efaminare, riconofceremo, che in quei dodici piedi di profondità d'inerte materia, vi c̀ l'origine della fpecial natura d'ogni Animale, vi è la forgente della falute, e de'morbi, vi fono le cagioni delle variazioni del viver noftro, vi è il germe dell inclinazioni particolari, e dalle umane paffioni, e vi è ancora nafcofta la principal caufa di molte operazioni degli Uomini .

Non fappiamo peró fe la Terra ufciffe dalle mani del Creatore con quefta defcritta crofta, o fuperficie carica d'innumerabilì, e diverf mifti; il fatto è affai difficile a verificarf, effendoci totalmente ignote le rivoluzioni del noftro Globo. Pù̀ effere avvenuto, che nel correr de'tempi fia la medefima a poco a poco talmente crefciuta, e che continuamente fia anch per crefcere, per le ragioni feguenti. L' efalazioni dalla Terra ognor fi diftaccano a forza del calor del Sole, e del fotterraneo fuoco avunque ditperfo; i vapori parimente fi efaltano di continuo nell' atmosfera; allorchè dunque dalla loro gravità fpecifica forza è che cadano, avranno potuto con fucceffione di tempo contribuire alla coftituzione di quella. Le acque piovane ancora, che da'luoghi più elevati fvellono le materie, e feco loro le portano, dove le circoftanze il permettono,

$$
\text { Q q2 l'avran- }
$$


l' avranno pofcia depofitate ne' refpettivi luoghi, e farà accrefciuta in tal guifa la fuperficie; e quefta è forfe una delle ragioni, che vi può avere avuta la più gran parte. Almeno di fatto così può fuccedere, e gli efempi pare, che non vi lafcin dubbiezza; de'quali per non addurne moltiplicità, un folo ne porteremo, con cui fenfibilmente fi vede, ch'in pochiffimo tempo capaci fono di riveftirfi di nuova crofta $\mathrm{i}$ terreni. La liquefatta materia, of fian le Lave, che fgorgano dal Vefuvio, allorchè furiofamente árde, e che rigogliofo nel fuo interno bolle, coprono alcune volte fpaziofe pianure, $e$ formano quegli ftrati sì comuni, e noti in quei contorni; ma fegue, che dopo qualche anno per le nuove fue accenfioni, cadendo fopra a quelle Lave della cenere, e delle altre terreftri parti, e per le nuove effervefcenze fotterranee ricadendovi l' efalazioni, ficcome le confuete acque, e vapori, ben prefto quelle Lave di nuova fuperficie $f$ conprono; e quefta inoltre ricompenfa coll' eluberante frutto $i$ danni apportati da quelle Lave fterminatrici. Ercolano ancora Città nota, e nuovamente foperta era un cumulo di pietre, e frantumi di Edifizj, ricoperti da molti Atrati di Lave; ma la medefima addotta cagione ricolmata l' avea col tempo, e ricoperta di fuperficie; coficchè quello fpazio, che fu una volta occupato dall' atmosfera della Città, l'abbiam vifto ai tempi noftri convertito in una campagna fertile, ed ameniffima. Per la qual cofa, fe nel primitivo Globo applicar dobbiamo un confimile andamento; cioè fupponendo, che la Natura per produrre gli ftefli effetti, fi vaglia fempre di alcune fole, e determinate caufe, parrebbe che prima foffe ftata creata 
la Terra, come il Nucleo, e pofcia; che fopra di effa crefceffe, con proporzionata fucceffione di tempo la fua crofta, o vogliam dire quel comun depofito, in cui vi è il germe d’ogni fertilità, e d'ogni naturale vicenda, come poco anzi fu detto.

I foli primitivi, ed alpettri monti fono della medefina affatto fpogliati, e perciò fterilifimi ; ed invece di quella altro non ci prefentano, che fcofcefe balze, e dirupi. Gli altri monti di altezza minore ne fono più, o meno coperti, fecondochè le circoftanze locali richiedono. Nón è, che fopra di effi non poffa egualmente crefcervi; ma la maggiore azione dei venti, i geli, e le nevi, che con più facilità vi agifcono, le acque che precipitofe vi fcorrono, o non danno luogo, che di quella fi coprano, ovvero coperti con più facilità gli fpogliano: Le fotterranee effervefcenze debbon dunque perfezionarli agevolmente nei monti; devono quindi eccitarfi più frequenti in effi i fenomeni; e farà perciò una delle potiflime ragioni, per cui nei foli monti nafcono $i$ Vulcani, ficcome avremo occafion di vedere.

Sotto quefta defcritta fuperficie vi è, come fi diffe, la feconda regione della Terra, ma differentiffima dalla prima ne' fuoi componenti, e ftruttura; ̀̀ deffa formata a Arati, ovvero letti; o fedimenti di diverfe materie compotti, feguendo uno dopo dell' altro, ma ciafcheduno in ifpecie, compofto per lo piu in tutta la fua eftenfrone, e profondità delle foftanze medefime. Sono tutti quefti Atrati paralelli all' Orizzonte, o almeno poclifinmo all' Orizzonte inclinati. Da molti, e celeberrimi Autori vengono accuratamente deścritte le mate- 
rie fpeciali componenti ciafcheduno di loro; e noi a fuo luogo farem menzione foltanto di alcune di effe, in quanto che hanno una neceffaria relazione con $i$ Vulcani.

Ognun fa quanto diverfe frano le oppinioni de' Filofofi full' Origine, e ful come fiano quefti Atrati cosi regolarmente formati. Quella però che più fodistá, e feguitata dai moderni Naturalifti, corroborata dalle offervazioni fu le rifpettive materie componenti, e da altre offervazioni ful Globo, ella è. la feguente. Convengono, che due fpecie di monti fia d' uopo riconofcere fopra la Terra; alcuni, che primitivi fi dicono, perchè nati infieme col Globo, ed altri chiamati monti nuovi, perchè con fucceffo di tempo crefciuti. Quei primitivi vengon riguardati, rifpetto al tutto delle altri parti terraquee, ch' abbiano gli fteffi ufi, che i folidi del corpo noftro hanno colle altre parti feco loro affociate; e quindi fiegue, come d' affoluta neceffità, che aveffero con quelle parti contemporanea origine. Per l'altezza maggiore, per effer di fuperficie fpogliati, per la figura piramidale, per la ftruttura interiore, $e$ per le qualità di materie, che al di dentro contengono, e finalmente per la loro unione in Giogaje, o catene continuate, credono poterfi quefti diftinguere $\mathrm{da}$. gli altri monti moderni, fucceffivamente nati.

Credono inoltre, che l' acqua del Mare abbia coperta per numerofa ferie di anni quafi la faccia tutta della Terra, fuorchè le primitive montagne già dette. Se vi era Mare, vi era ancora per le immutabili leggi della Natura, il Fluffo, e rifluffo, e gli altri movimenti dell' acque, i quali da ambedue neceffariamente dipendo: 
pendono. Sicchè le acque col lor moto dovevano diftaccare dalla fuperficie di que'monti, e dal fondo fterfo del Mare diverfe materie terreftri. I corpi urganizzati parimente, gli alberi, e piante della Terra faran fervite ancora in que' tempi di preda all' onde burafcofe del Mare, ficcome oggidi pur fegue. Frangendofi poi quefti corpi da i replicati urti, e molti di loro fciogliendo! ne' primi componenti a forza dell' aqueo umor diffolvente, fi depofitarono in feguito, dove le forze motrici, e la ragion del fito lo permettevano; diedero in tal guifa origine a quelli ftrati, o fedimenti fuddetti; i quali poi foprapponendor l'uno all'altro, ereffero $\mathrm{i}$ nuovi monti, e le nuove altre parti del Continente. Frattanto, o fia avvenuto ciò naturalmente, o per rivoluzioni, che cambiar faceffero l' inclinazione dell' Affe della Terra, e con effo il di lei centro, e per confeguenza fi mutafe l'antico letto del Mare; l'acque fi ritirarono, lafciando allo fcoperto. le depofitate materie, come per appunto avviene allorchè dal lido ritirafi la procella. E ficcome prima nuotavano, e difpofte erano a feconda dell'ondeggiamento, e del moto dell' acque, che per loro natura tendono a livellar$f_{1}$, ed equilibrarfi egualmente per tutto, così lafciarono quelle depofizioni, o letti collocati orizzontalmente nella forma, che oggidì offerviamo. Comparvero prima $i$ nuovi monti, o fu i primitivi appoggiati, o pur da fe foli ifolati; comparvero le colline, fpuntarono l' Ifole, pofcia le valli, e pianure; e coll' andare de'fecoli s'acquifto quefta gran parte di Continente, che poffediamo, cangiandoli gli algofi abituri di Moftri marini, in 
quei fuperbi Palazzi, ch'oggidi fanno il fafto, e la gloria de' Grandi .

Egli è vero altresi, .. che il tempo di quefte fiffatte depofizioni, ficcome quello del ritiramento dell' acque marine, non può cffere ftato che lunghiffimo, come ognun ben vede; ma la maggiore, o minor durata di tempo fufficiente argomento non è per abbatter la ragion d'un Filofofo. Ragion perfuade, che l' acque furono al certo quelle, che accumularono uno fopra l'altro gli ftrati, perchè il meccanifmo defcritto egli è quel naturale, che. l'acque fteffe producono; dovrem dunque a fpefe di verità cosi luminofe, limitarci ad una riftretta durazione di tempo? non lia mai vero.

Quefti tali ftrati così orizzontalmente difponti, ftabilifcono convincente prova, che generalmente $i$ monti non poffono effere ftati formati da' Vulcani, conforme alcuni con poco accorgimento afferifcono. Verifimo $\grave{e}$, ch' avvene alcuno, ma di piccola altezza, o vogliam dire come un nulla confiderato rifpetto al compleffo degli altri, il quale realmente da' fuochi fotterranei è ftato creato; ed il monte nuovo di Pozzuoli, ed alcun altró che riferir tralafciamo, entrano in quefto numero. Ma égli è da notare, che la loro interna ftrutcura differifce effenzialmente dalla ftruttura degli altri monti. I Vulcani con forze difuguali, e fenz ordine sbalfano in aria quelle materie terreftri, ch' arfe, ed abbruftolite fi ravvifano; onde è che non fono cosi difpolte regolarmente a Atrati orizzontali, qualmente ne' monti naturali fi vedono; ma apparifcon bensì gli ftrati fcompoAi, o circolari, o troncati; reguendo appunto quella 
direzione, che la violenza del fuoco fotterraneo richiedeva; conforme a vifta di chiunque fono efpofti gli Atrati del furriferito monte nuovo di Pozzuoli. Per lo che quei monti si pochi di numero, che dai Vulcani ebter l'origine, in vece di addur prova, ch'in tal gui$f_{a}$ il refto delle montagne della Terra poteva fimilmente formarfi, a contrario con evidenza dimoftrano, che quelle non nacquero, mai da' Vulcani. 'S'aggiunga ancor di più, che per cofa indubitata tutti $i$ Vulcani del Globo hanno avuta l'origin loro da'monti ; or dunque fe $\mathrm{i}$ monti fon quei, che producono $i$ Vulcani, è forza di ragione, che noi crediamo effere $\mathrm{i}$ monti anteriori di tempo ai Vulcani; qualora non G voleffe pervertito l'odine invariabile della Natura, che quelli pofteriormente nati deffero vita a chi loro precede; o fivvero che gli effetti foffero in ragion di tempo ariteriori alle loro cagioni.

Eccoci per tanto arrivati alla materia da cui tirano la forgente loro $i$ Vulcani; Son quelli Atrati di materie diverfe, da' quali ancora prendono la forza della loro durata, ficcome l' ampiezza della loro eftenzione; e ciò in proporzione della maggiore, o minor copia di corpi difpofti agli incendj. I Bitumi, gli Olj, ed i mincrali tutti fon le foftanze piu opportune, tanto per eccitare, che per comunicare le accenfroni, come pure per proccurare delle altre foftanze tèrreftri la combuftione. Quefti minerali ttanno alla rinfufa fparfi nelle vifcere de'monti, comecchè più in efli, che in qualunque altra parte della Terra fi generino, per alcune ragio$n i$, che ci difpenferemo quì riportare. Sono eziandio $i$ 
minerali promifcuamente involti in ogni maffa; fon difpofti fenza ordine in quelli ftrati, e fe ne trovan anche degli Atrati intieri, i quali, o probabilmente furon creati col Globo, oppure pofteriormente dalle nuove depofizioni fopraddefcritte. Ne abbiamo un efempio nella Solfatara di Pozzuoli, poco diftante da Napoli; quefta comprende lungo tratto di paefe fra valli, e colline, le quali altro non contengono, che monti; o ftrati fopra Atrati di puro Zolfo; quale è giuftamente la ragione; per cui da tempo immemorabile, vedonti in quei contorni dell' effervefcenze, ed accenfioni fotterranee, 'che poi con bizzarra fimetria formano ivi come tanti fotterranei cammini, continuamente fumiganti.

Però non folo quefte materie incendiarie de' Vulcani, ma qualifia natural corpo, s'e é non foffe da un primo agente moffo, e fcompofto, tutto refterebbe in perpetua quiete, nè avrebbemo mai in natura fenomeno alcuno; e quefta terreftre mole, che prefenta ogni di tante variazioni, e vicende, le quali difpongono quel bello, e maravigliofo del Mondo fifico, e che hanno la maffima parte ancora negli eventi del Mondo morale, in tal cafo altra moftra di fe non farebbe ', che d' un anmaffo inutile d'inerte materia. Porta feco ogni corpo, dall iftante della fua creazione, certe effenziali proprietà, o vogliam dire forze inerenti, capaci d'agire; ma affinchè entrino in azione, e forger-facciano quell' armonia, che il meccanifmo loro richiede, fembra che ne' primi componenti penetrar vi debba un general motore, che promuova il moto, che fepari, e difgiunga le parti coftituenti, e che in fomma avvenga di quei medefimi corpi una totale fcompofizione. Scio- 
Sciogliere dunque, e fcomporre neceffariamente fi devono quelle materie terreftri, che come parti d'un monte, forman pofcia un Vulcano.. Per ben intendere d'un tal fenomeno il principio, profeguimento, e fine, farà d'uopo feguir la Natura nell'opra; coficchè lei fola prendendo di mira, ci sforzeremo d'intenderne il fuo procedere, feguendone fedelmente le traccie.

L'efperienze d' accreditati, e moderni Filofofi han fatto vedere, che quel principio, il quale dà l'unione alle parri coftituenti ogni corpo, altro non è, ch'un principio volatile, e fugzitivo; ed è giuftamente quell' aria detta fiffa, e fparfa in ogni foftanza corporea a noi nota, la quale fta ivi in ripofo, fenza energia, e fenza attuale azione delle fue forfe elaftiche, fino a tanto che da qualche motore fia di colà fprigionata. Ella è come un legane, che per fua natura richiama all' unione fcambievole le particelle de' mifti, e prefta con ciò opportunità alle forze d'attrazione inerenti nei corpi, affinchè col loro efercizio produchino pofcia la neceffaria coefione. Sicchè l' aria fifla è quel principio, che fa fcaturire da' corpi le forze attraenti, e però fi conferveranno le parti tutte aderenti fra loro, in quanto che s'impedifce l' efito di tal principio, che le tira all' unione. Di fatto cosi fuccede; replicate fperienze confermano, che tofto che da qualche cagione venga quell' aria fprigionata dall' intima telficura de' corpi, riacguifta la fua naturale elaficità, divide, e rome con effa i legami delle particelle unite; e le forze dé corpi allora entrando in efercizio, trafportano in diverfe combinazioni le parti fuddette, fi movono quindi in varie guife; velocemente fl agitano; ed oprando vie piu infieme, è forze ela-

ftiche 
Atiche dell' aria fviluppata, e forze agenti de'corpi, ne rifulta una certa agitazione in ogni minima parte de'loro componenti.. Vi è in ogni maffa frammifchiato dell'aqueo umore, ficchè ajutando egli la difgregazione, per la fua naturale fluidità, ecço finalmente, che da tutto ciò entra in ogni particella corporea una tal quale veemente agitazione, o vogliam dire moto inteftino. Quefto inteltino moto, fe parlafi de'corpi animali, e vegetabili infreme, fi chiama dai Chimici Fermentazione; Se poi fi parli di minerali di differente fpecie, fi dice allora Effervefcenza. L'effervefcenza dun. que ella è deffa, che fa al cafo noftro; ed è quel primo paffo, che direttamete fa la Natura, per convertire $i$ monti in Vulcani.

Ma quale effetto mai produrrebbe l' effervefcenza, fe non vi entraffe di mezzo qualch' altro corpo movente, che totalmente fcomponeffe ogni parte integrante, e coltituente $i$ corpi, e che colla fua efficacia, e attività rifultar faceffe ulteriori fenomeni? Null' altro credo io, che quella continuata agitazione, bollore, o gorgogliamento delle parti molfe, ed in quefto avrebber forfe fine le fcompofizioni in Natura. Ma con fomma provvidenza creò Iddio un general motore nell' Univerfo, il quale ficcome fa parte componente i mift, così egli è la principal caufa della loro fompofizione né primi componenti. Quefto è l'Elemento del fuoco, o vapore Elettrico, come altri crede, fe pure foftanzialmente non fieno ambedue la cora medefima. Si vuole dai Filofofi, ch' il fuoco fia quel folo fluido per effenza, ch' egli rimanga perciò continuamente in moto; e che fia fola cagione della fluidità degli altri; che manten: 
ga pur anche difunite le particelle d'ogni corpo, affinchè il tutto non fi riduca ad un' intiera maffa omogenea, e di perfetta durezza.

Vien confiderato quefto Elemento da' Chimici moderni in due differenti thati, ch'egli affume fra la prodigiofa quantità de' corpi dell' Univerfo; il primo c̀ quello d'allorchè entra in qualità di principio, o di parte coftituente nella compolizione de' corpi ; e forma perciò colla fua combinazione, ed unione, quella parte infiammabile, o combuttibile d'ogni- foftanza; ed allora vien detto comunemente Flogiftico. Il fecondo ftato, in cui fi ritrova è $d$ 'allor quando non coftituifce alcuna parte de' compofti, ma che fpazia liberamente colla fua natural mobilità, come padrone dell' Univerfo, per tutta la Macchina mondana; fi move per ovunque, penetra con attività incredibile ogni corpo più denfo; e duriflimo, e nel tempo fteflo, ch' ogni foftanza al fuo dominio è foggetta, non ritiene egli parzialità per alcuna, pofciachè fi diftribuifce egualmente in tutti $i$ corpi a noi cogniti. Si chiama allora fuoco elementare, fuoco libero, o fivvero in azione. Sicchè vedefi dunque, ch' uno, e folo vien creduto quefto Elemento, ma che riguardafi poi in differenti afpetti ; l'uno d'allorchè fta in quiete combinato cogli altri elementi; l'altro di quando movefi, penetrando, $c$ vagando per la materia, e pel vacuo egualmente; da' quali pofcia differenti ftati, differentiffimi ne provengon gli effetti; poichè nel primo compone cogli altri elementi $i$ cor$\mathrm{pi}$ in natura, e nel fecondo gli dillipa, e fcompone.

Dagli foazj celefti, fino alle più profonde vifcere della Terra, fcorre per ogni dove quefto mobilifimo. Ele-

mento, 


\section{8 \\ A $\quad T \quad r \quad I$}

mento, confiderato come fuoco libero, o fuoco in azione. Egli è deffo, che produce in noi la fenfazion del calore; ma quefta non fi ravvifa quando che refta quello diffufo nella fua totale eguaglianza in tutti $\mathrm{i}$ corpi . Subito che però da qualfifia cagione venga determinato a fcomporfi dal fuo equilibrio; per correre verfo un qualche corpo, o determinata parte, in certa data maggior quantità, o di accrefcer ivi maggiore azione, allora è ch' eccita in noi la fenfazion del calore, e fi manifefta parimente coll' altre proprietà, alla di lui natura fpettanti. Varij fono i modi, e procacciati dalla Natura, e dall'arte, per i quali puó raccorfi, ed aumentarfi quefto fuoco libero, fparfo ugualmente; ma fra quefti quel folo ricorderemo, che al propofito noftro conduce. L' effervefcenza, che confifte nel moto inteftino di varj corpi, ficcome poco anzi abbiam detto, neceffariamente racchiude un continuo attrito, o sfregamento di parte con parte. Ed ecco uno de' più efficaci-mezzi in Natura, per raccorre, ed accrefcere quefto elemento, o fia per neceffitarlo a correre in maggior copia, e con altrettanta azione fopra de"corpi ; ed eccoci giunti parimente alla vera forgente delle accenfori, e de'fenomeni tutti, che prefentano alla faccia della Terra i Vulcani.

Eccitata per tanto l' effervefcenza fra le materie opportune nelle vifcere de'monti, fon preparate quelle in tal guifa, e difpofte per effere dal fuoco fterminatore arfe, e diftrutte, col meccanifmo quì fopra indicato. Ma e chi mai farà, ch'il primo legger moto abbia impreffo in quelle opportune foftanze terreftri, affinchè l'effervefcenza rifulti ; c cioè affinchè l'aria racchiufa dia 
principio al fuo fviluppo? Quefto è ciò che brevemente confidereremo, per abbracciare l'intiera teoria delle accenfioni vulcaniche, vale a dire per bene intendere tutti $\mathrm{i}$ paffi, che la Natura move in queft' opra. La Chimica coll' arte fua a forza di acidi, e di laltri diffolventi, mifchiati nelle preparate materie, moltiffime ne eccita delle effervefcenze, fjccome è notiffimo. La fagace Natura non farà meno induftriofa dell' arte, ed in modi eguali, e forfe in molto maggiori ne ecciterà ancor ella moltifịme a fuo talento.. Ma per appigliarci ad una delle maniere più ovvie, e comuni nelle naturali vicende, ricorreremo all' acque fteffe cadute dal Cielo fu la fuperficie dé monti, le quali nell' interno di quelli facilmente penetrano, filtrano, e fi diffondono per ogni parte, conforme a tutti è notiffimo.

Sicchè offerviamo adeffo in un punto di vifta il meccanifmo intiero, che idalle invariabili naturali leggi rifulta, per convertirf una groffa mole di terra, in una infernal fucina di vivaciffimo fuoco. $\mathrm{Vi}$ hanno ne'monti degli Atrati intieri di minerali , vi hanno fra quelli fpecialmente fparfi de' Zolfi ; Bitumi e degli olj, e fali d'ogni forta, fecondo che venne fopra indicato, vale a dire, che vi hanno delle: materie -talmente dalla Natura preparate, per follevarfi in effe dell' attiviffime effervefcenze. L', acqua in varie 'guife caduta dal Cielo penetra in quelle fortanze, e per la gravità, e fluidità infreme s'infinua intimamente ne' refpettivi compofti, e maffe, e diffocia così le parti unite, ch'incontra. L' aria fifla ivi racchiufa da principio a! fuo fviluppo, e riprendendo la naturale elanticità, coopera infieme alla difunione delle parti anzidette; le quali 
quali refe ancor più fluide dall' umore acquofo, pafferanno $d^{\prime}$ azione in azione, da forza in forza, e da difunione in difunione, dall' efercizio infieme ajutate delle forze a loro inerenti. Quefte replicate vicende fe faranno comunicate ad una notabile quantità di materia, avremo il cafo d' una quantità proporzionata di moto inteftino di varj corpi, cioè una notabile effervefcenza; e quindi con effa un proporzionato attrito, o sfregamento di parte con parte. Ed ecco ch'il fuoco libero fparfo egualmente in quei compofti, accorre a quella parte, acquifta da ciò un' azione maggiore, e comincia a manifeftarfi coll' eccitato calore. Prende pofcia il calore il neceffario aumento, per la continua azione, e reazione delle materic, e perciò trapaffa ad infiammare le particelle oleofe, e bituminofe, che più dell' altre faciliffime fono a concepire la framma; da che fprigionandofi dalle materie infiammabili, o fia dal flogittico, altro fuoco elementare, e puro, trapaffando in tal guifa dallo ftato di fiffazione in quello di libero, ed in azione, fe ne accumula così copia maggiore, e tutto infieme efercitando le forze elaftiche, o vogliam dire crefcendo la fua azione, e coll' altra dell' aria già fprigionata affociandof, dilata, fquarcia, e rompe ogni teffitura di corpo, e paffa il copiofo fuoco ad inveftire, ardere, e diffipare ogni parte componente; e giunti fram finalmente a veder concepita nella maffa totale una grandiofa accenfione.

Se notabilmente debba infiammarfi però una maffa effervefcente, fa di meftieri, ch'abbiavi il concorfo dell' aria libera ; infegnandoci cosi l' efperienza, la quale fa vedere, che qualunque fiafi di ciò la ragione, necella. 
ceffariflima ella è per la combuftione de' corpi. Le caverne dei monti, le fenditure, che fpeffe in quelli ritrovanfi, danno facilmente l' acceffo a queft' aria; onde $\grave{e}$, che non vi ha dubbio, ch' effa non fia difperfa nel loro corpo in varj luoghi, dove le circoltanze il permettono. Or qui brevemente riflettendo agli andimenti della Natura nel meccanifmo delle divifate accenfioni, ci dà luogo ad ammirare la femplicità del difegno, e l'uniformità delle fue leggi, fémpre coftanti, e femplici del pari nell' opre fue ; imperciocchè di quei principj, ch'agli occhj noftri fembra ella valerfi nella compofizione de' corpi, di quelli egualmente, con ammirabile femplicità, $f_{1}$ vale per la loro fcompofizione.

Abbiam fuppofto in una più natural maniera, che l' acqua porga occafione, ed opportunità alle preparate materie de monti, per la loro prima difunione, o fivvero per difgregarfi l'aria fiffa, e quindi follevarfi l'effervefcenza; ma non fi afferma con ciò, ch'altro movente effer non vi poffa in Natura, il quale o unitamente coll' acqua fteffa, c feparatamente ancora, poffa egli cffer cagione di un qualche leggier moto nelle materie medefime, e produrfi nel modo divifato, ed effervefcenze, ed accenfioni. Il fuoco libero, per efempio, che fi tramanda continuamente dal Sole, e che colla fua attivita, e forza produce quelle vicende tutte ovvie in Natura, fe mai addiviene, che raccolto in una quantità nei raggi folari, penetri per qualche fenditura, o caverna dei monti, dove abbianvi a calo preparate le foftanze incendiarie, potrà al certo effer valevole, colla fua naturale mobilità, e calore, di promuovere l'effervefcenza; e quindi dall' effervefcenza forgerà l'accenfione. 


\section{A T T 3}

Similmente ancora l' altro fuoco libero, che nelle vifcere della Terra fcorre ovunque a piacere, può coll'ordinario fuo moto diftaccare molte efalazioni, né luoghi fpecialmente di minerali fecondi; fe poi quelle s'incontrino coll'aria libera, ed elaftica nelle caverne difperfa, ovvero che s'urtino, e reagifcano fra loro tteffe, facil cofa è che sinfiammino; ed incontrando materie bituminofe, ed oleofe, potranno attaccarvi alle medefime il fuoco, e forger talmente un incendio. D' efempj confimili d'effalazioni infiammate, ne abbiamo moltiffimi nelle miniere, come pure de' tuoni fotterranei fpeffo exccitati da quelle fteffe infiammate efalazioni.

Pertanto per generarfi i Vulcani quelti furriferiti mezzi fono i più confacenti alle naturali leggi, e dalle offervazioni di fperimentati Filofofi confermati ; ma ficcome non vi è fatto in Natura, che come recede un poco dalle cognizioni ordinarie degli Uomini, non dia luogo a mille favolofi racconti; così appunto per l'origine de' monti ignivomi, tanto gli antichi, che i moderni Scrittori hanno inventate diverfe, e favolofe ragioni .

Autori di grido, feguẹndo l' antico Geografo Strabone, han creduto, che $i$ Venti furiof, i quali per Io più fpirano dal Mare, a guifa d'un Mantice foffino per canali fotterranei in quelle maffe di minerali, e materie combuftibili, e ch' in tal forma quelle fi accendano; non riftettendo quefti, che dove non avvi prima l' attual fuoco, inutil riefce ogni foffio; e mercè della divina Provvidenza, che così avvenga, altrimenti avrebbero vifto più volte gli Uomini da' furiof, Venti ardere, e confumare la faccia del Globo. 
I Fiumi di fuoco attuale, immaginati da alcuni fcorrere per fotterranei canali; i Fulmini, che fcoppian dal Cielo, e che nelle caverne de' monti cader poffono; l'urto accidentale di pietra con pietra, occorfo per avventura nelle montane caverne, fono le altre maniere, credute con qualche ficurezza da molti, attiviffime per rifvegliare $i$ vulcanici incendj.

Nel bizzarro fiftema di Votward, le acque, che pone nel grande Abiffo, e ripiene di fuoco, colla loro agitazione ne poffono fprigionare gran copia, il quale. pofcia per quei fuoi ideati fotterranei paffaggi, può dentro i monti accendere la materia terreftre, atta alla combuftione.

Gli Aftrologi pure, come foliti a metter le mani ne' Cieli per ifpiegare gli eventi terreni, vi hanno mifchiati cogli incendj fuddetti, gli afpetti, e congiunzioni de' loro Pianeti; e quindi ne han trovata la vera cagione nell' Eforbitanza dell' ottavo circolo fopra il centro di Ariste, e della Libra, con altre fimili ciance, che puerilità farebbe il riferirle.

Tutti i Vulcani del Globo, tanto gli eftinti, che quei a' giorni noftri accefi, fono originati ne' monti; egli è farto incontraftabile, certificato dall' autorità, e dall' efperienza di tutti $\mathrm{i}$ tempi, e d'ogni, e qualunque luogo. Vero è che dell' effervefcenze, e dell' accenfioni, e quefte di varie fpecie, ne nafcono realmente ancora ne' luoghi piani : ma propriamente incendj, e incendj durevoli, e tali per la fpecialità de' fenomeni, che fotto il nome di Vulcani s'intendano, ella è certamente una fola privativa de' monti . Se la compofizione, e l'interna ftruttura della Terra, quella a noi

$$
\mathrm{S} \mathrm{S}_{2} \text { nuta, }
$$


nota, non foffe tutta formata a Arati, fenza èccezzione di pianura, o di monte, farebbe in pronto faciliffima la ragione di quelt' accennata diverfità dei provenienti effetti; ma troppe fon l' efperienze, e le prove, ch'addur quì tralafcio, le quali apertamente moArano, che tanto $i$ monti, quanto le valli, e pianure, tutte egualmente fono piantate con $i$ foliti ftrati, o fedimenti. Or dunque un effetto cosi coftante deve avere eziandio una ragione fempre fola, e coftante; e la quale nell' afpetto di tutte le altre vicende della Natura, eguali da per tutto nella Terra, si nella Atruttura interiore, che negli avvenimenti delle ftagioni, non pare, che poffa effer altro, che quella ai foli monti comune; quale è la loro altezza, o fia d'un ammaffo di terreftre materia, al di fopra delle altre parti del Continente, elevato.

Il celeberrimo M. Buffon Filofofo, e Naturalifta di primo ordine, ch' un tal punto da fuo pari mette ad efame, apporta di ciò una ragione, che forfe non farà fola a produr la fingolarità del fenomeno nei foli monti ; ma bensì però unitamente coll altre, può, a mio credere, concorrere mirabilmente. Quella è, che nei monti , per lo più effendo efpofta la loro fuperficie all' ingiurie dell' aria, e dell' acque, vi hanno per neceffità delle aperture, per dove le acque fteffe fcolano, e generano nel corpo di quelli $i$ Zolfi, ed altri minerali, corpi attivifimi a produrre effervefcenze, vale a dire a rifvegliare accenfioni.

Appunto così è; la circoftanza d'effere i monti fo. pra le altre parti. della Terra elevati, gli efpone neceffariamente ad un'attività maggiore dell' intemperie delle 
ftagioni. Ciò produce, che facilmente fi fpogliano della crofta, o fuperficie, che fopra offervammo, e perciò ne rifultano due principali effetti, i quali fi convertono pofcia in cagioni per eccitare $i$ Vulcani. Son quefti l'ingreffo dell' aria libera, e l'ingreffo dell' acqua nel ventre di detti monti. Perciocchè l' acque, $\mathrm{ch}^{\prime}$ in effi cadono trovando fcoperte le antiche naturali fenditure, e del pari altre formandone col loro precipitofo corfo, penetrano dentro di effi, e s'infinuano; e collo fcorrere, fcavano eziandio delle caverne, maffimam ente per gli ftrati di arena, che fra loro tanto compatti non fono; ed eccovi che l'aria libera dell' ambiente vi ha facilmente l'ingreffo, vi fpazia, e vi domina, per fervire di neceffario, ajuto alle combuttioni. L' acqua medefima puó confervarfi in molte delle: mentovate caverne, ed unitamente con l'altra, che per altre parti filtra, e per le fenditure continuamente trapaffa, - penetra ovunque, e fino alle radici fteffe de' monti; per lo che avremo con quella un mezzo da movere l'effervefcenze, in quella maniera già fopra efpofta. Se a tutto ciò fi aggiunga la facilità maggiore di formarf nelle fenditure, ed altrove i minerali, ficcome il prelodato Filofofo giuftamente riflette, raccorremo un compleffo di ragioni, le quali, come ognun vede, o poco, o nulla militar poffono per $\mathrm{i}$ piani Continenti. Poichè ricoperti quefti di fuperficie maggiore per i fedimenti; che I' acque vi portano, ed efpofti meno all' ingiurie del Clima, fon per lo più privi di fenditure, o caverne c qualora in alcuni ancor ve ne abbiano, dovendovi concorrere unitamente le materie cpportune, ovvero la continuata generazione di minerali, non facilmente s'in- 
contra il concorfo di tutte quefte ragioni, neceffariftme infieme per eccitare i Vulcani. E perchè ne' monti più alti, in parità di circoftanze, più agevolmente $f_{1}$. adattano le quì addotte ragioni ; così offerviamo, che i Vulcani ordinariamente non nafcono, che nei monti d'un'altezza fra le maggiori.

Domandano alcuni fe i Vulcani fieno tanto antichi, quanto è antico il Mondo; a me par di sì. Se la Storia piu antica confultiamo e facra, e profana, in ciafchuna di effe commemorazione chiariffima ritrovali dei Vulcani. La facra Bibblia in più luoghi gli accenna, parlando dei monti fumiganti, ed ignivomi della Terra. Omero fpeffe volte ricorda i Vulcani dell' Ifole Eolie, oggidi appellate $l^{\prime}$ Ifole di Lipari, poco dalla Sicilia diftanti. Ognun fa dall altra parte, che gli Uomini ne' loro ritrovati hanno apprefo moltiffimo dalle operazioni della Natura; quindi può inferirfi ragionevole congettura, che dai Vulcani imparaffero a perfezzionare il ferro, o fia parte dell'arte metallurgica. Quelt' arte poi quantunque per la complicazione, e difficoltà de’ fuoi precetti, fia piu di qualunque altra difficile, non oftante l'abbiamo dalla foria, d'antichiffima invenzione; ficchè effendo addattabile la rifleflione, certo farebbe, che d'antichiffima data fono i Vulcani. Dai noftri antecedenti difcorfi : ricavafi ancora, ch' effendovi fopra la-Terra i monti, efsere vi potevano egualmente i Vulcani ; e quindi farà ragionevol cofa, fe porteremo credenza, che contemporanei fiano al Globo ftefso; o almeno che poco dopo poffano effere inforti, colla fola dilazione di tempo, che le circoltanze richiedevano, per eccitarfi in quelli ed effervefcenze, ed accenfioni. A chi di fatto per 
un poco ha offervate le materic efteriori, ed interiori, che fir ritrovano in molti dei monti, ed in altre parti della Terra, non ignora, ch'in qualunque luogo fi cerchi, fi vedono manifeftamente i faffi naturali, le terre, e le materie abbruftolite, e liquefatte, e corrofe dal fuoco; vale a dire, che quafi per tutta la Terra fi fcorg ono reliquie di abbruciamenti, e Vulcani, de' quali non refta nelle Storie, nè nella memoria degli uomini alcun veftigio, perchè in tempi a noi remotiffimi eftinti.

Il Prete Inglefe Burnet, che per fervirmi dell' efpreffioni del lodato Signor Buffon, aveva rifcaldato il cervello, ci fa una fingolar defcrizione della Terra avanti il Diluvio; e con tanta ficurezza, e precifione l'efpone, che pare fenz' altro, ch' ei fia ftato uno di quei miferi antidiluviani periti nella comune fciagura. Per fuccintamente quì accennarla, egli dice, ch' era la Terra allora d' egual fuperficie, uniforme, ed unita, fenza montagne, e fenza mari, é fenza ineguaglianze; ma che ttette però così per foli fedici fecoli. Immagina dunque tu che Terra mai effer quella doveva; non cadevan dal Cielo le pioggie, Giove non feriva coi Fulmini, e quelle apparenze, e vicende, che dai monti, e dai mari dipendono, le quali fon forfe le maggiori, ch'avvengano nel noftro Globo, dovevano effere affatto incognite allora. Dunque fu quefta opinione non vi farebbero ftati avantiil Diluvio $\mathrm{i}$ Vulcani, perchè non portava monti la Terra, dai quali abbiam detto aver quelli l'origine, e perchè l'altre cagioni, dipendenti da varie naturali vicende, non erano allora in Natura. Era però un eccellente fuolo, che mirabilmente ferviva alla 
alla delicatezza di que' Popoli Antidiluviani, e felicifimi frattanto vivevano; nell' immaginazione almeno del Prete Inglefe, di cui lafceremo la rifcaldata Fantasia, per tener dietro alle cofe vere, e reali delle Natura.

\section{$S \quad E \quad Z \quad I \quad O \quad N \quad E \quad I I$.}

\section{Dello fato prefente, ed antico del Vefuvio, fue Accensioni, e Fenomeni.}

T'Effere il Vefuvio a vifta, ed in vicinanza di Città così illuftre, quale è Napoli, e quindi a portata d' effere efaminato da quei fagaci Concittadini, $e$ dal copiofo numero degli eruditi Viaggiatori, che colà vanno per vedere le delizie, che il Cielo profufe, $e$ che l'arte induftriofamente raccolfe in quella grandiofa, e fra le Città d'Europa popolatiffima, ella è la principal cagione, perchè egli folo rammentato fia dagli antichi, e moderni Scrittori, più di quel che lo fono tutti infieme prefi i Vulcani del Globo.

Sole otto miglia di perfetta pianura vi corrono dalla Città, fino a dove ha principio la bafe del monTab. 4. te, il qual refta precifamente all' Oriente di quella. Tale è la di lui pofitura, che quafi da ogni angolo della Città fi fcorge rilevato dal fuolo, nella figura d' un perfettiflimo Cono troncato nel vertice, da una fezione orizzontale, o poco almeno inclinata all'Orizzonte; conforme può vederfene tutto il profpetto nella Tavola anneffa, coll'indicazione dell' altre parti, che ora defcriveremo. Il vertice fuo in tal guifa troncato viene perciò a formare un ambito circolare, per cui in molti 
luoghi vi fi può commodamente camminare; ed il fuo giro 's eftende in circa a piedi $5624 .$, fecondo le più accurate mifure. Quefto circolar fuo giro coftituifce poi come un Orlo d'uno fpaziofo Bacino, o Cratere, conforme volgarmente lo dicono, il qual nient' altro è, che un largo fcavo interiore del monte, per cui dall'Orlo fteffo vi fi feende fino al fuo fondo, per il tratto di piedi I 40. all' in circa. Egli è quefto fcavo, o Cratere opera del fuoco confumatore, il quale ha bruciata, ed efpulfa la materia terreftre, ch'ivi contenevafi, e però non è uno fcavo ordinato, ma di figura irregolare, e di varia mifura nella fua difcefa. Nelle di lui pareti vi fi fcorgono gli ftrati orizzontali troncati, e divifi, per la violenza del fuoco, dall'altre materie terreftri, delle quali era ripieno un tal vacuo; onde è ch' a ben ragionare, era ancora il Vefuvio compofto a ftrati paralelli, come tutti gli altri monti lo fono; e perció farà mal fondata opinion di coloro, conforme più a baffo vedremo, che penfano effere egli apparfo qual nuovo monte dall' accozzamento di pietra con pietra, di cenere fopra cenere, e da tutt' altro, che la violenza del fotterraneo fuoco vi abbia gettato.

Nel fondo del Cratere fuddetto egli è per appun: to dove fono le bocche, o aperture dell' orrende caverne, che nell' interno, o vifcere del monte fi nafcondono; caverne convertite al dì d'oggi in un Abiffo di fuoco, o infernal Fucina, feconda di perpetue accenfioni, c di ftrepitofiflimi incendj. Quefto tal fondo fpefto fi muta, e comparice fempre come un piano irregolare, dalle materie abbrultolite, dalle Lave, e dai faffi naturali efpulfi nelle furie dell' accenfioni. Vi fi accum'sI. lano 
lano talvolta delle materie in gran quantiti, e poi ben prefto fparifcono; vi forge un monte, e dopo non molto tempo fi difperde; e così difcorrendola, rara cofa è, ch' in ogni incendio tu non lo trovi notabilmente alterato, sì per la qualità, che per la quantità de’ corṕi varj gettati .

Dell' efterna regolar figura del monte, fimiliffima al Cono, nè Strabone, nè Vitruvio, nè gli altri antichi Scrittori, ch' il Vefuvio ricordano avanti lo ftrepitofo incendio del 79. dell Era Crittiana, che fu il primo a noi noto, ne hanno fatta parola alcuna. Forfe non parve loro oggetto degno di nota, o forfe che non era in quei tempi un Cono sì regolare, come in oggi fi offerva; perciocchè le Lave fcorfe nelle fuffeguenti accenfioni, che come liquida materia dirigono il corfo ai luoghi più baffi, e quindi congelandofi pofcia fi arreftano, avranno coll' andare dei tempi riempiti gli fcavi, e le valli della montuofa fuperficie, e mutata così quell' antica fua faccia in quefta conica figura, ch' ai di noftri ritiene.

Dalla parte, che riguarda verfo il Golfo di Napoli, la quale è quella, ch'al Vefuvio gli refta a Ponente, puo dirfi, ch' ei ftenda le fue radici fino al lido del Mare; poichè dalla fpiaggia principia una dolce falita, che poi vie più erta, e penofa fi rende per poggiar fino al Vertice. Principiando da quefta fteffa parte, e profeguendo il cammino verfo le altre del Mezzogiorno, e Levante, rimane diftaccato colla fua bafe, e cosi folo rilevato dal fuolo; ma dalla parte, che reftagli a Settentrione ha egli le radici, o bafe comune con altri due monti, uno detto in oggi di Somma, e d'Ot- 
d'Ottajano l'altro. Si elevano dunque unitamente dal terreno nella comun bafe, quando poi fi perviene ad una certa altezza, fi diftaccano, dividendofi in due parti; una delle quali è quella che coftituifce giuftamente il Vefuvio, e l'altra forma $\mathrm{i}$ due furriferiti monti ; e da quella divifione di bafe, principia parimente il Vefuvio dalla parte Settentrionale ancora, a prender la figura di un Cono.

Gli altri monti poi fin dal principio della loro divifione $s$ innalzano con dirupi perpendicolari, le quali cingono lo fteffo Vefuvio in forma di mezza Luna, con un giro, che s'eftende da Settentrione verfo a Levante, da due miglia e mezzo in circa. Quefto tal giro forma come un gran Vallone, che divide giuftamente i fuddetti monti dal monte del fuoco; ed è largo per tutto quafi egualmente, in circa a piedi 2220 , dirigendofi in forma di femicircolo per tutta la lunghezza già detta, a feconda della pofizione delle rupi defcritte.

Il giro della tafe del Vefuvio può confiderarfi in due efpetti ; cioè e prendendolo dal livello del Mare, col cinger tutta quella bafe, ch' a lui, ed agli altri monti è comune; e prefo poi quel folo circuito, da dove fi diftacca da quelli. Allor quando nell' ultima Sezione ci proponiamo di mifurare la di lui folidità, avremo occafione di vedere, a quanto s' eftendano quefte due diverfe mifure, e vedremo infieme qual fia il perpendicolo prefo da ambedue le differenti altezze. Cosi fuccintamente defcritta la pofizione, e ftato prefente del Vefuvio, vedremo ora con breve efame qual foffe il fuo ftato antico, avanti la prima ricordata accenfione dell'anno 79. , fopra di che non convengono gli Scrittori fra loro.

$$
\mathrm{T}_{2} \text { L'AL- }
$$


L'Autore dell' erudita, ed elegante Storia di quel memorabile incendio del $\mathbf{1 7 3 7 .}$, incendio pari in grandezza a quanti fin' ora ne ricordan le ftorie, penfa in primo luogo, che quel che allora, cioè avanti il 79., dicevafi Vefuvio, comprendeffe un fol Monte, da capo a fondo, e con un fol Vertice, infieme cogli altri due in oggi diftinti, e poco anzi ricordati d'Ottajano, e di Somma. Secondariamente ei crede, che il Vallone, quale in oggi fepara, e diftingue $i$ due predetti monti dal Vefuvio, fia nato da qualche precipitofo fubbiffamento, per opera del fuoco negli incendj fuffeguenti, dopo il primo accennato del 79 . In terzo luogo vuole, che quel folo monte, ch' ai dì noftri lo chiamiamo il Vefuvio, fia nato dopo quefta cataftrofe di ruine, -e dalle materie efpulfe dall' accenfioni, e dal rigonfiamento della Terra, prodotto parimente dalla forza, ed energia del fotterraneo fuoco.

Ch'il Vallone defcritto fia ftato originato dalla violenza del fuoco, anzichè in effo foffero le fucine, $e$ voragini, da dove una volta faturiva un Vulcano, lo credo egualmente ancora io, e fenza efitazione alcuna. lo credo. Le materie abbruftolite di quelle alte rupi, che il Vallone circondano; la patina fulfurea, e bituminofa, che le ricopre; le buche interpofte, e dimezzate in quei perpendicolari fcogli, e le quali fenz' altro, formal non potevanfi fenz appoggio d'altra materia terreftre, e che fono, a mio credere, i recipienti dove il fuoco accendevafi; gli ftrati, che full altezza di quelle balze patentemente fi fcorgono, ed $i$ quali cosi per aria, e privi di punto d'appoggio, non pare, che formar fi poteffero nel fopra efpolto fiftema, e ch' an- 
cor di più evidentemente moftrano d'effere fati troncati, e dai medefimi fi ravvifa effere fvelte, e diftaccate altre parti di terreftre materia; fon tutti argomenti quafi certi, che una volta non eran quelle, come fono in oggi, rupi, e balze pendenti, ma bensì parti unite ad altra terra, dal cui compleffo probabilmente forgeva un fol monte, formato allora dai tre in oggi divifi, Ottajano, Somma, e Vefuvio; e quindi è ragionevole il credere effere il ricordato Vallone un prodotto dell' accenfioni vulcaniche. Ed è ancor vero, che le Lave fcoperte alla Madonna dell' Arco, riportate dal citato Autore in conferma di fua opinione, fono argomenti d'indubitate prove, che dove ora è Valle, foffe una volta Vulcano; poichè fe Vulcano era allora il prefente Vefuvio, e che da effo quelle Lave doveffero fino a colà deviarfi, impoffibil cofa egli era, come impoftibile è, ch'i liquidi per loro natura falgano ai monti. Per ridurre poi il fatto a convincente dimoftrazione, fol baAterebbe, che verfo la cima de' due fudderti monti'd'Ottajano, e di Somma, fi tentaffero fcavazioni, qualora ivi fi ritrovaffero gli ftrati delle Lave, come nel pendìo del prefente Vefuvio fi trovano, fvanirebbe certamente ogni dubbio, ed avremmó con affoluta: certezza, ch'il Vallone fú una volta la Fucina d'un Vulcano; non effendovi altra Atrada, per cui in quefta fuperficie dei detti due monti poteffero fcaricarfi le Lave.

Il tempo pero, in cui avvenne queita sì nocabilè variazione di continente, non farà fato dal 79 . in quà, ficcome opina il lodato Autore... Egli lo crede, perchè l' antico Geografo Strabone, che defcrive il Vefuvio prima, che appariffe quefto incendio, ficcome pure Dio- 
ne Sifilino, neffun di loro riccrda, nè $\mathrm{i}$ due monti fuddetti, nè il Vallone fopra defcritto; e quindi fonda la fua ragione per credere, che niuno di quefti allora vi fofle, e che debbanfi perciò aver tutti in conto di nuove apparenze, dopo il primo Incendio del 79 . Potrebbe tutto ciò effer vero, ma io tengo opinione di no; e mi perfuado, che molti Secoli prima dell' apparfo incendio del 79., una tal cataftrofe avveniffe.

Già abbiamo, che Vitruvio, Diodoro Siciliano, lo fteffo Strabone, ed altri Autori antichi, che avanti il 79 . fcriffero, accennano, ch' ai loro tempi fi riconofcevano nel Vefuvio le reliquie, ed avanzi del fuoco, e concordemente credono effervi ftato ne'fecoli remoti un Vulcano; ficchè non è lontano dal credere, che nelle accenfioni più antiche produceffe gli effetti accennati. E realmente il filenzio di Strabone nulla mi par che giovi a ftabilir conclufioni; ei defcrive da Geografo, e defcrive il Vefuvio come un monte di qualche confiderazione; dunque non doveva far parola nè del Vallone, nè di quei monti meno notabili, e feparati dal medefimo, anzi per lungo tratto divifi. Dione poi, che fcriffe nel, 228. dell' Era Criftiana, cioè molto tempo dopo del primo fuddetto incendio, egli pure defcrive il folo monte del fuoco, racconta i fuoi effetti, e le ruine di quello fteflo Incendic, nella Vita di Tito Imperatore, e niente ha che defcrivere intorno al Vallone, nè intorno agli altri monti circonvicini ; anzichè fe dobbiamo ftare ai fuoi racconti, che per incidenza riporta intorno al Vefuvio, pare a me, che lo dipinga tal quale ai giorni noftri apparifes; e per confeguen$\mathrm{za}$, che tutt' altro ancora fteffe in quello ftato, $\mathrm{ch}^{\prime}$ al di d’oggi veggímo. 
Si può eziandio foggiungere, che fe it filenzió degli Scrittori ftabiliffe una prova; con maggior ragione potrebbé afferirfi, che dal 79. in quà, tutto è rimafo nel fuo primiero ftato, nè alcuna variazione di monti, nè di Valle è per avventura accaduta; perchè tútti gli Autori, $\mathrm{i}$ quali han defcritte le fuffeguenti accenfioni, e che ci hanro parimente lafciato fcritto ogni fenomeno, ed ogni più minuto, e fpeciale accidente, non fanno mai alcuna menzione di fterminio così notabile, nè di fconvolgimento di monti, nè di un tratto di terra così ampio, fubbiflata, quantunque ciò forfe una parte neceffaria alla loro Storia.

Le Lave fteffe della Madonna dell' Arco a ben confiderarne le circoftanze, ed i luoghi, fe provano, che dal Vallone Vulcano ebbero una volta l'origine, ficcome coftantemente credo, dimoftrano altresì poi la remotiffima antichità di quefto avvenimento. Le Fabbriche, e Monaltero che ivi fono, contano 500. anni almeno, dal principio; ch' ivi furon gettati i lor fondamenti; e fotto di quelli fi ritrovan le Lave; per fino alla profondità di palmi napoletani $300 . ;$ or dunque offervate qual tempo mai deve effere fcorfo, che furon già fuperficie di quel fuolo. In Ercolano ricoperta di cenere nel primo incendio accennato, e ne' fuffeguenti ricoperta di ftrati di Lave, le più profonde non fi ritrovano, che di palmi 6o. fotterra; non oftante che fia Ercolano efpofta maravigliofamente alle falde del Vefuvio, per foffrire gli effetti d'ogni fua furiola accenfione. Sicchè dunque le Lave della Madonna dell'Arco, ch' è un luogo dove certamente da gran tempo non vi arrivano, un luogo, il qual niente è efpofto ad effer ricolmato da' getti del 


\section{$33^{6} \quad$ A $\mathbf{T}$ I}

del.Vefuvio,"e da altre Atraordinarie cagioni, non pare; che non volendole fcorfe prima di quelle d'Ercolano. debbano effer poi fotterra palmi 300 .; Anzichè in forza di quefte rifleftioni, dovrebbero effere molto piu fuperficiali delle 'Lave d' Ercolano, che però non effendo tali, anzi ftraordinariamente più profonde, vuol ragione, che fi credano effer le medefime d'antichità remotiffima.

Minori ragioni vi abbifognano poi per provare l' incongruente opinione d' efferfi formato di nuovo il prefente Vefuvio dalle pietre efpulfe; ed infieme accozzate, e dal rigonfiamento della Terra, per forza delle accenfioni, fecondo che facilmente fi perfuade il prelodato Scrittore della Storia. Fu fopra dimoftrato quanto inverifimil fia, ch'i Vulcani abbian prodotto e pollan produrre monti confiderabili. Fu anche in ifpecie notato, che gli Atrati paralleli, de' quali fon conpolti, indicano, che con altra opera, ed arte fon contrutti, diverfflima da quella, di cui fi vale il fuoco; $\mathrm{i}$ quali Atrati così orizzontali componendo ancora il Vefuvio, per quanto qui fopra fu detto, terremo per ficurn adunque, ch' il medefimo egli è al par degli altri antichiffimo monte, e che non deve già la fua nafcita al fuoco, che tutto anzi diftrugge. Nella Storia citata s'afferifce in piu luoghi dall' Autore, che il monte fenfibilmente fi fcema nel Vertice, e che dentro di effo fi forman voragini, perchè il fuoco confumatore dentro, e fuori lo logra. Se dunque così è, come egli è realmente, non fo come poi da ciò ne confegua, ch'il monte fi generi dal fuoco, mentre ei pone, ch'il fuoco continuamente il diftrugga. Olervi pertanto l'Autore, ch' egii 
afferma, non volendo, ciò ch' ha intenzion di negare; perchè fe il fuoco rode le vifcere, e vi produce caverne, torna lo fteffo, ch'il dire, che la materia naturale del monte già tempo prima efifter doveva; poichè fe non vi era, non poteva confumarla il fuoco, nè lafciarvi in luogo d'effa caverne. Inoltre niuno Autore ha mai parlato di quefta nuova creazione di monte, la quale al certo non doveva fcappar della Terra in un momento; anzi tanto è lontano che dopo il primo già detto incendiu fiafi il Vefuvio di nuovo formato, che tutti gli Storici, che ne hanno fucceffivamente parlato, notano efpreffamente lo sbaffamento, e confumo del fuo Vertice. Non mi do pena di citar gli Autori, per non accrefcer tedio a chi legge.

$\mathrm{Ma}$ di quefto non piu; ed all'offervazioni, e fenomeni degli incend;, ch'il Vulcano produce, trapafferemo frattanto. E un fatto d' efperienza, che dentro il Vefuvio vi hanno de' minerali, e dello Zolfo fpecialmente in gran copia, e del pari vi abbondano i bitumi, ed olj di fpecie qualunque; l'oculare infpezione lo moftra, e $l$ ' analifi minutiffima, che de' corpi efpulf negli incendj, molti Scrittori ne han fatta, non lafcia luogo a dubbiezze. Perciocchè abbiamo il monte ripieno d'attiviflme materie, e facili al fommo per concepire effervefcenze, ed egualmente accenfioni. Subito che dunque pronta fia occafione per rifvegliare in quelle mafre il moto inteftino, che con tanta attivita accrefce azione, e richiama a quella volta copiofilfimo il fuoco, faremo giunti al termine di vedere fcoppiar dal Vulcano l'incendio. Rifvegliata l' effervefcenza col meccanifmo, che fopra abbiamo divifato, vede ognuno, che quanto più $\mathrm{U}$ u 
farà dilatata neil' interno del monte, tanto maggiormente l'accenfione che nafce farà poffente. Per le cagioni poi, che neceffariamente devon concorrere ad eccitar finalnente l'incendio, fi comprende agevolmente, che facil cofa non è affegnare il di lui principio, e nemmeno il tempo della fua durazione. Si conofcono all' ingroffo i fatti della Natura, ma i progreffi dipendenti da una complicazione di caufe, difficiliflimi fono a ravvifarfi nel fuo tutto dalla noftra limitata intelligenza. Sappiamo per efempio, che vi ha di bifogno della difpofizione delle materie, che vi vuole un mezzo per eccitarvi il movimento, che vi ha neceffario di foftanze fpeciali per concepire dall' eccitato calore la fiamma, ma per affegnare $i$ precifi termini de' progrefli, e dell efecuzione d'ogni vicenda, tant' oltre non arrivano $i$ noftri giudi$z j$. Vera cofa è però, che affinchè fegua l' incendio concorrer vi devono le fopraccennate cagioni, e dalla mancanza d'alcune di quelle, giuftamente ne feguon le variazioni, che poi negli effetti fi offervano; e perciò de' fecoli intieri è ftato fenza ardere il Vefuvio, a fegno di far credere la fua totale eftinzione; e pofcia a contrario per lunga ferie di anni non fi è dato ripofo alcuno; ed ai noftri tempi fpecialmente può dirfi, ch'ei non lafci un momento, in cui, o fia nel poco, o nell' affai, non moltri ch'il continuo fuoco gli bolle fempre nel feno.

La prima Arepitofa accenfione a nui nota del 79., e più volte fopra accennata, e mirabilmente defcritta da Plinio il Giovane; da gran tempo doveva nelle di lui vifcere prepararfi, ficcome credo, che d'ogni altra deile piu notabili avvenga. Una grolla mole, che deve 
deve infiammarfi, deve concepir prima un movimento proporzionato, ed una fiamma egualmente del pari attiva deve inveftirla; vicende tutte, che richiedono fucceffione di tempo. In fatti gli effetti, che prevengono $i$ più ftrepitofi incendj, fono $i$ Terremoti; vale a dire, che $f i$ ode avanti uno fcuotimento, o moto enorme della maffa effervefcente, prima che fi renda attiva a concepire il gran fuoco. Prevennero realmente i Terremoti, per molti anni prima, quel primo si deplorabile incendio. Seneca, che fcriffe 17., o I8. anni avanti di quello, racconta, che la Campagna felice, ed i luo: ghi adjacenti al Vefuvio continuamente erano devaftati dai tremori della Terra. E la Città di Pompej poche miglia dal medefimo diftante, ed ora nuovamente fcoperta per la Reale Munificenza del Re delle due Sicilie Ferdinando IV, fu in quei tempi fteffi dai Terremoti diftrutta. Le ftoric degli altri incendj remarcabili ci annunziano parimente $i$ Terremoti melfaggieri. La fpiegazione di quetti Terremot: Vulcanici, che colle accenfioni tirano una, e fola origine, avremo occalione d'efporla in feguito, allorchè tutti $i$ fenomeni; ch' accompagnano gli incendi partitamente noteremo.

Era per l'avanti il Vefuvio un deliziofo, ed ameno monte, ficcome Virgilio lo defcrive, e con effo diverfi altri antichi Scrittori; ma cangiò ben prefto la fua fertilifima fuperficie, in quella di fteriliffimo fuolo, $e$ del pari fi trasformó tutto in un afpetto lugubre, tofto che ivi apparfe il Vulcano. Ogni incendio, ch' in quello avvenga prefenta tempre quafi gli fteff fenomeni, o poco almen fra lor fon differenti; fe non in ruanto che le circoftanze ammettono l'avvenimento di

$$
\text { U แ } 2
$$


qualche fatto ipeciale, il qual parimente ricorderemo; ficchè efaminando in generale $l^{\prime}$ accenfione, come pure adattandovi a que' fatti fpeciali la fpiegazione, avremo per ogni incendio un' affoluta teoria.

Se. l'acque, che penetrano dentro del monte debbano effer la prima cagione del moto inteltino fra le materie terreftri, o fivvero dell' effervefcenza; e fe l'aria libera, che neceflaria troviamo per la loro combuttione, deva avervi luogo, immaginando i fatti nella maniera più naturale, e poffibile, e ftando tutte le altre cofe eguali, par fenza dubbio, che la materia fuperiore dell'interno del monte debba effer la prima ad accenderfi; vale a dire, che verfo la di lui cima s'ecciti il fuoco, e non già nelle più profonde vifcere della Terra, ficcome hanno alcuni opinato. Il primo indizio, ch' il fuoco la già inveftite le opportune foftarize, fi manifeita nel nero, e denfo fumo, fcagliato con violenza; che dalle aperture, o bocche del vertice efce in gran copia. S'innalza quefto rettamente ad una gran diltanza, fu la figura d'una fterminata colonna, che per nominarla col vocabolo degli antichi, $c$ moderni Scrittori, raffomiglia al Pedano d'un Pino. Concepifce quefta figura per la violenza con cui dalla maffa ardente fi fcaglia, il quale poi neceflitato a paftare per le anguite bocche del monte, s' unif́ce talmente in quella forma cilindrica. Segue pofcia, che la forza d'efpulfione perdendofi, più in alto quello non fale, ma a poco a poco ricadendo nel vicino ambiente, dilatandof, ed equilibrandofi infieme, forma con particolar fimetria, come tante frondi, e rami, e rapprefenta tutt' infeme la reale figura del Pino. La feconda forgente perennemen- 
te tramanda dell' altro fumo, e quello, che fu il primo ad ufcire, fucceflivamente fi fpande nell' Atmosfera, arreftandofi in diverfi luoghi, a feconda della fua gravità fpecifica, e riempie cosi il Cielo circonvicino di nubi grandiffime, o montagne fpaziofe di fumo, offufcando gli ftefli raggi folari; i quali, benchè di bel mezzo giorno, reftan talvolta così ottenebrati, che fembra quafi coprirfi tutto d'ofcuriflima notte.

Il primo fumo così efpulfo egli è fempre foltiflimo, e neru; non contenendo foltanto vapori, ed efalazioni non infiammate, di cui per la maggior parte fi compone ogni fumo ma vi fon frammifchiate pure molte particelle d'altri corpi, e fpecialmente delle parti oleofe in gran copia, le quali gli danno giuftamente quella nigredine, e quel fuliginofo, per effere di loro natura attiviffime ad afforbire la luce. La gran violenza del fuoco, che fcompone tumultuariamente l' intiera maffa, diftacca talmente quefti varj corpi, fenza dar loro agio d'effer dal medefimo depurati, ed arfi; e perciò al fubito apparire l' enunciata figura del Pino, fi pronoftica funefto augurio dagli Abitanti di quei Paefi. E con ragione, perchè le particelle componenti non ben depurate, e la forza con cui viene fofpinto in alto quel fumo, fon fegni non dubbiofi, che la maffa ardente dentro il Vulcano ella fia di fterminata mole, e del pari da copiofifimo fuoco inveftita; e quindi null' altro vi è da afpettare, che uno de' più portentofi incendj. Nel profeguimento poi, penetrando vie più il vivo fuoco nella maffa ardente, e le olecfe particelle ce vertendofi in pura fiamma, e gli altri corpi fifli depuran. i, ed abbruciandofi, efce allora il fumo men carico, c.u? 
più chiaro, e trafparente; ed in proporzione prende il fuo aumento l'incendio. Se le accenfioni a contrario fiano in origine di minori foftanze, e per confeguenza di minore attività, e polfanza, fanno fcaturir quel fumo non così raccolto, nè tanto fuliginofo; ma bensì a guifa di chiara Nube s'innalza, dilatandofi nel principio della fua ufcita dal vertice. Ai giorni noftri in ifpecie, quafi fempre così addiviene, quantunque l'accenfione fia talvolta delle maggiori; perchè le caverne, nelle quali ha la fua fede il fuoco, divenute ormai così profonde, e fpaziofe, non può in primo luogo la forza d'efpullione folpingerlo tanto in alto; fecondariamente poi la di lui concepita forza quafi fi annulla negli urti replicati, ai quali la fpaziofità, e l'altezza del luogo lo neceflitano, prima che efca al di fuori. Quefta medefima ragione della maggior profondità del luogo delle accenfioni è parimente caufa, che le fiamme, le quali in antico in ogni incendio ufcivano dalla fommità del monte, ficcome raccontan gli Storici, di raro ai tempi noftri s' offervino ufcir dalla cima, fuorchè nel cafo di qualche fpeciale incendio, concepito nelle parti più proffime al vertice del monte; poichè ficcome nient' altro è la fiamma, che le parti combuttibili attualmente ardenti, quando che debbano per lungo fpazio diftenderfi, non giungono così inframmate tant alto da poterfi vedere; perchè $f_{1}$ ardon frattanto, e le particelle flogiftiche a noi vifibili fi confunano; onde il fuoco combinato abbandonandole allora, fi rifolve nell' altro libero, ed elementare, e fi occulta in tal guifa ai noftri fguardi.

Fra quel primo fumo, che coftituifce il pedano, e rami del Pino, fovente vedefi quel fingolar fenomeno 
del Lampo, che ferpeggia velocemente con lucida fiamma, e fvanifce allorchè efce dalla nugola del fumo, ed entra nell' aria libera, ed aperta. Tanto è fimile ai Lampi inforti nei temporali nell' Atmosfera, che direfti non effer quella una nuvola di fumo, ma di vapori, ed efalazioni, non diffimile in niente alle nuvole delle pioggie; e come appunto in quefte vi hanno $i$ Tuoni contemporanei ai Lampi, non diverfamente in quella $i$ Tuoni feguono ai Lambi, benchè peró molto più deboli dei foliti, inforti nei temporali. Tali Lampi Vulcanici feguendo fpeffo nell' antichiflimo Vulcano dell' Etna in Sicilia, han dato forfe luogo a' Greci favoleggiatori, di collocarvi in quelle fucine il Dio Vulcano, che con $i$ fuoi Artefici fabbrichi i Fulmini al gran Giove.

Siccome i Lampi dell' Atmosfera è già provato altro non effere, che il Vapore Elettrico, cosi parimente fari ancor effo quello, che i noftri Lampi Vefuviani produce; il qual Vapore fi difacca da quei minerali del Monte, e fpecialmente dallo Zolfo per la viva azione del fuoco. E ficcome per fua natura, fi manifefta con i fegni elettrici, allorchè dal corpo dove è in abbondanza fi comunica agli altri, che ne hanno mero, per così equilibrarfi a foggia dei fluidi, onde avviene, che nella nuvola del fumo, dove avvene meno, vi fi diftribuifce l' ecceffo, che da quei corpi naturali fcaturifce; come per appunto fiegue nell' incontrarfi due nuvole nell' Atmosfera, che una comunica all' altra il fuo ecceffivo Vapore, manifettandofi nei Lampi, e negli altri eletrici fegni. Svanifce poi nell' entrare dalla nuvola nell' aria, perchè come è noto, l' aria refifte al Vapore, nè fi diffonde molto lungi a traverfo della medefi- 
defima; cioè l'Aria è elettrica per origine, o corpo Coibente, come altri il dicono, e quindi non fi elettrizza; il che torna lo fteffo, ch'il dire, che contenendo effa pure del Vapore non poco, non le viene perciò comunicato tanto ecceffo dalla fumigante nube, per manifeftarfi' nei foliti fegni .

Quetti Lampi faturiti in tal guifa dalle materie fompofte dentro del monte, fan vedere, che da fotto terra efce il vapore elettrico ancora, quando che da qual fi fia cagione venga diftaccato dai corpi; e pero fono una conferma, che parimente i fulmini poffono ufcire, e fcoppiar di fotterra, fecondo il confentimento di accreditati Filofofi.

Quel fentirfi ancora i Fulmini, e Tuoni cosi frequentemente, $e$ in ogni ftagione anco più cruda, nel Clima di Napoli, e di tutta la Sicilia, può derivar giuftamente dai gran Zolfi, e Minerali, che racchiufi fono nelle terre di quelle Provincie, ed unitamente da quelle si fpeffe effervefcenze, e dai Vulcani, che colà fono; perchè fprigionandofi l' elettrico vapore, e pofcia difperdendofi nell' Atmosfera, s' ecciteranno così frequenti tali fenomeni.

Fralle ricordate Nugole di denfo funo vi fon fempre mefcolate delle ceneri, o polveri finifime; e talvolta fono fi abbondanti, che nel cadere ricuoprono tutti $\mathrm{i}$ contorni del monte, s'alzano fopra $i$ veftiti degli Abitanti, abbronzifcono gli alberi, e danneggiano non poco le campagne adjacenti. Anco feparatamente dal fumo fon le medefime aftai volte follevate in alto, componendo grolfi Nuvoloni per l'aria, dove fi mantengono, finchè dalla loro gravita fpecifica non fiano necef- 
neceflitate a cadere; le quali dopo cadendo a guifa di pioggia, moftrano cosi uno fpettacolo, che come raro nelle ordinarie vicende della Natura, move la curiofità, ed ammirazione infieme degli Offervatori. Plinio nella prima accenfione già detta, ricorda la portentofa nuvola di cenere, che poi fi fcaricò fopra a Capri, Ifola 30. miglia diftante da Napoli. Nello Atrepitofo incendio del $\mathrm{r} 63 \mathrm{I}$. raccontano parimente, che coperte furono dalle pioggie di ceneri le ftrade tutte, e Laftrici della, Città, i quali fono quei Piani di calciftruzzo battuto, che fervon di Tetto a tutte le cafe, e Palazzi di Napoli. In altri incendj parimente fi fon vifte le Atrade ricoperte d'un fuolo di cenere; e nella noftra Memoria delle Mofete inferita nel Tomo IV. di quefti Atti, fi fa menzione della nera polvere, che nell' incendio del 1766 . cadde in grande abbondanza.

Tal cenere così fina, che fi mantiene perciò per la fua leggierezza gran tempo fofpefa nell' aria, eccita fempre una folta caligine, ch'ingombra talvolta tutto l'ambiente, e moftra varie apparenze nell' aria, giufta la qualità, e quantità fua. A me fteflo occorfe una volta vedere effere ingombrato il Cielo di una roffa caligine; da cui tutti $i$ corpi fembravano illuminati, e tinti di un roffo cupo, ficcome l'aria Ateffa d'un color di fuoco, ed il Sole parimente compariva un globo infuocato $\mathrm{di}$ un color cupo, e alquanto fmorto; lo che veramente formava tutto infieme una fingolare apparenza. Procedeva tutto ciò giuftamente da quella rofla polvere fofpefa nell' aria, nella quale refrangendofi i raz̧gi folari, i foli di color roffo ne rifultavano, che refpinti poi alla vifta, rapprefentayan perciò così rofleggiante egni corpo.

$$
\mathrm{X} \times \quad \text { E per- }
$$


E perchè anche in quella $f_{i}$ raccoglievano, accrefcevafi perciò alla Stagione eftiva, che allora dominava, un eccelfivo calore.

Quefte efpulfe ceneri, o finiffime polveri altro non fono che l'avanzo di varj corpi, i quali contenevano la materia combuftibile, di cui effendone fpogliati dall' azione del fuoco, rimangon foli ne' loro refpettivi principj-Son le prime per ordinario ad effer gettate, venendo come fi diffe trafportate infieme col fumo, $o$ pure da fe fteffe raccolte; perchè effendo corpicciuoli minuti, e fciolti, la comunicazion del moto, per le leggi meccaniche, con effl è più pronta; e però in virtù della reazione, e forza della maffa ardente, con facilità fi follevano, e sbalzan fuori, e di poi per l'aria lungo tempo $f($ arreftano.

Gli Storici dell' Eruzioni Vefuviane fpeffo raccontano, che per lunghi tratti di paefe fono fate quelle trafportate; e che per fino in Coftantinopoli, ed Egitto fieno qualche volta arrivate, vi è $\mathrm{ch}^{\text {i }}$ il crede. Se il 'fatto foffe abbaftanza certificato, non occorrerebbe andáre indagando, fe fia in Natura poffibile; ma talmente non effendo, farà dunque lecito efaminare, fe nel meccanifmo ordinario delle naturali leggi, ciò poffa avvenire. Io crederei con qualche ficurezza di nò ; e le Storie, che lo raccontano, mi perfuado effer più efagerate, che vere. Altra ftrada non vi è, per cui poffän quefte ceneri giungere a sì fterminate diftanze, fe non che quella de' Venti. Or noi ne' climi d'Italia, che foli Venti ftraordinarj, e vaghi abbiamo, che come è notiffimo, lunghifimi tratti di paefe non percorrono, non arrivando forfe, benchè di Atraordinaria pof- 
polfanza, alla diftanza di miglia 200 ., diremo dunque; ch' impoffibile egli è, che da Napoli fieno arrivate a Coftantinopoli. Lo potretbon forfe fare i Venti generali, ch' in altre parti coltantemente fpirano, pofciachè a gran diftanza fi eftendono; pofto ancora, che non incontraflero montagne cofte \&c., per depofitarle, e lafciarle nel loro cammino. Sappiamo, ch' ogni corpo. più grave in ifpecie dell' aria, come fono al certo quel. le ceneri, e polveri, fi forpende in effa per lungo tempo, attefa la piccolezza del fuo volume, la forza di tenacità dell' aria, ed altre forze attive; ma che finalmente la fua maggior fpecifica gravità lo richiama a cadere; nè per l'aria può molto fpaziare fe il movimento dell' aria medefima, feco non lo trafporti. Dunque per dare un' aria di verifimiglianza al fatto, bifognerebbe dire, che s'uniffero infieme a tal uopo molti ftraordinarj Venti ; cioè che uno terminando il fuo corfo, depofitafle le ceneri nell' ali dell' altro, il quale già il fuo corfo principia, e cosi fucceffivamente difcorrendola ; il qual meccanifmo fembrando effer piuttofto un concepimento ideale, che un atto reale della Natura, potremo perciò ragionevolmente dubitare dell' avvenimento del fatto.

E' fucceffo talvolta, che alcune ceneri, o perchè fran compofte di corpicciuoli di maggior volume delle altre, oppure che tanta non fia la forza d'efpulfione da poterle follevare nell' aria, fono fgorgate dalle bocche del monte, a foggia di rapido fiume, mefcolate con arene, ed altri avanzi del fuoco. Nel quarto notabile incendio, defcritto da Caffiodoro, al tempo di Teodorico Re d'Italia, l'anno 512. tanto s'innalzó la ce$\mathrm{X} \times 2$ 
nere fgorgata a guifa di fiume, che giunfe a ricoprir le cime degli alberi in quelle fottopotte pianure. Era ancora rovente; ed agitata dall' interno calore, e dalla raccolta mole, e dalla figura, ed infieme dalla pendenza de' luoghi ajutata, fcorreva mirabilmente come un naturaliffimo fluido. Non è quefto un fatto però, che fpeffo fucceda, anzi che ne' fuffeguenti incendj non fi ritrovan memorie d'un accidente confimile, ed una delle ragioni farà forfe la maggior profondità delle caverne fucceffivamente crefciuta: Apporta certamente la loro profondità maggiore', difficoltà maggiore eziandio per l' efpulfioni, e fpecialmente di quelle materie, ch' in piccoli corpicciuoli fon difunite; per lo che refteranno adeflo confufe, e fciolte infieme colle Lave, e con efse avranno il loro efito. Alcuni han pretefo che nei primi incendj, in vece delle Lave non ufciflero altro che quefte ceneri, a guifa di fiume; e fe le caverne non eran tanto profonde da potere effere facilmente efpulfe, e da non reftare perciò lungo tempo in effe a liquefarf, non è tanto improbabile il fatto; Certo è che $\mathrm{la}$ cenere allora fola fu; la quale nel primo incendio già detto del 79. fommerfe la Città d'Ercolano; fituata all' eftremità della bafe del monte. Si ritrova nello fcavare, fopra $i$ diruti Edifizj, pofata a frati; fi ritrova ancora - feparata in gran monti, fe ne trova fciolta nello ftato fuo naturale, $e$ trovafene pur anche della concreta in fafli d'enorme grandezza.

Quelti faffi cosi concreti di cenere fono una riprova delle concrezioni, che quotidianamente opera la Natura nelle vifcere della Terra. Le acque, ed i fali, che con effe filtrano, e pcuetrano dentro 'terra, vaglio. 
no quanto una colla, per unire le parti terreftri difciolte, e farne in tal guifa un maffo; o altro corpo concreto. In Pompej ancora ai di noftri difotterrata, vi fi ritrovano quefti faffi così naturali d'una non ordinaria mole, e dentro $i$ luoghi ftefli, che prima della rovina, erano Camere, e Cafe, o altri luoghi d' abitazione; per la qual cofa non vi refta dubbio, che di dopo non frano così crefciuti, e confolidati; e pero al mio avvifo, per analogia inferiremo, che le altre pietre dentro terra, ch'ogni metallo dentro le miniere, ed altro corpo, ch'ha l' abitazione fotterra, poffa generarfi, e crefcere per le cure, che di quei prendeli la $\mathrm{Na}$ tura, a fimiglianza che fa nafcere, e vegetare le piante nella fuperficie.

Sbalza ancora al di fuori il Vefuvio ammaffi prodigiofi d'arene, quantunque non così frequenti come le ceneri, nè sì lontano trafportate per aria, per effer molto più gravi in ifpecie della cenere, nè da poterfi percic̀ lungo tempo foltenere in alto, come di quella fuccede. La cima del monte refta quafi fempre in ogni accenfione ricoperta di quefte arene, ed havvene talvolta di quelle si bianche, che fanno apparire il di lui vertice, come ricoperto di Neve. Quefte faran quelle arene, che rifiedono in qualche fito del monte, 0 in qualche ftrato, di cui è compofto; le quali effendo corpicciuoli tra lor difuniti, in una violente fcoffa della materia fottopolta già ardente, faranno alla rinfufa in tal forma fcacciate. Potrà egli effere ancora, ch' effe fieno parti minutiflime terreftri, diftaccate dagli altri corpi, agitati, e commoffi dal fuoco; poichè veggiamo, che le pomici, e le fcoric, i fatte naturali \&c. qualo- 
ra fi urtino, ed infieme fi sfreghino, riduconfi realmente in quelle minutifime parti, fimili a quefte arene; come appunto fon nate le arene delle fpiaggie marine, cioè dall'azione, reazione, e sfregamenti, dal moto dell' acque verfo gli fcoglj, ed altri corpi diverf, che nel Mare nuotano, e dimorano. La fola differenza vi è fra l' arena del Mare, e quella del Vefuvio, che queft' ultima moftra fpeffo un certo grigio colore, che quanto è a dire, ch' ella è Aata toccata dal fuoco.

Di mano in mano che la maffa ardente concepifce energia, o forza maggiore, efpelle fucceftivamente il Vefuvio gli altri corpi più gravi. Le pietre, la Ghiaja, $i$ Ciottoli, e varj rottami d'altri corpi fcaglia talora in gran copia, e con violenza, che nella loro caduta, direfti venir dal Cielo una furiofa tempefta, fimile a quella della grandine, ma ben diverfa ne' corpi cadenti; ed a feconda, che le materie gettate ingroffano di volume, fempre più annegrite apparifcono, perchè maggior tempo dimorano nelle voragini, attefa la diffcoltà nell' efpellerfi, cagionata dalla loro maggior gravità. Getta infiem parimente le pomici, o pietre fpugnofe, e porofe, con altri fafi di teflitura affai fragile; cutto effendo in origine faffi naturali del monte, ne' quali arfa la materia combutibile, e corrofi dal fuoco, rimangono cosi fpugnofi, e forati in tal guifa, e quindi poi facilmente fi rompono. Vitruvio, ed altri molti Scrittori han confufe quefte pomici, colle fcorie, o rofticci; ma le prime, come abbiam detto, fon pietre naturali del monte di fpecie diverfe, che poi ridotte a calcinazione, mirabilmente fervono per cemento, non diverfamente che la calce ufuale; $i$ rofticci a contrario nafcono $e$ riful- 
rifultano dalla maffa già fciolta, e liquefatta in quello fterminato Calderone di fuoco; e perciò fono concrezioni fimili in tutto, e per tutto a quelle fcorie, che da'metalli fufi fi feparano; come fi offervano comunemente nelle fucine de' Fabbri: Hanno quel fuono fimile ai frantumi de' Vetri, allorchè fi urtano infieme, e fon fimilmente neri, e fuliginoli dagli Olj, e Bitumi a loro attaccati dalla fiamma, e dal fumo. Nel bollire, e fcioglierfi la portentofa maffa infuocata dentro del monte, fi feparano quelle particelle forfe più delle altre leggiere, ed alla fuperficie s' innalzano, le quali efpofte al contatto dell' aria, che nelle caverne ha l'ingreffo, $f$ rappiglieranno in tal forma; e fubito che accade qualche sforzo, o urto dell' ardente materia, vengono talmente al di fuori cacciate.

II fuoco allorchè più s' infinua; fempre più fquarcia, e fvelle di quei corpi naturali del monte, e fuo. ri gli efpelle. Sicchè dei faffi naturali, e delle pietre, fvelte dall' interna terreftre materia, ne getta il Vefuvio in ogni eruzione, che poi cadendo, e rotolando per le pendici del monte, bruciano ovunque paffano. Sono gettati 2 maggiori diftanze, che delle arene, e delle ceneri, avvegnachè l' impeto di projezione, in parità di circoltanze, s' imprima maggiormente ne' corpi piu gravi, che negli altri più leggieri; per quello che dalle meccaniche leggi è notiffimo. Alcuni di quefti faffi ve ne ha talvolta d' una mole sì fterminata, che fe la quotidiana efperienza non lo facefie ocularmente vedere, parrebbe al certo favolofo racconto. Il Paragallo nella fua naturale Storia del Vefuvio racconta, ch'effendof dato il penfiere di far pefare un di quei grofli 
groffi macigni a lunga diftanza sbalzato, divider facendolo in parti per confeguire l'intento, lo ritrovò tutt' infreme di cinque mila libbre di pefo. Se ne offervano quà, e là fparfamente moltiffimi in quei contorni; fon di varie grandezze, e polti a lontananze varie, e per eftere fopra la terra fcommoffi, e per la patina, che veAtono di Zolfi, e di bitumi abbronziti, non lafcian dubbio di non effere realmente pietre eruttate dal Vefuvio in furore.

Quefti tali corpi diftaccati dalle vifcere del mon: te vaglion di prova, che le accenfioni or quà, ed or là ferpeggiano; che le caverne ogni dì più fpaziofe divengono, e confeguentemente poi, che la materia naturale interna fi confuma fucceffivamente; e quindi può con filofofico ragionamento inferirfi, che quella materia la quale $f i$ arde, e fi efpelle ne' refpettivi incendj, fia attualmente nelle vifcere del medefimo, fenza aver noi di bifogno farla colà venire da altre parti, e remote Provincie, come alcuni vaneggiando afferifcono; di che avremo occafione favellare al fuo luogo.

Oltre all' efpulfioni fin qui ricordate, apparífono eziandio varj altri fenomeni, nell' accenderfi quelle foAtanze, i quali accompagnan talvolta il getto de corpi anzidetti, e talora feparatamente fi offervano. Il più comune fra quefti egli è quel fibilo, o fremito, ch'odefi nelle cupe caverne, quando il fuoco colla fua azione invefte que' corpi; è un effetto comuniffimo a tutti $i$ monti ignivomi, fe fpecialmente fia di gran poffanza l'accenfione. Raccontano le ftorie, ch' in ifpecie, il monte Hecla in Islanda ci fia particolare per quefti fremiti, e fu i quali fta il Popolo attaccatiffimo nella 


\section{Dell' Accademia.}

credenza d'effer quei, gli urli, e' le ftrida de' miferi Abitatori dell' Inferno, le quali per quelle infernali foci a noi fi tramandano. E' ftata ancora una credenza co. mune d'ogni altro Popolo; poichè ficcome fiffatta gente è portata fempre a credere cofa foprannaturale tutto ciò, che avviene fuori della sfera della fua intelligenza, ne fegue pofcia, che molcolar vi deva la vana fuperftizione. Il Popolo però merita $f_{c u f a}$, e meno la meritano a contrario alcuni altri, che pur fi reputano :comprefi nella fchieta de' Dotti, i quali confeffano naturaliffimi gli effetti provenienti dal noftro Vulcano; $\mathbf{m a}$ perchè fono uomini forettieri nelle Provincie della $\mathrm{Na}$ tura, il loro orgolio gli fpinge a credere, ch' ogni, e qualunque altro Uomo fia affatto all' of curo délle cagioni; che gli producono: Ed io ne ho di quelti. fpeffo fentiti; e ne ho anche degli altri con pazienza fentiti, deridere generalmente i moderni Fifici,' c tacciargli. infreme di vanità, perchè tanto s'intereffano indagar le cagioni d' ogni naturale vicenda, le quali nafcofte fono nell' intricato laberinto della Natura. E' lin-. guaggio per verità, che molto fente de'barbari Secoli, e non già de'notri, dalla buóna Filofofia illuminati. E' che forfe molte cagioni non fi poffon da noi conofcere in Natura, benchè vero fia, che moltiffime ne ignoriamo? E qual vanità ella è mai l'andare in cerca di ciò, che non ci è ben noto, $f e$ il cercare quel che ci. ¿ noto, perfuade ragione, ch' egli è affatto fupertluo ? Ma lafciamoli nelle tenebre,' e feguitianó.

Ognun vede, che a qualunque corpo, a cui il fuoco fi attacchi, ftride fubitamente, e fibila in proporzione della quantità accefa, e della qualità de' conpoY y

nenti 
nenti lo fteffo corpo. Quel mobilifimo Elemento tutto penetra, e fcompone, e fepara le parti integranti dei corpi colla fua energica azione; e allora l'aria fiffa, che fi fprigiona, acquifta nucvamente la naturale elafticità, quale è vie più accrefciuta dall' azione del fuoco; e peró fi slancia, e confufamente fi move, ed agita in tal guifa l'altra aria libera, che le fovralta, per ogni parte la commuove, e fimultaneamente ovunque la fpinge; ed è appunto allor quando che nafce quello ftridore, e quel fibilo; perchè urtando nelle caverne del monte, e ripercuotendo in quei tortuofi giri, a feconda della di lei commozione, comunica nell ambiente il fuo moto, da cui ne nafce la fenfazione di quel fremito. Il Vapore elettrico ancora, che dalle materie. fulfuree, e da altri-minerali fi fviluppa, colla fua velocità incredibile fi fcạglia nell" aria libẹra, e s'unifce come cagione a commuoverla con maggior gagliardia, e quindi a rendere più efficace il rumore. Lo ftridore, che dalle materie arfe ne fuochi noftrali fi eccita, egli è un paragone per poter concepire quefti ricordati fremiti del Vefuvio; qualora che nell' immaginazione fi accrefca il valore ai piccoli effetti, portandogli al fonmo grado, in cui rifaltar gli fanno i fatti forprendenti della Natura.

Nel monte Hecla, in cui fono sì fpeciali quefti ftridori, avverrà facilmente, che l'aria libera fommamente compreffa, e vibrata abbia l' efito per alcuni angufti, e tortuof giri del monte, non diverfamente che offerviamo, fe per avventura accada, ch' un furiofo Vento fpinga l'aria, e la neceffiti a paftare per riftrette aperture. Veggiamo fimilmente, che la fiamma slanciata con vigore 
gore dalla maffa infuocata, ed il gran fumo eziandio, allorchè abbiano l' efito per un qualche Atretto luogo, ftridono anch'effi, ed eccitano un certo cigolamento nell' aria; onde è, ch' in maniera confimile avvenir potrà nelle caverne vulcaniche. Dalla fomma rarefazione dell' acqua, che per accidente cada nel fúoco, faranno grandemente rinforzati quelti fremiti; perchè fprigionandofi da quella lo fpirito volatile, rifveglia effo pure uno fpeciale fridore, ficcome a tutti e palefe.

Son peró differenti quefti fibili tanto negli effetti, che nella cagione, che gli commuove, da quei scapi rimbormbi, che odonfi talora dentro del monte. Gli fco gli, e macigni pendenti entro le fpaziofe voragini, i quali ocularmente in varie occafioni furono da molti offervati, debbono fpeffo cadere nelle accenfioni, perchè sfiancati vengono dall'azione del fuoco; ficchè cadendo rifvegliano quell' orrido fuono; offufcato rimbombo.

L'accenfione quando avrà liquefatta tutta la maffa rovente, 0 pure quando che attualmente la fcioglie in quelle fpaziofe fucine, immaginiamoci qual bollore mai, e qual gorgogliamento deve quella produrre; or eccó altra cagione valevole al certo di tifvegliare $i$ gia detti offufcati rumori. Nell' eruzione del 1766 . da me brevemente defcritta nel Tomo IV.di quefti Atti, fi fa menzione di quel continuato fragor fotterraneo, il quale per molte ore, fenza interruzione alcuna, rumoreggiava fotterra, come nelle Nubi rumoreggia il fragore del Tuono, e fimilmente come ftrepita l' unda marina nelle furiofe tempefte. Quel bollimento della fciolta materia era di ciò la fola cagione, e quel rauco fuono; il qual fi eccita allorchè $i$ liquidi bollono, prefenta $\mathrm{Y} \mathrm{Y}_{2}$ all im- 
all' immaginazione un' idea di quel fotterraneo bollore della maffa difciolta. E'talvolta si poffente quel bollimento, che con $\mathrm{i}$ fuoi violenti urti commuove la Terra, e la fcuote non differentemente che i Terrenoti; ficcome di fatto avvenne nell' enunciata eruzione.

Con ragione M. Buffon chiama i Vulcani Cannoni, ma Cannoni della Natura ; perchè di fatto frequentiflime fono le cannonate, o tuoni, che con la projezione delle materie, nel tempo che le medefime fi accendono, icarica fempre il Vefuvio, e prefentano inoltre uno de' fuoi piu bizzarri fenomeni; a chi fente, e vede da lungi pero, ed a contracio incomodi di foverchio a chi di troppo fi apprefla. Comparifce prima copiofo, e folto fumo con violenza sbruffato; vi ha non di rado la fiamma con effo, confufa, fe tanta è da poter forpaffare la cima del monte; ne fegue contemporaneamente lo fcoppio, a cui vengon poi dietro l' efpulfe materie, come per appunto avviene nell' accenfione d'un mortaro da Bomba. Se ciò fucceda di notte, vedonfi per aria rotolare $\mathrm{i}$ corpi fcagliati, e roventi, come appunto rotolano pel Cielo le palle da Bomba. Di giorno poi il fumo, che fuol coprir fempre il vertice del Vefuvio, confonde la vifta di quefti corpi ardenti per aria, e fpeffo fegue che efpofti fono i curiofi Offervatori, a gravi pericoli. Fra i corpi gettati avvi bene fpeffo de' grofli macigni, che non arrivano all' altezza delle voragini, per l' enorme loro gravità, e quindi a baffo ricadono; e pero fpeffo fentefi, dopo lo fcoppio, un altro cupo rimbombo cagionato dalla loro caduta . Sono quefte cannonate cosi potenti, che rifvegliano molte volte qualche tremore negli Edificj circon- 
vicini; da che han confuf molti Scrittori quefti fquotimenti, prodotti dall' agitazione dell' aria, con quei veri Tremuoti del Vefuvio, i quali da altre cagioni dipendono, come occorrerà di vedere.

$\mathrm{Nel}$ incendio del 1766 , di cui abbiam fatto altre volte menzione, non paffava nemmeno un momento, in cui furiofamente non replicafte il Vefuvio le fue fcariche. Racconterò per digreflione, ch' efaminandone in tale occafione, e fuor d'ogni pericolo gli andamenti, ritrovai verfo la cima del monte due Oltramontani, e comprefi dal loro ardore, che pieni di coraggio affrontar volevano l'inimico nelle proprie trincèe, cioè che accoltar fi volevano alle furiofe foci del fuoco. Credei atto d'umanità l' avvertirgli che tanto non s' innoltraffero, perchè fcagliando quantità di fafli mifchiati fra il denfo fumo, e perciò invifibili, un ficuro rifchio correvano d'effer dannificati non poco . Gradirono cortefemente l' avvertimento, ma dai lor moti m' avviddi, che lo ftimarono un effetto di fola mia pufil.. lanimità, e quindi coraggiofi fi avvicinaron più oltre: Gli afpettava anfiofo al ritorno, e vennex già, ma meno lieti, e coraggiofi di prima; e con fommo mio difpiacere offervai, che un di loro portava appefo al collo un braccio troncato dalla percoffa d' un faffo, che inafpettatamente gli parve cadere dal Cielo.

$\mathrm{Da}$ una fola, e principal cagione fi ripete la fuiegazione della gran violenza, con cui fono efpulfi $\mathrm{i}$ corpi fopra defcritti, e delle cannonate, o tuoni, che l' efplofione accompagnano; e dopo l' invenzion della Polvere, veggiamo totto degli occhj, benchè in picçolo, gli effetti medefimi. Han cercato i Filofof con in- 
numerabili efperienze, da qual caufa dipenda la violenza della Polvere, con cui fcaglia i corpi nell' accenderfi ; e generalmente la ripetono tutti da un fluido fommamente agitato, e commoffo, il quale fi fviluppa da' piccoli granelli a forza det fuoco : Che fia dunque la vera cagione quefto fluido, ftabilmente da tutti $f_{1}$ crede; foltanto divife fon le opinioni full' affegnare quale egli fia quel fluido cosi attivo, e poffente. Il gran Newt on crede, che la materia fteffa della Polvere dalla fubitanea, $e$ veemente rarefazione per opra del fuoco, fi converta in vapori, l' azione de quali poi fi manifefta con quella forza incredibile.

Ad altri piace piuttofto, che prendendo foco la Polvere, fi fviluppi l'aria fiffa nei piccoli grani raccolta, la quale riacquiftando la naturale elafficita fa nafcere gli effetti già detti. Autori graviffimi non credonó fufficiente quefta elafticità dell' aria a produrre effetti cosi notabili, e per accrefcerle forza, vi mefcolano l'umore aquofo fommamente rarefatto, ed elaftico per l'azione del fuoco.

Ridurendo ciò che conduce al propofito noftro nelle fcariche del Vefuvio, non potremo definir certo quale effer poffa quefto fluido, che con tanta violenza, ed efficacia opera, avvegnachè difficil fia fare efperienze, ficcome nella Polvere le hanno fatte i Filofofi. Siam ficuri però, ch' il fuoco mobiliffimo di fua natura, e fommamente elaftico, poffa colla fua azione imprimer forza ne' corpi, e commuovergli. L'aria fiffa parimente meffa in azione, e fprigionata dalle refpettive foftanze, acquifta forprendenti forze, per quanto che le replicate efperienze $c^{\prime}$ infegnano; ed il fimile diremo dell' umo- 
re aquofo, convertito dal fuoco in fpirito fottile, e vo. latile. Quindi certo effendo che nelle accenfioni de' Vulcani tutti quefti fluidi abbianvi luogo , potremo dunque ammettere, ch' unitamente contribuifcano con la loro energia, a produrre gli effetti accennati. Se il Vapore Elettrico fia un fluido dal fuoco diverfo, egli pure colla tua fomma attività, fciolto ch' ei fia da quei lacci, ch' in quei minerali lo coftringono, accrefcerà a' mentovati fluidi la lor violenza, unendo con effi la fua mirabile energìa. Sicchè penetrato dunque il vivo fuoco nelle combutibili fortanze del monte; colla fua mobilità, ed elafticità naturale, sforzerà per ogni parté, fvellendo, e diftaccando ogni materia, ajutandone l' efpulfione. L' attività indicibile dell' Elettrico 'Vaporè opererà nella guifa fteffa del fuoco; ed oltre all' unirfy ambedue colla loro violenza ad efpeller que' corpi, mirabilmente poi difgregheranno dai medefimi quell' aria fiffa, ed arreftata, che fenza azione, ed efercizio ivi ripola.

Queft' aria di fatto a ragionevolmente parlare, credo effer quel fluido, che più d'ogni altro agifce nei portentofi effetti dell' efpulfioni. Già fi è detto, ch' ogni corpo ne contiene una gran parte intimamente inviluppata, che per la fua fiffità, velte come una forma di folido. Lunga cofa farebbe, fe riferir voleffimo l'efperienze, colle quali fi prova, che fia quefta un fluido finilifimo, e forfe lo fteffo dell' altra aria libera atmosferica. Or quanto l'aria colla fúa azione, ed elafticità operi nelle naturali vicende, e qual molla fia, e quante forze ecciti allorchè dalla condenfazione paffa al dilatarfi, vale a dire ch' entra in azione collo fpanderf, o rarefarfi 


\section{A $\quad$ T T I}

farfi, fono ormai a tutti comuni gli fperimenti della moderna Fifica. Per lo che meffa in azione, e fprigionata dalla forza del fuoco, diverrà al certo un mobile efficaciffimo, per quindi produrre gli effetti dell' efpulfioni accennate. Saran tali effetti proporzionati alle di lei forze, alla velocità con cui fi fviluppa da' corpi; ed alla quantità fviluppata in un tempo medefimo; e però ftando ella prima condenfata, e poi dal fuoco difgregandofi, e rarefacendofi, tira gli effetti proporzionati allo ftato, in cui era prima, a quello che dopo paffa, ed alla prontezzá, e veemenza, con cui fegue tal fucceffione di ftato. Nell' efercizio, ch' ei 'fa di quefte fue mirabili forze, allora appunto è , che feguono unjtamente i due effetti dello froppio, e dell efpulfione de' corpi. Il primo di quefti, ficcome è noto, fi produce dalla commozione, e collifione dell' aria libera, ed atmosferica; la 'quale al fatto noftro con ecceffiva velocità viene fpinta, e commoffa dalle particelle dell'altra aria già fviluppata; che tanto è a dire, che s'eccita quel fragore, e quello fcoppio, per la refiftenza, che quella efterna oppone all' altra aria if prigionata, che veloccmente l'urta, e commuove, e perciò a cofe egua$\mathrm{li}$, in proporzione di refiftenza dell'aria. efterna, maggiori fi fanno gli fcoppi.

Il fecondo effetto dell' efpulfioni fegue pofcia dalle forze medefime delle particelle dell' aria fiffa, e fviluppata, la quale meffa in azione dal vivo fuoco, ed acquifta ndo la naturale fua elafticità, rarefacendofi, $\mathrm{e}$ dilatandofi in proporzione della fua natura, e dell' azione del fuoco, move così, ed efpelle quelle foftanze, che alle di lei forze fi oppongono. 
Tanto più forprendenti rifultano gli effetti annunziati, quanto che, a cofe eguali, in copia maggiore: fi contiene vincolata ne' corpi di queft' aria. Nel Vefuvio i minerali d'ogni forta vi abbondano; e noi fappiamo altresi, che quefte fpecie di corpi molta ne conterigon racchiufa, clie probabilmente imbevuta l' avranno nella loro generazione. Molta ne contiene il Nitro, moltiffima il Tartaro, ed in generale tutti gli fpiriti acidi; il che giuftamente produce, ch' effendo rifcaldati dal fuoco fcoppiano efficacemente. Confiderando per tanto, che moltifima aria refti vincolata in quelle foftanze, e riguardandola in oltre più dell'ufato condenfata, e che quindi da vivaciffimo fuoco venga rarefatta, e con violenza vibrata, ritroviamo in tutto quefto apparato di ragioni, la vera forgente degli effetti fopra defcriti .

Si condenfa di fatto l'aria ne'corpi in forprendente maniera'; e di ciò non vi rimane alcun dubbio. In un folo granello di polvere da Schioppo la trovò il gran Bernoulli condenfata in maniera, ch' occupava uno fpazio 5o. volte meno d'allorchè reftituita in libertà; nè noi limitereno la Natura a non faper fare altrettanto nelle fue opere, anzi: a non far cofe maggiori. Siccome fi condenfa, così del pari fi rarefa in eccedente proporzione; e fe reftando nel fuo ftato naturale arriva la moderna Fifica a dilatarla, a forza de'fuochi noftrali, da r 4000 . volte di più, che diremo della forprendente dilatazione, che foffrir deve dal fuoco del Vefuvio, imcomparabilmente più grancle de' noftri? E che diremo ancor di più, fe racchiufa ftia in que' corpi no nel fuo tato naturale, ma eftremamente condenfata? Allora la fua forza, ed attività nel dilatarfi efce 


\section{$3^{6} 2$ \\ A $\mathrm{T}$ I $\mathrm{I}$}

fuori di proporzione, quanto efce fuori di proporzione la maggior poffanza del fuoco, che la dilata, e la maggior compreflione, che quella foffre. Sono i di lei effetti proporzionati, come fopra abbiam detto, allo ftato in cui ella era prima, a quello che quindi ne paffa, cioè allo ftato di condenfazione a quello di rarefazione. Arrivò coll' arte il gran Boyle a conofcere, che dall' eftrema reftrizione all' eftrema fua dilatazione, vi pafrava la differenza, ch'è fra l' I. al 520000 . Bifognerebbe dunque che la differenza di quefto ftato d'aria condenfata nelle foftanze terreftri del Vefuvio; e d'aria dilatata da quel fuoco, foffe a noi perfettamente nota, per conofcere in qualche maniera l' energìa delle fue forze, fe pure potelfimo colla noftra immaginazione adequatamente concepirla.

Sicchè confiderando in un colpo di vifta tutti $\mathrm{i}$ fenomeni, che ci prefenta il Vefuvio, offerviamo che la principal cagione almeno ella è virtualmente ripotta nell' Elemento dell' aria . Quella è, che nel fiffarfi vincola infieme le parti integranti de' mifti, e mette affieme per così dire i materiaii per l'accenfione. Secondo che poi, per qualunque frafi cagione fi fviluppa dai corpi, rifveglia ne'varj compotti l' effervefcenza, e divien perciò la cagione efficiente, per cui corra a quella volta il mobilifimo elemento del fuoco, richiamato, e meffo in azione dall'inteftino moto, ficcome fu fopra offervato. Gli prefta ancora ajuto per inveftire la maffa effervefcente, lo fpinge, e lo necelfita perchè manifefti il fuo calore, e perchè poi trasformi quella maffa terreftre in una maffa di fuoco, cioè che forga una grandiofa accenfione. L'Accenfione che invefte le refpetti- 
- foltanze, con veemenza, e fimultaneamente difcion glic queft' aria da'lacci fuoi, e per l'azione, che efercita, moffa con violenza dal fuoco, replića gli urti nell' aria libera dell' Ambiente, e quefta colla fua commozione porta al noftro udito i. Tuoni, i Fremiti, e Sibili, chiabbiam fopra defcritti. Giunta ch'è final, mente a tal grado di forze, allora appunto $\grave{e}$, che colle medefine efpelle dalle vifcere del Vefuvio con quella violenza incredibile tutti que corpi.

La quotidiana efperienza fa vedere, che l'aqueo umore ridotto anch' egli in fpirito volatile dall' azione del fuoco, talmente $f_{i}$ dilata in fluido fottile, ed ela. ftico, che rifveglia forprendenti effetti per la fua forza d'effulfione. Potrebbe effer forfe, che tale fpirito niente altro fia, che l'aria medefima vincolata, e riftretta nei primi elementi di quefto fluido ; e perció ad un folo principio fi ridurrebbe allora la provenienza di tutti gli eftetti. Sono alle volte fgorgate dalle bocche del vertice mole portentofe d'acqua bollente, convertendofi le fo. ci del fuoco in forgenti viviftime d'acqua. Per non lafiare indietro fatto alcuno, farem parola ancor di quelt' acque, poichè égli è ftato un oggetto, che molto ha dato da filofofare ad Autori di non ordimario no: me.. Nell' incendio del 1631. , il quale fu st terribile per la fua poffanza, da paragonarfi con qualunque altro de' portentofi, ed uno ancora de' più lagrimevoli per la defolazione apportata all' adjacente Paefe, e per i danni ftraordinari arrecati alle Perfone; gettò il montc dalla fua cima frabocchevoli frumi di acque, o per meglio dire, produffe un' inondazione si luttuofa, che per la fua eftenfione, e copiofità, e per le ceneri feco por-

$$
\mathrm{Z}_{2} 2 \text { tute }
$$




\section{$3^{64}$ \\ A $T$ T i}

tate calde, e roventi, devaftó ogni luogo, a cui toccò la mala forte riceverla.

Fatii fimili non mancano relativamente al celebre Vulcano dell Etna in Sicilia, e ne'. Vulcami d' America fpeffo è ciò avvenuto; ma rifpetto al Vefuvio non abtiamo certezza, che prima, o dopo del divifato incendio, un fimil fatto avveniffe. Quefta è circoftanza notabile per render vie piu inverifimile l' opinione d'alcuni, che credono avere avuta quelle acque l'origine loro dal Mare; poichè effendo il Mare una forgente inefaufta, non par verifimile, che prefentar doveffe tali fenomeni, fra tempi cosi diftanti fra loro, per fino da fcorrere r 500. anni prima di comparire, ficcome rifpetto al Vefuvio è fucceffo. Opinano dunque quefti Filofofi, che per canali fotterranei ftabili, e permanenti, ovvero in particolari circoftanze creati, fieno falite l' acque marine nelle caverne Vefuviane, le quali poi dall' azione del vivo fuoco fi fcarichino dalle di lui bocche, nella forma fuddetta. Come tale effetto feguir poffa il Signor Abate Nolet, nelle Memorie dell'Accademia delle Scienze di Parigi del 1750. lo fpiega col comune efperimento dell' Eolipyla ; cioè rarefatta l' aria nelle fucine del Vulcano, e facendofi perciò come un vacuo, l' aria atmosferica, che preme la fuperficie del Mare neceffita quell' acque a falire, per quell' immaginati fotterranei canali, e quindi poi è faciliffimo fpiegare la loro ufcita per la potente azione del fuoco. Si fa Atrada il Signore Abate preparando il Lettore a ben confiderare gli andamenti della Natura, la quale opra fempre colle medefime leggi, tanto nel piccolo, che nel grande. Non $\mathrm{v}^{\prime}$ ha dubbio effer quefta una 
verità incontraftabile, ma relativamente a noi, i quali veder non poffiamo il compleffo delle ragioni ne' fatt; forprendenti, e di lor natura complicati, come le offerviamo nel piccolo, può molte volte condurci a ma.nifettiflimi errori. Per efempio; nè perchè una goccia d' acqua fta rotonda come una palla fopra un mattone, fi può arguire, ch' un Baril d'acqua faccia lo fteffro, ficcome fu replicato al gran Galileo. Ma lafciando a parte quelte confiderazioni, ed accoftandoci al fatto, non è già vero, ch' il Vefuvio ardente fia come l'Eolipyla rifcaldata dal fuoco; manca l' effenzialità del fatto per poterlo giuftamente paragonare : Nell' Eolipyla, rarefatta fommamente l' aria dal fuoco, li fa come un vacuo, e perciò potrà forfe falire l'acqua dell' altro vafo, premuta dalla preffione dell' aria libera, conforme crede il dotto Scrittore; ma quefto vacuo nelle caverne del Vefuvio fiam molto lontani ad averlo; poichè l' aria atmosferica vi ha continuamente l' ingreffo, vi fpazia liberamente, e ceffato l'impulfo vi fubentra immediatamente, e fecondo la fua natura fi ricompone all' equilibrio. Nè perchè facilmente fi concepifce lo fcorrer dell: acque per i canali, col vederle per effi fcendere al baffo, ella è facil cofa poi trafportar giuftamente il fatto, nel farle per i medefimi falire; poichè il primo fegue per loro natura, quanto è il fecondo contro natura. Oltracciò i canali fotterranei faran fommamente commodi per concepire un tal fatto, ma dubito forte che fian piuttofto immaginati che veri . Ma e perchè dobbiamo inventare fiftemi per conplicare fatti fempliciffimi, e naturali in un cumulo di difficolta? L' acqua fgorgata dal Vefuvio eravi dentro del 
monte nel tempo dell' accenfione, e la mafia ardente colla fua veemenza, con cui efpelle ogni altra materia, diede a quefta parimente l'ufcita.

Era da un Secolo che il Vefuvio più non ardeva, talchè credevafi ëftinto; e però clie quefte acque dalle pioggie colà raccolte vi foffero, lo perfuade ragione, perchè l' efterior fuperficie del Cratere molte ne raccoglie, e dà loro l'ingreffo per le aperture, e per i meaii, e per le di lui ampie bocche. L' efperienza inoltre convince; poichè il Braccini, che fa la ftoria di quell' incendio, accenna che fendendo dentro delle caverne qualche anno prima dell' accenfione, ne ritrovò tre delle medefime vaftiffime, e tutte d'acqua ripiene. Fece anzi il faggio, ed una ne conteneva della falfedinofa, l'altra della bollente, e della tepida l'altra. Si aggiunga che la ftagione di quell' anno fu molto piovofa, e nel tempo fteffo dell' accenfione dirottamente pioveva, a fegno che non folo le adjacenti campagne del Vefuvio, ma tutti i circonvicini luoghi foffrirono Atrepitofe inondazioni. Comparvero quefte acque dofo un fieriffimo Tremuoto, cioè dopo lo fconvolgimento della materia interna del monte. Si diffeccarono dopo ancora le forgenti tutte, ch' erano alle falde del Vefuvio, ficchè fon circoftanze da convalidare il fatto, d' effere fate quell' acque ripofte prima nelle caverne, e non da altrove allora venute.

Altri groffolanamente han penfato, che nel feguito Terremoto fi fquarciaffero le vifcere del monte, e che le acque marine nel tempo fteffo faliffero; ma effi ron $f i$ fon dati pena di fpiegare con qual forza mai una mole d'acqua cosi fterminata poteffe colà falire, 
ed io non ne fo abbaftanza per poter ciò efaminare. Le Mofete, e le Fumarole accompagnano fempre gli incendj i più notabili; ma avendo noi, e dell' une, e dell' altre ragionato nell' altra ricordata memoria di quef' Atti, trapafferemo ed altro, e farem parola d' uno de' più comuni, e fpaventevoli effetti, che quafi in ogni incendio avviene, che fono $i$ Terremoti.

Seguono i Terremoti in ogni parte del noftro Globo, fenza apparenze d'accenfioni vulcaniche; e fra queAt havvene alcuni, che a fterminate diftanze fi propagano. Avran deffi forfe l'origine dalle cagioni medefime, ch' eccitano i Vulcanici Terremoti, ma però altre ve ne avranno eziandio con quelle congiunte, non effendo le medefime valevoli a fpiegare una comunicazione di moto, che a migliara di miglia quafi iftantaneamente fi comunica. Ma di quefta fpecie di Terremoti non è noftra ifpezione parlare, e ci reftringeremo foltanto ad efaminar quei Terremoti Vulcanici, dall' effervefcenze, ed accenfioni originati, i quali ordinariamente non molto lungi fi eftendono.

I Terremoti dunque fi fanno fentire, o allorchè le materie terreftri ftanno in attual movimento per una potente effervefcenza, e forfe concepita infiammazione prima d'apparire l' incendio al di fuori, o fivvero dopo un manifeftato incendio. Qualora fia quefto prevenuto da fpeff Tremuori, indizio manifefto è, che farà l' incendio uno de' piu nutabili; la ragione è per fe chiara, e l'efperienza il comprova, fecondo che le ftorie di tutti gli incendj riferifcono. Le cagioni che rifvegliano l'effervefcenze fono parimente le immediate cagioni de'Terremoti, e le accenfioni, che a quelle feguano fono il 
mezzo, per cui fi comunica il Terremoto alle aitie parti terreftri. Concepita dunque l'accenfione nella fopra divifata forma, fi fciolgono, e feparano le particelle fra lor vincolate; la forza efpanfiva in properzione fi accrefce, e dalla reazione delle parti fteffe fconvolte, e con violenza agitate, fi comunica alla Terrá il tremore, cioè ne provengono i Terremoti. Se l'accensione della mafla non è tale, che colla fua forza sbalzi fuora le materie inveftite dal fuoco, fi odono i tremori, ma non comparifce l'incendio, il qual forfe per mancanza di circoftanze fpeffo fi eftingue. Seguitando poi a comunicarfi nelle materie infiammabili il fuoco, fuccedono quafi ad ogni momento gli fquotimenti ; ed apertafi finalmente la ftrada colla fua violenza, vi ha l'ingreflo l'aria libera, ed allora è che la maffa ardente refta totalmente dal fuoco invertita, ed il VulGano, che internamente agiva, al di fuori $f_{1}$ manifefta. Nel diffonderfi l'accenfione del già aperto Vulcano, fezuono ancora $i$ tremiti della Terra, ma con minore energì, poichè le inveftite materie dal fuoco meno refiftenza incontrano, e la lor violenza fi perde, e però la reazione in confeguenza è minore, vale a dire che gli fcuotimenti effer dovranno in proporzione minori. Succedono per lo più allora dopo feguito lo fcoppio, cioè nella refiftenza, che le parti oppongono alla violenza, o forza efpanfiva delle materie infiammate. Non è vero ch' in ogni accenfione fi fentano $\mathrm{i}$ Terremoti; perchè forfe le materie infiammabili effendo fuperficiali, non trovano refiftenza per accrefcer la reazione; ovvero facilmente quelle difgregandofi, non concepifcono violenza tale per comunicarla alle altre parti aderenti. Se riflettiamo. per-

tan- 
tanto alla violentiffima forza efpulfiva di quelle ardenti foftanze, capaci di produrre colla loro reazione fquotimenti tali da rovefciar la Terra, e rovinare intiere Provincie, non ci farà più maraviglia quel vedere con quanta forza sbalzi in aria tutti que' corpi, e diftacchi dalle vifcere del monte que' groffi faffi, e macigni, che fopra abbiamo indicati.

L'aria dell' ambiente fpinta con violenza, e rarefatta da qualche fubitanea accenfione, rifveglia fpeffo un certo tremore con $\mathrm{i}$ fuoi replicati urti negli Edifizj circonvicini, e tremano non diverfamente che fcoffi dal Terremoto; ciò che ha fatto credere a molti; ficcome fopra accennammo, effer quello un vero Terremoto. Hanno fpeffe volte quefti tali fquotimenti rotte le vetriate ne' Palazzi di Napoli; il che di fatto non fa mai ordinariamente il vero Terremoto, il quale agifce per replicati tremori comunicati alla Terra, e non per urti, - fpinte direttamente, come cagionate da altro eftraneo corpo, conforme fuccede al cafo noftro da quell' aria commoffa .

Dopo che la mafla ardente fi accofta alla fufione; e che bolle, e gorgoglia, fi dilata con forza proporzionata fino alla fua maggiore eftenfione, ficcome è legge d'ogni corpo, che fi liquefaccia. Avviene allora parimente che dallo sforzo, ed urto di quelle foftanze nel dilatarfi, fl ecciti un irregolare fcuotimento al Tremoto confimile, accompagnato però da quel cupo rimbombo, fopra accennate, che nell' eruzione del i 766 . fu si notabile. Nel celebre incendio del 1537 . dell' Etna in Sicilia, raccontano parimente, che per I 2. giorni continui non cefiarono mai quefti fquotimenti, e fotterra- 
nei mugiti; che tutto poi ebbe fine nello fgorgar della Lava, allora ciò̀ che cefsò la cagione d'ambedue quelli effetti.

Per anni continuati fpuntavano dal vertice del Vefuvio interrottamente delle fiamme copiofe d'un color quafi ceruleo, fenza altra mefcolanza di fumo, e vapori, le quali fervivan anche mirabilmente per Fanale ai Naviganti nell' ofcure notti, in quei Mari circonvicini . Ceffavano poi all' apparire di qualche incendio, ficcome io fteffo ho veduto. Una continua accenfione di minerali, e di zolfi in ifpecie doveva mantener viva la forgente di quefte fiamme. Il color ceruleo, la chiarezza, e la lunga loro durata confermano l'opinione, poichè la Chimica colla mefcolanza di fpiriti oleofi, e bituminof, ed altri fali diverfi, ci prefenta in piccolo molti efempi confimili.

Merita d' effer qui ricordata un'opinione venuta per coftante tradizione, ed abbracciata dalla maggior parte degli Scrittori delle Vefuviane Eruzioni. Si dice adunque, che nelle poffenti accenfioni l' acqua del Mare fi fcofti dal Lido, e che talvolta ha lafciati in fecco i Vafcelli, ed i Pefci anelanti fuor d'acqua. L'accenfione come accenfione, è fuor di dubbio, che non può produrre un effetto fimile, perchè in quella non fappiamo trovare una forza così repellente, da fpingere contro natura un immenfo cumulo d'acque. Potrebbe forfe piu tofto chiamarle a $\mathrm{fe}$, col diroccare qualche interna parte del monte, ed aprir loro cosi una frada per deviarfi, ch'è appunto quel mezzo, con cui molti han fpicgato quefto pretefo profciuganento; $\mathrm{Ma}$ e chi non vede, che talmente effendo, crefcerebbero anzi le. 
acque nel Lido, per la copiofa corrente, che moverebbe la fterminata mole dell' acqua del Mare, colla di lei preffione, verfo quel nuovo aperto fcolo nelle vifcere della Terra, oppur del monte medefimo? Sicchè l'accenfione o niente può conferire, o fe mai, per fuppofto molto lontano conferifca, opererebbe in contrario.

Quando anco fi fupponga fenza ragione, ch' un' ampia voragine le può afforbire, e cagionare il loro decremento dal Lido, oltre ad effere il Mare un' inefaufta forgente, a cui non mancherebbero le acque da fomminiftrare i difetti, non può al certo avvenir mai, ch' il Lido fi afciutti, fe non fi diminuifca ancora in proporzione l' intiera mole dell' acque ; come non fi afciutta mai, nè diminuifce l' acqua verfo l'orlo d'un vafo, o verfo la fponda di un fiume, fe ancor non fi diminuifca proporzionatamente tutta la mole dell' acqua contenuta nel vafo, o nel letto del Fiume; cioè non fi diminuifca ancora in altezza. Dunque cosi effendo, tutto il Mare adjacente, e ancora l' Oceano fcemar d'altezza proporzionatamente dovrebbe. Ma e chi ardirebbe afferire fimili puerilità?

I Terremoti, che per effetto della comunicazione del noto, ch' imprimono all' acque, e che ftraordinariamente le commuovono come un ammaffo di folida. materia, per la indicibile velocità, che a quelle imprimono, faran forfe la cagione del ritiramento loro dal Lido, ficcome alcuni han pretefo fpiegare il fuppofto fenomeno, e con qualche più probabil ragione. Se il Terremoto abbia la fede verfo l'oppofta parte del Mare, cioè dalla parte, che fta il Vefuvio, e ch' imprima delle fiere fcoffe all' acque vicine del Lido, fi conA a a 2

cepi- 
cepifce allora, che vi è di bifogno d'un impeto di ftraordinaria polfanza, per ifpingere indietro un Mare intiero; ma che però può effer poffibile il fatto, di reftare per poco tempo afciutta la fpiaggia, fino a tanto che la forza impreffa è tale da fuperare la refiftenza della groffa mole dell' acque, che fi sforzano di ricomporfi per loro natura a livella. Succederebbe in tal cafo il fluffo del Mare, fimile a quello cagionato dall' attrazione della Luna, per cui fon tirate l'acque fuor del loro letto, e feguirebbe inoltre il refluffo confimile, per cui dalla veemenza, colla quale ritornano, ufcirebbero fuori, e formonterebbero l'ordinario lor polto. Ecco il meccanifmo più naturale, che avvenir potrebbe da $i$ Terremoti del Vefuvio, e forfe farà in qualche tempo accaduto; non potendo noi prefcrivere la veemenza, che quei aver poffono. Ma è chiaro altresì che nè le Navi, nè i Pefci poffon mai reftare in fecco alla fpiaggia ; perchè in tal cafo le colonne dell' acqua fmofte dalla concepita velocità, e velocità forprendente, come fe folide foffero, fcorrendo, neceffariamente feco trafportano tutti que' corpi, ch' incontrano .

Per non impugnare un fatto da tanti conteftato, diremo dunque così; che i Terremoti del Vefuvio avendo impreffa qualche fiera fcoffa a quell' acque, e fatte vacillare, come è loro natura, e forfe anco dalla fpiaggia recedere alquanto in quel fubitaneo impeto comunicato, abbia ciò dato motivo agli Scrittori di efagerare il fatto, e di alterare poi la fantasia dell' idiota gente, che tutto annunzia come prodigiofo.

L' ultimo che refta a notarfi de' fenomeni del nofro monte è quello giuftamente, ch' in tutto il tempo 
della fua accenfione, a poco a poco ce lo prepara; ed c̀ quello fpettacolo sì funefto di quei famigerati fumi ài pafta infuocata, detti volgarmente le Lave.

Egli è certifimo che fpecialmente dopo il fopra memorato incendio del I $\sigma_{3} \mathrm{I}$. fi è convertito il Vefuvio in una forgente inefaulta di quefti fiumi di fuoco, c niuno anno forfe paffa, che non fi rinnovino sì lugubri fpettacoli. L'intiera interna maffa fconvolta da quella portentofa accenfione, le aperture probabilmente di nuovo formate, altri nuovi ftrati fcoperti di minerali, avran forfe aperta una perpetua forgente alle accenfioni. In fette groffi rami di Lave dalle, bocche del vertice fcaturite, fi divife il rigogliofo fume di fuoco in quefto incendio. Eran prima le collinette dèl monte ameniffime, e le coltivazioni fino alla fommità fi eftendevano; ma fi muto ben prefto in lugubre afpetto tutta la di lui faccia; inondarono le Lave le deliziofe Campagne, devaftarono Ville, e Palazzi, ed intieri Villaggi, e reftaron vittima de'lor furori da 10000. perfone. Gli Storici, che raccontano quefto eccidio, movono a compaffione colle loro patetiche defcrizioni. La gran copia della cenere, e le montagne di nero fumo, che coprivano il Cielo, i turgidi fiumi di fuoco che defolavan la Terra, e le altre apparenze tutte, ch' il Vulcano prefenta, intimorirono talmente i Popoli di quei contorni, che annunziavano per le ftrade lo Aterminio del Mondo.

L'idea in fatti, che prefenta alla vifta la Lava non può al vivo rapprefentarfi, nè fenza vederla è poffibile poterla adequatamente concepire. 
La terreftre materia fciolta, e liquefatta nelle fpaziofe voragini, forma dunque la Lava, la quale nel crefcere all' eitremo grado della fua dilatazione, o rompe in alcuna parte, e fi procaccia l' ufcita; o fe la refiftenza oppofta è fuperiore alla forza d'efpanfione, col rigonfiarfi, e crefcere, efce allora dalle fuperiori bocche del Vertice; ciò che fempre faceva in antico, e che fa di rado al prefente; per la ragione che confumatafi l'interna materia, e refe più fpaziofe, e profonde quelle voragini, non fi dilata talmente da forpaffare la loro altezza, e affai più facile le refta il fuperare colla fua forza la refiftenza del già indebolito corpo del monte.

Ella è pertanto quefta Lava la materia dell interno del Vefuvio inveftita dal fuoco, e convertita in un fluido; ficchè qual debba effere l' energì di quel vivo fuoco, che converte in un liquido, macigni, pie tre, ed ogni altra fpecie di terreftre materia, fi può appena colla forza del noftro intelletto immaginare. Il gran Borelli nella fpiegazione della Lava, o fia Ghiaja dell' Etna, s' ingegna di dimottrare, che ficcome nel vetro fufo, così vi debbono effere anco con effa mefcolate le fabbie, e fali fiffi, affinchè ne fegua la fufione. Ma e chi prefcrive regole alla Natura? II fuoco del Vefuvio efce della sfera de' fuochi nottrali, e perciò gli effetti corrifpondenti ufciran fuori delle proporzioni cogli effetti di quelli.

Quella fterminata mole, che bollente, e fluida gorgoglia fotterra, fubito ch' ha l'efito all' aria libera, comparifce un piano di pietre irregolari, e fpugnofe,

tutte 
tutte di colore fuliginofo, e nero. Vorrei portarvi un efempio, per farvi in un colpo d'occhio concepire queft' ampia eftenfione ricoperta di Lave; nè fo più adequato trovarlo, ch in un campo arato di frefco, $r i$ coperto con quelle zolle fmolfe, e irregolari, e di diverfa figura, veftite d' un color terreo cupo, che fi approffima al nero. Si vede muovere quefta intiera maffa, fe un fi pone da fronte, d' un moto a riprefe a guifa dell' onde marine; nè fcorre già fluente come un fiume, ficcome per lo più ognun penfa, indotto dalle defcrizioni di efla . Con i fuoi ondeggiamenti va a feconda del moto inteftino impreffo nelle parti interne, che fluide fono, e fcorrevoli; ed incontrando ancora il declive del monte, la Gravita infieme, e la forgente, che continuamente ne fomminiftra, contribuifcono talmente alla medefima il moto.

Si vedono pure le pietre della fuperficie lentamente camminare, fenz' accorgerfi da chi fiano trafportate, e nel loro moto cadono, e s'urtano infieme, producendo quel fuono, che $i$ rolticci, o frantumi di vetro cozzati infieme tramandono. E'. la fluida materia, che tale confervandofi fotto quefta fuperficie di pietre, continuamente fcorre, e tutto feco trafporta ; e fe fra pietra, e pietra $f($ offervi, $f i$ vede ocularmente fcorrere a guifa di fufo metallo. Se veduta di giorno altro non ci rapprefenta, ch' una campagna coperta di glebe, e di pietre fuliginofe, ben diverfo è poi l' afpetto, che ci moftra di notte. Comparifce allora come un lago di fuoco, o una campagna ardente, o roffeggiante, qualora però fia di poco tempo dal monte fgorgata. Non offufcando allora $i$ raggi del giorno la fua tramandata 
luce, il rolfeggiante colore, che tra pietra, e pietra trapaffa, fmorza la loro nigredine, ed $\mathrm{i}$ foli raggi lthcenti, e roffi fi rimandano alla noftra vifta, per cui un' intiera campagna di fuoco ci contparife. Se la di lei eftenfione fia d'una confiderabil grandezza, quel fuo roffo cupo, e vivace, fi riflette, e refrange nell' Atmosfera; e vedefi allora nell ambiente una legittima $\mathrm{Au}$ rora Boreale; $e$ però nel vedere fu la Terra una Campagna di fuoco, e poi nel Cielo quel roffo colore, che le fovrafta; immaginatevi che colpifce la fantasia, come uno de piu inufitati fpettacoli.

Degna è di rifleflione la trasformazione di quella liquida pafta in quella moltiplicità di pietre fuperficiali, fubito che comparifce al contatto dell' aria. Molti varie cofe. han dette fopra di ciò; ma che il contatto dell' aria fia la ragion potiffima, ficcome ha creduto ancora il Borelli, facilmente lo perfuade ragione - Portiamoci frattanto coll' immaginazione a vedere fgorgare dal monte quefta liquefatta materia; Il contatto dell' ambiente efterno colla fua elafticità, e preffione fcacciar deve dalle di lei parti efteriori il fuoco; cioè fi comunica quello nell' aria che tocca, e fi. difperde, ed in proporzione ne perde altrettanto la Lava, ficcome è notiffimo per le Leggi Fifiche; che tanto è a dire, che la Lava nella fua fuperficie fi raffredda. Si comprime, e reftringe per confeguenza, nel paffaggio dallo ftato di calore a quello di freddo. Senza diftaccarfi parte con parte, non puó feguire tal reAtrizione, ovvero diminuzione nel fuo volume; ed ecco che avremo la divifione di quella fuperficie in varj, e diverfi modi, fecondochè più, e meno militano lé ragio- 
gioni della di lei reftrizione. Scorre ancora irregolatmente per le pendici del monte; e quel moto irregolare comunicato all' intiera mafta, ajuta la divifione già principiata di quelle parti, ed efficacemente conferifice alla loro frazione, per le fcoffe, ed urti, che vicendevolmente foffrono. Ci porta cosi il difcorfo a veder gia convertita una fuperficie levigata, in tante diftinte pietre di diverfa figura, e grandezza, delle quali per appunto vien ricoperta la Lava. L' acqua che ne rigidi tempi d'Inverno fcorre per piani irregolari, e ch' incontra infieme l'ambiente frigidiflimo; la cera parimente liquefatta ne' Torchj accefi, allorchè fcorrendo perde il calore, e che tanto l'una, che l'altra fi unifce in quei cannelletti, o gruppi irregolari, fono triviali efempj, ma ci fan concepire nel piccolo, un meccanifmo confimile a quello della formazione delle pietre già dette. Si forman quefte poi cosi fcabre, ed irregolari, e di varia confiftenza fra loro, attefa la difformità nei principj componenti, e dai quali pure anche dipende quella loro si difforme porofità; perchè effendo un compofto di parti eterogenee, non han quelle percio l'attività medefima nel raffreddarfi, e reftringerfi in un tempo medefimo, nè di adottare la fteffa figura.

Egli è fatto coftante, ch'ai giorni noftri getta il Vefuvio continuamente del fumo dalla fua cima; ed è ancor vero, che le fue accenfioni fpeffifime fono, in paragone di quelle de' tempi addietro. Bifogna dunque dire, ch'il fuoco fi mantenga accefo continuamente la dentro, giacchè fenza interruzione efce del fumo. Noi difficilmente fappiam concepire un fuoco perenne, ma fe perenne è $\mathrm{B}$ b b 
il fumo, deve effervi ancora l'attual fuoco non potendo quello fenza di quefto fuffiftere.

E'dunque certifimo per la coftante offervazione, che per lo fpazio d'un fecolo confervafi il vivo fuoco dentro del monte; vale a dire dopo quel lacrimevole Incendio fopra annunziato del 1631 . Lo fconvolgimento dell' interna maffa del monte avrà forfe allora aperta una nuova forgente, ovvero un alimento durevole al fuoco. La fterminata mole della fciolta materia, ch' in qualche accenfione non può avere facilmente l' efito, attefa la profondità, ed eftenfione dell' ampie caverne, fi dovrà mantenere colà dentro infuocata, e per confeguenza eruttare quel continuo fumo, e prefterà altres̀ facilità fomma per rifvegliare nuove effervefcenze. Molti di fatto han vifta quefta Lava infuocata, e bollente allorchè non era il Vefuvio in furore; e fe conferva per degli anni il calore fgorgata che fra all' aria aperta, conofcendof dal fumo, ch'or quà, ed or là dalla medefima fcaturifce, confideriamo dunque, per quanto tempo mai potrà colà dentro confervarlo:

$\mathrm{Da}$ quefto continuo ardere del Vefuvio, e dalle fue eruzioni potrebbefi forfe inferire una valida ragione della variazione dell' ambiente, o Clima di Napoli, che alcuni Vecchj della Citta dicono efferfi da un fecolo in quà notabilmente alterato. Vi vorrebbero al certo moltiffime offervazioni, lunga ferie di anni, e fcrupolofità filofofica, per poter con certezza afferire $\mathrm{i}$ differenti ftati dell' Atmosfera, o fia la di lei variazione, in tempi molto diftanti fra loro; però la ragione ci affife per creder variato un Clima qualunque, fe abbianvi luo- 


\section{Dell Accademia.}

go alcune determinate circoftanze, ficcome par che ve lo abbiano rifpetto al Clima di cui fi parla.

Concediamo che una nuova forgente d'efalazioni fi efalti nell' Atmosfera, ecco che avremo il di lei ftato alterato, o per meglio dire effenzialmente mutato. Son comuni gli efempj de' Climi ch' hanno acquiftate qualità diverfe, e ancor maligne per cagione di Terremoti, d'Alluvioni, e cole fimili. Siccome è altresi comune il total cambiamento, per il profciugamento di ftagni, e paludi, che quanto è a dire per efalazioni introdotte, o detratte dall' aria. Sicchè ammeffo il continuo fluffo di particelle bituminofe, e di fali d'ogni forta trafportati nel Cielo di Napoli, per mezzo delle accenfioni vefuviane, con piu forte ragione potremo credere efferfi mutata la natura del Clima.

$\mathrm{E}^{\prime}$ pofta la Città di Napoli fu la fpiaggia del Mare quafi ful mezzo d'un Golfo, voltata totalmente al mezzogiorno. Dalla parte di Settentrione è circondata dai monti, e da collinette ameniffime, che formano come un femicircolo, il quale va a feconda del Golfo, e che comprende con una delle fue eftremità tutta la Latitudine ortiva, e coll'altra l'óccidua del di lei Orizzonte; di maniera che da tal pofizione ha prefo motivo alcuno di motteggiare i Napoletani, che troppo efaltano il loro paefe, e di foverchio difprezzan l'altrui; rinfacciando loro, che vedendo nafcere il Sole ad una eftremità del Golfo, e full' altra tramontare, fi credono, che folamente per le loro Terre, e non per altre Provincie del Globo, fparga gli infuifi fuoi quell' Aftro benigno. Quefta tal defcritta pofizione pertanto, che taglia il corfo ai Venti Settentrionali, non permette fa$\mathrm{Bb}_{2}$ 


\section{$380 \quad A$ T $T$ I}

cilmente alle efalazioni efaltate dai minerali del Vefuvio di lungamente trafportarfi, benchè $i$ venti Meridionali vi regnino con poffanza; e però ripofando di continuo nell' Ambiente vengono a naturalizzarfi con l'aria, e la trafmutano con il loro mifcuglio in altra diveria da prima.

Comunque foffe in antico quefto Clima, verifimo è ch' al prefente è talmente variabile, che non credo darfi in altra parte di Terra il confimile. Più volte mi fon prefo il penfiere di efaminar col Termometro, e Barometro alla mano, le variazioni del caldo, e del freddo, ficcome della diverfa preffione dell' aria, e per replicate oflervazioni in giorni diverf, vi ho notato variar per fino nove volte in un fol giorno le refpettive altezze del Mercurio; corrifpondendo ancora le mutazioni nell' Atmosfera con caldo, con freddo, colle burafche, e col tempo chiaro, fuccedendo ognun di loro a vicenda. Dei Venti, che giuftamente fono la principal cagione della mutabilità dell' Ambiente, accadeva fimilmente lo fteffo; ed il fumo, che continuamente efce dall' erta cima del Vefuvio, egli è per appunto quello, che con particolar fimetria indica dei Venti, ogni benchè piccola mutazione.

Non oftante l'alterazione, che poffa aver fofferta quefto Clima, e non oftante che comparifa talmente variabile, egli è però di fua natura falubre. La Gente del paefe, e di quei contorni è faniflima, robufta, e vivace; indizio certo dell'aria felice che refpira. Le particelle fulfurec in gran copia, e gli altri volatili fpiriti de' minerali incorperati coll'aria, contribuiranno forfe alla di lei elafticita; tanto neceffaria per la refpira- 
zione, ed infpirazione de' Viventi. L' aria f́pecialmente di quella popolofa Città, faturata di vapori fuliginofi, per la gran gente, che la refpira, molto perderebbe nel fuo Elaterio, fe quefte fopraddette particelle non le fomminiftraffero forza per mantenerlo, e reftituirlo infieme, allorchè venga diminuito. Si diffe fopra, ch'il Clima è un potentifimo influffo, e che ha la gran parte in ogni umana vicenda; e chi fa dunque, che quell' attività delle furriferite fottiliflime particelle faline, naturalizzate coll'aria, non fia l'unica ragione, o almeno la principale, di quel brio, e vivacità, e di quella tanto connaturale variabilità di quei Popoli? Ma pafliamo ad altro.

Le confiderazioni fopra $\mathrm{i}$ defcritti incendj del. Vefuvio ci tirano per incidenza a far breve digreffione fo. pra lo fterminato, e perpetuo incendio del Dífo Solare, ch'alcuni giuftamente pretendono altro non effere ch' un' immenfa mole ardente da un quafi infinito numero di Vulcani. Di fatto è naturale il credere', che quel che rifcalda fia fuoco, giacchè la Natura è fempre limile nell' opre fue; Sicché una portentofa. Maffa infuocata riconofceremo nel Sole, e che fia del pari dal continuo fuoco inveftita, giacchè di continuo trafmette quel fuo calore, e vivaciffima luce, ad immenfe diftanze.

Le portentofe macchic, che di tanto intanto lo coprono, altro non faran forfe che quelle ttupende montagne di Fumo, e caligine, che col continuo ardere diftacca dalla maffa il vivo fuoco, ficcome ne'Vulcani fuccede; e da quel fuo moto di vertigine; compito intorno al fuo alle in giorni 25. e mezzo, l'oflervazioni ban 
fatto vedere, effer fimile in ogni parte, cioè ch egli fia un Globo inveftito dal fuoco per tutto egualmenre. La luce rifleffa nei Pianeti, e nelle Comete, Ie quali alla rinfufa fon difperfe nel Cielo, prova parimente, che trafmette, come fuol dirf, in isfera il fuoco, $c$ con effo la Luce. Egli è perciò il Difco Solare, fecondo il Newton, quattro volte men denfo del noftro Glo. bo Terraqueo; e ragione il perfuade, qualora fia, come fi diffe, una maffa infuocata, dovendo quella dilatarfi per le note leggi de'corpi penetrati dal fuoco: $\mathrm{C}_{i}$ porta il difcorfo a vedere, chªlterazioni non poche deb. bono effere avvenute, ed avvenir dovranno rifpetto a quefta fterminata mole di fuoco. Gran vicende né fuoi afpefti, varj gradi di calore, e varj pure gli ftati comparir devono della fua luce; ficcome realmente alcuni Aftronomi han pretefo dall' offervazioni riconofcere in quella, notabiliffima alterazione.

Noi fiam fempre Fanciulli, che tutto dobbiamo apprender noi fteffi, relativamente al governo, $\mathrm{e}$ agli andamenti, dei quali, da tempo immemorabile, ha per ufo valerfi la Maetra Natura; che fe i fuoi fatti fi perpetuaffero, per mezzo delle offervazioni nell' intelligenza degli Uomini per migliara di fecoli, allora è, che dai paragoni vedremmo le notabili differenze, $e$ gli ftati diverfi, in cui è paffato quefto Aftro luminofiffimo.

Supponiamo pertanto di riftringere in un circolo daterminato tutte le vicende, ed alterazioni del gran Vulcano del Sole, o fia d' una maffa ardente di foco. Quefta dopo effere ftata penetrata, e fcompofta da quell' attiviffimo elemento, deve a poco a poco convertirf in fufione 
fulione, cioè in una mole intiera di Lava. Spariranno allora le montagne intiere di fumo, o fian le macchie, e fi dilaterà all' eftremo grado, crefcendo per confeguenza di diametro, ed apparirà perciò luminare maggiore di prima, e dotato forfe di maggior vivezza di luce. A tal grado pervenuto compier deve le fue viceffitudini, e quel calore, che acquiftò dalla veemenza del fuoco, lo deve a poco a poco perdere raffreddandofi, ed unitamente in proporzione perdere la fua Luce. Vero è, che fram molto lontani nel vedere avvenute vicende sì formidabili, di cangiarfi tutto in un cieco abiffo di fole tenebrofe nótti che fe la Terra noftra fuppofta un Globo infuocato foltanto, fi trova per le leggi Fifiche, che non perderebbe il fuo total calore, e manterrebbe perciò la luce proporzionata; per lo fpazio di anni so. mila, maggiormente pói il Dif́co Solare dobbiam credere poterfi matenere infuocato, :o tivvero Aftro luminofo, per lunga ferie di migliara di fecoli, ritenendo la di lui grandezza; ovver Volume, benchè men denfo, al Volume, o grandezza del Globo terraqueo, quella proporzione, che ha il millione all' unità. Se da femplice Globo ardente trapaffic quindi il Sole alla liquefazione, fecondòchè le Leggi. Naturali richiedono, forpaffa allora la noftra immaginazione il confiderare, per quante migliara e $^{2}$ migliára di fecoli puó naturalmente fervire a far l'ueffizio di primo Luminare nel: Cielo, a cui fu dalla divina Provvidenza déftinato.

La Natura è un libro fempre aperto, in cui dal divino Artefice vi furono fcritte sab éterno alcune perpetue, ed invariabilideggi, eldelibeati i fatti, che nel corfo dei Secoli necoraciamente da quelle dipendono: Non paja dun- 


\section{$3^{84} \quad$ A $\quad$ r $\quad$ T $\quad I$}

dunque a taluno inutile diftrazione di fpirito, fe poitiamo ardimento nel rintracciare in quefto aureo libro qualche pezzo di delineata Storia de'Secoli futuri. s

\section{$S E$ I O NE I I I.}

Dove fi efamina fe la materia cbe efpelle negli Incendj

ibl Le fuvio venga da iltre parti, le fe quella cbe:

in $\int e$ contiene sia fufficiente per mantenergli.

A Spellono i Vulcani dalle loro vifcere, came abbiãmo vifto, copiofità di materie nelle refpettive accenfioni, ed il Vefuvio per le tante Lave, ed altra forta di corpi dal fuo ventre cacciati, ha fatto penfare a molti, chegli non poffa effer capace per fe folo di racchiudere tanta, ce si fatta materia eruttata finora; perciò fi credono forzati a credere, che da altre parti vi fia cola portatà; ch' è giuftamente tutto ció, ch' anderemo efaminando nella prefente Sezione.

Appagati da quefta prima apparenza, fenza rifletter piu oltre con qual meccanifmo; e qual moto fe come trasferir $\mathrm{fl}_{\mathrm{i}}$ poffa in un determinato luogo un corpo qualunque; e fenza efaminar feriamente, fe quella montuofa mole poffa effere ftata da per fe fufficiente a fupplire alla efpulfa materia, fonovi ftati alcuni che groffolanamente han creduto, ch'i fiumi di fotterraneo fuoco fian quelli, che depofitano le refpettive materie nelle voragini del Vefuvio, ed in quelle ancora di tutti i Vulcani del Globo:

Altri han creduto piò ragionevole il dire; che fia il Mare quel mezzo deferente le refpettive foftanze dentro $i$ Vulcani, indotti da un'apparente ragione, ch'i uss mede- 
medefimi fian tutri collocati in vicinanza del Mare; come $f e$, pofto vero anco il fatto, il trafportarli un corpo da luogo in luogo, dipendeffe dalla fola vicinanza, e non piuttofto dalle meccaniche leggi, colle quali fi efeguifcono $i$ movimenti in Natura.

Vedremo dunque, ch' il fupporre la venuta de' corpi eftranei dentro $i$ Vulcani da altre parti, e provin: cie, non è alle naturali leggi conforme; e vedrem poi, ch'il corpo del Monte tanta materia in fè contiene da fupplire, non folo agli incendj finora paffati, ma agli altri ancora, che per piu fecoli avverranno in futuro. La cofa è per fe manifefta, e non parrebbe. meritare attenzione per efaminarla, ma ficcome Autori gravi negano nei monti quefta fufficienza di materia, e la comune opinione, rifpetto al Vefuvio, fi uniforma con effi, crediam perciò noftro debito non trafcurare nè anco dì un tal punto l'efame.

Ella è la materia per fe fteffa fenza moto, che fe non vi concorrono alcune forze in natura, non può certamente dentro i Vulcani portarfi ; e però immaginati fi fono quei fotterranei fiumi di fuoco attuale, $i$ quali paffeggiando il fotterraneo Mondo per incognite ftrade, induftriofamente poi confegnano $i$ corpi ardenti nelle Fucine Vulcaniche. Gaffendo, uomo per altro illuminato, concepifce che quefti fumi paffando fotto il Vefuvio, fcorrano poi per fotterranei tragitti fotto $\mathrm{Ma}$ re, e col monte Etna in Sicilia comunichino; e di là parimente trafportando le accefe materie fotto l' ampio fpazio del Mare, pervengono in fino nella Siria, trapaffano l'Arabia felice, e facendo capo nella fotterranea Etiopia, comunicano col monte Semo, Vulcano C c c fimil- 
fimilmente rinomato, e così difcorrendola, paffeggiano il fotterraneo Globo, per fomminiftrare ai Vulcani tutti le materie opportune agli Incendj.

Di fatti così belli, e fpeciali, egli è un danno; che non nè abbiamo, non dico una prova, ma nemmeno un leggiero indizio, fe non che in qualche Romanzo, e nelle poetiche fantasie, le quali non par che debbano avere feriamente luogo nelle vere, e reali cofe della Natura. Il folo fatto fervirebbe a fmentir l'opinione, perchè nel Vefuvio avanti il $163 \mathrm{r}$. efaminato dentro le. fue caverne, non vi offervarono nè paffaggio di fiumi, nè indizio di fotterranee comunicazioni; ma paffando alle ragioni, vorrei domandar ai fautori di quefti fiumi, perchè incontrino difficoltà a trovare tanta materia fufficiente agli incendj nei monti, e niuna difficoltà a trovarla fufficiente poi al mantenimento de' fiumi? E fe per mezzo dei fiumi tanta fe ne porta dentro i Vulcani, faprei rolentieri, chi fia poi che porti tanta materia dentro dei fiumi; poichè cosi non fi toglie, ma fi volge altrove la difficoltà. E volendo che la materia infocata corra da per fe verfo dei fiumi, diremo che da per fe parimente corre verfo dei monti; ed ecco che non avrem bifogno dei fiumi. Si ammettono quefti fiumi di fuoco;, fpecialmente ancora perchè rendon faciliflima la Spiegazione dell' origine delle accenfioni Vulcaniche, non ben ravvifando., dicono quefti, diverfamente per le leggi meccaniche, il loro principio; come fe perfettamente intendeffero poi, qualmente ne' fuppofti fumi quelle materie theffe principiano l'incendio ; ta dunque fempre ferma la difficoltà, e nulla profitta perciò Ia loro intelligenza,

$$
\text { E'co. }
$$




\section{DéLI'ACCADEMIA.}

E' cofa a tutti nota che molti de' Vulcani s'eftinguono coll' andare de'tempi, è che altresi molti nuovamente ne inforgono; or dunque effendo $i$ frumi, che contribuifcono a quelli le loro materie, e che del par ri fervon di principio ai loro incendj, dovranno perciò deviare il corfo folito da' Vulcani già eftinti, e volgerlo a contrario verfo de' nuovi, giacchè le vicende fono üniformi fra loro; ed ecco ch' avrem neceffario d'ammettere altri incongruenti fuppofti. Bifognerà fupporre ancora, che appoftatamente, anzi con certa induftria. fenpre fotto dei monti fcorrano, e depofitino l'accefe materie, formando i Vulcani, e mai fuor ' $d^{\prime}$ effi, nèi luoghi piani, nè nelle valli ; poichè non abbiamo nemmeno un fatto nella ftoria della Natura, che ci indichi effere fgorgati, colle loro efcrefcenze nei luoghi di pianure, nè che abbianvi mài genérati Vulcani; quando che naturalmente parlando, nelle Valli piuttonto, o pianure dávrebbero metter foce, per incontrare ivi, meno che fotto la groffa mole dei monti, la refiftenza della foprappofta terreftre materia. E ficcome talmente fi gonfiano, che al dire dei loro Autori, per la forte concepita dilatazione dal fuoco $\vec{s}$ alzano fino alle alte cime de' monti, per dove fcaricano $i$ corpi accefi, e liquefatti, e che producono eziandio con tal dilatazione tutti i Terremoti del Globo; parrebbe dunque che giunti a tal violenza, rompere doveffero, e diroccare la Terra, dove meno fr oppone alla loro dilatazione, la refiftenza. Ma la Dio mercè farem femprè liberi da quefti difaltri, avvegnachè liberi framo da fimili fotteranei pericoli ; e quantunque Cicerone, e Serreca fautori di quefta opinione, ci annunzino, she par mezzo dei Terremoti,

$$
\mathrm{C}^{2} \mathrm{C} i
$$

rifve- 
rifvegliati dai fotterranei fiumi di fuoco, vedremo diroccata una volta, 0 fubbiffata la Terra, vivrem non oftante quieti, e ficuri, lafciando all' autore della $\mathrm{Na}$ tura diriger la Macchina Mondana, a feconda del fuo mirabil difegno.

Una valida congettura che efiftano quefti fiumi fotterranei di comunicazione con i Vulcani; la tirano dall' erronea credenza, che le accenfioni degli fteffi Vulcani frano contemporanee. Fra gli antichi, Strabone adduce gli efempj dell' Incendj dell' Etna, inforti nel tempo fteffo con quelli dell' Ifole di Lipari.

Gaffendo vuole anzi, che nel tempo delle Vefuviane accenfioni accadano fimilmente quelle dell' Etna in Sicilia, e del monte Semo in Etiopia; e fulle pedate di quefti, molti de' noftri moderni afferman lo fteffo; ma il gran Borelli però, che attentamente prefe conto del tempo degli Incendj del Vefuvio, e che offervò altresì quelli dell' Etna, fi ride di quefta pretefa corrifpondenza, ed io m' accordo con effo, perchè con quegli incendj da me notati nel. Vefuvio, non hanno mai e poi mai corrifpofto l'accenfioni dell' Etna.

E realmente fe le pretefe contemporanee accenfioni ftabiliffero l'efiftenza di quefti fiumi, come pure la loro comunicazione con i Vulcani, dovrebbe dirfi che il noftro Vefuvio per mezzo di quefti fiumi comunica col monte Hecla in Islanda, con i monti della Cordegliera in America; con i Vulcani delle Molucche, delle Filippine, del Giapponefe, ed in fomma, con tutti i Vulcani della Terra; perchè effendo da un Secolo che egli di continuo arde, or con più veemenza, ed or con meno, deve naturalmente effere avveruto, che dentro quefto fpazio d'anni, 
abbiano i fopraddetti Vulcani parimente generati Incen$\mathrm{dj}$; e però un per uno, ovver tutt' infieme avranno contemporaneamente con il Vefuvio prodotti gli Incendj. Si ftabilirà dunque da ciò, che efftano l'immaginati fiumi, e la loro comunicazione dell immenfo giro di tutto il Globo? = Sogni d'Infermi, e fole di Roman$z^{i}=$ Reca però maraviglia come Lomini illuminati ancora dei gionni noftri s'appaghino di quefta comunicazion di Vulcani, originata dai fiumi, per la fola fuppofzione de'loro contemporanei Incendj. Come mai puffon credere, che data ancora quefta uniformita di tempo nelle accenfioni, poffa ciò derivare da un meccanifmo di comunicazione fra loro, e non piuttofto da un mero accidente? Affinchè per la fuppofta comunicazione fi ecciti fra luoghi diftanti un incendio nel medefimo tempo, farà neceffario che la forza d' efpanfione della fciolta materia in quei fotterranei Alvei, principii ad efercitare la fua energì da un certo, e determinato punto, come Centro delle forze, e che con mifurata, e precifa regola imprima moto tale a quella fcorrevole materia infocata, a fegno che, mefle a computo le varie refiftenze che incontra, e gli fpazj diverfi da fcorrerfi, la porti, e la conduca a diverf Vulcani, per iftrade diverfe, nel tempo medefimo: In fomma fi deve prendcre un punto intermedio fra gli alvei di comunicazione, dal quale data la fpinta alla materia accefa, colle fue forze d'efpanzione imprefte, fia capace di fuperare, a mifura che incontra le refiftenze, e di fcorrere a puntino li neceffarii, e diverfi fpazii, per ritrovarfi in un tempo medefimo dentro $i$ varj Vulcani, benchè fieno a lontane diftanze, e talvolta in parte tutta

oppo- 
oppofta fra loro, così richiedendo me meccaniche leggi dei movimenti. Tutto quefto così ben regolato meccanifmo potrebbe in fatti avvenire, non avendo noi ra. gioni di provarlo impoffibile, ma refterà ancóra difficilifimo il provare, che realmente fucceda.

Il Mare porterà dunque le materie opportune densro i Vulcani, ficcome piace a molti, ed a Filofofi ancor di grido. Prima che gli Studiofi della Natura aveffero fcoperto, che le acque cadute in varie guife dal Cielo, foffero fufficienti a mantenere le forgenti tutte del Globo, ricorrevano a fpiegarne l' origine colli acqua del Mare. Finalmente acqua era quella della forgente, ed acqua era quella del Mare; ficchè col foccorfo di pochi altri fatti, e ragioni, inferite dalle nozioni fifiche, potevafi render probabile una tale opinione. Ma la faccenda è moltó diverfa rípetto alle forgenti di fúoco dei Vulcani ; le materie che le alimentano fon corpi duri, fon minerali, fon bitumi, tutti dall' acque differentiffimi; e però crefceranno molto le difficoltà, nel volere affegnare per forgente, ed alimento del fioco l' acque marine.

Conticne egli è vero l' acqua del Mare delle parzicelle terree, oleofe, e bituminofe, e fali $d^{x}$ ogni forsa, mifchiati con effa; e fi può col fuoco ridurre l' acqua marina ad una depofizione, da cui efaltati i vapori, reftino le altre particelle di corpi, opportune agli incendj; ma è manifelto altresi, che a raccorre in tal guifa tanta folida materia, quanta ne erutta il Vefuvio in una fola delle fue Lave, poco men ci vorrebbe, che Ia diftillazione di tutta. l' acquá del grande Oceano. Oltre di che bifogna anco fupporre, che queft acqua del Ma- 
re entri realmente dentro il Vefuvio; fuppofizione, che incontra informontabili difficoltà, conforme fopra abbiam vifto.

Siccome però in tempo di furiofe burrafche; diceva Strabone, e con effo replicando altri feriali Filofofi, trafporta il Mare verfo del Lido varj, e diverfi corpi, cosi egualmente dentro il Vefuvio trafmette i medefimi per fotterranee Atrade, per fervir poi di neceffario pafcolo al fuoco.

Quefte materie che dall' onde fi voglion là trafportate, non poffon condurfi per fottiliffimi canali, o tubi capillari, come ognuno conofce; ficchè dovremo neceffariamente ammettere de' canali di qualche luce confiderabile. Se così effer deve, ficcome quei corpi foli fenz' acque punto camminano, unitamente dunque - con effe entreranno dentro del monte; ed eccovi che avremo fempre in gran copia dell' acque nelle Caverne, ed aver per confeguenza dovremmo fpeffe le forgenti d' acque dalle cime del monte, in vece delle forgenti di fuoco; cofa che per migliara d'anni non è mai accaduta, fe fi eccettui una fola accidental volta, da noi fopra notâta. Seguirebbe oltracciò, che i canali, i quali dal Mare portano dentro il monte quell' acque, che doveffer portare ancora dentro del Mare il fuoco, e la Lava fabbricata dentro del monte, allorchè fpecialmente gorgoglia, e furiofamente bolle, a fegno di traboccare dal Vertice; non effendovi maggior ragione, che $\mathrm{i}$ canali trafportino $\mathrm{i}$ corpi dal Mare al monte, che dal monte al Mare. E pure non è a memoria d'uomini avvenuto mai un tal fatto; perchè, credo io, non è mai ftato vero, che fra il Mare, ed il monte fiavi comuni- 
cazione. Di fatto è quafi impofibile, che quefti alvei comunicanti efiftano nello ftato di cofe del Vefuvio ardente ; perchè contenendo dentro le fue voragini liquidiffima materia, e fcorrevole in tempo d'Incendio, quella deve neceffariamente infinuarfi, e chiudere ogni apertura, pofto che mai accidentalmente fiavi ftata; e però a chi ben ragiona; Lava dentro del monte, e comunicazione fra il monte, e il Mare, porta in fe manifeftiffima contraddizione.

Se a cafo mancaffe la terreftre materia dentro del monte, ben vedo che ragionevol faretbe $l$ indagare per quali ftrade colà $f_{1}$ porti, per mantenimento del fuo co; ma non fo intender poi perchè fi abbiano a mal comporre fiftemi, per provvedere il monte di tutto ciò, di cui foprabbonda, ed a tal fegno di racchiuder materia dentro fe fteflo, fufficiente a mantenere per migliara d'anni gli Incendj futuri, oltre a tutta quella già confunta finora; che quefto è ciò che refta da fare adeffo brevemente vedere.

Pertanto mifureremo in piedi cubici la naturale terreftre materia, che contener fi poteva in quel Circuito interno del monte, dove l'accenfioni fi effettuano. Prenderemo pofcia la mifura dei piedi cubici di Lava fgorgata dal monte, principiando dal primo Incendio del 7.9. a noi noto, fino a quello del 1766., da me efaminato; ne faremo il paragone, e vedrem. cosi con evidente dimoftrazione, fe il Vefuvio conteneva tanta mole terreftre in fe fteffo da fupplire all' Incendj. Sicchè principiando dal primo punto, la díforro così. Il monte è un Cono troncato nel Vertice da una fezione orizzontale, la quale coftituifce l'orlo del Cra- 
tere; 'o fa d'un vuoto fatto dal fuoco, per avere arfa, o efpulfa yuella materia. Affumeremo quenta Sezione per la bare d' un Cilindro di Terrà, il quale riempiva un tal vacuo. Sorco il fondo di quefto vuoto, -o Cratere, vi fono le roragini, dove hanno la fedele accenfioni, e pero prolungheremo l'altezza di detto Cilindro fino al fondo delle medefime, per comprender così la mifura di tutta quanta la materia terreftre, che poteva fare nell circuito, dove fi fon generati, le an. cor fi generano gli Incendj: Non pare che ci cada fallacia nel prender la mifura 'd' un Cilinidro di Terra, che ha la bafe fulla cima del monte, e fi profonda-in alcezza fino alle di lui voragini; poichè di fati ella è una mole di Terra', che contener fi poteva dentro del monte; e fulla quale vi hanno realmente le accenfioni operato; anzichè reftringendofn 'a confiderare follanto quefto Cilindro, ci determiniamo a prendere meno di fpazio, é per confeguenza a mifurar meno mole di materia, di quella che realmente fi racchiuda nel monte $\mathrm{Da}$ to danque il giro, o circuito della fuddetta Sezione, che è di piedi parigini 5624 ; per la ragione del Diametro alla circonferenza di $7 \cdot$ a 22 . ritrovo il Diametro di piedi 1789 . e mezzo, ed il femidiametro piedi 804 . e mezzo, più un quarto; e prendendo la merà del detto circuito, o fezione, e moltiplicandola in quefto femidiametro, ritrovo piedi quadrati i 5 16037., che formano giuftamente la bafe cercata del noftro $\mathrm{C} i$ lindro. L' altezza del Cratere fino al fuo fondo; prefa la mezzana, ella ̀̀ di piedi r 40. ; e quella dal fondo fino alla profondità delle voragini, piedi $543 \cdot$ prendendola ancora meno del vero, le quali fommate ci danno poi, pie- 
di 683. ; che tanti faranno per la totale altezza del Cilindro da mifurarfi. Ora moltiplicando queft' àltezza nella bafe foprannotata'; rifulterà la folidità del Cilindro in piedi, cubici 1718453271 .

$S$ avverta adeffo che noi abbiamo confiderata nella noftra mifura l'altezza del monte, tal quale ella è al prefente, ma ognun vede che affai più di materia effer vi doveva prima degli Incend $j_{j}$ e quella in punto che ftava fopra alla fezione del noftro Cilindro, e formava nel primiero, ftato del monte, la di lui Cima. In fatti gli Storici tutti convengono che in ogni eruzzione fiafi notabilmente abbaffato. Il Padre Torre dalle loro relazioni ricava, che confiderata la di lui fommità poco diffimile di figura ad un Cono, e che abbia per bafe la fezione defcritta, o bafe fteffa del noftro Cilindro, poteffe effere la folidità di quefto Cono, ora mancante, piedi cubici 209669750. Noi ci varremo delle medefime fue pofizioni, ficcome ci prevagliamo delle fue mifure, diligentemente ricavate; e perciò aggiuntando quelta folidita all' altra fopra ritrovata del Cilindro, avremo piedi cubici 192812302 I. E quefta farebbe la quantità totale di materia naturale del monte, contenuta nel folo interno Cilindro, e nel Cono già confumato; pofto fopra alla fezione, e che prima degli Incendj doveva terminare la fommità del monte.

Per ricavare quanta materia abbia efpulfa nei fuoi Incendj il Vefuvio, fi prenderà la mifura della Lava ufcita nel terribile Incendio del 1737 . efattamente mifurata dal Signor Serrao, e riferita nella fúa Storia. Quefta fu piedi cubici 31.9658 I61. Se tanti piedi cubici di materia naturale terreftre, coftituiffe altrettanti piedi 
piedi cubici di Lava, farebbe facilifimo il confronto; ma un piéde cubicol di Lava, inon è già del medefimo pefo d'un piede cubico di roba terreftre del Monter; cioc̀ la quantità di materia 'd' un piede cubico di Láva, non è eguale alla quantità di materia del piede cubico di Terra, perchè lla Lava è molto più fpecificamente leggiera, per effere dilatata al fommo dalla violenza del fuoco. E' ricoperta in oltre di guelle fcorie, - pomici, che fopra accennammo, le quali fecondo il giudizio del furriferito Padre Torre, faranno da tré quinti di tutto il Corpo della Lava, e che paragonate con egual volume di materia naturale del Monte, egli fterfo le crede da 30. volte più leggiere. Onde compafte quefte ragioni, fi potrebbe fenza errore ftabilire, che un piede cubico di Lava contenga dieci volte meno di materia, ch' un piede cubico di terreftre materia del monte.

Ma per foprabbondanza di ragioni, fupporremo con effo, che la Lava fia folo dilata cinque volte di piì; quindi per ridurla al fuo ftato di. Térra" naturale, prenderemo la fola quinta parte della Lava foprannotata, ufcita nell' Incendio del 1737. ed avremo piedi cubici 63931632.; ed ecco ch' abbiamo ridotta la Lava d'un Incendio, al medefimo pefo, o quantità di materia terreftre contenuta déntro del monte. Dal primo Incendio fino a quello del 1:766. da me offervato, ricavals dalle. Storie, che 26 . "ne ton feguiti, almeno dei più norabili. Onde è che confiderandoli tutti eguali, -moltiplicheremo per 26. la Lava qui fopra ridotta, ed avremo il totale della Lava, o fivvero della materia naturale ufcita dal Vefuvio finora; e farà piedicubici i 662222432 . Quella che vi era nel mifurato Cilindro, e nel Cono $\mathrm{D}$ d d 2 foprap- 
foprappofto, fu, come fopra abbiam vifto, piedi cubici. 1928123021 . ; onde fottraendo da quefta l' efcita finora, vi rimane ancora d' avanzo, piedi cubici 265900589. Refta dunque evidente, che vi era tanta mole di Terra da. fupplire agli Incendj paffati, e vi è di refiduo ancor di più, l'avanzo qui efpofto.

Si noti inoltre, che in quefto avanzo; la Lava ufcita nel 1737. a vera materia terreftre ridotta, vi entra 4 . volte, e vi rimangono ancora piedi cubici ro $17406 \mathrm{I}$. Sicchè per 4. Incendj confimili reftavi ancora di materia terreftre dentro il Cilindro, trafcurando ancor di più l'avanzo reftatovi. Ora fupponendo ch'il Vefuvio in avvenire proceda con i medefimi andamenti tenuti in paffato, farà lecito argomentare così. Se 26 . Iricendj portano lo fpazio di anni 1687. fcorfi dal primo del 79. fino al 1766. , i quattro Incendj futuri mi daranno un corfo di anni 259. ; cioè tanti anni ancora dovran paffare, prima che refti confunta la terreftre matéria del noftro Cilindro.

Or quì giova riflettere primieramente, che noi abbiamo fopra meffo a conto effer la Lava dilatata fole. cinque volte di più dello ftato naturale di Terra, quando che farà almeno 10 . volte più rarefatta; dunque abbiam polto che la metà di più di Terra naturale fiafi convertita in Lava, e fgorgata dal monte; vale a dire che abbiam fottratta la metà più di materia dal noftro Cilindro.

Secondariamente fi noti, che non è già vero, che tutti $\mathrm{i}$ paftati 26 . Incendj fieno fati cosi terribili, ed uniformi a quello notato del I 737., anzi deducefi dalle Storie, che nè meno 5 . in quefto lungo fpazio d'an- 
ni heno avvenuti a quello confimile; ma per efcludere ogni dubbio, e per mettere a conto ancora quanto che di materia terreftre poffa effere ftata gettata in altre guife; e negli altri minori Incendj, porremo che di 26 . la metà di effi fieno ftati a quello fimilifími; egli ̀̀ un concedere largamente più del bifogno, ma ciò nulla importa.

Avendone fopra pertanto meffi a computo 26 . tutti eguali, vienfi a fupporre che altra metà di più, di quel che è realmente di materia terreftre, fiafi convertita in Lava, e fgorgata fuor del Vefuvio. Sicchè la metà di più fi ammeffe rifpetto alla dilatazione, e la metà di più l' ammettiamo rípetto al numero degli Incendj; dunque la metà della metà di più di materia naturale abbiam fottratta dal noftro Cilindro, la quale non fi doveva di fatto fottrarre. Non oftante tutto ciò, ficcome abbiam vifto, rimane ancora di folidità tanti piedi cubici da fupplire per più anni in avvenire all' Eruzzioni, oltre alla confunta finora; onde parrebbe che a forza doveffero perfuaderfi i contrarj, che il Vefuvio non ha punto bifogno, che o i Fiumi fotterranei, o il Mare gli portino l'eftranee materie dentro il fuo corpo.

Se fi facefle la leggiera objezione, che non è la fola Lava da noi computata, quella materia terreftre, che efpulfa viene dal monte; ma che fonovi pure anco le ceneri, le diverfe pietre fcagliate, vi è il fumo, vi fono le efalazioni, corpi turti da noi non meffi a conto d'ufcita, e che realmente fon parte di materia, da doverfi detrarre dalla folidità mifurata; Rifponderei che tutto ciò è vero, ma francamente ancor direi, che tutte quefte fuddette efpulfe materie, in ogni refpetrivo In- 
cendio, non giungono mai ad effer la millionefima parte di materia; in confronto di quelle fterminate campagne di Lava, che fi eftendono a qualche miglio in lunghezza, ed altrettanto in larghezza, ed a braccia non poche di profondità; e però non ci dilontaneremo mai dal vero, $f e$ in paragon di quelle, confidereremo tutti $i$ furriferiti corpi come un nulla; e quindi le Lave meffe a conto, debbon tutti fvanire, riducendofi a zero. $\mathrm{Vi}$ fono inoltre da fare alcune rifleffioni a tal propofito, affinchè refti affatto dileguata l'objezione.

Per efempio, l'acqua che entra nelle compofizioni di tutti corpi, e genera molte delle concrezioni in ifpecie, continuamente ha fcolato in abbondanza nel Vefuvio, e non è irragionevole, che vi abbia accrefciuta della materia. Inoltre è da fapere, che le mifure prefe tanto delle larghezze, che delle altezze, rifpetto al mifurato Cilindro, e Cono foprappofto, l'abbiamo affunte fempre minori del vero; che ciò vuol dire, che molto meno di quella ch' in fatto è, abbiamo affunta la materia terreftre, efiftente dentro del monte. Molto maggiore del vero abbiamo poi a contrario ftabilito efferne ufcita in Lava, ficcome abbiam fopra notato; $E$ finalmente fi noti che noi non abbiam meffo a computo nè meno Ia centefima parte di materia, la qual di fatto è efiftente, anzi coftituifce il monte ftello, e fulla quale pu* re ferpeggiano, e fi effettuano vifibilmente le accenfioni. La femplice, e fola mole di terra Cilindrica, che entra dentro il Vefuvio, ed anco ad una tal quale determinata profondità, o fivvero altezza, è quella che fola abbiamo valutata, per paragonarla con la Lava finora efcita; ma è chi non vede quant' altra di più ancor ve ne refta nella refi- 
refidua terreftre maffa del monte? ficchè fvanifca pure ogni dubbio, fe a cafo non aveffimo giuftamente conteggiato ogni corpo, che, oltre alle Lave, foffe ufcito nelle furie delle accenfioni.

Per conferma di quanto dicefi, voglio che non altro brevemente facciamo, fe non che di profondarne l'altezza del mifurato Cilindro, fino al Livello del Mare, - fia prefo nell' affoluta altezza del monte, fino a dove in fatto poffono eftenderfi le accenfioni; fenza nè anco valerci dell'opinione, che giungano quelle ad immenfe profondità, e per fino vi è chi vuole, al Centro della Terra. Voglio ancora che prendiamo l' altezza del monte, come lo è al prefente, trafcurando tutto quel di più, di cui fi è negli Incendj fcemato nel Vertice, per forza del fuoco, e che al dir degli Storici, non è un confumo di sì poca confiderazione. Pertanto avendo l'affoluta altezza del monte, o del noftro Cilindro di piedi 1677 , moltiplicandoli nella folita bafe, fuperiormente defcritta, ricaviamo per la di lui folidità, piedi cubici 4219394046.

La Lava fgorgata nel fuddetto Incendio del $1737 \cdot$, ridotta a terra naturale di piedi cubici 6393 I631 tra in quefta folidita del Cilindro $6_{5}$. volte, e vi reftano ancor d'avanzo piedi cubici 63837969 . Onde 65 . Incendj confumili può egli mantenere; e 26. effendo, già accaduti, ve ne refteranno per gl'anni avvenire $43 \cdot$; i quali fupponendoli fuccedere col medefimo ordine, e poffanza dei paffati, argomenteremo al folito, e diremo; che fe 26. Lave han fervito per anni 1687 , le 43 . che ancor vi reftano, fuppliranno per anni 2790. in futuro. 
Che fi direbbe poi fe eftendeffimo l'immaginazione a computare la folidità totale dell' intiero monte, riguardato, come di fatto è, fimiliffimo ad un Cono? Il fuo giro, il quale circonda la bafe dalla divifione dei monti fopra mentovati d'Ottajano, e Somma, egli è di miglia 6.e mezzo; o fivvero piedi 37089 ., e l' altezza refpettiva di piedi 797. ; e prendendo poi il vero circuito della fua bafe, comune con $i$ detti monti, rifulta di miglia 24., e l' altezza affoluta quella fin qui fopraddetta, di piedi 1677 . Or fe con quefte mifure fe ne ricavaffe l' intiera folidità, forpaffa l'immaginazione la quantità della materia naturale, che ancor vi refta, per fupplire agli Incendj in avvenire.

Non ci daremo il penfiere di mettere fotto degli occhj $\mathrm{i}$ calcoli da noi fatti in quefta fuppofizione, per non tediar da vantaggio i Lettori; ma porremo fine ai noftri ragionamenti coll' infinuare ai mali avveduti penfatori, che non $f$ becchino il Ccrvello per inveftigare le Atrade, per le quali fi trafportino nel Vefuvio i Corpi eftranei, perchè tanta, c sì tanta inole terreftre in fe contiene quel monte, che a folo confiderarne la quinta parte per confumo del fuoco, la già efpulfa finora rimane quafi un nulla, in paragone dell'altra, ch'ancor vi refta per mantener vigorofe le accenfioni, a centinara di Secoli futuri. 



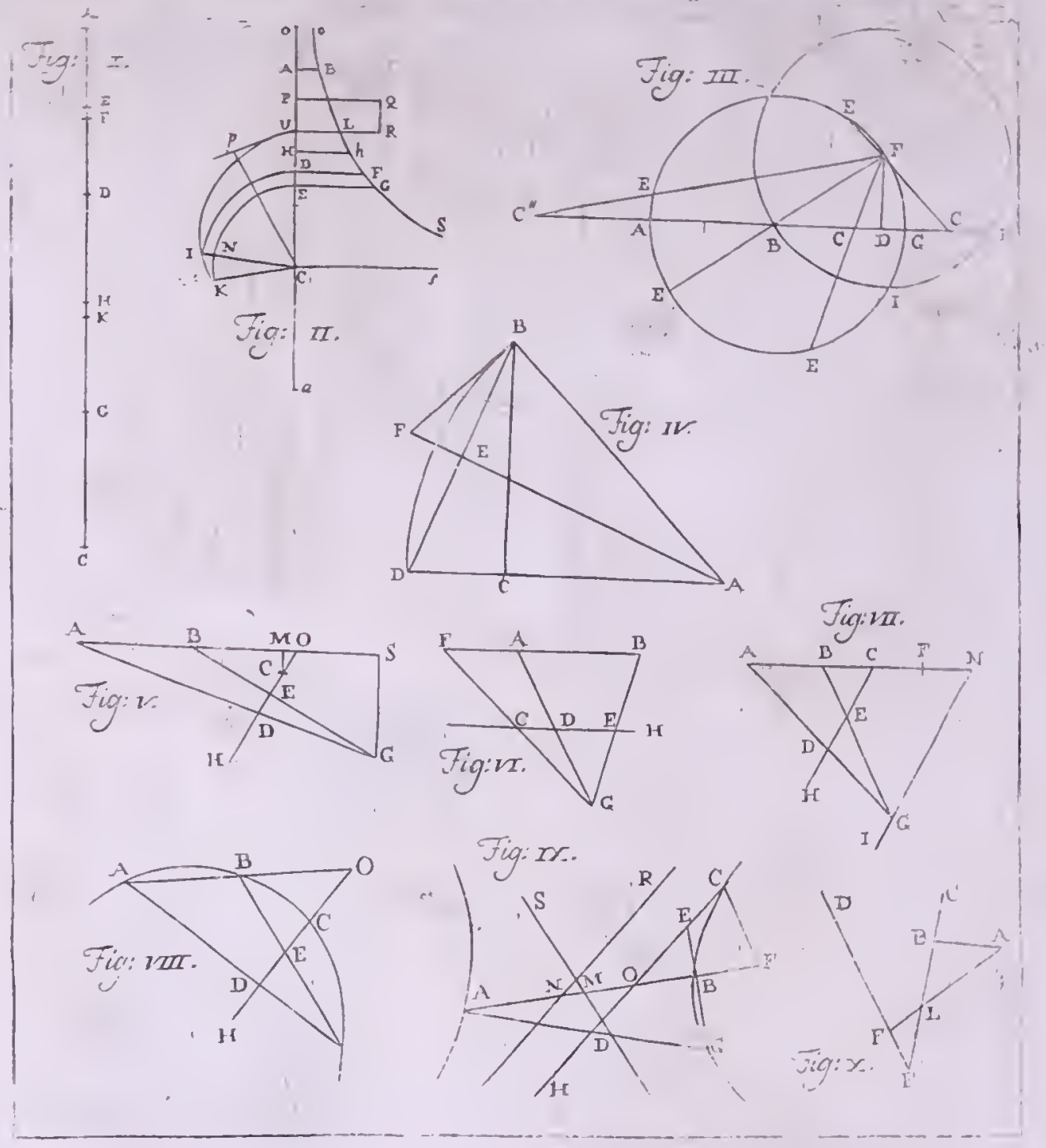




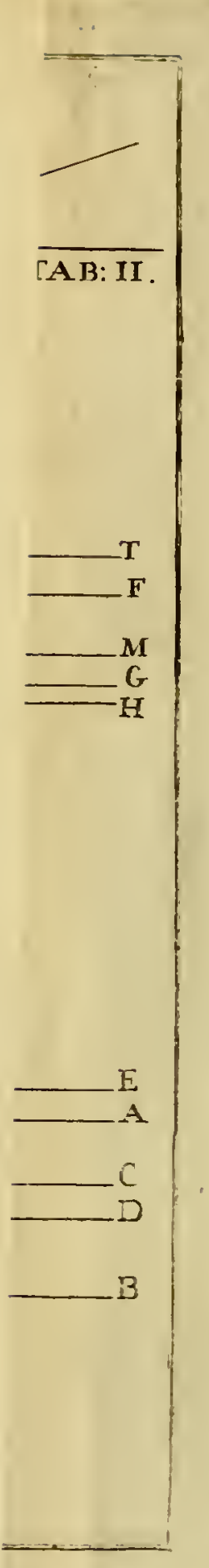




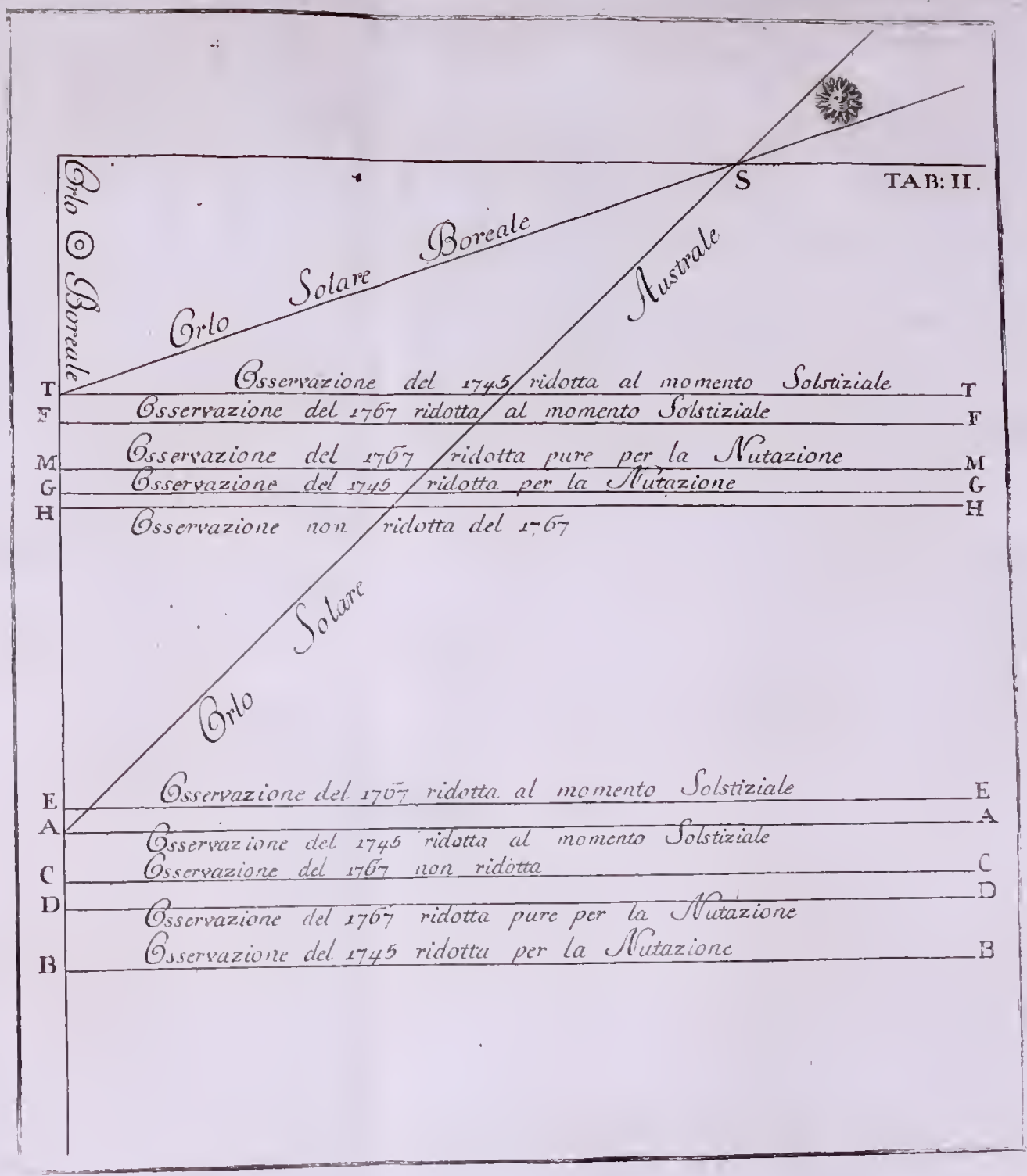




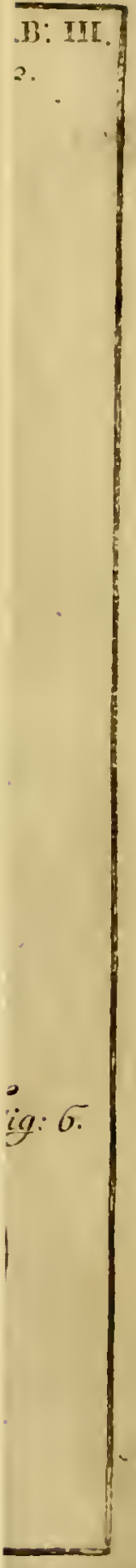




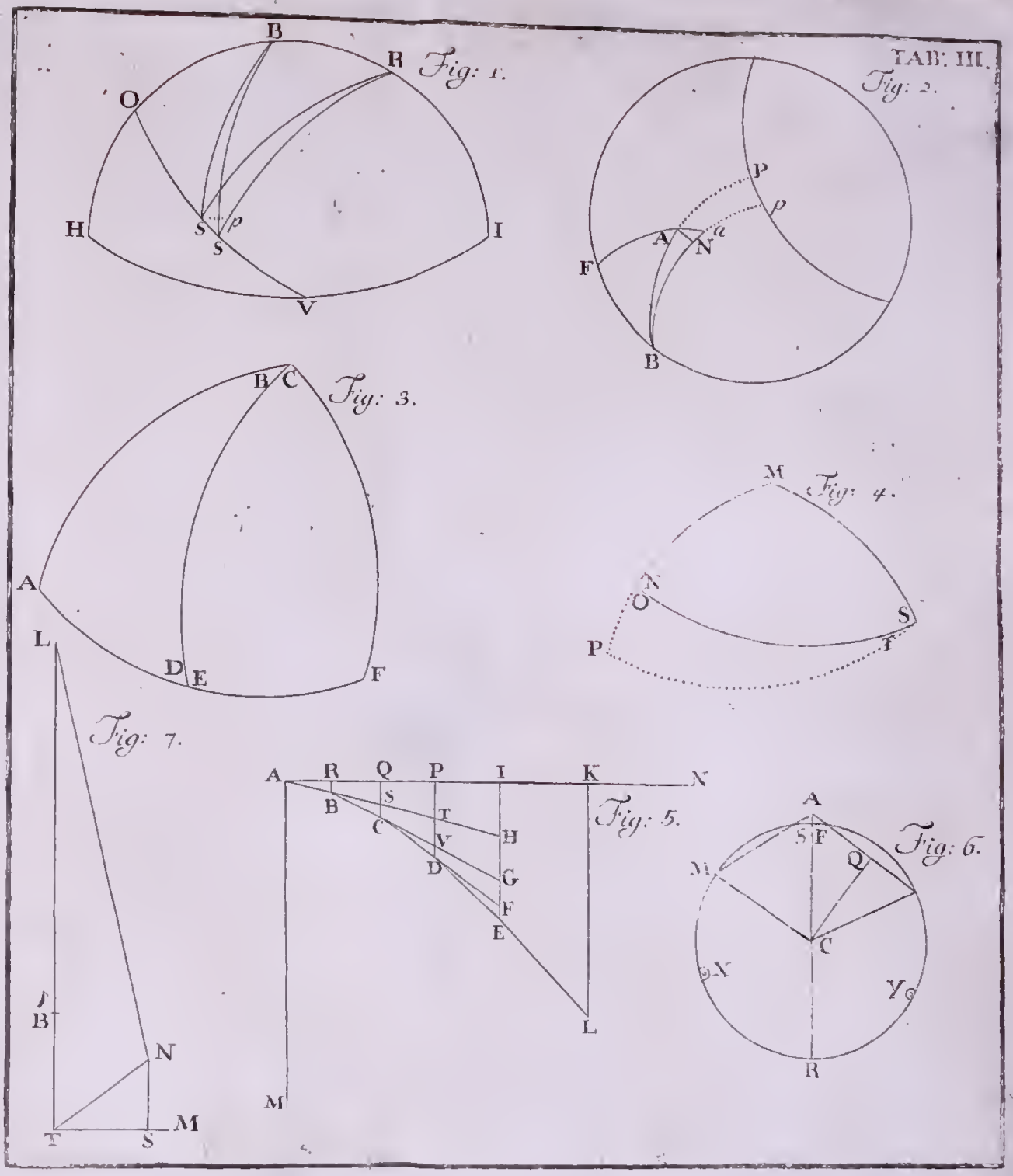




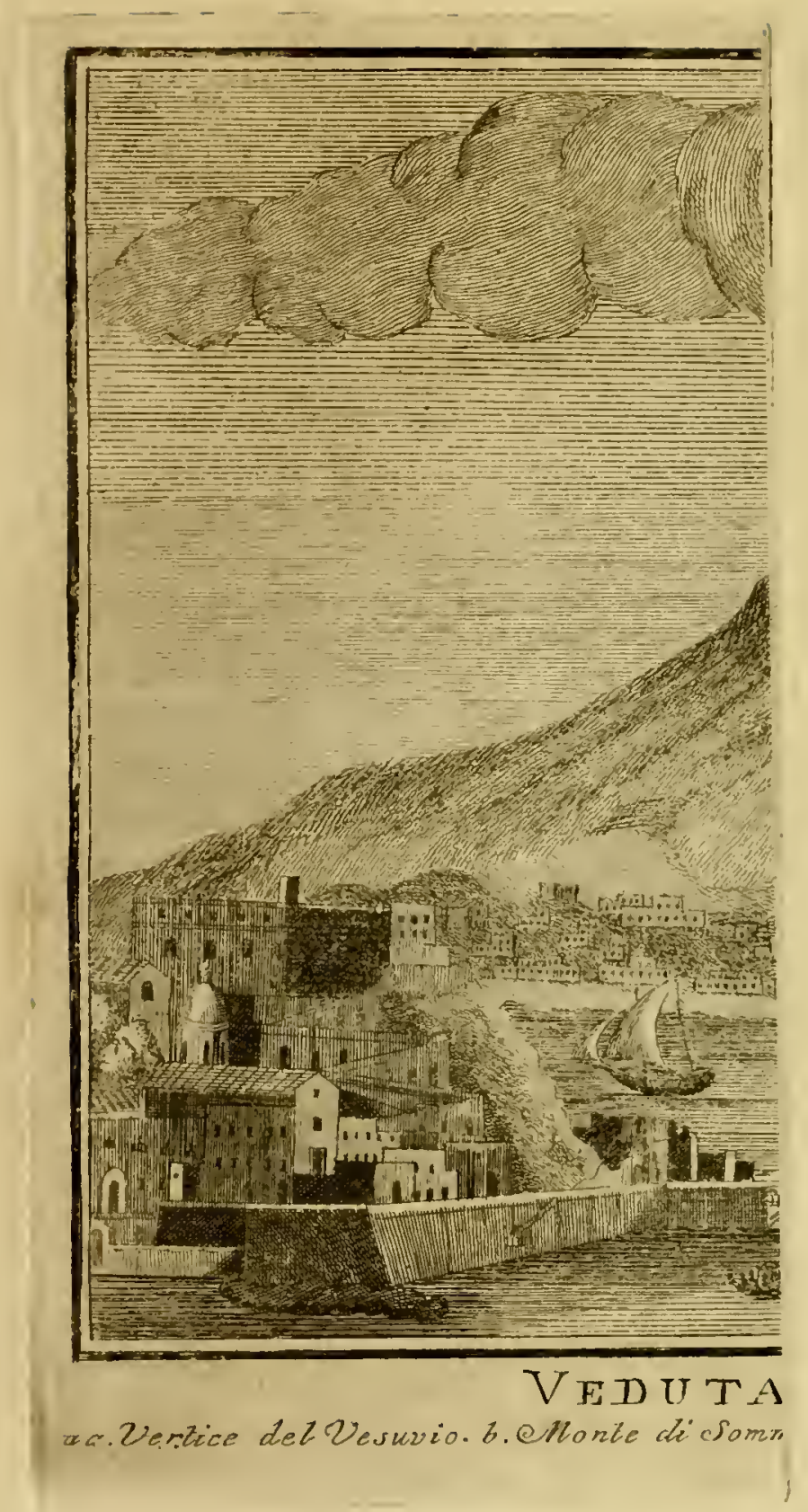




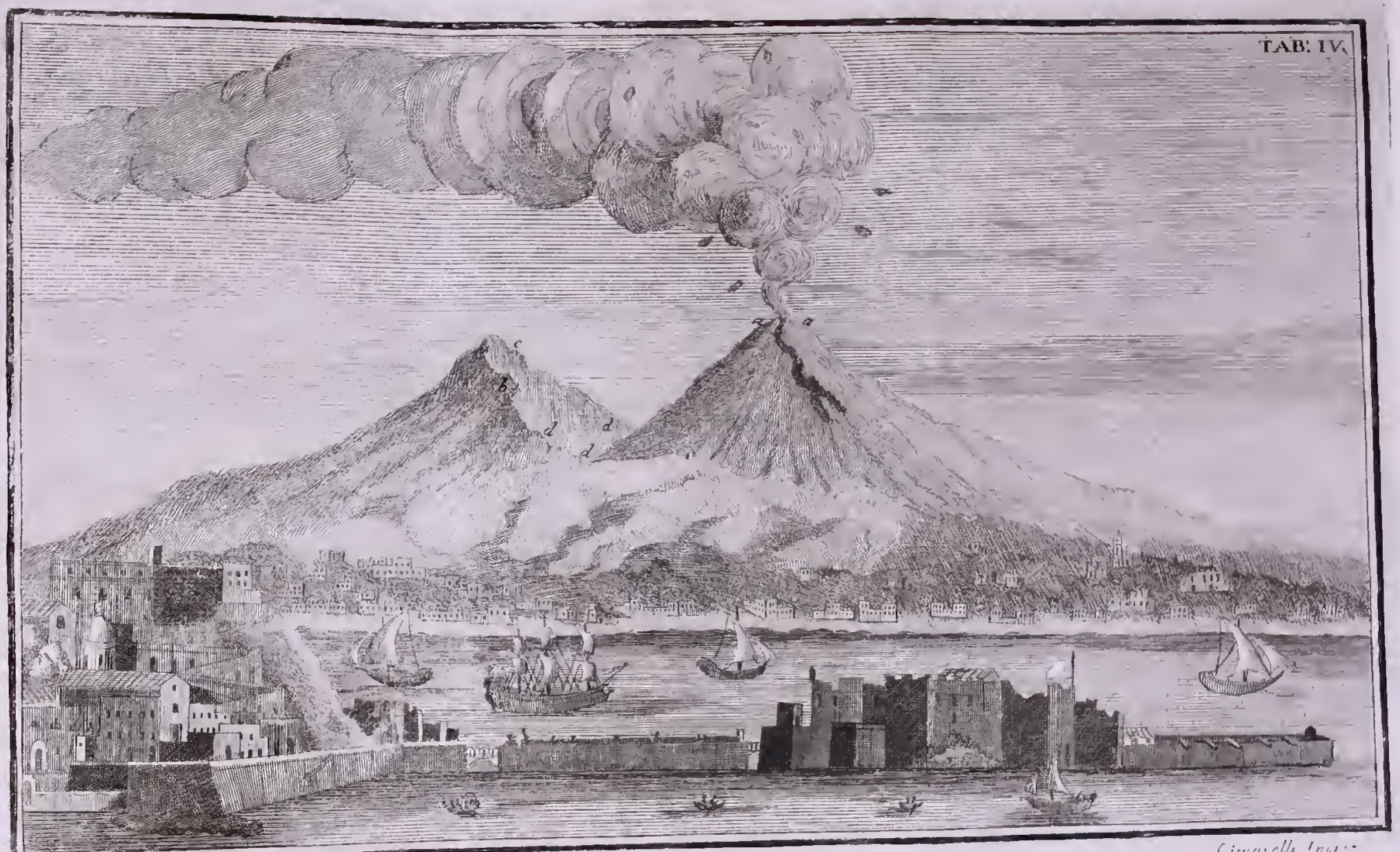

VEDUTA DEL VESUVIO DA POSILITO

- Vertice det Wesuvio. b. ellonte díçomma. 






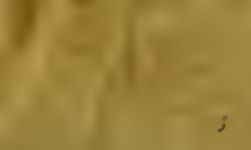

$x=-1=x-y=14=$

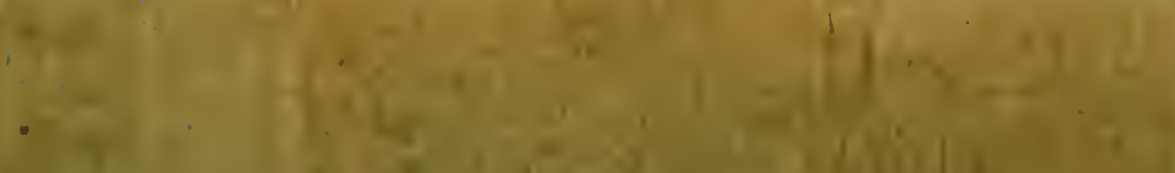

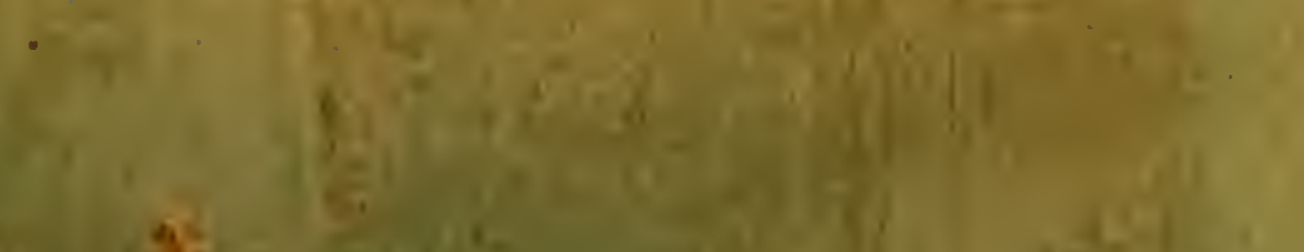

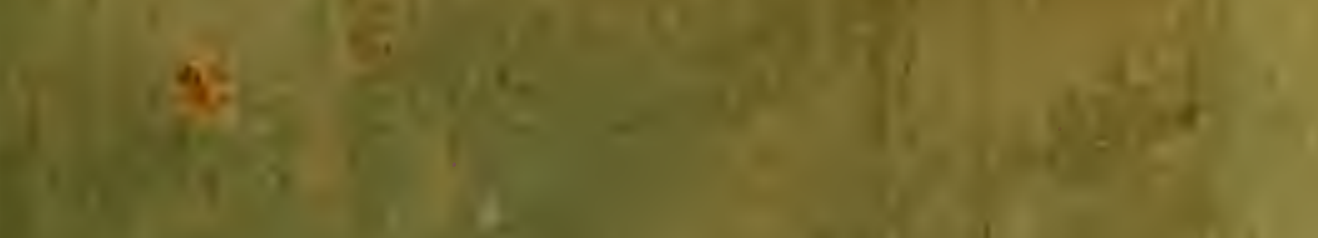

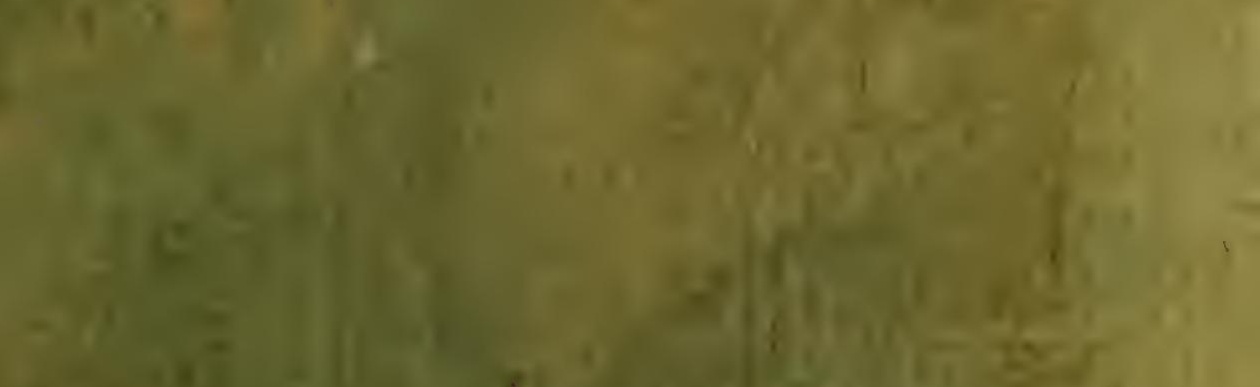

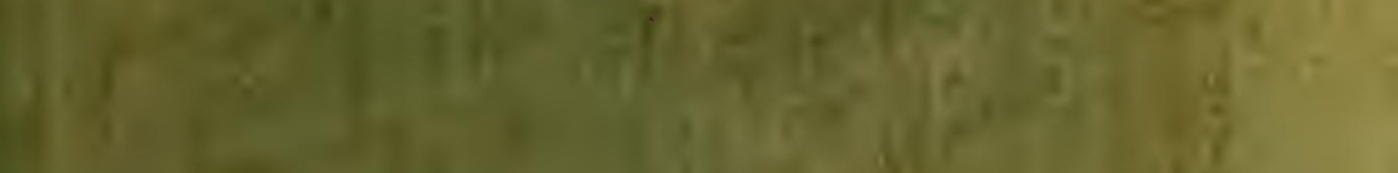
( $1, x$ 2612,92

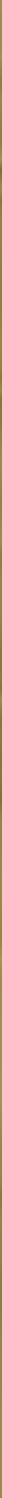


$+4,0+1$

$r$

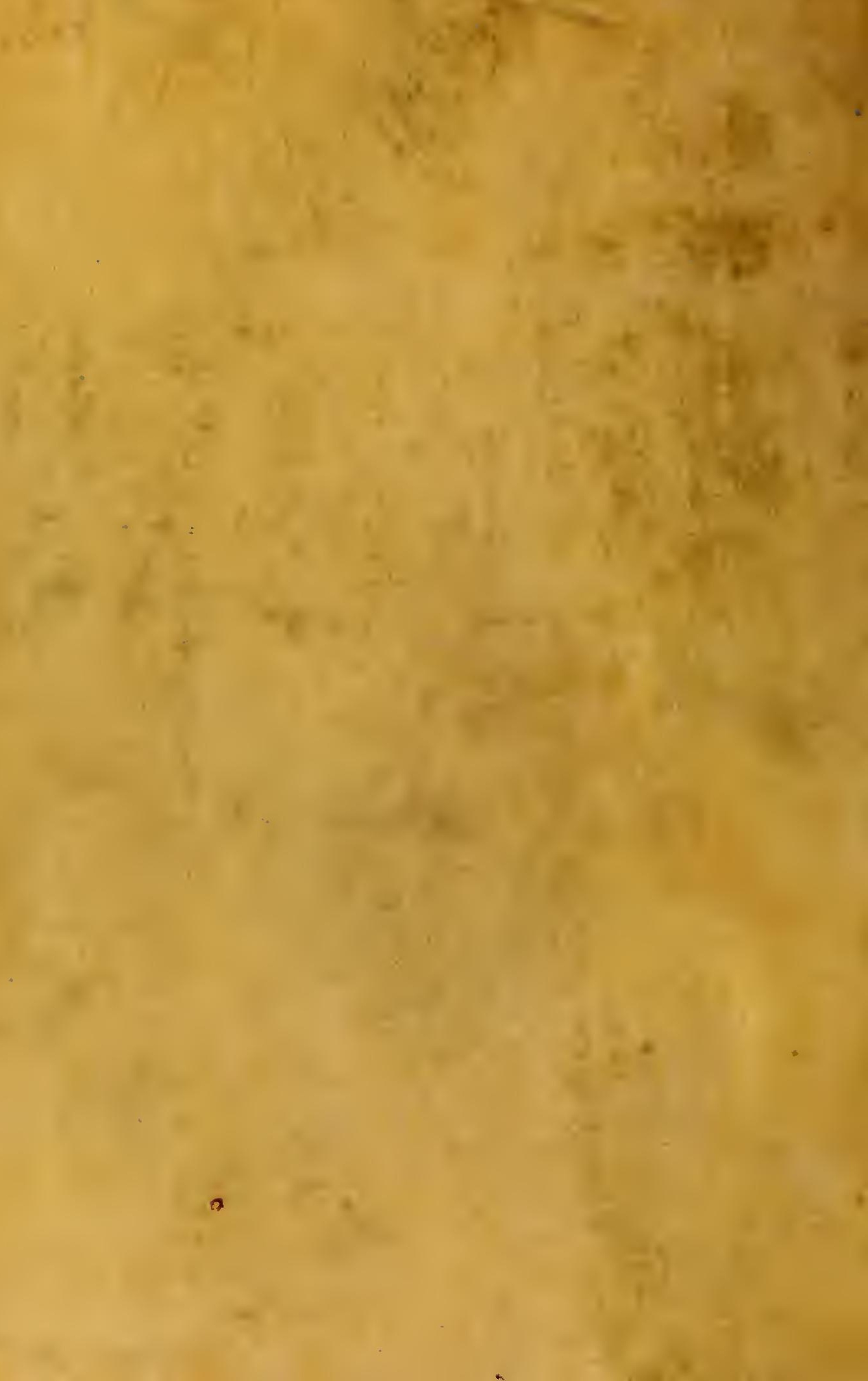

